

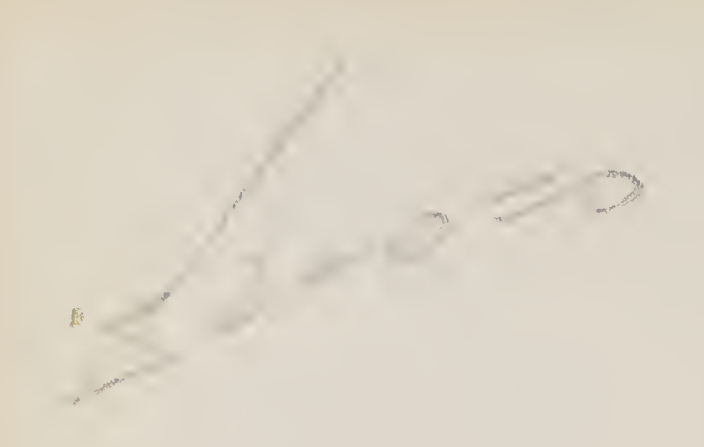






\section{SPECTRUM ANALYSIS.}


S 



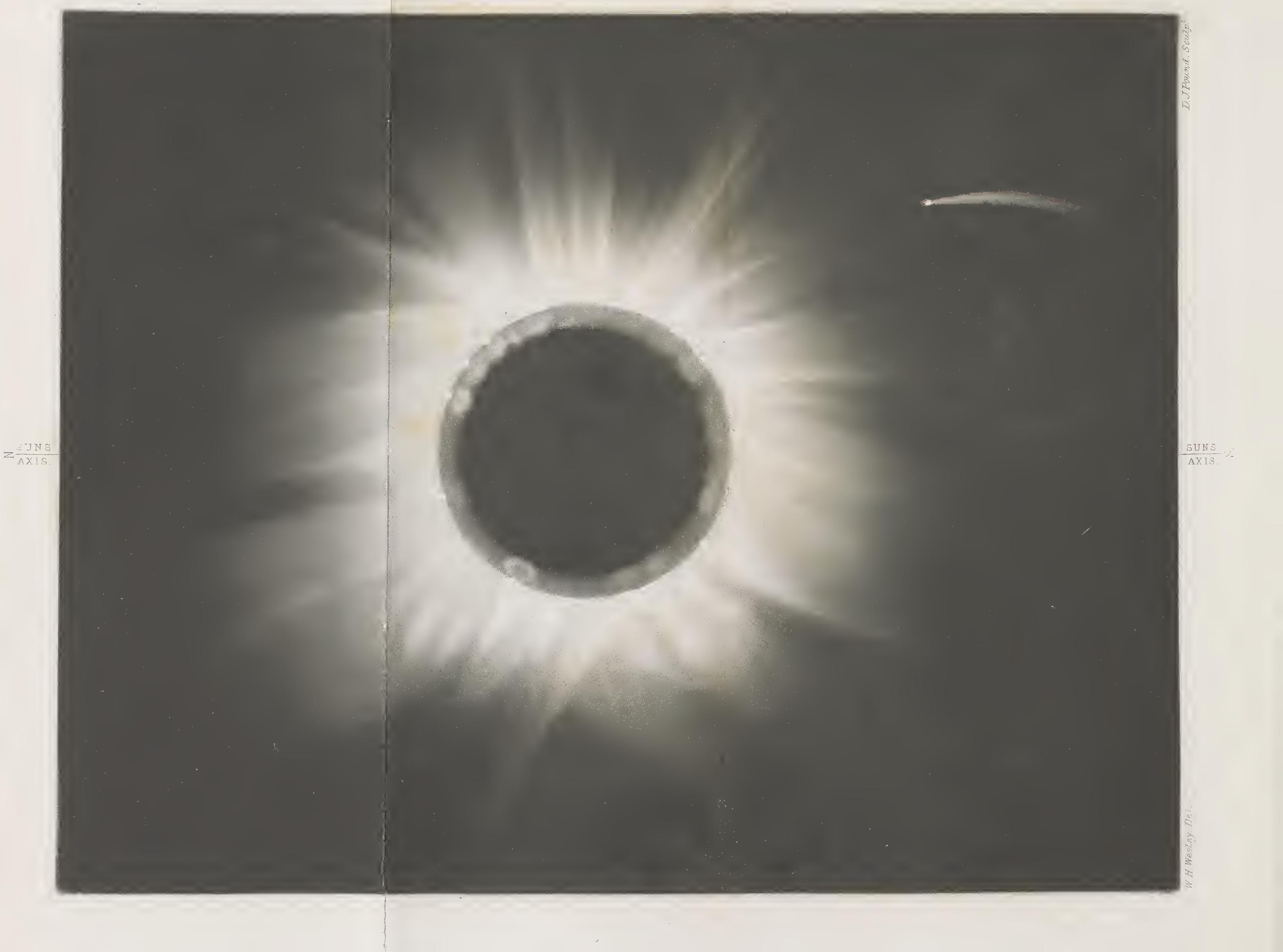





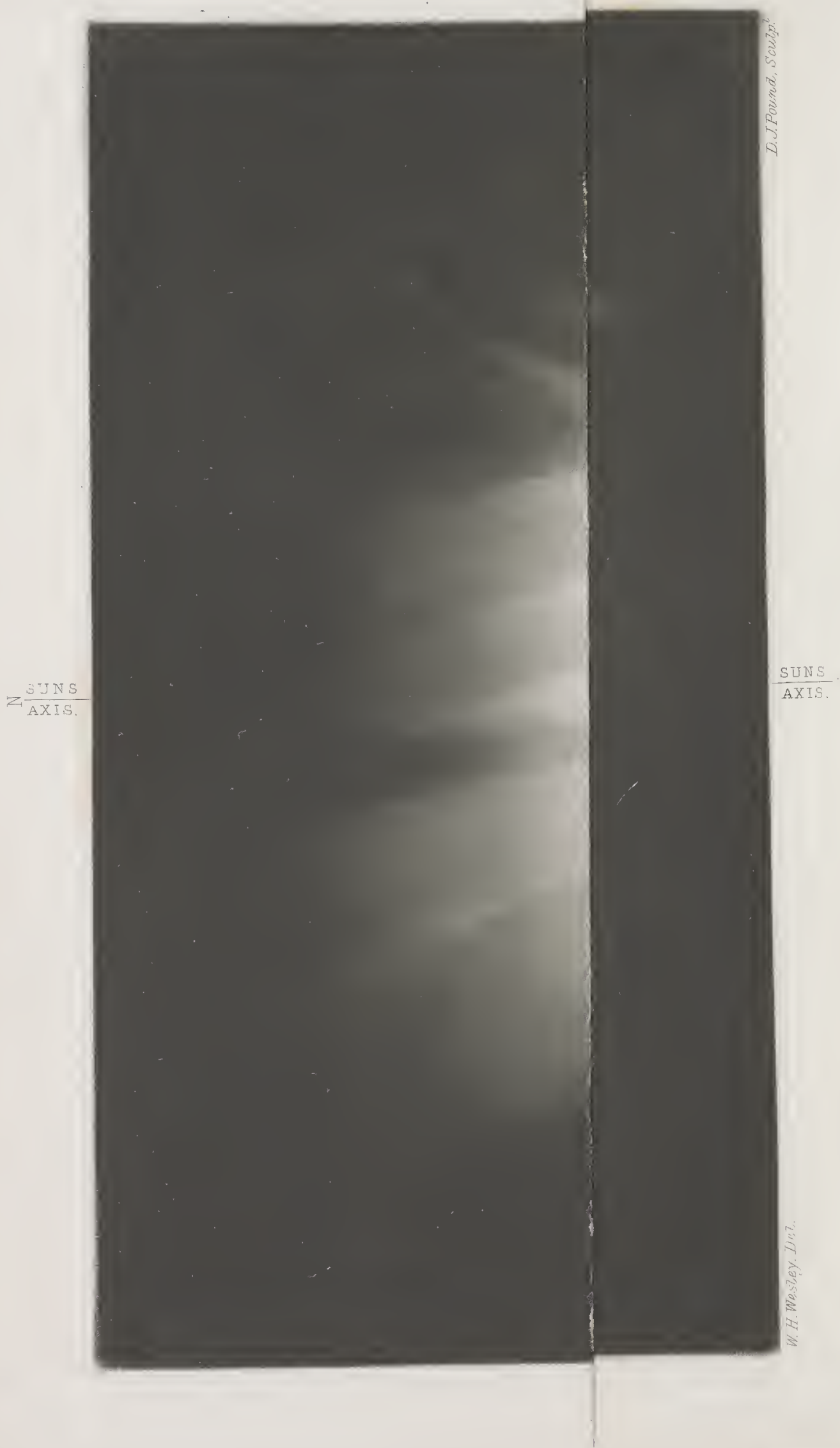




\title{
SPECTRUM ANALYSIS
}

\section{SIX LECTURES}

\section{DELIVERED IN 1868 BEFORE THE SOCIETY OH APOTHECARIES OF LONDON}

BY

SIR HENRY E. ROSCOE LL.D., F.R.S.,

PROFESSOR OF CHEMISTRY IN THE OWTNS COLIEGE, VICTORIA UNIVERSITY, MANCHESTER

FOURTH EDITION REVISED AND CONSIDERABLY ENLARGED BY THE AUTHOR

\section{AND BY}

ARTHUR SCHUSTER PH.D., F.R.S.,

PROFESSOR OF APPLIED MATHEMATICS IN THE OWENS COLIEGE, VICTORIA UNIVERSITY

WITH APPENDICES COLOURED PLATES AND ILLUSTRATIONS

\author{
ifonton: \\ MACMILLAN AND CO. \\ 1885 .
}


Richard Clay and Sons,

BREAD STREET HILL, LONDON, E,C. and Bungay, Suffolk. 


\section{PREFACE TO THE FOURTH EDITION.}

IN preparing this wholly revised and almost entirely rewritten Edition of the Lectures on Spectrum Analysis no effort has been spared to give a popular and concise account of the most important discoveries in this branch of science which have been made since the year 1873. Care has been taken, in so doing, to select such portions of this wide subject as have already been worked out with approximate completeness. Many questions of rising interest have thus necessarily been omitted as not lending themselves in the present state of our knowledge, to popular treatment. Even whilst these pages have passed through the press several new discoveries have been made. Of these the outbreak of the new star in Andromeda, and the researches of Crookes, Lecoq de Boisbaudran, and Von Welsbach on the spectra of the rare metals Didymium and Samarium are 
perhaps the most important. For information on these, the reader may consult the original memoirs, references to whieh are found at the end of the index.

H. E. Roscor.

Arthur Schuster.

Manahestier, November, 1885.

\section{PREFACE TO THE FIRST EDITION.}

Is publishing the following Lectures I have endeavoured to preserve the elementary character whieh they naturally assumed in delivery, thinking it best to give further detail in a series of Appendices. If the book thus assumes less of the eliaracter of a complete treatise than might be desirable, it gains in value for the general reader, inasmuch as the scienee of Speetrum Analysis is at present in such a rapid state of growtl that much of the subjeet is incomplete, and, therefore, necessarily unsuited to the public at large. I hope, however, that the addition of many extracts from the most important. Memoirs on the subject may prove interesting to all, as it will certainly be useful to those 
specially engaged in scientific inquiry, as indicating the habits of exact research and accurate observation, by which alone such striking results have been attained. For the permission to reproduce exact copies of Kirchhoffs, Ångström's, and Huggins' maps, together with the Tables of the positions of the dark solar and bright metallic lines, I liave to thank the above-named gentlemen. These maps will render the work valuable to the student for reference, whilst the elromolithographic plates of the spectra of the metals of the alkalis and alkaline earths, and of the spectra of the stars, nebulæ, and non-metallic elements, serve to give some idea of the peculiar beauty of the real phenomena thus represented.

Since last summer, wher these Lectures were delivered, our knowledge of the constitution of the sun especially lias made giant strides; and although I have been unable to introduce these rewest facts into the text of the Lectures, I have still brought forward the most important of these discoveries in the Appendices to Lecture V.

As the latest news on this subject, I may mention the arrangement contrived by Mr. Huggins, by which the wonderful changes of the red solar prominences 
can all be viewed at once; changes so enormously rapid that Mr. Lockyer has observed one of these red solar flames, 27,000 miles in length, disappear altogether in less than ten minutes. $\mathrm{Mr}$. Lockyer has also succeeded in seeing in the flames the red (c) line of hydrogen, as well as the line in the violet, which he finds corresponds to the line marked 2796 on Kirchhoff's map, and not, as was supposed, identical with Fraunhofer's line G.

H. E. R.

Madchester, Apl il, 1869. 


\section{CONTENTS.}

\section{LECTURE I.}

Introduction.-Newton's Discovery of the Composition of White Light, 1675.-Properties of Sum Light as regards Heating Power, Luminosity, and capability of producing Chemical Action.-The Solar Radiations.-Prismatic and Diffraction Spectra.-Position of Maxima.-Illustrations of these Radiations.-Means of obtaining a Pure Spectrum._Fraunhofer's Lines.-Planet and Moon Light._Star Light . . . . . . . . . . . . . . . . . 1

Appendix A.-Extracts from "Newton's Opticks" . . . . . . . . 40 Appendix B.-Burning Magnesium Wire, a Source of Light for photographic purposes. . . . . . . . . . . . . . 50 Appendix C.-On the Chemical Action of the constituent parts of Solar Light . . . . . . . . . . . . . . . 52 Appendx D.-Description of a Chemical Actinometer . . . . . 58

\section{LECTURE II.}

Continuous Spectrum of Incandescent Solids.- Effect of Increase of Heat.-Broken Spectrum of glowing Grs.-Application to Cliemical Analysis.-Spectra of the Elementary Bodies.-Construction of Spectroscopes.-Means of obtaining Substances in the state of glowing Gas.-Examination of the Spectra of Coloured Flames.Mapping Spectra, according to Bunsen.-Delicacy of the Spectrum. Analytical Method and its application to Physiological Research.Spectra of the Metals of the Alkalis and Alkaline Earths.Historical Sketch.-Discovery of New Elements by means of Spectrum Analysis. - Caesium, Rubidium, Thallium, Indium, Gallium

Appendix A.-Description of the Spectrum Reactions of the Salts of the Alkalis and Alkaline Earths . . . . . . . . . .

Appendix B.-Bumsen and Kirchhoff on the Mode of Using a Spectroscope..................... 103

ApPendix C.-Bunsen on a Method of mapping Spectra . . . . . 106 Appendix D.-Spectrum Reactions of the Rubidium and Caesium Compounds 


\section{LECTURE III.}

Spectra of the Heavy Metals.-Examination of the Light of the Electric Discharge. - Faraday. - Wheatstone. - Volatilization of Metals in the Electric Arc.-Kirchhoff, Ångström, Thalén, and Huggins. - Stokes's Ultra-violet Rays. - Spectra of the Non-metallic Elements.-Line Spectra and Spectra of Fluted Bands.-Connection between these Spectra and the Molecular Constitution of the Elements.-Lockyer's Method of Examining the Spectra.-Long and Short Lines.-Widening of the Lines.--Spectra of Compound Bodies.-Conchtsion . . . . . . . . . . . . . 113

ApPENDIx A.-Contributions towards the History of Spectrum Analysis. By G. Kirchhoff . . . . . . . . . . . . . . . . . 140

APPENDIX B.-On the Effect of Increased Temperature upon the nature of the light emitted by the vapour of certain Metals or Metallic Compounds. By H. E. Roscoe and R. B. Clifton (1862). Kirchhoff on the Variation of the Spectra of certain Elements (1862)

Appendix C.--On Clanges in the Spectrum of Oxygen. By Professor Arthur Schuster, F.R.S.

Appendix D.- On the Influence of Temperature and Pressure on the

Spectra of Gases; being an abstract of a Report presented to the

British Association in 1880 by Professor Schuster, F.R.S. . . . . 154

APPENDIX E.-On the Genesis of Spectra. Abstract of a Report drawn up by Professor Schuster for the British Association in 1882

\section{LECTURE IV.}

Selective Absorption.--Coloured Vapours.-Atmospheric Absorption. Iodine.-Bromine.-Chloride of Iodine.-Nitrous Fumes.-Metallic Vapours. - Liquids and Colonred Solutions. - Blood Bands.Chlorophyll. - Connection between Absorption Spectra and Chemical Constitution.-Selective Absorption by Solid Bodies.-Didymium Salts. - Applications of Spectrum Analysis. - Blood Poisoning.-Colouring Matters.-Spectrum of the Bessener Flame. -Fluorescence and Phosphorescence.-Construction of High Power Spectroscopes.-Arrangement for Diffraction-Spectra . . . . . 183

A prendix A.-On the Spectrum of the Bessemer Flame . . . . . 215 Appendix B.-Spectroscopic Measurements and Appliances . . . . 222 Appendix C.-On the Spectra of Erbium and Didymium, and their Compounds . . . . . . . . . . . . . . 


\section{LECTURE V.}

Foundation of Solar and Stellar Chemistry.-Examination of the Solar Spectrum. - Fraunhofer, 1814 -- Kirchhoff, 1861. _ Coincidence of Dark Solar Lines with Bright Metallic Lines.-Reversal of the Bright Sodium Lines. - Kirchhoff's Explanation. - Constituents of the Solar Atmosphere.-Lockyer and Janssen's Researches.-Phenomena observed during Total Eclipses.-Constitution of the Red Solar Prominences; their gaseous Nature.-The Chromosphere.-Physical Constitution of the Sun.-The Corona.Sun-spots and Faculæ . . . . . . . . . . . . . 229

Appendix A.-Professor Balfour Stewart on the Equality of Radiation and Absorption . . . . . . . . . . . . . . 265 Appendix B.-On Maps of the Solar Spectrum . . . . . . . . . . 272 ApPendix C.-On Lockyer and Janssen's Discovery . . . . . . . . 274 Appendix D.-Results of Recent Solar Eclipse Observations . . . . 281 AfPENDIX E.-Professor C. A. Young's Investigations on Chromospheric Lines.

Appendix F.-Professor S. P. Langley on Sun Tight and the Earth's Atmosphere . . . . . . . . . . . . . . .

\section{LECTURE VI.}

Planet- and Moon-Light.-Planetary Spectra.-Stellar Chemistry.Hnggins and Miller.-Spectra of the Fixed Stars.-Difficulties of Observation.-Methods employed.-Variable Stars.-Double Stars. - Temporary Bright Stars.-Photographs of Star Spectra. - Nebulæ -Comets.--Motion of the Stars (Huggins).-Determinations of Velocity of Solar Storms (Lockyer)

Appendix A.- Extract from a Memoir "On the Spectra of some of the Fixed Stars" . . . . . . . . . . . . . . .

Aprexdix B.-On the Spectrum of Mars, with some Remarks on the Colour of that Planct . . . . . . . . . . . . . 347

Appendix C.--On the Occurrence of Bright Lines in Stellar Spectra, and on the Spectra of Variable Stars . . . . . . . . . . 352

Appendix D.-Further Observations on the Spectra of some of the Stars and Nebule, with an attempt to determine therefrom whether these bodies are moving towards or from the Earth, also Observations on the Spectra of the Sun and of Comet II. 1868 . . . . . . 353 Appendix E.-On the Spectra of the Great Nebula in Orion, and on the motions of some Stars towards or from the Earth . . . . . 392 Appendix F.-Photographic Spectra of Comets and Nebulæ . . . . 411 Appendix G.-On the Spectra of Lightning . . . . . . . . . 420 INDEX . . . . . . . . . . . . . . . 4 427 



\section{LIST OF ILLUSTRATIONS.}

FIG.

1.-Facsimile of Diagram in Newton's "Opticks" . . . . . . 2

2.-Ditto . . . . . . . . . . . . . 4

3.-Ditto . . . . . . . . . . . . . . 5

4.-Coloured Rotating Disc . . . . . . . . . . 6

5.-Diagram illustrating the Diffraction of Light . . . . . . 11

6.-Ditto . . . . . . . . . . 13

7.-Diagram illustrating the appearance of Decomposed Solar Light. 16

8.-Ditto . . . . . . . . . . . . . . 16

9.-Diagram illustrating the difference between Prism and Grating Spectrum . . . . . . . . . . . 18

10.-Diagram giving the Distribution of Heat as shown by the Prism, with the maximum effect in the Infra-Red . . . . .

11.-Ditto, ditto, reduced to the wave-length scale, with the maximum in the Yellow ............... 21

12.-Tyndall's Experiments on Calorescence . . . . . . . . 23

13.-Explosion of Chlorine and Hydrogen by Magnesium Light . . 25

14.-Chemical Action of Blue Rays shown. . . . . . . . . . 26

15.-Curve of Chemically Active Light in Solar Spectrum . . . . 28

16.-Experiment of Photography in the Blue Rays . . . . . . . 29

17.-Ditto . . . . . . . . . . . . . 30

18.-Curves of Chemical Intensity of Total Daylight for Kew, $1866 \quad 33$

19 \& 20.-Ditto, for Para, April, 1866 . . . . . . . . . . 34

21.-Facsimile of Fraunhofer's Map (1814) of the Dark Lines in the Solar Spectrum . . . . . . . . . . . 37

22.-Repetition of Newton's Diagrams . . . . . . . . . . . 45

23.-Ditto . . . . . . . . . . . . . . . . 47

24.-Ditto . . . . . . . . . . . . . . . . . . 49

25.-Diagram of Roscoe's Actinometer as modified by Mr. Darwin 60

26.-Ditto . . . . . . . . . . . . . . 61

27.-Arrangement of Electric Lamp, Prism, and Screen . . . . . 66

28.-Arrangement of Lamp, Lens, and Prisms . . . . . . . . . 67

29.-Bunsen's Flame coloured by Alkaline Salt . . . . . . . . 68

30.-Bunsen's Old Form of Spectroscope . . . . . . . . . . 72

31.-Steinheil's Improved Form of Spectroscope . . . . . . . . 72

32.--Arrangement of Slit of Spectroscope . . . . . . . . 73 
FIG.

32a.-Arrangement of Slit of Spectroscope for Two Flames . . .

33. Kiveh offs Delicate Spectroseope .

34.-Bunsen's Map of the Spectra of the Alkalis and Alkaline Earths 77

35.-Repetition of Steinheil's Form of Speetroscope and Slit . . . 103

36.-Arrangement of Slit of Spectroscope . . . . . . . . . . 101

37.--Repetition of Bunsen's Maps of the Spectra . . . . . . . . 107

38.-Wheatstone's Metallic Lines . . . . . . . . . . . . 116

39.-Large Spectroscope with Nine Prisms . . . . . . . . . . . 117

40.-Arrangement for obtaining Spectra of the Heavy Metals . . . 119

41.-Electric Spark in different Gases . . . . . . . . . . . . . 124

42.-Geissler's Tubes, experimental form . . . . . . . . . 125

43.-Photograph of the Discharge in Vacuum Tubes . . . . . . 126

44.-Ditto . . . . . . . . . . . . . 126

45.-Boisbaudran's Spectrum of Nitrogen . . . . . . . . . 129

46.-Ditto, of Carbon . . . . . . . . . . . . . . 130

47.- - Lockyer's Photograph of the Ultra-Violet Bands in the Carbon

Spectrum . . . . . . . . . . . . . 131

48.-Band Spectrum of Oxygen at Negative Pole . . . . . . . 132

49.- Spectrum of Lithium and Strontium seen with intense Spark . 132

50.-Arrangement adopted by Lockyer in his Spectroscopic

Investigations . . . . . . . . . . . . . 133

51.-A grotup of Zinc Lines examined by Lockyer's method . . . . 134

52.- Spectrim of the Strontium in Electric Arc . . . . . . . . 137

53.- Spectra of the Chloride, Bromide, and Iodide of Barium . . 180

54.- Selective Absorption by Iodine Vapour and by Nitrous Fumes 184

55.-Distribution of Atmospheric Lines as Mapped by Brewster and Gladstone . . . . . . . . . . . . 185

56.-Janssen's Map of the Solar Spectrum from C to D . . . . 186

57.-Chromium Absorption Spectrum . . . . . . . . . 190

58.-Potassium Permanganate Absorption Spectrum . . . . . 190

59.-Diagram of some of the more interesting Absorption Spectra . 191

60.-Ditto . . . . . . . . . . . . . . 192

61.-Stokes's Blood Bands . . . . . . . . . . . . . . . 193

62.-Browning's Micro-Spectroscope . . . . . . . . . . . 195

63.-Section of the Micro-Spectroscope . . . . . . . . . . . 196

64.-Arrangement for Bessemer Steel-making . . . . . . . . . . 199

65.-Repetition of Maps of Bessemer Spectrum . . . . . . . 203

66.-Comparison of Bessemer Spectrum with that of Manganese Oxide 204

67.-Becquerel's Phosphoroscope . . . . . . . . . . . . 207

68.-Spectra of Phosphorescent Bodies . . . . . . . . . . . 208

69.-Repetition of Large Spectroscope with Nine Prisms . . . . . 203

70.-. Section of Large Spectroscope . . . . . . . . . . 210

71.-Repetition of Kirchhoff's Spectroscope. . . . . . . . . . 211

72.-Diagram of a Diffraction Grating fitted for observation . . . 213 
73.-Repetition of Map of Bessemer Spectrum . . . . . . . . 217

74.-Repetition of Fraunhofer's Map . . . . . . . . . . 230

75.-Ultra-Violet Rays, from Photograph . . . . . . . . . 232

76.-Spectrum of Burning Sodium compared with that of the Soda Flame . . . . . . . . . . . . . .

77.-Experiment showing Production of the Black Sodium Absorption Flame . . . . . . . . . . . . .

235

236

78.-Bunsen's Apparatus for obtaining a Constant Black Sodinm Flame . . . . . . . . . . . . .

79.-Coincidence of some of the Bright Jines of Iron with some of Fraunhofer's lincs . . . . . . . . . . . .

80.-Diagram of Sun-spot on the Slit of a Spectroscope . . . . . 247

81. - Spectrum of a Sun-spot as observed at Greenwich . . . . . 248

82. -Appearance of Red Solar Prominences in the Total Eclipse of 1860 . . . . . . . . . . . . . 249

83.-Ditto . . . . . . . . . . . . . 249

84.-Eclipse of 1869 observed in America . . . . . . . . . . . 250

85.-Lockyer's Drawings of Bright Lines in Solar Chromosphere . 253

86.-Forms of the Red Solar Prominences (Zöllner) . . . . . . . 255

87.-Ditto . . . . . . . . . . . . 256

88.-Ditto . . . . . . . . . . . . . . . . . 256

89.-Ditto . . . . . . . . . . . . 256

90.-Ditto . . . . . . . . . . . . . . . . 256

91.-Drawing of the Corona during the Total Eclipse of the Sun of 1870 . . . . . . . . . . . 258

92.- Solar Corona seen during a Sun-spot Minimum, 1878 . . . . 259

93.-Drawing of an intermediate state of the Corona between Maximum and Minimum, 1871 . . . . . . . 260

94.-Portion of Solar Spectrum surrounding the principal Coronal Line when seen with great dispersion . . . . . . . . 262

95.-Young's Drawing of the Solar Spectrum near $b$. . . . . . 262

96.-Photograph of Spectrum of the Corona . . . . . . . . 263

97.-Distribution of Energy in the Solar Spectrum at Sea Lcvel and at various Altitudes . . . . . . . . . . . . 299

98.-Absorption Spectrum of Uranus . . . . . . . . . . 307

99.-Huggins's Star-Spectroscope . . . . . . . . . . . . . 309

100.-Maps of the Lines in Aldebaran and $a$ Orionis . . . . . . . 311

101.-Photograph of Differcnt Star Spectra, showing chiefly the Hydrogen lines . . . . . . . . . . . . .

102.-Comparison of Nebular Spectrum with the Bright Lines of known substances .............. . . 318

103. -Nebula in Aquarius . . . . . . . . . . . . . . . 319

104.-Nebula 18H IV. . . . . . . . . . . . . . . . . . . . . 319

105.-Nebula in Andromeda. . . . . . . . . . . . . 320 
106.--Comparison of Nebular Spectrum with the Bright Lines of known substances . . . . . . . . . . . 32

107.-Nebula in the Sword-handle of Orion . . . . . . . . 323

108.-Photograph of the Spectrum of this Nebula . . . . . . . 323

109.-Comparison of Cometary and Nebular Spectra with those of Carbon and other known substances . . . . . . . 325

110.-Comet II 1868 . . . . . . . . . . . . . 326

111.-Apparatus for ascertaining the presence of Carbon in the Comet . . . . . . . . . . . . . . .

112.-Photograph of the Comet Spectrum between the Iines F and a 113.-Spectrum of Comet II. 1881 . . . . . . . . . . . . 114.-Spectrum (F Line) of Sirius compared with the Spectra of Hydrogen and the Sun . . . . . . . . . . . .

115.--Deviation of the $\mathrm{F}$ Line in a Spot-Spectrum (Lockyer) . . . . 116.--Shifting of the $\mathrm{F}$ Line in the Chromosphere . . . . . . . . 117.-Diagram of Two Red Lines displaced towards the Violet in the neighbourhood of a Sun-spot . . . . . . . . . . .

118.-An improved Spectroscope with Three Prisms . . . . . . . . 119.-Apparatus for ascertaining the Presence of Carbon "in the Comet . . . . . . . . . . . . . .

\section{PLATES.}

Total Solar Eclipse. Esgypt, 1882 . . . . . . Frontispiece. Chromo-lithogriph of the Spectra of the Metals of the Alkalis and Alkaline Earths, from the Drawings of Bunsen and Kirchhoff. facing 63

Forms of Eruptions and Clond-like Prominences as observed and drawn by Professor Zöllner . . . . . . . . . . . . facing 256 Appearance of the Corona of the Sun as photographed a few weeks before the Total Eclipse of 1883 . . . . . . . . . facing 265

A Diagrammatic Drawing of Ditto, from a Photograph taken at Caroline Island, one of the South Sea Islands, by Messrs. Lawrance and Woods................. . facing 265

Chromo-lithograph of the Spectra of the Stars and Nebulæ compared with the Solar Spectrum and the Spectra of some of the NonMetallic Elements . . . . . . . . . . . facing 305 


\section{ON SPECTRUM ANALYSIS.}

\section{LECTURE I.}

Introduction.-Newton's Discovery of the Composition of White Light, 1675.-Properties of Sun Light as regards Heating Power, Luminosity, and capability of producing Chemical Action.-The Solar Radiations. -Prismatic and Diffraction Spectra.-Position of Maxima.-Illustrations of these Radiations.-Means of obtaining a Pure Spectrum.Frainhofer's Lines.-Planet and Moon Light.-Star Light.

Appendix A.-Extracts from "Newton's Opticks."

Appendix B.-Buruing Magnesium Wire a Source of Light for photographic purposes.

Appendix C.-On the Chemical Action of the constituent parts of Solar Light.

Appendix D.-Description of a Chemical Actinometer.

Amongst all the discoveries of modern science none has deservedly attracted more attention, or called forth more general admiration, than the results of the application of Spectrum Analysis to chemistry. Nor is this to be wondered at when we remember that a new power has thus been placed in the hands of the chemist, enabling him to detect the presence of chemical substances with a degree of delicacy and accuracy hitherto unheard of, and thus to obtain a far more intimate knowledge of the composition of terrestrial matter than he formerly enjoyed. So valuable a means of research has this new process of analysis proved itself to be that since its first establishment, in 
1860 , no less than five new chemical elements have by its help been discovered.

Not only, however, have we to consider the importance and interest which attaches to the subject as evidenced by the discovery of these new elementary bodies, but we are forced to admit that by the application of the simple principles of spectrum analysis the chemist is able to overstep the narrow bounds of our planet, and, extending his intellectual powers into almost unlimited space, to determine, with as great a degree of certainty as appertains to any conclusion in physical science, the chemical composition of the atmosphere of the sun and far distant fixed stars. Nay, he has even succeeded in penetrating into the nature of those mysteries of astronomy, the nebulæ; and has ascertained not only the chemical composition, but likewise the physical condition, of these most distant bodies.

It does, indeed, appear marvellous that we are now able to state with certainty, as the logical sequence of exact observations, that bodies common enough on this earth are present in the atmosphere of the sun, at a distance of ninety-three millions of miles, and still more extraordinary that in the stars the existence of such metals as iron and sodium should be ascertained beyond a shadow of doubt. We thus see that the range of inquiry which the subject opens out is indeed vast, and it is well to bear in mind that as the discoveries in this branch of science are so recent, they are necessarily incomplete, so that we must expect to meet with many facts and observations which still stand alone and require further investigation to bring them into harmony with the rest. The advance in these new fields of research is, however, so rapid that, as time rolls on, and our range of knowledge widens, new facts quickly come to support the hitherto unexplained phenomena, and thus our theory becomes more and more complete. It will be my duty in the Lectures which I have the honour of delivering in this Hall to endeavour to explain to you that these results, apparently as marvellous as the discovery of the elixir vitæ or the philosopher's stone, are the plain and necessary deductions from exact and laborious experiment, and to show how two German philosophers, quietly 
working in their laboratories in Heidelberg, obtained this startling insight into the processes of creation.

The only means of communication which we possess with the sun, planets, or far distant stars, or by which we can ascertain anything respecting their chemical constitution, is by means of the life-supporting radiation which they pour down upon the earth, producing the effects which we call light and heat. It will, therefore, be our business, in the first place, to investigate the composition of the radiations which these bodies give off, and next gradually to notice, as our field of observation enlarges, the applications to which the properties of the light thus emitted lead us. One cannot help regretting that when, as at present, the sunlight is shining so brightly, we are unable to

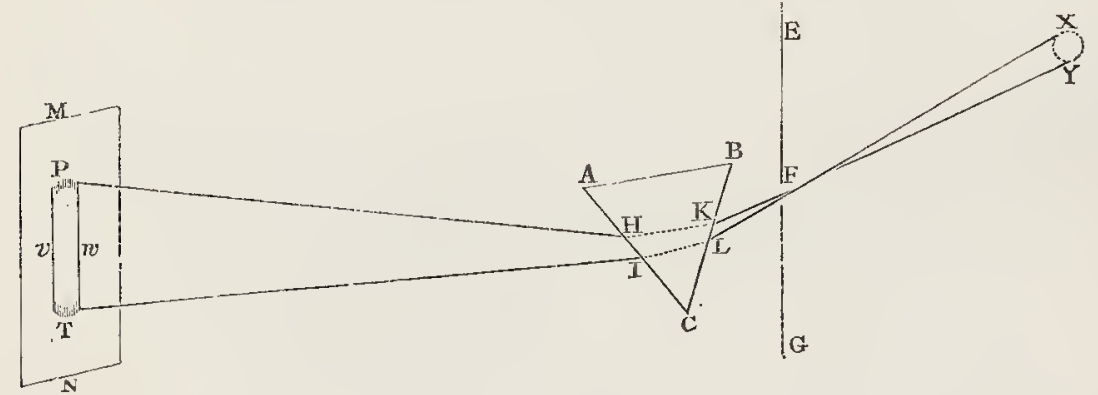

FIG. 1.

utilize it, and illustrate by experiments made with the sunlight itself the points which we wish to explain. In this climate, however, even if we could conveniently do it, the sun slines so intermittently, and it is so doubtful if we can have it just when it is required, that we have to make use of other means, especially of this bright light of the electric arc, which, if less perfect than sunlight, is more under our control.

In the year 1675, Sir Isaac Newton presented to the Royal Society his memorable treatise on Optics. ${ }^{1}$ In this treatise, which contains a large number of experimental and theoretical investigations, one point especially attracts our attention: it is the discovery of the decomposition of white light. We have here (Fig. 1) a fac-simile of the drawing illustrating Newton's

1 For extracts from "Newton's Opticks," see Appendix $\Lambda$. 
experiments on this subject. He heads the first paragraph in his memoir, written in the year 1675, with the words, "lights which differ in colour differ also in refrangibility." Newton allowed the sun to shine through a round hole (Fig. 1, F) in a shutter, and he then examined the character of this light by means of a triangular piece of glass (A B C) called a prism. Ho found that the white light, after passing through the prism, was bent or refracted out of its course, and split up into a coloured band ( $P$ T) which, when received on the white screen (M N), exhibited all the colours of the rainbow in regular succession, passing from red through all the shades of orange, yellow, green, indigo blue, to violet. Newton termed this coloured band the

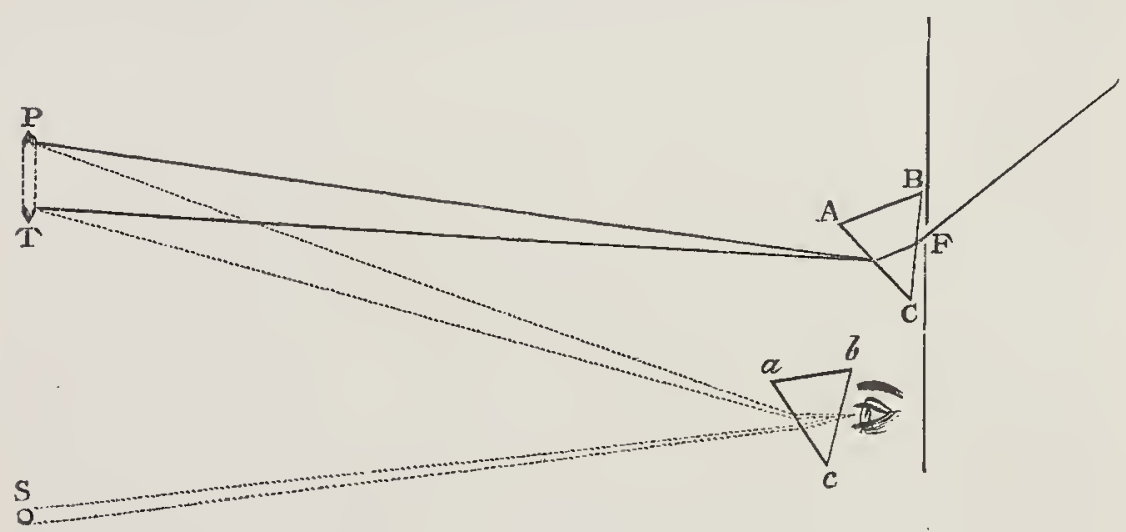

FIG. 2.

Solar Spectrum, and came to the conclusion that the light of the sun consists of rays of different degrecs of refrangibility. He also showed that all the various portions of this coloured band, when again brought together, produce upon the eye the effect of white light. This experiment (represented in Fig. 2) Newton performed by simply allowing the light passing through the round hole ( $F$ ) in the shutter to fall on a prism (A B C), producing the solar spectrum, and then on looking at this coloured band through another prism $(a b c)$ placed in the same direction, instead of seeing a coloured band he observed a spot of white light, thus showing that the whole of these differently coloured rays, when brought together by means of the second 
prism, produce on the eye the effect of white light. Here we have the intensely white electric light, and by means of these two prisms you observe that I can split the light up into its various constituent parts, and we obtain this splendid coloured band, the spectrum of the electric arc. Now I shall endeavour to show you the second effect which Newton observed. For this purpose I have only to reverse the position of one of the prisms; for if I allow this band of coloured light emitted from the first prism to pass through the second prism, placed in the opposite direction to the first, I shall again bring these coloured rays together, the second prism neutralizing altogether the effects of the first, and we obtain the bright white image of the slit.

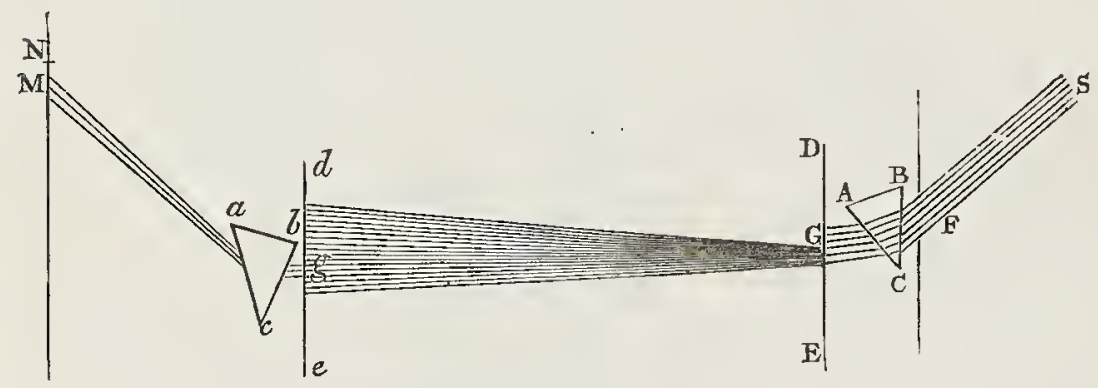

Frg. 3.

I should like next to show you a third part of the experiment made by Newton. If I cut off, by means of this screen, a portion of the spectrum which has passed through the first - prism, say the yellow or the green or the red, you will see that another refraction through the second prism does not alter this coloured light. Here, for instance, I take the green, and bring my prism into a proper position: we shall find that I only get the same green light on the screen behind, proving that the green ray cannot again be split up by further refraction. The mode in which Newton performed this experiment is seen in Fig. 3. The green rays $(g)$ in the spectrum $(d e)$, when refracted through a second prism $(a b c)$, appear as a green band (N M) on the second screen.

Now the reason why all these different coloured lights, when 
they reach the eye, produce the effect of white light, is a physiological question which we cannot explain. We find that not only do all the colours of the spectrum, when they are brought upon the eye, produce the effect of white light, but that several mixtures of only a few out of all these different colours have the same power of producing upon the eye the effect of white light.

Thus Helmholtz and Maxwell have shown that the following mixtures of two complementary colour's when brought together into the eye produce the effect of whiteness:-Violet and greenish

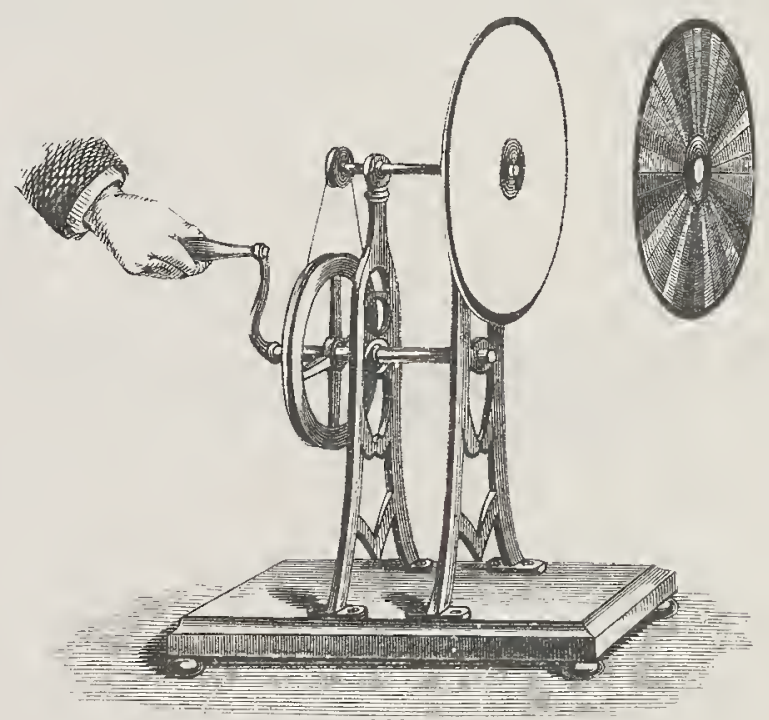

FIG. 4.

yellow; indigo and yellow; blue and orange; greenish blue and red. This I may readily illustrate to you by taking a revolving disc (Fig. 4), upon which segments of various colours have been painted. When I turn this disc-which you observe contains, to begin with, all the colours of the spectrum in due proportion -and then, when it is revolving, exhibit it to you by the light of burning magnesium, you will notice that the effect produced upon the eye is that of a uniformly white disc. Let me now substitute for this painted disc another one, which only contains the three colours, red, yellow, and blue. You will still perceive 
that when quickly revolved the disc appears white; and so I might substitute any of the above mixtures of colours, all of which produce on the eye the sensation of white light by the rapid succession of the images of the different colours, the effect of the one colour not having time to disappear from the retina before that of the other comes into play. If I illuminate the rotating disc by means of an instantaneous electric spark, you will see that all the colours of the disc become noticeable at once. This is because the period of illumination is so exceedingly short that the disc appears as if stationary. It was indeed at one time supposed that the various shades of colour in the solar spectrum were produced by an overlapping, as it were, of three distinct coloured spectra, - one red, the second yellow, and the third a blue spectrum,- the maxima of which are situated at different points, that of the red and blue at the extremes, and that of the yellow in the middle of the visible spectrum. This theory of Brewster's has, however, been proved to be fallacious, for Helmholtz has shown that the green ray, for example, is not made up of blue and yellow light superposed, and we cannot separate anything else but green out of it. Hence we conclude that each particular ray has its own peculiar colour, and that light of each degree of refrangibility is monochromatic. But, on the other hand, although physically, and in the actual spectrum, there is no such thing as a superposition, or overlapping of different spectra, yet it is likely that the retina is mainly sensitive to three impressions, and these are probably red, green, and violet.

In noticing the physical properties of the variously coloured light obtained when the sunlight or white light is decomposed into its constituent parts, we must remember, in the first place, that light is due to the undulations of the elastic medium pervading all space, to which physicists have given the name of luminiferous ether. As the undulations of the particles of water, causing the waves of the sea, differ in length (as from crest to crest), and in amplitude (as measured by the extent of the vibration of each particle), sometimes being minute and shallow like the ripples on the surface of a pond, sometimes rising and 
falling into the gigantic crests and valleys of the storm-ridden ocean, so also the undulations of the ether-producing light differ in wave-length and amplitude, giving rise to the different effects of colour and intensity to which we have referred.

Let us now compare the power of the ear and the eye, the one to receive the vibrations of the air called sound, and the other the vibrations of the ether termed light.

The human ear has the power of distinguishing variations in sounds differing widely in wave-length and in rapidity of vibration. Thus, for instance, the musical note, the deepest in the bass of all those that can be heard by the human ear, is produced by the regular succession of impulses occurring about 16 times in each second, whereas the highest continuous sound which is perceptible to the ear is caused by about 38,000 vibrations per second. Hence the range of audible notes extends over about 11 octaves. Let us next examine what is the range to which the eye is sensitive. It is found that we can observe, ordinarily, in the solar spectrum from the position indicated by a fixed dark line in the red portion termed A (see frontispiece of the Solar Spectrum) to a line $\mathrm{K}$ in the violet or most refrangible portion of the visible spectrum. Now the difference in the number of vibrations from $\mathrm{A}$ to $\mathrm{K}$ is but slight; it is indeed not one octave. The length of the undulation of each of these waves is excessively small, whilst the number of the vibrations of the ether which take place every second is enormously large. By certain methods of exact measurement, physicists have been enabled to determine with accuracy the length and the duration of these various waves of light; and they have come to the conclusion that the wave causing this red ray which is only just visible to the eye has a length of the $\frac{303}{1000000}$ th part of an inch, or the $\frac{77}{100000}$ th part of a centimetre, and that in one second of time no less than 390 millions of millions of these vibrations occur-whereas near the line $\mathrm{K}$ at the extreme end of the violet, the length of a wave is the $\frac{154}{10000000}$ th part of an inch or the $\frac{39}{100000}$ th part of a centimeter, and the number of vibrations is 770 millions of millions per second. Hence the difference is only from 390 to 770 , and you see that the rapidity 
of the vibration at the one point is not quite twice as great as it is at the other, and we are correct in stating that we can hear about 11 octaves, but that we see only about a single octave.

So long as the waves of light pass through interstellar space they all travel with the same velocity, but when they pass through a substance like glass, the shorter waves are propagated more slowly, and it is due to this fact that Newton was enabled to separate the differently coloured rays by means of the prism. Even in our own atmosphere, waves of light travel at different speed according to their lengths, but the difference is very small, and is generally neglected. Newton's method of separating the different rays of light is not the only one, and we must now discuss another one which has of late years received important applications in Spectrum Analysis. If we throw two pebbles into a pool of water, not too far from each other, and if we watch the waves, as they spread from the two points where the stones have touched the water, we shall see that as the waves cross, at some places the crests of one train fall exactly over the crests of the other train of waves. At such places we shall have a wave of double height, or, as we say, of double amplitude. At other places the crests of one set of waves fall over the hollows of another, and here the two waves exactly counteract each other. A piece of cork if swimming over such a spot would neither rise nor fall. This visible action of the waves upon one another can be produced with the invisible waves of light, and two sets of waves can be made to overlap in such a way as to produce darkness at some places and light of double amplitude at others. We then say that the waves have interfered, and the whole phenomenon is called the interference of light. There is another phenomenon called the diffraction of light, and to explain its nature we call to aid not the waves of water, but the waves of sound. Supposing we have a hole in a wall through which sound can freely enter, and supposing that the instrument which emits the sound is sufficiently far away to send the sound through the hole in one definite direction only, then a person placed in the room would hear the sound wherever he stands, thus proving that the waves of sound which have entered the room in one 
definite direction, namely, in the straight line joining the source of sound to the opening, do not after their passage through the hole pursue that straight line only, but spread about in every direction, just as if the opening itself was the source of sound. This is the diffraction of sound. If now we make a similar experiment with light, that is, if we admit light through a hole in a shutter, we shall obtain apparently an altogether different result, for the light does not seem to spread out as the sound did, but to pursue uninterruptedly its original direction. Thus at first sight there seems to be no diffraction of light, and this was found to be a great difficulty when the wave theory of light was first proposed. As a matter of fact, however, our two experiments, though apparently made in the same way, have differed in a very important point. The hole which admitted the sound was small compared to the length of a wave of sound, whilst the hole which admitted the light was very large compared to a wave-length of light. If we take care to make our experiments really comparable, that is to say, if we use a hole which shall really be sufficiently small, we shall find that waves of light, like waves of sound, can be diffracted. Light is also diffracted whenever a ray falls on to a sharp edge of an opaque body. Two very simple experiments illustrate this diffraction of light.

Take a piece of glass, those who are in the habit of wearing spectacles can conveniently make use of them, press your finger slightly on it and describe an approximately straight line. The glass will now present a striated appearance where tbe finger has passed over it, then bring it close to your eye and look at a candle, when rays of light will seem to diverge from the candle in a direction at right angles to that described on the glass by the finger. The finger has here rendered some parts of the glass less transparent than others, and the light has been diffracted at the edge of the more transparent parts. The openings only having been made small in one direction, light has only been diffracted in one direction. Another and still simpler experiment is the following. Bring your two eyelids close together, so as only to admit the light through a narrow slit, then look at a bright surface such as the opal globe of 
a lamp at night or a window in daytime. The light will now be diffracted at the eyelids, and streaks will be seen to come from the bright surface in a vertical direction. Thus a window will appear to be longer both at its upper and at its lower ends.

Let us now see how the diffraction and interference of light can produce effects similar to those of a prism. I have here a diagram which I hope will make my explanation clear (Fig. 5). Let $A, B, C$, and $D$ be four points at equal distances sending

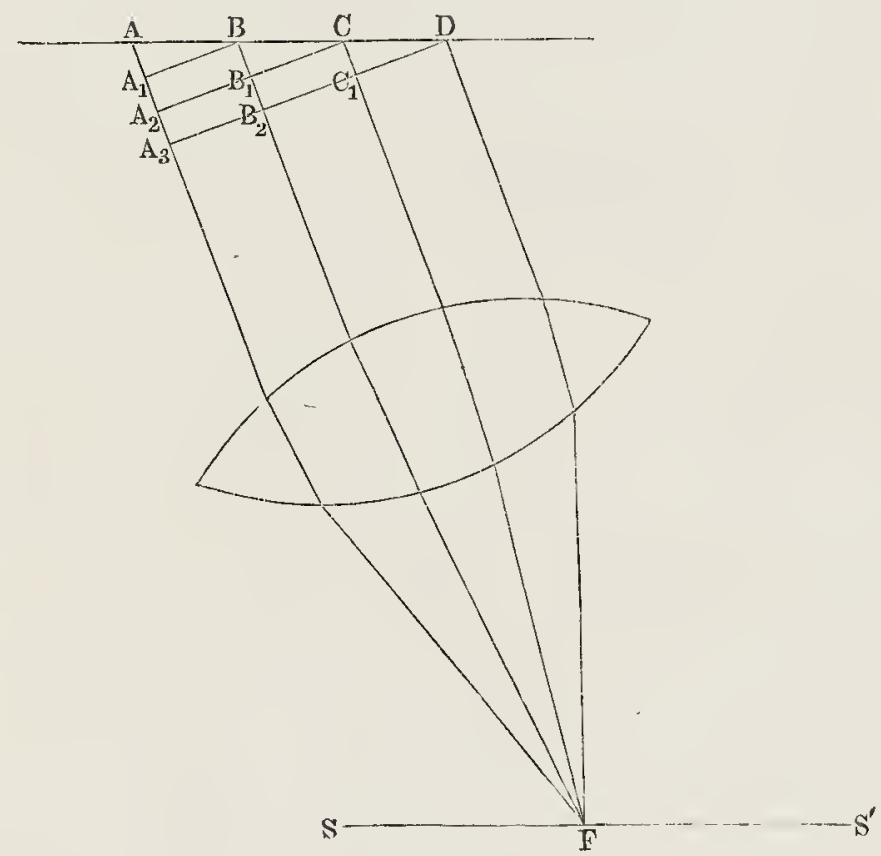

FIG. 5.

out light of the same wave-length, and let them vibrate in such a way that when a crest starts from $A$, a crest will also start from the other points. The points are then said to vibrate in the same phase. The waves will diverge from each point in all directions, but let us confine our attention to one direction only, and to fix that direction let us draw straight lines along it from the four points. Let us also draw from B, C, and D lines at right angles to that direction. These lines will, as shown on the diagram, cut off certain lengths from 
the rays, namely $\mathrm{CC}_{1}, \mathrm{BB}_{1}, \mathrm{~B}_{1} \mathrm{~B}_{2}, \mathrm{AA}_{1}, \mathrm{~A}_{1} \mathrm{~A}_{2}$, and $\mathrm{A}_{2} \mathrm{~A}_{\tilde{5}}$. As the four points are at equal distances, these lengths will, by an elementary proposition of geometry, be all equal; now suppose that these are just equal to the length of a wave of light, as a crest of a wave passes over $A$, another will pass over $A_{1}$, another over $A_{2}$, and another over $A_{3}$, as these points are exactly at the distances of the length of a wave. The same is true for $B, B_{1}$, $\mathrm{B}_{2}$, and $\mathrm{C}, \mathrm{C}_{1}$. All these points will have the crests pass over them simultaneously. At the moment a crest then leaves $D$ a crest will pass over $\mathrm{C}_{1}, \mathrm{~B}_{2}$, and $\mathrm{A}_{3}$, and these four points are on a straight line which is at right angles to the direction in which the wave is propagated. If now a lens is interposed which brings all the four rays together at one point $F$, then, by a law of optics, the times which the waves take to go from $A_{3}, B_{2}, C_{1}$, and $D$, to $F$, will be the same, and as the waves were at the same phase at these four points, they will come together with the same phase, their crests will overlap, and the point $F$ will be illuminated. But if we had supposed that the distance $\mathrm{AA}_{1}$ and with it all the other distances previously named were not equal to a wave-length, but only to half that length, we should have found that to a crest at $\mathrm{A}$ would correspond a hollow at $\mathrm{A}_{1}$, another crest at $\mathrm{A}_{2}$, and another hollow at $A_{3}$. It is in that case easily seen that when a crest will leave $\mathrm{D}$ a hollow will pass over $\mathrm{C}_{1}$, a crest over $\mathrm{B}_{2}$, and a hollow again over $A_{3}$. When the four rays therefore reach $F$ the ray from $D$ and that from $\mathrm{C}$ will be in opposite phases and will destroy each other, and similarly the rays from A and from B will mutually destroy each other, and in this case there would be darkness at $\mathrm{F}$. We see therefore that light or darkness at $F$ depends on the question whether the perpendiculars from $B, C$, and $D$ on to the direction of the rays, cut off portions equal to that of a wave-length or to that of half a wave-length. How this depends on the direction of the rays will be better seen by the inspection of this other diagram (Fig. 6). I have here twenty points emitting light, and these twenty points we suppose to be all at equal intervals and to vibrate always in the same phase. They send out rays in all directions, but not to 
confuse the diagram, I have drawn the rays of different directions as coming from different points.

Let us first consider the rays which leave at right angles to the line on which the points are situated. The points $9,10,11$, 12 are drawn as sending out such light. If we draw a line anywhere at right angles to that direction, all the points in that line will evidently be equidistant from the luminous points and therefore in the same phase. If, by means of a telescope, I collect all these rays I shall see light. Examine next the light sent out in the direction $\mathbf{B B}^{\prime}$, and suppose that the per-

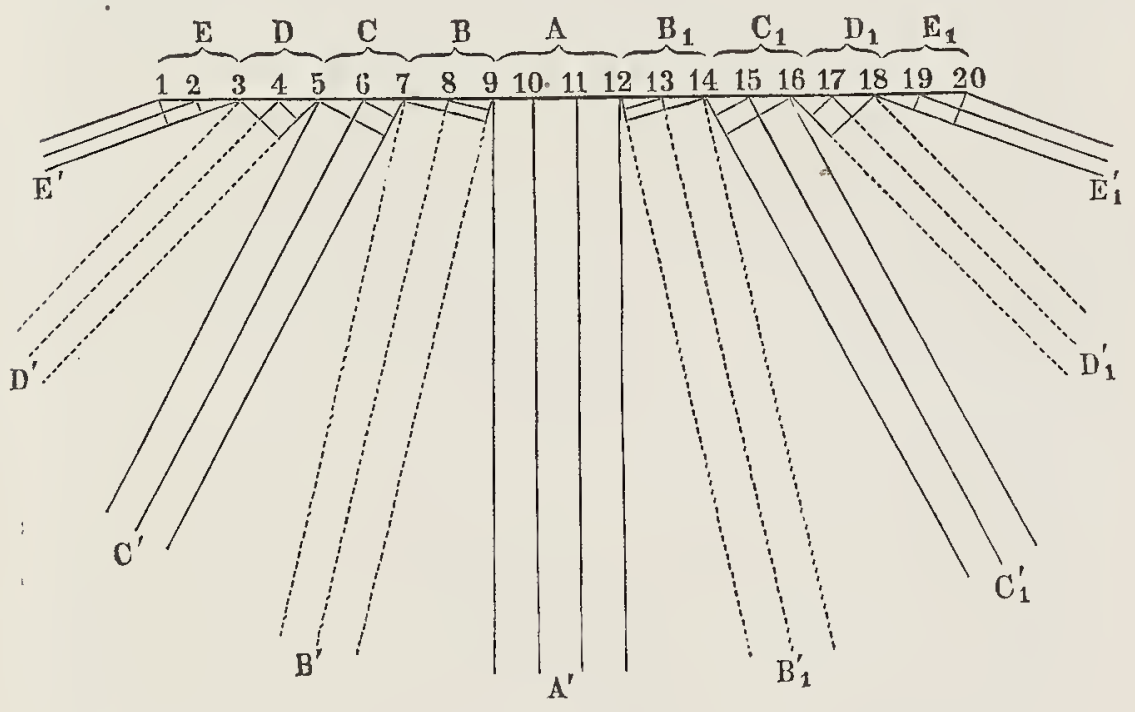

Fig 6 .

pendiculars drawn from 7 and 8 on that direction cut off distances equal to half a wave-length, then, by what $I$ have said, the rays will mutually destroy each other, and I shall not have any light by looking in the direction $\mathrm{BS}^{\prime}$. A.s I gradually incline the telescope more and more I shall come to a direction like $\mathrm{CC}^{\prime}$, in which the perpendiculars cut off distances equal to a whole wave-length, and in that direction again I shall see light. Going round still further I come to a direction $\mathrm{DD}^{\prime}$, in which the distances cut off are equal to a wave-length and half. Here also no light will be produced, for the crest of one ray 
will again come on to the hollow of another. Light, however, will be seen again in the direction $\mathbf{E E}^{\prime}$, in which the perpendiculars cut off distances equal to two wave-lengths. By moving the telescope round the other side I shall similarly come to directions in which light and darkness will be alternately produced. The number of times light and darkness will alternate depends chiefly on the distance between the luminous points.

Supposing now I look in directions intermediate between those drawn on the diagram in which there is neither complete agreement nor complete disagreement between the waves. It would seem as if here I ought to see neither as inuch light as in the directions in which there is agreement, but yet more than where there is disagreement. Such is really the case if the number of luminous points be but small, but it can be shown by calculation that, as the number of these points is increased, the directions in which any light at all is seen gradually restrict themselves more and more, and, finally, I shall see light solely in the directions such as $\Lambda \mathrm{A}^{\prime}, \mathbf{C C}^{\prime}$, and $\mathbf{E E}^{\prime}$, in which the perpendicnlars cut off distances either equal to a whole wave-length or' to some multiple of it; and this is the method by means of which we have been able to measure the length of a wave of light, small as that wave is; for if we know the distances between the points and the direction $\mathrm{BB}^{\prime}$, in which light first appears, we can calculate the perpendiculars which ineasure the length of a wave. At the same time you see how we can separate the different colours, for the directions in which light will appear are clearly different for waves of different lengths. But now I inust describe to you how I can produce a series of equidistant luminous points, whose distances at the same time shall be small compared to the length of a wave of light. Points really luminous I cannot employ, for neither could I bring them sufficiently near together, nor, if I could do so, would they be in the same plase of vibration, for the plase of luminous points is constantly changing in an irregular way. I must call to aid the previous phenomenon to which I have alluded, viz., the diffraction of light. If I scratch on a surface of glass a series of lines very close together 
with a diamond, I render some parts of the surface opaque, and the parts which transmit light will be separated by equal spaces, so that if these parts are small a beam of light passing through them will be diffracted and spread about in all directions from each opening. If the beam of light, moreover, has originally been at right angles to the surface of the glass, the light diverging from the openings will all be in equal phases. It is true the light does not diverge from a series of points as we have assumed, but from a series of small surfaces; but calculation shows that this does not make any difference as far as the phenomena we are here discussing are concerned, if I take the distances of the openings to be that of their middle points. If the beam of light is not incident at right angles, the direction in which light is seen will be different, but the general appearance will be the same.

A surface of glass ruled with a number of lines is called a diffraction grating. Fraunhofer first constructed such diffraction gratings, but their improvement is greatly due to Nobert, whose diffraction gratings, all ruled by hand, were until recently unsurpassed in their accuracy and excellence. Rutherfurd, however, has succeeded in making gratings by machinery, and has also ruled them on speculum metal instead of glass. 'The gratings on speculum metal act by reflection instead of by transmission; they give more light and are therefore more useful for spectroscopic purposes. The most powerful of Rutherfurd's gratings have over 17,000 lines ruled on the inch. Rowland quite recently has improved even on Rutherfurd's gratings, and has especially been able to cover a larger surface; so that it is possible now to have a surface of several inches square ruled with lines which are only the 20,000th part of an inch apart.

The general appearance presented by solar light decomposed by the grating is illustrated in Figs. 7 and 8. Let the light enter the grating at right angles to the surface, in the direction of the arrow in Fig. 7, when the grating is ruled on glass, or in the direction of the dotted arrow, when the grating is ruled on speculum metal. If we look in the direction from which the light comes, a bright white spot of light is seen, this is drawn 
on the figure as a point; as we move the eye to the right or left, we shall see the rainbow colours of white light decomposed by the grating, the violet light having the smallest wave-length will appear nearest to the bright central spot. This first spectrum on either side stands out clear from the remainder. If we look in directions gradually more inclined to the perpendicular of the grating a new spectrum is seen; this is due to the crest of one wave falling over the crest of another which was two wave-lengths in advance. Here again the violet will be least deflected, but the red will not stand out clearly for it will overlap the violet of the third spectrum; and as we go on changing the direction in which we look towards the
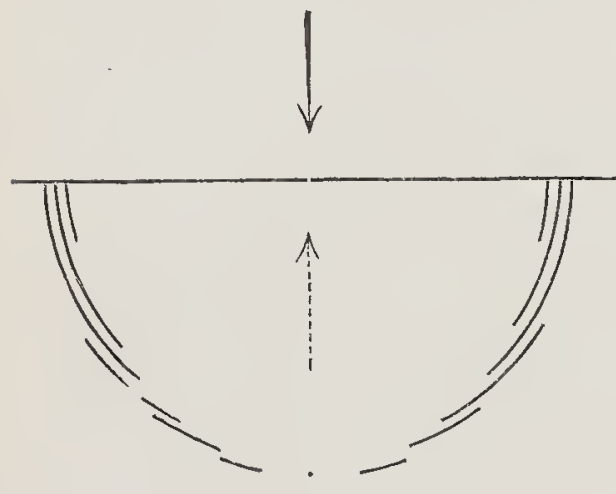
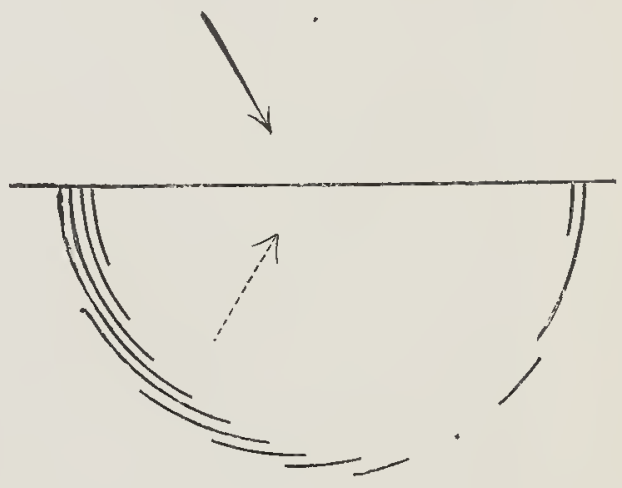

Figs, 7 and 8 .

grating we shall see more and more spectra and they will overlap more and more. The first spectrum, or the spectrum of the first order as it is called, shows the rays with least dispersion, while the greater the order of the spectrum, the greater is the dispersion. If we want to observe them we must separate the one we want to see from those which overlap, and I shall have to explain to you in a subsequent lecture how this can be done. In the figure each spectrum is drawn a little above the one preceding it, so as to avoid confusion, and if as is assumed in the drawing there are 400 lines on each millimeter, we shall see three complete spectra on either side, and the violet ends of three further ones. If we incline the grating to the direction of 
the rays, the appearance is illustrated by Fig. 8. The briglit spot of white light is no longer in the centre but towards one side; and the spectra on the two sides become unsymmetrical, but I can now see as many as five complete and four more incomplete spectra on one side, and this is a great advantage when I want the spectra of higher orders for great dispersion.

The weak point of these gratings consists in the light being broken up and separated, not into one spectrum, as in the case of a prism, but into several distinct spectra; the light in each is naturally weakened, and whenever we have therefore to deal with sources of light not very strong in themselves, the method of separation by a grating is difficult. Gratings are not likely for this reason ever to replace the ordinary one-prism spectroscopes such as I shall describe in my next lecture; but for the examination of solar light, and whenever we want and can employ high dispersion, gratings give a better result than the batteries of prisms which have until lately been used for the purpose.

There is one great difference between spectrum produced by means of a prism and that by means of a grating; while the grating separates out two waves of different length in such a way that their distance is always very nearly proportional to the difference in their wave-length, the prism spreads out the blue rays much more than the red ones. To give an example, let us take, as is usually done, as our unit of length, the ten millionth part of a millimeter, and let us call this length a tenthmeter. Consider two rays which shall have a wave-length 7,000 and 7,010 respectively, and also two rays with a wavelength of 4,000 and 4,010; the first set would look dark red to our eyes while the second set would be violet. The difference in wave-length of the rays in each set is 10 tenthmeters; accordingly, the grating will show the rays separated through almost exactly equal distances. But if the same rays are separated by means of a prism, the two violet ones would have more than five times the distance than the two red ones.

Fig. 9 (I. and III.) is intended to illustrate this distinction. In (III.) we have the rays with a wave-length of $4,000,4,500$, and so on up to 7,500 as spread out by means of a grating; and 
we see that to equal differences in wave-length correspond equal distances between the rays. On the other hand a prism would spread out these same rays as indicated in (I.), and we see how near the red end, on the right of the figure, the same difference in wave-length is separated to a much smaller extent than in the violet. If we had a dispersion spectrum and a diffraction spectrum, the first would show the red end much compressed together, while the blue and violet would occupy a much larger space than in the second. I shall presently have occasion to remind you of this important difference, but after this somewhat long digression I must now pass on to the consideration of another matter, and remind you that rays exist which do not make any luminous impression on our eyes, but which still play a most

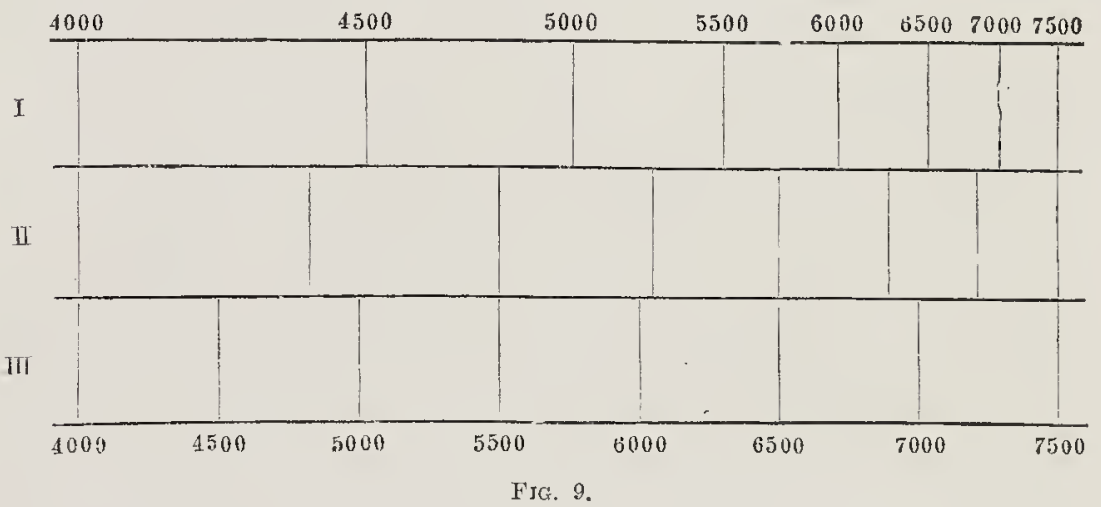

important part in the nature of the solar light, and of other sources containing an even greater amount of invisible rays. Amongst the various devices, by means of which we can make ourselves aware of the existence of these rays, by far the most valuable is photography. And on calling this most beautiful art to our assistance, we find that what we see is only a small part of the whole spectrum, which in reality extends both far beyond the red into what is called the infra-red, and beyond the violet into the ultra-violet. The total length of the spectrum which has been observed up to the present time, contains between three and four octaves instead of the one octave which affects our eyes; and every new improvement in our methods and apparatus 
shows a fresh extension of the rays emitted by luminous substances. The heating power of the rays sent out by selfluminous bodies is not confined to the luminous parts of the spectrum. What we call the heating power, in fact really measures the intensity of the ray. The loudness of a sound cannot be compared with the brilliancy of a ray of light as seen with our eyes, but rather with the heating power of the ray. Hence every ray must have some heating power, and indeed the infra-red rays were first discovered by means of their thermal effects. Suppose for instance that we produce a solar spectrum by means of a prism, in the manner which I have indicated, and suppose by means of a thermometer, a thermopile, or otherwise, we measure the heating effects of different parts of this solar spectrum. As we move the thermometer from the red end of the spectrum towards the violet, we find that a gradual diminution of heating power occurs. But if we move it from the red end in the opposite direction, that is into the regions in which we can no longer see any light, we shall at first observe an actual increase of heating power, and afterwards in the more remote portions a diminution will be noticed. It was by means of a thermometer and an experiment made exactly in this manner, that Sir William Herschel first discovered the infra-red rays in the year 1800. The experiment seemed to justify him and those who came after him in saying that the maximum heating power of the solar rays was in the invisible parts of the spectrum beyond the red. But this result depends altogether on the manner in which the experiment is performed. If instead of employing a prism we separate the rays by means of a grating we shall observe an altogether different effect. The maximum heating effect will no longer be in the infra-red but in the yellow or orange part of the spectrum. In order to examine a little more closely on what this curious difference depends, let us once more consider the different way in which the waves of light are separated in the grating and in the prism. Suppose for instance we were measuring the heating effect in a diffraction spectrum such as Fig. 9 (III.) and in a refraction 
spectrum Fig. 9 (I.), and let us assume that our thermometer was of such a size as to cover in the diffraction spectrum, the distance between the equidistant lines in the figure, then I might perhaps find that for the interval from 7,000 to 7,500 I should get the same heating effect as for the interval from 5,000 to 5,500 . Let us then move the thermometer into the spectrum produced by refraction. We see by the figure that the wavelengths in the red are so near together that the thermometer now will allow more than double the number of waves to fall upon it, that is all wares between 7,500 and 6,500 will fall into it, and consequently the heating effect here in the extreme red will be much greater when the spectrum is produced by a prism, than when a grating is used. On the other hand the green

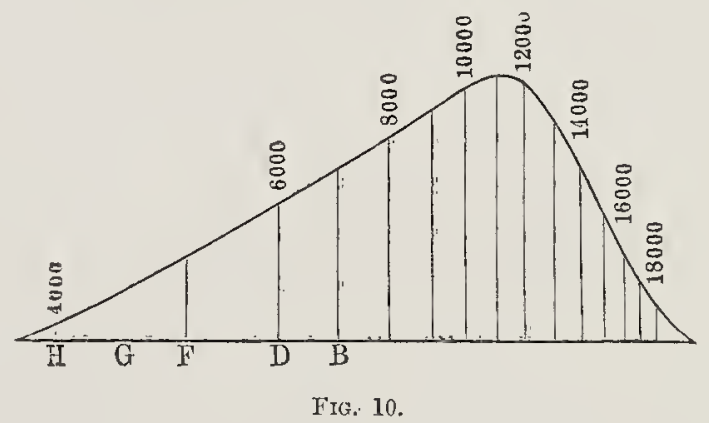

rays from 5,000 to 5,500 occupy a larger space with a prism than with a grating, the thermometer will therefore not receive so many and would not rise so high.

It is clear, therefore, that in the red, and $\hat{a}$ fortiori in the infra-red, the prism will show too large a heating power as compared with the grating, while in the green, and still more in the blue and violet, the prism will show a smaller thermal effect. It was the merit of Draper to have drawn attention to this important difference, and to have shown that when the spectrum is spread out according to a wave-length scale, the maximum heating effect of the solar rays is in the visible part instead of in the infra-red. But his instruments were insufficient for accurate measurements, and some of his conclusions were vitiated by his incorrect but otherwise laudable 
opinion, that the distribution of light and heat was the same in the solar spectrum. The mere existence of infra-red rays ought to have shown him that this is not the case. The first attempt to find accurately distribution of heat in the solar spectrum was made by J. Muiller, and published by him in 1858. The observations were made with a prism of rock-salt which does not absorb appreciably the infra-red rays, but were afterwards reduced to wave-lengths as well as the means of the author allowed him to do so. The results were plotted down in a curve given in Figs. 10 and 11, of which the first gives the distribution of heat as shown by the prism, having a maximum effect in the infra-red, whereas the second exhibits the same reduced to the wave-length scale and gives the maximum heating power in the yellow. As Miiller himself obseryed, the weak point of his

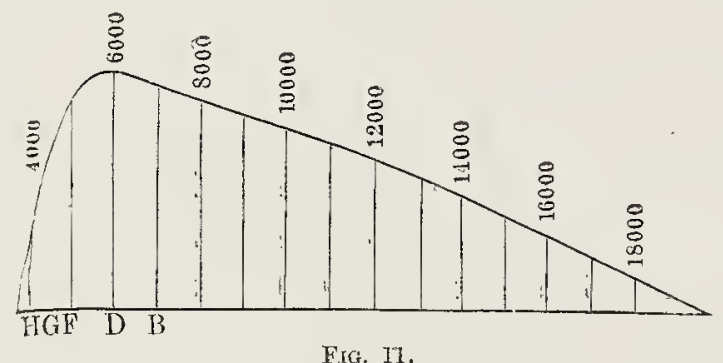

Fig. 11.

results consisted in the uncertainty of his wave-lengths. Modern researches have partly overcome this difficulty, and thanks to Prof. Langley we have now a fairly complete knowledge of the distribution of heat throughout the solar speetrum. His interesting series of experiments was made with an instrument termed a bolometer. ${ }^{1}$ This consists of very thin strips of metal which form the arms of an electric balance; if one of these arms be heated ever so slightly, while the temperature of the other remains unchanged, a movement of the galwanic needle included in the circuit is noticer. To give an idea of the delicacy of this measurer of sunlight I may state that a change of temperature of $\frac{1}{10000}$ of a degree centigrade is easily noted, and that the heating effects of the ultra-violet rays can be detected in less

I Bo $\lambda \grave{a} \iota \dot{\eta} \lambda \hat{l o v}$, sunbeams. 
than ten seconds, though the same radiation is so weak that falling uninterruptedly for over one thousand years on a kilogram of ice at $0^{\circ} \mathrm{C}$. it would not wholly melt it (Langley).

By what I have said you see that I get an altogether different curve of heat distribution according as I plot down on the horizontal line refractive indices, as I do with a prism, or wave-lengths as I do with a grating; and there can be no doubt that the curve obtained with the wave-length scale is to be preferred, as it is fixed and absolute, while prisms of different substances will not disperse the light in exactly the same way. But I can prepare scales as absolutely correct as that of the wavelength scale and still obtain different curves of heat distribution. Supposing for instance we take on a horizontal line equal distances, not for equal differences in wave-lengths but for equal differences in time of vibration of different waves, $I$ should obtain a distribution of rays in the spectrum given in Fig. 9 (II.) which you see is intermediate in its dispersive properties between the prism and the grating. If then I measure for each wavelength the heating power along a vertical line, I should get a curve somewhat intermediate between that shown in Fig. 10 and in Fig. 11. Such a curve would show its highest point somewhere in the extreme red. We see by all these examples that the results I obtain for the distribution of heat or of energy in the solar spectrum are somewhat arbitrary, for they depend on the way in which the spectra are obtained. To say that the maximum heating effect lies in the infra-red or in the yellow, or in the red, has no real meaning unless $I$ add in what way the results have been obtained and reduced. This was first clearly pointed out by Lord Rayleigh.

I will now endeavour to show you by an experiment the fict of the existence of these dark heating rays of really invisible light, and to do this I will employ the beautiful arrangement by which Dr. Tyndall first accomplished the separation of which I have just spoken. I have for this purpose placed in the dark box (D, Fig. 12) an electric lamp (L), which gives us a very bright light, and by means of this mirror (M) I can bring the rays to a focus at any desired point. Here is a cell (c) which 
Dr. Tyndall very expressively calls a ray-filter, by which I can filter out the whole of the luminous rays, by passing them through this opaque solution of iodine in disulphide of carbon, whilst the invisible heating rays are transmitted, and will soon

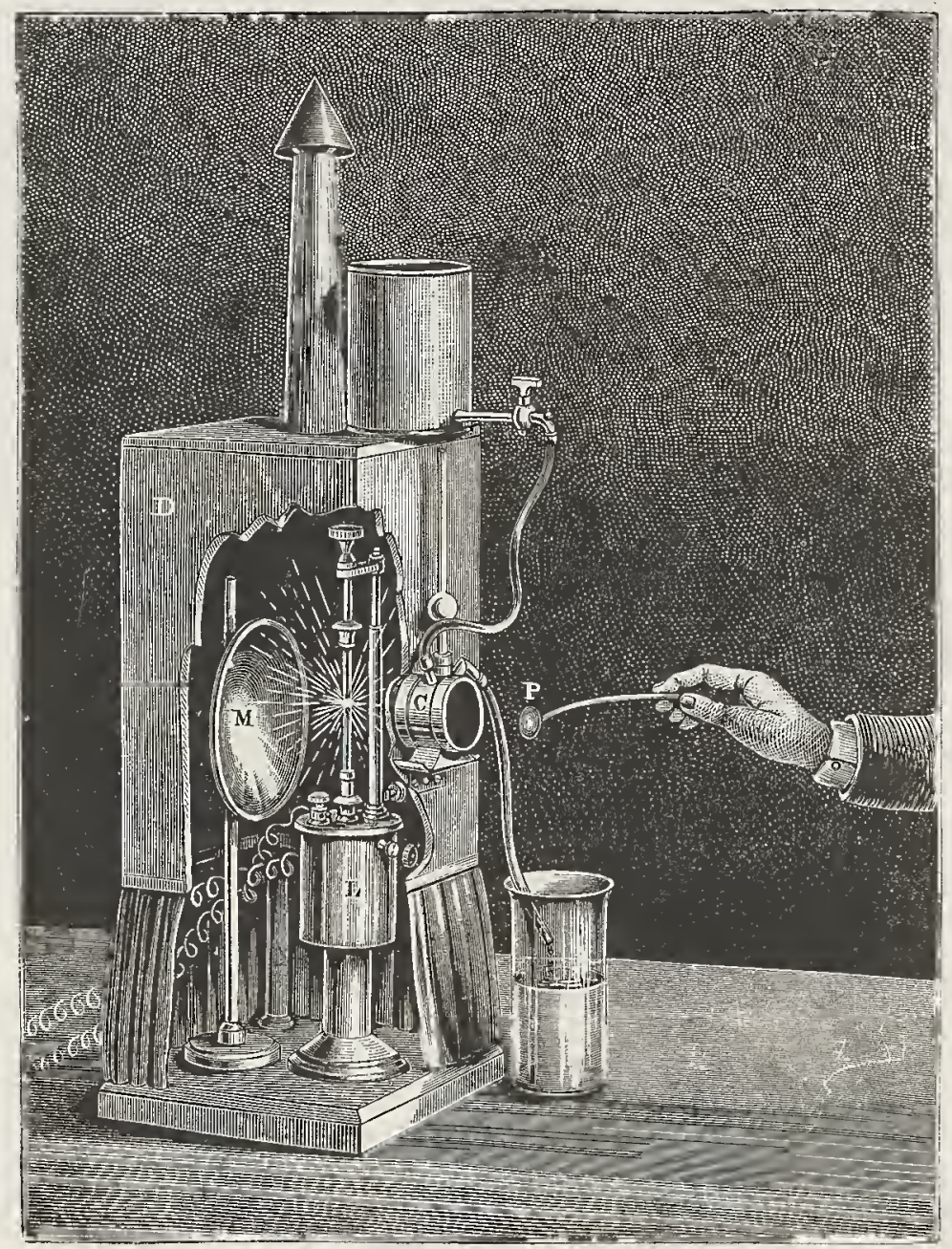

FIG. 12 .

render themselves evident to you. A current of cold water circulates through a double jacket on the outside of the cell, to keep the volatile disulphide cool. Now I think you will observe that no rays of light come through, but if I take a 
piece of black paper, and place it in the focus of our mirror, you see the paper is ignited, owing to the presence of these dark heating rays. I may now do the same with a piece of blackened platinum (P, Fig. 12); you see that this also is heated to redness. I can show you this again in a variety of forms. Here is some gunpowder strewed on this paper; you observe that it at once explodes when brought into the focus of the dark rays. Here I have some blackened gun-cotton, which instantly catches fire. I may vary the experiment by lighting a cigar; and here you see the brilliant scintillations of charcoal burning in oxygen, having been heated up to the temperature of ignition in the focus of the dark rays. Dr. Tyndall has measured the proportional amount of the entire heating rays which, pouring forth from this incandescent carbon, has passed through this dark filter, and he has found that this consists of $\frac{7}{8}$ of the whole amount; so that only $\frac{1}{8}$ of the radiation is really visible. Another, and perhaps still more striking, method of showing the existence of these infra-red rays is that employed by Captain Abney, who has been able to prepare sensitive films capable of receiving photographic impressions of these invisib!e vibrations, and thus the notion, previously held, that only the more refrangible portion of the solar radiation was capable of producing chemical action, has now to be given up. Another, and third, method of investigating these infra-red rays has been founded by $\mathrm{M}$. Becquerel on the properties of certain bodies with respect to phosphorescence and fluorescence. I must, however, defer a description of this until, in a subsequent lecture, I shall speak of these phenomena.

Let us now examine a little more closely the power of the luminous vibrations to produce chemical action. It still remains quite true that for most cases of chemical change, and those which are perhaps the best known, it is the more refrangible rays at the blue end of the spectrum which effect this chemical action. Allow me to illustrate this fact to you by an experiinent, and in order to render the illustration more perfect I will first make an experiment with reference simply to white light, to show you that the brilliant light which is emitted by this 
burning magnesium, and is almost too dazzling for the eye to bear, contains a very large proportion of the rays which we are about to investigate. ${ }^{1}$

I have here a thin glass bulb containing a mixture of equal volumes of two gases, chlorine and hydrogen. These gases when exposed to a bright light combine together, and form hydrochloric acid gas. If I were to throw this bulb out into the sunlight, so rapid would be the combination, and so great the consequent evolution of heat and sudden expansion, that this little bulb would instantly be shattered into a thousand

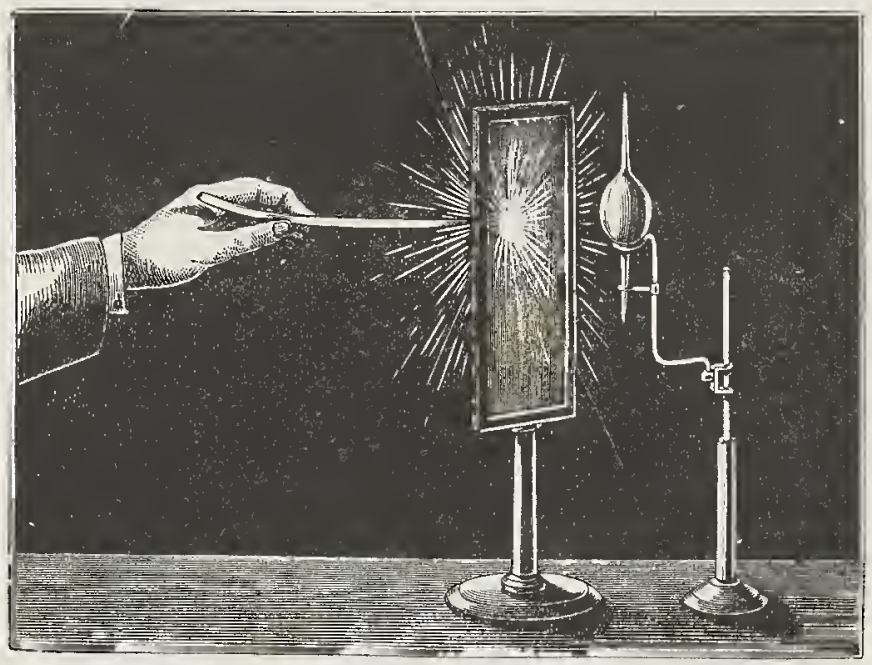

FIG. 13.

fragments. Almost as sudden an effect would be produced if I simply burn a bit of magnesium wire in the neighbourhood of the bulb (Fig. 13); it explodes with a pretty loud report, the bulb is shattered, and the gases have been combined by virtue of the blue rays contained in this kind of light. In order to convince you that the red rays cannot produce the action which the blue ones are able to effect, I here burn a piece of phosphorus, first in a red globe full of oxygen gas, when, as you see, an intense light is emitted wholly without action on our little bulb of sensitive gas. Next I burn a similar piece

1 See Appendix $B$ for the measurement of the chemical intensity of magnesium light. 
of phosphorus in oxygen contained in this blue globe, and as soon as the intensity of the light attains its maximum, the bulb explodes with a loud report. I will next show you in another way that it is the blue rays which thus act chemically. This lantern (Fig. 14) contains panes of different coloured glass,--here a white one, there a yellow one; here a red one, there a blue one. I am going to put another of these little bulbs filled with chlorine and hydrogen in the inside of this lantern, and then I will produce, not by magnesium wire, but by another means,

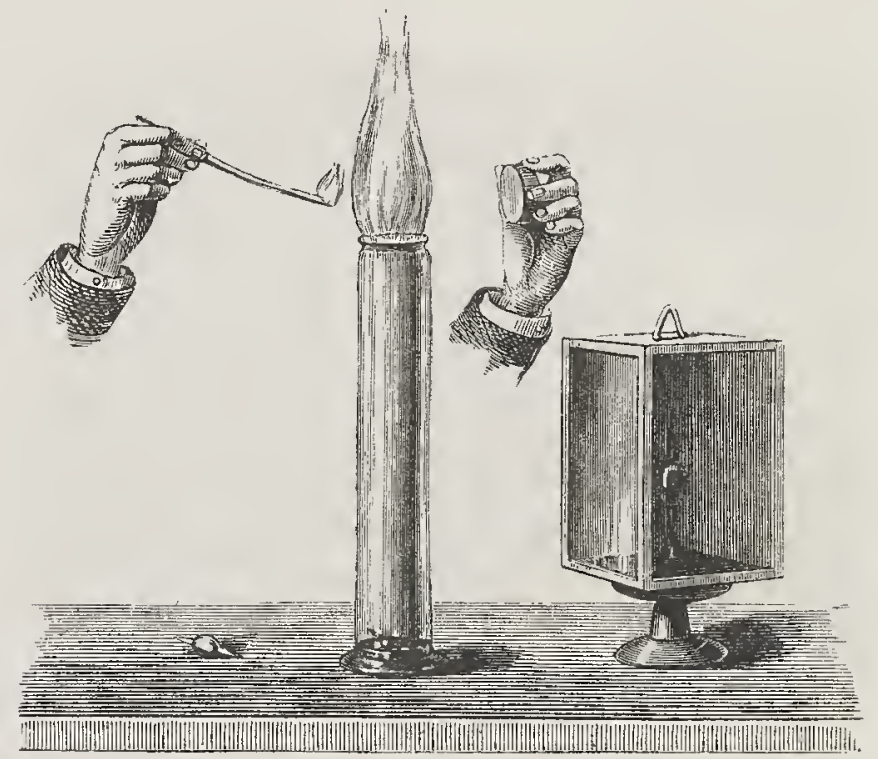

FIG. 14.

a very bright blue light, a light which contains these chemically active rays in great quantity. I will first allow this blue light to shine upon the bulb through the red pane of glass. Here I produce a very bright flame, by throwing some carbon disulphide into a tall cylinder full of nitric oxide gas, and igniting the mixture. There you have the bright flash, but you have noticed no explosion of the bulb, for all the chemical rays have been lield back: filtered off by this red glass, they cannot pass through; and the consequence is, there has been no action on my bulb. I will now allow another of these flashes of light to pass through the blue glass, which being of course trans- 
parent to the blue rays, my little bulb will be shattered into a fine powder, as you observe. Here then we have asccrtained by experiment that the blue rays act chemically on a mixture of chlorine and hydrogen, whilst the red rays are the most intense, as shown by their heating power.

This sensitive mixture of chlorine and hydrogen, which, as you have seen, explodes when the chemical activity of the light is great, may be used as a most delicite means of measuring the amount of light of slight intensity. The combination of the gases then occurs slowly, and may be rendered evident by allowing the hydrochloric acid thus formed to be absorbed by water, when the consequent diminution in bulk of the gas accurately represents the chemical action effected.

The varying power of the different parts of the solar spectrum to produce the chemical combination of chlorine and hydrogen has been carefully investigated. The accompanying figure (Fig. 15) exhibits the chemical action effected by the various portions of the spectrum on the sensitive mixture for one particular zenith distance of the sun. The lines marked with the letters of the alphabet from $\mathrm{A}$ to $\mathrm{W}$, at the bottom of the figure, represent the fixed dark lines. which exist in the solar spectrum, of which I shall have much to say in the subsequent lectures. They serve as landmarks by which to ascertain the position of any given point in the spectrum. The greatest amount of chemical action is noticed between the line in the indigo marked $\mathrm{G}$, and that in the violet marked $\mathrm{H}$. In the direction of the red end of the spectrum, the action becomes imperceptible about $\mathrm{D}$, in the orange (the maximum of visible illumination); whilst towards the other end of the spectrum the action is found to extend as far as the line marked $U$, or to a greater distance beyond the line $\mathrm{H}$ in the violet than the total length of the ordinary visible spectrum. For other chemical substances, such as the salts of silver, which are capable of undergoing decomposition when exposed to the light, different curves of sensitiveness are obtained. All these bodies are acted upon by the blue rays, but some have their

1 See Appendix C for description of method. 
maximum action at one part, some at another part of the spectrum.

The fact that the more refrangible rays act more energetically upon the ordinary photographic plate can be illustrated by showing that I can photograph with these blue rays, whereas I fail to produce the same effect with the red rays. I will coat a plate with collodion, and then darken the room, with the exception of this yellow monochromatic flame, produced by the volatilization of soda salts, which is incapable of acting chemically, and with which we may work without

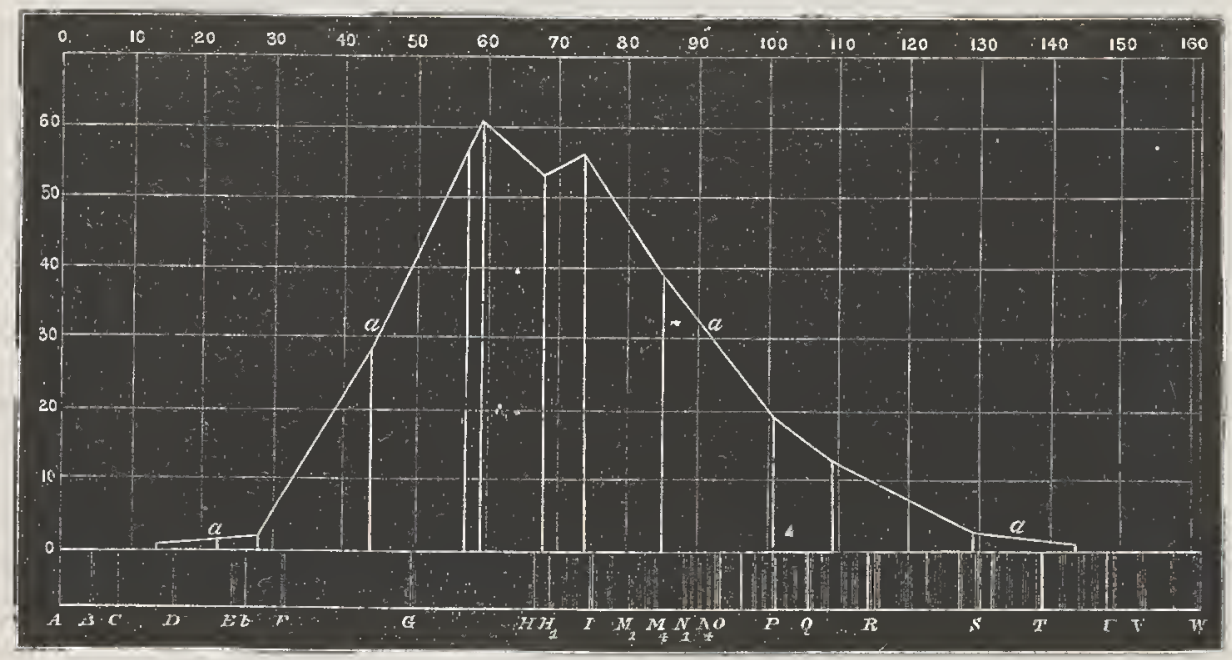

FIG. 15

at all affecting our photographic plate. Now I have coated a plate with collodion, and sensitized it in the silver bath. I shall next expose this to the action of the light of the spectrum of the electric lamp. Let me first show you that $I$ have here (Fig. 16) a negative photograph, of which I am about to take a print by means of the blue rays of the electric lamp. You will observe that there are two figures upon the negative, one marked $V$ and the other marked $R$ : these letters being intended to signify Violet and Red. The one figure marked V, I propose to place in front of my sensitized plate in the blue or violet ray, and the one marked $\mathrm{R} I$ shall open in the red ray, and I hope 
to be able to produce a chemical effect on that portion of my sensitized plate which has been exposed to the blue, whilst we shall get no corresponding effect on the portion exposed to the red ray. I next place my plate with its face downwards on the negative; we now start our electric lamp, using a small spectrum in order to have the action rather more distinct. I then expose half my plate in the red rays for about twenty seconds, and afterwards expose the other half, with the $V$ upon it, for about the same length of time, to the violet light. I will now develop and wash the photograph, and throw the image

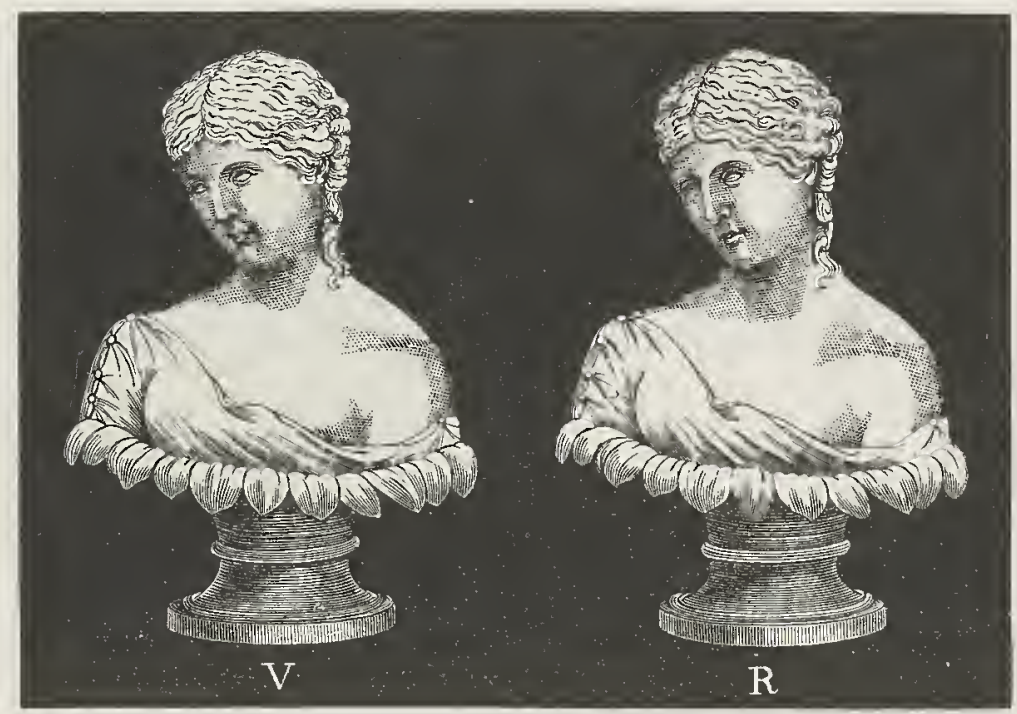

FIG. 16.

produced on the screen, when you will observe (Fig. 17) a very marked difference between the two halves, the one showing that no action has been produced on the sensitive plate exposed to the red rays, and the other giving us the perfect picture with the $V$ upon it.

Now let me bring before you evidence of our extended knowledge on this subject. I have here a collodion film plate prepared by Captain Abney's method, and with it I will perforra exactly the same series of operations which you have just witnessed with the ordinary film. Here I expose part of it in 
the red, and now I open the other part of the plate in the violet light from my electric lamp. Now I will develop the plate, and you see that the image is visible on the side marked $R$, although it is also seen that an action has also taken place on the side marked $V$. How can this remarkable difference be explained? A simple experiment will, I think, serve to do this. Here I have an ordinary dry collodion plate, such as I used in the first of the two experiments. I place this in front of our electric lamp, and you observe that we have an orange or red coloured image thrown on the screen. In other words, the

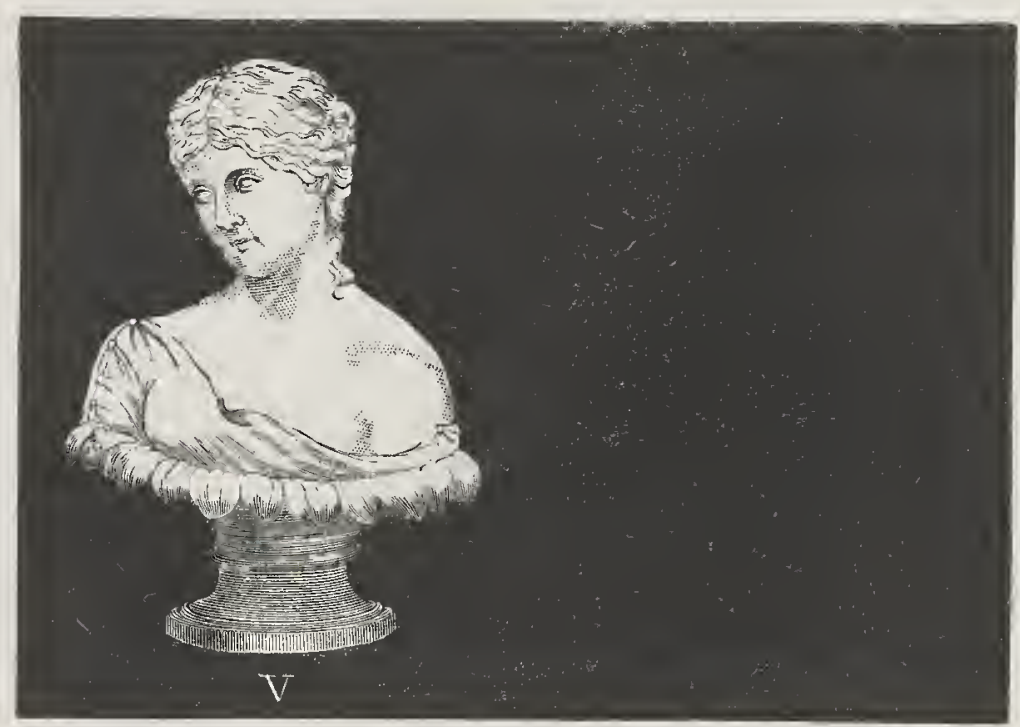

Fia. 17.

blue rays of the white electric light have been absorbed, whilst the red and orange rays pass through the film. Now I replace this ordinary plate by one prepared by Captain Abney's process. This you see throws a blue image on the screen; it absorbs the red and orange rays, although it has not lost all its power of absorbing the blue, and this is the plate, you will remember, which possesses the capability of photographing the red and infra-red portions of the spectrum. Here, then, we have an explanation of this diverse action; for in order that a chemical change shall be effected, the molecules of which the compound 
is built up must be broken up if a decomposition takes place, or the constituent atoms of the elements must be brought into contact if a combination occurs. This can only take place when the vibrations which constitute light are taken up by the molecules or atoms, and in this case the light ceases to exist as such, and is transformed into molecular motion. So if the molecules have the power of absorbing the blue rays, as you have seen is the case with the ordinary photographic plate, and with the yellow mixture of chlorine and hydrogen, these blue rays will have the power of bringing about either the decomposition of the silver salt or the combination of chlorine and hydrogen. If, on the other hand, another kind of molecule has the capacity of taking up the red rays, and therefore of allowing the blue rays to pass, as you saw in Abney's plate, then we find that it is the red rays which are capable of acting chemically upon this molecule. Thus you will understand that we can no longer speak of specifically actinic or chemically active rays, for every ray is eapable of acting on a certain chemical compound, and all may, therefore, be said to be chemically active. So far, indeed, has Abney increased the sensitiveness of lis film for the infra-red rays, that he has not only succeeded in obtaining photographs of the invisible solar rays, but has even taken a picture, in the dark, of course, of a kettle of boiling water.

Another very interesting example of the chemical changes effected by the more refrangible rays, recently studied by Professor Tyndall, ${ }^{1}$ is the decomposition which the vapours of certain organic bodies undergo when exposed to the concentrated solar rays, or to the concentrated beam of the electric light. To investigate these remarkable changes Tyndall passes a very small quantity of the colourless and invisible vapours of such a liquid as nitrite of amyl, together with air, into a: long glass tube closed at each end by glass plates; on allowing a beam of parallel rays to fall through the length of the tube, the space at first appears to be empty and dark, but after a few seconds singularly beautiful, coloured cloud-forms make their appearance, and as

1 "On the Action of Rays of high Refrangibility unon Gaseous Matter," by J. Tyndall, F.R.S., Phil. Trans., 1870, p. 333 . 
these are not seen when the tube contains only air, we thus learn that a chemical cliange has taken place in the vapour itself. Of the exact nature of the changes here produced we are as yet ignorant, but in many cases it is probable that an oxidizing action is going on. The delicacy of these reactions is remarkable, even by the side of the phenomena of spectrum analysis, for the quantity of matter capable of producing these actinic clouds is found to be extremely small.

Even the impression produced by the rays of light on the retina seems to be a kind of photographic action, for Professor Kühne has found that the purple film, termed the visual purple, which covers this delicate menbrane, loses its colour on exposure to light. He has, indeed, proved that it is possible to obtain upon this film a so-called optogram, or visible image fixed on the retina, of the object at which the eye may be directed. In the living eye the sensitive surface is quickly being renewed, and consequently the eye constantly recovers its power. ${ }^{1}$

The rays which the sun constantly pours down upon our earth, doubtless exert, by their chemical action, a most important influence on the fauna and flora of a country, and it becomes a matter of some importance to measure accurately their varying intensity with the changing seasons, and at different parts of the globe. It may not be uninteresting if I point out some of the results which have already been obtained by measurements of this kind, made by a method proposed by Professor Bunsen and myself, and depending on the darkening of a sensitive paper prepared with chloride of silver. The principles upon which the method is based are: (1) that a photographic paper can be prepared which always shall possess a constant degree of sensitiveness; and (2) that the darkening effect produced by the light on this paper is constant when the product of the intensity of the light into the time of exposure is also constant. Measurements according to this method have been carried out at Kew Observatory, and an interesting series of curves obtained, showing the variation of the chemical action

1 See "On the Photochemistry of the Retina and Visual Purple," Macmillan and Co., 1878. 
of the daylight with the seasons. The curves (Fig. 18) show the rise and fall of monthly chemical intensity with the hour of the day, from 6 A.M. to 6 P.M., for the year 1866. We see from these curves that the maximum amount of chemical action occurs at 12 o'clock, and that this action is equal at hours equidistant from noon; we learn also that if the chemical intensity in July 1866 be represented by the number 100, that in January is represented by 14 ; or in mid-summer the chemical intensity of the sun is more than seven times as great as in mid-winter. ${ }^{1}$

Still more interesting is it to ascertain the distribution of the chemically active rays on the earth's surface, about which we
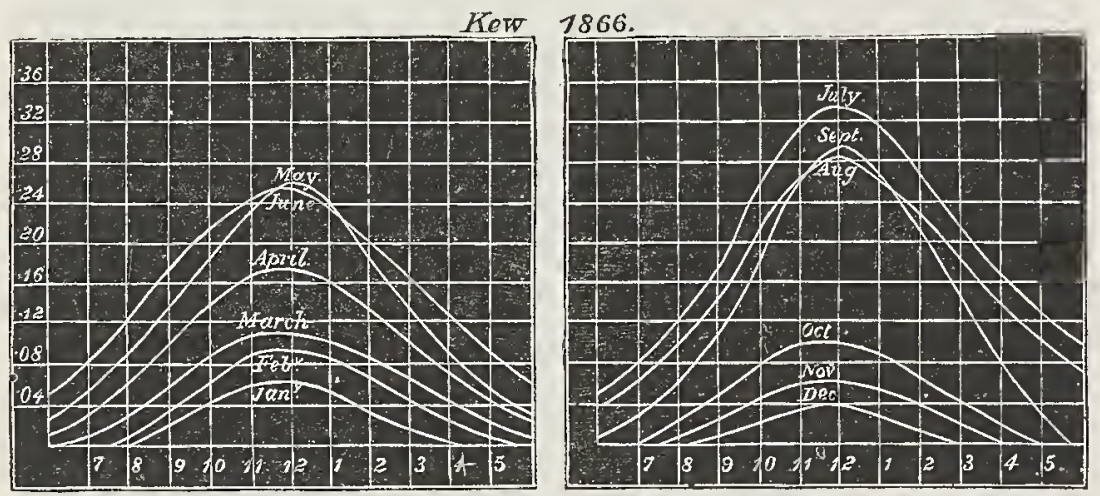

Fi'g. 18.

as yet know but little. According to the vague observations of photographers, it would appear that in advancing from England towards the equator, the difficulty of obtaining good pictures is increased, and more time is said to be needed to produce the same effect on the photographic plate under the full blaze of a tropical sun than in the gloomier atmosphere of London. It has likewise been stated that in Mexico, where the light is very intense, from twenty minutes to half an hour was required to produce photographic effects which in England occupy only a minute; and it is said that travellers engaged in copying the

1 (Roscoe) Bakerian Lecture, Phil. Trans., part ii. 1865, p. 605; and also (Roscoe) Phil. Trans. 1867, p. 555. 
antiquities of Yucatan have on several occasions been obliged to abandon the use of the photographic camera, and have had to take to their sketch-books! In order to test the validity of these statements, it becomes a matter of great importance to determine directly the intensity of the chemically active rays in the tropics; and, thanks to the zeal and ability of my friend Professor Thorpe, I am able to show you the results of such measurements, made at Pará, situated nearly under the equator, in the northern province of the Brazils, and lying on a branch

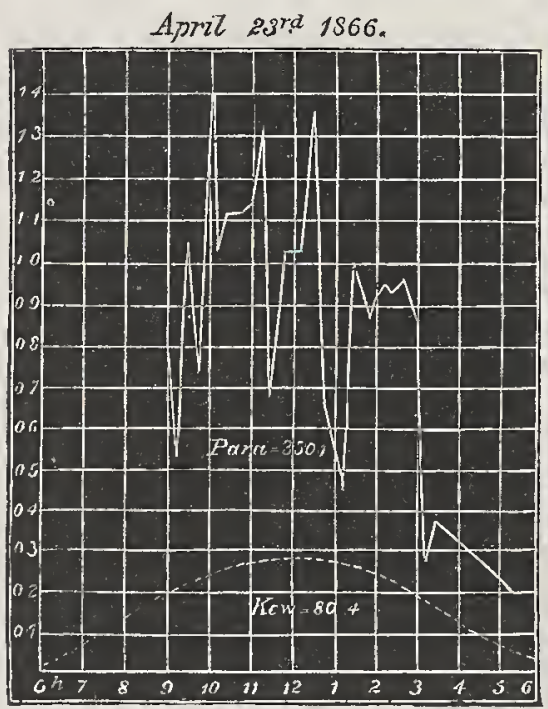

Fig, 19.

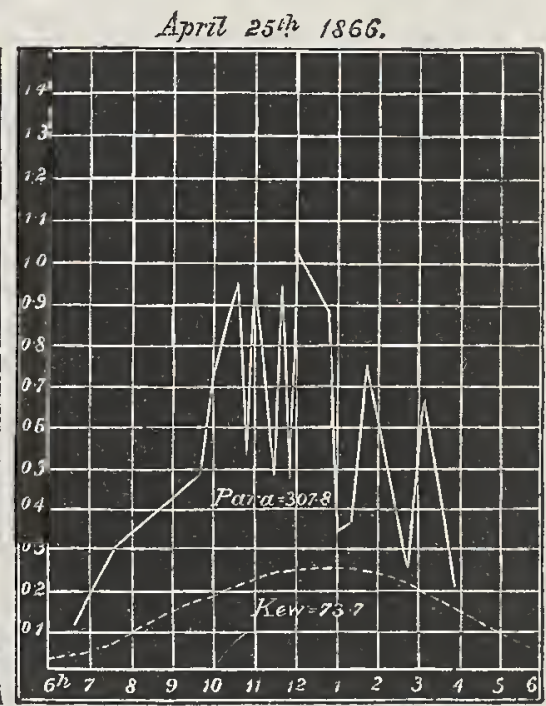

FIG, 20.

of the Amazons. Owing to the rainy season having commenced when the experiments were made, the changes in the chemical intensity as observed from hour to hour, and even from minute to minute, are very sudden and remarkable, and are well seen on the curves (Figs. 19 and 20); and these, compared with the dotted lines below indicating the corresponding action on the same day at Kew, show the enormous variation in chemical intensity which occurs under a tropical sun in the rainy season. Regularly every afternoon, and frequently at other hours of the day, enormous thunder-clouds obscure the sky, and, discharging 
their contents in the form of deluging rain, reduce the chemical action nearly to zero. The storm quickly passes over, and the chemical intensity rapidly rises to its normal value. By comparing the curves for Pará and Kew on the same days, we obtain some idea of the energy of chemical action at the tropics, and it is at once evident that the alleged failure of the photographer cannot at any rate be ascribed to a diminution in the sun's chemical intensity as thus measured, which in the month of April 1866 was nearly seven times as great at Pará as at Kew.

The absorptive effect of the earth's atmosphere on the direct rays of the sun is apparent to everybody looking at the brilliant red colours of sunrise and sunset, these colours being due to the absorption and scattering of the blue rays. Occasionally, however, the red rays are absorbed more than the refrangible ones, and in that case we have the remarkable phenomenon of a green sun.

The whole subject of the effects of our atmosphere on the solar radiation, both at the red and blue end of the spectrum, has recently been investigated by Professor Langley, who finds that to an observer placed above the atmosphere the sun would appear of a decidedly blue colour.

I have in conclusion to point out to you that the solar spectrum differs in certain respects from that beautiful spectrum of the electric arc with which we have been working; and it differs in this way, that the solar spectrum consists, not of a continuous band, passing without break or interruption from red to violet, through all the shades of colour which we know as the rainbow tints, but that in the solar spectrum we find, interspersed between these colours, certain dark lines which we may regard as shadows in the sunlight, spaces where certain rays are absent. The first person who observed these dark lines was Dr. Wollaston. ${ }^{1}$ Newton did not observe them, and for the good reason that he allowed the light to fall on a prism from a round hole in the shutter. In this way he did not obtain what is termed a pure spectrum, but a series of spectra, one overlapping the other, owing to the ligltt coming through different parts of the round

1 Phil. Trans. 1802, p. 378. 
hole. If he had allowed the light to pass through a fine vertical slit, and if this slit of light, if we may use such a term, had then fallen upon the prisms, placed so that the edge of the refracting angle is parallel to the slit, he would have observed that the solar spectrum was not continuous, but broken up by permanent dark lines. Dr. Wollaston, making use of a fine slit of light, discovered these fixed dark lines in the solar spectrum. Newton does, indeed, state that advantages accrue if a "hole shaped like a long parallelogram an inch or two long, but a tenth or twentieth of an inch broad or narrower," be used instead of a round hole, but this width of slit is far too great to allow of the fixed lines being seen.

I invite your attention to the drawings of these lines in this very imperfect sketch of the solar spectrum (see coloured diagram on Frontispiece). These dark lines are always found in the same position in the sunlight, whether you take direct, diffused, or reflected sunlight. The exact mapping and observation of these lines in the solar spectruin is a matter of as great importance to astronomy and to physical science generally as the mapping of the stars in the heavens, because by knowing exactly the position of these dark lines in the solar spectrum we can ascertain that iron, sodium, and other well-known substances exist in the solar atmosphere.

I will now show you a diagram illustrative of this fact, and remind you that we are indebted for the first careful examination of these lines to a German optician, Fraunhofer, whose name has been attached to these lines. Fraunhofer mapped no less than 576 of these lines in the year 1814. ${ }^{1}$ This is an exact reduced copy of his map (Fig. 21). On the left is the red and on the right is the violet portion of the visible spectrum. You observe how this spectrum is shaded. Notice, if you please, the immense number of lines which intersect and almost appear to make the solar spectrum dark. Fraunhofer employed the letters of the alphabet to designate some of the principal lines, beginning with $\mathrm{A}$ in the red and passing on to $\mathrm{H}$ in the violet. Many of these lines are as fine as the finest spider's web, so that they

1 Denkschriften der Münchener Akademie, 1814. 


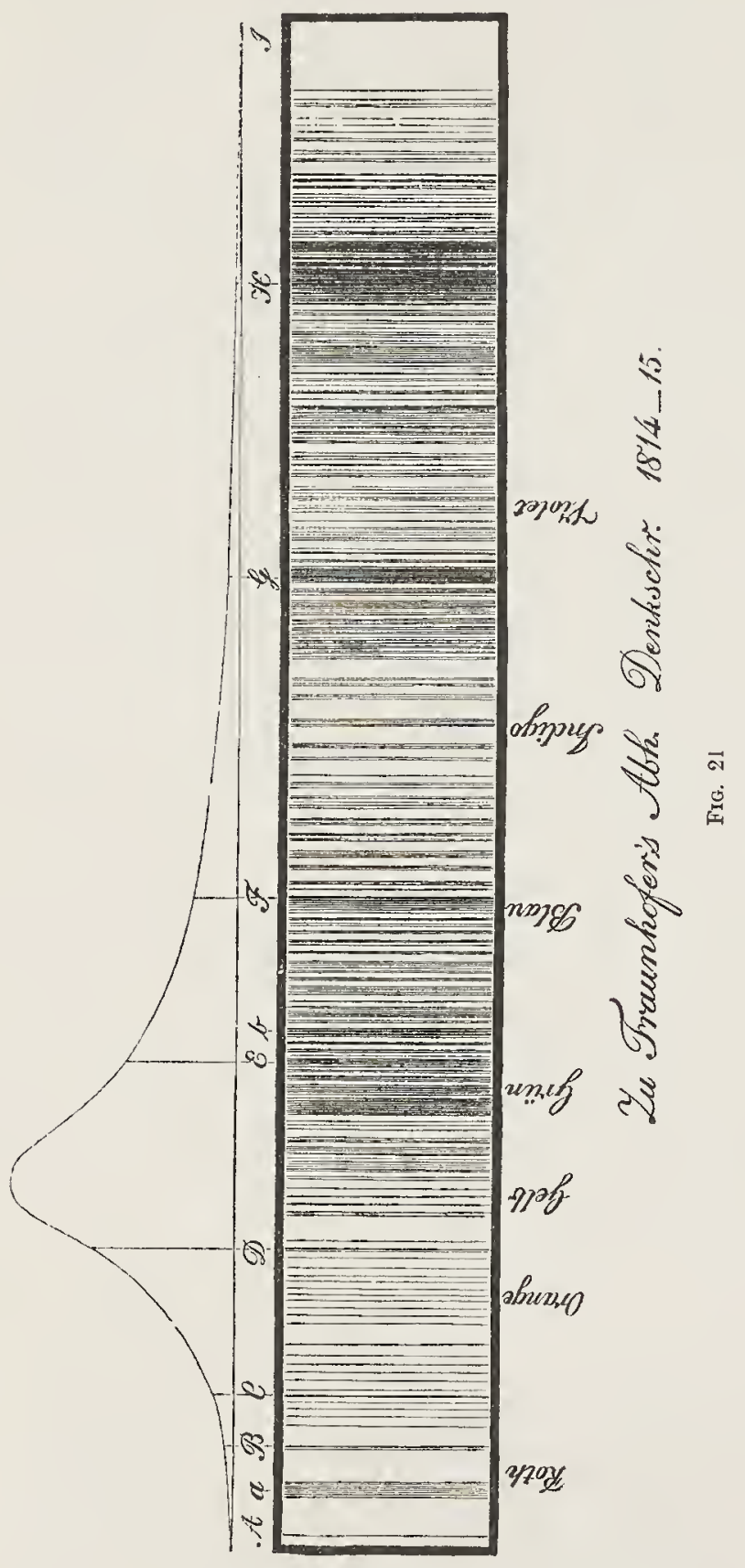


occupy but a small portion of the whole area of the spectrumthat is, the portion which is filled with light is far greater than the portion filled with these shadows, although the number of these shadows is so exceedingly large. The curve in the upper part of the figure gives Fraunhofer's estimate of the intensity of the visible rays at different parts of the spectrum, showing that when the light is spread out by means of a prism, the maximum visual intensity occurs in the yellow.

Fraunhofer first ascertained that these lines are present in every kind of sunlight, and that moonlight, as well as the light of the planet Venus, exhibits the same dark lines. He likewise measured the refractive indices and the wave-lengths of these lines,- - that is, determined their relative positions in the solar light; and he found that the relative distances between any given lines remained constant, whether he took direct sunlight, or sunlight reflected from the moon or planets.

Since Fraunhofer's time more accurate determinations of the wave-lengths of the various dark solar lines have been made. Of these the most exact are those observed by Professor Angström,1 of Upsala; and the numbers obtained by him for the wave-lengths of the most important solar lines are usually taken as the standard measurements in this branch of science. In one column of the following table you will find these standard wave-lengths in Angström's numbers expressed in tenth-metres (or in ten-millionths of a millimetre); in the other column are seen the numbers obtained by Fraunhofer in 1814 for the wave-lengths of the same dark lines.

Name of Line.

A.

$B$.

C.

D.

E.
Ảngström's Wave-lengths. Fraunhofer's Wave-Iengths. $7604^{\circ} 00$

$6867 \cdot 00$

$6562 \cdot 10$

$5892 \cdot 12$

$5269 \cdot 13$

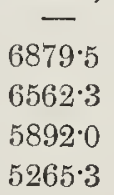

1 Ångström, "Recherches sur le Spertre Solaire, Spectre Normal du Soleil," p. 25. Upsala, 1868. 
Name of Line.

F.

G.

$\mathrm{H}_{1}$.

$\mathrm{H}_{2}$.

Ångström's Wave-lengths. Fraunhofer's Wave-lengths. (In ten-millionths of a millimetre.) $4860 \cdot 72$ $4849 \cdot 5$

$4307 \cdot 25$

$4293 \cdot 3$

$3968 \cdot 19$

$3945 \cdot 0$

$3933 \cdot 00$

Another important observation was made by Fraunhofer,namely, that the light from the fixed stars, which, as you know, are self-luminous, also contains dark lines, but different lines from those which characterize the sunlight, the light of the planets, and that of the moon; and hence in 1814 Fraunhofer came to this remarkable conclusion: that whatever produced these dark lines-and he had no idea of the cause-was something which was acting beyond and outside our atmosphere, and not anything produced by the sunlight passing through the air.

This conclusion of Fraunhofer has been borne out by subsequent investigation, and the observations upon which it was based may truly be said to have laid the foundation-stone of solar and stellar chemistry. 
LECTURE I.-APPENDIX A.

LXTRACTS FROM “NEWTON'S OPTICKS," 1675.

BOOK I. PART I.

Prop. I. THEOR.-Lights which differ in colour, differ also in degrees of refrangibility.

\section{THE PROOF BY EXPERIMENTS.}

Exper. 1.-I took a black oblong stiff paper terminated by parallel sides, and, with a perpendicular right line drawn across from one side to the other, distinguished it into two equal parts. One of these parts I painted with a red colour and the other with a blue. The paper was very black, and the colours intense and thickly laid on, that the phenomenon might be more conspicuous. This paper I viewed through a prism of solid glass, whose two sides through which the light passed to the eye were plane and well polished, and contained an angle of about $60^{\circ}$ : which angle I call the refracting angle of the prism. And whilst I viewed it, I held it and the prism before a window in such manner that the sides of the paper were parallel to the prisin, and both those sides and the prism were parallel to the horizon, and the cross line was also parallel to it; and that the light which fell from the window upon the paper made an angle with the paper, equal to that angle which was made with the same paper by the light reflected from it to the eye. Beyond the prism was the wall of the chamber under the window covered over with black clotl, and the cloth was involved in darkness that no light might be 
reflected from thence, which in passing by the edges of the paper to the eye might mingle itself with the light of the paper, and obscure the phenomenon thereof. These things being thus ordered, I found that if the refracting angle of the prism be turned upwards, so that the paper may seem to be lifted upwards by the refraction, its blue half will be lifted higher by the refraction than its red half. But if the refracting angle of the prism be turned downward, so that the paper may seem to be carried lower by the refraction, its blue half will be carried something lower thereby than its red half. Wherefore in both cases the light which comes from the blue half of the paper through the prism to the eye, does in like circumstances suffer a greater refraction than the light which comes from the red half, and by consequence is more refrangible.

Exper. 2.-About the aforesaid paper, whose two halves were painted over with red and blue, and which was stiff like thin pasteboard, I lapped several times a slender thread of very black silk, in such manner that the several parts of the thread might appear upon the colours like so many black lines drawn over them, or like long and slender dark shadows cast upon them. I might have drawn black lines with a pen, but the threads were smaller and better defined. This paper thus coloured and lined I set against a wall perpendicularly to the horizon, so that one of the colours might stand to the right hand, and the other to the left. Close before the paper at the confine of the colours below, I placed a candle to illuminate the paper strongly: for the experiment was tried in the night. The flame of the candle reached up to the lower edge of the paper, or a very little higher. Then at the distance of six feet and one or two inches from the paper upon the floor I erected a glass lens four inches and a quarter broad, which might collect the rays coming from the several points of the paper, and make them converge towards so many other points at the same distance of six feet and one or two inches on the other side of the lens, and so form the image of the coloured paper upon a white paper placed there, after the same manner that a lens at a hole in a window casts the images of objects abroad upon a sheet of white paper in a dark room. 
The aforesaid white paper, erected perpendicular to the horizon and to the rays which fell upon it from the lens, I moved sometimes towards the lens, sometimes from it, to find the places where the images of the blue and red parts of the coloured paper appeared most distinct. Those places I easily knew by the images of the black lines which I had made by winding the silk about the paper. For the images of those fine and slender lines (which by reason of their blackness were like shadows on the colours) were confused and scarce visible, unless when the colours on either side of each line were terminated most distinctly. Noting therefore, as diligently as I could, the places where the images of the red and blue halves of the coloured paper appeared most distinct, I found that where the red half of the paper appeared distinct, the blue half appeared confused, so that the black lines drawn upon it could scarce be seen; and on the contrary, where the blue half appeared most distinct, the red half appeared confused, so that the black lines upon it were scarce visible. And between the two places where these images appeared distinct there was the distance of an inch and a half: the distance of the white paper from the lens, when the image of the red half of the coloured paper appeared most distinct, being greater by an inch and a half than the distance of the same white paper from the lens, when the image of the blue half appeared most distinct. In like incidences therefore of the blue and red upon the lens, the blue was refracted more by the lens than the red, so as to converge sooner by an inch and a half, and therefore is more refrangible. . . . .

Scholium.-The same things succeed notwithstanding that some of the circumstances be varied; as in the first experiment when the prism and paper are anyways inclined to the horizon, and in both when coloured lines are drawn upon very black paper. But in the description of these experiments, I have set down such circumstances by which either the phenomenon might be rendered more conspicuous, or a novice might more easily try them, or by which I did try them only. The same thing I have often done in the following experiments; concerning all which this one admonition may suffice. Now from these experiments 
it follows not that all the light of the blue is more refrangible than all the light of the red; for both lights are mixed of rays differently refrangible, so that in the red there are some rays not less refrangible than those of the blue, and in the blue there are some rays not more refrangible than those of the red; but these rays in proportion to the whole light are but few, and serve to diminish the event of the experiment, but are not able to destroy it. For if the red and blue colours were more dilute and weak, the distance of the images would be less than an inch and a half; and if they were more intense and full, that distance would be greater, as will appear hereafter. These experiments may suffice for the colours of natural bodies. For in the colours made by the refraction of prisms this proposition will appear by the experiments which are now to follow in the next proposition.

Prop. II. Theor. 2.-The light of the sun consists of rays differently refrangible.

\section{THE PROOF BY EXPERIMENTS.}

Exper. 3.- In a very dark chamber at a round hole about one third part of an inch broad made in the shut of a window I placed a glass prism, whereby the beam of the sun's light which came in at that hole might be refracted upwards towards the opposite wall of the chamber, and there form a coloured image of the sun. The axis of the prism (that is, the line passing through the middle of the prism from one end of it to the other end parallel to the edge of the refracting angle) was in this and the following experiments perpendicular to the incident rays. About this axis I turned the prism slowly, and saw the refracted. light on the wall or coloured image of the sun first to descend, and then to ascend. Between the descent and ascent when the image seemed stationary, I stopped the prism and fixed it in that posture, that it should be moved no more. For in that posture the refractions of the light at the two sides of the 
refracting angle, that is, at the entrance of the rays into the prisn and at their going out of it, were equal to one another. So also in other experiments, as often as I would have the refractions on both sides the prism to be equal to one another, I noted the place where the image of the sun formed by the refracted light stood still between its two contrary motions, in the common period of its progress and regress; and when the image fell upon that place, I made fast the prism. And in this posture, as the most convenient, it is to be understood that all the prisms are placed in the following experiments, unless where some other posture is described. The prism therefore being placed in this posture I let the refracted light fall perpendicularly upon a sheet of white paper at the opposite wall of the chamber, and observed the figure and dimensions of the solar image formed on the paper by that light. This image was oblong and not oval, but terminated with two rectilinear and parallel sides, and two semicircular ends. On its sides it was bounded pretty distinctly, but on its ends very confusedly and indistinctly, the light there decaying and vanishing by degrees. The breadth of this image answered to the sun's diameter, and was about two inches and the eighth part of an inch, including the penumbra. For the image was eighteen feet and a half distant from the prism; and at this distance that breadth, if diminished by the diameter of the hole in the window-shut, that is by a quarter of an inch, subtended an angle at the prism of about half a degree, which is the sun's apparent diameter. But the length of the image was about ten inches and a quarter, and the length of the rectilinear sides about eight inches, and the refracting angle of the prism whereby so great a length was made was $64^{\circ}$. With a less angle the length of the image was less, the breadth remaining the same. If the prism was turned about its axis that way which made the rays emerge more obliquely out of the second refracting surface of the prism, the image soon became an inch or two longer, or more; and if the prism was turned about the contrary way, so as to make the rays fall more obliquely on the first refracting surface, the image soon became an inch or two shorter. And therefore, in trying this experiment, I was as curious as I could 
be, in placing the prism by the above-mentioned rule exactly in such a posture that the refractions of the rays at their emergence out of the prism might be equal to that at their incidence on it. This prism had some veins running along within the glass from one end to the other, which scattered some of the sun's light irregularly, but had no sensible effect in increasing the length of the coloured spectrum. For I tried the same experiment with other prisms with the same success; and particularly with a prism which seemed free from such veins, and whose refracting angle was $62 \frac{1}{2}^{\circ}$. I found the length of the image $9 \frac{3}{4}$ or 10 inches at the distance of $18 \frac{1}{2}$ feet fiom the prism, the breadth of the hole in the window-shut being $\frac{1}{4}$ of an inch, as before. And because it is easy to commit a mistake in placing the prism in

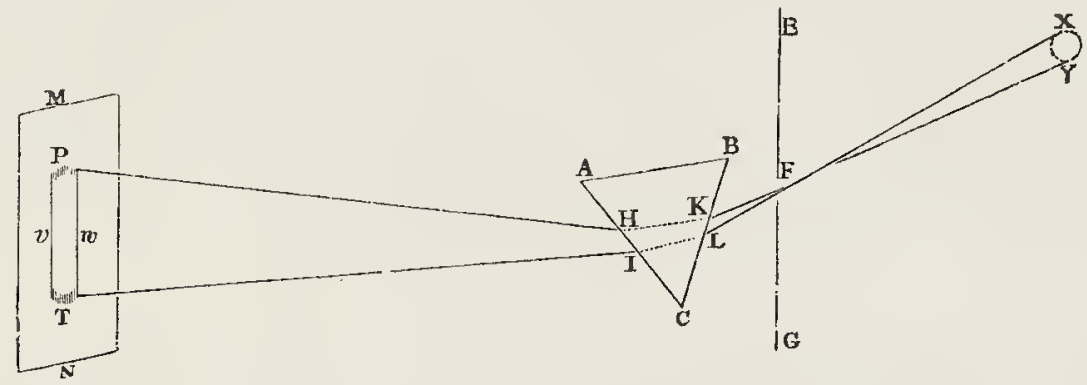

FIG. 22.

its due posture, I repeated the experiment four or five times, and always found the length of the image that which is set down above. With another prism of clearer glass and better polish, which seemed free from veins, and whose refracting angle was $63 \frac{1}{2}^{\circ}$, the length of this image at the same distance of $18 \frac{1}{2}$ feet was also about 10 inches, or $10 \frac{1}{2}$. Beyond these measures for about $\frac{1}{1}$ or $\frac{1}{3}$ of an inch at either end of the spectrum the light of the clouds seemed to be a little tinged with red and violet, but so very faintly, that I suspected that tincture. might either wholly or in great measure arise from some rays of the spectrum scattered irregularly by some inequalities in the substance and polish of the glass, and therefore I did not include it in these measures. Now the different magnitude of the hole 
in the window-shut, and different thickness of the prism where the rays passed through it, and different inclinations of the prism, to the horizon, made no sensible changes in the length of the image. Neither did the different matter of the prisms make any: for in a vessel made of polished plates of glass cemented together in the shape of a prism and filled with water, there is the like success of the experiment according to the quantity of the refraction. [After giving a rigorous proof that the rays in different parts of the spectrum are differently refracted, Newton proceeds.]

This image or spectrum P T was coloured, being red at its least refracted end $\mathrm{T}$, and violet at its most refracted end $\mathrm{P}$, and yellow, green, and blue in the intermediate spaces, which agrees with the first proposition, that lights which differ in colour do also differ in refrangibility. The length of the image in the foregoing experiments I measured from the faintest and outmost red at one end, to the faintest and outmost blue at the other end, excepting only a little penumbra, whose breadth scarce exceeded a quarter of an inch, as was said above.

Prop. III. THEOR. 3.-The sun's light consists of rays differing in reflexibility, and those rays are morc reflexible than others which are more refrangible.

This is manifest by the ninth and tenth experiments, for in the ninth experiment, by turning the prism about its axis until the rays within it, which in going out into the air were refracted by its base, became so oblique to that base as to begin to be totally reflected thereby; those rays became first of all totally reflected which before at equal incidences with the rest had suffered the greatest refraction. And the same thing happens in the reflexion made by the common base of the two prisms in the tenth experiment. 


\section{BOOK I. PART 2.}

Prop, V. Theor. 4.-Whiteness and all grey colours betwecn white and black may be compounded of colours, and the whiteness of the sun's light is componuded of all the primary colours mixed in a duc proportion.

Exper. 11.-Let the sun's coloured image P T (Fig. 23) fall upon the wall of a dark chamber, as in the third experiment of the first book, and let the same be viewed through a prism $a b c$, held parallel to the prism $\mathrm{A} B \mathrm{C}$, by whose refraction that image was made, and let it now appear lower than before,-suppose in the place $\mathrm{S}$ over against the red colour $\mathrm{T}$. And if you go near to the image $\mathrm{P} \mathrm{T}$, the spectrum $\mathrm{S}$ will appear oblong and coloured

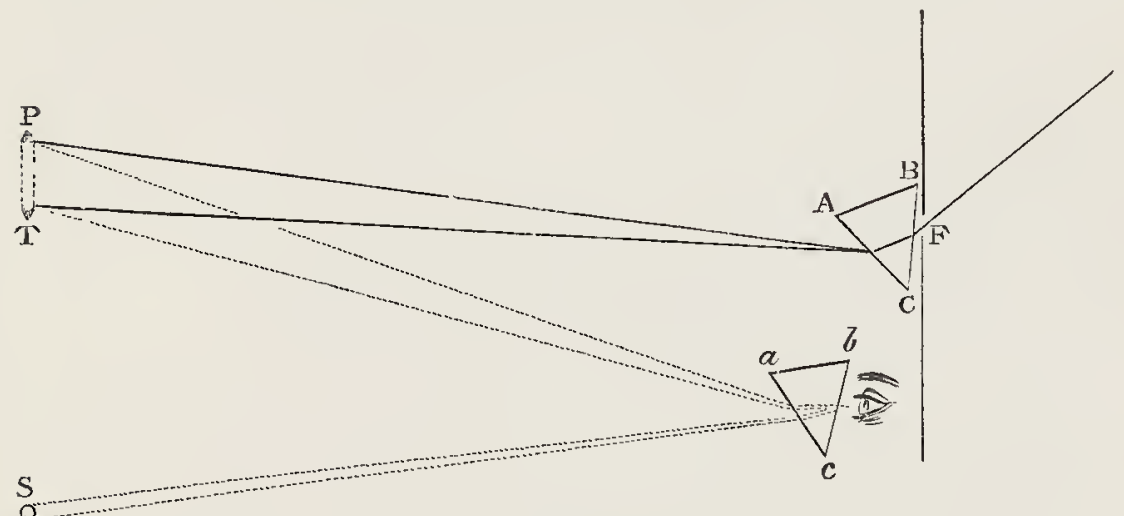

FIG. 23.

like the image $\mathrm{P} \mathrm{T}$; but if you recede from it, the colours of the spectrum $s$ will be contracted more and more, and at length vanish, that spectrum $s$ becoming perfectly round and white: and if you recede yet farther, the colours will emerge again, but in a contrary order. Now that spectrum s appears white in that case when the rays of several sorts which converge from the several parts of the image $\mathrm{P} T$, to the prism $a b c$, are so refracted unequally by it, that in their passage from the prism to the eye they may diverge from one and the same point of the spectrum $\mathrm{s}$, and so fall afterwards upon one and the same point in the bottom of the eye, and there be mingled. 
And farther, if the comb be here made use of, by whose teeth the colours of the image P T may be successively intercepted, the spectrum $\mathrm{S}$ when the comb is moved slowly will be perpetually tinged with successive colours; but when, by accelerating the motion of the comb, the succession of the colours is so quick that they cannot be severally seen, that spectrum s, by a confused and nixed sensation of them all, will appear white.

Prop. II. THeor. 2.-All homogeneal light has its proper colour, answering to its degree of refrangibitity, and that colour cannot be changed by reflexions or refractions.

In the experiments of the fourth proposition of the first book, when I had separated the heterogeneous rays from one another, the spectrum $p t$ formed by the separated rays did, in the progress from its end $p$, on which the most refrangible rays fell, unto its other end $t$, on which the least refrangible rays fell, appear tinged with this series of colours, violet, indigo, blue, green, yellow, orange, red, together with all their intermediate degrees in a continual succession, perpetually varying. So that there appeared as many degrees of colours as there were sorts of rays differing in refrangibility.

Exper. 5. - Now, that these colours could not be changed by refraction, I knew by refracting with a prism sometimes one very little part of this light, sometimes another very little part, as is described in the twelfth experiment of the first book (see Fig. 24). For by this refraction the colour of the light was never changed in the least. If any part of the red light was refracted, it remained totally of the same red colour as before. No orange, no yellow, no green or blue, no other new colour was produced by that refraction. Neither did the colour anyway change by repeated refractions, but continued always the same red entirely as at first. The like constancy and immutability I found also in the blue, green, and other colours. So also if I looked through a prism upon any body illuminated with any part of this homogeneal light, as in the fourteenth experiment of the first book is described, I could not perceive any new colour generated this way. All bodies illuminated with compound light appear through prisms con- 
fused (as was said above), and tinged with various new colours, but those illuminated with homogeneal light appeared through prisms neither less distinct, nor otherwise coloured, than when viewed with the naked eye. Their colours were not in the least changed by the refraction of the interposed prism. I speak here of a sensible change of colour : for the light which I here call homogeneal, being not absolutely homogeneal, there ought to arise some little change of colour from its heterogeneity. But if that heterogeneity was so little as it might be made by the said experiments of the fourth proposition, that change was not sensible, and therefore in experiments, where sense is judge, ought to be accounted none at all.

Exper. 6.-And as these colours were not changeable by refractions, so neither were they by reflexions. For all white,

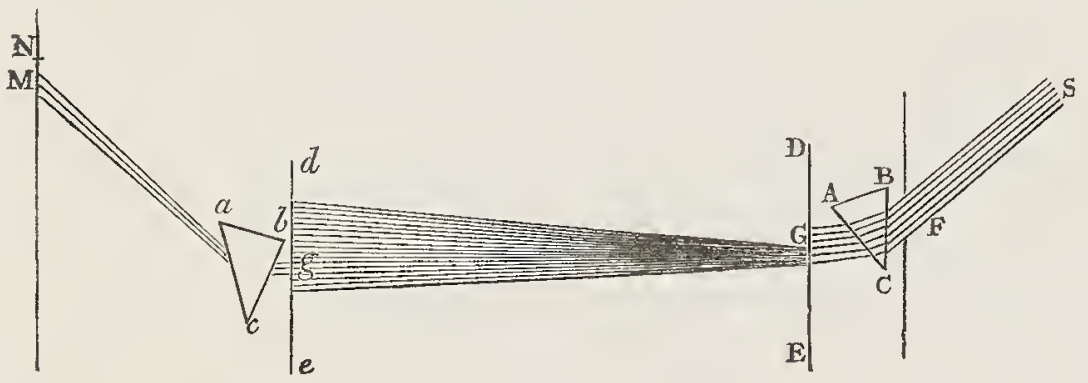

FIG. 24.

grey, red, yellow, green, blue, violet bodies,-as paper, ashes, red-lead, orpiment, indigo, bise, gold, silver, copper, grass, blue flowers, violets, bubbles of water tinged with various colours, peacocks' feathers, the tincture of Lignum nephriticum, and such like,-in red homogeneal light appeared totally red, in blue light totally blue, in green light totally green, and so of other colours. In the homogeneal light of any colour they all appeared totally of that same colour, with this only difference, that some of them reflected that light more strongly, others more faintly. I never yet found any body which by reflecting homogeneal light could sensibly change its colour.

- From all which it is manifest, that if the sun's light consisted of but one sort of rays, there would be but one colour in the 
whole world, nor would it be possible to produce any new colour, by reflexions and refractions, and by consequence that the variety of colours depends upon the composition of light.

\section{DEFINITION.}

The homogeneal light and rays which appear red, or rather make objects appear so, I call rubrific or red-making; those which make objects appear yellow, green, blue, and violet, I call yellowmaking, green-making, blue-making, violet-making, and so of the rest. And if at any time I speak of light and rays as coloured or endued with colours, I would be understood to speak not philosophically and properly, but grossly, and according to such conceptions as vulgar people in seeing all these experiments would be apt to frame, For the rays, to speak properly, are not coloured. In them there is nothing else than a certain power and disposition to stir up a sensation of this or that colour. For as sound in a bell or musical string, or other sounding body, is nothing but a trembling motion, and in the air nothing but that motion propagated from the object, and in the sensorium 'tis a sense of that motion under the form of a sound; so colours in the object are nothing but a disposition to reflect this or that sort of rays more copiously than the rest, in the rays they are nothing but their dispositions to propagate this or that motion into the sensorium, and in the sensorium they are sensations of those motions under the forms of colours.

\section{APPENDIX B.}

\section{BURNING MAGNESIUM WIRE, A SOURCE OF LIGHT FOR PHOTOGRAPHIC PURPOSES. ${ }^{1}$}

Another interesting practical application of our knowledge concerning the properties of the kind of light which certain bodies emit when heated, is the employment of the light evolved by burning magnesium wire for photographic purposes. The

1 Professor Roscoe on Spectrum Analysis, Royal Institution of Great Britain Proceedings, May 6, 1864. 
spectrum of this light is exceedingly rich in violet and ultraviolet rays, due partly to the incandescent vapour of magnesium and partly to the intensely-heated magnesia formed by the combustion. Professor Bunsen and the speaker in 1859 determined the chemically active power possessed by this light, and compared it with that of the sun; and they suggested the application of this light for the purpose of photograplry. They showed ${ }^{1}$ that a burning surface of magnesium wire, which, seen from a point at the sea's level, has an apparent magnitude equal to that of the sun, effects on that point the same chemical action as the sun would do if shining from a cloudless sky at a height of $9^{\circ} 53^{\prime}$ above the horizon. On comparing the visible brightness of these two sources of light it was found that the brightness of the sun's disc, as measured by the eye, is 524.7 times as great as that of burning magnesium wire, when the sun's zenith distance is $67^{\circ} 22^{\prime}$; whilst at the same zenith distance the sun's chemical brightness is only 36.6 times as great. Hence the value of this light as a source of the chemically active rays for photographic purposes becomes at once apparent.

Professor Bunsen and the speaker state, in the memoir above referred to, that "the steady and equable light evolved by magnesium wire burning in the air, and the immense chemical action thus produced, render this source of light valuable as a simple means of obtaining a given amount of chemical illumination; and that the combustion of this metal constitutes so definite and simple a source of light for the purpose of photochenical measurement, that the wide distribution of magnesium becomes desirable. The application of this metal as a source of light may even become of technical importance. A burning magnesium wire of the thickness of 0.297 millimetre evolves, according to the measurement we have made, as much light as 74 stearine candles of which five go to the pound. If this light lasted one minute, 0.987 metre of wire, weighing 0.120 gramme, would be burnt. In order to produce a light equal to 74 candles burning for ten hours, whereby about 20 lbs. of stearine are consumed, $72 \cdot 2$ grammes ( $2 \frac{1}{2}$ ounces) of magnesium would be required. The 1 Phil. Trans. 1859, p. 920. 
magnesium wire can be easily prepared by forcing out the metal from a heated steel press having a fine opening at bottom: this wire might be rolled up in coils on a spindle, which could be made to revolve by clockwork, and thus the end of the wire, guided by passing through a groove or between rollers, could be continually pushed forward into a gas or spirit-lamp flame in which it would burn."

It afforded the speaker great pleasure to state that the foregoing suggestion had now been actually carried out. Mr. Edward Sonstadt has succeeded in preparing magnesium on the large scale, and great credit is due to this gentleman for the able manner in which he has brought the difficult subject of the metallurcy of magnesium to its present very satisfactory position.

Some fine specimens of crude and distilled magnesium weighing 3 lbs. were exlibited as manufactured by Mr. Sonstadt's process, by Messrs. Mellor and Co. of Manchester.

The wire is now to be had at the comparatively low rate of $3 d$. per foot; and half an inch of the wire evolves on burning liglit enough to transfer a positive image to a dry collodion plate; whilst by the combustion of 10 grains a perfect photographic portrait may be taken; so that the speaker believed that for photographic purposes alone the magnesium light will prove most important. The photochemical power of the light was illustrated by taking a portrait 1 during the discourse. In doing this the speaker was aided by Mr. Brothers, photographer, of Manchester, who was the first to use the light for portraiture.

\section{APPENDIX C.}

ON THE CHEMICAL ACTION OF THE CONSTITUENT PARTS OF SOLAR LIGHT. ${ }^{2}$

The chemical action effected by the several portions of the solar spectrum depends not only upon the nature of the refracting body, but also upon the thickness of the column of air through which the light has to pass before decomposition. In

1 Of Professor Faraday.

2 Bunsen and Roscoe, Phil. Trans. 1859. 
the following experiments we have employed prisms and lenses of quartz, cut by Mr. Darker of Lambeth, instead of glass prisms, which, as is well known, absorb a large portion of the chemically active rays. In order to render our experiments as free as possible from the irregularities arising from variation in the atmospheric absorption, the observations were made quickly one after the other, so that the zenith distance of the sun altered but very slightly.

A perfectly cloudless day was chosen for these observations, and the direct sunlight reflected from the speculum mirror of a Silbermann's heliostat through a narrow slit into our dark room. The spectrum produced by the rays passing through two quartz prisms and a quartz lens fell upon a white screen, which was covered with a solution of sulphate of quinine to render the ultra-violet rays and the accompanying dark lines visible. In this screen a narrow slit was made, through which the rays from any wished-for portion of the spectrum could be allowed to pass, so as to fall directly upon the insolation vessel,1 situated at the distance of from four to five feet. A finely divided millimetre scale was also placed on the screen, by means of which the distance between the Fraunhofer lines could be accurately measured, and the portion of light employed thus exactly determined.

In order to recognize with accuracy the various portions of the spectrum, we employed a map of the dark lines prepared by Mr. Stokes, which he most kindly placed at our disposal. The figure (Fig. 15) contains a copy of Mr. Stokes's map, with the distance measured by him, and letters given according to his notation. We have divided the space between the letter $\mathrm{A}$ in the red to the last ray Stokes observed, $w$ in the lavender rays, into 160 equal parts, and we represent the position and breadth of the bundle of rays which effected a given action upon the insolation vessel as follows:-If a bundle of rays lying between,

1 This vessel was filled with the sensitive mixture of ehlorine and hydrogen gases together with water. The chemically aetive rays effected a union of the gases, and the resulting hydroehloric acid gas being absorbed by the water, gave a diminution of volume, direetly proportional to the intensity of the acting chemical rays. 
the abscissæ 20.5 and 34 in Fig. 15, page 28, had to be represented, we should call the edge of the bundle towards $A, \frac{1}{2} \mathrm{DE}$, and that towards $\mathrm{W}, \frac{1}{4} \mathrm{FG}$, whilst the middle of the portion of the spectrum, which produces the action, we call " $\frac{\mathrm{I}}{2} \mathrm{D} \mathrm{E}$ to $\frac{1}{4} \mathrm{FG} . " \quad$ The breadth of this bundle of rays in which the insolation vessel was completely bathed was $\frac{8}{100}$ of the total length of the spectrum.

The following table gives the direct results of a series of obscrvations made by perfectly cloudless sky at Heidelberg on the 14th of August, 1857, under a barometric pressure of $0.7494 \mathrm{~m}$. The first colunin gives the numbers of the observations in the order in which they were made; Column II. the times of observation in true solar time; Column III. the portion of spectrum under examination; and Column IV. the action corresponding to this portion.

\begin{tabular}{|c|c|c|c|c|}
\hline r. & II. & III. & & IV. \\
\hline & H. M. & & & \\
\hline 1 & 1054 A. M. & From $\frac{3}{5} \mathrm{CH}$ to I , & . & $48 \cdot 80$ \\
\hline 2 & $1058 \mathrm{~A} \cdot \mathrm{MI}$. & From $\frac{1}{5}, \mathrm{DE}$ to $\mathrm{E}$ & . & $1 \cdot 27$ \\
\hline 3 & 114 A.M. & From $\mathrm{C}$ to $\frac{1}{2} \mathrm{DE}$ & . & $0 \cdot 47$ \\
\hline 4 & 118 А.м. & From $N_{1}$ to $\frac{3}{4} Q R$ & & $18 \cdot 28$ \\
\hline 5 & 1113 A M. & From $\frac{1}{2} \mathrm{RS}$ to $\frac{2}{3} \mathrm{ST}$ & & $2 \cdot 03$ \\
\hline 6 & 1141 A.M. & From $\frac{3}{4} \mathrm{ST}$ to $\frac{2}{3} \mathrm{UV}$ & & $1 \cdot 27$ \\
\hline 7 & 1147 A 3. & From $\frac{1}{2} \mathrm{~N}_{4} \mathrm{Q}$ to $\frac{1}{3} \mathrm{RS}$ & & $11 \cdot 73$ \\
\hline 8 & $1150 \mathrm{~A} \mathrm{MI}$. & From $\frac{3}{4} \mathrm{ST}^{4}$ to $\frac{2}{3} \mathrm{UV}$ & & $1 \cdot 02$ \\
\hline 9 & 1154 A.M. & From $\frac{4}{5} \mathrm{JM}_{3}$ to $\mathrm{N}_{4}$ & & $37 \cdot 87$ \\
\hline 10 & 1157 А.М. & From $\mathrm{H}_{1}$ to $\frac{3}{4} \mathrm{IM}_{1}$. & & $57 \cdot 42$ \\
\hline 11 & 0 I'P.M. & From $H_{1}$ to $\frac{3}{4} I M_{1}^{2}$. & & $52 \cdot 30$ \\
\hline 12 & $0 \quad 4$ P.M. & From $\frac{1}{5}$ GH to $\mathrm{H}^{2}$. & & 6138 \\
\hline 13 & 07 P.M. & From $\frac{1}{5} \mathrm{FG}$ to $\mathrm{G}$ & . & $27 \cdot 64$ \\
\hline 14 & 016 P.M. & From $\frac{1}{5} \mathrm{FG}$ to $\mathrm{G}$ & " & 2874 \\
\hline 15 & 020 P.M. & From $\frac{3}{4} \mathrm{DE}$ to $\mathrm{F}$ & 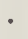 & $1 \cdot 39$ \\
\hline 16 & 025 к... & From $\frac{1}{2} \mathrm{~N}_{4} \mathrm{Q}$ to $\frac{1}{3} \mathrm{RS}$ & & $13 \cdot 19$ \\
\hline 17 & 032 г.M. & Erom $\frac{1}{2} \mathrm{~N}_{4} \mathrm{Q}$ to $\frac{1}{3} \mathrm{RS}$ & & $12 \cdot 41$ \\
\hline 18 & 040 Р $\mathrm{M}$. & From G to $\frac{4}{5} \mathrm{GH}$ & & $53 \cdot 78$ \\
\hline 19 & 042 г.м. & From $\frac{1}{5} \mathrm{GH}$ to $\mathrm{H}$. & • & $58 \cdot 74$ \\
\hline 20 & 045 Р М. & From $\frac{3}{5}$ GH to I . & & $53 \cdot 9$ \\
\hline
\end{tabular}

If the refraction of the unit amount of incident light which is reflected from the mirror of the heliostat at the commencement and at the end of the series of the experiments be 
calculated, we get the numbers 0.644 and 0.642 , which differ so slightly that the variations brought about by the reflection may be neglected without overstepping the observational errors. At the times of observation on the 14th of August, 1857, the sun's zenith distance was as follows:-

$$
\begin{aligned}
& \text { At } 10 \mathrm{~h} . \check{5} 4 \mathrm{~m} \text {. A.M. . . } 37^{\circ} 35^{\prime} \\
& \text { At } 0 \quad 0 \quad \text { A.M. . . } 3513 \\
& \text { At } 0 \quad 45 \quad \text { P.M. . . . } 3616
\end{aligned}
$$

The chemical intensity of the sun's rays at these various periods may be calculated by formula (14). They are in the proportion of the numbers 1.002, 1.000, and 1.016. Although the differences between these numbers are but small, we have reduced all the observations to that chemical action which would have been observed if they had all been made at 12h. 0m. A.M. upon the day in question. The following table contains the numbers thus reduced, the mean value having been taken of those observations which occur more than once:-

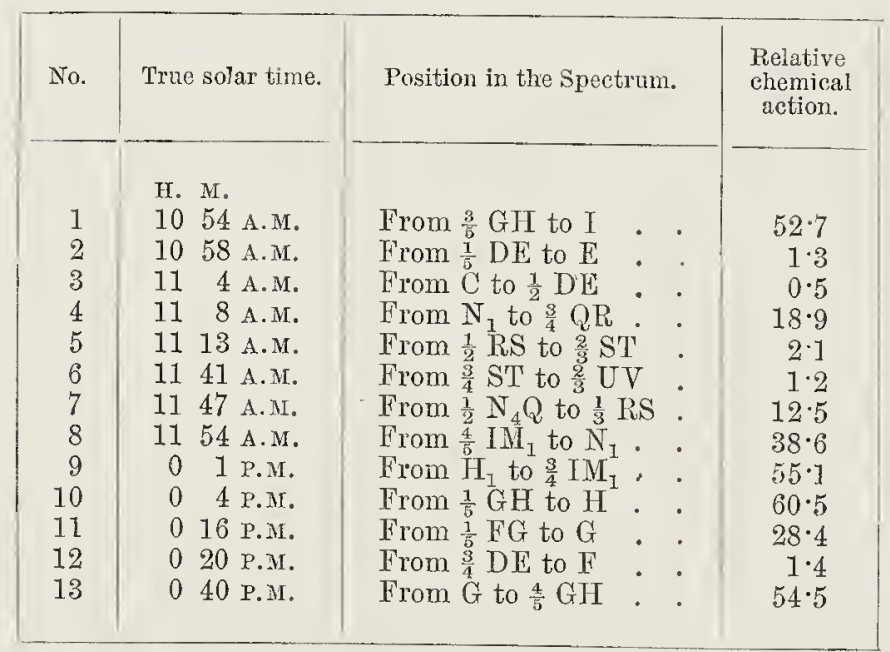

The lines a a a a (Fig. 15, page 28) give a representation of the relative chemical action which the various parts of the spectrum, the rays of which have only passed through air and quartz, effect on the sensitive mixture of chlorine and 
hydrogen. It is seen that this action attains many maxima of which the largest lies by $\frac{1}{5} \mathrm{GH}$ to $\mathrm{H}$, and the next at $\mathrm{I}$, and also that the action diminishes much more regularly and rapidly towards the red than towards the violet end of the spectrum.

The sun, when it was employed for these experiments, was $35^{\circ} 13^{\prime}$ removed from the zenith. If the atmosphere were throughout of the density corresponding to $0.76 \mathrm{~m}$. and $0^{\circ} \mathrm{C}$., the perpendicular height which, during our experiment, it would have possessed, is

$$
\frac{0 \cdot 7491}{0.000495084}=7,881 \text { metres. }
$$

The depth of atmosphere through which the rays had to pass in this experiment was, however,

$$
\frac{7881}{\cos 35^{\circ} 13^{\prime}}=9,647 \text { metres. }
$$

We have stated in one of our previous communications, ${ }^{1}$ that the solar rays which at different hours of the day pass through the same column of chlorine are altered in a very different manner. This shows that rays of different chemical activity are absorbed in very different ways by the air. The above results are therefore only applicable for sunlight which has passed through a column of air, measured at $0.76 \mathrm{~m}$. and $0^{\circ} \mathrm{C}$. of 9,647 metres in thickness. For rays which have to pass through a column of air of a different length from this, the chemical action of the various constituents of the spectrum must be different. The order and degree in which the chemical rays are absorbed may be obtained by repeating the observations according to the above method from hour to hour during a whole day. Such a series of experiments we have unfortunately as yet been unable to execute, owing to the variability of the weather in our latitudes. One very imperfect series of observations we can, however, quote, and they suffice to show that the relation between the chemical action of the spectral colours is perceptibly altered when the thickness of air through which the rays pass changes from 9,647 to 10,735 metres.

1 Phil. Trans. 1857, p. 617, \&c. 
These experiments were likewise made on August 14th, 1857 , in the short space of time from $9 \mathrm{~h} .44 \mathrm{~m}$. to $10 \mathrm{~h} .19 \mathrm{~m}$. A.M., and gave the following numbers reduced to the zenith distance $\left(42^{\circ} 46^{\prime}\right)$, corresponding to $10 \mathrm{~h} .0 \mathrm{~m}$. A.M. They were, however, made with a bundle of rays of a different thickness from the former experiments, and therefore caunot be compared with those.

\begin{tabular}{|c|c|c|c|}
\hline No. & Time. & Portion of Spectrum. & $\begin{array}{l}\text { Relative } \\
\text { chiemical } \\
\text { action. }\end{array}$ \\
\hline $\begin{array}{l}1 \\
2 \\
3 \\
4 \\
5 \\
6 \\
7 \\
8\end{array}$ & 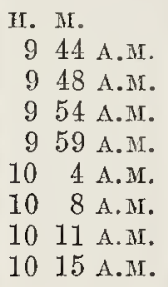 & $\begin{array}{l}\text { From } \frac{3}{5} \mathrm{GH} \text { to } \mathrm{I} \\
\text { From } \mathrm{N}_{3} \text { to } \mathrm{P}_{2} \\
\text { From } \frac{1}{1} \mathrm{R}_{2} \mathrm{~S} \text { to } \frac{1}{5} \mathrm{ST} \\
\text { From } \frac{1}{2} \mathrm{ST} \text { to } \mathrm{U} \\
\text { From } \mathrm{G} \text { to } \frac{4}{5} \mathrm{GH} \\
\text { From } \mathrm{F} \text { to } \frac{3}{4} \mathrm{FG} \\
\text { From } \delta \text { to } \frac{1}{2} \mathrm{FG} \\
\text { From } \frac{1}{2} \mathrm{DE} \text { to } \frac{3}{4} \mathrm{EF}\end{array}$ & $\begin{array}{r}14 \cdot 5 \\
10 \cdot 1 \\
2 \cdot 4 \\
0 \cdot 0 \\
13 \cdot 0 \\
7 \cdot 1 \\
3 \cdot 2 \\
0 \cdot 4\end{array}$ \\
\hline
\end{tabular}

From this it is seen that the relation of the chemical action of the spectrum from the line $\mathrm{E}$ to the line $\mathrm{II}$ undergoes a considerable alteration when the rays have to pass through a column of air 10,735 metres in height instead of 9,647 metres.

An extended series of measurements of the chemical action of the several portions of the solar spectrum under various conditions of atmospheric extinction may prove of great interest, if, as we can now scarcely doubt, the solar spots appear at regular intervals and our sun belongs to the class of fixed stars of variable illuminating power. It is possible that such observations, made during the presence and during the absence of the solar spots, may give rise to some unlooked-for relations concerning the singular phenomena occurring on the sun's surface. Whether, however, the atmospheric extinction can ever be determined with sufficient accuracy to render visible the alteration in the light which probably occurs with the spots, is a question which can only be decided by a series of experimental investigations which must extend far beyond the scope of any single observer. 


\section{APPENDIX D.}

Description of Roscoe's ACtinometer, ModIfied by Mr. Horace Darwin, and manufactured by the Cambridge SCIEntufic Instrument Company.

The measurement of the chemical effect of total daylight is made in this instrument according to Roscoe's method by exposing a piece of sensitized paper to the light for known intervals of time at each hour. To effect this the piece of sensitized paper is fixed round a drum. This drum can turn on a horizontal axis, and the frame which supports it can slide horizontally. A piece of thin sheet brass is fixed over the drum and a hole is cut through it. It is so arranged that it presses down lightly on the top of the drum, thus a small piece of the sensitized paper is always exposed to the daylight, and the rest of the paper is in total darkness. Thus by the horizontal movement of the frame and the turning of the drum every part of the sensitized paper can be brought under the hole. We will suppose the instrument to be working. The frame which supports the drum has a slow and continuous movement in a horizontal direction. Between the hours the drum does not turn, and it only moves slightly in a horizontal direction. At the hour the drum turns suddenly for a small part of a revolution, thus exposing a fresh piece of paper under the hole. After 2 seconds it again turns suddenly as before, exposing that piece of paper for 2 seconds. Similarly fresh pieces of paper are exposed for $2,4,6,10,20,40,90$ seconds, and after this the drum does not rotate again till the next hour, when a similar set of movenents takes place. Hence, between the hours there is a piece of paper exposed for 57 minutes, and which is useless on account of the long exposure. Owing to the horizontal movement of the drum a fresh piece of paper is exposed when one complete revolution of the drum has taken place. 
The mechanism by which this movement is effected is as follows :

To the barrel of a clock (i.e., the barrel round which the gut from the weight is coiled and which drives the clock) a pinion is fixed. This pinion gears into a tooth-wheel, and on the arbor of this wheel a small barrel is fixed. When the clock is wound up this small barrel turns, and in so doing winds a piece of chain on it, and as the weight of the clock goes down this small drum turns slowly in the opposite direction, and the chain is slowly unwound from it. The other end of this chain is fixed to the frame which supports the drum, and which can slide horizontally. Thus if the chain is always kept taut, the drum will slowly move horizontally in one direction as the clock goes, and when the clock is wound up it will be pulled back into its original position. This chain is kept taut by a piece of gut, which is fastened to the other end of the frame, and thence passes over a pulley and is attached to a weight. The horizontal movement of the drum is thus given, and it only remains to explain the arrangement for the rotatory movement.

See Fig. 25. A is an arbor which has a tendency to turn in the direction of the arrow. The mechanism for doing this is not shown in the sketch, but is arranged thus: On the arbor is a pinion into which a wheel gears; this wheel is made to turn by means of a weight attached to a string which is wound round a small barrel fixed to the same arbor as the wheel. $\mathrm{B}$ is a ratchet-wheel, which is not fixed to the arbor $\mathrm{A}$, but turns freely on it in the direction of the arrow once in 3 minutes, by being geared to a wheel on the scape-wheel arbor of the clock. This gearing is not shown, in order not to complicate the sketch. An arm $\mathrm{C}$ is fixed to the arbor A. D is a pin fixed to the end of this arm, on this pin a piece E can turn, a pin $F$ projects from the side of the piece $E$, and is so arranged that it can engage in the teeth of the ratchet-wheel B. A spring $\mathrm{G}$ tends to turn the piece $\mathrm{E}$, so that if not prevented the pin $F$ will be forced down between the teeth of the ratchetwheel. We have seen that the arbor A tends to turn and carry 
the arm $\mathrm{C}$ with it; in the position shown in the sketch this turning is prevented by the hooked end $\mathrm{H}$ of the lever $\mathrm{H} \mathrm{K}$, this hook catches in the end of the piece $\mathrm{E}$, and as the other end of this piece comes against a stop $\mathrm{L}$ the arm $\mathrm{C}$ is held in the position shown in the sketch, this stop is fixed to the arm $\mathrm{c}$ and projects from its side. In this position the pin $\mathrm{F}$ does not engage in the teeth of the ratchet-wheel $\mathrm{B}$. The weight tending to turn the arbor A will keep the arm in this position. At the hour the hook $H$ is raised, thus releasing the piece $\mathrm{E}$, the spring $G$ will then turn the piece $\mathrm{E}$, so that the pin $\mathrm{F}$ will engage in the teeth of the ratchet-wheel. We have seen that

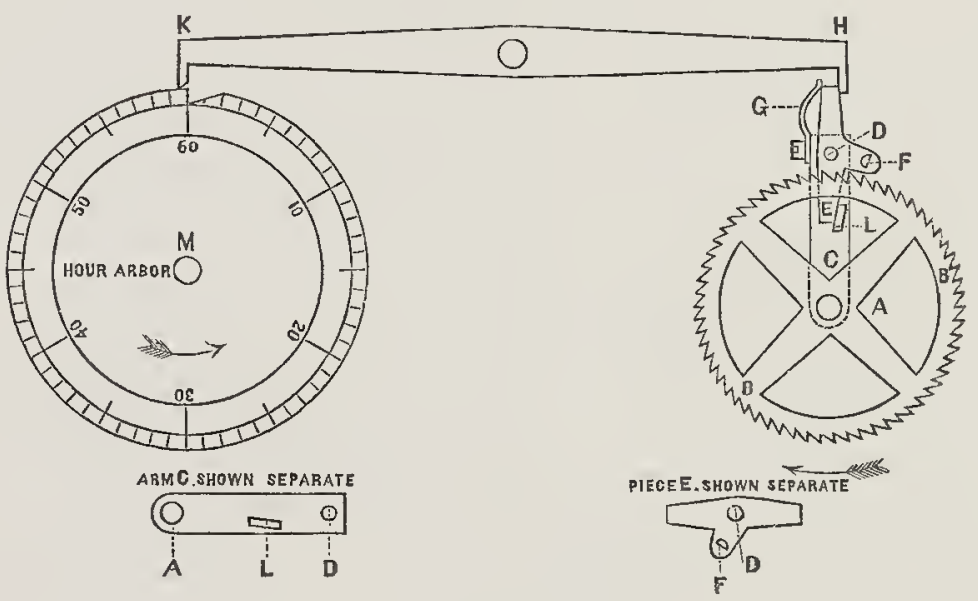

Fita. 25,

the ratchet-wheel is always turning at the rate of one turn in 3 minutes, and as the arbor $\mathrm{A}$ tends to turn in the same direction it will now turn at the same rate. Before the arm $\mathrm{C}$ has time to get round again to its original position the hook $H$ has come down again, and thus the outer end of the piece $\mathrm{E}$ will hit against it, but the arm will go on for a short time till the other end of the piece $\mathrm{E}$ comes against the stop $\mathrm{L}$. This movement throws the pin $\mathrm{F}$ out of gear with the ratchetwheel and again holds the arbor A. Thus at each hour the arbor $\mathrm{A}$ takes one complete revolution in 3 minutes, and then remains at rest till the next hour, and so on. The manner in 
which this regulates the turning of the drum will be explained further on. The lever $\mathrm{H} \mathrm{K}$ can turn about its centre, and its end $\mathrm{K}$ rests on the edge of the disc M. This disc turns once an hour, and has a nick cut in its edge so that at the hour the end $K$ falls into the nick and thus the end $H$ is raised. The slanting side of the nick again raises $\mathrm{k}$, and so lowers $\mathrm{II}$ before the end of the 3 minutes, at the end of which time the arm $\mathrm{C}$ returns to its original position; the disc $\mathrm{M}$ is divided into 60 divisions, and can be set like the hands of a clock, i.e. it can turn with a stiff joint on the hour arbor; thus it can be set so that the nick will come uppermost exactly at each hour.

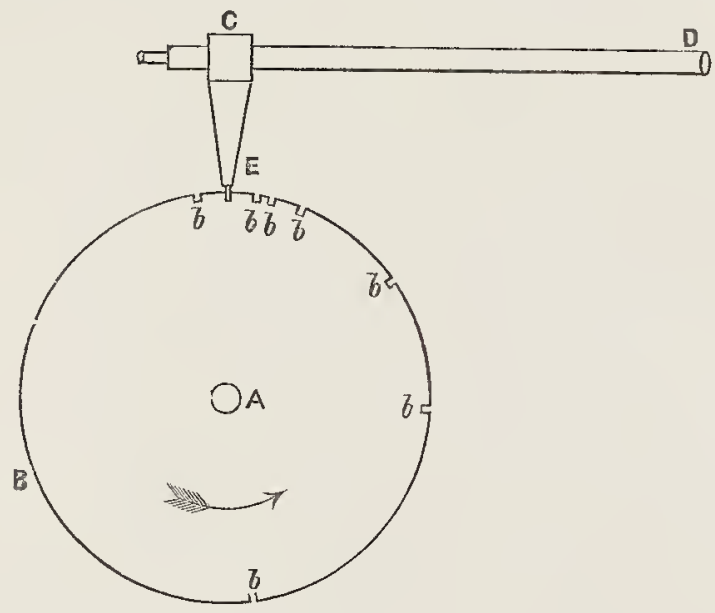

FIG. 26.

In Fig. 26, A represents the same arbor as A in Fig. 25, but a separate sketch is given in order to make them more clear. $\mathrm{A}$ disc $\mathrm{B}$ is fixed to the arbor. Thus at each hour this disc makes one complete revolution in three minutes. On the axis of the drum to which the paper is fixed, there is a small barrel, round which a cord is wound and a weight attached to the cord. Thus the drum tends to turn. This barrel is connected by a train of wheels with the arbor $\mathrm{C} \mathrm{D}$, which thus also tends to turn. For one turn of this arbor the drum turns through the right amount to expose a fresh piece of paper. Fixed to this arbor is a small arm $\mathrm{C} \mathrm{E}$. The point of this arm is made 
small, and rests against the side of the disc. At varying intervals round the disc nicks $b b$ are cut through its edge. The point of the arm $\mathrm{C} \mathrm{E}$ is made so as to pass through these nicks. During the hour this point rests against the side of the disc between two nicks. When at the hour this disc turns, every time a nick comes opposite the point of the arm, the arbor C D gives one turn, and thus the drum turns through a fraction of a revolution, and a fresh piece of paper is exposed. This disc turns with jerks at each second as the clock ticks; the point E passes through a nick during one of these jerks while the disc is moving comparatively quickly. Thus the intermittent movement of the drum takes place with considerable accuracy. Any required lengths of exposure can be given by putting the nicks in the corresponding places. The nicks are cut diagonally across the edge of the disc. This is done in order to prevent the possibility of the points $\mathrm{E}$ coming round so quickly as to pass through the same nick twice.

The accompanying sketches must be taken merely as diagrams to show the principle of the mechanism.

The above instrument is analogous in principle to that described by Professor Roscoe in the Philosophical Transactions for 1874, a modified form of which has now been continuously at work at South Kensington for several months.

Mr. Horace Darwin's arrangement is in several particulars superior to the original form of the actinometer, and especially in requiring a smaller quantity of the sensitized paper. It has been tested by Prof. Roscoe and found to work perfectly. The instrument is now set up at South Kensington, and gives regular and reliable results.

A special reading-off apparatus has been designed by Captain Abney, which greatly facilitates the readings, and renders the daily operations as simple and rapid as possible. 


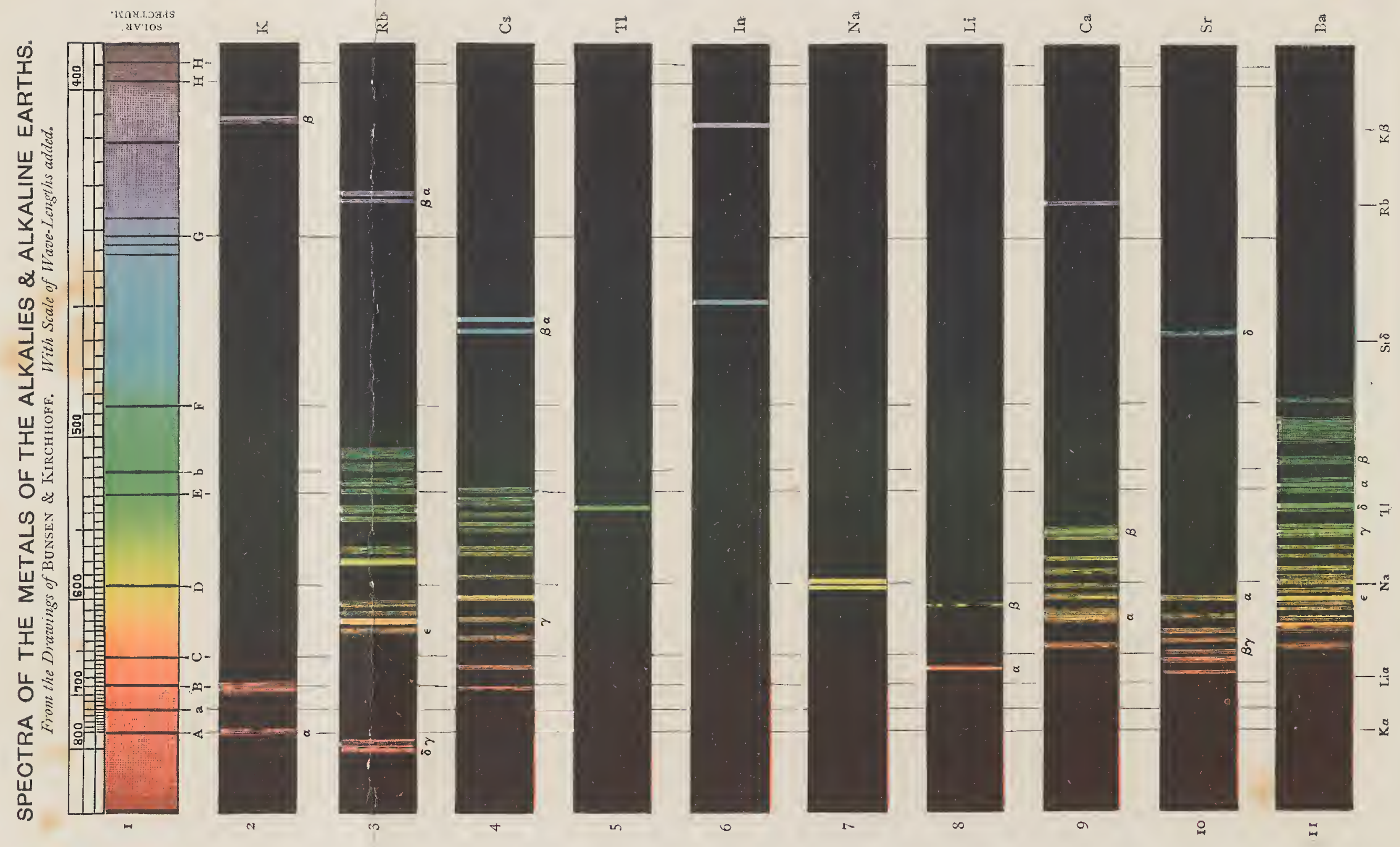





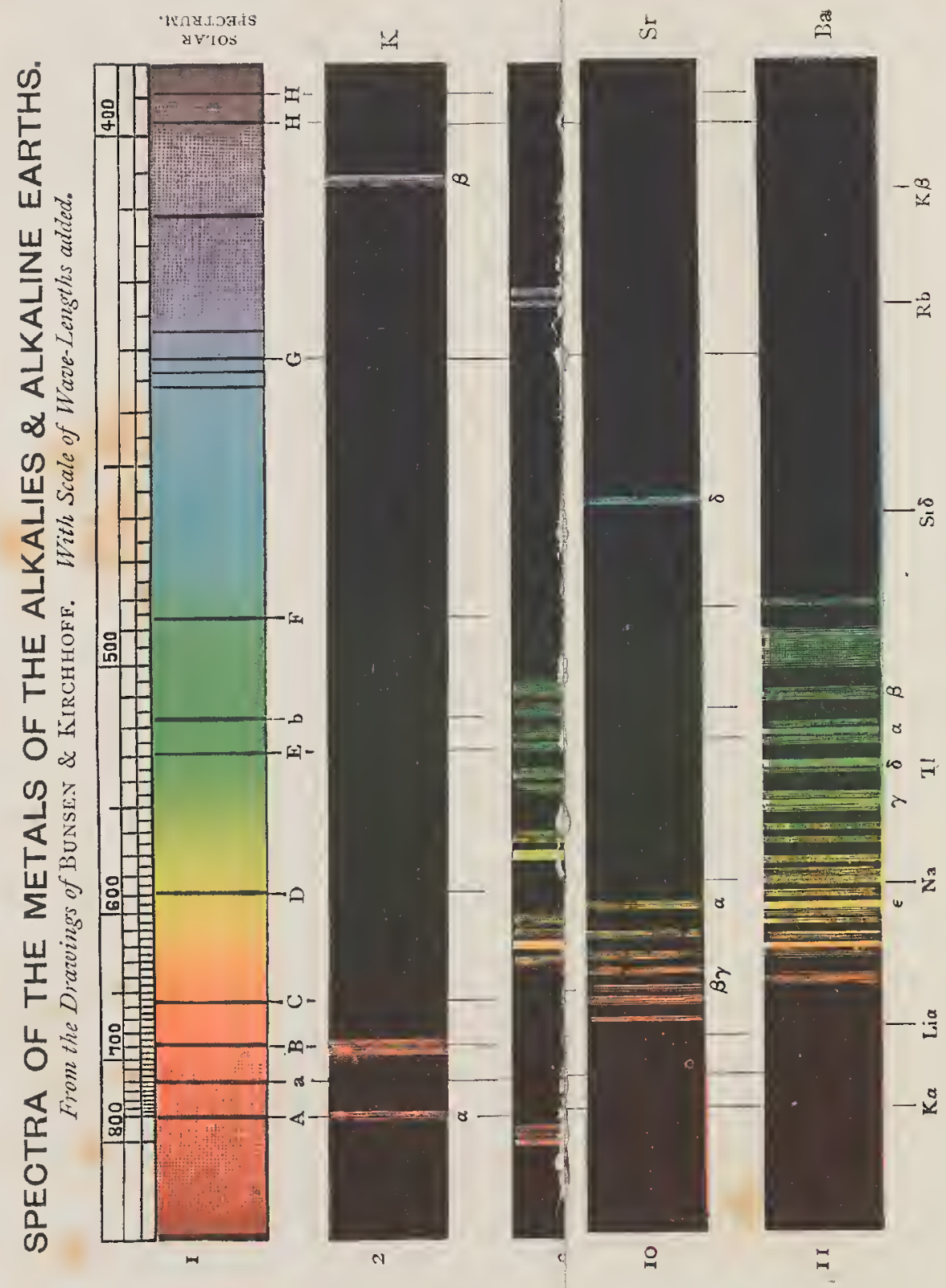




\section{LECTURE II.}

Continuous Spectrum of Incandescent Solids.--Effect of Increase of Heat. -Broken Spectrum of glowing Gas.-Application to Chemical Analysis. - Spectra of the Elementary Bodies.-Construction of Spectroscopes.Means of obtaining Substances in the state of glowing Gas.-Examination of the Spectra of Coloured Flames.-Mapping Spectra, according to Bunsen.-Telicacy of the Spectrum Analytical Method and its application to Plysiological Research.-Spectra of the Metals of the Alkalis and Alkaline Earths.-Historical Sketch.-Discovery of New Elements by means of Spectrum Analysis.-Caesium, Rubidium, Thallium, Indium, Gallium.

Appendix A.-Description of the Spectrum Reactions of the Salts of the Alkalies and Alkaline Earths.

A.ppendix B.-Binsen and Kirchhoff on the Mode of using a Spectroscope. ApPexdix C.-Bunsen on a Method of mapping Spectra.

APPENDIX D.- Spectrum Reactions of the Rubidium and Caesium Compounds.

A PPENDIX E.-Contributions towards the History of Spectrum Analysis by G. Kirchhoff.

In the last lecture I pointed out to you some of the chief properties of the light with which we are now, I am glad to say, illumined-the light of the sun. I explained that the white sunlight can be divided up into a large number of different coloured rays by means of the prism; that these differently refrangible rays possess different properties; that we find the heating rays chiefly situated at the red end, or in the least refrangible part. I showed that we could separate out by certain means the light rays from the less refrangible ultra-red rays, and obtain at the dark focus of these rays the phenomena of incandescence and of combustion, showing that these rays 
which do not affect the eye, are capable when brought together of producing ignition. We also saw that at and beyond the other end, the blue end, of the spectrum we have rays which have especially the power of effecting the decomposition of ordinary silver salts such as are commonly used in photography, though we must bear in mind that chemical compounds exist which can be decomposed by the green, yellow, and red rays, and that, therefore, the chemical rays, as distinguished from those which affect the eye or from those which produce heat, cannot be said to exist.

We proceed to-day in the examination of the action of heat upon terrestrial matter in so far as it evolves light. The question may very properly be asked, "What has all this to do with chemical analysis?" It might be said, "It is true you have pointed out the difference between the various parts of the solar spectrum; but how is this connected with the analysis which we expect to be told about-with the method by means of which chemical substances may be detected or examined with a degree of accuracy beyond anything that has hitherto been attained?" In order to enable you to answer this question, let us begin by examining the action of heat upon terrestrial matter, and, in the first place, upon solid bodies. I have here the means of heating a long piece of platinum wire, first of all to redness, and by diminishing its length I shall be able to increase the temperature of the wire gradually until I raise it to the melting point of platinum. The first thing we observe when a solid body, such as this wire, is heated, is that it becomes red-hot; and that as we increase the temperature, the light which it gives off increases in refrangibility, so that it ends by emitting light of every degree of refrangibility. I cannot show you on the screen the spectrum which this heated wire yields, simply because the intensity of light which it emits is insufficient for the purpose; but if I were to allow the light to fall into my eye through a prism, I should see that the red rays become first visible, and that then a gradual increase in the refrangibility of the light occurs, and that successively yellow, green, blue, and violet rays will be emitted as the temperature 
is increased up to a white beat, when all the rays of light are given off.

I will endeavour to render this fact visible to you in a rougher way by heating the wire gradually up to whiteness, and allowing the light to pass through these coloured glasses placed between you and the wire. At first, when it is red-hot, the glowing wire will be visible only through the red glass, none of the rays being able to pass through the blue glass; or, in other words, there is no blue light given off: when the temperature is increased, blue rays begin to be given off, and these can pass through the blue glass, as you now plainly see when I raise the temperature of the wire. Here I can increase the temperature of the wire until we get at a point at which I have no doubt you will be able to see that the blue rays are emitted; and if I continue this and go on until the wire becomes intensely white-hot, you will see it through this blue glass perfectly well.

Such then is the action of increased temperature upon solid bodies. If I had taken any other substance which I could have heated in the same way, I should have produced the same effect: for it has been found that all solid and liquid substances act in this same way with regard to increase of heat; they all begin to be visibly hot at the same temperature, and the spectrum thus produced is in every case a coutinuous one. ${ }^{1}$ I may remind you that this is the case by again throwing on the screen the spectrum of the white-hot carbon points heated in the electric arc. Here we have this grand continuous band of light. The arrangements for producing this are simple enough. We require to connect the terminal wires from about sixty pairs of Grove's or Bunsen's cells with the carbon electrodes of a Duboscq's lamp (E, Fig. 27) contained inside this lantern. The light passes through a narrow vertical slit (s), and by means of the moveable lens (c) a distiuct image of the slit it thrown upon the screen ( $w$ w). A hollow prism filled with bisulphide of carbon $\left(p_{1}\right)$ is now introduced at the distance of about two feet

I This law was discovered by Draper (Phil. Mag. 1847). The only known exception to this law is glowing solid Erbia; whose spectrum exhibits bright liues; see Appendix F to Lucture IV. 
frorn the lens; next the lamp, with the arm carrying lens and prism, is turned round until the coloured band falls upon the screen, and the prism then adjusted to the angle of minimum deviation for the yellow rays. A second prism $\left(p_{2}\right)$ is then interposed, and the lamp and arm again turned so as to allow the lengthened spectrum to fall on the screen. A drawing of lamp, lens, and prisms, thus placed, is shown in Fig. 28.

How does the case stand with respect to that important form of matter termed the gaseous? Do gases when they become incandescent all emit the same kind of light, like solids, or does

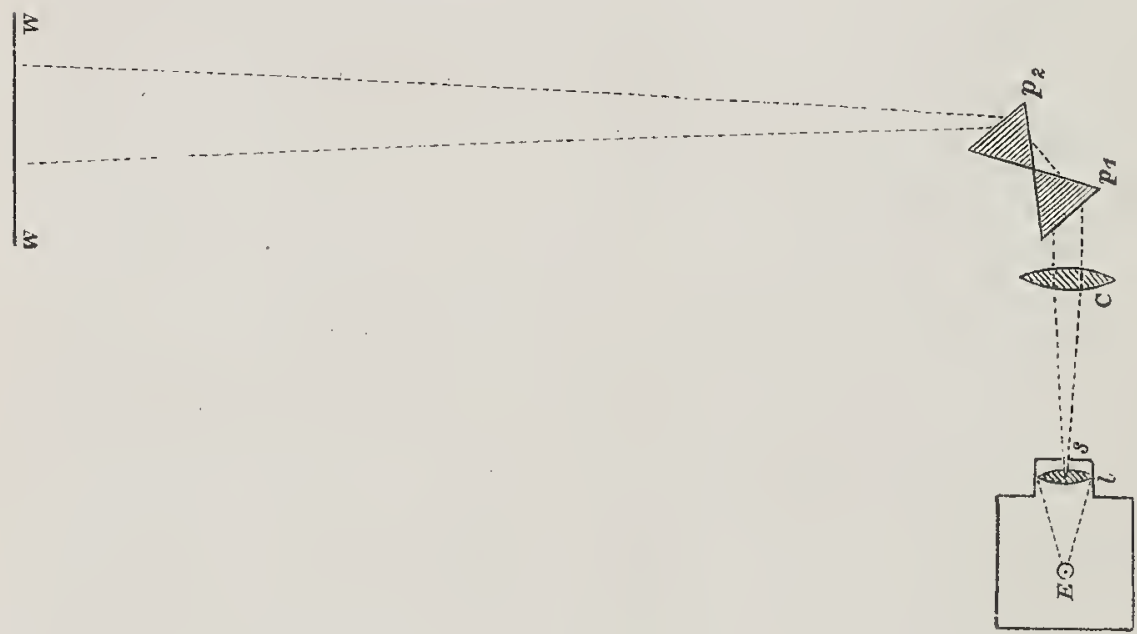

FเE. 27 .

each chemically different gas emit a characteristic and peculiar kind of light? I purpose now to show you that every different chemical element in the state of gas, when heated until it becomes luminous, gives off a peculiar light, so that the spectrum of every element in the state of glowing gas is totally different from that of any solid body, inasmuch as, instead of giving a continuous spectrum, it presents a broken or discontinuous one containing bright bands or lines, indicative of the presence of the particular elementary gas in question. ${ }^{1}$ I will illustrate

1 Under peculiar circumstances to be mentioned hereafter, certain dense incandescent gases give continuous spectra. 
this fundamental difference to you by means of the following experiment. It has long been known to chemists that certain substances have the power when brought into a colourless flame of producing peculiar tints. Thus, for instance, if we bring

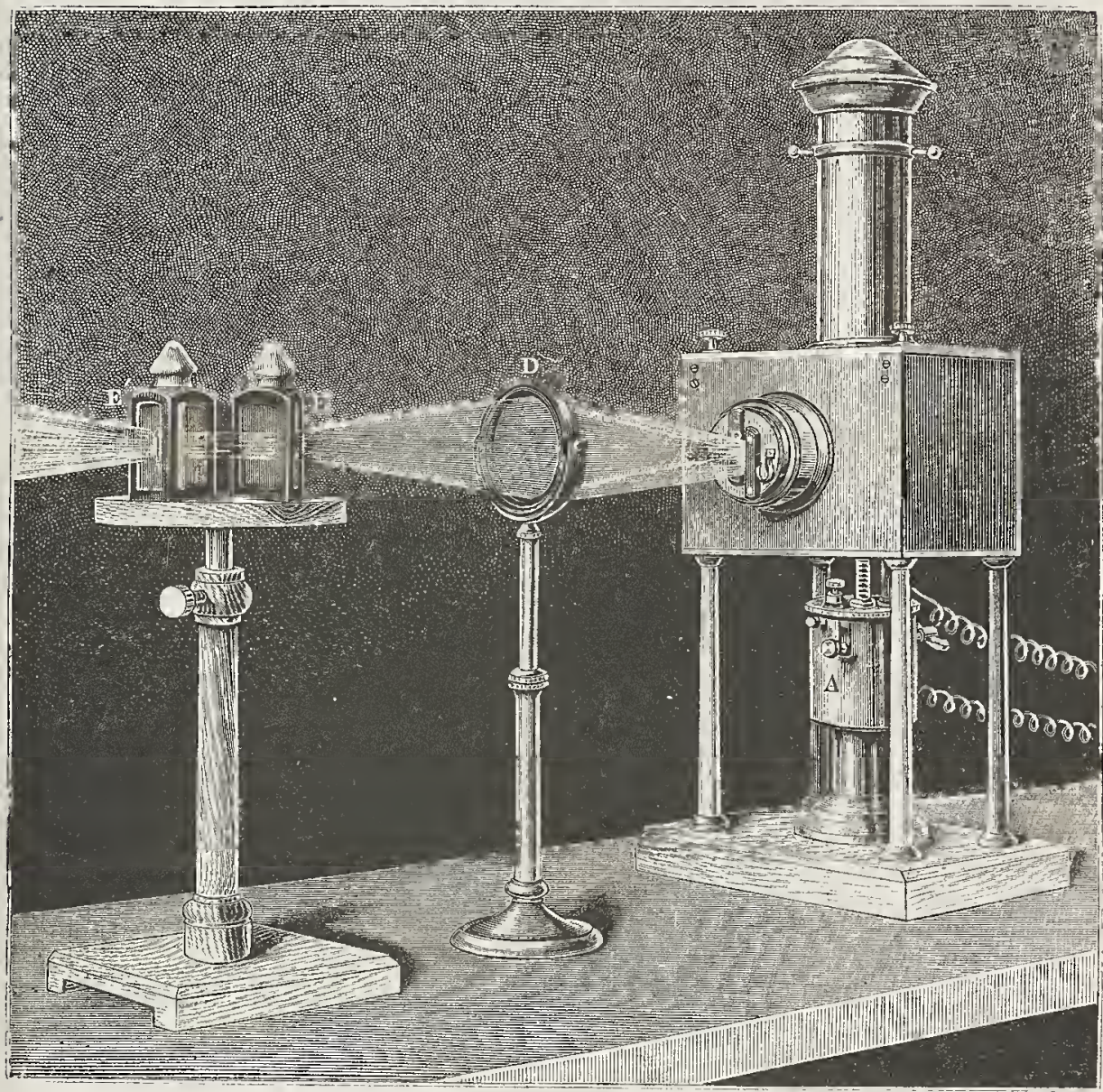

Fir. 28.

various bodies into the flame, such as the alkalis soda and potash, we observe that the flame becomes coloured in the first case of a bright yellow, and in the second of a pale violet tint; whilst the salts of strontium colour the flame crimson, and those of barium produce a green tint, and calcium compounds impart 
a red culour to the flame. Here we have the beautiful nonluminous gas-flame produced by the combustion of coal gas mixed with air, in what we know as the Bunsen burner. The air and gas mix in the chimney, the gas issuing from a jet at the centre of the foot, and the air entering by holes at the side; the mixture burns with a light blue flame, which we can tinge with the peculiar colours of the alkalis by bringing a small fused bead of salt into the cuter mantle of the flame on the loop of thin platinum wire (Fig. 29). Here is another substance called lithium; if we bring the slightest trace of this

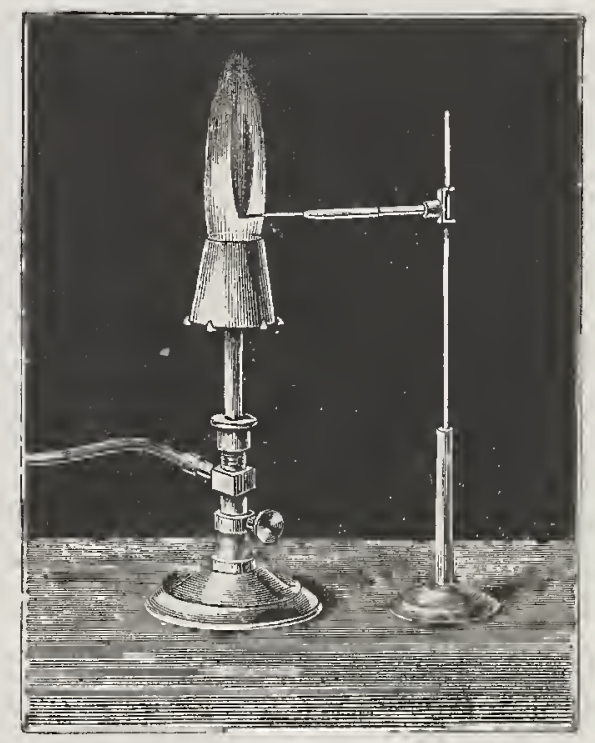

FiG. 29.

lithium salt into the flame, you perceive the magnificent crimson tint which it at once imparts to the flame: whilst in these other burners we see the colours due to the salts of potassium, calcium, strontium, and barium.

A most important observation has now to be made, namely, that all the salts of sodium give off this yellow light when brought into the flame; so, too, all the lithium compounds tint the flame crimson; and this property of emitting a peculiar kind of light is one of the means by which the presence of 
these various chemical substances can be detected. Here I will produce a peculiar blue flame by a substance which differs entirely from the foregoing in properties, viz. the non-metallic element selenium: it is a very volatile substance, and the blue flame lasts only for a short time. In these other flames we see the characteristic green colours communicated to flame by salts of copper and boracic acid.

I will next show you the same thing in other ways; for instance, I can here produce a much larger flame, and show you the colour of the same salts. I liave a large gas burner which, when urged by this blowpipe, gives us a colourless flame three feet long. If I hold in this flame pieces of pumice-stone moistened.with solutions of the chlorides of sodium, potassium, lithium, barium, strontium, and calcium, the colours imparted by these substances will be rendered evident. Again, I have another illustration in these gun-papers, which lave been soaked in solutions of the chlorates of these metals and then dried. The combustion is rather quick, but by reflection on the white screen their peculiar colours come out well. Here you have the violet potash tint; here the bright green colour characteristic of the barium compounds. The common fireworks of the stage are further illustrations of the peculiar colours produced by certain chemical substances. I may imitate the red fire by igniting some chlorate of strontium in coal gas; we must melt the salt and then plunge it into the jar of burning coal gas, when we get this splendid combustion of oxygen in coal gas coloured crimson by the ignited vapour of strontium salt.

We have already seen that the quality of the light emitted by solid bodies varies with difference of temperature. The quality of the light emitted by gaseous bodies, however, with certain exceptions-about which I shall have to speak subsequently-does not vary under change of temperature. Here I have the means of igniting some sodium salt at various temperatures. There, in the first place, is the bluish flame of burning sulphur, one of the coldest flames we can obtain, the temperature being about $1,820^{\circ}$ Centigrade; then I noxt ignite 
the flame of burning carbon disulphide, having a temperature of $2,229^{\circ} \mathrm{C}$. Here we see the flame of coal gas burning mixed with air: if I cut off the air, we get the common luminous flame of coal gas; but if I allow the air to mix with it before it burns, then we have this beautiful non-luminous flame. The temperature of this flame has been calculated to be $2,350^{\circ} \mathrm{C}$ Here I have another jet from which the blue flame of carbonic oxide gas (the body which produces the blue lambent flame frequently seen in coal fires) is seen burning in oxygen: the temperature of this flame is somewhat higher, and has been found to be over $3,000^{\circ}$. If I bring a little common salt (sodium chloride) into these flames, you observe that in all cases we get them coloured yellow. I have here the beautiful purple flame of cyanogen gas, which possesses a temperature of $3,300^{\circ} \mathrm{C}$, and you see, when we bring the sodium salt into it, we have the same yellow colour produced: in other words, we cannot get sodium vapour either red-hot or blue-hot, it always remains yellow-hot; that is to say, the first moment that the sodium vapour becomes luminous, it gives off this particular and peculiar yellow light, and if we heat it more, the effect is not to alter the refrangibility of the rays, but merely to increase their intensity.

Thus we see that when a body becomes gaseous, the light which it gives off is of a particular kind, and does not undergo any gradual alteration when we increase the temperature. Another experiment may indicate this to you still more clearly, and this I can make by means of the electric spark, which I have here the means of producing. The temperature of this electric spark is so high that it has never been measured, but it is certainly very much higher eren than the temperature of any of the flames which we have just now used. Still, if I bring this piece of sodium salt into the electric spark, I find that the same thing occurs-I get the same yellow-coloured light; and if I take some other substance, such as lithium, and bring a small trace of this substance first into the different flames and then into the electric spark, the permanent red colour which lithium vapour gives off will in each case be clearly seen. 
Now the methods by means of which we can obtain bodies in the state of luminous gas vary with the nature of the substance; but I would beg you to understand that the property which we have noticed with regard to sodium and the other alkalis is not confined to those bodies which have the power of being volatilized in such a flame as I have burning before me. This property belongs to matter in general; it belongs to every chemical element; and if we can by any method get the vapour of a chemical element so hot as to become luminous, we find that the light emitted by it is peculiar to itself, and is distinctive of that special body, whether under the ordinary circumstances the element be gaseous, solid, or liquid. Hence you see that we have at last reached the principles upon which the science of spectrum analysis is based, by means of which we can detect the presence of any of the elementary bodies when they can be obtained in this state of glowing gas.

We must now pass on to the consideration of the various methods by which the elements can be obtained as luminous gases. I purpose to confine our attention in this lecture to the method by which we can detect the presence of the metals of the alkalis and alkaline earths. Let me, however, first point out to you the kind of spectrum which we obtain when we look at any one of these variously-coloured flames through a prism or spectroscope, the construction of which we will now briefly consider.

The simplest form of spectroscope which Bunsen adopted in his first experiments is represented in Fig. 30. It consists of a common hollow prism (F) placed in a box; a telescope (C) is fixed at one side of the box, and a slit is placed at one end of a tube having a lens at the other end, in order to obtain a pure spectrum, and to render the rays parallel; this is called a collimator. This collimator (B), the employment of which was first proposed by Professor Swan of St. Andrews so long ago as 1856, is fixed at the other side of the box. The substance to be examined is placed in the non-luminous Bunsen's flame, and the light passing through the slit falls upon the prism, and having been split up into its constituent parts, the differently coloured 
rays pass through this telescope, are magnified, and then fall upon the retina. In Fig. 31 we have the more perfect form of

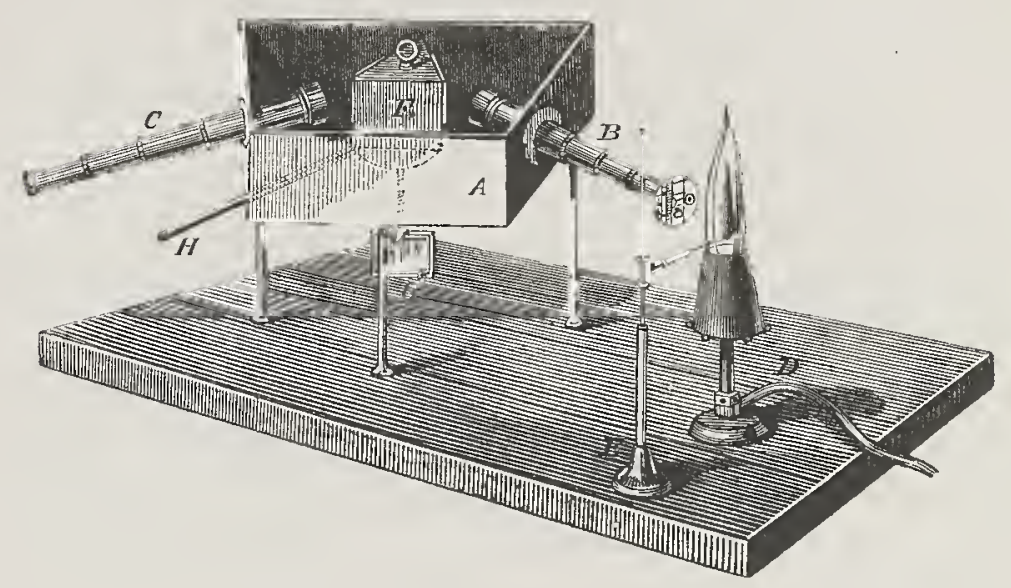

FIG. 30.

the instrument represented, as made by Steinheil of Munich. ${ }^{1}$ With this we are enabled to use two flames, and the apparatus

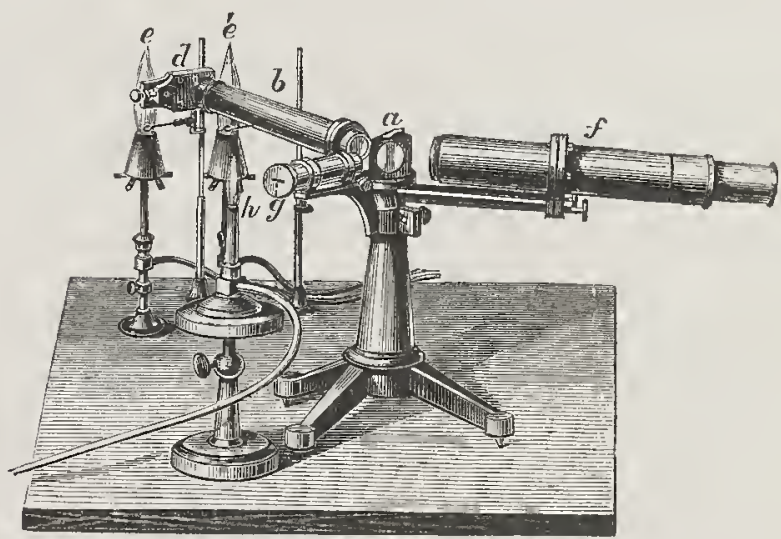

FIx. 31 .

is so arranged that we can see the two spectra placed one above the other. The rays from one of the flames pass direct through

1 This instrument consists of a prism ( $(\iota)$ fixed upon a firm iron stand, and a tube $(b)$ carrying the slit $(d)$, seen on an enlarged scale in Fig. $32 \alpha$, through which the rays from the coloured flames ( $c$ and é) fall npon the prism, being rendered parallel by p'issing through a lens. The light having been refracted, is 
the upper part of the slit, whilst those (A) from the other flame, placed on one side, are reflected (by total internal reflexion) from the surface of the prism through the lower part of the slit in the direction indicated by Fig. 32. The object of this superposition of the spectra is evident: it is to enable us to see whether the substance under examination really is the body which it is supposed to be. For instance, putting a small quantity of the substance we know to contain sodium in this flame, we place a substance supposed to contain sodium in the other flame, and then by means of a small reflecting prism placed on the end of the slit, we have the spectra of these two flames sent into the telescope one above the other, so that we see at the same time the spectrum of the pure sodium and the spectrum supposed to

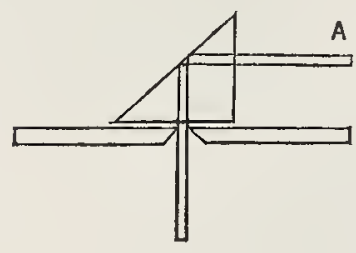

FIG. 32 .

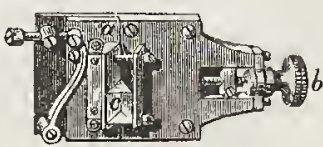

Fig. $32 a$.

be that of sodium; and we can readily observe whether the lines coincide. If they coincide, and the two spectra have these lines exactly continuous one below the other, then we are quite certain that sodium, or any other substance which we may have been investigating, is present. Another arrangement for facilitating the comparison of spectra consists in the illuminated millimetre scale contained in the tube $g$ (Fig. 31), a magnified reflexion of which is thrown into the telescope from the surface of the prism. The illuminated scale is thus seen between the

received by the telescope $(f)$, and the image magnified before reacling the eye. The rays from eaeh flame are made to pass into the telescope $(f)$; one set through the upper uneovered half of the slit, the other by refleetion from the sides of the small prism $(c)$, Figs. 32, 32a, through the lower half; thus bringing the two spectra into the field of view at once, so as to be able to make any wished for eomparison of the lines. The small luminous gas flame (h), Fig. 31, is placed so as to illuminate a fixed scale contained inside the tube $(g)$ : this is reflected from the surfaee of the prism $(c)$ into the telescope, and serves as a means of measuring the position of the lincs. 
two superimposed spectra, and the position of any line or lines can be accurately determined. The further arrangements-mechanical and optical-of these instruments I need hardly trouble you with in detail. I have here a variety of spectroscopes kindly lent to me by a maker, Mr. Browning; one with one, one with two, one with three, and one with four prisms. The more prisms we employ, of course the greater dispersion we get, the more is the light drawn out into its special varieties, and the greater also is the intensity of the light which it is necessary to employ in order to get the rays to pass through this greater number of prisms.

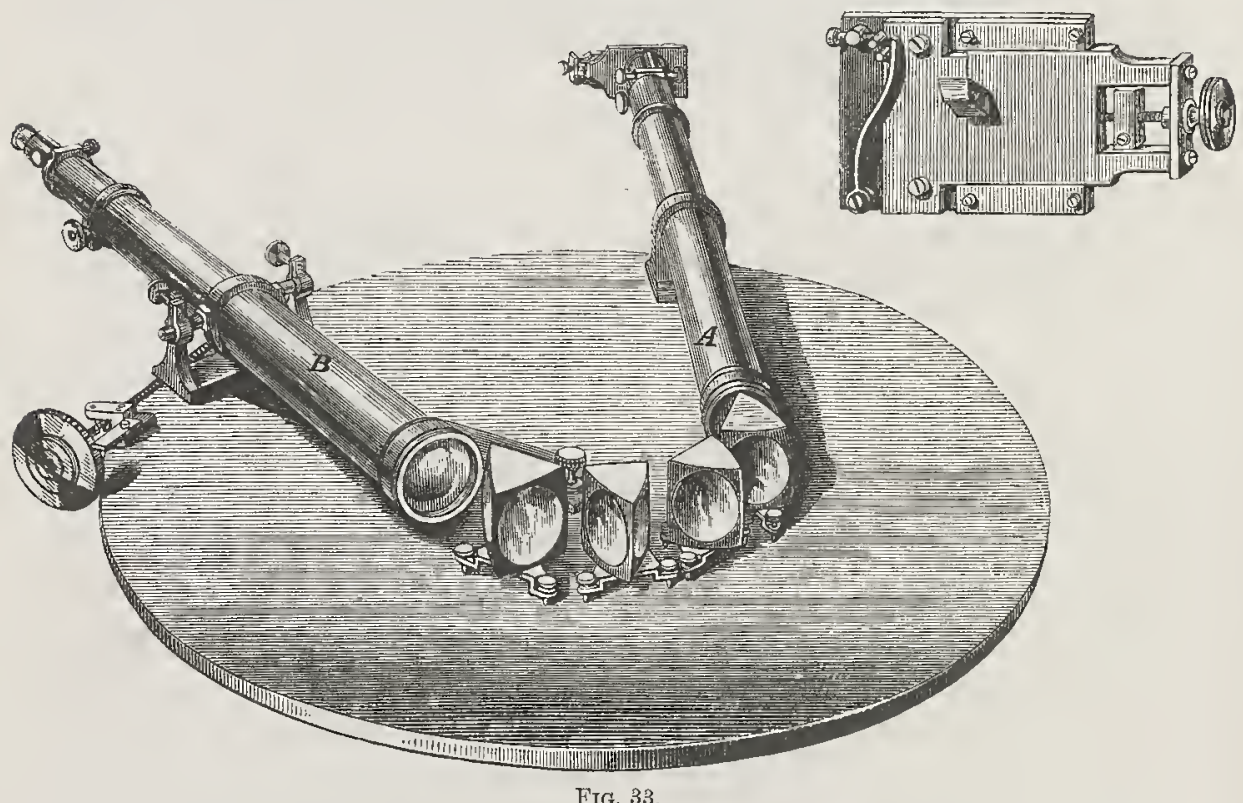

Fig. 33.

I will next show you a drawing of the actual arrangement used by Kirchhoff (Fig. 33). There you see the prisms employed, four in number, placed one behind another on a horizontal table of cast iron. The light passes through the slit at the end of this tube. Here (top of Fig. 33) is an enlarged representation of the slit, the breadth of which can be altered at pleasure by means of the screw; on this slit is placed a small reflecting prism to enable us to get two superposed spectra. The 
light passes through the fine vertical slit, the rays are rendered parallel by the leas fixed at end of the tube (A); it then passes through these four prisms, and the rays thus split up into constituent parts fall on to the telescope (B), at the end of which the eye is placed. This, then, gives you the simplest, and at the same time the most delicate and complete form of spectroscope.

We have here representations as truly painted as possib?e (see Frontispice) of what is seen when we allow a light from such coloured flames as those which have been burning to fall on to the retina through a spectroscope properly arranged.

At the top of the diagram (No. 1) is a drawing showing a solar spectrum, and underneath we have the spectra of the alkalis and alkaline earths, potassium (No. 2), sodium (No. 7), and litbium (No. 8), calcium (No. 9), strontium (No. 10), and barium (No. 11), together with the two new metals, rubidium and cæsium (Nos. 3 and 4), discovered by Bunsen, about which I shall afterwards speak; also the spectra of thallium and indium (Nos. 5 and 6), two other new metals, the first of which was lately discoverer by our countryman Mr. Crookes, and the second by two German chemists, Messrs. Reich and Richter. You will perceive in the first place that each of these spectra is different from the others, although they all possess the common characteristic of containing bright lines or bands, which occur in various portions of the spectrum and indicate the peculiar kind of light which these various bodies, when brought into a state of glowing gas, emit. The sodium flame, when observed by means of the spectroscope exhibits only one bright double yellow line together with a faint continuous spectrum; in other words, this light is monochromatic, or nearly so : almost all the light which glowing sodium vapour gives off is light of one degree of refrangibility, and the spectrum is confined to one very narrow yellow band. The red light, which we saw was due to the presence of lithium, when seen through a prism gives this beautiful red line, together with this paler orange line. I need not describe the more complicated spectra of strontium, calcium, 
and barium: suffice it to say that each yields peculiar bright bands, perfectly characteristic of the metal in question, as is seen at once by reference to the drawings. ${ }^{1}$

For the purpose of enabling an observer unacquainted with the spectra to identify with certainty the presence of any of the foregoing metals by means of their bright lines, and to lay down their positions in his own instrument, the following method of mapping the spectra has been devised by Bunsen. The millimetre scales (Fig. 34) represent the illuminated divisions seen with the scale of the spectroscope ( $g$, Fig. 31): the exact position of the bright lines in any spectrum is shown by the black marks below the divisions; whilst their breadth, intensity, and gradation are indicated by the breadth, depth, and contour of these blackened surfaces. When the spectrum contains a continuous portion of light, this is shown by a continuous black band above the divisions. The positions of the fixed solar lines are given on the first horizontal scale, and those of the most prominent bands in several of the elements are placed as fiducial points at the bottom of the map. ${ }^{2}$

Having thus made ourselves acquainted with this new mode of chemical analysis, we may ask, "What improvement is this upon our ordinary chemical methods? What benefit is it to us that barium gives us these peculiar bands, that strontium yields certain different bands, that calcium produces others again? We know already that the chemical reactions of these bodies are very different, and we can detect these substances by ordinary chemical analysis." The answer to this is, that the new method is far more delicate than anything which we have hitherto employed, so delicate indeed as almost to pass belief, so that we have hereby obtained a means of examining the composition of terrestrial matter with a degree of exactitude hitherto unknown.

I will try to give you some idea of the delicacy of these spectrum reactions. I can show that the reaction for sodium is so sensitive that we can detect the presence of this element

1 For the special description of these spectra see $\Lambda$ plendix $\Lambda, 1.94$.

2 For further information see Appendix C, p. 106. 


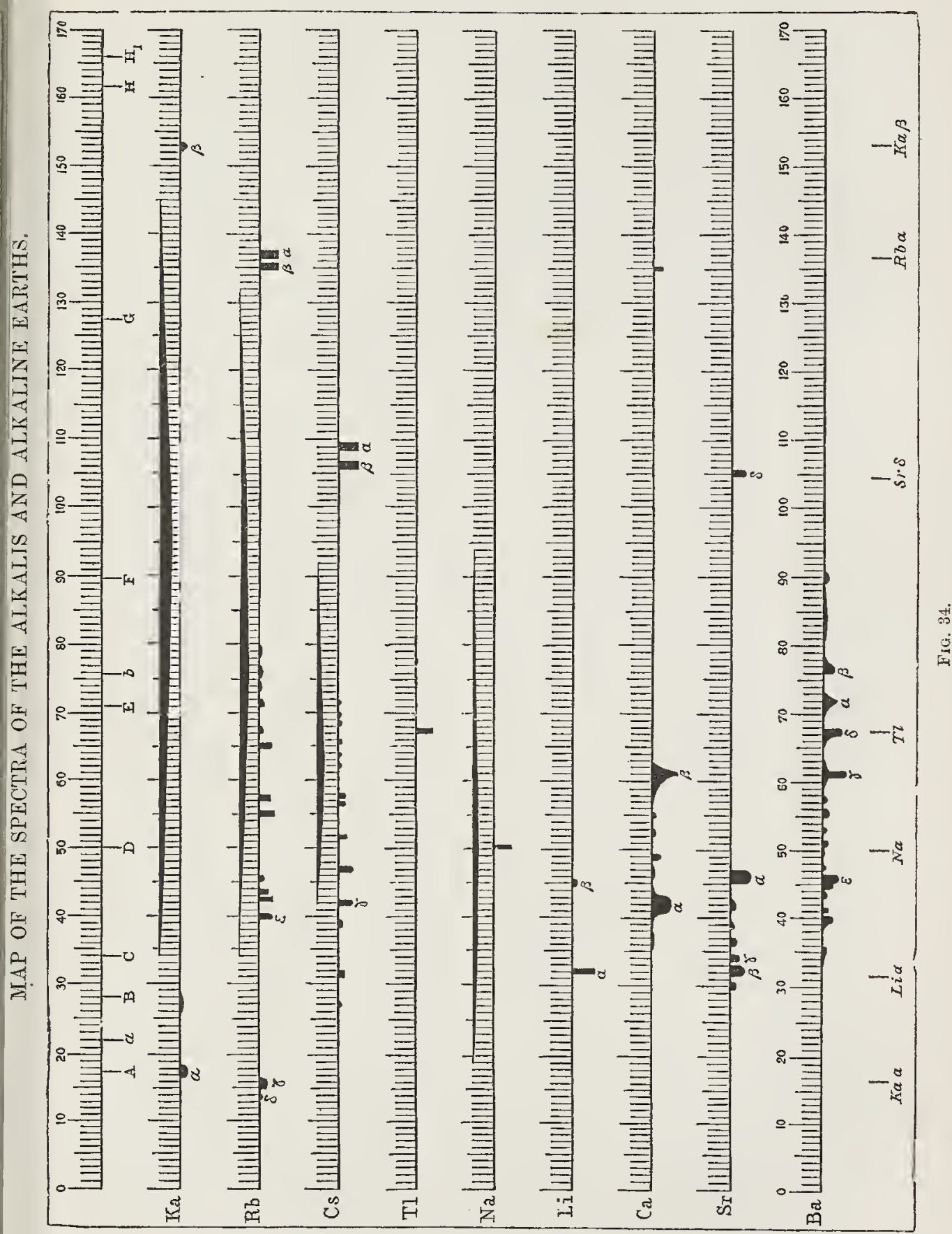


everywhere. There is not a speck of dust or a mote seen dancing in the sunbeam which does not contain chloride of sodium. Sodium is a prevailing element in the atmosphere; we are constantly breathing in portions of the compound of this elementary substance together with the air which we inhale. Two-thirds of the earth's surface is covered with salt water, and the fine spray which is continually being carried up into the air by the dashing of the waves evaporates, leaving the minute specks of salt which we see dancing as motes in the sumbeam. If I clap my hands, or if I shake my coat, or if I knock this dusty book, I think you will observe that this flame becomes yellow, and this not because it is the hand or coat of a chemist, but simply because the dust which everybody carries about with him is mixed with sodium compounds. When I place in the colourless flame this piece of platinum wire, which has been lying on the table for a few minutes since I heated it red-hot, you see there is sodium in it; there, we have for one moment a glimpse of a yellow flame. If I heat the wire in the flame, the sodium salts will all volatilize, and the yellow tinge will quite disappear; but if I now draw the wire once through my fingers, you observe the sodium flame will on heating the wire again appear. If I draw it through my mouth and heat it again, it will be evident that the saliva contains a very considerable quantity of sodium salts. Let me leave the wire exposed here, tied round this rod, so that the end does not touch anything, for ten minutes or a quarter of an hour; I shall then obtain the sodium reaction again, even if the wire be now perfectly clean. This is because sodium salts pervade the atmosphere, and some particles of sodium dust flying about in the air of the room settle on the wire, and show their presence in the flame.

In the short historical sketch of the subject which I hope presently to lay before you, we shall see that this constant reaction of sodium puzzled the old observers very much. They thought this reaction must be due to the presence of water, for there was no other substance which was so widely diffused; and it is only recently that this yellow reaction has been recognised as being due to this metal, sodium. 
To refer for a moment to the distribution of lithiun compounds: we must remember that this substance, giving the beautiful red flame which you saw just now and the spectrum exhibiting the one bright red line, was until lately only known to exist in three or in four comparatively rare minerals. The moment, however, we come to examine substances by the method of spectrum analysis, we find that the brilliant red line, which is characteristic of the presence of lithium, occurs very frequently. And why, then, was not the red flame noticed before? Because when the light was examined by means of the eye alone, its red colour was masked by the presence of sodium salts, and other substances affecting the flame, so that the red tint produced by the small quantity of lithium was unseen. But when we examine the flame with the prism, then all these lines range themselves into due order, no one interfering with the other. The presence of lithium may be thus easily detected, though it may be mixed with ten thousand times its bulk of sodium compounds, because, as you see by reference to this chart, the sodium line occurs in a different position to the lithium line, according to the differences in their refrangibilities. We now learn that this substance supposed to be so rare is found to be most widely distributed-not, it is true, in very large quantities, but still that it is one of the most widely diffused of the elementary bodies. Lithium not only occurs in very many minerals, but also in the juice of plants, in the ashes of the grape, in tea, coffee, and even in milk; in human blood, and in muscular tissue. It has also been found by Dr. Bence Jones in many varieties of fruit and vegetables, in all the different kinds of wine, in ale and porter, and in bread. So who can tell what part this hitherto rare substance may not play even in the animal economy? It has been also found in meteoric stones, in the water of the Atlantic Ocean, as well as in that of most mineral springs and many rivers. It is present in the ashes of tobacco, and, if we hold the end of a cigar in the colourless flame, we may always notice the red lithium line when the light is examined with a spectroscope. Dr. W. Allen Miller found lithium in very large quantities in the water of a spring 
in the Wheal Clifford Mine in Cornwall. ${ }^{1}$ This water contains 26 grains of lithium chloride in one gallon, and the spring flows at such a rate as to pour forth 800 lbs. of this salt every twentyfour hours!

Here, at the risk of some repetition, I may refer you to a diagram indicating concisely some of the most striking discoveries, and at the same time showing the great delicacy of the methods of spectrum analysis :-

1. Sodium. $\frac{1}{3000000}$ part of a milligramme, or $\frac{1 \overline{1} 0000000}{180 \mathrm{p} t}$ of a grain, of soda can easily be detected. Soda is always present in the air. All bodies exposed to the air show, when heated, the yellow soda line.

2. Lithium. $\frac{1}{10000}$ part of a milligramme, or $\frac{1}{800000}$ part of a grain, can easily be detected. Lithium was formerly only known to exist in four minerals: it is now found by spectrum analysis to be one of the most widely distributed elements. It exists in almost all rocks, in sea and river (Thames) water, in the ashes of most plants, in milk, human blood, and muscular tissue.

3. Strontium. $\frac{6}{10000}$ of a milligramme, or $\frac{1}{1000000}$ of a grain of strontia is easily detected. Strontia has been shown to exist in very many limestones of different geological ages.

4. Calcium. $\frac{6}{100000}$ of a milligramme, or $\frac{}{5000000}$ of a grain, of lime can be easily detected.

5. Cosium and Rubidium. These new alkaline metals were discovered by Bunsen in the mineral waters of Baden and Dürkheim. Forty tons of mineral water yielded 200 grains of the salts of the new metals.

6. Thallium. A new metal discovered by Mr. Crookes in 1861 distinguished by the splendid green line which its spectrum exhibits. It is found in iron pyrites, and resembles lead in its properties.

7. Indium. Discovered in zinc blende by Professors Reich and Richter in 1864: found in very minute quantities. It is distinguished by one blue and one indigo coloured band seen in its spectrum.

8. Gallium. Discovered in blende from the Pyrenees by M.

1 Chem. News, x. 181. 
Lecoq de Boisbaudran in 1875. Its spectrum consists of two bright violet lines which are best seen in the electric spark spectrum.

I will now endeavour to illustrate, by means of the electric lamp, the fact that all these bodies give off coloured lights, and that each of these coloured lights is of a peculiar kind ; and I would wish first to show you that when we bring a small fraction of a grain of common salt, chloride of sodium, on to the lower carbon of the lamp, we obtain a distinct yellow band which was not seen before, for previously, you will remember, we had a perfectly continuous spectrum. This yellow band is due to the presence of sodium. You will probably see that there are other bands present as well as the sodium band, because it is impossible to obtain any carbon which is perfectly free from other chemical salts, and the minute amount of impurities which exist in the carbon comes out as evidence against us on the screen; yet I think you will see that we have the sodium line more distinctly visible than anything else.

No other metal but sodium gives this yellow band; still I must beg you to understand that this rough representation is not exactly that which you would see if you were to look at the yellow soda flame through a prism, by means of an accurate spectroscope. I would wish you to remember that this yellow line is in reality double when examined with a perfect optical arrangement, and that these lines are very fine, placed close together, and become finer as the slit is narrowed. It is only because the arrangements I have to employ here for the purpose of exhibiting these lines on the screen are, optically considered, very crude and rough, that we get any appreciable breadth of this line.

Now allow me to show you the light which the substance lithium gives off. For this purpose I will bring a little lithium salt on the same carbon, for by taking a new one we should not gain much, because, as I have said, all these poles are more or less impure. Here you observe the red line, which was not noticeable before. This splendid red band is due to the presence of lithium; and when we see it through an accurate instrument, it appears, like the sodium lines, as fine as the finest slit of 
light which we can take. This bright red line is always found exactly in the same position; and the fixity of these lines is in fact the most important principle involved in our inquiry: they are unalterable in refrangibility.

I have next to direct your attention to the blue line which is now visible on my right. This is also caused by lithium, for when we heat up lithium vapour beyond a certain point, as high as I am now doing with the electric lamp, this blue band also becomes visible; but it is not visible when the temperature of the incandescent lithium vapour is lower. The blue ray may perhaps always be given off, even at lower temperatures; for if light requires to be of a certain intensity before it can affect the retina and become visible, and if, in order that the intensity of the light nlay thus increase we must heat the vapour to a ligher point; we have a complete explanation of the appearance of the blue line. It is important to notice that the positions of the red and of the orange lines seen at the lower temperature never shift or change the least when the temperature is changed. Hence the appearance of this red line is proof positive of the presence of lithium. In this lithium spectrum you will also notice the sodium line. We can never get rid of our friend sodium, he always remains steadfast to us; in fact, we should be sometimes glad to dispense with his presence, but it is not an easy matter to induce liim to leave us.

As an interesting application of spectrum analysis, we must not forget the examination of the rate of circulation of the blood in the animal body lately made by Dr. Bence Jones and Dr. Dupré, by means of observations of the red lithium line. Experiments made upon animals showed that when any lithium salt is taken in with the food, this metal can be detected very shortly afterwards in the most distant parts of the body, even where no circulation of blood exists, as for instance in the lens of the eye, proving the extraordinary rapidity with which the chemical circulation in the body goes on. Thus twenty-four minutes after injecting three grains of lithium salt under the skin of a guinea-pig the lithium was found to be present in the lens and in every part of the body, it only being necessary to burn a 
portion of the animal tissue in a colourless flame in order to see the bright red line of lithium; ten minutes after the injection. lithium was found in small quantities in the lens but plentifully everywhere else; whilst four minutes after the injection the lithium was not found in the lens but plentifully in the aqueous humour of the eye, and in the bile.

The same rapid diffusion of the lithium salts was shown to exist in the human body; thus, for example, the lenses of several persons who had been operated upon for cataract were examined, the patients having previously partaken of lithia water. It was thus seen that in the human body 20 grains of carbonate of lithium will in three and a half hours penetrate through every part of the body, and be capable of detection in each particle of the lens. The rate of passage of salts out of the body can likewise be satisfactorily examined by the spectroscope, and thus spectrum analysis may be made materially to aid the physiologist in his researches into the chemistry and physics of the body.

I shall next show you the spectra of metals of the alkaline earths. I will first bring a small quantity of strontium salt on to the pole, and we find that the strontium spectrum is characterized by a series of red lines, and also by a beautiful blue band almost identical, but not exactly so, with the blue line of lithium which I had the pleasure of showing you an instant ago. What a large number of bands we have here, especially in the red! Those red and blue bands are the ones to which I beg to draw your attention. These red lines now come out very distinctly; and we have here also the strong blue line flashing out brightly. This then is the strontium spectrum. In like manner I may show you the beautiful and characteristic spectrum of barium, with its five green bands; and that of calcium, exhibiting special orange and green lines together with a purple band in the more refrangible part of the spectrum.

Now let us suppose that we have a mixture of compounds of all the abovesubstances which are capable of being volatilized, namely potassium, sodium, lithium, barium, strontium, and calcium, and let us expose this mixture to such a temperature that all the salts become volatilized, one after another; we shall see, in the 
first place, that the bands of those substances which are the most volatile appear first; that then, when these have burnt out, those next in order of volatility make their appearance; and that those which are the least volatile come out last. Thus we have the beautiful appearance of what may be called a natural dissolving view.

I place on the carbon poles a mixture containing a few grains of salts of all the above-mentioned metals. You see in the first place that the sodium line comes out at once, and afterwards the lines of the other metals gradually make their appearance. We have thus simply to place the smallest fraction of a grain of such a mixture as this before the slit of our spectroscope, and with the merest trace of substance we can in a moment obtain absolute and decisive evidence of the presence of all these elements, the lines coming out, as I said, like a dissolving view, one after another; and the quantity which we can thus detect is, we must remember, most minute. Here we have this splendid series of variegaled bands, exhibiting the superposed spectra of all the substances I have mentioned. There you see the lithium red line; here the less refrangible red line of potassium; there the orange band of calcium and the red strontium bands: observe, if you please, the two blue bands, one due to strontium and the other to lithium.

But although Bunsen and Kirchhoff are the real discoverers of this method, because they carried it out with all due scientific accuracy and placed it on the sure foundation upon which it now rests, yet we must not suppose that the ground was before their time absolutely untrodden. No great discovery is made all at once. There are always stepping-stones by which such a position is reached, and it is right to know what has been previously done, and to give such credit as is their due to the older observers.

So long ago as 1752 , Thomas Melville, while experimenting on certain coloured flames, observed the yellow soda flame, although he was unacquainted with its cause. In 1822 Brewster introduced his monochromatic lamp, in which the soda light is used; the first idea, however, being due to Melville. A 
simple experiment will prove to you the nature of this monochromatic soda light. I have here the means of producing a very intense soda flame, and $I$ will throw the light on to this screen with different coloured letters. You will observe that no colour is noticeable in these letters. They appear in various degrees of shade or intensity, but no difference of colour is visible, because the light falling upon them is of a pure yellow tint. Now, if I throw a small quantity of magnesium powder into the flame, you will at once notice how brightly the various colours come out. We have here white light containing rays of every degree of refrangibility; hence the different colours are rendered visible, each being able to reflect its own peculiar rays.

Sir John Herschel, in the year 1822, investigated the spectra of many coloured flames, especially of the strontium and copper chlorides, and of boracic acid, and he writes in 1827 as follows: "The colours thus contributed by different objects to flame afford in many cases a ready and neat way of detecting extremely minute quantities of them."

Fox Talbot, whose name is so intimately associated with the origin of the beautiful art of photography, makes the following suggestions respecting these spectra. Writing in 1826, he says: "The red fire of the theatres examined in the same way gave a most beautiful spectrum, with many light lines or maxima of light. In the red these lines were more numerous, and crowded with dark spaces between them" (these are the strontium lines which you see on the diagram), "besides an exterior ray greatly separated from the rest, and probably the effect of the nitre in the composition" (this is really the red potassium line caused by the nitre). "In the orange was one bright line, one in the yellow, three in the green, and several that were fainter." The blue line which he mentions is the blue strontium line which we saw so plainly. "The bright line in the yellow" (our friend sodium) "is caused without doubt by the combustion of sulphur." Talbot got wrong there, like many of the early observers. They did not suppose that so minute a trace of sodium could produce that yellow light; and even 
Talbot says that the yellow line must be caused in certain cases by the presence of water. $\mathrm{He}$ continues: "If this opinion" (about the cause of formation of these lines) "should prove correct, and applicable to the other definite rays, a glance at the prismatic spectrum of a flame might show it to contain substances which it would otherwise require a laborious chemical analysis to detect." We cannot even now express the opinion entertained at the present moment more concisely than Talbot did in the year 1826. These early observer's did not, however, determine the exact nature of the substance producing the colour, inasmuch as the extreme sensitiveness of this sodium reaction put them off the scent: they conld not believe that sodium was present everywhere.

Both Herschel and Brewster found that the same yellow light was obtained by setting fire to spirits of wine diluted with water, and Talbot also mentions cases in which no soda was, as he thought, present, and yet this yellow line always made its appearance. Hence he says, "The only matter which these substances have in common is water," and he throws out the suggestion that this yellow line is produced by the presence of water. In February 1834 Talbot writes: "Lithia and strontia are two bodies characterized by the fine red tint which they communicate to the flame. The former of these is very rare, and I was indebted to my friend Mr. Faraday for the specimen which I subjected to the prismatic analysis. Now it is very difficult to distinguish the lithia red from the strontia red with the naked eye, but the prism betrays between them the most marked distinction which can be imagined. The strontia flame exhibits a great number of red rays well separated from each other by dark intervals, not to mention an orange and a very definite bright blue ray. The lithia exhibits one single red ray. Hence I hesitate not to say, that optical analysis can distinguish the minutest portions of these two substances from each other with as much certainty as, if not more than, any known method." Stiil Talbot says further on, that "the mere presence of the substance, which suffers no diminution in consequence, causes the production of a red and green line to appear in the spectrum." 
Professor William Allen Miller next made some interesting experiments in $\mathbf{1 8 4 5}$ on the spectra of coloured flames produced by the metals of the alkaline earths, and came still nearer to the result which we now find Bunsen and Kirchhoff arrived at in 1861. Diagrams of these spectra accompany the memoir, but they are not characteristic enough to enable them to be used as distinctive tests for the metals, owing to the fact that a luminous flame was used. Hence the investigations of Miller in 1845 attracted less attention than they deserved. ${ }^{1}$ The first person who pointed out this characteristic property of sodium was Professor Swan, in 1856, and it is to him that we owe the examination and first accurate determination of the very great sensitiveness of this sodium reaction. So much then for the history cf the method as applied to the detection of the wellknown alkalis and alkaline earths.

We will now pass on to the consideration of the new elements which have been discovered by spectrum analysis. And, in the the first place, I would direct your attention to the new alkali metals discovered by Professor Bunsen in 1860. Shortly after he made his first experiments on the subject of spectrum analysis, Bunsen happened to be examining the alkalis left from the evaporation of a large quantity of mineral water from Dürkheim in the Palatinate. Having separated out all other bodies, he took some of these alkalis, and found, on examining by the spectroscope the flame which this particular salt or mixture of salts gave off, that some bright lines were visible which he had never observed before, and which he knew were not produced either by potash or soda. So much reliance did he place in this new method of spectrum analysis, that he at once set to work to evaporate so large a quantity as forty-four tons of this water in which these new metals, which he termed ccesium and rubidium, were contained in exceedingly minute quantities.

In short, he soon succeeded in detecting and separating the two new alkali metals from all other elements, and the complete

1 See extract in Appendix E. from Kirchhoff's "Contributions to the History of Spectrum Analysis," Phil. Mag., Fourth Series, vol. xxv. p. 250, 1863. 
examination of the properties of their compounds which he made with the very small quantity of material at his disposal remains a permanent monument to the skill of this great chemist. Both these metals occur in the water of the Dürkheim springs. Bunsen's analysis of the mineral waters of Dürkheim and of Baden-Baden will be found in the Second Memoir on Spectrum Analysis, published in 1861.

The quantity of the new substances contained in the water from the Dürkheim springs is excessively small, amounting in one ton to about three grains of the chloride of cæsium and about four grains of the chloride of rubidium; whilst in the BadenBaden spring we have only traces of the crsium chloride, and a still smaller quantity than in the other spring of the rubidium chloride. From the forty-four tons of water which he evaporated down, Bunsen obtained only about 200 grains of the mixed salts. You will easily appreciate the delicacy and accuracy of a method by which the presence of so minute a trace of the new metals as that contained in the water could be so readily detected.

Let me show you, in the first place, the colours produced by these two new metals when brought into a non-luminous gas flame. We have already noticed the beautiful violet tint which the potash flame exhibits. The tint yielded by these two metals is very similar to this, and in fact, not only in the character of the light which they emit, but in all their chemical properties, the compounds of both the new bodies resemble potassium compounds very closely. Here I bring a small quantity of rubidium salt into the flame, and you observe the beautiful purple colour with which the flame is tinged. Now I throw in a little cæsium salt, and you notice we get a very similar kind of tint, rather more red, but still scarcely to be distinguished from the violet potash flame burning alongside.

If I next show you the spectra of cæsium and rubidium on the screen, and compare them with the spectrum of potassium (see Frontispiece, Nos. 2, 3, and 4), you will see that the spectra of these three metals exhibit (in accordance with their 
correspondence in other chemical properties) a striking analogy. Each of the three metals possesses a spectrum which is continuous in the middle, showing that under certain circumstances gases may emit light of every degree of refrangibility, and decreasing in intensity towards each end. In the case of potassium the continuous portion is most intense, in that of rubidium less intense, and in the cæsium spectrum this luminosity is least. In all three we observe the most intense and characteristic lines towards both the red and blue ends of the spectrum. The metal rubidium, as its name inplies, is characterized by two splendid deep red lines (see Frontispiece, No. 3), both less refrangible than the potassium red line; but the two violet lines are even more characteristic, and serve as the most delicate test of the presence of the metal. No less than the 0.0002 part of a inilligramme of rubidium can be detected by the spectrum reaction. The cxsium spectrum is chiefly characterized by the two blue lines from which it derives its name; they are remarkable for their brilliancy and sharpness of definition: while it is singular that cæsium exhibits no red lines whatever.

Since the discovery of these two bodies by Bunsen in 1860, chemists have been on the look-out for them, and have found both of them in very different situations; one of them, rubidium, being comparatively widely distributed. The celebrated French waters of Bourbonne-les-Bains contain $0.032 \mathrm{grm}$. of chloride of crsium and 0.010 grm. of chloride of rubidium in one litre of water; whilst in the well-known mineral springs of Vichy, Gastein, Nauheim, Karlsbrunn, and many more, either one or both of the new metals has been discovered. And here the thought strikes one, that the presence of these metals, even in such minute quantities, may possibly exert a not unimportant influence upon the medicinal qualities and effects of the waters. Rubidium has been found to be very widely diffused; it has been found in beetroot, in tobacco, in the ash of the oak (the Quercus pubescens), in coffee, in tea, and in cocoa: indeed of the new metals it is only rubidium which is found in vegetables and in vegetable products; whilst 
both new metals are found in tolerably large quantities in certain minerals, especially in lepidolite and petalite.

One very interesting example of the occurrence of the metal cæsium has been observed in a mineral termed pollux, which was analysed in the year 1846 by the well-known chemist Plattner, and supposed to contain potassium. In calculating out the results of his analysis Plattner invariably found a considerable loss, the cause of which he was unable to account for. Spectrum analysis has now explained this anomaly, for since the discovery of the two new metals it las been found that it was not potassium, but the new metal cxsium, which was present, of the oxide of which no less than 34 per cent. is contained in this mineral. The want of agreement of the former analysis is therefore wholly attributable to the difference of the combining weights of these two bodies; that of potassium being only $39 \cdot 1$, whilst cesium is 133; and if we use this last number in the calculation, we find that Plattner's analysis comes up exactly, as it ought to do, to 100 parts. So closely indeed are cæsium and potassium allied in their chemical characters, that it is only by the discriminating power of spectrum analysis that we have been able to ascertain even the existence of the new metal.

Having once proved the existence of these two new elementary bodies, Bunsen was of course easily able to find the means of separating them accurately one from the other and from the well-known substance potassium; and at the present day the chemical history and characters of these two metals and their compounds are as well known as those of the common alkalis.

The reaction by which Bunsen separated the new metals from potassium can easily be rendered visible to you. I have here a small quantity of rubidium chloride in solution, and here again I have a solution of the double chloride of potassium and platinum.

The chloride of rubidium and platinum is much less soluble than the corresponding potassium compound, and hence, if I add the potassium double-chloride to this rubidium salt, I shall have a precipitation of the double chloride of rubidium and 
platinum; and this will indicate to you the mode by which Bunsen separated these two metals from each other.

Here you observe by pouring in this solution the liquid at once becomes turbid, and we get a very considerable quantity of a heavy yellow granular precipitate of the new rubidium compound.

It is unnecessary now to enter into the analytical methods by which cæsium can be separated from rubidium; it is sufficient to state that one of these methods is based upon the different solubilities of the tartrates of the new metals, the acid tartrate of cæsium being much more soluble than the corresponding rubidium salt.

The isomorphous relations between the salts of rubidium and cæsium and those of potassium also point out the striking chemical analogy subsisting between these interesting bodies.

Shortly after the discovery of these two new alkaline metals the existence of a third new elementary substance was made known by our countryman Mr. Crookes. In the year 1862 he sent to the Exhibition a very small portion of a substance which he stated was a new element obtained from a certain seleniferous deposit from a sulphuric acid manufactory at Tilkerode in the Hartz. This body gives a most beautiful green tint to flame. If I bring a small quantity of this element into the flame, you see that it produces this exquisite green colour. And this was the reaction by which it was discovered. Mr. Crookes proved that this green light was due to some new elementary body ; then he separated out the substance, and gave to it the name thallium, from thallus, a green twig.

Now the spectrum of thallium is very distinct and specific, consisting of one bright green line. I will show it to you with the electric lamp. Here you see this magnificent green band (Frontispiece, No. 5). The spark-spectrum of thallium is rather more complicated, as it exhibits five other lines in addition to the bright one in the green. ${ }^{1}$ The line Tl $a$ possesses a wave-length of 5349 ten-millionths of a millimetre, and when examined with a one-prism instrument appears to be coincident with one of the barium lines. This apparent

$$
1 \text { Miller, Proc. Roy. Soc., 1863, p. } 407 .
$$


coincidence is, however, resolved when examined by a higher magnifying power. The chemical properties of this substance are very remarkable. It stands about half-way between lead and the alkalis, resembling in many of its characters the metal lead, and in others potassium, so that it has been well described by Dumas as the "ornithorhynchus" amongst the metals. Thallium can, however, be perfectly separated from the alkalis and lead by means of the insolubility of its chloride and the solubility of its sulphate. The specific gravity of thallium is 11.8 ; its combining weight is a very high one, 204; and it acts as a monad metal, forming an oxide having the formula $\mathrm{Tl}_{2} \mathrm{O}$, and a chloride $\mathrm{TlCl}$.

The properties of thallium have been examined by a French chemist, M. Lany, as well as by Mr. Crookes. It has been found to exist in very large quantities in certain varieties of iron pyrites, a substance from which we manufacture almost all our sulphuric acid.

The metal thallium can be easily obtained in the metallic state from its salts. This I can readily render evident to you all. We can here decompose a solution of the sulphate of thallium by a current of electricity, and then we shall observe the metallic thallium shooting out as a beautiful arborescent growth on the screen. Here you see the crystals of metallic thallium stretching out their long branches all over the screen.

The soluble salts of thallium act as a cumulative poison: they have been found in large quantities in animals which have been poisoned by this substance. The method for determining or detecting the presence of thallium in such a poisoned animal by means of spectrum analysis is extremely simple. If we had such a means of detecting some of the other metallic poisons as readily as that of thallium, the work of the toxicologist would be extremely easy; except that under these circumstances the very delicacy of the test becomes in itself a danger, as the most minute trace of the poisonous metals which might by chance be present would in this way be as easily detected as a larger quantity.

There are still two other elementary bodies of which I have to speak, namely, the metals indium and gallium. 
Indium was discovered in 1864 by two German professors, Reich and Richter, of the celebrated Mining School of Freiberg. It also was detected by the peculiar spectrum which consists simply of two indigo-coloured lines. These lines are best seen when a bead of an indium compound is held between two electrodes from which a spark passes. The lines In $a$ and In $\beta$ fall respectively upon divisions 107.5 and $140 \mathrm{~mm}$. of the photographic scale of the spectroscope, when $\mathrm{Na} a=50$ and $\mathrm{Sr} \delta=100 \cdot 5$. (See Frontispiece, No. 6.) It was discovered in certain zinc ores, and has only been found in small quantities. Its chemical and physical characters have recently been carefully examined. It is a silver-white metal, soft, ductile, and compact, melting at $176^{\circ} \mathrm{C}$. Its combining weight is 113.4 $(\mathrm{O}=16)$, and its specific gravity is $7 \cdot 421$; and it forms definite compounds, amongst which the trichloride $\mathrm{InCl}_{3}$, the yellow trioxide $\mathrm{In}_{2} \mathrm{O}_{3}$ and the corresponding nitrate and sulphate, are the most characteristic. Hence you will see that indium belongs to the triad family of metals.

I can here show you the indigo colour which indium compounds impart to the flame; and you now see on the screen that the spectrum of indium consists of two bright indigo-coloured lines, one situated in the blue and one in the violet portion of the spectrum. Another example of the power of spectrum analysis in unravelling the secrets of nature is the discovery by M. Lecoq de Boisbaudran in 1875 of a fifth new metal, and patriotically called by him gallium, because it was first found in zinc blende from the Pyrenees. The spectrum of gallium consists, like that of indium, of two violet lines, but the position of these lines in the spectrum, or, to speak more correctly, their wave-length, differs entirely from that of the indium lines, for whilst the indium lines have the wave-length In a 4,509 tenmillionths of a millimetre, and In $\beta 4101$, the wave-lengths of the gallium lines are 4040 and 4170 respectively. I need scarcely say that there is as yet no notion of any practical employment of any of these new substances, though chemists never can tell what important applications of their most recondite discoveries may not arise even in the immediate future. 


\section{LECTURE II.-APPENDIX A.}

EXTRACT FROM BUNSEN AND KIRCHHOFF'S FIRST MEMOIR ON THE SPECTRUM REACTIONS OF THE SALTS OF THE ALKALIS AND ALKALINE EARTHS. ${ }^{1}$

WE now proceed to describe the peculiarities of the several spectra, the exact acquaintance with which is of practical importance, and to point out the advantages which this new method of chemical analysis possesses over the older processes.

\section{SODIUM.}

The spectrum reaction of sodium is the most delicate of all.

The yellow line $\mathrm{Na}$ a (see Chromolith. Table, No. 7), the only one which appears in the sodium spectrum, is coincident with Fraunhofer's dark line D, and is remarkable for its exactly defined form and for its extraordinary degree of brightness. If the temperature of the flame be very high, and the quantity of the substance employed very large, traces of a continuous spectrum are seen in the immediate neighbourhood of the line. In this case, too, the weaker lines produced by other bodies when near the sodium line are discerned with difficulty, and are often first seen when the sodium reaction has almost subsided.

The reaction is most visible in the sodium salts of oxygen, chlorine, iodine, bromine, sulphuric acid, and carbonic acid. But even in the silicates, borates, pliosphates, and other nonvolatile salts, the reaction is always evident. Swan ${ }^{2}$ has already remarked upon the small quantity of sodium necessary to produce the yellow line.

The following experiment shows that the chemist possesses no reaction which in the slightest degree will bear comparison

1. From Kirchhoff and Bunsen's first Memoir on Analysis by Spectrum Observations (Phil. Mag. vol. xx. 1860).

2 Trans. Roy. Soc. Edin. vol. xxi. part iii. p. 411. 
as regards delicacy with this spectrum-analytical determination of sodium. In a far corner of our experiment room, the capacity of which was about sixty cubic metres, we burnt a mixture of three milligrammes of chlorate of sodium with milk-sugar, whilst the non-luminous colourless flame of the lamp was observed throngh the slit of the telescope. Within a few minutes the flame, which gradually became pale yellow, gave a distinct sodium line, which, after lasting for ten minutes, entirely disappeared. From the weight of sodium salt burned and the capacity of the room, it is easy to calculate that in one part by weight of air there is suspended less than $\frac{3}{2000000}$ of a part of soda smoke. As the reaction can be observed with all possible comfort in one second, and as in this time the quantity of air which is heated to ignition by the flame is found, from the rate of issue and from the composition of the gases of the flame, to be only about 50 cub. cent. or $0.0647 \mathrm{grm}$. of air, containing less than $\frac{1}{2000000}$ of sodium salt, it follows that the eye is able to detect with the greatest ease quantities of sodium salt less than 3000000 of a milligramme in weight. With a reaction so delicate, it is easy to understand why a sodium reaction is almost always noticed in ignited atmospheric air. More than twothirds of the earth's surface is covered with a solution of chloride of sodium, fine particles of which are continually being carried into the air by the action of the waves. These particles of sea water cast thus into the atmosphere evaporate, leaving almost inconceivably small residues, which, floating about, are almost always present in the air, and are rendered evident to our eyesight in the sunbeam. These minute particles perhaps serve to supply the smaller organized bodies with the salts which larger animals and plants obtain from the ground. In another point of view, however, the presence of this chloride of sodium in the air is of interest. If, as is scarcely doubtful at the present time, the explanation of the spread of contagious disease is to be sought for in some peculiar contact-action, it is possible that the presence of so antiseptic a substance as chloride of sodium, even in almost infinitely small quantities, may not be without influence upon such occurrences in the atmosphere. 
By means of daily and long-continued spectrum observations, it would be easy to discover whether the aiterations of intensity in the line $\mathrm{Na} \alpha$ produced by the sodium in the air have any connection with the appearance and direction of march of an endemic disease.

The unexampled delicacy of the sodium reaction explains also the well-observed fact, that all bodies after a lengthened exposure to air show the sodium line when brought into a flame, and that it is only possible in a few salts to get rid of the line even after repeated crystallization from water which had only been in contact with platinum. A thin platinum wire, freed from every trace of sodium salt by ignition, shows the reaction most visibly on allowing it to stand for a few hours in the air: in the same way the dust which settles from the air in a room shows the bright line $\mathrm{Na} a$. To render this evident it is only necessary to knock a dusty book, for instance, at a distance of some feet from the flame, when a wonderfully bright flash of yellow band is seen.

\section{LITHIUM.}

The luminous ignited vapour of the lithium compounds gives two sharply defined lines; the one a very weak yellow line, Li $\beta$ and the other a bright red line, Li $a$. This reaction exceeds in certainty and delicacy all methods hitherto known in analytical chemistry. It is, however, not quite so sensitive as the sodium reaction, only, perhaps, because the eye is more adapted to distinguish yellow than red rays. When nine milligrammes of carbonate of lithium mixed with excess of milk-sugar were burnt, the reaction was visible in a room of sixty cubic metres' capacity. Hence, according to the method already explained, we find that the eye is capable of distinguishing with absolute certainty a quantity of carbonate of lithium less than $\frac{9}{1000000}$ of a milligramme in weight: $0.05 \mathrm{grm}$. of carbonate of lithium, burnt in the sanie room, was sufficient to enable the ignited air to show the red line Li $a$ for an hour after the combustion had taken place. 
The compounds of lithium with oxygen, iodine, bromine, and chlorine are the most suitable for this peculiar reaction; still the carbonate, sulphate, and even the phosphate, give almost as distinct a reaction. Minerals containing lithium such as triphylline, triphane, petalite, lepidolite, require only to be held in the flame in order to obtain the bright line in the most satisfactory manner. In this way the presence of lithium in many felspars can be directly detected; as for instance, in the orthoclase from Baveno. The line is only seen for a few moments, directly after the mineral is brought into the flame. In the same way the mica from Altenburg and Penig was found to contain lithium, whereas micas from Miask, Aschaffenburg, Modum, Bengal, Pennsylvania, \&c., were found to be free from this metal. In natural silicates whıch contain only small traces of lithium this metal is not observed so readily. The examination is then best conducted as follows:-A small portion of the substance is digested and evaporated with liydrofluoric acid or fluoride of ammonium, the residue moistened with sulphuric acid and heated, the dry mass being dissolved in absolute alcohol. The alcoholic extract is then evaporated, the dry mass again dissolved in alcohol, and the extract allowed to evaporate on a shallow glass dish. The solid pellicle which remains is scraped off with a fine knife, and brought into the flame upon the thin platinum wire. For one experiment $-\frac{1}{10}$ of a milligramme is in general quite a sufficient quantity. Other compounds besides the silicates, in which small traces of lithium require to be detected, are transformed into sulphates by evaporation with sulphuric acid or otherwise, and then treated in the manner described.

In this way we arrive at the unexpected conclusion that lithium is most wideiy distributed throughout nature, occurring in almost all bodies. Lithium was easily detected in forty cubic centimetres of the water of the Atlantic Ocean, collected in $41^{\circ} 41^{\prime} \mathrm{N}$. latitude and $39^{\circ} 14^{\prime} \mathrm{W}$. longitude. Ashes of marine plants (kelp), driven by the Gulf Stream on the Scotch coasts, contain evident traces of this metal. All the orthoclase and quartz from the granite of the Odenwald which we have 
examined contained lithium. A very pure spring water from the granite in Schleierbach, on the west side of the valley of the Neckar, was found to contain lithium, whereas the water from the red sandstone which supplies the Heidelberg laboratory was shown to contain none of this metal. Mineral waters, in a litre of which lithium could hardly be detected according to the ordinary methods of analysis, gave plainly the line $\mathrm{Li}$ a even if only a drop of the water on a platinum wire was brought into the flame. ${ }^{1}$ All the ashes of plants growing in the Odenwald on a granite soil, as well as Russian and other potashes, contain lithium.

Even in the ashes of tobacco, in vine leaves, in the wood of the vine, and in grapes, ${ }^{2}$ as well as in the ashes of the crops grown in the Rhine plain near Wäghausel, Deidesheim, and Heidelberg, on a non-granite soil, was lithium found. The milk of the animals fed upon these crops also contains this widely diffused metal. ${ }^{3}$

It is necessary to say that a mixture of volatile sodium and lithium salts gives the reaction of lithium alongside that of sodium with a precision and distinctness which are hardly perceptibly diminished. The red lines of the former substance are still plainly seen when the bead contains io $\frac{1}{1000}$ part of lithium salt, and when to the naked eye the yellow soda flame appears untinged by the slightest trace of red. In consequence of the somewhat greater volatility of the lithium salt, the sodium reaction lasts longer than that of the other metal. In those cases, therefore, in which small quantities of lithium have to be detected in presence of large quantities of sodium salt, the bead must be brought into the flame whilst the observer is looking through the telescope. The lithium lines are often only seen during a few moments amongst the first products of the volatilization.

1 When liquids have to be brought into the flame, it is best to bend the end of the platinum wire, of the thickness of a horsehair, to a small ring, and to beat this ring flat. If a small drop of liquid be brought into this ring, eaough adheres to the wire for one experiment.

${ }_{2}^{2}$ In the manufactories of tartaric acid the mother-liquors contain so much lithium salts that considerable quantities can thus be prepared.

3 Dr. Falwarezny has been able to detect lithium in the ash of human blood and museular tisste by the help of the line Li $\alpha$. 
In the production of lithium salts on the large scale, in the proper choice of a raw material, and in the arrangement of suitable methods of separation, this spectrum analysis affords most valuable aid. Thus it is only necessary to place a drop of mother-liquor from any mineral spring in the flame and to observe the spectrum produced, in order to show that in many of these waste products a rich and hitherto unheeded source of lithium salts exists. In the same way, during the course of the preparation any loss of lithium in the collateral products and residues can be easily traced, and thus more convenient and economical methods of preparation may be found to replace those at present employed. ${ }^{1}$

\section{POTASSIUM.}

The volatile potassium compounds give, when placed in the flame, a widely-extended continuous spectrum, which contains only two characteristic lines,-namely, one line, Ka $a$, in the outermost red, approaching the ultra-red rays, exactly coinciding with the dark line $\mathrm{A}$ of the solar spectrum; and a second line, $\mathrm{Ka} \beta$, situated far in the violet rays towards the other end of the spectrum, and also identical with a particular dark line observed by Fraunhofer.

A very indistinct line, coinciding with Fraunhofer's line B, which, however, is only seen when the light is very intense, is not by any means so characteristic. The violet line is somewhat pale, but can be used almost as well as the red line for the detection of potassium. Owing to the position of these two lines, both situated near the limit at which our eyes cease to be sensitive to the rays, this reaction for potassium is not so delicate as the reaction for the two metals already mentioned. The reaction became visible in the air of our room when one gramme of chlorate of potassium mixed with milk-sugar

I We obtain by such an improved method from two jars (about four litres) of a mother-liquor from a mineral spriug, which by evaporation with sulphuric acid gave 1.2 kil. of residue, half an ounce of carbonate of lithium of the purity of the commercial, the cost of which is about 140 florins the pound. A great number of other mineral-spring mother-liquors which wo examined showed a similar lichness in compounds of lithium. 
was burnt. In this way, therefore, the eye requires the presence of $\frac{1}{1000}$ of a milligramme of chlorate of potassium in order to detect the presence of potassium.

Caustic potash, and all compounds of potassium with volatile acids, give the reaction without exception. Potash silicates, and other non-volatile salts, on the other hand, only produce the reaction when the metal is present in very large quantities. It is only necessary, however, to melt the substance with a bead of carbonate of sodium in order to detect potassium even when present in a very small quantity. The presence of the sodium does not in the least interfere with the reaction, and scarcely diminishes its delicacy. Orthoclase, sanidine, and adularia may in this way be easily distinguished from albite, oligoclase, labradorite, and anorthite. In order to detect the smallest traces of patassium salt, the silicate requires only to be slightly ignited with a large excess of fluoride of ammonium on a platinum capsule, after which the residue is brought into the flame on a platinum wire. In this way it is found that almost every silicate contains potasli. Salts of lithium diminish or influence the reaction as little as soda salts. Thus we need only to hold the end of a burnt cigar in the flame before the slit in order at once to see the yellow line of sodium and the two red lines of potassium and lithium, this latter metal being scarcely ever absent in tobacco ash.

DETECTION BY SPECTRUM ANALYSIS OF THE CONSTITUENTS OF

A MIXTURE CONTAINLNG ALL THE METALS OF THE ALKALIS AND ALKALINE EARTHS.

A mixture of the chlorides of potassium, sodium lithium, calcium, strontium, and barium, containing at the most $\frac{1}{10}$ of a milligramme of each of these salts, was brought into the flame, and the spectra produced were observed. At first the bright yellow sudium line, $\mathrm{Na}$ a, appeared with a background formed by a nearly continuous pale spectrum: as soon as this line began to fade, the exactly defined bright red line of lithium, Li $a$, was seen; and still further removed from the sodium line 
the faint red potassium line, Ka $\alpha$, was noticed; whilst the two barium lines, $\mathrm{Ba} a, \mathrm{Ba} \beta$, with their peculiar form, became visible in the proper position. As the potassium, sodium, lithium, and barium salts volatilized, their spectra became fainter and fainter, and their peculiar bands one after the other vanished, until after the lapse of a few minutes, the lines Ca $a$, $\mathrm{Ca} \beta, \operatorname{Sr} \alpha, \operatorname{Sr} \beta, \operatorname{Sr} \gamma$, and $\operatorname{Sr} \delta$ became gradually visible, and, like a dissolving view, at last attained their characteristic distinctness, colouring, and position, and then, after some time, became pale and disappeared entirely. The absence of any one or of several of these bodies is at once indicated by the nonappearance of the corresponding bright lines.

Those who become acquainter with the various spectra by repeated observation do not need to have before them an exact measurement of the single lines in order to be able to detect the presence of the various constituents ; the colour, relative position, peculiar form, variety of shade and brightness of the bands are quite characteristic enough to ensure exact results even in the hands of persons unaccustomed to such work. These special distinctions may be compared with the differences of outward appearance presented by the various precipitates which we employ for detecting substances in the wet way. Just as it holds good as a character of a precipitate that it is gelatinous, pulverulent, flocculent, granular, or crystalline, so the lines of the spectrum exhibit their peculiar aspects, some appearing sharply defined at their edges, others blended off either at one or both sides, either similarly or dissimilarly; or some again appearing broader, others narrower; and just as in ordinary analysis we only make use of those precipitates which are produced with the smallest possible quantity of the substance supposed to be present, so in analysis with the spectrum we employ only those lines which are produced by the smallest possible quantity of substance, and require a moderately high temperature. In these respects both analytical methods stand on an equal footing; but analysis with the spectrum possesses a great advantage over all other methods, inasmuch as the characteristic differences of colour of the lines serve 
as the distinguishing feature of the system. Most of the precipitates which are valuable as reactions are colourless; and the tint of those which are coloured varies very considerably, according to the state of division and mechanical arrangement of the particles. The presence of even the smallest quantity of impurity is often sufficient entirely to destroy the characteristic colour of a precipitate; so that no reliance can be placed upon nice distinctions of colour as an ordinary chemical test. In spectrum analysis, on the contrary, the coloured bands are unaffected by such alteration of physical conditions, or by the presence of other bodies. The positions which the lines occupy in the spectrum give rise to chemical properties as unalterable as the combining weights themselves, and which can therefore be estimated with an almost astronomical precision. The fact, however, which gives to this method of spectrum analysis an extraordinary importance, is that the chemical reactions of matter thus reach a degree of delicacy which is almost inconceivable. By an application of this method to geological inquiries concerning the distribution and arrangements already mentioned, we are led to the unexpected conclusion, that not only potassium and sodium, but also lithium and strontium, must be added to the list of bodies occurring only indeed in small quantities, but most widely spread throughout the matter composing the solid body of our planet.

The method of spectrum analysis may also play a no less important part as a means of detecting new elementary substances; for if bodies should exist in nature so sparingly diffused that the analytical methods hitherto applicable have not succeeded in detecting or separating them, it is very possible that their presence may be revealed by a simple examination of the spectra produced by their flames. We have had opportunity of satisfying ourselves that in reality such unknown elenents exist. We believe that, relying upon unmistakeable results of the spectrum analysis, we are already justified in positively stating that, besides potassium, sodium, and lithium, the group of the alkaline metals contains a fourth member, which gives a spectrum as simple and characteristic as that of lithium, a metal which in our 
apparatus gives only two lines,-namely, a faint blue one almost coincident with the strontium line $\mathrm{Sr} \delta$, and a second blue one lying a little further towards the violet end of the spectrum, and rivalling the lithium line in brightness and distinctness of outline.

\section{APPENDIX B.}

\section{BUNSEN AND KIRCHHOFF ON THE MODE OF USING A SPECIROSCOPE. ${ }^{1}$}

The apparatus is represented by Fig. 35. On the upper end of a cast-iron foot a brass plate is screwed, carrying the flintglass prism (a), having a refracting angle of $60^{\circ}$. The tube $b$ is also fastened to the brass plate: in the end of this tube nearest the prism is placed a lens, whilst the other end is closed by

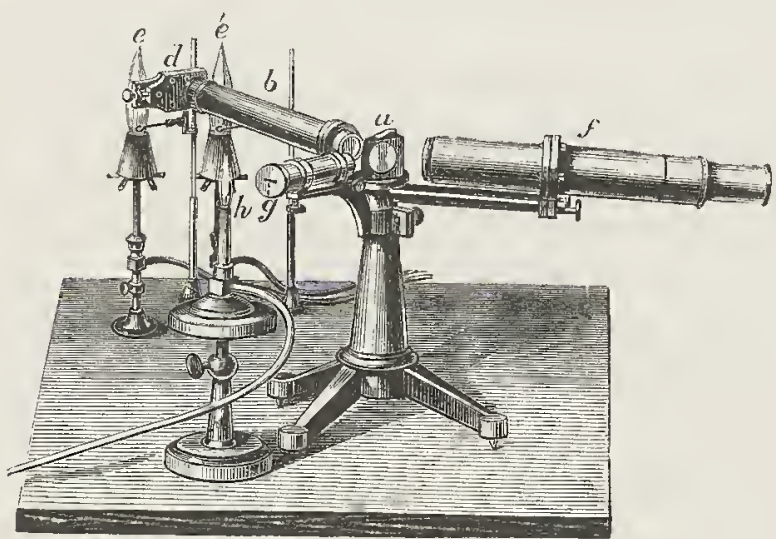

FIG. 35 .

a plate in which a vertical slit has been made. Two arms are also fitted on to the cast-iron foot, so that they are moveable in a horizontal plane about the axis of the foot. One of these arms carries the telescope $(f)$, having a magnifying power of 8 , whilst 498.

1 Second Memoir on Spectrum Analysis, Phil. Mag., vol. xxii. 1861, pp. 334-. 
the other carries the tube $(g)$ : a lens is placed in this tube at the end nearest the prism, and at the other end is a scale which can be seen through the telescope by reflection from the front surface of the prism. This scale is a photographic copy of a millimetre scale, which has been produced in the camera of about $\frac{1}{15}$ the original dimensions. ${ }^{1} \quad$ The scale is covered with tinfoil, so that only the narrow strip upon which the divisions and the numbers are engraved can be seen. The upper half only of the slit is left free, as is seen by reference to Fig. 36; the lower half is covered by a small equilateral glass prism, which sends by total reflection the light of the lamp é, Fig. 35, through the slit, whilst the rays from the lamp e pass

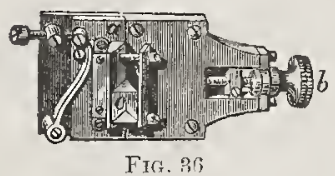
freely through the upper and uncovered half. A small screen placed above the prism prevents any light from é passing through the upper portion of the slit. By help of this arrangement the observer sees the spectra of the two sources of light immediately one under the other, and can easily determine whether the lines are coincident or not. ${ }^{2}$

We now proceed to describe the arrangement and mode of using the instrument.

The telescope $f$ is first drawn out so far that a distant object is plainly seen, and screwed into the ring, in which it is held, care being taken to loosen the screws beforehand. The tube $b$ is then brought into its place, and the axis of $\mathrm{B}$ brought into a straight line with that of $b$. The slit is then drawn out until it is distinctly seen on looking through the telescope, and this latter is then fixed by moving the screws, so that the middle of the slit is seen in about the middle of the field of view. After removing the small spring, the prism is next

1 This millimetre scale was drawn ou a strip of glass, covered with a thin coating of lampblack and wax dissolved in glycerine. The divisions and numbers, which by transmitted light showed bright on a dark ground, were represented in the photograph dark on a light ground. It would be still better to employ, for the spectrum apparatus, a scale in which the marks were light on a dark ground. Such scales are beautifully made by Salleron and Ferrier of Paris.

2 This apparatus was made in the celebrated optical and astronomical atelier of C. A. Steinheil in Munich. 
placed on the brass plate, and fastened in the position which is marked for it, and secured by screwing down the spring. If the axis of the tube $b$ be now directed towards a bright surface, such as the flame of a candle, the spectrum of the flame is seen. in the lower half of the field of the telescope on moving the latter through a certain angle round the axis of the foot. When the telescope has been placed in position, the tube $g$ is fastened on to the arm belonging to it, and this is turned through an angle round the axis of the foot so that, when a light is allowed to fall on the divided scale the image of the scale is seen through the telescope $f$, reflected from the nearer face of the prism. This image is brought exactly into focus by altering the position of the scale in the tube $g$; and by turning this tube on its axis it is easy to make the line in which one side of the divisions on the scale lies parallel with the line dividing the two spectra, and by means of the screw $\delta$ to bring these two lines to coincide.

In order to bring the two sources of light, $e$ and $e$ into position, two methods may be employed. One of these depends upon the existence of bright lines in the inner cone of the colourless gas flame, which have been so carefully examined by Swan. If the lamp e be pushed past the slit, a point is easily found at which these lines become visible; the lamp must then be pushed still further to the left, until these lines nearly or entirely disappear; the right mantle of the flame is now before the slit, and into this the bead of substance under examination must be brought. In the same way the position of the source of light $\varepsilon^{\prime}$ may be ascertained.

The second method is as follows:-The telescope $f$ is so placed that the brightest portion of the spectrum of the flame of a candle is seen in about the middle of the field of view; the flame is then placed before the ocular in the direction of the axis of the telescope, and the position before the slit determined in which the upper half of the slit appears to be the brightest; the lamp $e$ is then placed so that the slit appears behind that portion of the flame from which the most light is given off after the introduction of the bead. In a similar way the position of the 
lamp é is determined by looking through the small prism and the lower half of the slit.

By means of the screw the breadth of the slit can be regulated in accordance with the intensity of the light, and the degree of purity of spectrum which is required. To cut off foreign light, a black cloth, having a circular opening to admit the tube $g$, is thrown over the prism $a$ and the tubes $b$ and $f$. The illumination of the scale is best effected by means of a luminous gas flame placed before it : the light can, if necessary, be lessened by placing a silver-paper screen close before the scale. The degree of illumination suited to the spectrum under examination can then be easily found by placing this flame at different distances.

\section{APPENDIX C.}

\section{BUNSEN ON A METHOD OF MAPPING SPECTRA. ${ }^{1}$}

For the purpose of facilitating the numerical comparison of the data of various spectrum observations, we give in Fig. 37, p. 107, graphical representations of the observations which are taken from the guiding lines given in chromolithograph drawings of the spectra published in our former memoirs, and in which the prism was placed at the angle of minimum deviation. The ordinates of the edges of the small blackened surfaces, referred to the divisions of the scale as abscissæ, represent the intensity of the several lines, with their characteristic gradations of shade. These drawings were made when the slit was so broad, and the flame of such a temperature, that the fine bright line upon the broad Ca $a$ band began to be distinctly visible. This breadth of the slit was equal to the fortieth part of the distance between the sodium line and the lithium line $a$. For the sake of perspicuity, the continuous spectra which some bodies exhibit

1 Phil. Mag., Fourth Series, vol. xxvi. p. 247. 


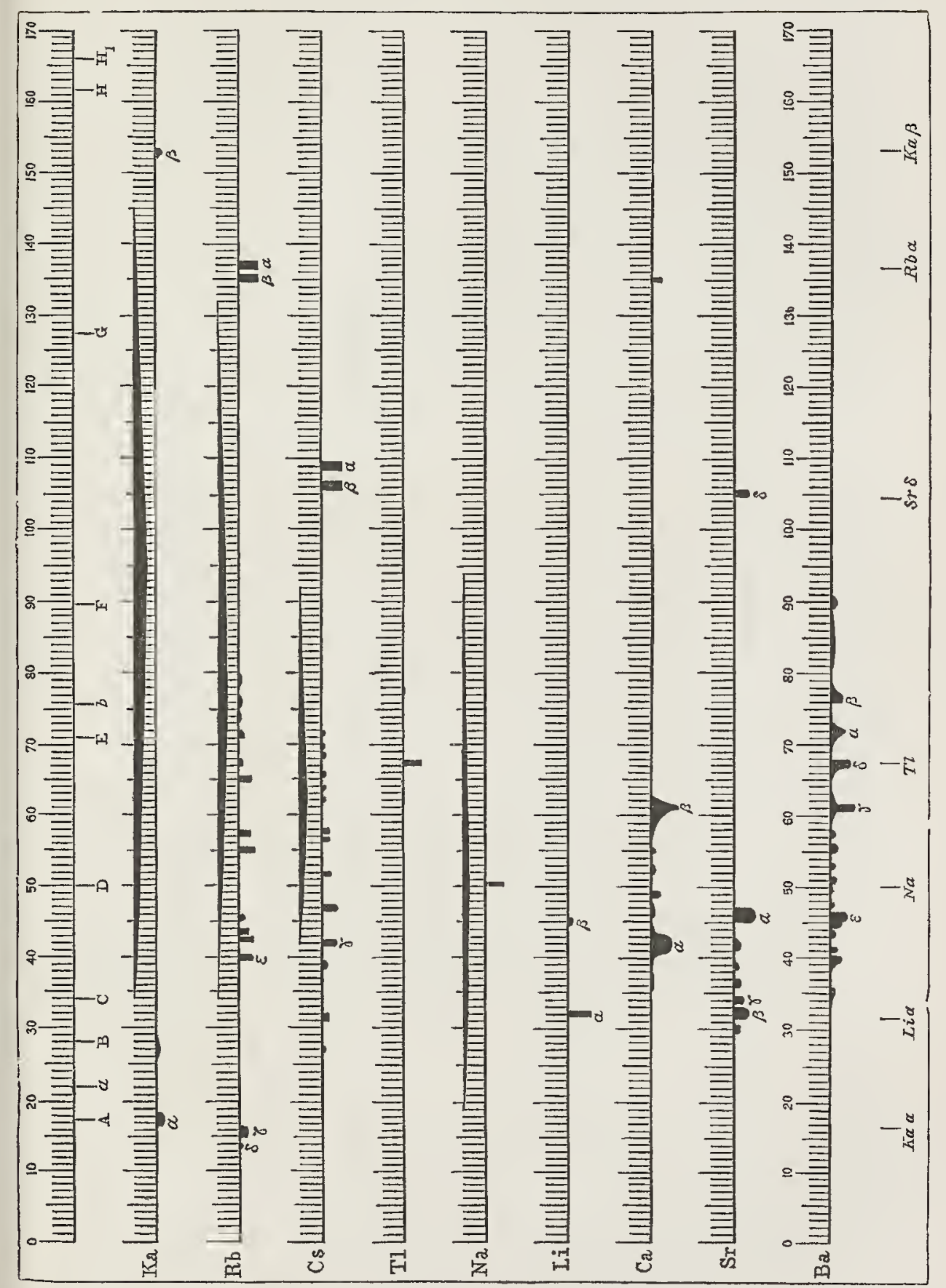


are specially represented on the upper edge of the scale, to the divisions of which they are referred as abscissæ. In order to render these drawings, which have reference to our instrument, applicable to observations upon the scale of any other apparatus, which we may call $\mathrm{B}$, it is only necessary to prepare a reduced scale, which is laid upon the several drawings, and used in place of the divided scale given in the figure. The lines marked at the bottom of Fig. 37 serve for the preparation of this new scale: these lines denote the distances between the lines $\mathrm{K} \alpha, \mathrm{Li} a, \mathrm{Na}, \mathrm{Tl}$, $\operatorname{Sr} \delta, \mathrm{Rb} a$, and $\mathrm{K} \beta$, measured according to the scale of our instrument. The position of each of these lines is determined by the edge of the line, which does not change its place on altering the breadth of the slit. The position of these same lines is read off on the scale of the instrument $B$, and the corresponding number written under each. A series of fixed points on the scale is thus obtained, and the complete divisions for the scale of the instrument $\mathbf{B}$ are got by interpolating the values of the portions of the scale situated between the fixed points.

The sodium line is then inserted in this scale, which is pasted upon a straight-edge, and the divisions numbered in tens and fives. If this measure be now laid upon any one of the drawings, so that the sodium line on the measure coincides with the division 50 on the drawing, the scale on the measure will give the position of all the lines in the particular spectrum exactly as they are seen in the photographic scale of the instrument $B$. When the position of the line under observation has in this way been ascertained, it is easy to assure oneself of its exact identity by means of the small prism on the slit of the spectroscope. 


\section{APPENDIX 1).}

\section{SPECTRUM REACTIONS OF THE RUBIDIUM AND CAEIUM COMPOUNDS. ${ }^{1}$}

Cæsium and rubidium are not precipitated either by sulphuretted hydrogen or by carbonate of ammonium. Hence both metals must be placed in the group containing magnesium, lithium, potassium, and sodium. They are distinguished from magnesium, lithium, and sodium, by their reaction with tetrachloride of platinum, which precipitates them like potassium. Neither rubidium nor cæsium can be distinguished from potassium by any of the usual reagents. All three substances are precipitated by tartaric acid as white crystalline powders; by hydrofluosilicic acid as transparent opalescent jellies; and by perchloric acid as granular crystals: all three, when not combined with a fixed acid, are easily volatilized on the platinum wire, and they all three tinge the flame violet. The violet colour appears indeed of a bluer tint in the case of potassium, whilst the flame of ruhidium is of a redder shade, and that of cæsium still more red. These slight differences can, however, only be perceived when the three flames are ranged side by side, and when the salts undergoing volatilization are perfectly pure. In their reactions, then, with the common chemical tests, these new elements cannot be distinguished from potassium. The only method by means of which they can be recognized when they occur together is that of spectrum analysis.

The spectra of rubidium and cæsium are highly characteristic, and are remarkable for their great beauty (Frontispiece, Nos. 3 and 4 ). In examining and measuring these spectra we have employed an improved form of apparatus (Fig. 35), which in every respect is much to be preferred to that described in our

1 Extract from Professors Kirchhoff and Bunsen's Second Memoir on Chemical Analysis by Spectrum Observations (Phil. Mag., vol. xxii. 1861). 
first memoir. In addition to the advantages of being more manageable and producing more distinct and clearer images, it is so arranged that the spectra of two sources of light can be examined at the same time, and thus, with the greatest degree of precision, compared both with one another and with the numbers on a divided scale.

In order to obtain representations of the spectra of cæsium and rubidium corresponding to those of the other metals which we have given in our former paper, we have adopted the following course :-

We have placed the tube $g$ (Fig. 35) in such a position that a certain division of the scale, viz. No. 100, coincided with Fraunhofer's line $\mathrm{D}$ in the solar spectrum, and then observed the position of the dark solar lines A, B, C, D, E, F, G, H, on the scale: these several readings we called A, B, C, \&c. An interpolation scale was then calculated and drawn, in which each division corresponded to a division on the scale of the instrument, and in which the points corresponding to the observations A, B, C, \&c. were placed at the same distances apart as the same lines on our first drawings of the spectrum. By help of this scale, curves of the new spectra were drawn (Fig. 37, p. 107), in which the ordinates express the degrees of luminosity at the various points on the scale, as judged of by the eye. The lithographer then made the designs represented in the Frontispiece from these curves.

As in our first memoir, so here we have represented only those lines which, in respect to position, definition, and intensity, serve as the best means of recognition. We feel it necessary to repeat this statement, because it has not unfrequently happened that the presence of lines which are not represented in our drawings has been considered as indicative of the existence of new bodies.

We have likewise added a representation of the potassium spectrum to those of the new metals for the sake of comparison, so that the close analogy which the spectra of the new alkaline metals bear to the potassium spectrum may be at once seen. All three possess spectra whicl are continuous in the centre, and decreasing at each end in luminosity. In the case of potassium 
this continuous portion is most intense, in that of rubidium less intense, and in the cæsium spectra the luminosity is least. In all three we observe the most intense and characteristic lines towards both the red and blue ends of the spectrum.

Amongst the rubidium lines, those splendid ones named $\mathrm{Rb} a$ and $\mathrm{Rb} \beta$ are extremely brilliant, and hence are most suited for the recognition of the metal. Less brilliant, but still very characteristic, are the lines $R b \delta$ and $R b \gamma$. From their position they are in a high degree remarkable, as they both fall beyond Fraunhofer's line A; and the outer one of them lies in an ultrared portion of the solar spectrum, which can only be rendered visible by some special arrangement. The other lines, which are found on the continuous part of the spectrum, cannot so well be used as a means of detection, because they only appear when the substance is very pure, and when the luminosity is very great. Nitrate of rubidium and the chloride, chlorate, and perchlorate of rubidium, on account of their easy volatility, show these lines most distinctly. Sulphate of rubidium and similar salts also give very beautiful spectra. Even silicate and phosphate of rubidium yield spectra in which all the details are plainly seen.

The spectrum of cæsium is especially characterized by the two blue lines Cs $\alpha$ and Cs $\beta$ : these lines are situated close to the blue strontia line $\operatorname{Sr} \delta$, and are remarkable for their wonderful brilliancy and sharp definition. The line Cs $\delta$, which cannot be so conveniently used, must also be mentioned. The yellow and green lines represented on the figure, which first appear when the luminosity is great, cannot so well be employed for the purpose of detecting small quantities of the cæsium compounds; but they may be made use of with advantage as a test of the purity of the cæsium salt under examination. They appear much more distinctly than do the yellow and green lines in the potassium spectrum, which, for this reason, we have not represented.

As regards distinctness of the reaction, the cæsium compounds resemble in every respect the corresponding rubidium salts: the chlorate, phosphate, and silicate gave the lines perfectly clearly. The delicacy of the reaction, however, in the case of 
the crsium compounds, is somewhat greater than in that of the corresponding compounds of rubidium. In a drop of water weighing four milligrammes, and containing only 0.0002 milligramme of chloride of rubidium, the lines $\mathrm{Rb} a$ and $\mathrm{Rb} \beta$ can only just be distinguished; whilst 0.00005 milligramme of the chloride of cæsium can, under similar circumstances, easily be recognized by means of the lines $\mathrm{Cs}_{\mathrm{s}} a$ and $\mathrm{Cs} \beta$.

If other members of the group of alkaline metals occur together with cæsium and rubidium, the delicacy of the reaction is of course materially impaired, as is seen from the following experiments, in which the mixed chlorides contained in a drop of water, weighing about four milligrammes, were brought into the flame on a platinum wire.

When 0.003 milligramme of chloride of cæsium was mixed with from 300 to 400 times its weight of the chloride of potassium or sodium, it could be easily detected. Chloride of rubidium, on the other hand, could be detected with difficulty when the quantity of chloride of potassium or chloride of sodium amounted to from 100 to 150 times the weight of the chloride of rubidium employed.

0.001 milligramme of chloride of cæsium was easily recognized when it was mixed with 1,500 times its weight of chloride of lithium; whilst 0.001 milligramme of chloride of rubidium could not be recognized when the quantity of chloride of lithium added exceeded 600 times the weight of the rubidium salt. 


\section{LECTURE III.}

Spectra of the Heavy Metals.-Examination of the Light of the Electric Discharge.-Faraday.-Wheatstone.-Volatilization of Metals in the Electric Arc._Kirchlıofi, Ångströın, Thalén, and Huggins.-Stokes's Ultra-violet Rays. - Spectra of the Non-metallic Elements-Line Spectra and Spectra of Fluted Bands - Connection between these Spectra and the Molecular Constitution of the Elements.-Lockyer's Method of Examining the Spectra.-Long and Short Lines.-Widening of the Lines. -Spectra of Compound Bodies.-Conclusion.

A ppendix A.-Contributions towards the History of Spectrum Analysis. By G. Kirchliofi.

Appexdix B.-On the Effect of Increased Temperature upon the nature of the light emitted by the vapour of certain Metals or Metallic Compounds; by H. E. Roscoe and R. B. Clifton (1862). Kirchhoff on the Variation of the Spectra of certain Elements (1862).

Appendix C.- On Changes in the Spectrum of Oxygen. By Professor Arthur Schuster, F.R.S.

APPENDIX D.-On the Influence of Temperature and Pressure on the Spectra of Gases ; being an abstract of a Report presented to the British Association in 1880 by Professor Schuster, F.R.S.

Appendix E.--On the Genesis of Spectra. Abstract of a Report drawn up by Professor Schuster for the British Association in 1882.

THE subject to which I would wish to-day in the first place to direct your attention, is the mode by which we can determine by spectrum analysis the presence of metals proper, or heavy metals. How, for instance, can we ascertain the presence of copper, or of gold, or of silver, or of zinc, or of iron? how can we volatilize these metals so as to make them give off the light which is peculiar to each one? I have here the means of doing this. We have again to employ our most valuable agent, electricity. By means of this battery and induction coil I can obtain an electric spark; 
and by means of the electric spark I can get wliat I require, namely, the volatilization of these metals. It is many years since the application of the electric spark to this particular branch of analysis was discovered. The first person who examined the nature of the electric spark was Wollaston, whose name I mentioned to you in my opening lecture as having first pointed out the existence of these very important dark lines in the solar spectrum. But it was Faraday who first declared that the electric spark consists solely of material particles. He speaks, in his experimental researches, of the electric spark as being produced by a current propagated along, and by, ponderable matter, and heated in the same manner, and according to the same laws, as a voltaic current heats and volatilizes a metallic wire. So that what we see and call the spark is really the ignition of the matter which lies in its path, and which we now know to be partly due to the electrodes. When we reflect how short a space of time the spark lasts we shall understand with how great a velocity these particles are driven forward, and we have then only to remember that a great velocity implies a high temperature, in order that we may be able to account for the brightness of the spark. If this be so, it is evident that, when we bring certain different metals in this arc, we must obtain different-coloured sparks : thus, if I bring a small quantity of strontium salt into the spark, we shall have a very intensely red light, due to the ignition of the peculiar body strontium which is volatilized between the poles; and when I take some thallium, we have the green colour characteristic of this metal.

If we examine the light of such a spark with a spectroscope, we shall find that two superimposed spectra here present themselves; the one spectrum produced by the parts lying close to the poles, and the other by the central portion of the spark. The spectrum of the bright points is, as we shall see, chiefly that of the metal present, whilst the light from the less luminous portion in the centre exhibits the spectrum of the incandescent air, and shows the particular lines produced by the gases existing in the atmosphere, viz. nitrogen, oxygen, and hydrogen (for in the atmosphere we have constantly the vapour of water present). 
Each gas gives us lines peculiar to itself; and in some cases, when the quantity of carbonic acid present in the air is considerable, we may even get the carbon lines.

It was Sir Charles Wheatstone, in the year 1835, who first pointed out that the spectra produced from the sparks of different metals were dissimilar; and he concluded that the electric spark resulted from the volatilization, and not from the combustion, of the matter of the poles themselves, for he observed the same phenomena in vacuô and in hydrogen, in which no combustion can occur; and in 1835 he writes as follows: "These differences are so obvious, that one metal may easily be distinguished from another by the appearance of its spark; and we have here a mode of discriminating metallic bodies more readily than that of chemical examination, and which may hereafter be employed for useful purposes."

You have here a copy of the diagram (Fig. 38) published in Wheatstone's paper, ${ }^{1}$ giving the lines which he saw in the metals. Subsequent research has shown that the number of the lines peculiar to each of these metals is very large, although on Wheatstone's diagram but a few of these are noticeable. On this drawing you see some of the bright lines of the metals mercury $(\mathrm{Hg})$, zinc $(\mathrm{Zn})$, cadmium $(\mathrm{Cd})$, bismuth (Bi), tin ( $\mathrm{Sn})$, and lead $(\mathrm{Pb})$. The letters placed above and below each bright line indicate its degree of intensity: very bright, bright, faint, very faint.

It was, however, chiefly through the experiments of the Swedish philosopher Angström, that we gained an intimate knowledge of the nature of the electric spark. In the year 1855 Angström investigated the matter very thoronghly, and pointed out the important fact which I have explained, that the spark yields two superimposed spectra; one derived from the metal of the poles, and the other from the gas or air through which the spark passes.

Perhaps I had better show you, first of all, the beautiful spectra of some of these metals, as I can exhibit them to you on

I For a reprint of Wheatstone's paper see Chemical News, vol. iii. p. 198 (1861). 
a screen; and then explain to you the nature of the spectra which we see when we look at the spark through such a train of prisms as you have in the large spectroscope on the table (see Fig. 39). I cannot show you these lines on the screen with anything like the amount of accuracy or delicacy with which we can see them when we throw the image on the retina itself, for then we notice

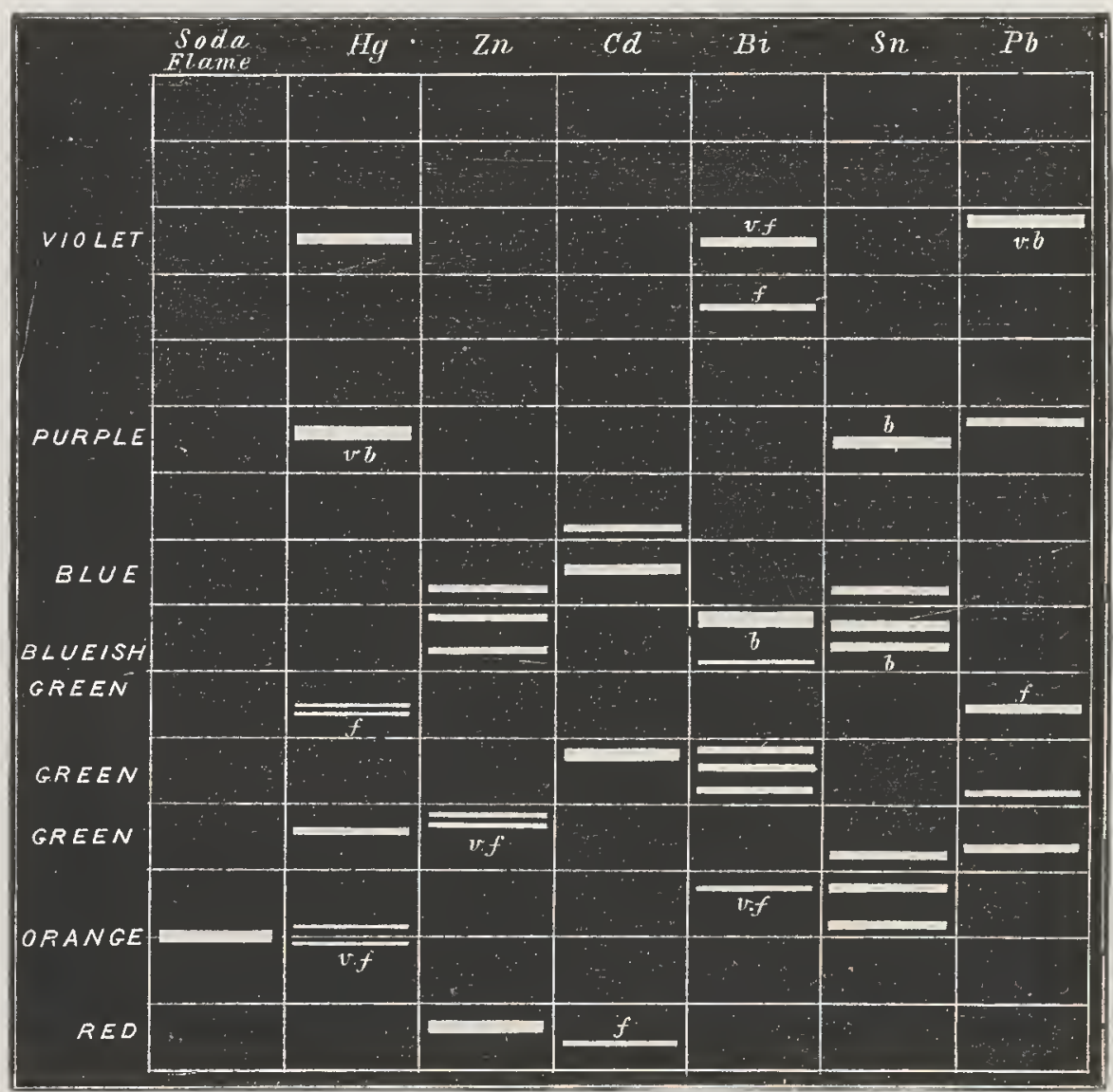

FIG. 33.

the true spectra. The lines then observed are excessively fine and extremely numerous, and each line possesses a fixed position, and does not interfere with the lines of any other metal. Still I can show you something very beantiful and interesting. I will endeavour by means of the electric lamp to throw the spectrum 
of metallic copper on the screen. I take a small piece of metallic copper and volatilize it between the incandescent carbon poles, and then you will see the green bands indicative of the presence of this metal. Here you observe these magnificent green bands, which are characteristic of copper; but when we examine the copper spark by throwing the image into the eye, we get a much more splendid effect, and see the way to a far more delicate method of detecting the presence of copper In the next place I will take another carbon pole, and bring a small piece of zinc into it, and we shall see that zinc also gives

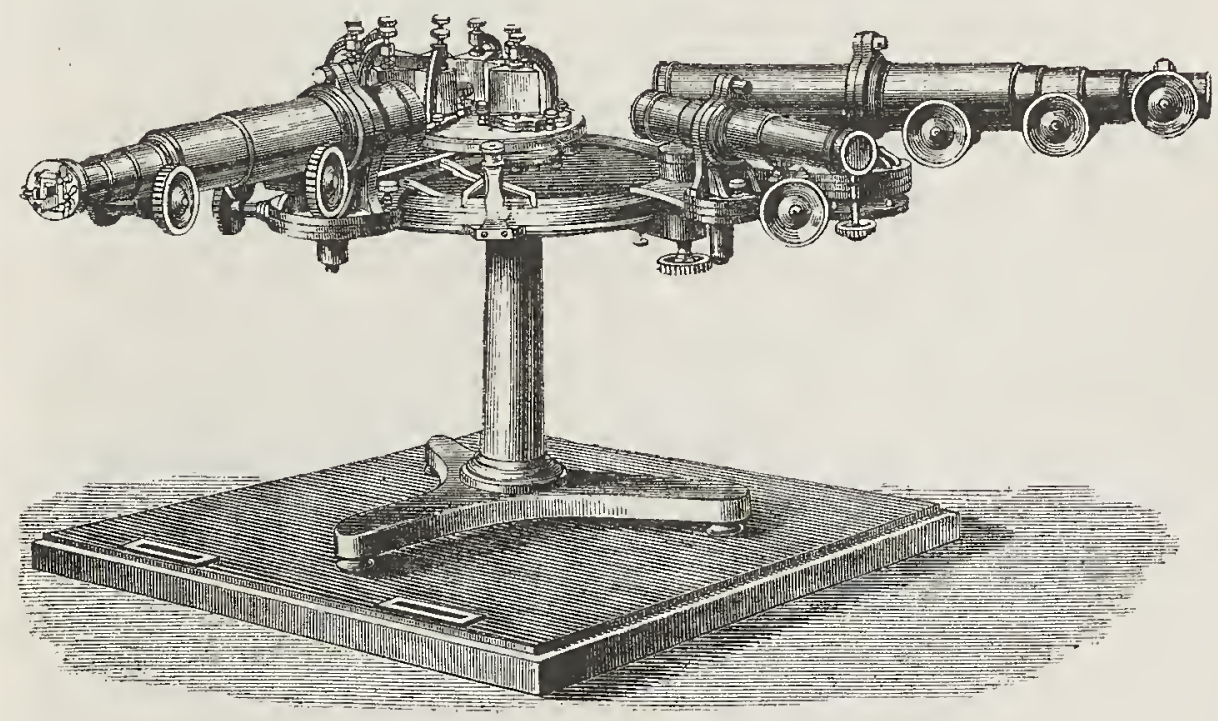

FIG. 39.

its peculiar and beautiful lines perfectly characteristic of this special metal. If we examine this light by means of an accurate spectroscope, these broad bands are seen to consist of masses of bright lines, each one as fine as the most gauzy spider's web.

If I now take a mixture of zinc and copper, such as brass, we shall not only get the lines of the zinc, but we shall also see the bright copper lines. I have put a small piece of brass on the pole, and when I make the contact I shall volatilize this brass and the result is a spectrum showing both the copper lines and 
the zinc lines. You will notice that what I have said with respect to the other metals, the alkaline earths, holds good with this, viz.: that the most volatile of the metals burns out first. Now we can still see the less volatile copper, but the zinc lines have died away. In the sme way I may show you that cadmium gives us a peculiar set of lines. If we volatilize some metallic cadmium, we shall have a series of lines somewhat resembling those of zinc, but not identical with them. There you observe three bands: these are the cadmium lines, something perfectly characteristic and distinct.

Fox Talbot observed these metallic lines in June 1834, by deflagrating thin sheets of metal by a powerful electric current; he says: "Gold leaf and copper leaf each afforded a fine spectrum exhibiting peculiar definite rays. The effect of zinc was still more interesting: I observed in this instance a strong red ray, three blue rays, besides several more of other colours."

I will next show you the spectrum of silver. Here you observe those splendid green lines, and the beautiful purple lines in the distance: the latter are only visible when you look at the most refrangible end for some time. Nothing, surely, can be more magnificent than these spectra! These green lines are quite different in position and in character from the green copper lines. To exhibit that difference to you, I will put a bit of copper into this silver-which is chemically pure-and I think you will be able to see that we get the green copper lines distinctly arranged alongside of the green lines of silver. Thus, then, by means of the electric lamp, many of these limes can be rendered visible, although to see others distinctly we must employ a delicate spectroscope, and throw the light directly into the eye of the observer.

By the examination of the spark-spectrum, chemists are now able to distinguish with the greatest ease between the rarest metals. We are able to detect the difference between erbium and yttrium, and didymium and lanthanum-metals which resemble one another in their properties so closely that it is extremely difficult to separate them from each other by ordinary chemical means. These substances all give distinct lines, and 
any one of these substances may be detected when mixed with the other: and we thus get a decisive answer as to their presence.

In order to examine with accuracy the spectra of the heavy metals, an arrangement, represented in Fig. 40, is necessary. This consists of a powerful induction coil used in conjunction with a delicate spectroscope, such as that used by Kirchhoff (see page 103). The light from the spark falls on to the slit, and is refracted by passing through the prisms.

For the purpose of intensifying the spark, the ends of the secondary coil are placed in contact with the coatings of a large Leyden jar. The electrodes, also of course connected with the

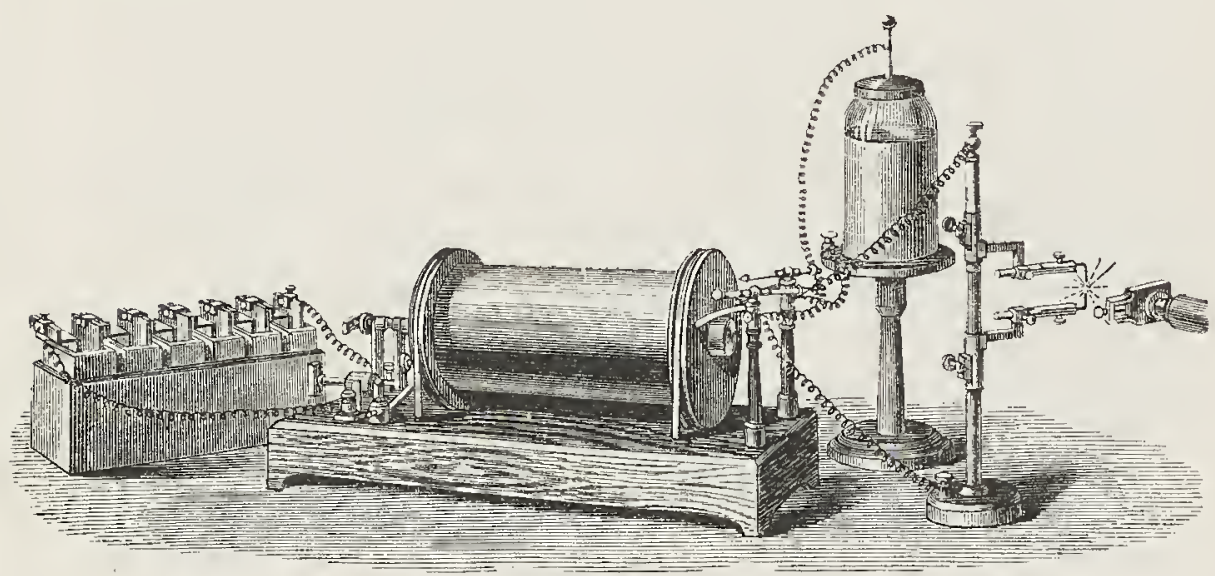

Fig, 40 .

poles of the secondary coil, consist of the metals under examination, either in the form of wire, or of irregular pieces held by forceps on a movable stand. Many precautions must be taken, especially with two sets of electrodes, as it has been found that currents caused by the rapid passage of air between the poles are sufficient to carry over to a second set of electrodes, placed at a distance of a few inches, a very perceptible quantity of the materials undergoing volatilization.

We are indebted to the labours of Professors Kirchhoff Angström, and Thalén, Lecoq de Boisbaudran, and Dr. Huggins, for the most accurate sets of maps of the metallic lines which 
we possess. The positions of the metallic lines have been arranged by Kirchhoff with reference to the dark solar lines, whilst Huggins has used the bright air lines as a constant scale upon which to note the positions of the metal lines; but both experimenters use an arbitrary scale of divisions by which the lines are designated. Owing to the very large number of the lines of each of the metals, very great care is needed in the discrimination of these spectra; still, when the eye has been trained, the detection of the individual metal is perfectly certain. The spectra of the following elements were mapped by Kirchhoff :-
1. Sodium.
9. Strontium.
17. Antimony.
25. Aluminium.
2. Calcium.
10. Cadmiun.
18. Arsenic.
26. Lead.
3. Barium.
11. Nickel.
19. Cerium.
27. Silver.
4. Magnesium.
12. Cobalt.
20. Lanthanun.
28. Gold.
5. Iron.
13. Potassium.
21. Didymium.
29. Ruthenium.
6. Copper.
14. Rubidium.
22. Mercury.
30. Iridium.
7. Zinc.
15. Lithium.
23. Silicon.
31. Platinum.
8. Chromium.
16. Tin.
24. Glucinum.
32. Palladium.

The spectra of the following metals have been drawn by Huggins :-

1. Sodium.

7. Thallium.

13. Antimony.

19. Lead.

2. Potassium.

8. Silver.

14. Gold.

20. Zinc.

3. Calcium.

9. Tellurinm.

15. Bismuth.

4. Barium.

10. Tin.

16. Mercury.

5. Strontium.

11. Iroul.

17. Cobalt.

6. Manganese.

12. Cadmium.

18. Arsenic.

21. Chronium.

22. Osmium.

23. Palladium.

24. Platinum.

A very interesting fact is noticed by all the observers, namely that several of the bright lines of different metals seem to coincide. When, however, these cases of apparent coincidence are narrowly observed, most of the lines are found to show real though slight differences of refrangibility.

Thalén has also published ${ }^{1}$ an interesting memoir upon the determination of the wave-lengths of the lines of the metal spectra. In Kirchhoff's and Huggins' maps the bright metal lines are arranged according to an arbitrary scale, but it is of great interest to know the absolute position of each line,

1 Aunales de Chemie et de Physiqque, Oct. 1869, sér. [4], vol. xriii. p. 202. 
and this can only be ascertained by the determination of its wave-length. ${ }^{1}$ The spectra of no less than forty-five metals are given in Thalén's tables, and amongst them are those of many rare metals not previously examined; thus we find the lines of the spectra of zirconium, erbium, yttrium, thorium, uranium, titanium, tungsten, molybdenum, and vanadium, most accurately mapped. You may judge of the accuracy and value of this method of examination from the fact that each of these rare metals possesses a distinct, and numerons set of lines, none of which lines coincide with any belonging to another element, and each of which may therefore be considered as a separate test of its presence.

Thus the extremely rare metal vanadium yields the following bright lines according to Thalén :-

\section{SPECTRUM OF VANADIUM.}

$$
\begin{gathered}
\text { WAVE IENGTH. } \\
\text { Orange }\left\{\begin{array}{l}
6240 \cdot 5 \\
6134 \cdot 4 \\
6119 \cdot 0 \\
6109 \cdot 5 \\
6089 \cdot 0 \\
6080 \cdot 0 \\
6039 \cdot 0
\end{array}\right.
\end{gathered}
$$

Violet, $4110 \cdot 0$

$$
\text { Blue }\left\{\begin{array}{l}
4881 \cdot 0 \\
4874 \cdot 5 \\
4864 \cdot 0 \\
4851 \cdot 0 \\
4843 \cdot 0 \\
4831 \cdot 5 \\
4593 \cdot 0 \\
4585 \cdot 0 \\
4579 \cdot 0 \\
45760^{\circ} 0
\end{array}\right.
$$

\begin{tabular}{|c|c|c|c|}
\hline WAVI & LENGT H, ${ }^{2}$ & WAVE & ENGTH. ${ }^{2}$ \\
\hline & $5786^{\circ} 0$ & & 52400 \\
\hline & $5725 \cdot 0$ & & $5233 \cdot 0$ \\
\hline & $5706 \cdot 0$ & Green & $5195 \cdot 0$ \\
\hline & $5702 \cdot 5$ & & $5191 \cdot 5$ \\
\hline & $5697 \cdot 5$ & & \\
\hline Yellow & $5668 \cdot 0$ & & \\
\hline & $5626^{\circ} 0$ & & \\
\hline & $5622 \cdot 5$ & & \\
\hline & $5414 \cdot 0$ & & \\
\hline & $5401^{\circ} 0$ & & \\
\hline & $4459 \cdot 0$ & & $4332 \cdot 5$ \\
\hline & $4407 \cdot 5$ & & $4329 \cdot 5$ \\
\hline & $4406^{\circ} 0$ & & $4310 \cdot 0$ \\
\hline & $4400 \cdot 5$ & & $4297^{\circ} 0$ \\
\hline & $4395^{\circ} 0$ & Indigo & $4292 \cdot 5$ \\
\hline Indigo & $4389 \cdot 0$ & & $4283 \cdot 5$ \\
\hline & $4384^{\circ} 0$ & & $4277 \cdot 0$ \\
\hline & $4379 \cdot 0$ & & $4272 \cdot 0$ \\
\hline & $4352 \cdot 5$ & & $4268 \cdot 5$ \\
\hline & 4340.5 & Violet & $4110^{\circ} 0$ \\
\hline
\end{tabular}

1 This has been accomplished for the above measurements by Dr. Marshall Watts in his Index of Snectra.

2 In ten-millionths of a millimetre. 
To give you a notion of the immense number of these lines, I should like to show you a drawing or two: for this purpose I will throw on the screen an enlarged image of one of Kirchhoff's maps. Professor Kirchhoff was the first to examine the exact character of these metallic lines, and he drew an accurate map, not only of these metallic lines, but of the dark lines in the spectrum of the sun; and this is a copy of one of his diagrams, to which I would now briefly allude. The dark lines here represent the dark lines in the sun. With these I have at present nothing to do. We shall devote a subsequent lecture to a detailed discussion of this most remarkable subject. To-day I would simply draw your attention to the short lines at the lower part of the diagram, which indicate to us the positions of the bright metal lines with regard to the fixed dark solar lines, these latter being taken as a sort of inch-rule, by which the positions of the other lines are reckoned. The lines which you see joined by a horizontal line, and marked $\mathrm{Fe}$ (for Ferrum), are the iron lines; and I beg you to notice the very large number and the very beautifully fine nature of these iron lines. On Kirchhoff's map each line is accompanied by a letter, being the chemical symbol of the element to which this one belongs; liere an aluminium line, here an antimony line, here a calcium line, here again a number of iron lines connected together; and so I might go through all these diagrams, showing the number of lines which Kirchhoff has mapped-and this for only a small portion of the spectrum. The one end of this diagram is in the yellow, and the other end in the green; so that we have, on this map, only a very, very small portion of the metal lines which would be seen if we were looking at the whole length of the visible spectrum.

I would next illustrate this fact by showing you another beautiful drawing of these metal lines, made by our countryman, Dr. Huggins; this map which is copied from Dr. Huggins' paper in the Philosophical Transactions for 1864, will also give you an idea of the very great number of these metal lines. We lave here about twenty metals, and each pair of these horizontal lines includes the spectrum of a particular 
metal. For instance, if we take silver, here is one line, here three, here two, here a number of other lines: thus we go on through the whole spectrum, and have altogether a great number of silver lines. On the right we have the red end, on the left the blue end of the spectrum, and at the top, for the sake of comparison, are the chief lines of the solar spectrum and the air lines. From this table you may not only form an idea of the large number of metal lines existing, but you will see that the lines of any one metal do not coincide or interfere with those of any other. For facilitating spectroscopic research, Dr. Marshall Watts has compiled a most useful set of tables in his Index of Spectra, in which all the reliable observations of the positions of the bright lines are mapped according to their wave-lengths.

The lines, which are thus easily visible, are by no means all the peculiar rays which such highly heated metallic vapours emit, for Professor Stokes was the first to show that the bright sparks from poles of iron, aluminium, and magnesium give off light of so high a degree of refrangibility, that distinct, bands are situated at a distance beyond the last visible violet ray, ten times as great as the length of the whole visible spectrum from red to violet! These bands cannot of course be seen under ordinary circumstances, but when allowed to fall on a fluorescent body, such as paper moistened by quinine solution, they can easily be rendered visible; or we may photograph them, and make them leave their impression on the sensitive film. In order that these highly refrangible rays may be seen, no glass lenses or prisms must be used, as the rays of the highest refrangibility cannot pass through glass; quartz, on the other hand, permits them to pass; hence all the lenses and prisms must be made of quartz.

I now pass to the description of spectra of the non-metallic elements and of the methods employed for obtaining them. We have seen that the light of the electric spark consists of rays emitted from the incandescent materials, first, of the poles from which the discharge passes, and in the second place, of the air or gas surrounding those poles. If the density of the 
gas through which the discharge passes be diminished to a certain point within a few millimetres of a vacuum, the electricity is able to pass through a longer column of gas than it can do under the ordinary atmospheric pressure; and we thus may obtain this beautiful phenomenon of the discharge in vacuó, for we see this tube ten feet long, on being rendered nearly vacuous by the air-pump, becomes filled with purple light; though if the whole of the gas be withdrawn, no electrical discharge whatever can pass. The character of the light thus emitted depends then upon the chemical nature of the gas through which the electric spark or discharge passes.

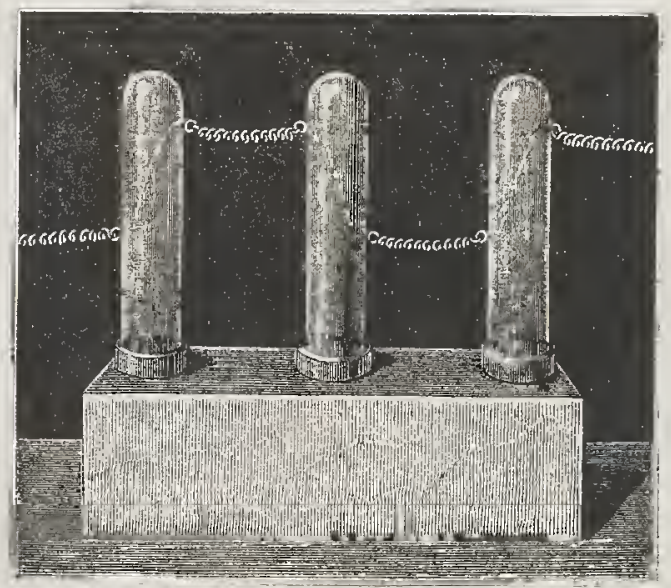

FIG. 41

If we seal up a quantity of hydrogen gas, of carbonic acid gas, and of nitrogen gas, in separate tubes and allow an electric spark to pass through these tubes (see Fig. 41), the spark which passes through the hydrogen has a red colour, and that which passes through the nitrogen has a yellow colour, while that which passes through the carbonic acid gas has a blue colour; and these differences of colour are due sinuply to the presence of the gas inclosed in the tube. I can vary the experiment by taking Geissler's tubes (Fig. 42), containing these gases only in very minute quantities, so that the electric discharge can pass through a longer capillary column of gas; 
we then find that the small quantity of gas in the exhauster tube becomes heated up to incandescence, and gives off its peculiar rays in lines of brilliantly coloured light.

I have here a hydrogen vacuum tube, next a tube containing a carbonic acid vacuum, then one containing nitrogen, then one containing chlorine, then one containing iodine. I have only to connect these with the induction coil, and the discharge will pass through the whole of these tubes; and at once you see the variety of bright colours obtained, entirely due to the small traces of the various gases which are here present in the tubes. If we examine the character of these lights by means of the spectroscope, we shall obtain the peculiar and characteristic spectra of each of these gases.

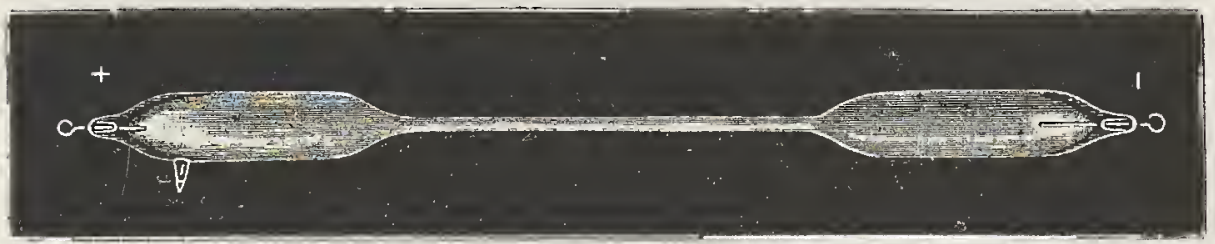

FIC, 42 .

Here are some large tubes, in which we can see the same effects of the ignition of the small quantities of these various gases by means of the electric spark; and you observe the beautiful striated appearance which the light exhibits-a phenomenon which physicists are at present quite unable to explain. Figs. 43 and 44 are copies of photographs of the discharge in vacuum tubes taken by Mr. De la Rue and Dr. Müller.

I regret that it is impossible to exhibit the spectra of these luminous gases on the screen, owing to the slight intensity of the light which they emit. I must ask you to be content with my references to diagrams to explain to you the exact character of the light which these gases give off.

Thus when we examine the peculiar red colour which this hydrogen-tube exhibits, we find that the spectrum consists of four distinct bright lines; one bright red line so intense as almost to overpower the others, one bright greenish-blue line 
and one dark blue or indigo line, whilst a fourth line appears in the violet, when the temperature of the gas is very high. These are exhibited to you in the diagram. (See Fig. of hydrogen spectrum, No. 8 on the chromolith. plate facing Lecture VI.) The bright red hydrogen line is always seen when an electric spark is passed through moist air : this is due to the decomposition of the aqueous vapour which the air contains. If the air

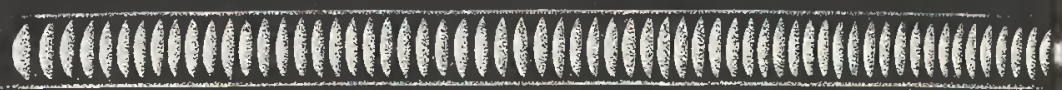

FIG.. 43

be carefully dried by passing it over hygroscopic substances, the red line disappears. Hence the spectroscope can be made a means of testing the presence of moisture.

A very remarkable fact, and one to which I shall have frequently to refer in the subsequent lectures, is that these four lines of hydrogen are found to be coincident with four well-known dark lines in the sun, of which I spoke to you in

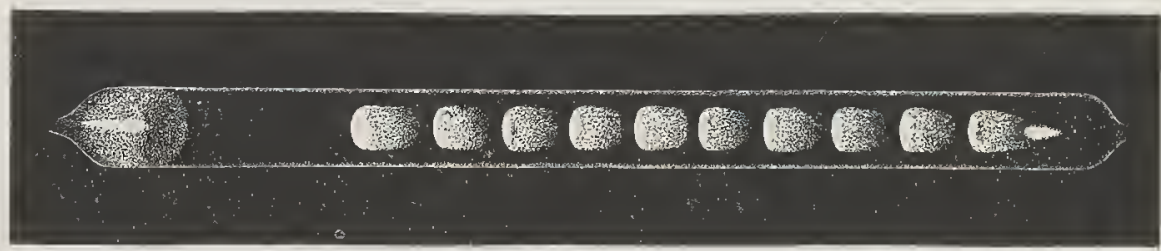

Frit 44.

the first lecture. This red hydrogen line possesses exactly the same degree of refrangibility as the dark line $\mathrm{C}$ in the solar spectrum; the green hydrogen line corresponds to the wellknown solar line $\mathrm{F}$; whilst the blue hydrogen line is identical in position with a dark line near $\mathrm{G}$ in the sun's spectrum, and the solar line coincident with the fourth line has been called (h) by Angström. We shall see in a subsequent lecture how such 
coincidences point out to us the existence of hydrogen and other elements in the solar atmosphere.

When a spark is passed through the air, the lines of both nitrogen and oxygen are seen. This air spectrum has been carefully mapped by Dr. Huggins, who employed it as a scale to which to refer the metal lines in his drawings. He observed the lines simultaneously given off from two sets of poles, one set being of gold and the other set of platinum (in order to eliminate any confusion arising from the presence of metallic lines): and he took those lines which were common to both these spectra as being those due to the components of the air. The spectrum thus obtained remains perfectly constant with reference to the position and relative characteristics of its lines when other metals are employed as electrodes. It is, however, found that the air spectrum varies as a whole in distinctness according to the volatility of the metal used as poles, the air being more or less replaced by metallic vapours in the neighbourhood of the electrodes. The bright hydrogen lines due to aqueous vapour are, as I have said, seen when the spark is passed through moist air, whilst the spectrum of lightning, as examined by Grandeau and Kundt, has been found to exhibit in addition to the nitrogen and hydrogen spectra, also the bright yellow sodium line.

The nitrogen spectrum is more complicated than that of hydrogen, but still perfectly definite and characteristic. (See No. 9 on the chromolith. plate in Lecture VI.)

In looking at these Geissler's tubes, whilst an electric current is passing, you may have noticed, that the colour of the spark varies in different parts of the tubes, and I might also have considerably altered it by changing the intensity of the current which in fact implies a change of temperature. These and similar appearances led Plïcker and Hittorf some years ago ${ }^{1}$ to results of considerable importance. They found, for instance, that the spectrum of nitrogen is altogether changed when the intensity of the current is sufficiently altered, and they explained this by supposing that nitrogen can exist in various allotropic

${ }^{1}$ Phil. Trans. 1865, 1. 
conditions resembling the cases of ozone and oxygen, their idea being, that changes in the intensity of the electric discharge may cause changes in the chemical condition of nitrogen, thus bringing about a variation in the appearance of the spectrum. A gool deal of discussion has since taken place, both as to the facts discovered by the above observers and as to their explanation, but spectroscopists at the present time generally accept their conclusions.

Pliicker and Hittorf believed that different types of spectra exist, and they spoke of spectra of the first and of the second order. These two types may, however, be more appropriately distinguished by the designation of "line" spectra and spectra of "fluted bands." The spectra of fluted bands when examined by spectroscopes of small dispersive powers appear to be made up of bands having a sharp boundary on one side and gradually fading away on the other. When observed by more powerful instruments, each band is seen to consist of a number of lines of nearly equal intensity which gradually approach one another as the sharp edge is reached. This sharp edge is seen where the lines appear to be so closely ruled that we can no longer distinguish the individual components. The edge is situated sometimes towards the red and sometimes towards the violet end of the spectrum. Occasionally, however, the fluted bands do not show any sharp edge whatever, but are simply made up of a number of lines which are, roughly speaking, equidistant. Fig. 45 shows a spectrum of fluted bands or, as they are sometimes called, of channelled spaces, when seen in a spectroscope of one prism only. It is the spectrum of nitrogen, as mapped by Lecoq de Boisbaudran, in his admirable charts of spectra. The wave-lengths of the different bands can readily be ascertained by reference to the upper half of the figure, the scale of which gives wave-lengths, and on which the position of the brightest parts of each band is indicated. It will bc seen, from the numbers on Figs. 45 and 46 , that the red end of the spectrum is placed on the left hand of the drawing, contrary to the prevailing practice.

In like manner the fluted spectrum of carbon as seen with one 
prism (Fig. 46) is found to be conposed of a number of bright lines when examined with a high power. This is well shown in the photograph obtained by Mr. Lockyer of the ultra-violet bands given by the carbon points of the electric arc (Fig. 47).

As an example of a band with no sharp erge seen under high dispersion we give in Fig. 48 a drawing of one of the bands appearing in tubes filled with oxygen near the negative pole.

The second type of spectra are the line spectra as pu'cviously described.

I have alrearly mentioned that the different spectra of one and the same element are attributed to different allotropic conditions; and the fact that compound bodics nearly always give us spectra of fluted bands poirts strongly to the conclusion that this type is due to a more complex molecular arrangement than the line spectrum. I have also stated that solid and liquid bodies generally give us continuous spectra, but there are exceptions to this rule, and even gases often yield continuous spectra, especially

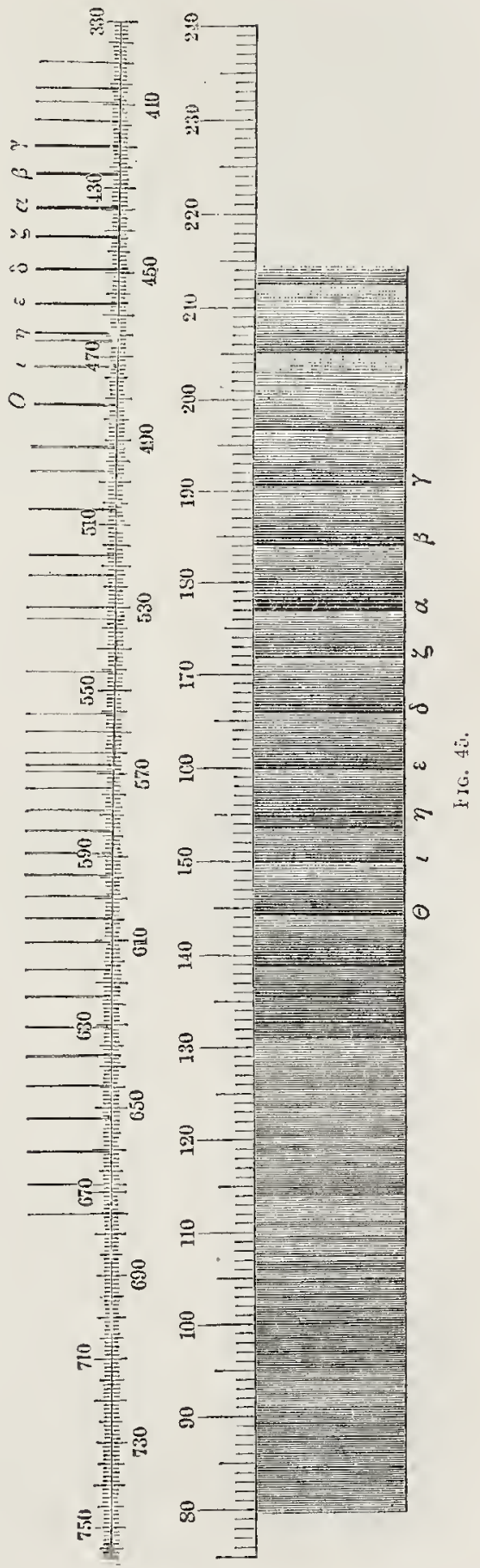

K 
when they are near their condensing points. Bearing all these facts in mind, it would seem that the state of aggregation has not so much to do with the nature of the spectrum, as the molecular arrangement and complexity. This view was first expressed by Mr. Lockyer, and there are many points which tell in its favour, thus it agrees with the views now generally adopted that the gaseous and liquid states of matter pass gradually one into the other.

Nearly all bodies which bave been examined with sufficient care have been found to exhibit both a band and a line spectrum, the band spectrum always belonging to the lower temperature. Oxygen even has as many as four different spectra. At the
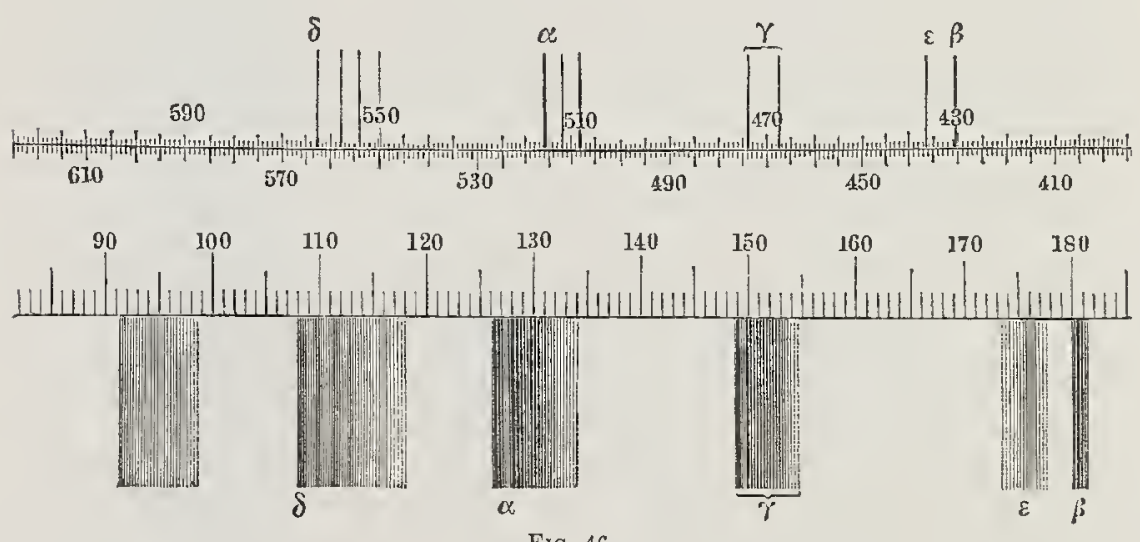

lowest temperature at which it becomes luminous it has a perfectly continuous spectrum; as the temperature is raised, this continuous spectrum gives way to another, corresponding to, though not altogether identical with, a spectrum of fluted bands. At the highest attainable temperature, the ordinary line spectrun. is seen, whilst the fourth spectrum is observed in vacuum tubes, at the negative pole only. One of the bands of this spectrum is represented in Fig. 48. Other gases like nitrogen show also peculiar spectra at the negative pole. This last phenomenon may probably be explained by the production of an allotropic modification of the substance at that pole.

These changes in type are, however, by no means the only 
changes which can be observed, when the temperature of the luminous body is altered. Line spectra especially, are subject to curious variations in the relative intensities of their lines whilst new lines are often brought out at high temperatures. Let me, in the first place, show you that new lines may make their appearance in the spectra of certain elements when the temperature is raised. Thus, for instance, if we heat lithium, either the metal or its salts, in the electric are, we obtain a splondid blue band having the wave-length of 4605 tenmillionths of a millimetre (see Fig. 49), in addition to the red and orange rays $a$ and $\beta$ seen in the flame spectrum, showing that the undulations in this particular set of vibrations have become more intense. The same phenomenon is observed in the case of the strontium spectrum, where no less than four new lines $(\epsilon, \eta, \kappa$, and $\lambda$, Fig. 49) make their appearance on increasing the temperature of the incandescent vapour of the metal. Also in the case of sodium four sets of new lines appear when the temperature is increased, in addition to the wellknown " $D$ " lines; and singularly enough, each of these four lines is double. Dr. Watts has shown that the first of these higher sodium lines (wave-length 5681 and 5687) become visible at a temperature of $2000^{\circ}$ C., whilst the next set (wave-length 5150 and 5152) appear when the temperature rises to about $3000^{\circ}$ C. The analogy between the
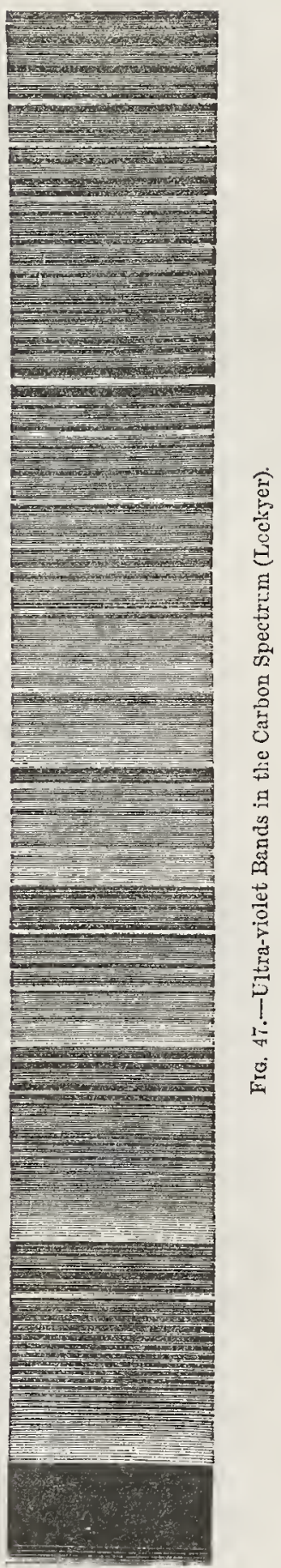
production of these more highly refrangible rays and that of the overtones or harmonics of a vibrating string will occur to all. The different behaviour of different lines under varying circumstances is well illustratcd by an experimental

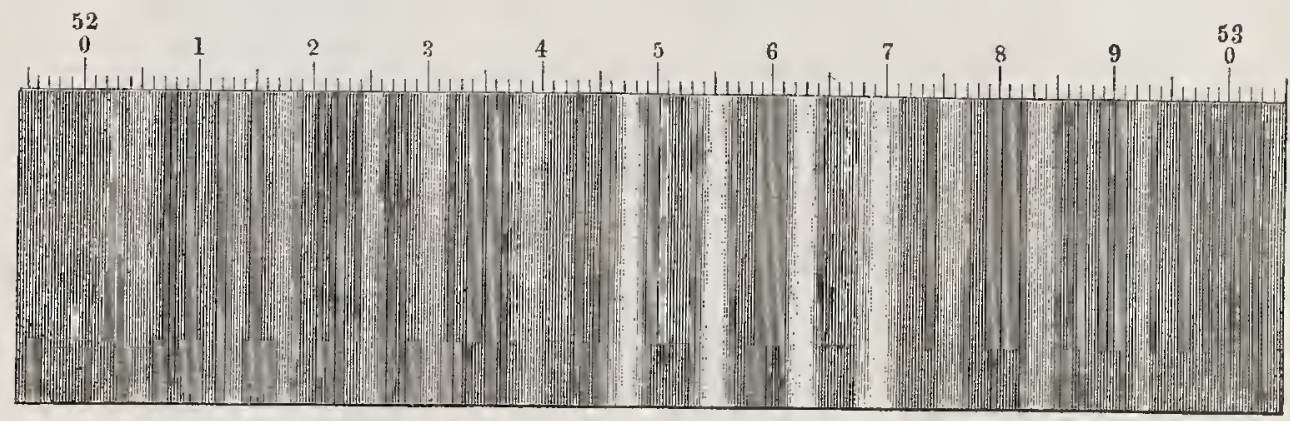

Fra. 43. - Band Spectrum of Oxygen at Negative Pole.

method, the importance of which was first pointed out by Mr. Lockyer. The arrangement adopted by him in his spectroscopic investigations is one of great utility. Instead of arranging the spark directly in front of the slit, he places it at some distance, interposing a lens which must be in such

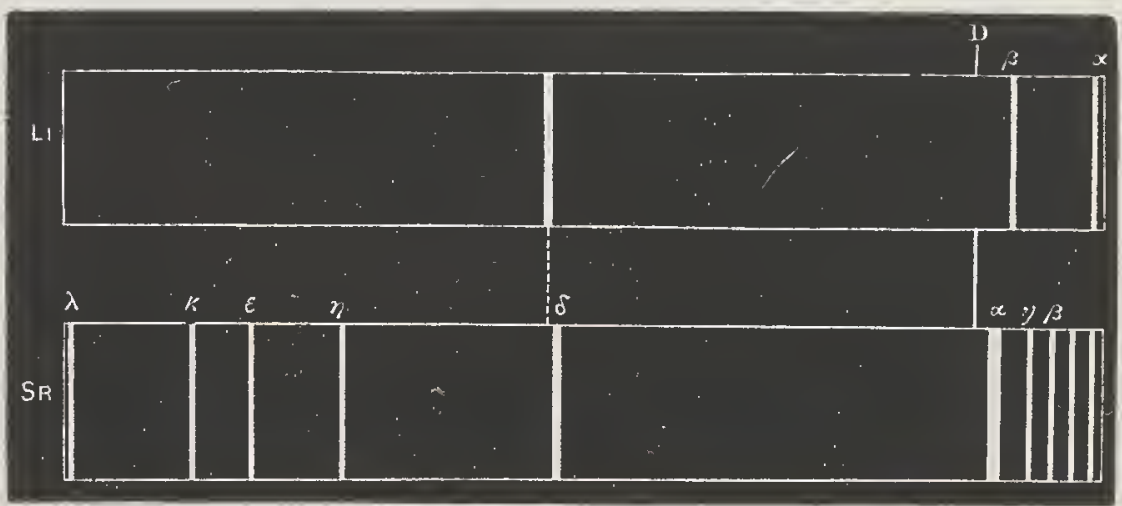

FIG. 49.

a position that it forms an image of the spark on the slit of the spectroscope. The advantage of this method lies in the fact that we can examine the spectrum, not only of the spark as a whole, but of any wished-for section of the spark. Thus, for instance, if 
we had two poles of different metals, the image of the two poles would be separated on the slit-plate, one pole appearing, say,

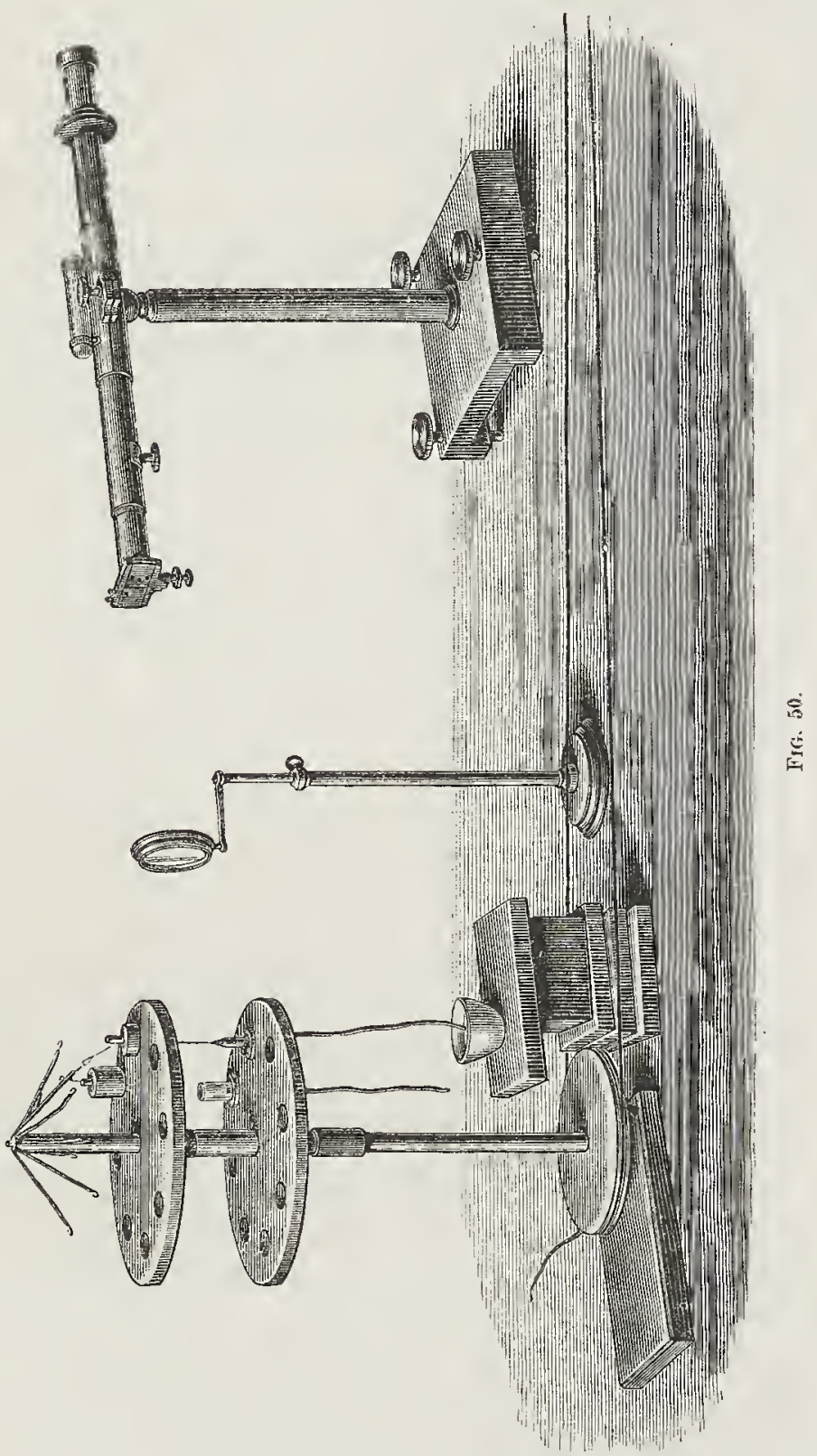

towards the upper end of the slit, while the other pole appears underneath. After the light has fallen through the prism, the 
two images would still be separate, and we should see the spectrum of each metal, one above the other. Fig. 50 represents Mr. Lockyer's arrangement. ${ }^{1}$

Now if we examine the spectrum of a metal in this way, we find that some of the lines are altogether confined to the neighbourhood of the poles, whilst others stretch nearly across the field; curiously enough it is by no means always the strongest lines which spread out the furthest, and this is an important point. It proves that the relative intensity of the lines is different in that part of the spark which is near the pole from that which is situated in the centre of the spark. Fig. 51 represents a group of zinc lines as seen when examined by this method. If we had simply placed the spark in front of the slit

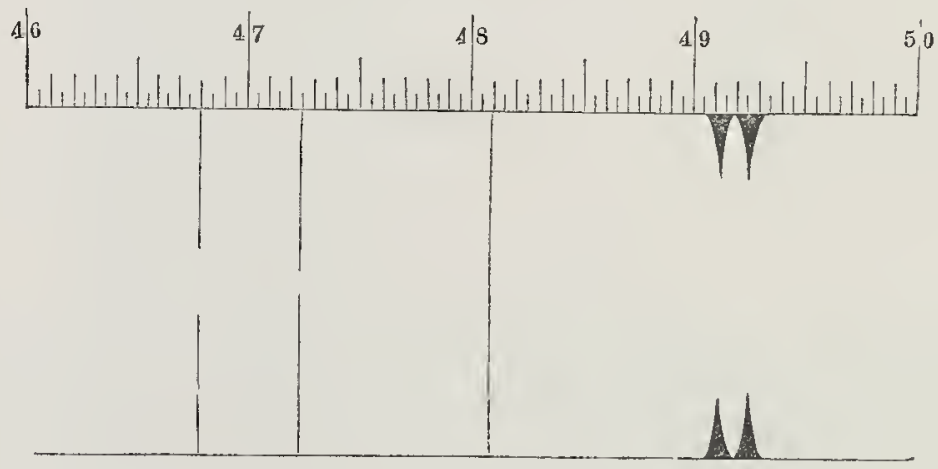

FIG. 51.

without the lens, the lines at 4924 and 4911 would have appeared stronger than any of the others, whilst examined by this method we see that although they are strong they are altogether confined to the neighbourhood of the pole whilst the line 4810 , for instance, though weaker stretches right across the field. The explanation which naturally occurs to any one considering these facts is, that the temperature of the spark is different near the pole, where it must be hotter, than in the centre between the poles where it is cooler, and that the relative intensity of the lines depends on the temperature. This explanation is well supported by facts, for we always find that these longest lines are those

I The construction of the spark-holder shown in this figure will be explained in Appendix B., Lecture JV. 
which are most persistent when the temperature of the spark is lowered.

In order to prove this we may, instead of heating a metal in the spark, render it luminous at the lower temperature of the Bunsen burner, and whenever we succeed in obtaining a spectrum in this way we find that the lines which appeared strongest in the centre of the spark now come out most clearly, while the lines which in the spark were strong near the pole are generally not seen at all at the lower temperature. Another way of lowering the temperature of the spark consists in reducing the pressure of the gas in which the spark is taken, and this method also gives us the same results.

This explanation, however, if it be so far the true one, is certainly not complete, for we find that even when the temperature of the spark is kept constant, the relative intensities of the lines may still undergo alteration. This was observed by Mr. Lockyer in examining the spectra of mechanical mixtures and alloys. By taking an alloy of zine and copper, he found that the same spark which with zinc alone gave the strong lines 4911 and 4924 near the poles, gave, in the case of the alloy, only the more refrangible lines which, as we have seen, are more persistent when the temperature is lowered. A number of observations bearing on this point have been made by Lecoq de Boisbaudran, who took the spark from liquid solutions in different states of concentration. He observed that with concentrated solutions the metallic lines often possess different relative intensities to those given by weak solutions.

When we compare the resilts thus obtained with the changes produced by an alteration of temperature, we are led to the remarkable result that a reduction of the quantity of material present in the spark has the same effect as a reduction of temperature. Accepting this conclusion we are able to explain a series of very complicated facts, and from the mere inspection of a spectrum examined by Mr. Lockyer's method, we can predict the changes which that spectrum will undergo, either on alteration of temperature or by an increase or decrease of quantity of incandescent substance. The change 
of relative intensity in the centre of the spark would then be due, not only to a change of temperature, but also to a reduction of the quantity of metallic vapour present. There is no theoretical difficulty in assuming that the relative intensities of the lines may vary when the tempetature is altered, and the molecular theory of gases furnishes us with a plausible explanation of the corresponding change when the relative quantities of the luminous elements in a mixture is altered. Mr. Lockyer has proposed a different explanation of the facts from that here given. According to him, a change of relative intensity always means a change of molecular complexity, and the lines which are strong near the poles would bear the same relation to those which stretch through the field of view, as a line spectrum bears to a band spectrum; but if we accept this view, almost every line must be due to a different molecular grouping.

The phenomena of long and short metallic lines are beautifully seen in the spectrum of the electric arc. Let me now place the arc, into which I have introduced traces of strontium and calcium, at right angles to the slit, and focussing the arc well on the slit, you will see a series of bright lines of different lengths (Fig. 52). The middle portion of each line corresponds to the centre of the arc, and the longest lines are those which pass into its outer portions.

There are still some minor changes to which I must refer. Thus the lines of some spectra, like those of hydrogen, are apt to widen when the pressure of the gas is increased; and when the pressure is sufficiently high this widening may become so great that the different lines fuse into each other, and the spectrum appears continuous. But a continuous spectrum thus obtained must be distinguished from that given by incandescent solids. Flames often give us a purely continuous spectrum; thus, for instance, the flame of carbonic oxide in oxygen. Again, Dr. Frankland has shown that the flame of hydrogen and oxygen, which under ordinary circumstances is almost invisible becomes luminous when burning under great pressure. It then gives a continuous spectrum, except when an excess of hydrogen is present, which brings out the hydrogen 


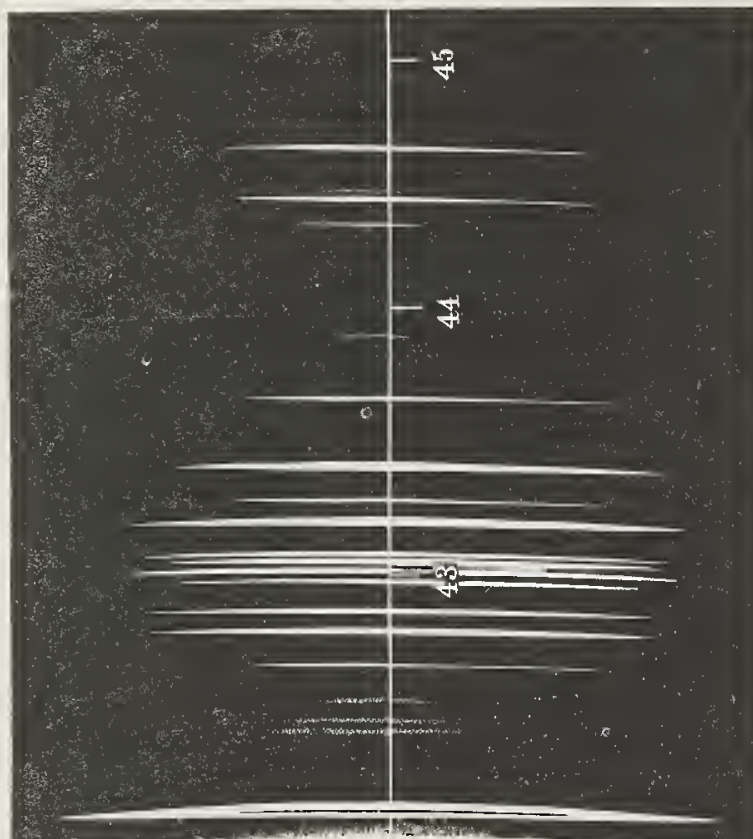

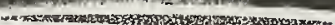

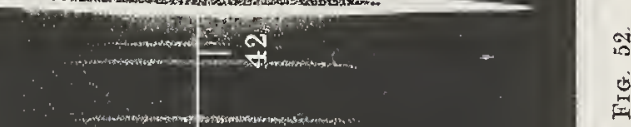


lines. The alterations which the spectrum of an element shows by a change of temperature can be made use of in the determination of the temperature of the sun and of the other heavenly bodies; but this branch of inquiry has not yet received the attention it deserves, and there is a great difficulty in fixing the temperature of an electric spark. An attempt has been made by Mr. Lockyer to found a quantitative analysis on the spectroscopic changes, which are due to variations in the quantity of the luminous material. Imagine, as an illustration of $\mathrm{Mr}$. Lockyer's method, that a spark is taken from an alloy of zinc and cadmium, and examined by means of the spectroscope. We should see in addition to the lines (Fig. 51) of zinc, the lines of cadmium, but the relative length of zinc and cadmium lines would depend on the quantities of these metals present in the alloy; for the metal which preponderates would show its lines larger than the other. If we then construct a table which tells us what for different composition of the alloys is the relative length of some lines, which are easily recognized, we should by a reference to the table be ablo to ascertain with considerable accuracy the composition of the alloy. Certain metals lend themselves more easily to this analysis than others, and $\mathrm{Mr}$. Lockyer has, in conjunction with Mr. Roberts, shown how especially in the case of alloys of gold and copper accurate results can be obtained.

Another matter of great importance is the investigation of the spectra of compound bodies, and here we must carefully distinguish the alterations in a spectrum which are due to an actual chemical decomposition from those which I have hitherto discussed. Thus, if we examine the spectrum of calcium chloride in the flame, we obtain different lines from those which we see if we bring a small quantity of the salt on an electrode. But here the spectrum which is seen in the flame is due to the incandescent vapour of the chloride of calcium, or rather to a mixture of the chloride and the oxide, while in the spark we see the spectrum of the metal itself, the temperature, as a rule, being sufficiently high to decompose the compound. Indeed, during the furst few years after the discovery 
of the principles of spectrum analysis, it was supposed that a compound gave the same spectrum as its elements, but we now know that each compound has a spectrum of its own. Whether the spectrum of a compound exhibits any relations to that of its elements is an important question. No decided connection has hitherto been traced, but Appendix D contains a short account of the attempts which have been made in this direction. The same appendix also discusses the question as to how far any numerical relation holds good between the lines of the same element.

In new and interesting subjects like those which now occupy our attention, the mind is very apt to be led away into speculations, which, however engrossing they may prove, are foreign to the spirit of the exact scientific inquirer. Such speculations might in this case have special reference to the possibility or probability of arriving, by the help of the observations of the bright lines which bodies give us, at some more intimate knowledge of the composition of the so-called elements. We might speculate as to the connection, for instance, between the wavelengths of the various bright lines of the metal and the particular atomic weight of the substance; or we might ask, Can we find out any relation between the spectra of the members of some well-known chemical family, as of the alkaline metals, potassium, sodium, cæsium, and rubidium? Such questions as these naturally occur to every one. At present, however, this subject is in such an undeveloped state that these speculations though useful in suggesting new lines of investigation are unsuited for general discussion; but doubtless a time will come when these matters will be fully explained, and a future Newton will place on record a mathematical theory of the bright lines of the spectrum as a striking monument of the achievements of exact science. 


\section{LECTURE III.-APPENDIX A.}

\section{CONTRIBUTIONS TOWARDS THE HISTORY OF SPEC'RUM} ANALYSIS. BY G. KIRCHHOFF.'

IN my "Researches on the Solar Spectrum and the Spectra of the Chemical Elements," 2 I made a few short historical renuarks concerning earlier investigations upon the same subject. In these remarks I have passed over certain publications in silence -in some cases because I was unacquainted with them, in others because they appeared to me to possess no special interest in relation to the history of the discoveries in question. Having become aware of the existence of the former class, and seeing that more weight has been considered to attach to the lutter class of publications by others than by myself, I will now endeavour to complete the historical survey.

Amongst those who have devoted themselves to the observation of the spectra of coloured flames, I must in the first place mention Herschel and Talbot. Their names need special notice, as they pointed out with distinctness the service which. this mode of observation is capable of rendering to the chemist. For a knowledge of their researches I am mainly indebted to Professor W. Allen Miller, who gave an extract from them in a lecture republished in the number of the "Chemical News" for 19th April, 1862. It is there stated that in the volume of the "Transactions of the Royal Society of Edinburgh" for 1822, at page $4 \check{5}$, Herschel shortly describes the spectra of chloride of

1 Communicated to the Phil. Mag., Fourth Series, vol. xxv. p. 250, by Professor Roscoe.

$\approx$ Published by Macmillan and Co., Cambridge and London, 1862. 
strontium, chloride of potassium, chloride of copper, nitrate of copper, and boracic acid. The same observer says, in his articlc on Light in the "Encyclopæedia Metropolitana," 1827, page 4.38 : "Salts of soda give a copious and purely homogeneous yellow; of potash, a beautiful pale violet." He then describes the colours given by the salts of lime, strontia, lithia, baryta, copper, and iron, and continues: "Of all salts, the muriates succeed the best from their volatility. The same colours are exhibited also when any of the salts in question are put (in powder) into the wick of a spirit-lamp. The co'ours thus communicated by the different bases to flame afford in many cases a ready and neat way of detecting extremely minute quantities of them. The pure earths when violently heated, as has recently been practised by Lieut. Drummond, by directing on small spheres of them the flames of scveral spirit-lamps, urged by oxygen gas, yield from their surfaces lights of extraordinary splendour, which, when examined by prismatic analysis, are found to possess the peculiar definite rays in excess which characterise the tints of flames coloured by them; so that there can be no doubt that thesc tints arise from the molecules of the colouring matter, reduced to vapour and in a state of violent ignition."

Talbot says:1 "The flame of sulphur and nitre contains a red ray which appears to me of a remarkable nature. This red ray appears to possess a definite refrangibility, and to be characteristic of the salts of potash, as the yellow ray is of the salts of soda, although, from its feeble illuminating power, it is only to be detected with a prism. If this should be admitted, I would further suggest, that whenever the prism shows a homogeneous ray of any colour to exist in a flame, this ray indicates the formation or the presence of a definite chemical compound." Somewhat further on, in speaking of the spectrum of red fire and of the frequent occurrence of the ycllow line, he says: "The othcr lines may be attributed to the antimony, strontia, \&c., which enter into this composition. For instance, the orange ray may be the effect of the strontia, sincc $\mathrm{Mr}$. Herschel found in the flame of muriatc of strontia a ray

1 Brewster's Journal of Science, vol. v. 1826 ; Chemical News, April 27, 1861. 
of that colour. If this opinion should be correct, and applicable to the other definite rays, a glance at the prismatic spectrum of a flame may show it to contain substances which it would otherwise require a laborious chemical analysis to detect." In a subsequent communication ${ }^{1}$ the same physicist, after a striking description of the spectra of lithium and strontium, continues: "Hence I hesitate not to say that optical analysis can distinguish the minutest portions of these two substances from each other with as much certainty as, if not more than, any other known method."

In these expressions the idea of "chemical analysis by spectrum observations" is most clearly put forward. Other statements, however, of the same observers, occurring in the same memoirs from which the foregoing quotations are taken (but not mentioned by Professor Miller in his abstract), flatly contradict the above conclusions, and place the foundations of this mode of analysis on most uncertain ground.

Herschel, in page 438 of his article on Light, almost immediately before the words quoted above says: "In certain cases when the combustion is violent, as in the case of an oil-lamp urged by a blowpipe (according to Fraunhofer), or in the upper part of the flame of a spirit-lamp, or when sulphur is thrown into a white-hot crucible, a very large quantity of a definite and purely homogeneous yellow light is produced; and in the latter case forms nearly the whole of the light. Dr. Brewster has also found the same yellow light to be produced when spirit of wine, diluted with water and heated, is set on fire"

Talbot says: "Hence the yellow rays may indicate the presence of soda; but they nevertheless frequently appear where no soda can be supposed to be present." 2 He then mentions that the yellow light of burning sulphur, discovered by Herschel, is identical with the light of the flame of a spirit-lamp with a salted wick, and states that he was inclined to believe that the yellow light which occurred when salt was strewed upon a platinum foil placed in a flame "was owing to the water of

. 1 Thil. Mag. 1834, vol. iv. p. 114; Chemical News, April 27, 1861.

2 Brewster's Journal, vol. v. 1826. 
crystallisation rather than to the soda: but then," he continues, "It is not easy to explain why the salts of potash, \&c., should not produce it likewise. Wood, ivory, paper, \&c., when placed in the gas flame, give off, besides their right flame, more or less of this yellow light, which $I$ have always found the same in its characters. The only principle which these various bodies have in common with the salts of soda is water; yet I think that the formation or presence of water cannot be the origin of this yellow light, because ignited sulphur produces the very same, a substance with which water is supposed to have no analogy." "It may be worth remark," he adds in a note, "though probably accidental, that the specific gravity of sulphur is 1.99 , or almost exactly twice that of water." "It is also remarkable," he continues in the text, "that alcohol burnt in an open vessel, or in a lamp with a metallic wick, gives but little of the yellow light; while, if the wick be of cotton, it gives a considerable quantity, and that for an unlimited time. (I have found other instances of a change of colour in flames, owing to the mere presence of the substance, which suffers no diminution in consequence. Thus, a particle of muriate of lime on the wick of a spirit-lamp will produce a quantity of red and green rays for a whole evening without being itself sensibly diminished)." 1

In a later portion of the memoir he attributes the yellow line in one place to the presence of soda salts, in another to that of sulphur. Thus, in the above-mentioned statement concerning the spectrum of red fire, he says, "The bright line in the yellow is caused, without doubt, by the combustion of the sulphur." 2

Hence we must admit that the conclusion that the aforesaid yellow line can be taken as a positive proof of the presence of sodium compounds in the flame can in no way be deduced from Herschel and Talbot's researches. On the contrary, the numerous modes in which the line is produced would rather point to the conclusion that it is dependent upon no chemical constituent

1 Brewster's Journal, vol. v. 1826.

2 A short statement of Herschel and Talbot's results, as here quoted, was made by me in a lecture at the Royal Institution on April 5, 1862, and reprinted in the Chemical News for May 10, 1862.-H. E. R. 
of the flame, but arises by a process whose nature is unknown, which may occur, sometimes more easily, sometimes with difficulty, with the most different chemical elements. If we accept such an explanation concerning this yellow line, we must form a similar opinion respecting the other lines seen in the spectrum which were far more imperfectly examined; and in this we should be strengthened by the statement of Talbot, that a piece of chloride of calcium by its mere presence in the wick of a flame and without suffering any diminution, causes a red and a green line to appear in the spectrum.

The experiments of Wheatstone, ${ }^{1}$ Masson, Angström, Van der Willigen, and Plicker, upon the spectra of the electric spark or electric light (to which I have already referred in my "Researches on the Solar Spectrum and Spectra of the Chemical Elements," p. 8, Macmillan, London, 1862, ) as well as those of Despretz, ${ }^{2}$ from which the physicist concluded that the positions of the bright lines in the spectrum of the light from a galvanic battery were unaltered by variation of the intensity of the current, might serve to support the view that the bright lines in the spectrum of an incandescent gas are solely dependent upon the chemical constituents of the gas; but they could not be considered as proof of such an opinion, as the conditions under which they were made were, for this purpose, too complicated, and the phenomena occurring in an electric spark too ill understood. The demonstrative power of the above experiments as regards the question at issue is rendered less cogent by the difference visible in the colour of the electric light in different parts of a Geissler's tube; by the circumstance noticed by Van der Willigen, who obtained different spectra by passing an electric spark from the same electrodes through gas of constant chemical composition, if the density of the gas was varied within sufficient limits; and lastly by an observation which Ångström cursorily mentions. This physicist says: ${ }^{3}$

1 Wheatstone not merely experinented with the spark from an electrical machine, but likewise with the voltaic induction spark. (Report of the British Association, 1835; Chemical News, March 23 and March 30, 1861.)

2 Comptes Rendus, vol. xxxi. p. 119 (1850).

3 Pogg. Am. vol. xciv. p. 150 (translated in Phil. Mag. for May 1855). 
"Wheatstone has already noticed that when the poles consist of two different metals the spectrum contains the lines of both metals. Hence it became of interest to see whether a compound of these metals, especially a chemical compound, also gives the lines of both metals, or whether the compound is distinguished by the occurrence of new lines. Experiment shows that the first supposition is correct. The sole difference noticed is, that certain lines were wanting, or appeared with less distinctness; but when they were observed, they always appeared in the position in which they occurred in the separate metals." In the following sentence, however, he states, "That in the case of zinc and tin the lines in the blue were somewhat displaced in the direction of the violet end, but the displacement was very inconsiderable." Had such a displacement, however small, really occurred, we must conclude either that the bright lines of the electric spark obey other laws than those of a glowing gas, or that these latter are not solely dependent on the separate chemical constituents of the gas.

The question at issue respecting the lines of incandescent gases could only be satisfactorily solved by experiments carried out under the most simple conditions-such, for instance, as the examination of the spectra of flames. Observations of this kind were made in the year 1845 by Professor W. Allen Miller, but they do not furnish any contribution towards a solution of the question. Dr. Miller has the merit of having first published diagrams of the spectra of flames; ${ }^{1}$ but these diagrams are but slightly successful, although, in a republication in the Chemrcal $N_{e w}{ }^{2}$ of the paper accompanying these drawings, Mr. Crookes remarks: "We cannot, of course, give the coloured diagrams with which it was originally illustrated; but we can assure our readers that, after making allowance for the imperfect state of chromolithography sixteen years ago, ${ }^{3}$ the diagrams of the spectra given by Professor Miller are more accurate in several respects than the coloured spectra figured in recent numbers

1 Phil. Mag. for August 1845.

2 Chemical News, May 18, 1861.

3 Prof. Miller's diagrams are not printed by chromolithography, but, as is seen on inspection, tinted by hand.-H. E. R. 
of the scientific periodicals." In reply to this "assurance" of Mr. Crookes I only have to remark that, by way of experiment, I have laid Professor Miller's diagrams before several persons conversant with the special spectra, requesting them to point out the drawing intended to represent the spectrum of strontium, barium, and calcium respectively, and that in no instance have the right ones been selected.

Swan was the first who endeavoured experimentally to prove whether the almost invariably occurring yellow line may be solely caused by the presence of sodium compounds. In his classical rescarch "On the Spectra of the Flames of the Hydrocarbons" (referred to both in my "Researches" and in the paper published by Bunsen and myself) Swan shows how small the quantity of sodium is which produces this line distinctly; he finds that this quantity is minute beyond conception, and he concludes: "When indeed we consider the almost universal diffusion of the salts of sodium, and the remarkablc energy with which they produce yellow light, it seems highly probable that the yellow line $R$, which appears in the spectra of almost all flames, is in every case due to the presence of minute quantities of sodium."

The strict subject-matter of Swan's investigation was the comparison of the spectra of flames of various hydrocarbons. "The result of his comparison has been, that in all the spectra produced by substance, either of the form $\mathrm{C}_{\mathrm{r}} \mathrm{H}_{\mathrm{s}}$, or of the form $\mathrm{C}_{\mathrm{r}} \mathrm{H}_{\mathrm{s}} \mathrm{O}_{\mathrm{t}}$, the bright lines have been identical. In some cases, indeed, certain of the very faint lines which occur in the spectrum of the Bunsen lamp were not seen. The brightness of the lines varies with the proportion of carbon to hydrogen in the substance which is burned, being greatest where there is most carbon. . . . . The absolute identity which is thus shown to exist between the spectra of dissimilar carbo-hydrogen compounds is not a little remarkable. It proves, Ist, that the position of the lines in the spectrum does not vary with the proportion of carbon and hydrogen in the burning body-as when we compare the spectra of light carburetted hydrogen, $\mathrm{CH}_{2}$,

1 Trans. Roy. Soc. of Edinburgh, vol. xxi. p. 414. 
olefiant gas, $\mathrm{C}_{2} \mathrm{H}_{2}$, and oil of turpentine, $\mathrm{C}_{20} \mathrm{H}_{8}$; and $2 \mathrm{dly}$, that the presence of oxygen does not alter the character of the spectrum: thus ether, $\mathrm{C}_{4} \mathrm{H}_{5} \mathrm{O}$, and wood spirit, $\mathrm{C}_{2} \mathrm{H}_{4} \mathrm{O}_{2}$, give spectra which are identical with those of paraffin, $\mathrm{C}_{20} \mathrm{H}_{20}$, and oil of turpentine, $\mathrm{C}_{20} \mathrm{H}_{8}$.

"In certain cases, at least, the mechanical admixture of other substances with the carbo-hydrogen compound does not affect the lines of the spectrum. 'Thus I have found that a mixture of alcohol and chloroform burns with a flame having a very luminous green envelope-an appearance characteristic of the presence of chlorine-and no lines are visible in the spectrum. When, however, the flame is urged by the blowpipe, the light of the envelope is diminished, and the ordinary lines of the hydrocarbon spectrum become visible."

In this research Swan has made a most valuable contribution towards the solution of the proposed question as to whether the bright lines of a glowing gas are solely dependent upon its chemical constituents; but he did not answer it positively, or in its most general form; he did not indeed enter upon this question, for he wished to confine his investigation to the spectra of the hydrocarbons, and was only led to the examination of this yellow line by its frequent occurrence in these spectra.

No one, it appears, had clearly propounded this question before Bunsen and myself ; and the chief aim of our common investigation was to decide this point. Experiments which were greatly varied, and were for the most part new, led us to the conclusion upon which the foundations of the "chemical analysis by spectrum observations" now rest. 


\section{APPENDIX B.}

ON THE EFFECT OF INCREASED TEMPERATURE UPON THL NATURE OF THE LIGHT EMTTTED BY THE VAPOUR OF CERTAIN METALS OR METALLIC COMPOUNDS, BY H. E. ROSCOE AND R. B. CLIFTON. ${ }^{1}$

In a letter communicated to the Philosophical Magazine for Jantuary last we stated that, in examining, with Steinheil's form of Kirchhoff and Bunsen's apparatus, the spectra produced by passing the induction spark over beads of the chlorides and carbonates of lithium and strontium, we had olserved an apparent coincidence between the blue lithium line, which is seen only when the vapour of this metal is intensely heated, and the common blue strontium line called $\operatorname{Sr} \delta$. We further stated that on investigating the subject more narrowly by the application of several prisms and a magnifying power of 40 , we came to the conclusion that the lithium blue line was somewhat more refrangible than the strontium $\delta$, but that two other more refrangible lines were observed to be coincident in both spectra. Having constructed a much more perfect instrument than we at that time possessed, we are now able to express a definite opinion on the subject, and beg to lay a short notice of our observations before the Society. Our instrument is in all essential respects similar to the magnificent apparatus employed by Kirchhoff in his recent investigations on the solar spectrum and the spectra of the chemical elements. It consists of a horizontal plane cast-iron plate, upon which three of Steinheil's Munich prisms, each having a refracting angle of $60^{\circ}$, are placed; and of two tubes fixed into the plate, one being a telescope having a magnifying power of 40 , movable with a slow-motion screw about a vertical axis placed in the centre of the plate, and the other being a tube carrying at one end the

\footnotetext{
1 Proc. Lit. Phil. Soc. Manchester, read April 1, 1862.
} 
slit, furnished with micrometer screw, through which the beam of light passed, and at the other end an object-glass for the purpose of rendering the rays parallel. The luminous vapours of the metals under examination were obtained by placing a bead of the chloride or other salt of the metal on a platinum wire, between two platinum electrodes, from which the spark of a powerful induction coil could be passed. In order to obtain a more intense, and therefore a hotter, spark than can be got from the coil alone, the coatings of a Leyden jar were placed in connection with electrodes of the secondary current respectively. When this arrangement was carefully adjusted, the two yellow sodium lines were observed to be separated by an apparent interval of two millimetres, as seen at the least distance of distinct vision.

The position of the blue line, or rather blue band, of lithium was then determined with reference to the fixed reflecting scale of Steinheil's instrument, by volatilizing the carbonate of lithium in the first place on a platinum wire between platinum electrodes, and secondly on a copper wire between copper electrodes. A bead of pure chloride of strontium was then placed on new platinum and copper wires between two new platinum and copper electrodes, and the position of the blue line $\mathrm{Sr} \delta$ read off upon the same fixed scale : a difference of one division on the scale was seen to exist between the positions of the two lines, the lithium line being the more refrangible. The salts of the two metals were then placed between the poles at the same time, and both the blue lines were simultaneously seen, separated by a space about equal to that separating the two sodium lines. When experimenting with this complete instrument, we were unable to observe any other blue lines in the pure lithium spectrum than the one above referred to; we have however noticed whe formation of four new violet lines in the intense strontium spectrum, and we now believe that the other two lithium lines mentioned in our letter to the Philosophical Magazine are caused by the presence of the most minute trace of strontium floating in the atmosphere, and derived from a previous experiment. We have convinced ourselves by numerous observations that the 
currents of air caused by the rapid passage of the electric spark between the electrodes are sufficient to carry over to a second set of electrodes placed at the distance of a few inches a very perceptible quantity of the materials undergoing volatilization. The greatest precautions must hence be taken when the spectra of two metals have to be compared; and no separate observations of the two spectra can be relied upon, unless one is made a considerable space of time after the other, and unless all the electrodes which have been once used are exchanged for new ones.

Kirchhoff in his interesting Memoir on the Solar Spectrum and the Spectra of the Chemical Elements, ${ }^{1}$ noticed in the case of the calcium spectrum that bright lines which were invisible at the temperature of the coal-gas flame became visible when the temperature of the incandescent vapour reached that of the intense electric spark.

We have confirmed this observation of Kirchhoff's, and have extended it, inasmuch as we, in the first place, have noticed that a similar change occurs in the spectra of strontium and barium; and, in the second place, that not only new lines appear at the high temperature of the intense spark, but that the broad bands, characteristic of the metal or metallic compound at the low temperature of the flame or weak spark, totally disappear at the higher temperature. The new bright lines which supply the part of the broad bands are generally not coincident with any part of the band, sometimes being less and sometimes more refrangible. Thus the broad band in the flame spectrum of calcium named $\mathrm{Ca} \beta$ is replaced in the spectrum of the intense calcium spark by five fine green lines, all of which are less refrangible than any part of the band $\mathrm{Ca} \beta$; whilst, in the place of the red or orange $\mathrm{Ca} \alpha$, three more refrangible red or orange lines are seen. The total disappearance in the spark of a well-defined yellow band seen in the calcium spectrum at the lower temperature was strikingly evident. We have assured ourselves, by repeated observations, that, in like manner, the

I Kirchhoff on the Solar Spectrum, \&c. Translated by H. E. Roscoc. (Cambridge : Macmillan, 1862.) 
broad bands produced in the flame spectra of strontium and barium compounds, and especially $\operatorname{Sr} a, \operatorname{Sr} \beta, \operatorname{Sr} \gamma, \mathrm{Ba} a, \mathrm{Ba} \beta$, $\mathrm{Ba} \gamma, \mathrm{Ba} \delta, \mathrm{Ba} \in, \mathrm{Ba} \mu$, disappear entirely in the spectra of the intense spark, and that new bright non-coincident lines appear. The blue Sr $\delta$ line does not alter either in intensity or in position with alterations of temperature thus effected, but as has already been stated, four new violet lines appear in the spectrum of strontium at the higher temperature.

If, in the present incomplete condition of this most interesting branch of inquiry, we may be allowed to express an opinion as to the possible cause of the phenomenon of the disappearance of the broad bands and the production of the bright lines, we would suggest that, at the lower temperature of the flame or weak spark, the spectrum observed is produced by the glowing vapour of some compound, probably the oxide, of the difficultly reducible metal; whereas at the enormously high temperature of the intense electric spark these compounds are split up, and thus the true spectrum of the metal is obtained.

In conclusion, we may add that in none of the spectra of the more reducible alkaline metals (potassium, sodium, lithium) can any deviation or disappearance of the maxima of light be noticed on change of temperature.

RIRCHHOFF ON THE VARIATION OF THE SPECTRA OF CERTAIN ELEMENTS : 1862.

"I close this section with the following remarks : The position of the bright lines, or, to speak more precisely, the maxima of light in the spectrum of an incandescent vapour, is not dependent upon the temperature, upon the presence of other vapours, or upon any other conditions except the chemical constitution of the vapour. Of the validity of this conclusion Bunsen and I have assured ourselves by experiments made for that special object, and I lave confirmed it by many observations made with the extraordinary delicate instrument just described. The 
appcarancc of the spectrum of the same vapour may, neverthelcss, be very different under different circumstances. Even the alteration of the mass of the incandescent gas is sufficient to effect a change in the character of the spectrum. If the thickness of the film of vapour, whose light is being examined, be increased, the luminous intensities of all the lines increase, but in different ratios. By virtue of a theorem which will be considered in the next section, the intensity of the bright lines increases more slowly than that of the less visible lines. The impression which a line produces on the eye depends upon its breadth as well as upon its brightness. Hence it may happen that one linc being less bright, although broader, than a second, is less visible when the mass of incandescent gas is small, but becomes more distinctly seen than the second line when the thickness of the vapour is increased. Indced, if the luminosity of the whole spectrum be so lowered that only the most striking of the lines are seen, it may happen that the spectrum appears to be totally changed when the mass of the vapour is altered. Change of temperaturc appears to produce an cffect similar to this alteration in the mass of the incandesccnt vapour. If the temperature be raised, no deviation of the maxima of light is observed, but the intensities of the lines increase so differently, that those which are most plainly seen at a high temperature are not the most visible at a low temperature."

\section{APPENDIX C.}

CHANGES IN THE SPECTRUM OF OXYGEN, ABSTRACTFD FROM A PAPER IN THE " PHILOSOPHICAL TRANSACTIONS." BY PROFESSOR SCHUSTER, F.R.S.

THE appearance of an oxygen tube when a spark passes while it undergoes gradual exhaustion is as follows:-

A.t first the spark has a ycllow colour, and the spectrum is perfectly continuous. Almost immediately, howcver, four lines 
are seen in the capillary part above the continuous spectrum. One of these lines is in the red, two are in the green, and one is in the blue. The discharge still passes as a narrow spark throughout the length of the tube. In the wide part the spectrum remains continuous, and it extends more towards the red and blue than in the capillary part. It seems as if the four lines had taken away part of the energy of the continuous spectrum. As the pressure diminishes, these lines increase considerably in strength; the spark spreads out in the wide part of the tube, and the intensity of the continuous spectrum is therefore considerably diminished, while it still forms a prominent part in the spectrum of the capillary part. When the pressure is small the continuous spectrum decreases in intensity. At the same time the negative glow with its own characteristic spectrum, gradually extends through the negative half of the tube into the capillary part. The continuous spectrum has now entirely disappeared; the bands of the negative pole and the four lines stand out on a perfectly black background. It is under these conditions that the change from the compound line spectrum to the elementary line spectrum is best studied. The mere insertion of a Leyden jar, I find makes hardly a difference; the jar does not seem to be charged at all. If in addition to the jar we insert a movable air-break, which can be opened or closed at will, while we look through the spectroscope, we shall be able to see alternately two perfectly distinct spectra. If the air-break is closed the four lines of the compound spectrum only are seen; if the air-break is opened these four lines will disappear entirely, and the elementary line spectrum will come out. We have here as complete a transformation as we have from the band to the line spectrum of nitrogen taking place under exactly the same circumstances. We have, therefore, the same right to consider the two line spectra of oxygen as two distinct spectra, as we have in the case of the two spectra of nitrogen. 


\section{APPENDIX D.}

ON THE INFLUENCE OF TEMPERATURE AND PRESSURE ON THE SPECTRA OF GA.SES. BY DR. SCHUSTER, F.R.S.

A sTUDY of the changes which may be observed in the spectra of gases, under varying circunstances, is of great importance, both from a practical and a theoretical point of view. We are here chiefly concerned with the practical side; and it is clear that a full investigation of all spectroscopic variations attending changes of physical conditions will ultimately lead to a science. which will aim, not only at a merely qualitative analysis, as the original spectroscopy did, but which will enable us to determine the exact physical state of a luminous body, at whatever distance from us that body might be placed.

There is some difficulty in arranging the great quantity of partially unconnected facts which we shall have to place before the reader. We shall endeavour, for clearness' sake, to arrange our material under five different heads. ${ }^{1}$ We shall first discuss what changes we have a right to expect in the appearance of a spectrum, if the quantity of luminous matter is increased, or if the temperature is raised, the absorbing properties of the gas remaining unaltered. We shall next speak of the widening of lines, which, as we shall see, often accompanies an increase of pressure. Then we shall treat of the different spectra given by one and the same body at different temperatures, and we shall see how far satisfactory explanations have been offered for their existence.

So far our road will be clear, but we shall find that these spectra of different orders, as they have been called, are only extreme cases of continuous changes which are nearly always going on. Very often we can refer these continuous changes to a gradual displacement of one spectrum by another; but often

1 In this Appendix only the two first heads are disenssed, as the important points contained in the remainder lave been noticed in the lecture itself. 
we shall not be able to prove the existence of a second spectrum. There is, à priori, nothing impossible, or even improbable, in the view that the relative intensity of different lines may be different at different temperatures, and often when we observe a variation we may equally well explain it by assuming the gradual appearance of a new spectrum, or an alteration only in the relative intensities of the lines. It becomes then a matter of extreme difficulty to decide which of the two suppositions is correct. In doubtful cases we may often be able to obtain important information by means of a method which is little understood, even by spectroscopists. It is the method which has first been extensively used and investigated by Mr. Lockyer, of projecting an image of the luminous source, spark, arc, or flame, on the slit of the spectroscope, and thus localising the spectra which are thrown and confused together, if the luminous source is examined directly without the interposition of a lens. We shall see how, by means of this method, we shall often at a single glance be able to tell how the body will behave at different temperatures and under different pressures. Many facts which have been quoted as remarkable might have been foretold by means of this method.

\section{Infuence of Thickness of Radiating Layer on the Spectra of Gases.}

Let $a$ be the coefficient of absorption for a certain wavelength of a layer of gas, of thickness and density equal to unity. Let $e$ be the radiation of a perfectly black body for the given wave-length, and at the temperature of the body, the radiation of which we are considering. Then the radiation $\mathrm{E}$ of a layer of thickness $a$ and density $\delta$ will be ${ }^{l}$

$$
\mathrm{E}=\left[1-(1-a)^{\alpha}\right] e
$$

We pass over some obvious consequences of this formula, which have been treated in detail in Zöllner's paper, but shall

1 Zöllner: Phil. Mag. xli. p. 190 (1871); Wüllner : Wied, Ann. viii. p. 590 (1879). 
discuss whether a mere increase in the thickness or density of the layer can alter the relative intensities of the lines. Put $a \delta=\sigma$ and let $\mathrm{E}_{1}$ be the radiation of the same body for another wave-length, $e_{1}$ being the corresponding radiation for a perfectly black body.

In the first place we remark that there can only be one finite value of $\sigma$, for which the two radiations can be equal; for the equation

$$
\left[1-(1-a)^{\sigma}\right] e=\left[1-\left(1-a_{1}\right)^{\sigma}\right] c_{1}
$$

has only two roots, one of them being $\sigma=0$, which case, of course, is excluded from our consideration. For an infinite thickness-

$$
\frac{\mathrm{E}_{1}}{\mathrm{E}}=\frac{e_{1}}{e}
$$

Let $e_{1}$ be larger than $e$; then, if for any given value of $\sigma$, say $\sigma^{1}, \mathrm{E}_{1}$ is larger than $\mathrm{E}$, it must be also larger for all greater values of $\sigma$, for if for any value larger than $\sigma^{1}, E_{1}$ could be smaller, it would have to be equal to $E$ for two values lying between $\sigma=\sigma^{1}$ and $\sigma=\infty$; which, as we have seen, is impossible. On the other hand, if, for any value of $\sigma, \mathrm{E}_{1}$ is smaller than $\mathrm{E}$, the relative intensities must be reversed by an increase of thickness, for an infinite value of $\sigma$ will make $\mathrm{E}_{1}>\mathrm{E}$.

We have been assuming that $e_{1}$ is larger than $e$; $e$ being the radiation of a perfectly black body. Now for all temperatures which we are considering, the radiation of a perfectly black body decreases in the visible part of the spectrum with the wave-length. Hence the wave-length, for which $\mathrm{E}_{1}$ is the radiation, must be larger than the corresponding wave-length for $\mathrm{E}$. Putting all these considerations together we arrive at the following laws :-

1. If the less refrangible of two rays is the stronger for any given quantity of luminous matter, no increase of that quantity can reverse the relative intensitics, but a decrease may render the more refrangible ray stronger. 
2. If the more refrangible of two rays is the stronger, a sufficient increase in the quantity of luminous matter will, in all cases, reverse the relative intensities, but a dccrease will ncver make the less refrangible ray stronger.

Zöllner, who was the first to draw attention to the fact that a reversal of relative intensity may be produced by an increase in the quantity of luminous matter, has failed to notice that this inversion can only take place if the less refrangible ray is the weaker of the two. ${ }^{1}$

When we come to look round for examples in which the effect of thickness of a layer can be clearly traced, we shall have difficulty in finding any. For most gases the values of $a$ are exceedingly small, and then, of course, the increase of quantity must be exaggerated to an enormous extent before any appreciable effect is produced. Even on the sun, the relative intensities of the lines is often the same as that we observe in our laboratory experiments, and where it is not, it does not show such changes as would be produced by a mere increase of the absorbing layer. In liquids, of course, and some vapours which have large absorbing powers, an effect of the thickness of the absorbing layer can be traced.

If the temperature of a radiating gas is increased, the absorbing power for each ray remaining the same, the radiation will vary in the same proportion as $e$; that is, as the radiation from a perfectly black body. It follows that the more refrangible rays will relatively gain more than the less refrangible rays, but it must be borne in mind that the absolute intensity of any given line can never decrease, unless the quantity of luminous matter decreases.

\section{Widening of Lines.}

In his celebrated paper, Optiska Undersökningar ${ }^{2}$ (1853), Ångström gives two drawings of the hydrogen spectrum. In neither of them are the lines sharp; but in one of them

I Appendix B shows that Kirchloff had previously considered this point, though he did not investigate the conditions under which an actual reversal of luminosity of two equally broad lines can occur.

2 Translated, Pogg. Ann. xciv. p. 141 (1855). 
especially they are drawn out into broad bands. 'The property of widening their lines under certain circumstances bas since been found to be common to all bodies, though some of them possess it to a much larger extent than others. Hydrogen and sodium are the best known instances of elements which widen their lines considerably, though in one of the spectra of oxygen the lines broaden even more easily. Wüllner ${ }^{1}$ has given a detailed description of the behaviour of the hydrogen lines under different pressures, both with the condensed and uncondensed discharge. The same author has also given us information as to the widening of some of the oxygen lines in the same paper, and his observations were confirmed and extended by the author of this report. ${ }^{2}$ Ciamician ${ }^{3}$ has described the widening of the lines of mercury, sodium, and some other gases. The papers of Lockyer and of Liveing and Dewar will also be found to contain many observations on the widening of lines. We shall refer presently to some of their most important experiments on the subject.

It is a fact which is often, though by no means generally, true, that if a spectrum widens its lines easily, the widening begins with the most refrangible lines. This was first noticed by Plücker and Hittorf in the case of hydrogen. They express themselves as follows:- 4

"Hydrogen shows in the most striking way the expansion of its spectral lines and their gradual transformation into a continuous spectrum. By increasing the power of the coil, $\mathrm{H}_{\gamma^{\prime}}$ (coincident with solar line near G) first expands, then $\mathrm{H} \beta$ (solar F). Let the aperture of the slit be so regulated that the double sodium line will separate into two lines, nearly touching; then the angular breadth of $\mathrm{H} \beta$ becoming two or three minutes, ${ }^{5}$ the breadth of $\mathrm{H}_{\gamma}$ is about double. $\mathrm{H} a$ remains almost unchanged after $\mathrm{H}_{\gamma}$ has passed into an undetermined hazy band, and $\mathrm{H} \beta$ extended its decreasing light on its two sides." A fourth hydrogen line, more refrangible than the others, was discovered by

1 Pogg. Ann. cxxxvii. p. 339 (1869).

2 Phil. Trans. clxx. p. 37 (1879).

3 Wien. Ber. (2) lxxviii. p. 867 (1878).

4 Phil. Trans. clv. p. 21 (1865).

5 The large Steinheil spectroscope was used. 
Angström, and as Goldstein ${ }^{1}$ has remarked, this line is the first to widen, thus following the general rule. It would be interesting to determine the behaviour of the ultra-violet lines of hydrogen which have recently been discovered.

In the case of oxygen, Plücker and Hittorf have remarked that the less refrangible lines widen most easily. But Plücker and Hittorf did not separate the two line-spectra of oxygen. The lines belonging to the lower temperature widen more easily perhaps than any other lines, with the exception of the blue line, which always remains sharp, and presents a striking contrast to the other lines. The two green lines belonging to this spectrum widen more easily than the red line; so that the lines which do expand follow the rule. The lines of the other line spectrum do not expand very much, though their edges lose their sharpness at high pressures. Plücker and Hittorf 'remark that the blue group widens more easily than the violet groups.

The more refrangible of the two double sodium lines $\left(\mathrm{D}_{2}\right)$ widens more than the less refrangible component. According to Ciamician's experiments, mercury follows the rule, and widens the most refrangible lines most easily. It has often been remarked that all lines lose the sharpness of their edges when the pressure is increased, ${ }^{2}$ but there is a great difference between the cases we have just mentioned and the lines, for instance, of chlorine, bromine, and iodine, or nitrogen, which may become fuzzy, but never spread over an appreciable part of the spectrum. Though the difference is one of degree only, it is very marked. We may say that, as a general rule, if a system of lines widens much or easily, the more refrangible lines of the system will be the first to widen, while if a system of lines shows the broadening to a small degree only, no general rule can be given.

When a line widens, it may do so either symmetrically on both sides, or the widening may be greater on one side. It is a remarkable fact that when a line widens chiefly towards one side, that side is in nearly all, if not in all cases, the less refrangible one.

\footnotetext{
1 Berl. Ber. p. 593 (1874).

${ }^{2}$ e.g. Cailletet, C.R. lxxiv. p. 1282 (1872).
} 
In the case of the hydrogen lines, it is of some importance to determine whether they widen symmetrically, because the small displacements of the line in stars which are referred to star motions, may be in part due to a one-sided widening. The Greenwich observers ${ }^{1}$ have, therefore, made a very careful series of measurements, in order to find out whether the centre of the $\mathrm{F}$ line shifts as it broadens. No shift was detected in a range of pressure from $3.0 \mathrm{~mm}$. to $500 \mathrm{~mm}$., the width of the line altering considerably within that range. J. J. Müller's experiments, giving an apparently different result, will be presently referred to.

The lines of sodium seem also to widen nearly equally on both sides. Zöllner ${ }^{2}$ has exanined these lines with his reversion-spectroscope-an instrument which is pre-eminently fitted for such an investigation. He gives the results in the following words: "In the more refrangible line, which, with increase of the vapour-density, was the most widened, no displacement was perceptible; meanwhile there appeared to take place in the other line, as the brightness increased, an extremely slight displacement in the direction of a diminution of the refrangibility."

But Zöllner does not seem himself to attach very much value to this observation, the displacement being very slight. Dr. J. I. Müller ${ }^{3}$ has had the curious idea of examining whether the rate of propagation of a ray of light in space depended on the amplitude of vibration. The wave-length was measured by means of interference fringes of long difference of path (Newton's rings), and he was incidentally led to inquire into the possibility of a displacement of the centre of the sodium lines, when larger quantities of sodium were introduced into a Bunsen flame; in which case it is known the lines are seen to widen. Muiller found such a displacement in the same direction as Zöllner, who refers to his experiments as corroborating his own results. But Miiller also found the effect he was looking for; that is to say, he found the centre of lines to shift

${ }^{1}$ Results of the Astronomical Observations made at the Royal Observatory, Greenwich, 1876, p. 118.

2 Phil. Mag. xli. p. 204 (1871).

"Leipg. Ber. (1871), and Pogg. Ann, el. p. 311 (1873). 
when the light was weakened after it had left the flame. Now, this latter part of the investigation has been subjected to a very careful examination by Lippich, ${ }^{1}$ who could discover no such effect. As there undoubtedly was a source of error in Müiller's experiments, which has not yet been pointed out, we must defer our judgment also on his other results. When he therefore nnds a very light shifting of the hydrogen lines, due to an alteration of the power of the spark, we cannot put his observations on an equal level with the subsequent negative results of the Greenwich observers.

Speaking of reversals in the voltaic arc, Mr. Lockyer ${ }^{2}$ adds the foliowing note: "The absorption-line does not always occupy the exact centre of the bright band. This point is occupying my attention, as it raises a very interesting question connected with molecular vibrations." In the plates accompanying the paper we find one at least of the aluminium lines between $H$ and $\mathrm{K}$ slightly more expanded on the less refrangible side.

Mr. Lockyer ${ }^{3}$ has referred to the same question in a recent paper, and mentions two examples in the silver spectrum. In one case $\left(4210^{\circ} 0\right)$ the line seemed to be 'much more widened on the more refrangible side of the absorption-line; in the other case (4054.3), it was more widened in the opposite direction. The rubidium line (4202) is also given as more expanded on the less refrangible side.

Profs. Liveing and Dewar ${ }^{4}$ mention that the magnesium lines (4703) (4354) widen more on the less refrangible side.

The lines of the lower temperature spectrum of oxygen widen considerably on the less refrangible side. This is very marked in the case of the more refrangible of the two green lines, and of the red line. The centre of the former line shifts through 2Xth metres. ${ }^{5}$ The less refrangible of the two green lines widens more symmetrically. According to Ciamician, ${ }^{6}$

1 Wien. Ber. (2) lxxii. p. 355 (1875).

2 Phil. Trans. clxiv. p. 805 (1874).

3 Proc. Roy. Soc. xxviii. p. 428 (1879).

4 Proc. Roy. Sac. xxviii. p. 367 (1879).

5 Wien. Ber. (2) lxxviii. p. 886 (1878).

${ }^{\circ}$ Nature, xvii. p. 148 (1877). 
most of the mercury lines show this one-sided widening, and with some of them it is so marked that they seem exclusively expanded towards one side only.

I have only come across two cases in which lines seemed to be more widened on the most refrangible side, and neither of them seems to be established beyond doubt. The first is the one in the spectrum of silver mentioned by Lockyer and quoted above. But Profs. Liveing and Dewar did not notice the reversal of the line in question, the wave-length of which they give as 4208. A new line, however, came out at 4211.3; that is on the less refrangible side. It is possible that the dark space between the two lines was taken by Lockyer for a reversal, and that consequently the greater intensity and width of the more refrangible line appeared as a one-sided development of the wings. The second case is not very clearly described by Ciamician. In the text of the paper he only mentions that when the double yellow mercury line becomes fuzzy, continuous light is seen to the right and left of it. In the drawing this is figured as a widening of the more refrangible line towards the violet, and of the less refrangible line towards the red. The description is too vague to allow any certain inference to be drawn, but it seems possible, as a simple optical fact, that, when a double line widens, the wings can be traced to a greater distance on that side of each component which is removed from the other.

We have now to discuss the causes which may produce the widening of lines. In the first place it might be suggested that in accordance with a formula which we have already given, an increase of the quantity of luminous matter would produce an apparent widening of the lines; for it follows from the formula that, unless the coefficient of absorption is absolutely zero for any given wave-length, the spectrum sent out by an infinite number of molecules in the line of sight is always continuous. A greater number of molecules will, therefore, cause the spectrum to approach the continuous state, and the widening of the lines may be due to the first stage towards this approach. To this we shall reply, that an increase in the number of molecules cannot be the 
primary cause of the widening of lines; for the lines of sodium, for instance, in the sun are comparatively sharp, though the thickness of the absorbing layer is greater than anything we can produce in our experiments. We can prove the same point more clearly in the case of hydrogen. If we inclose the gas in a tube of the form adopted by Mr. Monkhoven and Prof. Piazzi Smyth, so that we may look longitudinally through the capillary bore, we increase the thickness of the radiating layer to a very great extent; yet lines which are sharp when the tube is looked at transversely, will remain sharp when it is looked through longitudinally: although an increase in the pressure or in the intensity of the discharge will at once produce a widening. It must be remembered that the effect of an increased number of radiating molecules will only depend on the curve of intensity near the line. If a line is absolutely sharp, no increase in the number of molecules will ever increase its width, and two lines of the same brightness, which present the same appearance at their edges, must behave exactly in the same way when the thickness of the radiating laycr is increased. Yet, while we have some lines which widen easily and enormously, others, which present the same appearance, do not show any widening. That if a line is once widened, an increase in the number of radiating or absorbing molecules will increase the apparent extent of the widening is possible, but we must distinguish this effect from the original cause which has produced the widening.

It is, I believe, the almost unanimous opinion of spectroscopists that the widening is, in most cases, produced by an increase of pressure. This opinion was first put forward by Frankland and Lockyer. ${ }^{1}$ In the case of gases, the easiest way to produce the widening is by an increase in the pressure of the gas, and the metallic lines are also generally seen to widen when the density of the gas through which the spark is taken is increased. But we may also, in the case of hydrogen, for instance, widen the lines by an increase in the intensity of the discharge. ${ }^{2}$ Those

1 Proc. Roy. Soc. xvii. p. 288 (1869).

2 The widening observed by Stearn and Tee (Proc. Roy. Soc. xxi. p. 282-1873) is due to this cause. 
who believe that the widening of a line is clue to an increase of pressure, attribute to an increase in temperature, such as is produced by an increased discharge, an influence only in so far as it raises the pressure of the gas. This opinion is supported by the fact that the sodium lines widen rather more easily at low temperatiures.

According to the molecular theory of gases, the following explanation might be given for the widening of lines:

As long as a molecule vibrates by itself, uninfluenced by any other molecule, its vibrations will take place in regular periods. The lines of its spectrum will consequently be sharp. But if the molecule is placed in proximity with others, its vibrations will be disturbed by occasional encounters. The number and strength of these collisions will depend on the pressure of the gas. Ideas analogous to these seem to have been in the minds of many writers, and it is difficult to decide where they first occurred; but we may quote a short passage taken out of a paper by Lippich, ${ }^{1}$ in which similar views were, perhaps for the first time, clearly expressed, and in which the reference to Boyle's law is especially interesting :-

"If the pressure of a gas is increased, or if, as in the case of vapours, its properties are no longer those of a perfect gas; that is, if the length of the path during which a molecule is within the influence of another is no longer small compared to the length of the mean free path, changes in the spectrum will necessarily accompany the new state of things. ... New vibrations will arise, the intensity of which will be the smaller the further removed they are from the vibrations of the molecule in the ideal state. The lines of the spectrum will then appear with indistinct edges and expand the more the more the gas deviates from the laws of Mariotte and Gay-Lussac."

The behaviour of the two yellow sodium lines is in many respects remarkable. It has already been mentioned that the widening seems to take place more easily at a lower temperature, but it is obviously not due to a lowering of temperature, for Profs. Liveing and Dewar ${ }^{2}$ have observed that a lajer of sodium

1 Pogg. Ann. cxxxix. p. 465 (1870).

2 Proc. Roy. Soc. xxix. p. 482. 
vapour about $4 \mathrm{~cm}$. thick, at atmospheric pressure, gave sharp and narrow lines, at a temperature which was lower than that of a Bunsen burner; while in the Bunsen a much smaller quantity of sodium vapour will produce winged lines. Some of the experiments described in the paper to which we have just referred are not easily reconci'ed with the explanation given above for the widening of lines. Profs. Liveing and Dewar describe the effects of pressure thus :-

"The effects of compressing the vapour were very remarkable. As the pressure increased the channelled spectrum speedily disappeared, then the diffused edges of the $\mathrm{D}$ band contracted, the band itself likewise contracting until it became a very fine pair of lines, or if the amount of sodium present was not too much, $\mathrm{D}$ came out bright. On letting off the pressure, the phenomena recurred in the reverse order, and the whole could be repeated several times. After compression, as long as the pressure was sustained, the ID absorption remained permanently narrowed, but did not continue bright."

The general results of the investigation are summed up by Profs. Liveing and Dewar thus :-

"The phenomena attending the compression of the vapours, as well as those of the amalgams of varying pereentages, seem to indicate that the width of the $\mathrm{D}$ absorption is dependent on the thickness and temperature of the absorbing vapour rather than on the whole quantity of sodium present in it. Very minute quantities diffused into the cool part of the tube appear to give a broad diffuse absorption, while a layer of denser vapour of small thickness in the hottest part of the vessel gives but a very narrow absorption. This may, however, be due to the variation of temperature."

In a previous paper Profs. Liveing and Dewar ${ }^{1}$ had expressed themselves as follows on the widening of lines:-

"It is apparent that the expansion of lines so often observed when fresh materials are introduced, must be ascribed to increase in the density of the vapours, not to any increase in temperature. Moreover, the length of the tube, which reaches a very high

$$
1 \text { Proc. Roy. Soc, xxviii. p. } 370 .
$$


temperature in the experiments above described, is very sliort in the lime crucible, and still shorter in the carbon crucible, so that the reversing layer is also shoit in many cases."

There is one cause, which, as Profs. Liveing and Dewar mention, may have affected the results of the later paper: "The results of the foregoing experiments may have been complicated by the sodium vapour which diffused into the cool part of the vessel. We have attempted to overcome this complication by passing down into the bottle, when full or nearly full of sodium vapour, a platinum tube, closed at the top with a glass plate and filled with nitrogen, and observing the absorption through this tube. The nitrogen in the tube prevents, for a short time, the entry of the sodium vapour into tle tube, and so, by passing the tube to different depths, the thickness of the layer of sodium through which the observations were made could be varied. It was found, in this way, that a layer of sodium vapour, about $4 \mathrm{~cm}$. thick, at the atmospheric pressure at the temperature of our furnace, gave the $\mathrm{D}$ absorption sharp and very narrow; but as the sodium diffused into the tube the absorption extended until it produced a broad band with diffuse edges."

In these experiments, the light emitted by the bottom of the platinum vessel, in which the sodium was evaporated served as the source of light, the absorption of which on its passage through the vapour was observed. Now it is clear that, had the vapour been throughout of the same temperature with the vessel, the absorption would have exactly counterbalanced the radiation, and no effect would have been produced. The absorption which was produced was, therefore, entirely due only to the vapour in the parts of the tube which were cooler than the bottom. If, therefore, the effect of compression was to drive down the vapour into the hotter part of the tube, a thinning out of the absorption would be a necessary consequence, and no conclusions as to the effect of pressure can be drawn. On the other hand, it is difficult to see why, even in the compressed tube, the vapour should not have gradually diffused into the cooler parts. The disappearance of the channelled-space spectrum of sodium, however, in the compressed vapour, 
indicates a higher temperature, and consequently a diminished absorption.

Taking the whole of these experiments together, they do, I believe, indicate that the lines of sodium widen more easily at a comparatively low temperature; but, as they may also be seen very wide at high temperatures and narrow at low ones, they leave the original cause of the widening unexplained.

The fact that the sodium lines widen more easily at a comparatively low temperature is in accordance with the theoretical speculations we have given on the cause of the widening of lines. For in the passage quoted, Lippich has remarked that an increased widening of lines would go on simultaneously with an increased deviation from Boyle's law; and that deviation will be greater when the vapour approaches its temperature of condensation.

Moreover, the widening of the sodium lines seems to take place chiefly at the temperature at which the line-spectrum changes into the band-spectrum. It will be seen further on that, according to the opinion held by most spectroscopists, the band-spectrum is due to a molecule containing a greater number of atoms than that giving the line-spectrum. If this opinion is true, sodium vapour ought to show a change of vapourdensity as one spectrum changes into the other, similar to that which has recently been proved to exist in iodine by Victor Meyer: ${ }^{1}$ That at the moment when the atoms or molecules of sodium have a tendency to combine with each other, the molecular forces should be affected and disturbed in such a way as to produce a widening of lines seems perfectly intelligible. At the same temperature at which the band-spectrum of sodiun changes into the line-spectrum, Mr. Lockyer has observed some very remarkable phenomena. ${ }^{2}$ In some parts of the tube in which the sodium was volatilized, the lines seem to widen only towards one side, while in others they were widened towards the other side. It is, perhaps, worth mentioning, in connection with a remark by Lord Rayleigh on disturbed vibrations, ${ }^{3}$ that

1 Chem. Ber. xiii. p. 394 (1880). ${ }^{2}$ P'roc. lioy. Soc. xxii. p. 378 (1874). 
the two parts of the band-spectrum of sodium lie on the two sides of the $\mathrm{D}$ lines.

Referring again to the effect of pressure on the widening of lines, the question arises whether for a given temperature and pressure a line may be of different width whether the molecule is placed in an atmosphere of similar or dissimilar molecules. We shall have occasion to refer to this point again, and to show that such a difference in all probability exists, and that it is not due to a mere reduction or increase in the number of luminous molecules in the line of sight. We may mention here, for instance, that Mr. Lockyer ${ }^{1}$ has observed that the lines of oxygen or nitrogen may be obtained sharp at atmospheric pressure by mixing a small quantity of one gas with the other. The gas which is present in small quantities has its lines sharp. If, therefore, we observe that in putting larger quantities of sodium into a flame we widen the lines, we must take many questions into account, and not conclude merely that an increased thickness of the radiating layer has produced the result.

We finally refer to one cause which limits the sharpness of spectroscopic lines, and which was first pointed out by Lippich ${ }^{2}$ and later by Lord Rayleigh. ${ }^{3}$ The molecules of a gas are, in addition to their vibratory motion, endowed with a translatory motion. Those molecules which are moving towards us will, in accordance with Doppler's principle, send us light which is slightly more refrangible than that which would be sent out by a quiescent molecule or one moving at right angles to the line of sight. On the other hand, the molecules which are moving away from us will have the wave-length increased. The lines as they appear to us, and as they come from molecules moving: in all directions, must have a certain width. Lippich has pointed out how this limit of sharpness which cannot be surpassed may be made use of to determine which lines in a mixture of gases are due to each component; for the heavier

1 Phil. Mag. vi. p. 161 (1878).

2 Pogg. Ann. cxxxix. p. 465 (1870).

3 Nature, viii. p. 474 (1873) ; see also W. M. Watts, Chemical News, Oct. 7 , 1870 . 
gas will have its lines narrower than the lighter gas. As a rule, however, the lines of a spectrum are wider than the limit given, and especially the widening of lines which we have been discussing in this chapter is of a much higher order of magnitude.

\section{APPENDIX E.}

ABSTRACTED FROM A REPORT ON "THE GENESIS OF SPECTRA" DRAWN UP BY DR. ARTHUR SCHUSTER FOR A COMMITTEE OF THE BRITISH ASSOCLATION, APPOINTED FOR THE PURPOSE OF REPORTING UPON THE PRESENT STATE OF OUR KNOWLEDGE OF SPECTRUM ANALYSIS.

\section{Connection between the different Periods of Vibration of one Molecule.}

IN some acoustical systems the different periods of vibration are connected together by means of a very simple law, and it was a natural idea to trace the same law if possible in the luminous vibrations of molecules. If the law holds good, the periods of vibrations or the lengths of the waves of light sent out by molecules ought to be in the ratio of small integer numbers. The first published attempt to trace such a connection is due to Lecoq de Boisbaudran, who investigated the spectrum of ritrogen ${ }^{1}$ with special reference to this point.

The author had, however, only a one-prism spectroscope at his disposal, and the wave-lengths as determined by him could not possibly possess that accuracy which is necessary for an investigation of this nature. The more accurate measurements of 'Thalèn do not bear out Lecoq's results.

Inquiries such as those attempted by Lecoq can only be conducted with advantage when we have measured to the highest degree of accuracy which we can obtain in our best instruments, and many of the apparent harmonic ratios which

$$
{ }^{1} \text { C. R, 1xix. p. } 694 \text { (1869). }
$$


at one time were thought to hold good had to give way when subjected to a severer test. Professor Johnstone Stoney, ${ }^{1}$ realizing this fact, has, however, pointed out one set of harmonic ratios which seems to hold good to a high degree of accuracy. We know of four hydrogen lines in the visible part of the spectrum, and three of these are found to be in the ratios of $20: 27: 32$. The wave-lengths of these lines are amongst those best determined by Angström, and they were corrected by Professor Stoney for atmospheric refraction. The following table exhibits the very remarkable coincidence:

TABLE I.

\begin{tabular}{|c|c|c|}
\hline Observed Wave-length. & Calculated Values. & Differences. \\
\hline$h=4102 \cdot 37$. & $\frac{3}{32} \times 131277.14=4102.41$ & +0.04 \\
\hline$F=4862 \cdot 11$ & $\frac{1}{27} \times 131277 \cdot 14=4862 \cdot 12$ & +0.01 \\
\hline $\mathrm{C}=6563.93$ & $\frac{1}{20} \times 131277.14=6563.86$ & -0.07 \\
\hline
\end{tabular}

Other writers, as, for instance, Soret, ${ }^{2}$ have from time to time drawn attention to harmonic ratios in various spectra, and the author of this report has during the last ten years collected a large quantity of material bearing on the question. The results have, on the whole, not been favourable to the theory of harmonic ratios. In any spectrum containing a large number of lines it is clear that, owing to accidental coincidences, we shall always be able to find ratios which agree very closely with the ratios of small integer numbers. It is only by means of a systematic investigation that we can find out whether these coincidences are due to any real cause. We must, by means of the theory of probabilities, calculate the number of the coincidences, which we might expect to find on the supposition that the lines are distributed at random throughout the whole range of the visible spectrum. If, on calculating out all fractions which can be formed in a spectrum by any pair of lines, the number of ratios agreeing within certain limits with ratios of integer number greatly exceeds the most probable number, we should have reason to suppose that the lines are not distributed 
at random, but that the law suggested by Mcssrs. Lecoq de Boisbaudran and Stoney is a true one.

The results of a long investigation conducted in this manner, tend to show that the number of harmonic ratios is, if anything, smaller than was to be expected on the hypothesis of no connection.

There is one fact, moreover, which points very strongly to another yet undiscovered law which rules over the distribution of lines in spectra. It is often observed that the spectrum of a given body contains two or three lines in close proximity forming a characteristic group. Such doublets or triplets are often repeated several times in the spectrum; yet, though we might expect, if the harmonic law were true, to find some simple relations connecting the periods of these sets, such is not the case.

The most remarkable, perhaps, of all the groups of lines observed in the spectra of metals are the magnesium triplets. The well-known set of lines in the green is repeated three times in the ultra-violet; but Table II. shows that the resemblance is only a general one, and that the relative distances vary considerably in each set.

TABLE IJ.

\begin{tabular}{|c|c|c|c|}
\hline $\begin{array}{c}\text { Wave-length of least } \\
\text { refrangible line. }\end{array}$ & $\begin{array}{c}\text { Distance from first to } \\
\text { second line. }\end{array}$ & $\begin{array}{c}\text { Distance from seend } \\
\text { to third line. }\end{array}$ & Observer. \\
\cline { 1 - 2 } & $10 \cdot 9$ & $5 \cdot 3$ & Angström \\
$5183 \cdot 1$ & $6 \cdot 2$ & $2 \cdot 4$ & Cornu \\
$3837 \cdot 6$ & $4 \cdot 2$ & $3 \cdot 0$ &,, \\
$3334 \cdot 2$ & $3 \cdot 7$ & $1 \cdot 9$ &,$\%$ \\
$3095 \cdot 6$ & & & \\
\hline
\end{tabular}

It will be noticed that the groups come nearer and nearer together as they approach the violet, and that also the lines in each group are the closer together the more refrangible the set. 


\section{Relation of the Spectrum of an Element or of a Radical to that of its Compounds.}

There is perhaps no investigation connected with molecular vibrations which is of greater interest than that which tries to trace the connection between the spectrum of an element and that of its compounds. It was at first considered, as has already been mentioned, that an clement preserved its spectrum when entering into combination, so that, for instance, the oxide of a metal would show ouly the metallic lines except in so far as oxygen lines might be visible. This idea had to be given up, but the absorption spcctra of fluids were considered at first to be evidence in favour of the assumption of permanence of the spectrum of an element when combined with others. We owe the first systematic investigation on this point to Dr. Gladstone, ${ }^{1}$ who examined the absorption spectra of the solution of salts, each constituent of which was coloured. He came to the conclusion that, generally, but not invariably, the following law held good: "When an acid and a base combine, each of which has a different influence on the rays of light, a solution of the resulting salt will transmit only those rays which are not absorbed by either, or, in other words, which are transmitted by both."

Erhard ${ }^{2}$ has examined the absorption spectra of some salts in which chromium plays the part of a base. It may be said as a general rule that these salts absorb the yellow and yellowish green and also the violet end of the spectrum, transmitting the blue; the exact position of the maximum of absorption, however, and the intensity of the absorption band, vary considerably with different salts, and even for the same salt with different temperatures, and the results are complicated by the fact that heating the salts produces a permanent alteration in the absorption. The insoluble chloride of chromium shows a behaviour differing from that of the other chromium salts. It transnits the yellow and more of the violet than the other salts. Some of

1 Phil. Jag. xiv. p. 418 (1857).

2 Inaugural Dissertation, Freiburg (without date). 
the solid crystals of various chromium salts show fine absorption bands in the red which can also be traced in some of the solutions. There is, therefore, a general resemblance in the absorption of different chromium salts, but no identity.

Dr. Gladstone has also examined the effect of chlorine, bromine, and iodine when combined with different metals. The bromides of gold, platinum, palladium, and potassium give a spectrum which is identical with that of bromine water; the same applies to a concentrated solution of the bromide of copper, which, in addition, shows the red absorption characteristic of copper. A dilute solution of bromide of copper shows, however, no absorption which can be traced to the bromine. Similar results were obtained with the chlorides and iodides. In pointing out that it is generally, though not universally true that a base or an acid retains its absorptive properties in different combinations, Dr. Gladstone draws attention to the remarkable exception of ferric ferrocyanide, which when dissolved in oxalic acid transmits blue rays in great abundance, though the same rays are generally absorbed both by ferrocyanides and by ferric salts.

Nitric acid and the nitrates of transparent bases such as potassium, sodium, and ammonia show spectra, according to Soret, which are not only qualitatively but also quantitatively identical; that is to say, a given quantity of nitric acid in solution gives a characteristic absorption band of exactly the same width and darkness whether by itself alone or combined with a transparent base. It also shows a continuous absorption at the most refrangible side, beginning with each of the mentioned salts at exactly the same point. The ethereal nitrates, ${ }^{1}$ however, give different results.

Messrs. Hartley and Huntington have by photographic methods examined the absorption spectra of a great number of organic compounds. As their researches have already been referred to at length in these reports, ${ }^{2}$ by one of the authors, we need at present only mention one or two of the results which most interest us from our present point of view. The normal

I C. R. 1sxxix. p. 747 .

2 Brit. Ass. Rep. p. 55 (1880). 
alcohols were found to be transparent for the ultra-violet rays, the normal fatty acids less so. In both cases an increased number of carbon atoms increases the absorption at the most refrangible end. The fact that benzene and its derivatives are remarkable for their powerful absorption of the most refrangible rays, and for characteristic absorption bands appearing on dilution, led Professor Hartley to a more extended examination of some of the more complicated organic substances. He came to the conclusion that definite absorption bands are only produced by substances in which three pairs of carbon atoms are doubly linked together as in the benzene ring.

In most of the cases which we have hitherto discussed, the characteristic absorption of the substance under examination extended over a considerable range; the substance either blocked out altogether a large part of the spectrum, or at least showed absorption bands which were broad and increased considerably in width with increased concentration. When, however, absorption bands become narrower and more definite, so that they can be examined under high dispersive powers, their behaviour under different circumstances becomes more interesting, for we can trace smaller differences and more minute changes.

It was Bunsen ${ }^{\mathrm{I}}$ who first showed that such small changes do occur, and he thereby led the way in a line of research which promises to be of great importance. While examining the absorption spectra of different didymium salts, he found that though all the salts showed spectra so nearly identical that with the ordinary one-prism spectroscope they could easily be mistaken for each other, higher dispersive powers revealed some very interesting and characteristic changes. His conclusions are best quoted in his own words:

"Very remarkable and noteworthy are the small alterations in position which occur in the minima of brightness in the didymium spectrum, dependent upon the nature of the compound in which the metal occurs. These changes are too minute to be seen with the small, though seen with the large instrument. I

\footnotetext{
¿ Phil, Mag. xxxii. p. 177.
} 
have as yet only investigated them completely in the case of three didymium salts, viz., the chloride, sulphate, and acetate. It is, however, more than probable that the same phenomena will also be found to occur with other solutions, and with the absorption spectra of other crystals of didymium salts, and perhaps may be exhibited with the luminous spectra of the oxide and other compounds of didymium. . . . The atomic weight of didymium chloride is 959 , and that of the anhydrous acetate is 106.9 . It will be noticed that all the groups of bands in the case of salts under examination approach the red end of the spectrum in the order of their increasing atomic weights.

"These differences, here noticed in the absorption spectra of different didymium compounds, calmot in our present complete state of ignorance of any general theory for the absorption of light in absorptive media be connected with other phenomena. They remind one of the slight and gradual alterations in pitch which the notes from a vibrating elastic rod undergo when the rod is weighted, or of the change of tone which an organ-pipe exhibits when the tube is lengthened."

Some interesting cases of this shifting of bands in different compounds of the same body have been found by Professor Russell, ${ }^{1}$ who has subjected the cobalt salts to a very careful and most instructive examination. The anhydrous bromide of cobalt, for instance, was found to give an absorption spectrum strongly similar to that of the chloride, but there is a general displacement of all the bands towards the red corresponding to the increased atomic weight of bromine. The effect on the most refrangible band is stronger than that on the other two bands, which is contrary to what Bunsen has observed in the case of didymium acetate.

Captain Abney and Lieutenant-Colonel Festing's paper 2 "On the Influence of the Atomic Grouping in the Molecules of Organic Bodies on their Absorption in the Infra-red Region of the Spectrum," contains an account of investigations undertaken to throw light on the effect of chemical combination on molecular

1 Phil. Trans. p. 887 (1881, iii.).

2 Proc. Roy. Soc. xxxii. p. 258 (1881). 
vibrations. The importance of the results which they have obtained will justify a few verbal quotations. They distinguish a general absorption from the least refrangible end, and special absorptions which may consist of lines or bands.

"Regarding the general absorption we have nothing very noteworthy to remark, beyond the fact that, as a rule, in the hydrocarbons of the same series those of heavier molecular constitution seem to have less than those of lighter."

"A crucial test was to observe spectra of bodies containing hydrogen and chlorine, hydrogen and oxygen, and hydrogen and nitrogen. We therefore tried hydrochloric acid and obtained a spectrum containing some few lines. Water gave lines, together with bands, two lines being coincident with those in the spectrum of hydrochloric acid.

"In ammonia, nitric acid, and sulphuric acid, we also obtained sharply-marked lines, coincidences in the different spectra being observed, and nearly every line mapped found its analogue in the chloroform spectrum, and usually in that of ethyl iodide. Benzene again gave a spectrum consisting principally of lines, and these were coincident with some lines also to be found in chloroform. It seems then that the hydrogen, which is common to all these different compounds, must be the cause of the linear spectrum. In what manner the hydrogen anmihilates the waves of radiation at these particular points is a question which is, at present at all events, an open one, but that the linear absorptions, common to the hydrocarbons and to those bodies in which hydrogen is in combination with other elements such as oxygen and nitrogen, is due to hydrogen, there can be no manner of doubt."

"The next point that required solution was the effect of the presence of oxygen on the body under examination, and here we had ample material on which to make our observations. It appears that in every case where oxygen is present, otherwise than as a part of the radical, it is attached to some hydrogen atom in such a way that it obliterates the radiation between two of the lines which are due to that hydrogen." . . . If more than 
one hydroxyl group be present, we doubt if any direct effect is produced beyond that produced by one hydroxyl group except a possible greater general absorption; a good example of this will be found in cinnamic alcohol and phenyl-propyl alcohol, which give the same spectra as far as the special absorptions are concerned.

"Hitherto we have unly taken into account oxygen which is not contained in the radical ; when it is so contained it appears to act differently, always supposing hydrogen to be present as well. We need only refer to the spectrum of aldehyde, which is inclined to be linear rather than banded, or rather the bands are bounded by absolute lines, and are more defined than when oxygen is more loosely bonded."

Perhaps the most interesting passage is that which refers to the detection of the radical, and we therefore quote it in full :

"An inspection of our maps will show that the radical of a body is represented by certain well-marked bands, some differing in position according as it is bonded with hydrogen, or a halogen, or with carbon, oxygen, or nitrogen. There seem to be characteristic bands, however, of any one series of radicals between 1000, and about 1100 , which would indicate what may be called the central hydrocarbon group to which other radicals may be bonded.

"The clue to the composition of a body, however, would seem to lie between $\lambda 700$ and $\lambda 1000$. Certain radicals have a distinctive absorption about $\lambda 700$ together with others about $\lambda$ 900, and if the first be visible it almost follows that tho distinctive mark of the radical with which it is connected will be found. Thus in the ethyl series we find an absorption at 740 , and a characteristic band, one edge of which is at 892 , and the other at 920 . If we find a body containing the 740 absorption and a band with the most refrangible edge commencing at 892 , or with the least refrangible edge terminating at 920 , we may be pretty sure that we have an ethyl radical present. So with any of the aromatic group; the crucial line is at 867 . If that line be connected with a band we may feel certain that 
some derivative of benzene is present. The benzyl group shows this remarkably well, since we see that phenyl is present, as well as is also methyl. It will be advantageous if the spectra of ammonia, benzene, aniline, and dimethyl-aniline be compared, when the remarkable coincidences will at once become apparent, as also the different weighting of the molecule. The spectrum of nitro-benzene is also worth comparing with benzene and nitric acid. We should have liked to have said more regarding the detection of the different radicals, but it might seem presumptuous on our part to lay down any general law on the results of the comparatively few compounds which we have examined. In our own minds there lingers no doubt as to the easy detection of any radical which we have examined, but it will require more energy and ability than we possess to thoroughly classify all the different modifications which may arise.

"We may say, however, it seems highly probable by this delicate mode of analysis that the hypothetical position of any hydrogen which is replaced may be identified, a point which is of prime importance in organic chemistry.

"The detection of the presence of chlorine or bromine or iodine in a compound is at present undecided, and it may well be that we may have to look for its effects in a different part of the spectrum. The only trace we can find at present is in ethyl bromide, in which the radical band about 900 is curtailed in one wing. The difference between amyl iodide and amyl bromide, is not sufficiently marked to be of any value."

Alexr. Mitscherlich ${ }^{1}$ was the first to prove that compound bodies, when luminous, have a spectrum of their own, and do not simply show the superposed spectra of the elements. He followed up this important discovery by investigating the spectra of different compounds of the same metal, and he could not fail to be struck with the similarity which such spectra often present.

Many, for instance, will instantly recognize the spectrum of

1 sce, however, Appendix B, according to which Roscoe and Clifton, two years previous to Mitscherlich, have shown the difference between the spectrum of an element and that of its compounds. 
the oxide or chloride of calcium as that of a calcium compound, without being even aware that these spectra present certain well-marked differences. Comparing together the spectra of the fluoride, chloride, bromide, and iodide of barium, as they appear on Mitscherlich's map, we detect at once a strong similarity; we seem to have one spectrum shifted towards the red with increasing atomic weight of the metalloid. At the same time the least refracted bands seem to be most affected and, as a consequence, the bands appear nearest together in the fluoride and farthest apart in the iodide. In the calcium and strontium salts we notice the same increase of wave-length in corresponding bands with increasing atomic weight, but with these two metals the most refracted bands are most affected, so that the bands are nearer together the higher the atomic weight. Mitscherlich ${ }^{1}$ tried to find a numerical expression for these relations, and he expresses the law which, according to his observation, represents the facts, in the following way:

"It follows that in the haloid compounds of barium (excepting the fluoride) the distance between corresponding spectral lines is directly proportional to the atomic weight, and that in the haloid compounds of calcium and strontium (excepting again the fluoride) these distances are inversely proportional to the atomic weights. Further, that there is such a point in the spectrum of each metal that the bands keep their relative distances from it in the different compounds. Here also we must except the fluorides."

Mitscherlich's measurements were not sufficiently accurate to prove his statement satisfactorily, but we can easily test it by means of the more exact experiments of Lecoq de Boisbaudran, who has carefully mapped the spectra of the chloride (I), bromide (II), and iodide (III) of barium. A glance at Fig. 53, which represents these spectra according to Lecoq's measurements, will show their similarity.

We ought to inquire whether a connection can be traced between the lines of a metal and that of one of its compounds.

1 Mitscherlich's rule is not, however, confirmed by Lecoq's more accurate measurements. 
It would be possible; for instance, that the oxide should show its bands chiefly at such places at which we find lines in the metallic spectrum, and such a rule might be suggested by the examination of the calcium spectrum, which shows a characteristic group of lines exactly at the place which is filled by the green band of the oxide. No general rule can, however, be given, and in some cases even the metallic spectrum seems particularly free of lines in or about the place in which we tind the oxide bands. There is at present no hope whatever of directly connecting the spectrum of a metal and that of its compounds, though, as was seen, we may hope to gain an insight into the relations of the spectra of such similar compounds as the chlorides, bromides, and iodides, which may be supposed to have a similar constitution.

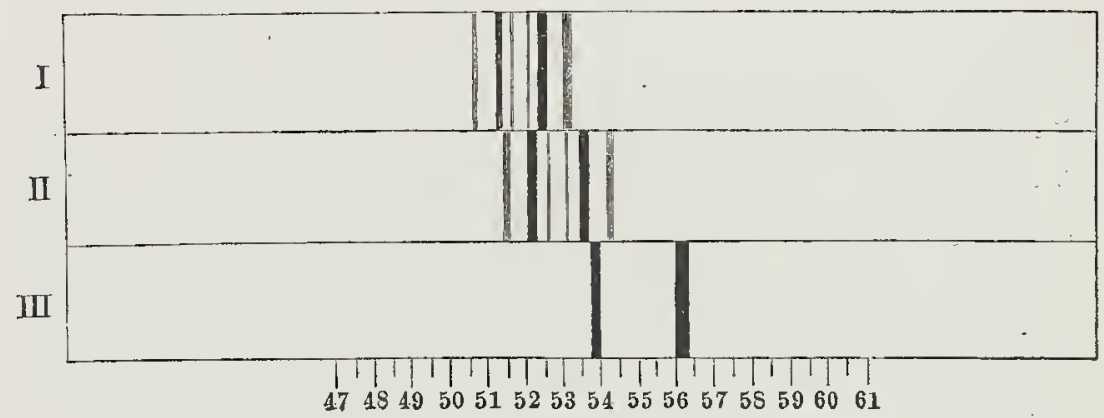

FIG. 53.

Some very interesting changes have been noticed in the position of absorption bands when certain colouring matters are dissolved in different liquids. We mention the names only of Hagenbach, Kraus, and Kundt as having studied the question in particular cases. Two papers by Kundt ${ }^{1}$ and Claes ${ }^{2}$ contain all that is known at present on the subject, but no value ought to be attached to the theoretical part of Dr. Claes's paper.

Kundt examined the position of the absorption bands of chlorophyll, anilingreen, cyanin, fuchsin, chinizarin, and of the colouring matter of the yolk of egg when dissolved in a number of liquids.

1 Wied. Ann. iv. p. 34 (1878).

2 Wied. Ann. iii. p. 389 (18r8). 
In a preliminary examination, ${ }^{1}$ Professor Kundt had come to the conclusion that solvents displaced absorption bands towards the red in the order of their dispersive powers. But a more complete investigation showed that no absolute law holds.

Professor Kundt therefore replaces his old conclusion by the less definite rule that "When a colourless solvent has a considerably larger dispersive power than another, the absorption band of a colouring matter dissolved in it, is placed more towards the red."

\section{Relations of the Speetra of different Elements.}

Various efforts have been made to connect together the spectra of different elements. The attempts in this direction generally assume that certain lines in one spectrum correspond to certain lines in another spectrum, and the question is raised whether the atom with the ligher atomic weight has its corresponding lines more or less refrangible. In the opinion of the writer of this report no definite judgment can as yet be given as to the success of these efforts; some of the relations traced no doubt are interesting and deserve further attention, but most of them are far-fetched, and will very probably be proved to have no foundation in fact. Lecoq de Boisbaudran has led the way in these speculations, and some of the similarities in different spectra pointed out by him are certainly of value. But whether his conclusion, that "the spectra of the alkalis and alkaline earths, when classed according to their refrangibilities, are placed as their chemical properties in the order of their atomic weights," will stand the test of further research, remains to be seen.

Ditte $^{2}$ has tried to find an analogy between the spectra of sulphur, selenium, and tellurium, and to establish that the spectrum is displaced towards the blue as we go from the metalloid to the metal, that is to say, from the lighter to the heavier element. An inspection of the spectra in question has not led the author of this report to confirm this statement. The spectrum of selenium seems more contracted than the

\footnotetext{
1 Poggendorff, Jubelband, 615.

2 C. R., lxxii. p. 622 (1871).
} 
spectrum of sulpliur, but we cannot recognize clearly any displacement towards the red or towards the blue. The spectrum of tellurium seems, if anything, more to the red than that of the other two metals.

Messrs. Troost and Hautefeuille ${ }^{1}$ have compared the spectra of carbon, boron, silicium, titanium, and zirconium, and they also come to the conclusion that from the metalloid to the metal the spectrum seems progressively to move towards the blue.

As far as the first tluree bodies are concerned the relation seems, at first sight at any rate, to have some foundation, for we have here to deal with three spectra which, on the whole, resemble each other in appearance, and which seem to be displaced according to the suggested law.

Ciamician ${ }^{2}$ has compared together the spectra of chlorine, bromine, and iodine, and has arrived at the following conclusion : "The spectrum of vapour of bromine at low pressures becomes the more nearly like that of chlorine the smaller the pressure, while the spectrum of condensed bromine more nearly resembles the spectrum of iodine. Todine, on the other hand, gives a spectrum resembling that of bromine at moderate pressures only; at very low pressures an analogy with the chlorine spectrum takes preponderance over that with the bromine spectrum. The spectrum of highly condensed iodine vapour cannot well be compared with that of the other two bodies. Chlorine, when it is highly condensed, gives a spectrum resembling that of bromine at large pressures and of iodine at moderate pressures; while at low pressures the spectrum of chlorine has no analogy to that of the other two elements.

"If we collect together for each body all the lines which appear separately under different circumstances, we can establish a complete correspondence between the lines of the three complete spectra."

We have quoted Ciamician's results, as his experiments seem well conducted, but we must reserve our opinion as to the reality of the relations pointed out by him.

${ }^{1}$ C. R., Ixxii. p. 620 (1871).

2Wien. Ber, Ixxriii. (1878). 


\section{LECTURE IV.}

Selective Absorption,-Coloured Vapours.-Atmospheric Absorption.-Iodine.-Bromine.-Chloride of Indine-_Nitrous Fumes.-Metallic Vapours.-Liquids and Coloured Solutions.-Blood Bands.-Chlorophyll.-Connection between Absorption Spectra and Chemical Constitution.-Selective Absorption by Solid Bodies.-Didymium Salts.Applications of Spectrum Analysis.-Blood Poisoning.-Colouring Matters. - Spectrum of the Bessemer Flame. - Fluorescence and Phosphorescence. - Construction of High Power Spectroscopes. Arrangement for Diffraction-Spectra.

Appexdix A.-On the Spectrum of the Bessemer Flame.

A ppendix B.--Spectroscopic Measurements and Appliances.

Appendix C.-On the Spectra of Erbium and Didymium, and their Compounds.

THE subject to which I beg your attention to-day is one of a somewhat different character from that which we have already considered. Hitherto we have confined our attention to the character of the light emitted by incandescent bodies; we find, however, that many substances, not only gases, but liquids, and even solid bodies, exert at the ordinary temperature of the air a selective absorption power upon white light when it passes through them. This effect can easily be rendered evident to you when I hold this globe filled with the red coloured vapours of the oxides of nitrogen before the slit of our electric lamp. We now see that the continuous spectrum of the carbon points is crossed by a large number of dark bands and lines, and observation has shown that these are as characteristic of and as peculiar to this gas as are the bright lines emitted by an 
incandescent vapour. For instance, if I replace the red nitrous fumes by this violet vapour of iodine you will observe another series of dark bands which are caused by iodine and by no other known substance. We can of course measure the position of these absorption bands exactly as we do that of the bright lines. For this purpose we have only to place the absorptive inedium in front of the slit of our spectroscope, and allow the light from a flame of coal gas or petroleum to pass through the coloured substance. Fig. 54 gives a rough representation of the absorption spectra thus produced, the dark bands being seen on a background of a continuous spectrum.

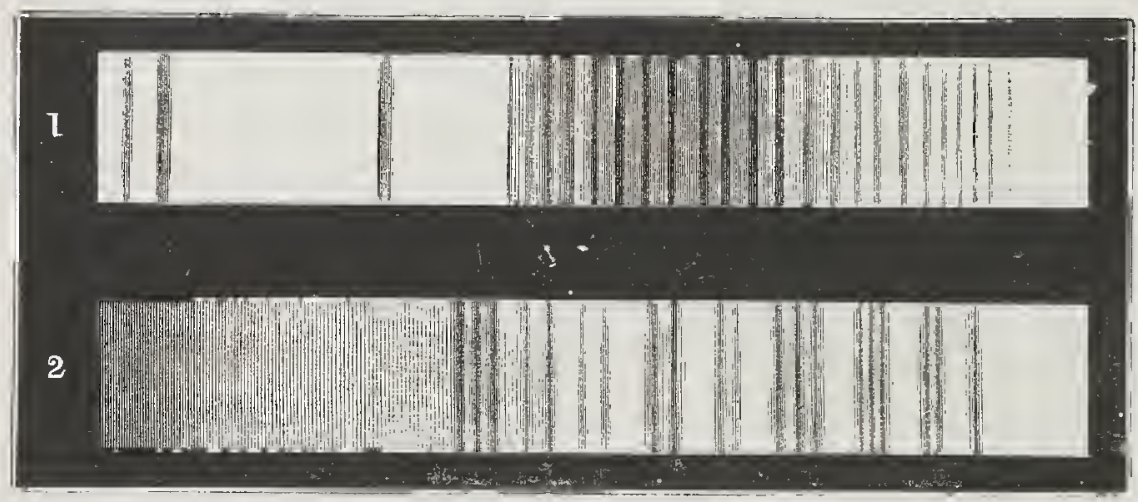

File. Es.

It is well to remember that the very fact of a gas bcing coloured indicates an unequal absorptive power for the various constituents of white light, and also that gases which are colourless in small bulk may prove to be coloured, and therefore, to give absorption bands, when the light passes through a thick stratum. Thus for instance ozone or modified oxygen is colourless when seen in small quantity but possesses a blue colour, and shows distinct bands when the light is allowed to pass through a long column of the gas. Moreover M. Janssen was able to observe the peculiar bands due to aqueous vapour, which is colourless when seen in small quantity, by passing a ray of white light through a tube 100 feet long filled with high-pressure steam. 
These bands are identical in position with certain broad dark lines seen in sunlight, especially when the sun is low on the horizon and when the earth's atmosphere is charged with aqueous vapour. Certain other dark lines, which also increase in intensity as the altitude of the sun diminishes, are probably due to the absorption of oxygen and nitrogen; both these classes of lines have been termed atmospheric lines because produced by the sun's rays passing through the earth's atmosphere. Fig. 55 gives a general idea of the distribution of these atmospheric lines, which were first observed by Sir David Brewster in 1833,

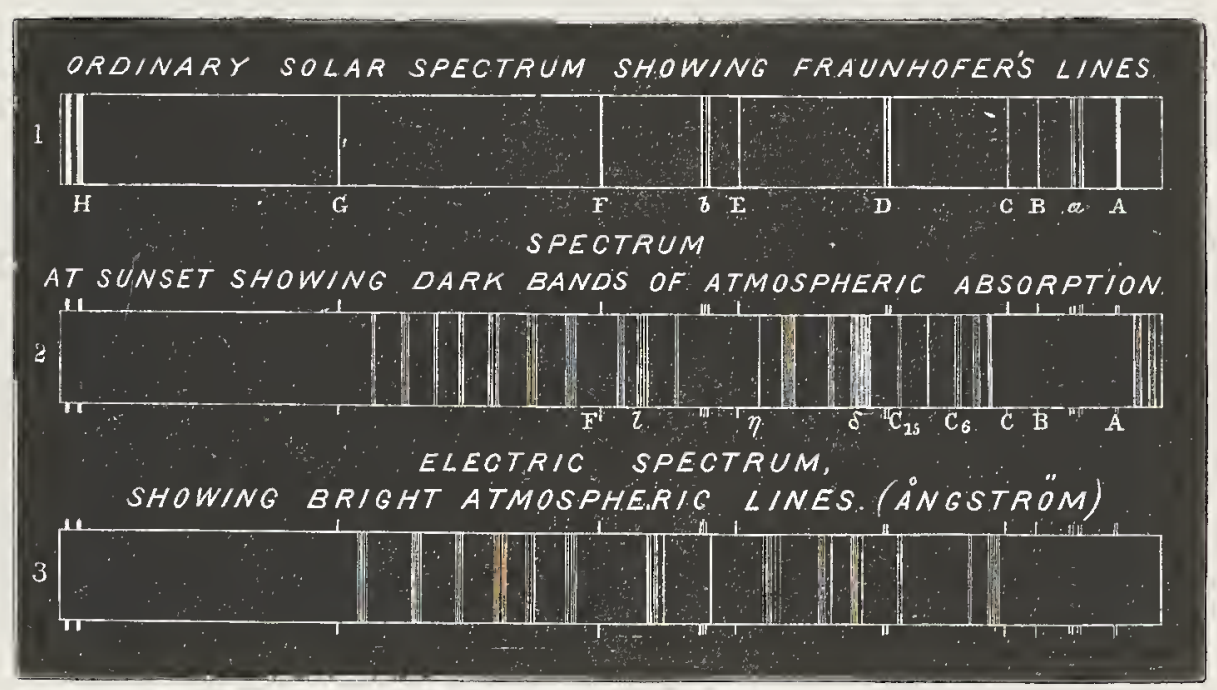

FiG. 55.

and afterwards nnapped by himself and Dr. Gladstone, and more recently by Prof. Angström. Fig. 56 is an accurate map of the telluric lines observed by Janssen in a column of steam. The important results of his researches are, (1) that Brewster's dark bands are resolvable into fine lines comparable with Fraunhofer's lines, and (2) that the terrestrial atmosphere produces in the spectrum a system of fine lines, so that the absorptive action exerted by our own atmosplere is analogous to that of the sun in spite of the enormous difference in temperature. All the dark lines seen in the lower, but not found in the upper spectrum 
(Fig. 56) have a telluric origin, and they have been designated by Greek letters, and are classed in groups according to their position with regard to well-known solar lines.

The telluric lines situated close to the $\mathrm{D}$ lines, but rather less refrangible, are seen to be considerably strengthened before a heavy shower of rain. In dry weather on the other hand they are but indistinctly seen, even when the sun is near the horizon. These lines in spectroscopes of small dispersive power are not resolved but appear as one dark band lying on the red side of the sodium D lines, and this has been called the "rain band." Another telluric band in the yellowish green is also seen, but this appears to be less affected by the changes in the weather. Attempts have been made, thus to predict coming rain by means of the spectroscope, and these seem to have been successful in the hands of experienced observers, particularly of Piazzi Smyth and Rand Capron. From the observations of

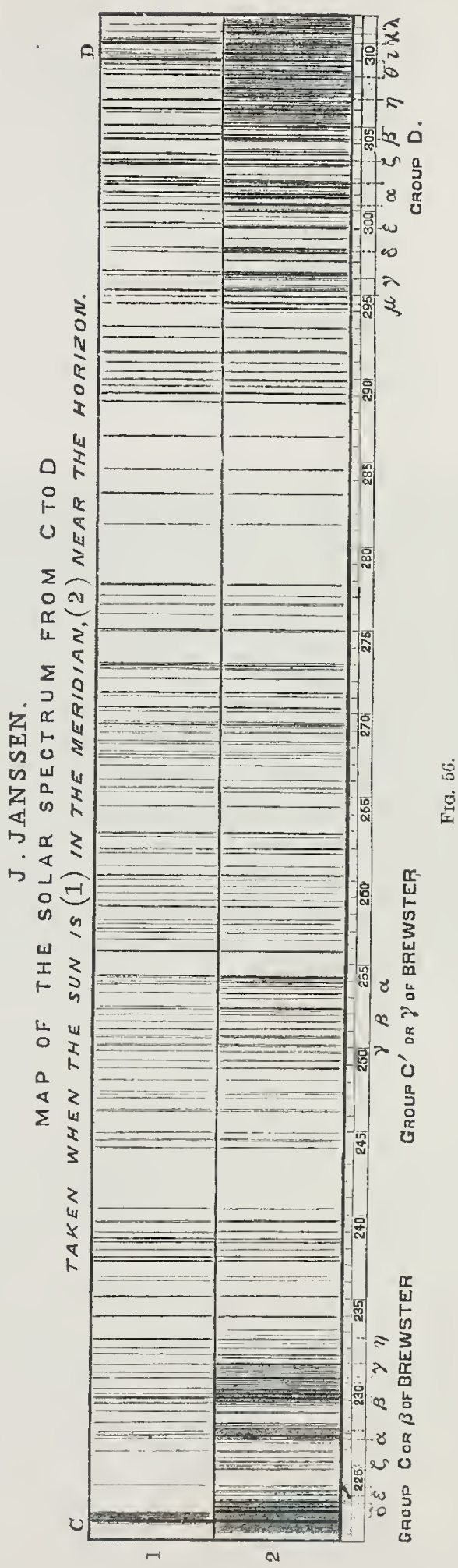


Professor Smyth spread over seven months, it appears that when the rainband was seen at its maximum of intensity, rain followed in 92 cases out of 100 ; whilst when at its minimum, fine weather continued in 79 per cent. of the cases. ${ }^{1}$

That this property of gases to exert selective absorption and to give peculiar dark band spectra may often be advantageously employed for the purpose of tracing the chemical composition or constitution of a gas may readily be illustrated. Here I have two glass globes each filled with a reddish brown-coloured gas; by the naked eye no difference can be perceived between them. Moreover many of their chemical properties are so closely allied that it is not very easy to show that they are in reality different substances, indeed a chemist of no less renown than Liebig liimself acknowledged that he had mistaken the one for the other. $^{2}$ This brown gas is the element Bromine; this one is a compound of the two elements Chlorine and Iodine. If we examine these gases by means of a spectroscope their essential difference at once becomes manifest, for we notice that whilst the spectra exhibit a resemblance at first sight, none of the bands in the one spectrum are found on examination to coincide with those of the other, and we see, moreover, other differences, such for instance as that in the bromine spectrum, six bands occur in the red beyond any found in the spectrum of the iodine mono-chloride. ${ }^{3}$

1 "The Rainband, How to Observe It." By H. R. Mill. Adam Hilger, 204, Stanhope Street, London, N.W. (1883).

2 "I know a chemist who, while at lireuznach, many years ago undertook an investigation of the mother-liquor from the salt works. He found iodine in it; he observed, moreover, that the iodide of starch turned of a fiery yellow by standing ovcruight. The phenomenon struck him, he produced a large quantity of the mother-liquor, saturated it with chlorine, and obtained by distillation a considerable amount of a liquid colouring starch yellow, and possessing the external properties of chloride of iodine, but differing in many of its reactions from the latter compound. He explained, however, every discrepancy most satisfactorily to himself, contriving for himself a theory on it (Er machtc sich eine Theoria daribber). Several months later he received the splendid paper of M. Balard, and on the samc day, he was in a condition to publish a series of cxperiments on the behaviour of bromine with iron, platinum, and carbon, for Balard's bromine stood in his laboratory, labelled "liquid chloride of iodine." Since that time he makes no theories unless they are supported and confirmed by unequivocal experiments : and I can positively assert that he has not fared badly by so doing " (Liebig).

3 Roscoe and Thorpe, "Absorption Spectra of Bromine and Iodine Mono. ehloride." Phil. Trans. 1877, part i. p. $20 \%$. 
Nor is the production of absorption spectra confined to nonmetallic gases; the vapours of certain metals when examined at comparatively low temperatures also exhibit definite absorption spectra. Thus for example this green coloured gas which I obtain by heating a small globule of the alkali-metal potassium in a glass tube filled with hydrogen, yields a complicated but perfectly definite series of dark bands, by help of which the presence of metallic potassium can be ascertained with as much certainty as when the bright red and violet lines of the emission spectrum are observed. So, too, the vapour of sodium shows a definite absorption spectrum quite distinct from that of potassium. In all probability the complexity of the gaseous molecule yielding these channelled-space spectra is greater than that of the molecule existing at higher temperatures when line spectra only are seen. ${ }^{1}$

A most striking instance of the selective absorption exerted by liquid and solid bodies is that of the salts of the rare metal Didymium. These possess the power of absorbing from white light certain definite rays, so that if I place some of the solution in the path of our continuous spectrum, we get the broad dark bands by which, as Dr. Gladstone has shown, the presence of didymium may be recognized when present even in very minute quantities. As in the case of aqueous vapour, so here it is true that, although these didymium absorption lines are so black, and serve as such a reliable test of the presence of this metal, yet the fraction of the total light which is absorbed is so small that the solution appears colourless. From the experiments of Bunsen on this subject we learn that the various didymium compounds do not exhibit exactly the same absorption lines, und that if light is allowed to fall upon a crystal, the dark bands also differ according to the direction in which the light passes through (see Appendix C).

"The differences thus observed," says Bunsen, "cannot as yet be connected with other phenomena. They remind one of the gradual alterations in pitch which the notes from an elastic rod undergo when the rod is weighted."

1 Roscoe and Schuster, Proc. Roy. Soc. xxii. 362. 
Some interesting cases of this shifting of bands in different compounds of the same body have been described by Professor Russell, ${ }^{1}$ who has subjected the cobalt salts to a very careful and most instructive examination. The anhydrous bromide of cobalt, for instance, was found to give an absorption spectrum very similar to that of the chloride, but there is a general displacement of all the bands towards the red colresponding to the increased atomic weight of bromine. The effect on the most refrangible band is stronger than that on the other two bands, which is contrary to what Bunsen has observed in the case of didymium acetate.

Remembering these changes, we must accept with great caution any conclusions as to chemical composition derived solely from variation in the absorption spectra. ${ }^{2}$ Indeed, it has been shown that even careful observers may be led to false conclusions by relying too implicitly on the complicated absorption bands which certain mixtures may yield. In experienced hands, however, the examination of the distinct absorption spectra yielded by the metals of the rare earths has proved to be of the greatest service as a means of distinguishing between these closely allied elements.

The solutions of many other coloured metallic salts possess a similar property of yielding definite absorption lines, and Dr. Gladstone finds that with very few exceptions all the compounds of the same base, or acid, have the same effect on the rays of light: thus the chromium salts (both green and purple) exhibit the same form of absorption spectrum (Fig. 57). Fig. 58 shows the bands produced by potassium permanganate solution contained in a wedge-shaped vessel. The right-hand corresponds to the red end of the spectrum, and the letters refer to the position of Fraunhofer's lines. The absorptive action of the solution is most powerful at the upper part of each drawing, which represents the spectrum seen where the layer of solution

1 Phil. Trans. (1881), p. 887, also Proc. Roy. Soc. xxxii. 258.

2 It has been found that the absorption bands attributed to a new metal contained in zircons are due to a mixture of salts of zirconium and uranium. This mixture appears to afford a most delicate means of detecting the presence of uranium, for bands appear in the mixture which are not seen when the two metals are examined scparately in much larger quantities (Sorby). 
was thickest, and diminishing towards the lower part of the figure.

Even some compounds which are usually considered as colourless, such as nitric acid and the nitrates of potash and soda, show spectra, which, according to Soret, are not only qualitatively, but quantitatively, identical, that is to say, a given quantity of nitric acid in solution gives a characteristic absorption band of exactly the same width and darkness whether observed by itself alone, or when combined with a transparent base.

In like manner Messrs. Russell and Lepraik have shown that colourless organic liquids when examined in columns, varying

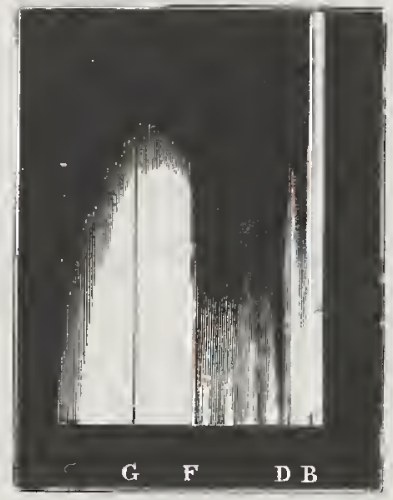

lif. 57.

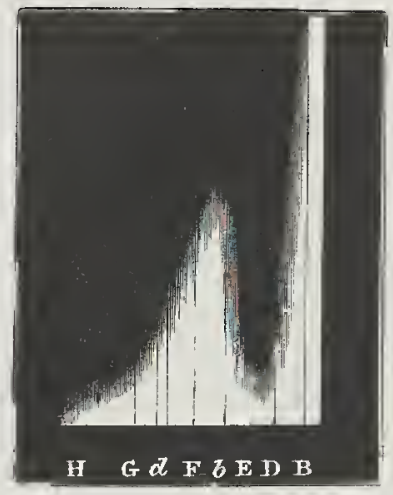

FiG. 58 ,

from two to eight feet in thickness, exhibit definite absurption bands by which the special compound can be recognized. ${ }^{1}$

A variety of other substances possess this selective power. Some of the more interesting absorption spectra are shown on Figs. 59 and 60 . In both of these, the position of the dark bands is referred to the fixed dark lines of the solar spectrum indicated by vertical straight lines and by the letter's of the alphabet. Spectrum No. 1, Fig. 59, is that of the violet-coloured solution of potassium permanganate; No. 2, that of arterial or oxidized blood; No. 3, that of venous or reduced blood; No. 4, that of a solution of didymium chloride; No. 5, that of one of

1 Chem. Soc. Journ. 1881 (1) 168. 
erbium chloride; No. 6, that of one of uranium nitrate, and No. 7, that given by glass coloured blue by oxide of cobalt. Fig. 60 exhibits the absorption spectra of the very interesting colouring matters contained in the madder root(Rubia tinctorum),

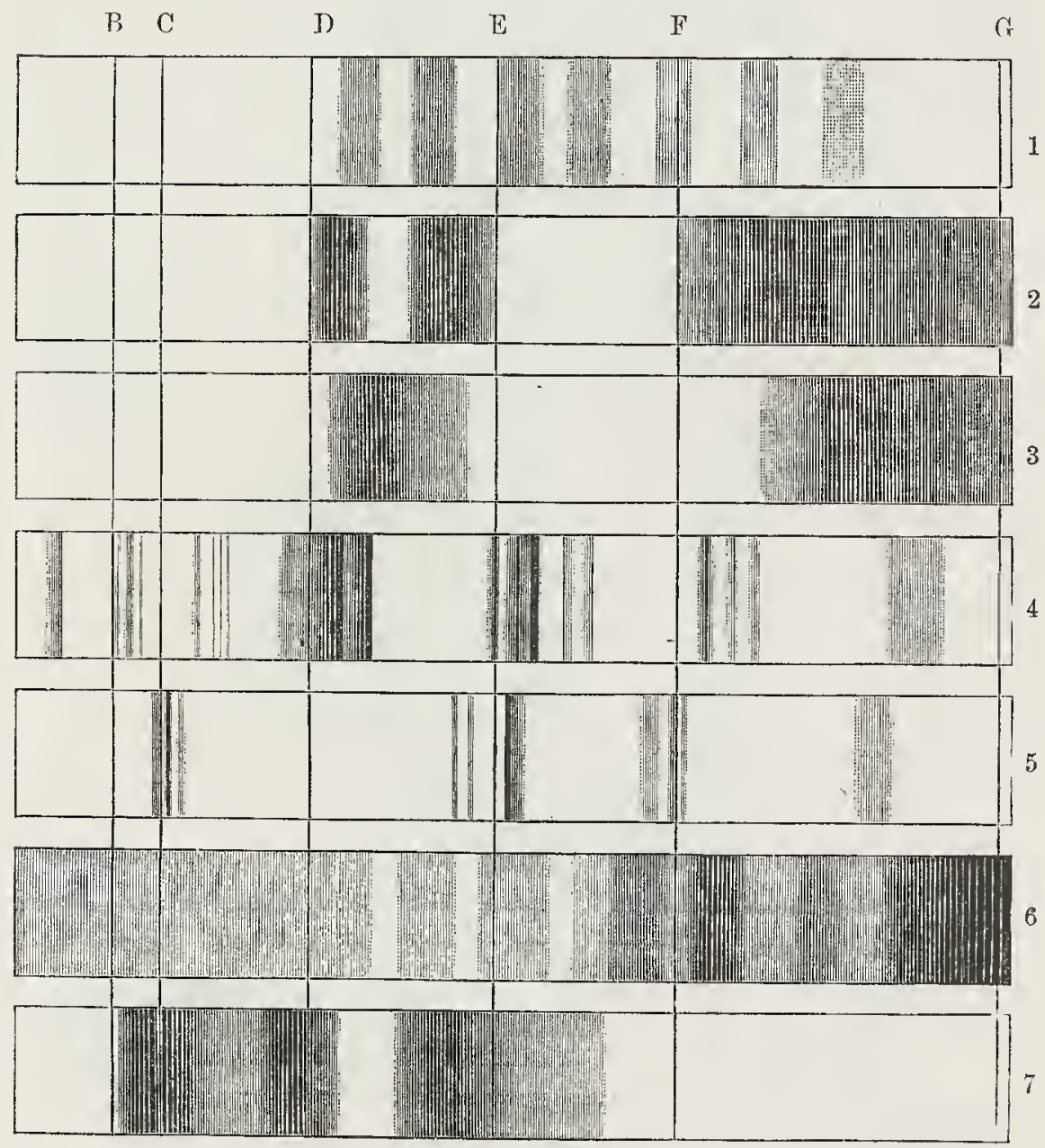

FIG. 59.

but now artificially prepared from coal-tar, and known as the alizarine colours. The selective absorption exerted by the artificial colouring matter is precisely the same as that observed in the case of the natural madder dye, and hence the perfect 
identity of the colours is placed beyond doubt. A remarkable difference is noticed between the absorption spectrum of the

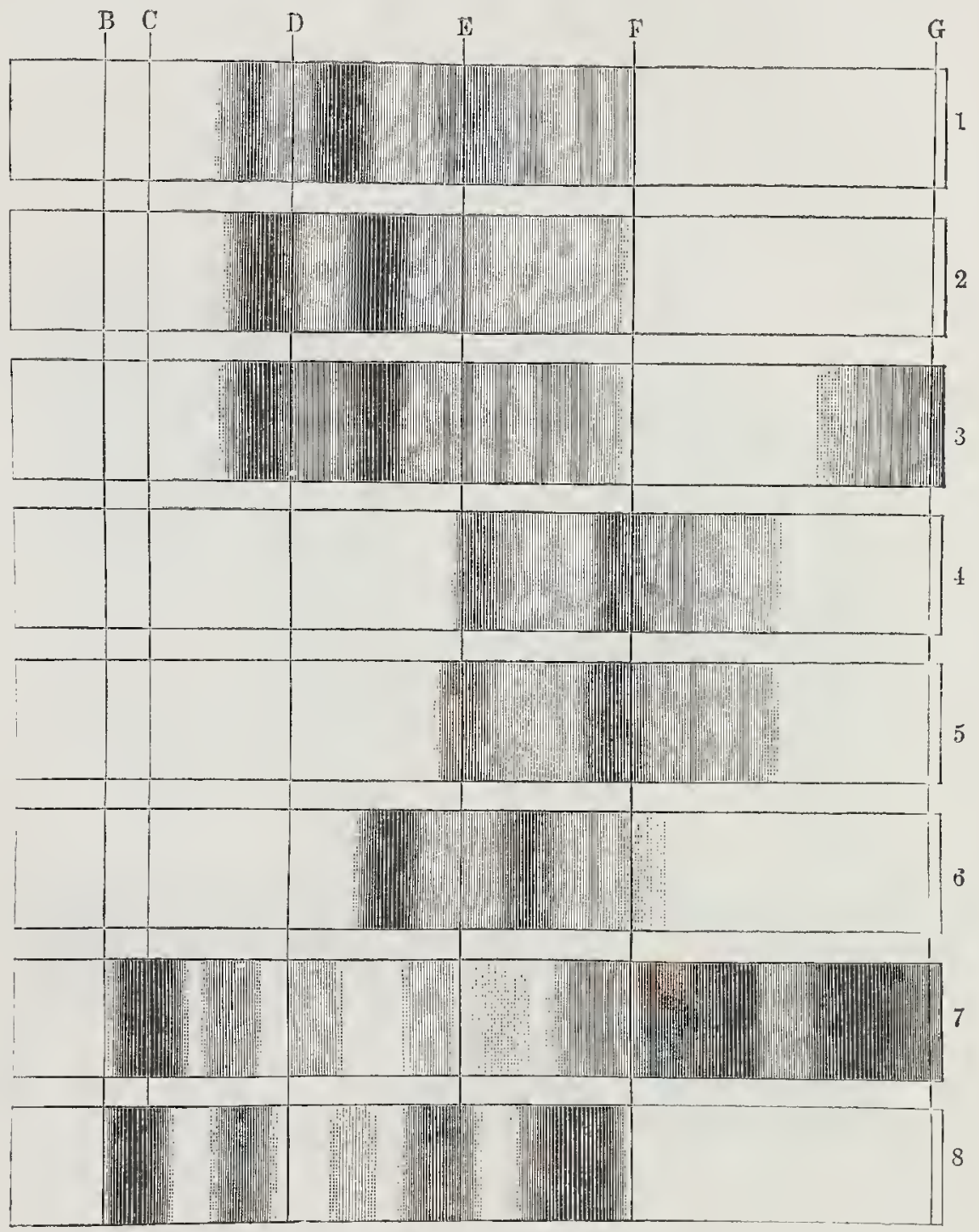

FIG. 60.

same substance according to the nature of the liquid used for its solution; thus Nos. 4,5 and 6, Fig. 60, distinctly different 
spectra, are all due to the one colouring matter purpurine, in the first instance dissolved in ether, in the second in carbon disulphide, and in the third in alkali. Spectrum No. 1 is that of an alkaline solution of alizarine; No. 2, that of flavopurpurine and No. 3, that of isopurpurine, whilst No. 7 is that of an alcoholic solution of chlorophyll, the green colouring matter of leaves, and No. 8 that of phyllocyanine, another colouring matter obtained from living plants. It is to be noticed, however, that frequently the spectrum of chlorophyll only exhibits one band, viz. that near $\mathrm{C}$, the other bands being visible when either a

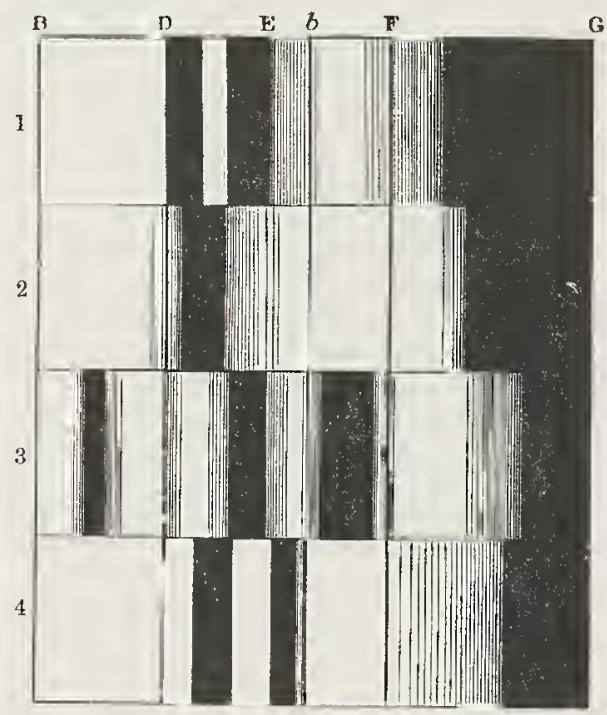

FIG. 61 .

concentrated solution or a thick layer of the chlorophyll is employed.

Professor Stokes, who has examined the spectra of these colouring matters, says: "The characters of these subjects are so marked that I do not know any substance with which either of them could be confounded, even if we restricted ourselves to any one of the solutions yielding the peculiar spectra. Not only so, but these properties enable us to detect small quantities, in the case of purpurine the merest trace, 
of the substance present in the midst of a quantity of impurities." 1

I may now show you that if I take a solution of blood, and place the cell containing it before the slit, we get these distinct dark absorption bands, due to the presence of the blood (Fig. 61). This (1) is the red blood; deoxidized blood gives a different appearance. Here (1) you see the two bands due to the oxyhæmoglobin; whilst this portion of deoxidized blood gives only one black band (2), somewbat similar, but not identical in position with the dark band in magenta which I now throw upon the screen. This subject was first examined by Professor Stokes, who published a paper on the subject in the Proceedings of the Royal Society for 1864. From this we learn that " the colouring matter" of blood, like that of indigo, is capable of existing in two states of oxidation, distinguishable by a difference of colour and a fundamental difference in the action on the spectrum." These two forms may be made to pass one into the other by suitable oxidizing and reducing agents, and they were termed red and purple cruorine, but are now known as oxyhæmoglobin and hæmoglobin.

I have here a drawing of Mr. Stokes's diagram of the blood bands. At the top (Fig. 61, No. 1) you see the position of the two bands of the scarlet cruorine, or oxyhæmoglobin. The deoxidized blood is seen in No. 2 to have only one dark band. By the action of an acid on blood the cruorine is converted into hæmatin, yielding a different absorption spectrum; and this hæmatin is capable of reduction and oxidation like cruorine. The absorption bands of hæmatin and reduced hæmatin are represented in Nos. 3 and 4. The more recent investigations of Soret have shown the existence of another blood band in the violet.

One very interesting point to which I must refer is the fact that the blood, when it contains very small quantities of carbonic oxide gas in solution, exhibits a very peculiar set of bands. And the poisoning by carbonic oxide-for, as is well known, the

1 Journal of the Chemical Society, vol. xii. p. 21. 
poison of burning charcoal is due to this gas-can be readily detected by the peculiar bands which the blood containing carbonic oxide in solution exhibits; and hence we have these absorption lines coming out as a most valuable aid in toxological research.

A valuable suggestion as to the mode of accurately measuring the position of these various absorption bands of the bloodcolouring matters has been made by Professor Ray Lankester, ${ }^{1}$ in referring to the numerous and well-marked absorption bands

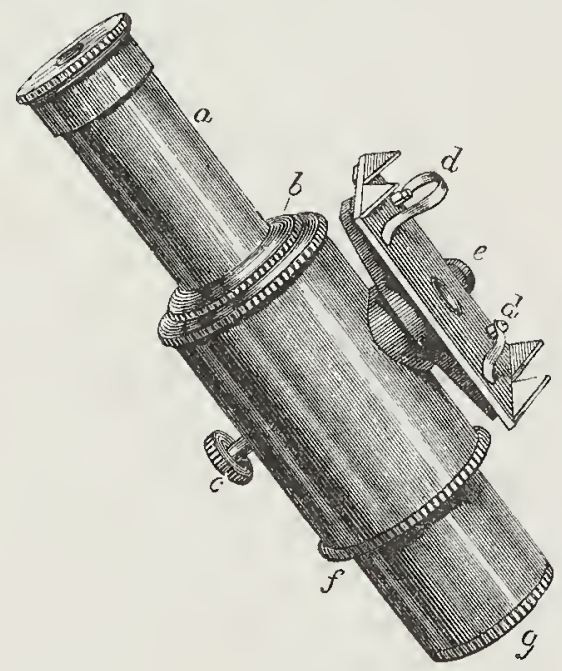

I1 1 (A. 62

of the red nitrous fumes $\mathrm{N}_{2} \mathrm{O}_{4}$ as a fixed scale upon which the places of the blood bands can be easily marked.

The instrument by which all these beautiful absorption phenomena can be observed with delicacy and accuracy is simply a spectroscope placed in connection with a microscope (Fig. 62). Here we have the instrument. ${ }^{2}$ The eyepiece contains prisms, so placed as to enable the refracted ray to pass in a straight line to the eye. Such spectroscopes are termed direct-vision

I Joumal of Anatomy and Physiology, vol. iv. p. 119.

W. Huggins, "On the Prismatic Examination of Microscopic Objects" ('Trans. Microscopical Socicty, May 10, 1865). 
instruments. This (Fig. 63) is a diagram showing the structure of the eyepiece which I hold in my hand. This is the first lens of the eyepiece; here is the adjustable slit, for we must have a line of light in order to get a pure spectrum. When the light passes through the second lens, the rays are rendered parallel; and then they pass through this compound prism, consisting of

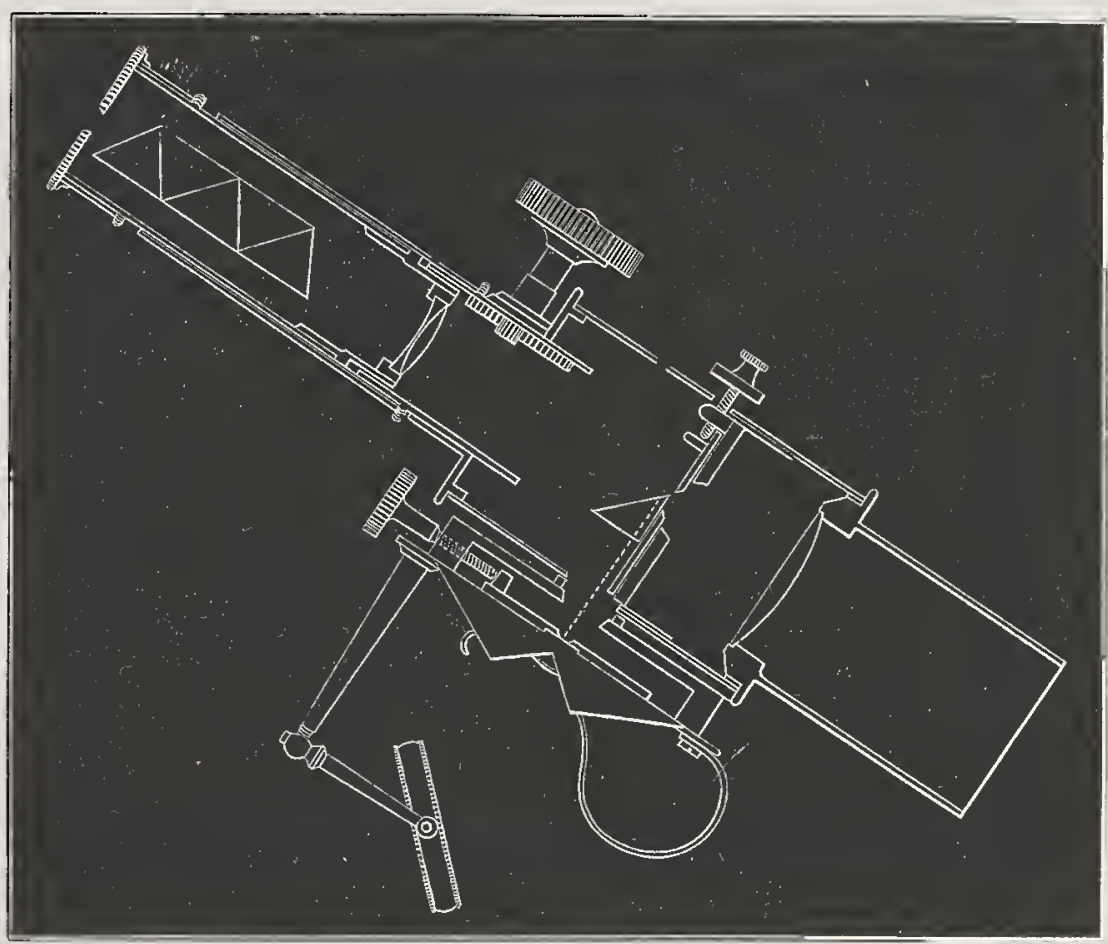

FiG, 63 .

three crown-glass prisms placed in one direction and two flintglass prisins placed in the opposite direction, so that we see the spectrum by looking straight at the source of light, or have a direct-vision spectroscope. In this way, then, the absorption bands can be very beautifully seen; and, what is important, we can, by means of this little moveable mirror, send a ray of light (shown in the dotted line) through the slit at one side of the instrument, which being reflected upwards passes through the 
prisms along with the other light which comes from the object under the microscope, and so observe the two spectra one above the other: and thus it is that we can detect, for instance, the presence of blood. Supposing we wish to know whether a substance is blood which we have in solution: nothing is easier than to place a small quantity of the liquid supposed to be blood on the table of the microscope, and to bring a small quantity of blood in a tube, placed before the slit in the side of the instrument, so as to compare the spectrum obtained from the body under examination with that of the body which we know is really blood. This instrument, which in the hands of Dr. Sorby has taught us how to detect $\frac{1}{1000}$ th part of a grain of the red colouring matter in a blood-stain, and by means of which I have seen the characteristic bands in the blood circulating in a frog's foot, is a most beautiful one, and the method of microscopic spectrum analysis must every year become a more and more trusted and valuable means of research in medico-legal investigations.

The application of photography to spectrum analysis, to a description of the methods of which I shall refer in a subsequent lecture, has also been of the greatest assistance in enabling us to obtain a knowledge of the arrangement of the absorption bands of chemical substances in portions of the spectrum which are invisible to the human eye. We have already seen (p. 29) that not only can an image of the invisible portions of the spectrum lying beyond the violet be imprinted on the sensitive photographic film, but also that those rays possessing a lower degree of refrangibility than the last visible red, can in like manner leave their impress on a film specially prepared by Abney's method. Both in the ultra-violet, and in the ultra-red portions of the spectrum definite absorption bands occur when different absorptive media, often colourless bodies, are placed in front of the slit of the spectroscope, and these bands serve as means of detecting the presence of given chemical elements or compounds, as do the bright bands of the emission spectra. Moreover this method of inrestigation enables us to employ spectrum analysis in the examination, at ordinary temperatures 
of organic liquids and vapours which are altogether decomposed at higher points, and thus opens out an entirely new and fertile field of research. Amongst the recent work done in this subject the experiments of Messrs. Hartley and Huntington in the most refrangible or ultra-violet portion, and of Messrs. Abney and Festing in the least refrangible or ultra-red rays, are well worthy of note. The results arrived at by the first of these observers may be generally stated to be that the power to yield definite absorption bands in the ultraviolet is confined to a particular class of organic compounds, viz., to those the constitution of which is usually considered by chemists as representerl by carbon atoms doubly linked together in the benzene ring. The researches of Abney and Festing are of great interest, inasmuch as they point the way to the establishment of a physical test of the existence in organic compounds of the so-called organic radicals which chemists suppose to form a kind of common nucleus in whole farnilies of allied bodies, and, therefore, giving the means of recognizing the chemical structure of an organic compound by means of the spectroscope. This effects for what I may term the organic metals, that which ordinary high temperature spectrum analysis does for the inorganic metals. Thus characteristic and distinct absorption lines and bands occur for eacl "organic metal." All the ethyl compounds show one "ethyl" band, the methyl compounds a special "methyl" band, and just as a glance at the luminous portion of the spectrum satisfies us of the presence of calcium or lithium, so a simple inspection of these infra-red photographs enables us to ascertain the presence of the various organic radicals. The future developments of this interesting branch of inquiry will be watched by all with great interest.

Allow me next to point out to you some of the more interesting practical applications of spectrum analysis. And first I may mention the application of spectrum analysis to the important branch of steel manufacture which has been introduced and is well known under the name of the Bessemer process. In this process ten tons of cast-iron are in twenty minutes converted into cast-steel. Steel differs from cast-iron in containing less carbon, and by the 
Bessemer process the carbon is actually burnt out of the molten white-hot cast-iron by a blast of atmospheric air. The arrangement employed for this purpose is shown on this diagram (Fig. 64).

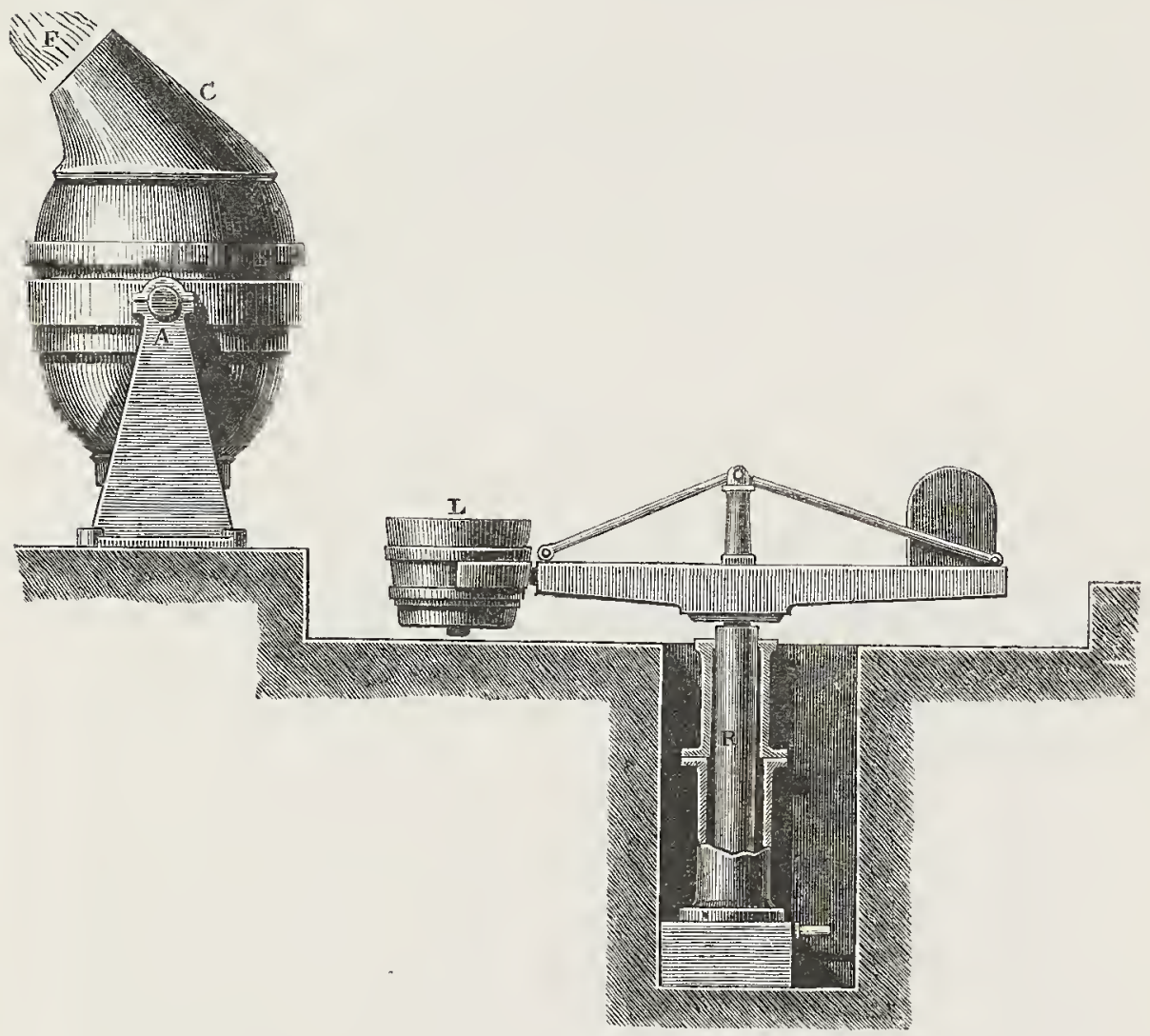

FTg. 64.

The exact nature of the chemical changes which occur in this most interesting process I cannot at present discuss, but mainly they are as follows:- In the first place the graphite which is contained in the pig-iron is converted into combined carbon; in the second place, we find that the silicon begins to burn off, and that afterwards the combined carbon is oxidized. The following 
analyses of portions of metal taken out during the operation show this gradual diminution in the silicon and combined carbon, until in the fourth sample we find that from one- to two-tenths of carbon remain, whilst only a few hundredths, or mere traces, of silicon are left.

\section{ANALYSES SHOWING THE CHEMICAL CHANGES OCCURRING IN THE BESSEMER CONVERTER.}

Samples of Metal taken out at various stages of the operation, viz. :

(1) Original Pig-iron.

(2) Taken out at end of first stage $(6 \mathrm{~min}$.)

(3) , , , boil (12 mim.)

(4) ," ," blow.

(5) After addition of spiegel.

Fliom the Atlas Works, Shefrield.

IRON.

(1)

Graphite . . . 2.573

Combined Carbon . . $1 \cdot 000$

Silicon . . . . 2*260

Phosphorus . . . 0.073

Sulphur . . . . 0.107

Manganese ... . 0.410
(2)

$\begin{array}{cc}3.040 & 1.640 \\ 0.955 & 0.470 \\ 0.070 & 0.070 \\ 0.091 & 0.098 \\ \text { trace } & \text { trace }\end{array}$

STEEL.

$\begin{array}{cc}- & - \\ 0.190 & 0.370 \\ \text { trace } & \text { traee } \\ 0.070 & 0.059 \\ 0.093 & 0.090 \\ \text { trace } & \text { trace }\end{array}$

The molten cast-iron is run into a large wrought-iron vessel, termed the converter (c), lined with refractory clay. The converter is capable of being turned round on a pivot (A), through which pivot passes a tube in connection with a powerful blowing apparatus, by means of which air can be thrown into the bottom of the vessel, through a sort of tuyère or blowhole into the molten iron. The oxygen of the air burns out the carbon and silicon which the cast-iron contains, and the heated gases issue in the form of a flame (F) from the mouth of the converter during the time that the molten iron is being burned. This flame varies in appearance, and it is of the utmost importance that the operation should be stopped instantly when the proper moment has arrived. If the blast be continued for 
ten seconds after the proper point has been attained, or if it be discontinued ten seconds before that point is reached, the charge becomes either so viscid that it cannot be poured from the converter into the ladle (L), from which it has to be transferred to the moulds, or it contains so much carbon as to crumble up like cast-iron under the hammer.

Those who are accustomed to work this process are able by the simple inspection of the flame to tell with more or less exactitude when the air has to be turned off. To those who are uninitiated in this peculiar appearance of the flame no difference at all can be detected at the point in which it is necessary to stop, but by the help of the spectroscope this point can be at once ascertained beyond shadow of doubt, and that which previously depended upon the quickness of vision of a skilled eye has become a simple matter of exact scientific observation. The light which is given off by the Bessemer flame is most intense, indeed a more magnificent example of combustion in oxygen cannot be imagined. A cursory examination of the flame spectrum in its various phases reveals complicated masses of dark absorption bands and bright lines, showing that a variety of substances are present in the flame in the state of glowing gas.

By a simultaneous comparison of the lines in the Bessemer spectrum with those of well-known substances I was able in the year 1863 to detect the following substances in the Bessemer flame: sodium, potassium, lithium, iron, and hydrogen, and others which I attributed to nitrogen and carbon. At a certain stage of the operation I found that all at once the lines supposed to be due to carbon disappeared, and we got a continuous spectrum. The workman by experience has learned that this is the moment at which the air must be shut off; but it is only by means of the spectroscope that this point can be exactly determined.

The following table gives you a good idea of the changes seen to take place in the flame, (1) as seen by the unaided eye, (2) as seen by the aid of the spectroscope. 


\begin{tabular}{|c|c|c|c|c|c|c|}
\hline STAGE. & \multicolumn{4}{|c|}{ Ттме. } & $\begin{array}{l}\text { Apritaranote of } \\
\text { Flame to Eye. }\end{array}$ & APPEAPANCE OF SPECTRUM. \\
\hline \multirow{3}{*}{ Ist. } & \multicolumn{4}{|c|}{$\begin{array}{l}\text { About } \\
0 \text { min. } 4 \text { see. }\end{array}$} & No flime seen. & $\begin{array}{l}\text { Faint eontinuous speetrum, due } \\
\text { to sparks of burning metal. }\end{array}$ \\
\hline & 4 & , & & , & Small pointed flame. & $\begin{array}{l}\text { Brighter, with yellow sodium } \\
\text { line flashing out. }\end{array}$ \\
\hline & 6 & , , & 8 & $"$, & $\begin{array}{l}\text { Unsteady flame, } \\
\text { with explosions. }\end{array}$ & $\begin{array}{l}\text { Bright eontinuous, with sodium } \\
\text { line permanent. Red lithium } \\
\text { line, red and violet potassium } \\
\text { lines. }\end{array}$ \\
\hline $2 \mathrm{~d}$. & 8 & , & 10 & , & $\begin{array}{l}\text { Very bright and } \\
\text { dense flame. }\end{array}$ & $\begin{array}{l}\text { Bright lines in red, green, and } \\
\text { blue, with preceding spec- } \\
\text { trum. }\end{array}$ \\
\hline & 10 &, & 14 &, & $\begin{array}{l}\text { Flame less dense, } \\
\text { but bright. }\end{array}$ & $\begin{array}{l}\text { Bright green lines more dis- } \\
\text { tinet. }\end{array}$ \\
\hline $3 \mathrm{~d}$. & 14 & , & 16 & , & $\begin{array}{l}\text { Flame diminishing } \\
\text { in size and in- } \\
\text { tensity. }\end{array}$ & Bright green lines beeome faint. \\
\hline & 16 &,, & 18 & , & $\begin{array}{l}\text { Flame drops; blow } \\
\text { ends. }\end{array}$ & $\begin{array}{l}\text { Bright green lines suddenly dis- } \\
\text { appear, leaving a continuous } \\
\text { speetrum. }\end{array}$ \\
\hline
\end{tabular}

No. 2, Fig. 65, represents the general appearanee of the Bessemer spectrum towards the close of the "blow," drawn according to the plan proposed by Bunsen (see page 107). The striking analogy between the flame spectrum and that of carbon (No. 1, Fig. 65) renders it at first sight probable that the principal lines of the Bessemer spectrum are due to carbon in some form; and this conclusion is strengthened when we find that the lines disappear at the moment when, by chemical analysis, we can show that the carbon has been burnt out. Still it has been stated that the lines are due to the presence of manganese, although they are brightly seen in the working of certain iron ores (such as the Ulverstone beds) which contain scarcely any of this metal. And, singular and at present unaccountable as it may appear, it has been shown beyond doubt by Dr. Marshall Watts, that the bright lines in the Bessemer flame are 
in reality not identical with carbon lines, as we had long believed, but with those of oxide of manganese. Fig. 66 on page 204 shows this singular fact clearly: No. 1 gives the iron spectrum; No. 2 that of the Bessemer flame; No. 3 that of oxide of manganese; and No. 4 the spectrum of the spiegel flame. Here again we must be content accurately to record ascertained facts, leaving the explanation for a future time. When the spiegeleisen is brought into the converter, a very bright flame issues from the mouth of the vessel, and this flame exhibits a spectrum (No. 4, fig. 66) which really contains the same lines as that of the Bessemer flame, although the general appearance of the spectrum is completely changed by the alteration of the relative brightness of the lines.

Those who are practically engaged in working this process would like spectrum analysis to do a great deal more; they

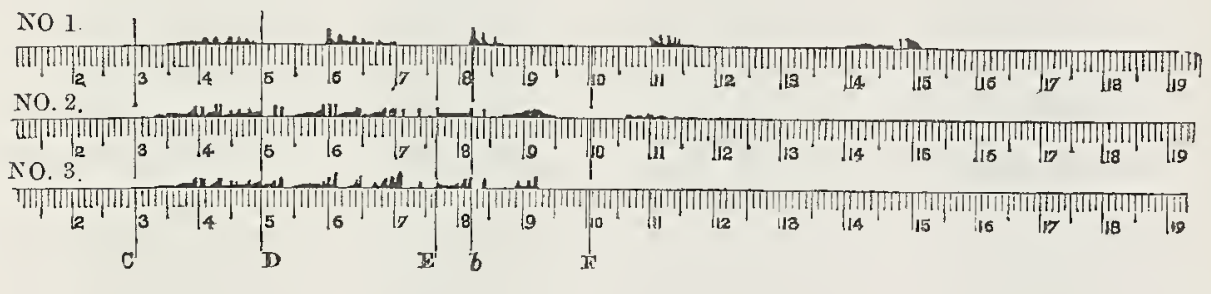

Fra. 65 .

would like to be told whether there is any sulphur, phosphorus, or silicon in their steel: questions which unfortunately at present spectrum analysis cannot answer, for this very good reason, that these substances do not appear at all as gases in the flame, but that they either remain unvolatilized in the molten metal, or swim on its surface in the slag of the ore; and consequently the lines of these bodies are not seen in the spectrum of the flame.

Another valuable application of the principles of spectrum analysis in addition to those already referred to, consists in the employment of absorption-spectra for the detection of adulteration in wines. It is well known that various colouring matters are used in the falsification of red wines; the presence of certain of these can at once be recognized by their peculiar 


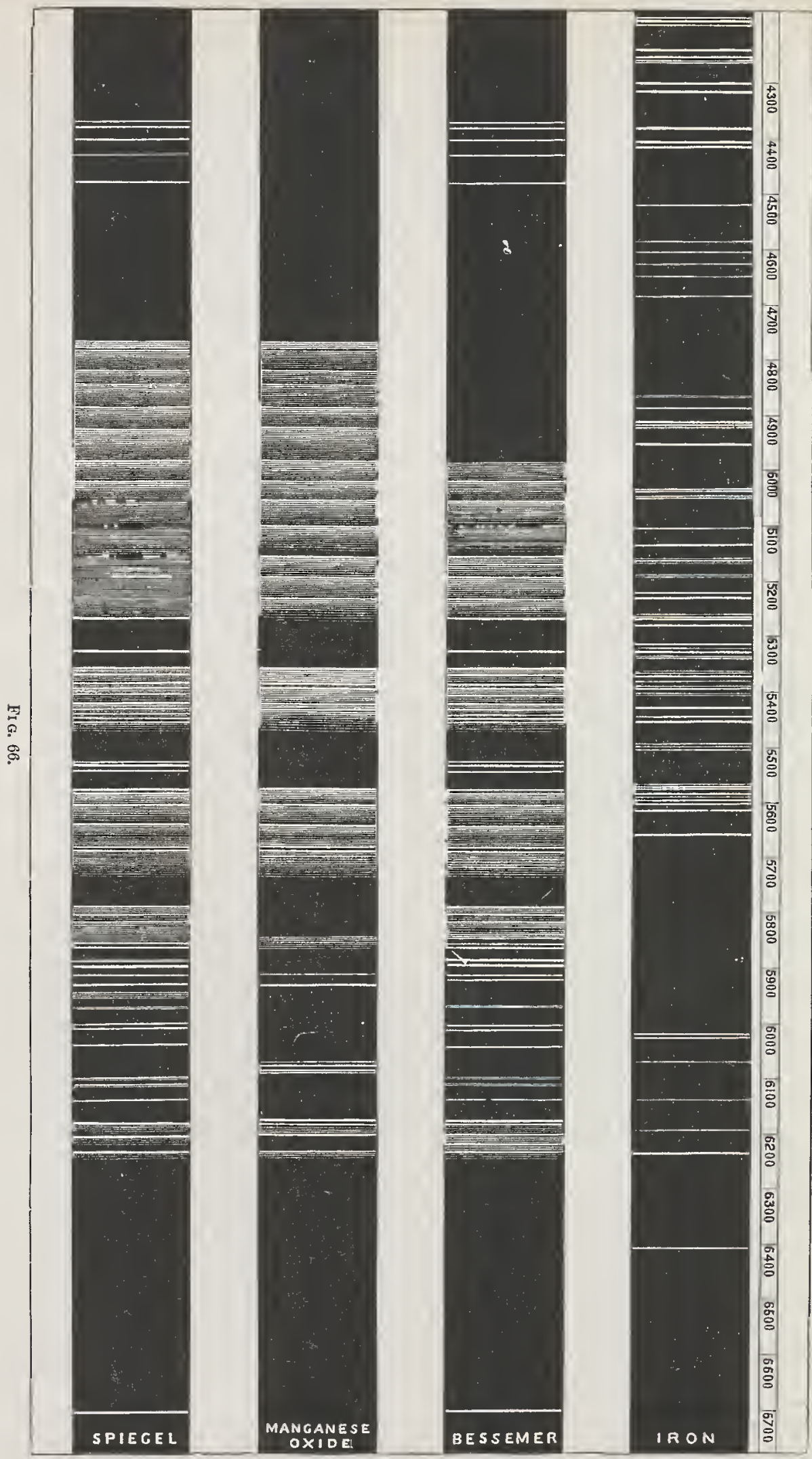


dark bands, whilst others can, in like manncr, be detected by the previous addition of certain reagents such as ammonia. ${ }^{1}$

We may now ask ourselves what becomes of the light absorbed by these various coloured substances. It is clear that the rays which a body absorbs are not destroyed, for there is no such thing as a destruction of energy in the universe. These rays when absorbed go to increase the temperature of the body. If a medium is absolutely transparent, it cannot bc heated by radiation, and the more absorbent a medium becomes, the more quickly can it be heated by rays passing through it. The energy of the incident light is, therefore, converted into intra-molecular motion, and into the radiation which the absorbent body emits. ${ }^{2}$

In the case which we are considering at present, namely the one in which the average temperature of the body is only slightly raised above that of surrounding bodies, an absorption of light must always be accompanied by a conversion of rays of higher wave-length into rays of lower wave-length, as the emissive power for the latter rays is relatively higher at a low temperature. As a rule that conversion takes p'ace very quickly, and as the rays of low refrangibility which are strengthened lie in the infra-red, we only observe a slightincrease of temperature in the absorbing body. There are some instances, however, in which the conversion takcs place more slowly, and, as it were, visibly. In such bodies a blue ray is not converted into an invisible infra-red ray, without having first been changed to a grcen, yellow, or red ray.

If such substances receive a ray of light which they have the power of absorbing, they are seen to become slightly luminous, emitting rays which are nearly always less refrangible than the

I (Vogel's Praktische Spsctral Analyse, 1877, p. 293). Sorby has also pointed out that the spectroscope may be used to ascertain the age of red wines, espccially of port, as changes in the absorption spectra of such wines can be traced from year to year, up to an age of six years.

2 The conversion of particular vibrations into those of different refrangibility has been occasionally quoted as an exception to Kirchhoff's law, which states that the ratio of the emissive to the absorbing power is independent of the nature of the body, and only depends on its temperature; but it is in reality a consequence of that law when it is applied to the case of rays coming from a hotter and passing through a cooler body. 
ray which has produced the luminosity. Sir David Brewster investigated some cases of this emission of light about the year 1835, terming the phenomenon "internal dispersion." Sir John Herschel also made a few experiments on the subject, which attracted the attention of Professor Stokes, to whom we owe the first and most thorough investigation of the phenomenon which he subsequently called "fluorescence." One of the principal laws which regulate the phenomenon is, according to Stokes, that the rays sent out by the fluorescent bodies are always less refrangible than those causing the fluorescence. This law has recently been doubted, and there is evidence that certain exceptions to it exist.

The fluorescence of bodies is closely allied to other phenomena to which the term phosphorescence is usually applied. These only seem to be cases in which the fluorescence does not disappear on the removal of the light causing it, but subsists for some sensible length of time. It has indeed been shown by Becquerel that nearly all bodies which are fluorescent remain luminous for a short time, and he was able to prove this by means of a very ingenious apparatus termed a phosphoroscope (Fig. 67). In order, for example, to show, with this apparatus, the phosphorescence of this small crystal of fluor-spar placed in the small stirrup (a) between the two screens (A B) which I can set into rapid rotation, and then allow our powerful electric light to shine upon the back of the closed cylindrical box. From the peculiar construction of the instrument, you cannot see the light when by means of the handle I rotate the perforated screens which move inside the fixed box; for these screens (P P and $\mathrm{M} \mathrm{M}$ ) are so arranged that when the front slit $(0)$ in the box is open that at the back is closed, and vice versâ. If I now cause the perforated screens to rotate, the crystal will be exposed to the light placed behind the box for a series of very short spaces of time, and will also be seen by you through the front hole for a series of equally short moments. If I turn the handle slowly, after darkening the room, you do not see the fluor-spar; but if I increase the speed of rotation so that the times of illumination and of observation do not exceed the $\frac{1}{1000}$ th part of a sccond, 
you will observe that the crystal glows with a very perceptible amount of light, or in other words it becomes phosphorescent This crystal of nitrate of uranium produces a more brilliant appearance. But many substances, such as sulphur, quartz, the metals, and liquids, cannot be made thus to phosphoresce.

Becquerel has carefully examined the spectra of these phosphorescent bodies, and he has found that the light emitted

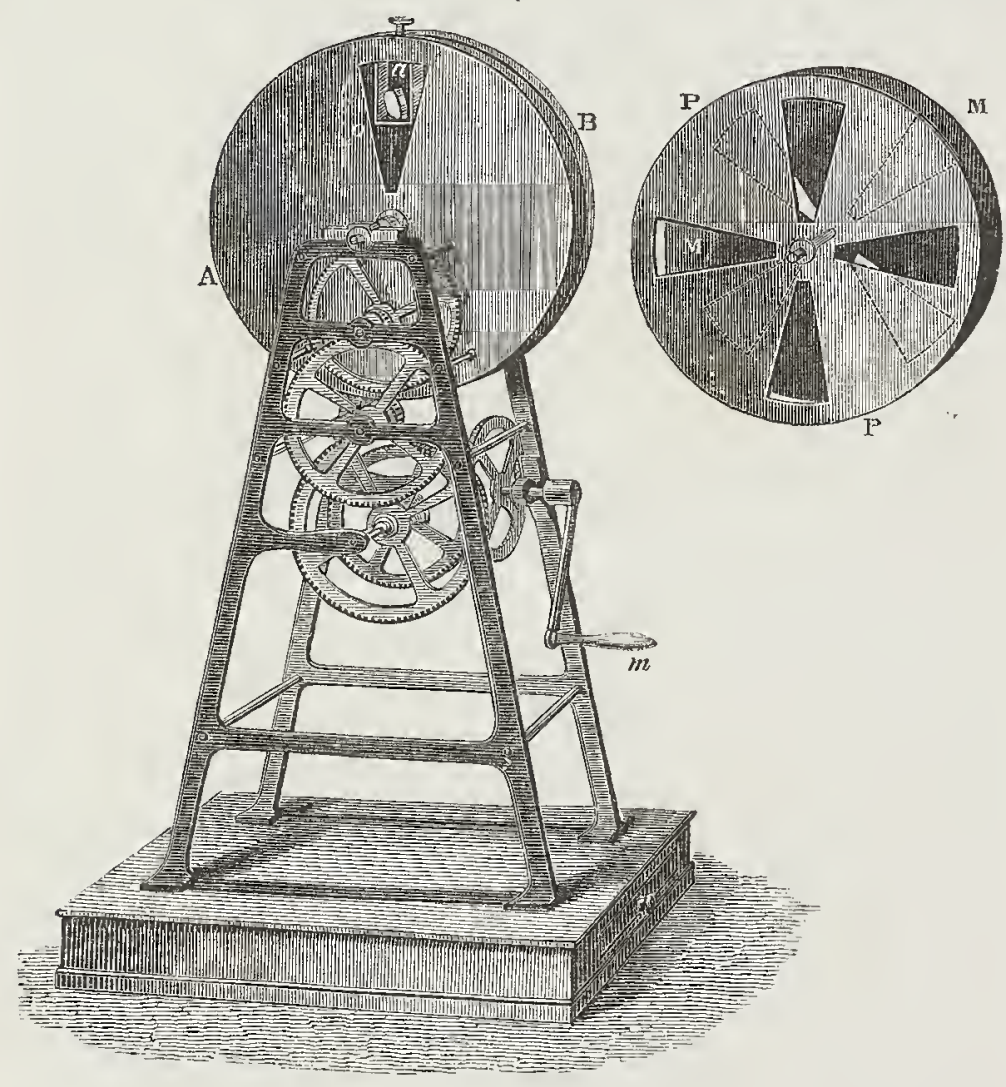

Fia. $6 i$.

by many of them is of a peculiar kind; that they, in fact, give broken spectra, or bands of differently coloured rays. Thus in Fig. 68 we have a representation of the pliosphorescent spectra of several substances: alumina, when phosphorescent, emits a red light, and its spectrum (No. 1) exhibits four bands between the lines $\mathrm{C}$ and $\mathrm{H}$ in the solar spectrum; diamond (No. 3) emits, 
when phosphorescent, light of many degrees of refrangibility, giving an almost continuous spectrum stretching from $\mathrm{B}$ in the

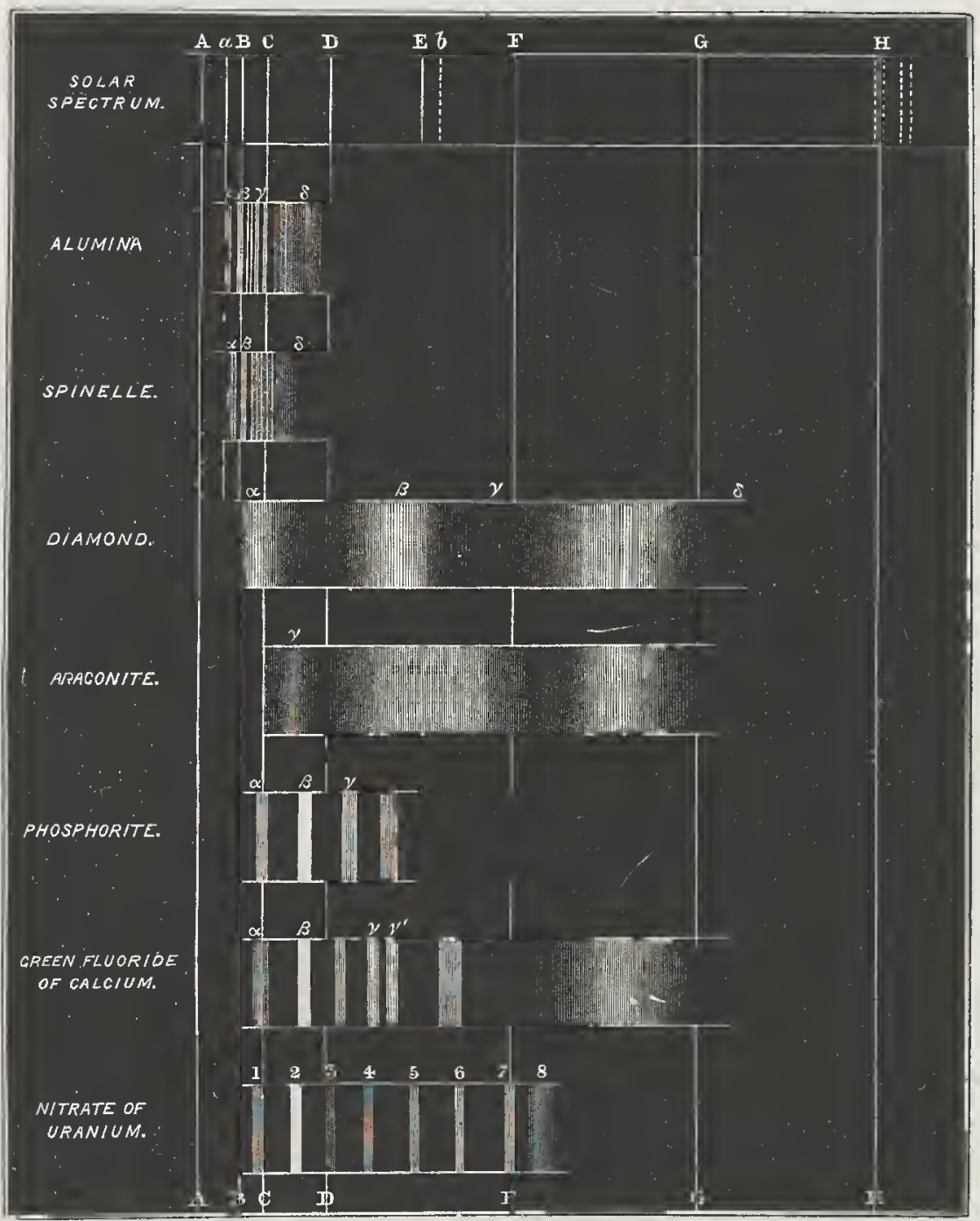

FIG. 68 .

red to beyond $\mathrm{G}$ in the indigo; arragonite (4) also gives a continuous spectrum; whilst native phosphate of lime (5), fluor-spa! (6), and nitrate of uranium (7), each phosphoresces with the 
emission of a peculiar light, as is seen in the varying character of the above spectra.

The different rays of the solar spectra possess a very different power of producing phosphorescence. By far the most powerful in this respect are the more refrangible rays. Phosphorescent bodies exposed to the so-called chemically active portion of the spectrum emit light which, as we have seen, varies from red to violet, and as a rule depends only upon the nature of the substance.

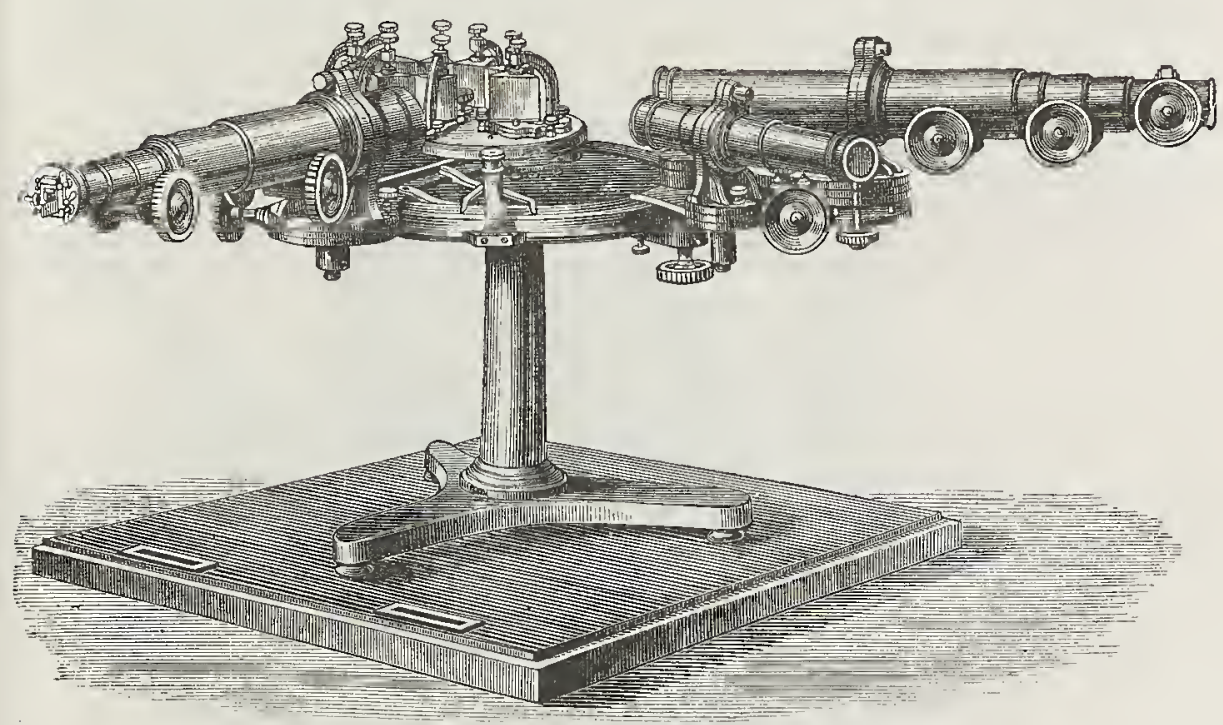

FIG. 69.

M. Becquerel has discovered a singular property of certain phosphorescent bodies. Thus it appears that whilst these bodies are rendered phosphorescent when exposed to ultra-violet rays, the infra-red rays have the power of rapidly destroying the luminosity thus produced. If then we place such a phosphorescent surface in a spectrum which contains infra-red, and therefore invisible, bands or rays, we shall notice that the phosphorescence is destroyed wherever the image of such a band is formed. Becquerel has applied this method with success to the study of the infra-red lines emitted by luminous gases, and his observations are found to agree well with the photographs obtained by Captain Abney's process. 
I will next show you that the property of phosphorescence is possessed by certain substances to such a high degree that they remain luminous for many hours after exposure to light. Thus the tubes which, when the room is darkened, you perceive to glow with red, yellow, green and blue light, were all exposed this morning for five minutes to the direct sunlight, and still each emits its peculiar light. This property, which is especially well marked in the sulphides of the metals of the alkalineearths, calcium, strontium and barium, has been made use of by the late Mr. Balmain for the production of the now well known

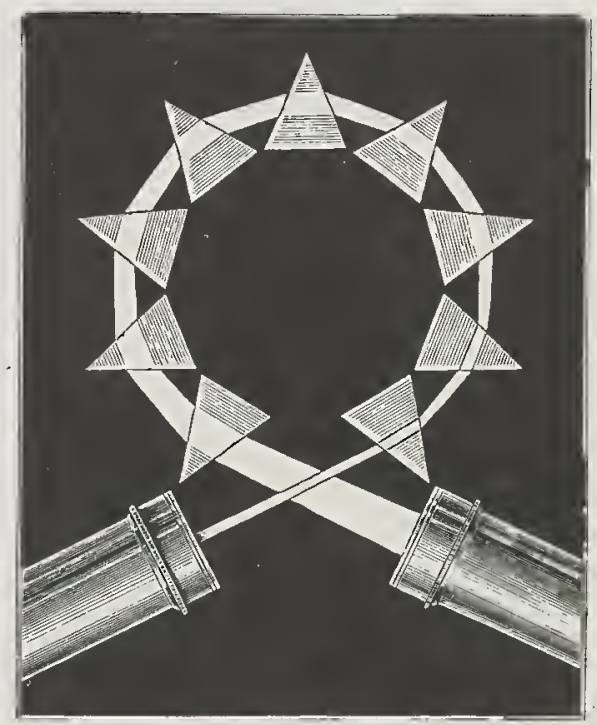

Fig 70 .

luminous paint. There remain, however, of course, other wellknown cases of phosphorescence like that of the glow-worm, and that produced by the slow combustion of phosphorus, but as these sources of light give continuous spectra they are of less interest to us at the present moment.

All the spectrum observations which I have hitherto mentioned can be made, and are best made with the smaller instruments, such as those I have described in my second lecture. But I shall soon have to draw your attention to more delicate observations 
which can only be carried on with instruments of high power. Fig. 71, shows, for instance, the spectroscope used by Professor Kirchhoff in his classical researches on the solar spectrum, which will form the principal subject of my next lecture. The light after passing through the collimator was successively refracted through four prisms, each prism increasing the length of the spectrum, which is observed through the telescope B. Kirchhoff's success encouraged other observers to use still larger instruments and still further to increase the number of prisms. In Fig. 69

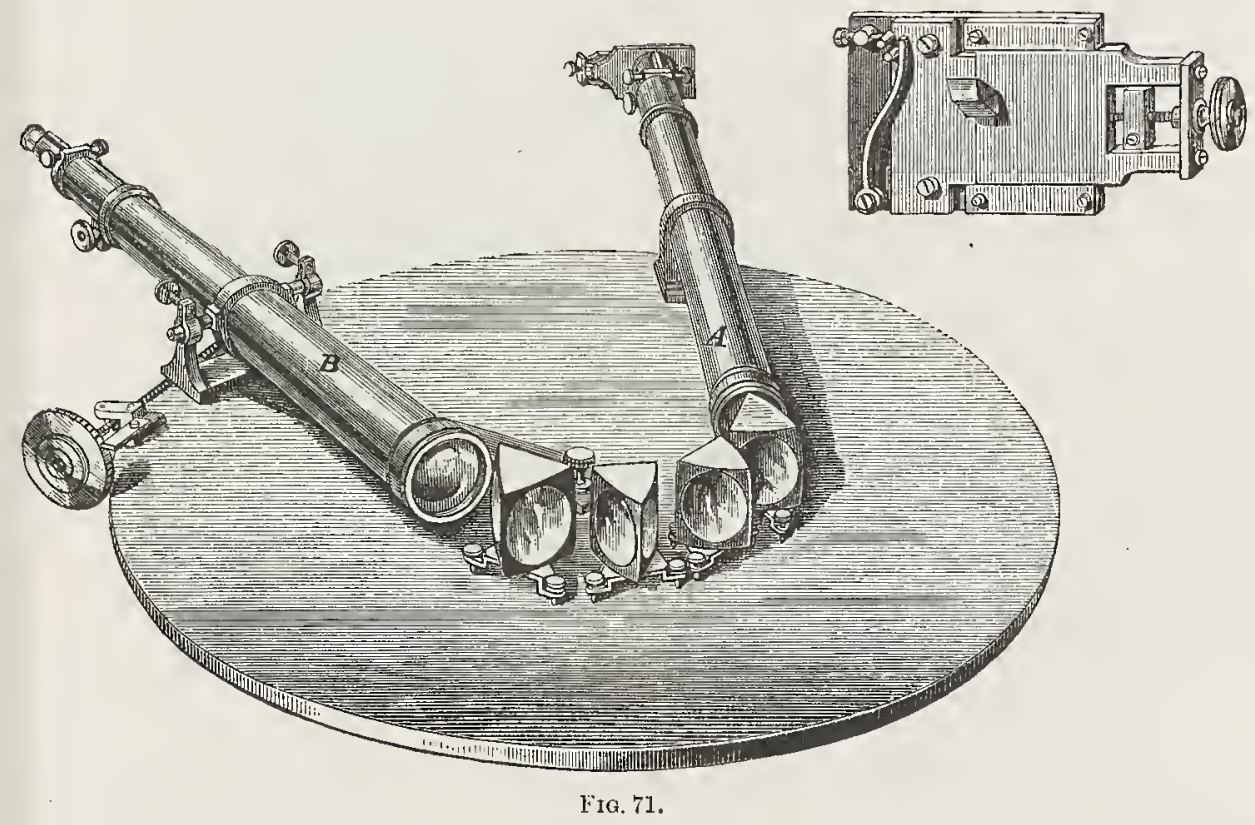

we have for instance a spectroscope made for Mr. Gassiot, in which there are nine prisms, and in which the light is actually bent round, so that the incident and emergent beams cross each other's path, as is seen in Fig. 70 giving a plan of the instruments, and showing the path of the light through the prisms. In some spectroscopes the light after having passed through a number of prisms is reflected back and passing once more through them has its dispersion doubled. Mr. Hilger has even. made one spectroscope in which the light passed six times in 
succession through the same train of prisms. There are, however, practical reasons why we cannot in this way indefinitely increase the dispersion; for not only is there a considerable loss of light at the surface of each prism, but as these surfaces are never perfectly plane, and as the glass is never perfectly homogeneous, the lines lose their sharpness rapidly by passing through a large thickness of glass. It thus often happens that though the dispersion is much increased in large instruments we really see less, owing to the loss of definition.

Attempts were therefore made to increase the dispersion in other ways. As we have in bisulphide of carbon a highly dispersive substance, hollow prisms have been used filled with that liquid. Such are the prisms which I used in my second lecture when I projected the metallic spectra on the screen (Fig. 28). For accurate observations these prisms have not come into use, but M. Thollon has constructed a cumpound prism $^{x}$ in which the dispersion is due to bisulphide of carbon. He obtained excellent results, and Professor Piazzi Smyth's large spectroscope, which is one of the most perfect in the world, is constructed witl a number of prisms nearly resembling those of Thollon. Unfortunately the refraction of bisulphide of carbon is much affected by changes of temperature, and great care must be taken to preserve uniformity of temperature throughout the liquid. This is difficult when strong light like that of the sun or of the electric lanip has to pass through them.

In the diffraction grating, however, we have an instrument which allows us to combine high dispersion with excellent definition. These gratings are now rapidly coming into use for the observation of all spectra which are sufficiently luminous. Fig. 72 shows a diffraction grating fitted up for observation. Collimator and telescope are mounted separately, and are carried on stands which admit of accurate levelling. The grating itself is carried on a circular table which can be revolved so as to bring the spectra of different orders into view. I have described at length the action of gratings in my first lecture, and I have there stated that one of the difficulties we have to meet on

\footnotetext{
1 See Appendix B.
} 
observing the higher orders consisted in the over-lapping of different spectra. This difficulty can be overcome, if we use a prism of small angle placed in such a way that the refraction takes place upwards. Instead of overlapping, the spectra will then be seen one above the other. The prism can either be placed in front of the slit or between the grating and the telescope.

The gratings which now are usually made of speculum metal ought to be protected, when used in laboratories, from noxious

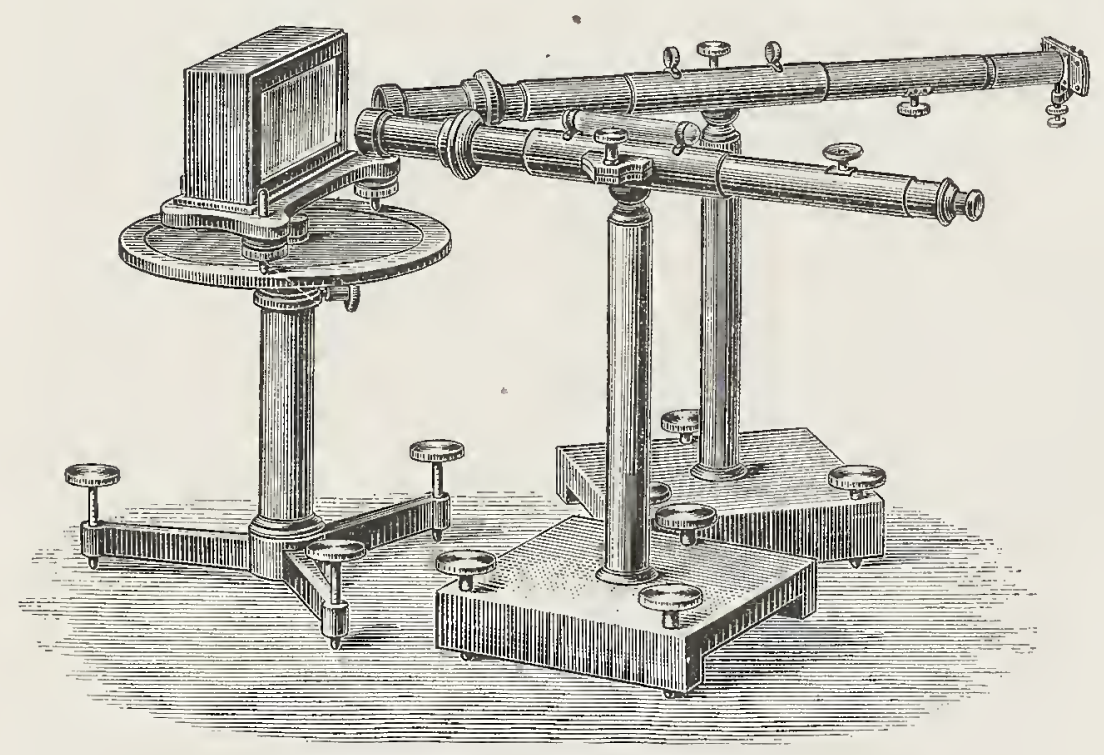

FIG 72.

fumes which rapidly tarnish their surface. They are best placed in an air-tight box with a front of plate-glass as is shown in the figure. I must not forget to mention that we owe to $\mathrm{Mr}$. Rutherfurd, in New York, the important improvements in the construction of gratings which render them now almost indispensable to spectroscopists. Professor Rowland of Baltimore, who has lately directed his attention to this subject, has also produced very excellent gratings and especially much improved their luminosity. He has also succeeded in ruling gratings on a 
concave surface of speculum metal. Such a concave grating can be used without either collimator or telescope, for it is in itself sufficient to form an image of the slit either on a sensitized photographic plate, or in the focal plane of the eyepiece which inust be used for eye observations.

In the next lecture we shall consider the application of these various methods to the study of solar physics. 


\section{LECTURE IV.-APPENDIX A.}

ON THE SPECTRUM OF THE BESSEMER FLAME.

BY W. M. WATTS, D.Sc. ${ }^{1}$

THE October number of the Philosophical Magazine contains translations of two Papers by Professor Lielegg, giving the results of his observations on the spectrum of the Bessemer flame. As these results are published as entirely new, and no mention is made of any prior observations, it is only right that attention should be called to the fact that as long ago as $\mathbf{1 8 6 2}$ the same results had been obtained by Professor Roscoe, and were published in the form of a short preliminary notice in the "Proceedings of the Manchester Literary and Philosophical Society" for February 24th, 1863. As the note is extremely short, I venture to transcribe it in full :-

"Professor Roscoe stated that he had been for some little time, and is still, engaged in an interesting examination of the spectrum produced by the flame evolved in the manufacture of cast steel by the Bessemer process, on the works of Messrs. John Brown and Co. of Sheffield. The spectrum of this highly luminous and peculiar flame exhibits during a certain phase of its existence a complicated but most characteristic series of bright lines and dark absorption bands. Amongst the former the sodium, lithium, and potassium lines are most conspicuous; but these are accompanied by a number of other, and as yet undetermined, bright lines: whilst among the absorption bands those formed by sodium vapour and carbonic oxide can be readily distinguished. Professor Roscoe expressed his belief that this

1 Phil. Mag. (4), xxxiv. 437. 
first practical application of the spectrum analysis will prove of the highest importance in the manufacture of cast steel by the Bessemer process, and he hoped on a future occasion to be in a position to bring the subject before the Society in a more extended form than he was at present able to do."

In a lecture delivered before the Royal Institution (May 6, 1864), a year later than the communication quoted above, Dr. Roscoe described the Bessemer spectrum more fully, and pointed out the existence of lines produced by carbon, iron, sodium, lithium, potassium, hydrogen, and nitrogen.

An important practical result of the observations on which these communications were based was the discorery that the exact point of decarbonization could be determined by means of the spectroscope with much greater exactitude than from the appearance of the flame itself, the change in which indicating the completion of the process is minute, and requires a lengthened experience to detect with certainty. This method of determining the point at which it is necessary to stop the blast was indeed at that time (1863) in constant use at Messrs. Brown's works at Sheffield, and has since been introduced with equal success by Mr. Ramsbottom (at the suggestion of Dr. Roscoe) at the London and North-Western Railway Company's steelworks at Crewe.

I was at that time acting as inspector to Professor Roscoe, and in that capacity conducted a lengthened examination of the Bessemer spectrum at the works at Crewe. The results of that investigation were not published at the time, on account of their incompleteness; and I have since then continued in Glasgow the same research, which has now extended itself into an inquiry into the nature of the various spectra produced by the carbon compounds. These experiments are still incomplete; but under the circumstances of the publication of Professor Lielegg's papers, I have put together a few of the more important results obtained in the examination of the Bessemer spectrum.

The changes which take place in the spectrum from the commencement of the "blow" to its termination are extremely interesting. When the blast is first turned on, nothing is seen 
but a continuous spectrum. In three or four minutes the sodium line appears flashing through the spectrum, and then becoming continually visible; and gradually an immense number of lines become visible, some as fine bright lines, others as intensely dark bands; and these increase in intensity until the conclusion of the operation. The cessation of the removal of carbon from the iron is strikingly evidenced by the disappearance of nearly all the dark lines and most of the bright ones.

The spectrum is remarkable from the total absence of lines in the more refrangible portion; it extends scarcely beyond the solar line $b$.

No. 2, Fig. 73, represents the general appearance of the Bessemer spectrum towards the close of the "blow," drawn according to the plan proposed by Bunsen (see page 107). It must

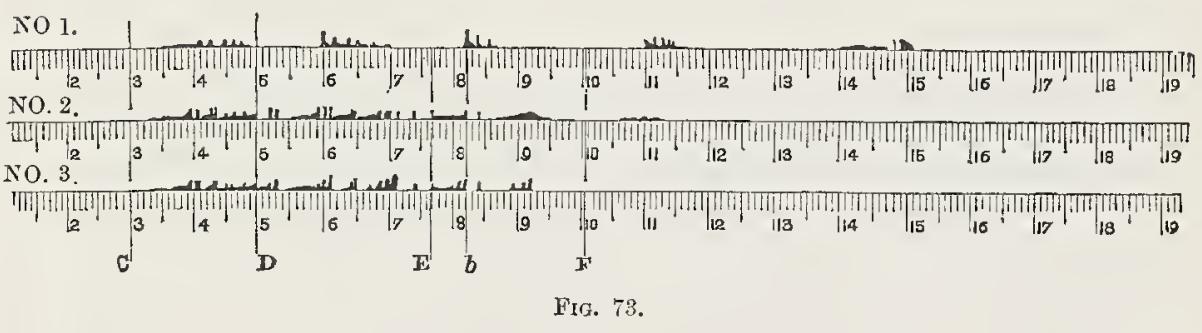

be remarked, however, that at the period of greatest intensity almost every bright band is seen to be composed of a great number of very fine lines.

The occurrence of absorption lines in the Bessemer spectrum is in itself extremely probable; and that this is the case appears almost proved by the great intensity of some of the dark lines of the spectrum. It was with this view that the investigation was commenced, with the expectation that the spectrum would prove to be a compound one, in which the lines of iron, carbon, or carbonic oxide, \&c. would be found, some as bright lines, others reversed as dark absorption bands. To a certain extent his anticipation has been verified; but the great mass of the lines, inclurling the brightest in the whole spectrum, have not as yet been identified. 
In dealing with a complicated spectrum like that of the Bessemer flame, it is indispensable that the spectrum should be actually compared with each separate spectrum of the elements sought. This was the plan actually pursued; the spectroscope was so arranged that the spectrum of the Bessemer flame was seen in the upper half of the field of view, and the spectrum with which it was to be compared was seen immediately below. In no other way can any satisfactory conclusion be obtained as to the coincidence or non-coincidence of the lines with those of known spectra.

The spectrum of the Bessemer flame was thus compared with the followering spectra:-

(1) Spectrum of electric discharge in a carbonic oxide vacuum.

(2) Spectrum of strong spark between silver poles in air.

(5) Solar spectrum. iron

(6) Carbon spectrum-oxyhydrogen blowpipe supplied with olefiant gas and oxygen.

The coincidences observed were, however, but very few, and totally failed to explain the nature of the Bessemer spectrum. The lines of the well-known carbon spectrum (given in No. 1) do not occur at all, either as bright lines or as absorption bands; nor was any coincidence observed between the lines of the Bessemer spectrum and those of the carbonic oxide vacuum tube.

The lines of lithium, sodium, and potassium are always seen, and are unmistakable.

The three fine bright lines, $73 \cdot 7,76 \cdot 8$, and 82 , are due to iron. The red band of hydrogen (c) is seen as a black band, more prominent in wet weather.

After the charge of iron has been blown, it is run into the ladle, and a certain quantity of the highly carbonized spiegeleisen is run into it. The effect of the addition of the spiegeleisen is the production of a flame which is larger and stronger when the blow has been carried rather far. This flame occasionally gives the same spectrum as the ordinary Bessemer flame; but 
more commonly a quite different spectrum (No. 3) is seen, which reminds one at first of the ordinary carbon spectrum, but differs from it very remarkably.

In the carbon spectrum, which is drawn in No. 1, each group of lines has its strongest member on the left (i.e. less refrangible), and fades gradually away towards the right hand: in the spectrum of the spiegel flame the reverse is the case; each group has its brightest line most refrangible, and fades away into darkness on the least refracted side. A comparison of the drawing of the spectrum of the spiegel flame (No. 3) with that of the Bessemer flame (No. 2) will show that they really contain the same lines; but the general appearance of the spectrum is completely changed by alteration of the relative brightness of the lines. This was shown by direct comparison of the actual spectra.

There can be no doubt that the principal lines of the Bessemer spectrum are due to carbon in some form or other. My own belief is that they are due to incandescent carbon vapour. The experiments in which I am at present engaged have already shown the existence of two totally different spectra, each capable of considerable modification (consisting in the addition of new lines), corresponding to alterations in the temperature or mode of producing the spectrum, and each due to incandescent carbon. It is possible that the Bessemer spectrum may prove to be a third spectrum of carbon, produced under different circumstances from those under which the ordinary carbon spectrum is obtained; and the intensity of the dark bands is more probably due to contrast with the extreme brilliancy of the bright lines than to their actual formation by absorption.

The most recent observations on the singular spectrum of the Bessemer flame, made by Dr. Marshall Watts, appear to throw entirely new light upon the cause of the lines therein seen. The following contains the result of Dr. Watts's observations, and from them we can now no longer doubt that the peculiar lines are due to the presence of a manganese compound, and not, as was previously and on good ground supposed, to that of carbon. 
FURTHER OBSERVATIONS ON THE SPECTRUM OF THE BESSEMER FLAME.

These observations were made by Dr. W. M. Watts, at the steel-works at Barrow. The "Barrow Hæmatite Iron and Steel Company " assisted the investigation in the most liberal manner, not only erecting an observatory on the works, but also contributing to the expense of the experiments.

The instrument employed was Browning's Automatic Spectroscope, of six prisms. The measurements were made by means of a micrometer tangent-screw, giving 2.94 turns between the lithium orange line and the least refrangible D-line. In some of the later measurements a micrometer eyepiece was employed, fur. nished with two pairs of cross wires. To separate the wires by the same interval requires 12.49 turns of the screw. The method of observation commonly employed was to throw an image of the flame on the slit of the spectroscope by means of a large lens of about 25 centimetres' focus; and just in front of the lens was placed a spark-discharger. The spectrum of the flame could thus be obtained simultaneously with that of any metal, or either spectrum could be obtained in the field alone. The wave-length of a line in the Bessemer spectrum was obtained by graphical interpolation (occasionally by calculation) from the known wave-lengths of two metal lines, selected so that the Bessemer line fell between them as closely as possible. The metals employed in the comparison were aluminium, copper, cadmium, iron, lithium, lead, magnesium, manganese, platinum, sodium, thallium, tin, and zinc; besides which, some lines of the air-spectrum were made use of.

The results of the observations are embodied in Fig. 66 (page 204), which shows the spectrum on the scale of wavelengths. With it are given, upon the same scale, the spectra of iron-of the flame obtained on adding the spiegel-and of oxide of manganese. A comparison of these drawings shows that the spiegel spectrum is essentially the same spectrum as the Bessemer spectrum-only further developed-and that the Bessemer 
spectrum, while containing a few lines of iron, sodium and lithium, is essentially the spectrum of oxide of manganese.

No lines certainly known to be produced by carbon have been observed.

It has been repeatedly affirmed that the Bessemer spectrum is produced by manganese-a conclusion which has been denied by many who have observed this spectrum (by Dr. Roscoe and myself, as well as by many others who have observed this spectrum), and upon what appeared to be very satisfactory evidence. It was stated loosely, that the spectrum was that of manganesewhich it certainly is not; for if the spectrum of the electric spark between manganese poles be observed together with that of the Bessemer flame, it is seen that there is absolutely no single coincidence. Moreover, the spectrum is obtained just as brightly in working iron, which contains scarcely any manganese at all, as with highly manganiferous iron, and is obtained from coke-fires and other furnace-flames in which only traces of manganese can be supposed to be present; and, most strange of all, repeated observation shows that in 99 blows out of 100 this spectrum disappears at the time when, as analysis shows, the carbon is completely removed from the iron, and the manganese is not. Nevertheless, simultaneous comparison of the Bessemer spectrum with the spectrum of manganese oxide shows the coincidence in a manner so entire as to completely convince any one who sees it, of the identity of the two spectra. This spectrum of manganese oxide is obtained faintly by bringing manganese chloride into a Bunsen flame; but best and very brilliantly by bringing manganese chloride, pyrolusite or any manganese compound, into the oxyhydrogen flame. Why this spectrum, which is not that of carbon, should disappear at the exact moment when all the carbon is burnt out, is very difficult to understand. Possibly, the point which it is necessary to hit is not that of the complete removal of carbon, but that at which the injurious oxidation of iron begins, viz. when the quautity of manganese becomes too small to combine with the excess of oxygen. 
APPENDIX B.

SPECTROSCOPIC MEASUREMENTS AND APPIIANCES.

THE ineasurements of position of the lines and bands in spectra are now nearly always expressed in wave-lengths. The methods employed for this reduction differ according to the dispersion which is employed and with the accuracy which is required. The following remarks may serve to give an idea of the several methods in use.

The small one-prism spectroscopes which are employed in chemical laboratories are usually fitted with an additional collimator containing in its focus a photographic scale which is thrown into the field of view (see Fig. 31). The tube containing the scale is adjustible, and is placed in such a position that the same number is always coincident with the sodium lines. Bunsen and Kirchhoff chose the number five for this purpose, and their example is generally followed, but Lecoq de Boisbaudran employs ten instead. When once the scale is adjusted, the lines of the elements most frequently occurring in chemical analysis, such as those of potassium, lithium, and calcium, are measured and used as reference lines; the bands of the carbon spectrum seen at the base of a Bunsen burner may also be employed with advantage. If the spectroscope be only employed for the detection of the alkalis and alkaline earths, the measurements made with the arbitrary scale can always be referred to and need not be reduced any further; but for the investigation of lines which occur less frequently and for all purposes of publication, the measurements must be reduced to wave-lengths. For this purpose some sharply-lefined lines, such as the lines of potassium, lithium, thallium, magnesium, and calcium are taken; and their positions according to the scals number are placed in theirproper relative positions on a horizontal 
line of a paper ruled in squares. The wave-lengths of the lines are then plotted down on the vertical lines, passing through the different points, and the points thus obtained joined by a curve. By means of this curve we can then in the usual way find the wave-lengths of any line if its scale-number be given. Instead of marking down on the vertical lines the wave-lengths of different lines we may also, following a suggestion of Professor Stokes, mark down the inverse squares of these numbers. An advantage is in this way gained, as the curve then obtained will be nearly a straight line and the interpolation curve can therefore be drawn with greater accuracy. The following is a list of some lines which can with advantage be used as reference lines to obtain the curve :

$\begin{array}{ll}6705 \cdot 5 & \mathrm{Li}\left({ }^{1}\right) \\ 6492 \cdot 4 & \mathrm{Ca}\left({ }^{(\prime)}\right) \\ 6399 \cdot 3 & \mathrm{Fe} \\ 6245 \cdot 6 & \mathrm{Fe} \\ 6135 \cdot 8 & \mathrm{Fe} \\ 6023 \cdot 2 & \mathrm{Fe} \\ 5895 \cdot 1 & \mathrm{Na}\left({ }^{1}\right) \\ 5889 \cdot 1 & \mathrm{Na}\left({ }^{1}\right) \\ 5781 \cdot 4 & \mathrm{Cu} \\ 5708 \cdot 4 & \mathrm{Fe}\end{array}$

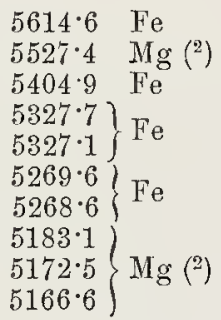

$\begin{array}{ll}5041 \cdot 3 & \mathrm{Ca}\left({ }^{2}\right) \\ 4923 \cdot 9 & \mathrm{Zn} \\ 4911 \cdot 3 & \mathrm{Zn} \\ 4809 \cdot 8 & \mathrm{Zn} \\ 4721 \cdot 5 & \mathrm{Zn} \\ 4679 \cdot 6 & \mathrm{Zn} \\ 4580 \cdot 9 & \mathrm{Ca}\left({ }^{2}\right) \\ 4454 \cdot 1 & \mathrm{Ca}\left({ }^{2}\right) \\ 4325 \cdot 2 & \mathrm{Fe} \\ 4226 \cdot 3 & \mathrm{Ca}\left({ }^{2}\right)\end{array}$

The lines marked ( ${ }^{1}$ ) can be obtained in a Bunsen burner; those marked ( $\left.{ }^{2}\right)$ are best seen in a spark taken from a solution; for all other lines a strong induction spark is necessary. If more reference lines are needed, they will generally be found in the spectra of iron and manganese.

If this method be employed to obtain wave-lengths distributed over a wide range of the spectrum, as, for instance, in the infra-red or ultra-violet, it becomes essential to use Professor Stokes's plan of plotting down inverse squares of the wavelengths.

In the ordinary chemical spectroscopes the curve can be drawn once for all, if care be taken always to bring the sodium line to its proper position. The measurements ought to be sufficiently accurate to give the wave-lengths to three figures; thus in tenth metres the sodium line may be taken as 5890 ; but the last figure in all such measurements will be unreliable. 
This method is not applicable in the case of spectroscopes of higher dispersive powers, which, of course, are only employed when greater accuracy is required. The optical parts of the instruments are not sufficiently fixed and steady to ensure the same dispersive power in every series of measurements. Even if the reading of the sodium line is the same, the other lines may have slightly different positions. In this case a separate interpolation curve must be drawn each time the instrument is used; and as greater accuracy is required, more reference lines are necessary. It becomes then most convenient to draw different curves for different parts of the spectrum, or an interpolation formula may be used. For very high dispersions the following will be found the most convenient method: The line or set of lines to be measured are well brought into the field of view, and some reference lines of known wave-length are chosen as near as possible to them, and at any rate are situated in the same field of view. If five figures are required, that is the tenth part of a tenth meter, it is necessary to employ as reference the most accurate standard which we possess, viz., the metal lines known to be present in the sun and found in Ångström's Atlas. The most easily obtained are those of iron, cobalt, nickel, copper, zinc and manganese. If reference lines can be found sufficiently close on either side it will be most convenient to employ the following interpolation formula:

$$
\frac{\Lambda_{2}(x-a)+\Lambda_{1}(b-x)}{(b-a)}=\Lambda
$$

$a$ and $b$ are the readings of the two reference lines, and $\Lambda_{1}, \Lambda_{2}$ the inverse squares of the corresponding wave-lengths; $x$ is the reading of the line to be measured; and $\Lambda$, the inverse square of the corresponding wave-length, will be found from the equation. Tables are in preparation which will give directly the inverse squares of all numbers between 1,000 and 10,000. The readings have to be taken either by means of a scale reflected into the field of view, in the manner described above, or by means of angular measurements of the position of the axis of the telescope, when the lines to be measured are made to 
coincide with the cross wires in the centre of the field of view. When a further degree of accuracy is required, a micrometer eyepiece, such as that used in astronomical measurements, becomes necessary, and working with such an eyepiece is in general less fatiguing than either of the other methods of reading.

For the greatest attainable accuracy and with large dispersion or when no reference line can be found near enough, the interpolation formula given above is not sufficient. But such cases are rare, and will only occur to skilled spectroscopists, who will have no difficulty in each particular case in choosing a suitable method.

When gratings are used instead of prisms, the interpolation becomes much simpler; as the curve connecting the angular reading with the wave-length is very nearly a straight line. This advantage is so great, that it will very often counterbalance the disadvantage of loss of light when measurements are to be taken.

The bisulphide of carbon prisms which are much used now for high dispersion are very convenient, and may be constructed so as to have a very high resolving power; but their refractive index varies so rapidly with the temperature, that they are very treacherous when measurements are to be taken, the lines shifting through a space equal to the distance between the two sodium lines by an alteration of only one-tenth of a degree centigrade. In order to ensure definition in these prisms differences of temperature in different parts of the prism have to be carefully avoided.

Lord Rayleigh has suggested the use of a stirrer for this purpose which, kept in constant motion, causes the different parts of the liquid to mix, and to equalise their temperature. The late Dr. Henry Draper also independently tried this method, and secured excellent definition. Other highly dispersive liquids have been suggested to replace the bisulphide, but each possesses certain disadvantages, and they have not come into general use.

When reference lines of different metals are to be used, it 
becomes a matter of importance to bring them into the field of view in rapid succession, as each series of measurements ought to occupy as little time as possible. The spark-holder, figured on page 133, has been found very convenient for this purpose. It may be constructed with an ordinary retort-stand. A tube carrying a pulley at its lower end is put over the upright bar of the retort-stand, and can revolve round it. It carries two wooden circular discs with a number of holes in each. The tube may be partly made of metal, but the two discs ought to be insulated from each other. The electrodes to be used are fitted into the holes by means of corks. One pole of the induction coil is permanently connected with the retort-stand, which is in metallic communication through connecting wires, shown in the figure, to all the upper electrodes. Flexible wires are attached to the lower electrodes, which are just sufficiently long to dip into a mercury cup, which is also permanently connected with the other pole of the coil. The spark will always pass through that set of electrodes which has a wire dipping into the mercury cup. The observer stationed at the eye-end of the spectroscope can by pulling at the string which is wound round the pulley, bring any required set of electrodes into such a position that the lens will throw an image of the spark on the slit of the collimator. The mercury contact may with advantage be replaced by a sliding contact.

It often becomes necessary in spectroscopic researches to take a spark from liquid solutions, and those who have no powerful coil at their disposal are almost exclusively driven to that expedient whenever the substance to be investigated does not volatilize in the Bunsen burner. Lecoq de Boisbaudran, to whom this method is principally due, used a glass tube witl a platinum wire sealed in at the lower end.

The spark is simply taken from the upper surface of the liquid, but this is inconvenient as the sides of the tube easily become wetted, and the spark passes towards the sides instead of directly to the liquid. Under the most favourable circumstances the spark is found to move about the liquid surface whenever the spark-distance is not very carefully adjustod to 
the spark-intensity. The liquid wetting the tube and rising up the sides by capillary attraction is also a source of annoyance, as the most luminous parts of the spark, uamely, those close to the liquid surface, cannot be examined. Some of these inconveniences have been avoided by Delachanel and Mermet, who have placed a capillary tube over the lower electrode, but they still viewed the spectrum through the glass walls of the tube containing the liquid, and therefore only avoided part of the disadvantages of Lecoq's arrangement. It is much more convenient to fill the tube very nearly with the liquid to be examined and to cover the electrode with a capillary of thick glass, sufficiently fine to make the liquid rise above the glass walls of the tube. There is then nothing interposed between the spark and the collimator, and the spark can be examined as conveniently as if taken from a metal pole. It is hardly necessary to remark that a few lines are introduced by the volatilization of the glass capillary, but these are easily recognized, and will only in exceptional cases interfere with the spectrum under examination. One of these tubes is shown in the figure, fitted into the spark-holder.

\section{APPENDIX C.}

\section{ON THE SPECTRA OF ERBIUM AND DIDYMIUM AND THEIR COMPOUNDS.}

BUNSEN ${ }^{1}$ has shown that the rare earth erbia is distinguished from all other known substances by a peculiar optical reaction of the greatest interest. This solid substance when strongly heated in the non-luminous gas-flame gives a spectrum containing bright lines, which are so intense as to serve for detecting this substance. This singular phenomenon does not, however, constitute any exception to the law of exchanges; for Bunsen has shown that the bands of maximum intensity in the emission

1 Ann. Ch. Pharm, cxxxvii, p. 1. 
spectrum of erbia coincide exactly in position with the bands of greatest darkness in the absorption spectrum. A similar inversion of the didymium absorption bands has also been observed by Bunsen. ${ }^{1}$

Some very interesting observations have also been made by Bunsen upon the absorption spectrum of didymium, ${ }^{2}$ from which we learn that the didymium spectrum, and also that of erbium, undergo changes if examined by polarized light according as the ordinary or extraordinary ray be allowed to pass through the crystal. These changes only become visible, however, when a powerful battery of prisms and a telescope of high magnifying power are employed. According to the direction in which the ray of polarized light is allowed to traverse the crystal of didymium sulphate is the position of the dark absorption bands found to vary; whilst the bands produced by the solution of the salt in water are again different. Very remarkable are the small alterations in the position of the dark bands of the didymium salts, dependent upon the nature of the compound in which the metal occurs. These changes are too minute to be seen with a small spectroscope, but are distinctly visible in the larger instrument. "The differences thus observed in the absorption spectra of different didymium compounds cannot, in our cornplete ignorance of any general theory for the absorption of light in media, be connected with other phenomena. They remind one of the slight gradual alteration in pitch which the notes from a vibrating elastic rod undergo when the rod is weighted, or of the change of tone which an organ-pipe exhibits when the tube is lengthened."

From experiments made with erbia and other earths Dr. Huggins and Professor Emerson Reynolds ${ }^{3}$ conclude that the bright lines seen in the spectra obtained by heating these earths in the oxylydrogen flame are due to the partial vaporisation of the heated substance. If this is really the case, the only exception to the law of solid bodies giving continuous spectra will disappear.

${ }^{1}$ Ann. Ch. Pharm. cxxxi. p. 255 ; Phil. Mag. vol, xxviii. p. 246.

${ }^{2}$ Phil. Mag. vol. xxxii. 126, p. 177.

3 l'roc. Roy. Soc. xviii. 546. 


\section{LECTURE V.}

Foundation of Solar and Stellar Chemistry.-Examination of the Solar Spectrum.-Framhofer, 1814.-Kirchhoff, 1861.-Coincidence of Dark Solar Lines with Bright Metallic Lines.-Reversion of the Bright Sodinu Lines.-Kirchhoff's Explanation.-Constituents of the Solar Atmosphere.-Lockyer and Janssen's Researches.-Phenomena observed during Total Eclipses.-Constitution of the Red Solar Prominences ; their gaseous Nature.-The Chromosphere.-Physical Constitution of the Sun.-The Corona.-Sun-spots and Faculæ.

Appendix A.-Professor Balfour Stewart on the Equality of Radiation and Absorption.

Appendix B.-On Maps of the Solar Spectrum.

ApPendix C.-On Lockyer and Janssen's Discovery.

Appendix D.-Resnlts of Recent Solar Eclipse Observations.

Appendix E.-Professor C. A. Young's Investigations on Chromospheric Lines.

Appendix F.-Professor S. P. Langley on Sunlight and the Earth's Atmosphere.

WE have in this lecture the somewhat formidable task set before us of endeavouring to explain the grounds upon which Professor Kirchhoff concluded with certainty, that in the solar atmosphere, at a distance of about 93 millions of miles, substances such as iron, sodium, magnesium, and hydrogen, which we know well on this earth, are present in a state of luminous gas.

In beginning to consider this matter, we shall, however, do well to remember that the subject is still in its youth; that it is only within the last twenty years that we have been at all acquainted with the chemistry of these distant bodies. We must not be surprised to find that some of our questions cannot be 
satisfactorily answered, and we may expect in several instances to meet with facts to which an explanation is still wanting.

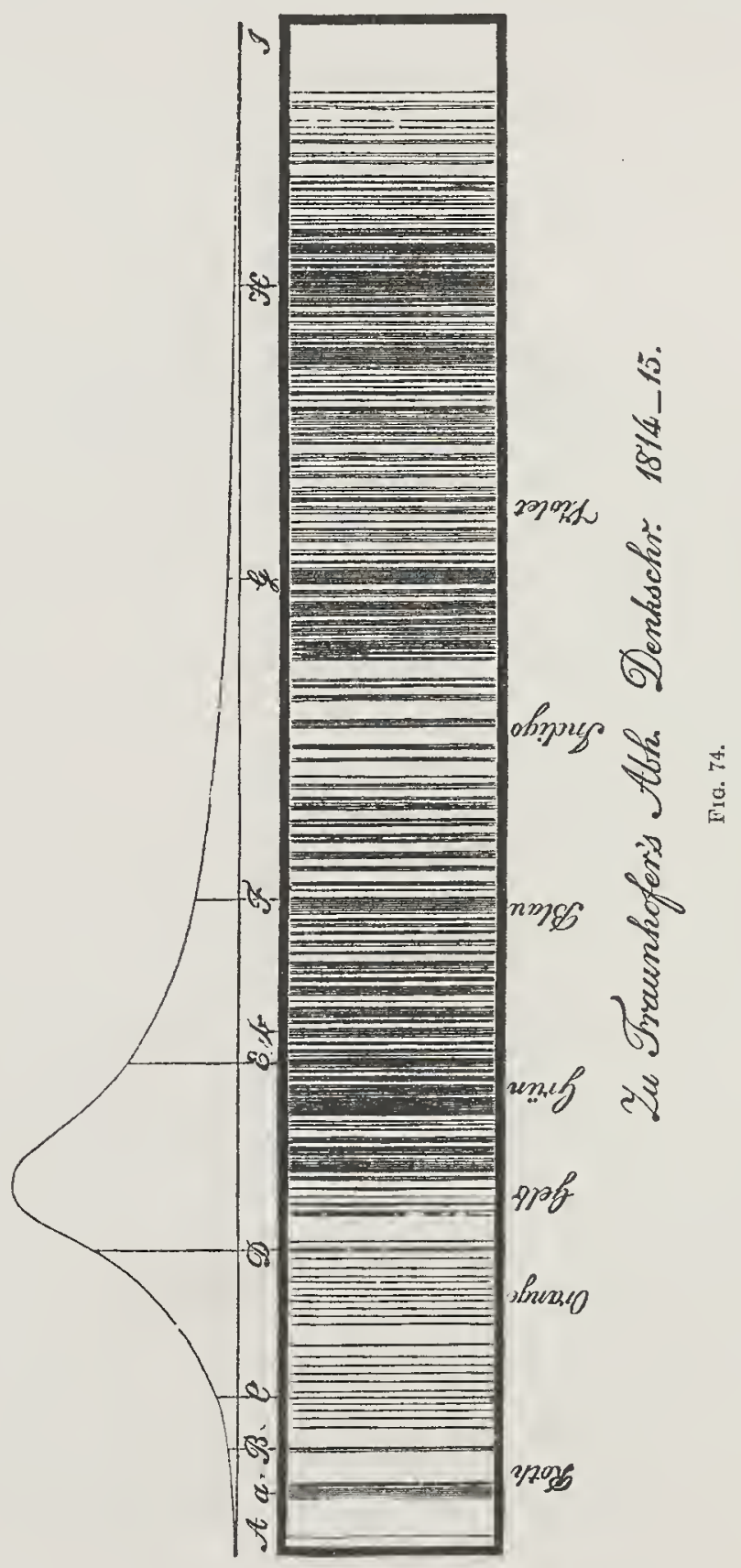


In the first lecture I pointed out to you that sunlight differs from the light given off by solid and liquid substances, as well as from the light given off by gaseous bodies. If we were experimenting with sunlight now, and if I could throw the solar spectrum on to the screen, instead of this continuous spectrum of the incandescent carbon poles, we should find that this bright band was cut up by a series of dark lines or shadows.

These lines I mentioned to you were discovered in 1814 by Fraunhofer-at least they were first carefully observed by him -and have since gone by the name of Fraunhofer's lines.

Fraunhofer measured the distances (see Fig. 74) between these fixed lines, and he found that the distance from $\mathrm{D}$ to $\mathrm{E}$, and from $\mathrm{E}$ to $\mathrm{F}$, remained perfectly constant in the sunlight, that these are fixed lines which always appear in sunlight; and, moreover, as I think I mentioned to you on a previous occasion, he examined the light from the moon and from the planet Venus, and observed that the same lines occur in moonlight and in planet-light, which is simply reflected sunlight, and he found that the relative distances between these lines were the same in light from these three sources. He then examined the light from some fixed stars, from Sirius and others, and he noticed that, although in some of these fixed stars certain lines exist which occur in sunlight, yet that other lines, always present in sunlight, are absent from the light of the stars: thus in Procyon and Capella he saw the double solar line D, but other well-known solar lines were wanting. Hence so long ago as 1814, Framnhofer concluded that these dark lines were caused by some absorptive power exerted in the star or in the sun.

The exact mapping of these lines becomes a matter of very great importance, and during the last few years much attention has been paid to this subject. I shall shortly have to draw your attention to some of the results of these investigations.

I will now content myself with projecting, by means of the oxyhydrogen light, a photograph of one of Professor Kirchhoff's maps upon the screen, in order to give you an idea of the great number of lines existing in the solar spectrum. Here is the 
line $\mathrm{D}$ in the yellow, which was noticed by Fraunhofer, and observed by him to be double. We see in addition a great number of lines varying in intensity, in depth of shade, as well as in breadth. Here we come to $\mathrm{E}$ in the blue. I might in the same way show you that throughout the whole length of the spectrum similar groups of dark lines occur. From these diagrams you will, however, form an estimate of the enormous number of these lines which exist in the solar spectrum.

I hope you will understand that these dark lines, betokening the absence of certain kinds of rays in the sunlight, not only exist in the visible portions of the spectrum, but also occur in the portions which, as I have already mentioned, do not affect

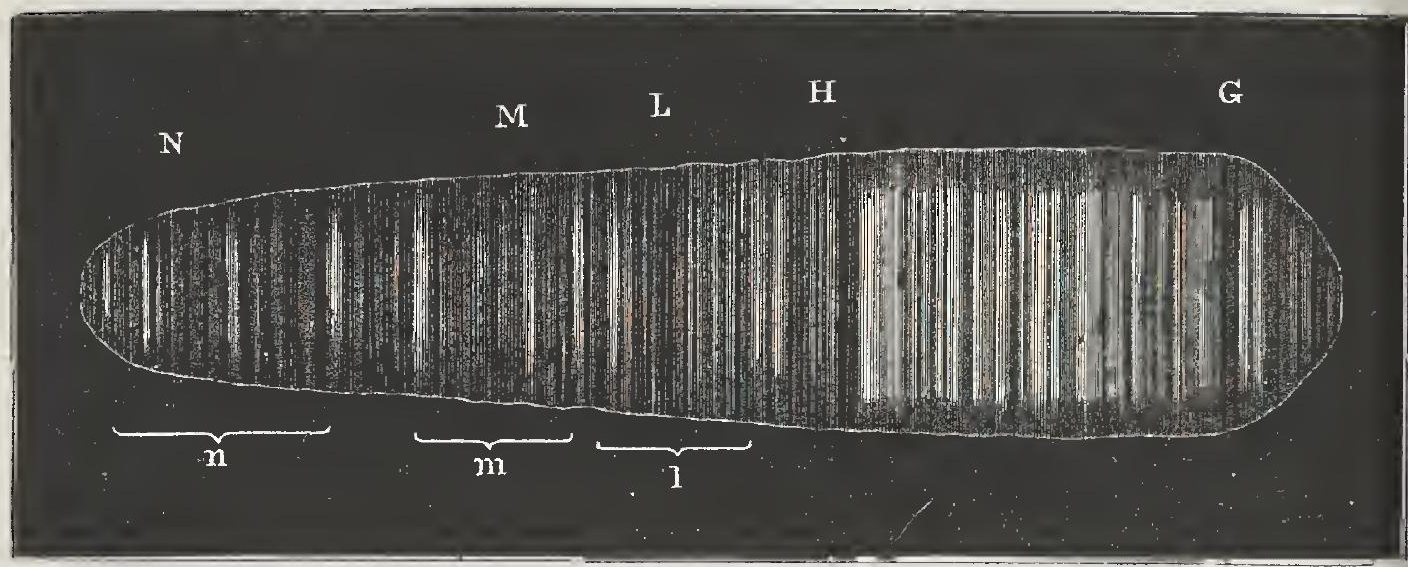

F̈IG. 75.

the retina, being either less refrangible than the red or more refrangible than the violet. Thanks, in the first instance, to the beantiful researches of Professor Stokes on fluorescence, and more recently to the labours of other observers, these lines have become perfectly well known (see Fig. 75). The diagram shows the effect produced on a film of sensitized collodion which was exposed to the action of these ultra-violet rays passing through quartz prisms. The shaded spaces indicate the positions in which the intensity of the rays is small; they are the Fraunhofer lines in the ultra-violet sunlight. You see that the lines stretch out a long way beyond the visible portion of 
the spectrum, that to which the eye is ordinarily sensitive, ending somewhere near the line $\mathrm{H}$.

Having mastered the facts concerning the composition of sunlight, I must now ask you to pass on to the examination of the first of Kirchhoff's discoveries by which the cause of these singular dark solar lines is explained. So long ago as 1814. Fraunhofer discovered that the dark lines D in the sunlight were coincident with the bright sodium lines. The fact of the coincidence of these lines is easily rendered visible if the solar spectrum is allowed to fall into the upper half of the field of our telescope, while the sodium spectrum occupies the lower lialf. The bright lines produced by the metal, as fine as the finest spider's web, are then seen to be exact prolongations, as it were, of the corresponding solar lines.

These facts, however, remained altogether barren of consequences, so far as regards the explanation of the phenomena, except to the bold niinds of Angström, Stokes, and William Thornson; the last two of whom, combining the facts with an ill-understood experiment of Foucault's made in 1849, foresaw the conclusion to which they must lead, and expressed an opinion which subsequent investigations have fully borne out. Clear light was, however, thrown upon the subject by Kirchhoff in the autumn of $18599^{1}$ Wishing to test the accuracy of this asserted coincidence of the bright sodium lines and the dark solar lines with his very delicate instrument, Professor Kirchhoff made the following very remarkable experiment, which is memorable as giving the key to the solution of the problem concerning the presence of sodium and other metals in the sun. "In order," says Kirchlıoff, for I will now give his own words, "to test in the most direct manner possible the frequently asserted fact of the coincidence of the sodium lines with the lines D, I obtrined a tolerably bright solar spectrum, and brought a flame coloured by sodium vapour in front of the slit. I then saw the dark lines D change into bright ones. The flame of a Bunsen's lamp threw the bright sodium lines upon the solar spectrum with unexpected brilliancy. In order to find out the

\footnotetext{
${ }^{1}$ Berlin. Akad. Bericht. 1859, 662 ; Phil. Mag. Fouth Serirs, xix. 192, xx. 1.
} 
extent to which the intensity of the solar spectrum could be increased without impairing the distinctness of the sodium lines, I allowed the full sunlight to shine through the sodium flame, and to my astonishment $I$ saw that the dark lines $D$ appeared with an extraordinary degree of clearness.

"I then exchanged the sunlight for the Drummond's or oxyhydrogen lime-light, which, like that of all incandescent solid or liquid bodies, gives a spectrum containing no dark lines.

"When this light was allowed to fall through a suitable flame coloured by common salt, dark lines were seen in the spectrum in the position of the sodium lines.

"The same phenomenon was observed if, instead of the incandescent lime, a platinum wire was used, which being heated in a flame was brought to a temperature near its melting-point by passing an electric current through it. The phenomenon in question is easily explained upon the supposition that the sodium flanie absorbs rays of the same degree of refrangibility as those it emits, whilst it is perfectly transparent for all other rays."

Kirchhoff had in fact, as far as he had gone, produced artificial sunlight, because he had obtained the two double dark lines in his continuous spectrum. I will try to show the formation of the dark lines of the sodium: for this purpose we will again eniploy our electric lamp, and I will throw the continuous spectrum of the carbon points on to the screen, and then I will bring into the lower carbon, which is shaped like a cup, a small quantity of metallic sodium: and we shall thus see that the vapour of the sodium has the power of absorbing the particular kind of light which it emits, and that in place of the bright sodium line we shall have a dark line. There you observe the dark sodium line. As a further illustration I have here a diagram (Fig. 76) representing what is seen when we look at the spectrum of burning sodium with an instrument such as that which Kirchhoff used. Below (No. 2) we have a drawing of the ordinary sodium spectrum, giving us these bright yellow double lines on a dark background, and above (No. 1) we see a drawing of the spectrum of burning sodium. 
Instead of two bright yellow lines, we here find we have two intensely black lines upon a bright continuous spectrum, the "D" light having been absorbed by the sodium vapour. The difference between the intensities of the lights on each side of these lines and in that particular part where the lines fall is so great as to give an actual shadow, which we see as a black line. There is a well-known experiment by which we cast a shadow with a luminous object, such as a candle flame: so here, although these black lines are not wholly devoid of light, yet the light is so much less intense than in the surrounding parts, that they appear dark to our eyes.

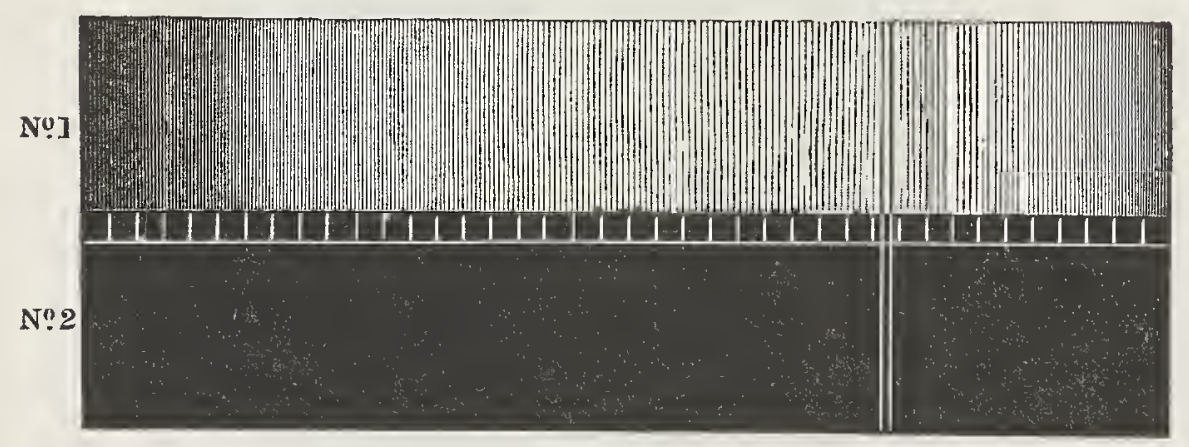

Fig 76.

I can illustrate this to you in another way. Here (Fig. 77) we have a large sheet of non-luminous gas flame $(b b)$ burning under a tall chimney $(c)$, and the flame I can colour by sodium. In front of this I am going to ignite a flame of hydrogen $(\alpha)$, and I will also place in the hydrogen flame some sodium compound; so that we shall have two sodium flames burning, one in front of the other. I want you to notice that the yellow rays passing from this large flame at the back through the hydrogen flame tinged with soda will be absorbed, and that the outer rim of this hydrogen flame, where the temperature is decidedly low, will appear dark; in fact, it will look just as if the hydrogen flame was smoky, as though we had a smoky candle burning in front of the large flame. There is no carbon in this, flame to produce a smoky appearance, as there is nothing but pure hydrogen burning. We will now light 
our hydrogen, but before you can see the phenomenon of absorption I must make a large soda flame. This I do by burning a little sodium, the fumes of which I waft into the flame. Now you see the large flame has turned yellow, and you will notice that in front we get a smoky flame. It is now very distinct. If, instead of a sodium compound, I next place some lithium salt in the flame, no black rim will appear. We shall get the red colour of the lithium flame, but it will not give us

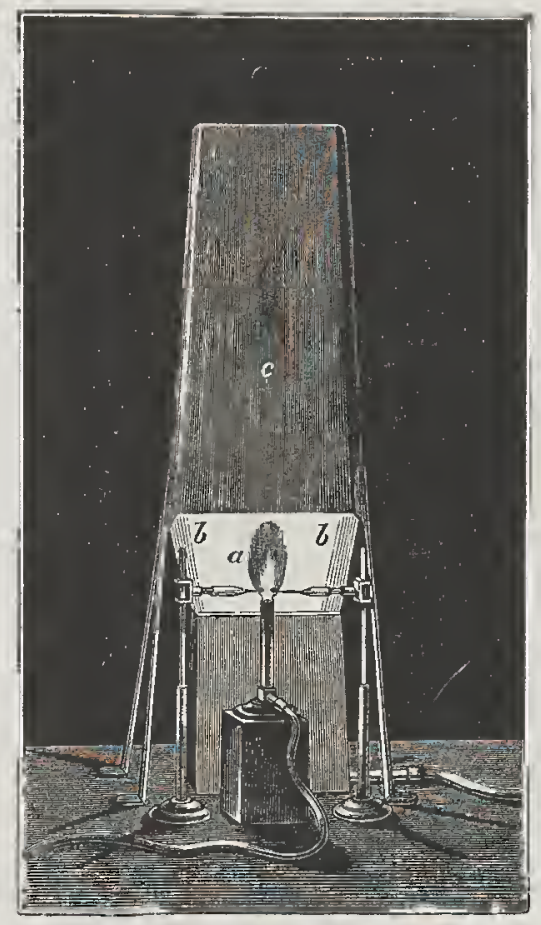

Frg. 77.

any black shadow, because it has no power of absorbing the yellow light. Hence we conclude that the smoky appearance was really caused by the absorption of the yellow " $D$ " light by the sodium vapour in a state of incandescence.

Here is another most ingenious apparatus, devised by my friend Professor Bunsen, for exhibiting a constant black sodium flame caused by its absorption of the rays of the same degree of refrangibility as it emits. The little cap of yellow flame (d) 
which floats from the first burner in front of the larger yellow soda flame $(g)$ absorbs the " $D$ " rays, and in consequence when the small flame is seen in front of the larger one we have the peculiar phenomenon of a constantly burning black sodium flame (Fig. 78).

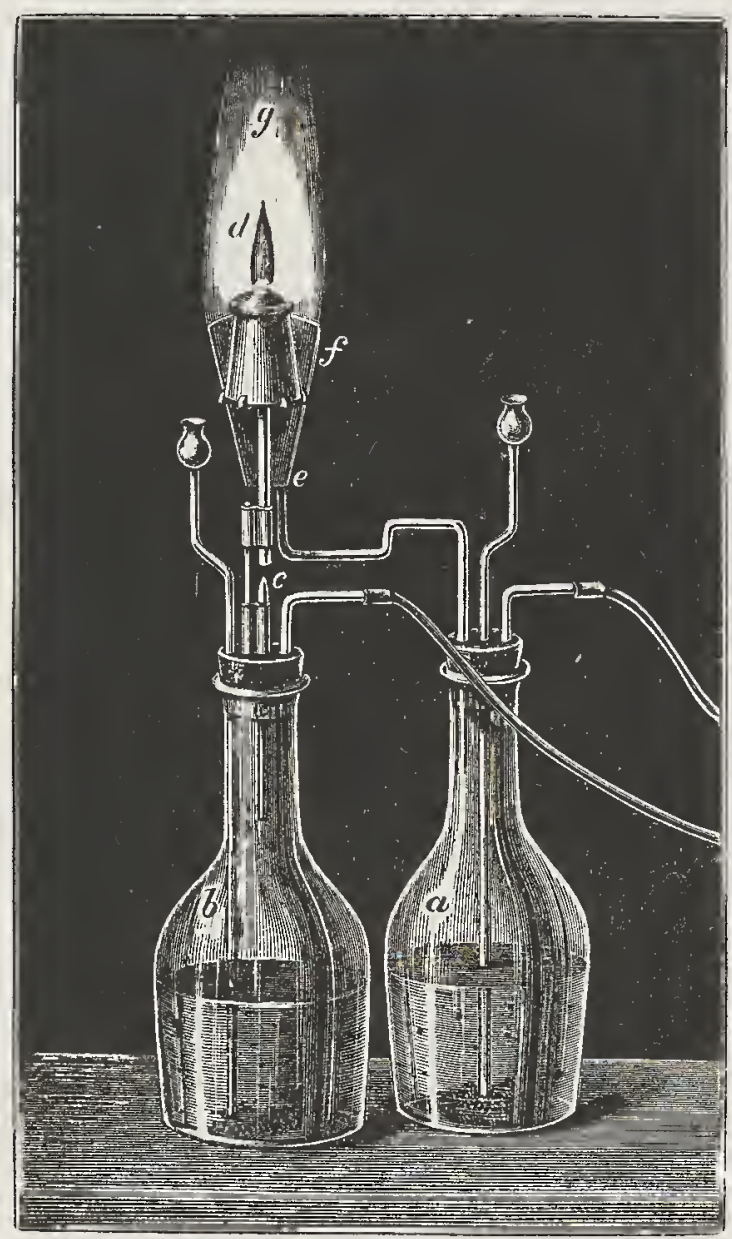

FIG. 75

I can also show you in a third way the fact that sodium vapour is opaque to the light which it gives off. I have prepared a tube containing some sodium which I can convert into vapour. By heating the tube as I am doing, it will become filled with sodium vapour, and you will see that the tube is 
transparent when we look at it by white sunlight; but when we look at it by means of the yellow sodium light it will appear to be opaque. We shall then see that the tube containing the sodium vapour throws a dark shadow on the screen. [The lights were turned down, and the screen was illuminated with a yellow sodium flame.] Now the tube looks black; we cannot see through it; it throws a dark shadow. [Light was again admitted.] Now, by the daylight, it is again colourless. This shows us, then, very distinctly, that the sodium vapour is opaque for the rays which itself can emit.

Thoroughly understanding, then, the nature of the phenomena with which we have to deal, let us follow Kirchhoff to the interesting conclusions which he draws from this experiment. $\mathrm{He}$ states that from this fact it appears likely that glowing gases have the power of especially absorbing rays of the same degree of refrangibility as those they emit; and that, therefore, the spectrum of such a glowing gas can be reversed, or the bright lines turned into dark ones, when light of sufficient degree of intensity, giving a continuous spectrum, is passed through it. This idea was further confirmed by substituting for the sodium flame the flame coloured by potassium, when dark lines appeared in the exact position of the characteristic bright lines of this metal. Bunsen and Kirchhoff have likewise succeeded in reversing the flames of lithium, calcium, strontium, and barium; whilst Dr. Miller, M. Cornu, Mr. Lockyer, and Professors Liveing and Dewar, have also reversed lines in the spectra of many metals. I can here show you the reversal of the red lithium line on the screen. For this purpose I bring on to the carbon pole of the lamp some salt of lithium, together with a piece of metallic sodium. The sodium will reduce the lithium salt to the metallic state, and I can then show you that we have got not only a dark sodium band, but a dark lithium band in the red part of the spectrum. Now the reversed lines of both these metals are clearly seen.

Generalizing from these facts, Kirchhoff has arrived, by the help of theoretical considerations which I am unable now to lay before you, at a law, previously partially enunciatcd by Prevost 
of Geneva and by Prevostaye and Dessains in France, and extended by Dr. Balfour Stewart in this country, which expresses the relation between the amount of heat of any wave-length which a body receives and that which it emits. This law has been called the law of exehanges. It asserts that the relation between the amount of heat emitted and that which is absorbed at any given temperature is eonstant for all bodies; and that the greater the amount of heat emitted, the greater must be the amount of heat absorbed. Kirchhoff has proved that the same law holds good for light as well as for heat; that it is as true of all rays and of each kind of rays : for instance, if we compare red rays emitted with red rays absorbed, or yellow rays emitted with yellow rays absorbed. From this we see that an incandescent gas which is giving off only certain kinds of light - that is, whose power of emission is limited to light of certain definite degrees of refrangibility-must have the power of absorbing those kinds of light, and those kinds only. This is what we find to be the case with the luminous' sodium vapour: it has a very high power of emission for the " $\mathrm{D}$ " rays, and it has a proportionately high power of absorption for that kind of light; but for it alone. And we see that every substance which emits at a given temperature certain kinds of light must possess the power, at that same temperature, of absorbing the same kinds of light. ${ }^{1}$

We must remember, however, that the emissive and absorptive powers of substances can only be compared at the same or nearly the same temperature. This is of very great importance, for it has been supposed that in some cases the law of exchanges does not hold good, the comparison between absorption and radiation not having been made at the same temperature. It must not be assumed that because the bright lines of the incandescent iodine spectrum, for instance, do not correspond to the dark absorption bands of the gas at a much lower temperature, therefore the law is faulty or incorrect. We must compare the lines at the same temperature.

I Report on the Theory of Exchanges, by B. Stewart (Brit. Assoc. 1861) ; Firchhoff on the History of the Analysis of the Solar Atmosphere (l'hil. Mag., Fourth Serics, vol, xxy. 1. 256). 
Now we know that the same kind of law holds good with the other vibrations known to us-the vibrations of the air which we call sound. We are all acquainted with what is called resonance. When we sing a particular note in the neighbourhood of a piano, that same note is returned to us. The particular vibrating string which can emit that note has the power also of absorbing vibrations of that particular kind, when proceeding in a straight line, and emitting them again in all directions. We are not therefore, without analogy, in the case of sound, for the absorption and emission of the same kind of undulation by the same substance. It was this analogy that first suggested to Professor Stokes the existence of a real connection between the dark lines $\mathrm{D}$ in the solar spectrum and the bright yellow sodium lines coincident with these dark lines.

We will now pass to the application of this principle of the reversibility of the spectra of luminous gases to the foundation of a solar and stellar chemistry. How does this principle assist us in our knowledge of the constitution of the solar atmosphere?

In order to map and determine the positions of the bright lines found in the electric spectra of the various metals, Kirchboff, as I have already stated, employed the dark lines in the solar spectrum as his guides. Judge of his astonishment, when he observed that dark solar lines occur in positions coincident with those of the bright iron lines which he saw in the spark. Exactly as the sodium lines were identical with Fraunhofer's lines D, so for each of the iron lines, of which Kirchhoff and Angström have mapped no less than 460, a dark solar line was seen to correspond. Not only had each iron line its dark representative in the solar spectrum, but the breadth and degree of shade of the two sets of lines were seen to agree in the most perfect manner, the brightest iron lines corresponding to the darkest solar lines.

To those who have not themselves witnessed this coincidence it is impossible to give an adequate idea, by words, of the effect produced on the beholder, when looking into the spectroscope he sees the coincidence of every one of perhaps a hundred of the 
iron lines with a dark representative in the sunlight, and the idea that iron is contained in the solar atmosphere flashes at once on his mind (see Fig. 79).

These hundreds of coincidences cannot be the mere effect of chance; in other words, there must be some causal connection between these dark solar lines and the bright iron lines. That this agreement between them cannot be simply fortuitous is proved by Kirchhoff, who calculates-from the number of observed coincidences, the distance between the several lines, and the degree of exactitude with which each coincidence can be determined - the fraction representing the chance or probability that such a series of coincidences should occur without the two sets of lines having any common cause: this fraction he finds to be less than $\frac{100000000000000000}{\text { : }}$ or, in other words, it is practically certain that these lines have a common cause. "Hence this coincidence," says Kirchhoff, "must be produced by some cause; and a cause can be assigned which affords a perfect explanation of the

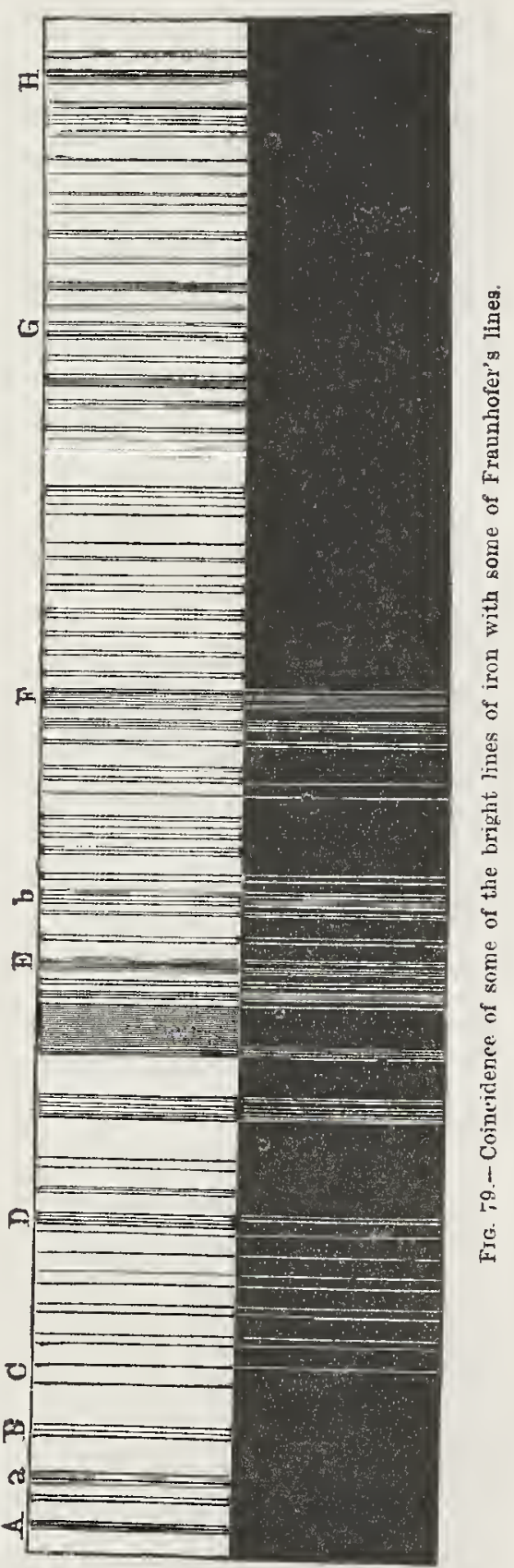


phenomenon. The observed phenomenon may be explained by the supposition that the rays of light which form the solar spectrum have passed through the vapour of iron, and have thus suffered absorption which the vapour of iron must exert."

"As this is the only assignable cause of this coincidence, the supposition appears to be a necessary one. These iron vapours might be contained either in the atmosphere of the sun or in that of the earth. But it is not easy to understand how our atmosphere can contain such a quantity of iron vapour as would produce the very distinct absorption lines which we see in the solar spectrum; and this supposition is rendered still less probable by the fact that these lines do not appreciably alter when the sun approaches the horizon. It does not, on the other hand, seem at all unlikely, owing to the high temperature which we must suppose the sun's atmosphere to possess, that such vapours should be present in it. Hence the observations of the solar spectrum appear to me to prove the presence of iron vapour in the solar atmosphere with as great a degree of certainty as we can attain in any question of natural science." This statement is, I believe, not one jot more positive than the facts warrant. For what does any evidence in natural science amount to, beyond the expression of a probability? A mineral sent to me from New Zealand is examined by our chemical tests, of which I apply a certain number; and these show me that the mineral contains iron: and no one doubts that my conclusion is correct. Have we, however, in this case, proof positive that the body really is iron? May it not turn out to be a substance which in these respects resembles, but in other respects differs from, the body which we designate as iron? Surely. All we can say is, that in each of the many comparisons which we have made, the properties of the two bodies prove identical, and it is solely this identity of the properties which we express when we call both of them iron.

Exactly the same reasoning applies to the case of the existence of these metals in the sun. Of course the metals present there, causing these dark lines, may not be identical with those we have on earth; but the evidence of their being the same is as 
strong and cogent as that which is brought to bear upon any other question of natural science the truth of which is generally admitted.

I do not think I can give you a more clear or succinct account of the development of this great discovery than by quoting from Kirchhoff"s admirable memoir the following passage : "As soon as the presence of one terrestrial element in the solar atmosphere was thus determined, and thereby the existence of a large number of Fraunhofer's lines explained, it seemed reasonable to suppose that other terrestrial bodies occur there, and that, by exerting their absorptive power, they may cause the production of other Fraunhofer's lines. For it is very probable that elementary bodies which occur in large quantities on the earth, and are likewise distinguished by special bright lines in their spectra will, like iron, be visible in the solar atmosphere. This is found to be the case with calcium, magnesium, and sodium. The number of bright lines in the spectrum of each of these metals is indeed small, but those lines, as well as the dark lines in the solar spectrum with which they coincide, are so uncommonly distinct that the coincidence can be observed with great accuracy. In addition to this, the circumstance that these lines occur in groups renders the observation of the coincidence of these spectra more exact than is the case with those composed of single lines. 'The lines produced by chromium, also, form a very characteristic group which likewise coincides with a remarkable group of Fraunhofer's lines: hence I believe that I am justified in affirming the presence of chromium in the solar atmosphere. It appeared of great interest to determine whether the solar atmosphere contains nickel and cobalt, elements which invariably accompany iron in meteoric masses. The spectra of these metals, like that of iron, are distinguished by the large number of their lines. But the lines of nickel, and sti'l inore those of cobalt, are much less bright than the iron lines; and I was, therefore, unable to observe their position with the same degree of accuracy with which I determined the position of the iron lines. All the brighter lines of nickel appear to coincide with dark solar lines; the same was observed with respect to 
some of the cobalt lines, but was not seen to be the case with other equally bright lines of this metal. From my own observations I consider that I am entitled to conclude that nickel is visible in the solar atmosphere. I do not, however, yet express an opinion as to the presence of cobalt. Barium, copper, and zinc appear to be present in the solar atmosphere, but only in small quantities; the brightest of the lines of these metals correspond to distinct lines in the solar spectrum, but the weaker lines are not noticeable. The remaining metals which I have examined-viz., gold, silver, mercury, aluminium, cadmium, tin, lead, antimony, arsenic, strontium, and lithium -are, according to my observation, not visible in the solar atmosphere."

Ångström and Thalen soon added hydrogen, manganese, aluminium, and titanium to the list of elements found by Kirchhoff. A considerable difficulty remained, however. It was found that some of the lines of an element were reversed in the sun, while others equally bright or even brighter did not appear as dark lines. In the passage quoted, Kirchhoff mentions that this is the case with the cobalt lines, and he thereby was led to consider the presence of cobalt in the sun as doubtful. The difficulty was overcome in great part by the series of experiments made by Mr. Lockyer, which we have already discussed in the third lecture. It had already been observed by several experimenters, and by M. Cornu amongst others, that if a metal is volatilized in the voltaic arc in sufficient quantity, the cooler vapour in front of the hotter often produced a reversal of the lines, and it was noticed that it was not al ways the brightest lines which were reversed in this manner, but those which seem longest when an image of the spark or arc is formed on the slit. Mr. Lockyer reasoned from this, that if an element is not present in the sun in sufficient quantity to have all its lines reversed, we must look for the longest lines and take them as an indication of the presence of the element rather than the strongest lines. In this way Mr. Lockyer could show that, for instance, in the case of cobalt the longest lines are reversed and the missing lines are short, so that we have very strong evidence 
of the presence of cobalt in the sun. Mr. Lockyer thus added a number more elements to the list of those already found present in the sun. Among them are : lead, cadmium, potassium, cerium, strontium, uranium and vanadium, probably also lithium, rubidium, caesium, tin, bismuth and silver. The numerous experiments of Messrs. Liveing and Dewar enable us to ascertain whether Mr. Lockyer's statement as to the easier reversibility of the longest lines is a general one, and the law may be said to have stood the test very well. There are, no doubt, some exceptions requiring further investigation, but in the great majority of cases the law holds good.

According to Mr. Lockyer the relative intensity of the dark lines in the solar spectrum is in some instances so different from any distribution of relative intensity which we can obtain in our laboratories, that he assumes an actual decomposition of the element in order to explain the facts. Whether his numerous experiments and observations admit of a less revolutionary interpretation is a question which is still much discussed by Chemists and Spectroscopists, but the majority of scientific men have not hitherto felt inclined to accept Mr. Lockyer's views without obtaining more convincing evidence than he has yet adduced.

Another subject which has received much attention, and which is closely connected with the preceding, is the apparent coincidence of certain dark lines in the sum with lines of two or sometimes even of three terrestrial elements. The fact of the apparent coincidences of lines of different elements has already been mentioned in a previous lecture, and it was there pointed out that the coincidences were in all probability apparent only, and that higher dispersion would probably always be able to show slight differences of position. This view is strengthened by the fact that in nearly every case where a dark solar line was found coincident with a line of more than one terrestrial element, that solar line has on closer exumination been found to be really a double or even a triple line. It does not necessarily follow from this, but still it is probable, that one of the components belongs to one element, while the other element has its representative 
in the other component. This view has in some instances been confirmed by the experiments of Professors Liveing and Dewar, who have actually shown which of the two components is due to the one and which to the other metal.

What may be called the average chemical composition of the sun is, however, by no means the only result of the application of the spectroscope. To obtain the solar spectrum Kirchhoff had only to allow the light of the sun to fall on his spectroscope, and nothing but a mirror, used to reflect the sunlight, was placed in front of his instrument; but if we add a lens to the mirror, and if we place the lens at such a distance from the slit that an accurate solar image is focussed on it, we may study, according to Lockyer's method already described, not the average light of the solar surface but the light sent out by any part of it; we may for instance compare the light of the central portions of the sun's disc with that sent out by the limb, and this observation has shown that no appreciable difference can thus be detected. But important differences have been discovered when, on the other hand, the image of a sun-spot is thrown on the slit.

Sun-spots, as you are well aware, have long ago attracted the attention of astronomers, and various theories have been given to account for them. Kirchhoff showed from general considerations that the temperature of a sun-spot must be lower than that of the remainder of the solar surface, and this has since been confirmed by spectroscopic evidence. On throwing the image of a sun-spot on the slit of a spectroscope and investigating the light after its prismatic decomposition, we find, in the first place, dark bands crossing the spectrum (Fig. 80). These bands tell us that there is within the sun-spot a region of general absorption affecting all the rays. This proves without a doubt, and in spite of recent assertions to the contrary, that the temperature of a sun-spot is lower than that of the surrounding regions of the solar surface. We also find that some of the dark lines are widened in the spot. Such a widening we know to be due to an increase of pressure, and hence the spectroscope tells us that the pressure in the nucleus of a sun-spot is higher 
than that on the surface of the sun. Fig. 80 represents the widening of the two yellow sodium lines, and some of the lines near it, as observed by Young. It is curious to note that the line which lies between the two sodium lines and which is due to nickel is not widened, but remains as thin inside the spots as on the bright parts of the sun. The question as to which lines are, and which are not widened in the sun-spots has lately received much attention. Mr. Lockyer especially has made a very careful and detailed series of observations on the lines which are most widened in spots at different times. Valuable and important results have already been obtained by him, as, for instance, that the lines of one and the same element which are most widened in a sun-spot may be different at different times,

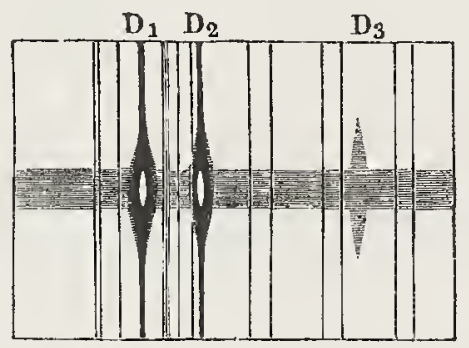

Fig. 80 .

and it seems likely that some connection between these phenomena and the general changing state of solar surface activity, as shown by the number of the sun-spots present, will ultimately be discovered. But it is only by a long series of researches that we shall gain a clear insight into these complicated phenomena. As yet it is too early to say more than that we shall in all probability thus arrive at facts of the highest importance both as regards the internal state of the sun and as regards the behaviour of the molecules of our terrestrial elements when they are subjected to the enormous temperatures and pressures which must exist on our luminary.

Sometimes, however, absorption bands and lines are observed in a sun-spot which are not seen at all on the sun under ordinary 
circumstances. It is probable that in these cases the temperature within the spot has been sufficiently lowered to produce changes in the spectrum such as have been discussed in the third lecture. Fig. 81 represents the spectrum of a sun-spot as observed at Greenwich, and the drawing exlibits a number of bands in the spot-spectrum which are not seen in the spectrum of the adjoining parts of the solar surface.

In the next lecture we shall see how the spectroscope can also give us information as to the motion of luminous bodies towards us or away from us, and how we thus become aware of the existence of violent commotion within the spot, consisting of upward or downward rushes of gas.

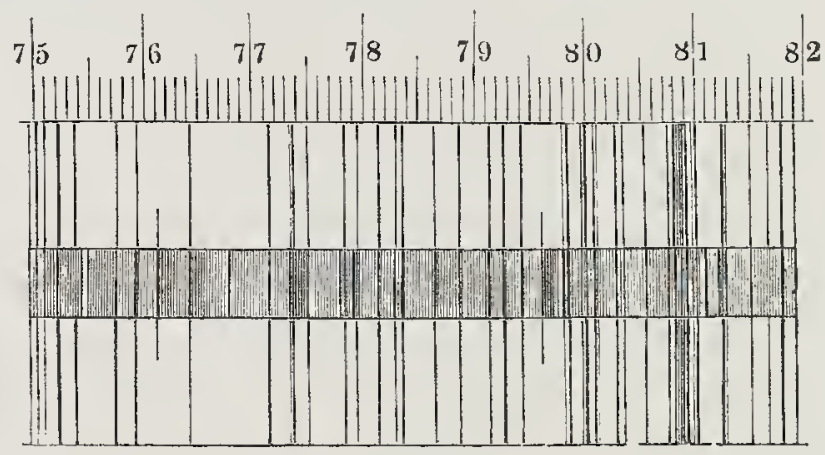

FIG. 81.

I cannot of course enter into an account of the numerous theories of sun-spots, but it seems certain that the blackest parts of the spot are cooler and subjected to greater pressure than the rest, and it has also been found that the level of these cooler gases is lower than that of the general surface of the sun, so that these spots, whatever they are caused by, are really depressions in the solar surface. The remarkable physical conditions which an examination of these sun-spots reveals will be referred to hereafter.

I now pass to another important application of the spectroscope to solar physics. The first step towards this application has been the examination of the light emitted by those remarkable protuberances, or red flames, which, during a total eclipse, are 
seen to dart out from the surface of the sun to the enormous height of some 80,000 to 90,000 miles. The appearance of the sun during the total eclipse of 1860 is represented by Figs. 82 and 83,

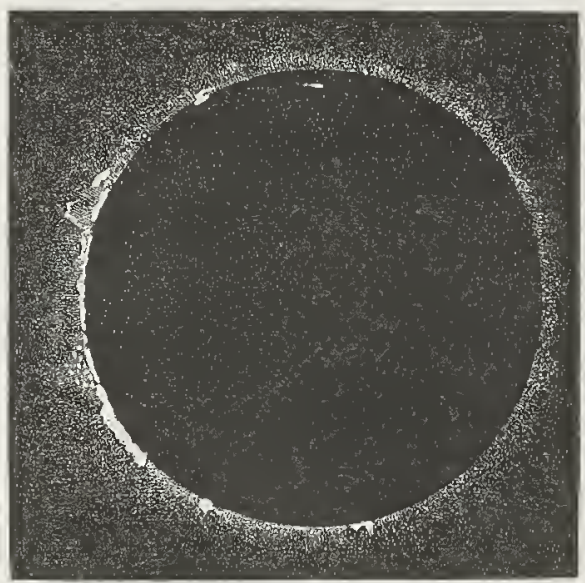

Fia. 82

which are copies of photographs taken by Mr. De la Rue in Spain during the eclipse. The first one of these was taken immediately after the total obscuration, and the second just

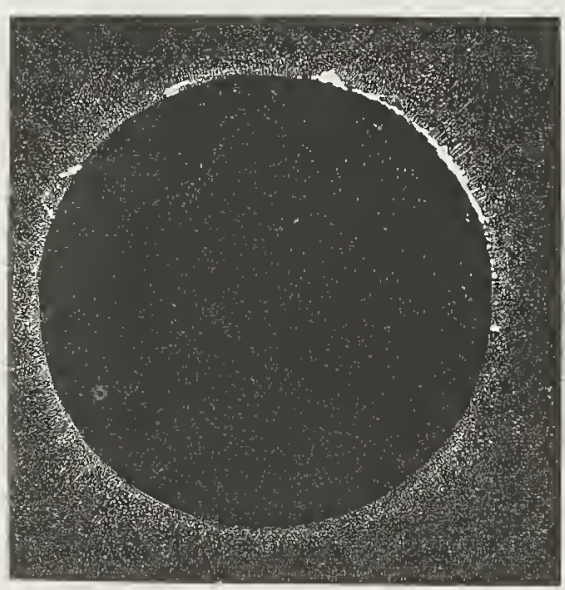

FlG. 83

previous to the reappearance of the sun. The existence of these flames proves that the sun's incandescent atmosphere extends to a very great height above the ordinary and usually 
visible portion, and it is a very remarkable fact, exp'ained, however, by recent observations, that certain protuberances which were not visible to the naked eye are found in the photograph; the flames emitting rays of a high degree of refrangibility so weak as not to act upon the retina, although strong enough to produce an image on the sensitive plate.

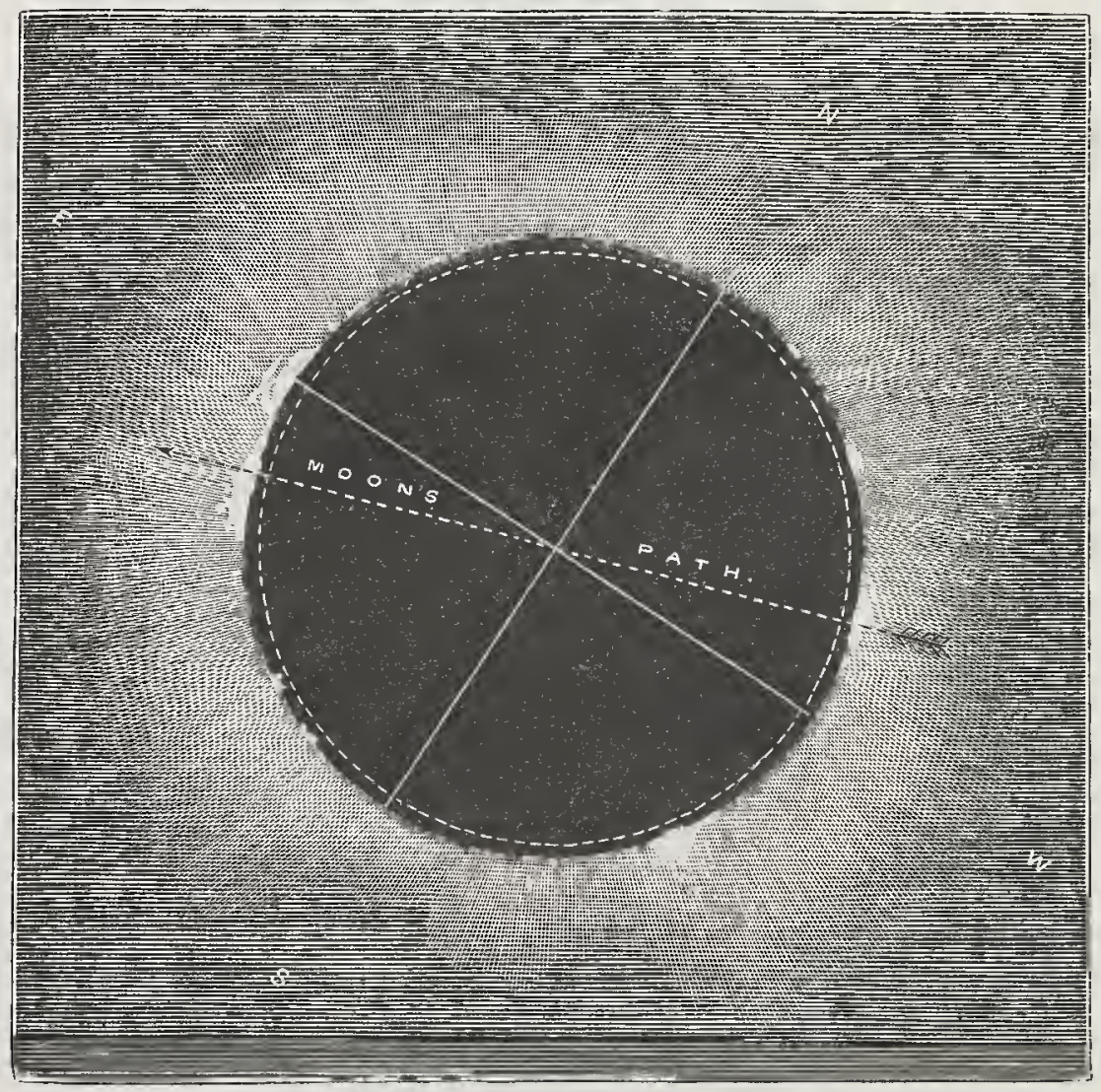

Frg. 84 gives a representation of the total eclipse of 1869 , copied from the photographic registrations taken at Burlington in the United States by Dr. Mayer. In this drawing both the prominences and the coronal rass are seen, and the peculiarly indented appearance of the moon's dark limb in the neighbourhood of the prominences is well shown.

A most striking feature of the discovery of the nature of the material composing these red protuberances is that it was made independently and nearly simultaneously, by two observers, many thousands of miles apart, namely, by Mr. J. Norman 
Lockyer in England, and by the French astronomer M. Janssen in India. In the autumn of 1866 Mr. Lockyer suggested ${ }^{1}$ that it might be possible, by the use of the spectroscope, to obtain evidence, under the ordinary conditions of the solar disc, of the red prominences which had hitherto only been seen during total eclipses. After many fruitless attempts Mr. Lockyer at last, on Oct. 20, 1868, succeeded in seeing the prominences with an unobscured sun, and he ascertained that the spectrum of the prominences is discontinuous, consisting of three bright bands, which he then gave as being-1, absolutely coincident with $\mathrm{C}$; 2 , nearly coincident ${ }^{2}$ with $\mathrm{F} ; 3$, near $\mathrm{D}$. The principle adopted by Lockyer, by which these bright lines, proving the gaseous nature of the prominence, were rendered visible, was that of employing a spectroscope with a heavy battery of prisms, and possessing a strong dispersive power. The light from the body of the sun, producing an almost continuous spectrum, was in this way much spread out and thereby weakened; whilst the luminous intensities of the monochromatic rays emitted by the glowing gas were but slightly diminished, and thus the light from the prominences became visible without being interfered with by that emanating from the body of the sun. A simple experiment will render this important point clear. If I throw the light from the incandescent carbon points on to the screen by passing the rays through a flint-glass prism, you will observe a short but very bright and perfectly continuous spectrum; if I next substitute for the glass prism two prisms filled with carbon disulphide, you see that the spectrum becomes very much elongated and its luminous intensity correspondingly diminished, so that now you can distinctly observe the narrow yellow sodium band and many other bright lines due to impurities in the carbon. These lines were present when the glass prism was used, but they were rendered invisible by the greater brightness of the continuous spectrum.

Whilst Mr. Lockyer was experimenting in England, M. Janssen had been sent out by the French Government to

1 Proc. Roy. Soc., Oct. 11, 1866.

2 Since shown to be actually coincident with it. 
Guntoor, in India, to observe the spectroscopic appearances presented by the sun on the total eclipse of August 18, 1868. On that occasion he saw and measured the position of the bright lines above referred to ; but, struck by their intensity, he likewise conceived the idea that they might be seen when the sun was un-eclipsed, and cried out, as he was looking through his telescope, "Je reverrai ces lignes-là!" On the next morning, as soon as the sun rose out of the bank of clouds which lay on the horizon, he succeeded in his endeavour, he saw the protuberances plainly, and was able to do what he failed to accomplish in the hurry and excitement of the eclipse; namely, to measure the exact position of the bright lines. "So that," he writes, "the last seventeen days have been to me like a perpetual eclipse."

The announcement of M. Janssen's independent observation was received by the French Academy on October 26, 1868, a few days after Mr. Lockyer's discovery had been made known to the Royal Society. As regards the claims of priority of this discovery, you will all, I am sure, feel inclined to agree with the following eloquent words of M. Faye when speaking on this subject in the French Academy on October 26, 1868 :-

"Mais au lieu de chercher à partager, et par conséquent à affaiblir le mérite de la découverte, ne vaut-il pas mieux en attribuer indistinctement l'honneur entier à ces deux hommes de science qui ont eu séparément, à plusieurs milliers de lieues de distance, le bonheur d'aborder l'intangible et l'invisible par la voie la plus étonnante peut-être que le génie de l'observation ait jamais conçue?"

By these observations of Lockyer and Janssen, therefore, it was proved that the prominences contained glowing masses of hydrogen, and since that time prominences and their spectra have received continuous attention and are daily watched in different Observatories.

Lockyer, soon after his first discovery, noticed the presence of the violet hydrogen line $(h)$; this only appears, however, at high temperatures, a fact which is of some importance, as by such observations we may ultimately hope to fix the temperature 
at different parts of the solar surface. In addition to the lines of hydrogen, those of other substances make their appearance especially in the lower parts of the prominences. A yellow line shown in Fig. 8.5, close to the sodium lines, though somewhat more refrangible (5875) seems always present and rises to the same height as the hydrogen lines. The same figure also exhibits the magnesium and sodium lines, which are however, confined to the neighbourhood of the sun's limb and may possibly, as we shall see, not belong to the prominences properly speaking. The photographic results obtained during the last three eclipses have proved, moreover, the presence in the prominences of calcium in large quantities. The lines of calcium which are especially strong are those known as in the violet $\mathrm{H}$ and $\mathrm{K}$, but as these lines are near the linits of

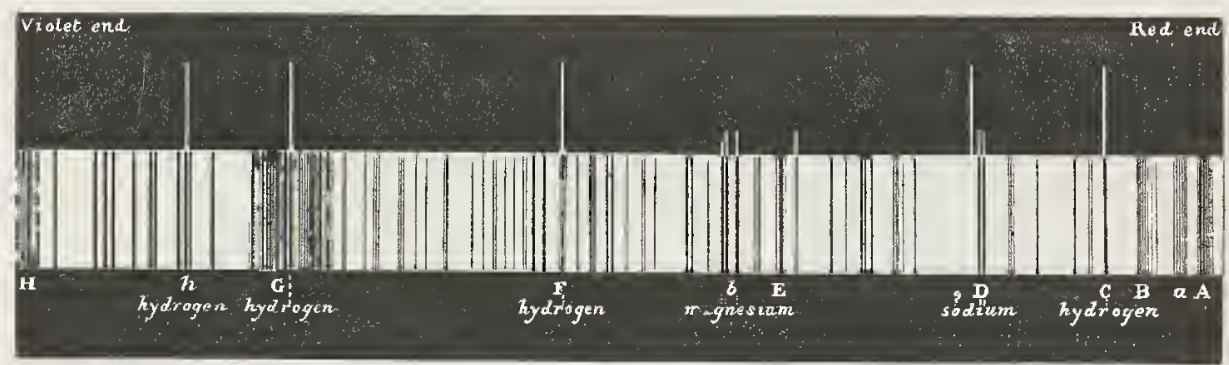

FIG. 85.

visibility they had for some time escaped notice. From the appearance of the photographs taken during the eclipses of 1875 and 1882 , it would seem that calcium forms perhaps even a larger part of the proininences than hydrogen.

A great step in the further investigation of these phenomena was made by Dr. Huggins, who first showed how by widening the slit we may observe not only the spectrum but even the actual shape and form of the prominences. To make his observations successful he had, however, to einploy a screen of ruby glass; but Lockyer and Zöllner afterwards showed that this can be dispensed with if a spectroscope of sufficient dispersive power be used. It is easy to see how the widening of the slit produces the required effect, for if it be sufficiently broad to take in the 
whole inage of a prominence, that image will be repeated as many times as there are elementary rays in its composition. The large dispersion is necessary, as before, in order to spread out and weaken the continuous band of light which also forms a regular part of the prominence spectrum; and as that continuous spectrum will appear more strongly with a wide than with a narrow slit, we easily see that a dispersion which may be sufficient to show the prominence lines with a narrow slit may not be large enough to show the whole prominence with a wide slit. It is usual in observing solar prominences to throw the image of the sun in such a way on the collimator that the slit shall be tangential to the solar $\operatorname{limb}$, as it is found that we can in this way more easily avoid the introduction of the spectrum of the sun itself, which by its intensity would increase the difficulty of seeing the prominences.

Lockyer describes some of these enormous flames of incandescent hydrogen as being 27,000 miles in height, and finds their changes to be so rapid that they totally disappear in less than 10 minutes! "During the last few days," he writes, "I have been perfectly enchanted with the sight which my spectroscope has revealed to me. The solar and atmospheric spectra being hidden and the image of the wide slit alone being visible, the telescope or slit is moved slowly, and the strange shadow-forms flit past. Here one is reminded by the fleecy, infinitely delicate cloud-forms, of an English hedge-row with luxuriant elms; here of a densely intertwined tropical forest, the intimately interwoven branches threading in all directions, the prominences generally expanding as they mount upwards, and changing slowly, indeed almost imperceptibly. By this method the smallest details of the prominences, and of the chromosphere itself, are rendered perfectly visible and easy of observation."

Zöllner has also made similar observations, and has published striking drawings of some of these protuberances, which I here l.ave the pleasure of showing you (Fig. 86), These drawings represent one and the same protuberance observed on July 1 , 1869, which underwent the singular changes here seen between the hours of $6 \mathrm{~h}$. $45^{\prime}$ and $7 \mathrm{~h} .8^{\prime}$ A.x., some of which remind one 
of the outbursts of a volcano, or the eruptive discharges of a geysir. ${ }^{1}$ In one case a flickering flame-like motion was observed to pass in a few seconds up and down, a horn shooting up to the height of 50,000 iniles (Fig. 87); whilst other protuberances formed clouds which seemed to have been shot upwards by a kind of explosion (Figs. 86-90). The chromolith on the opposite page, also one of Zöllner's drawings, shows still more strikingly the appearance of these prominences.
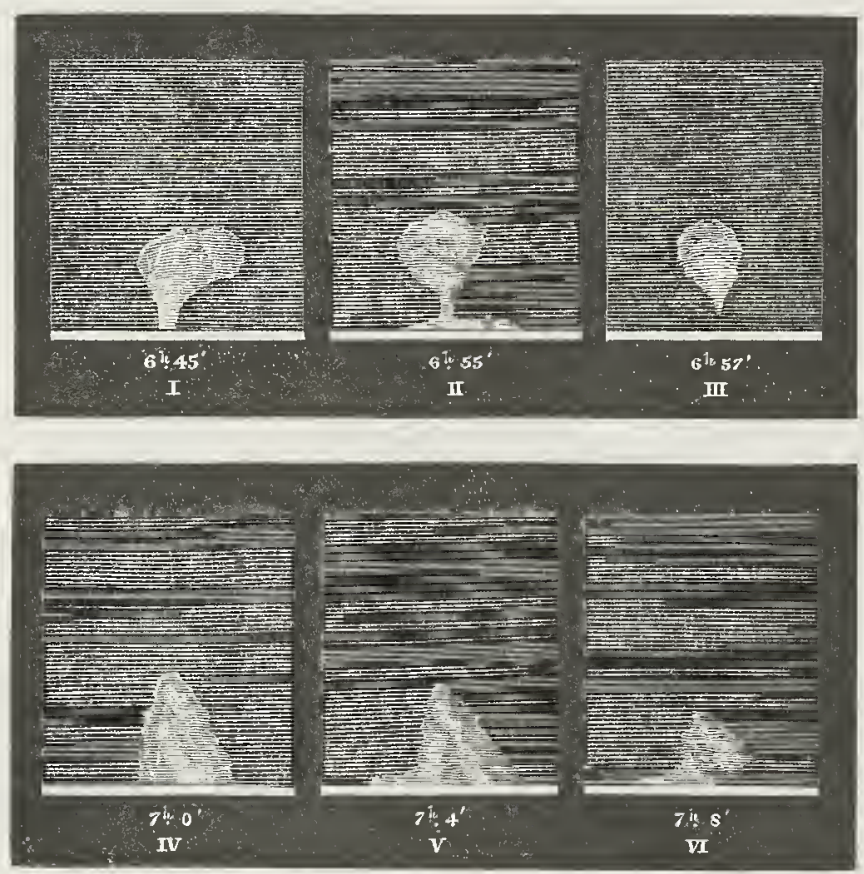

Fig. 80.

The startling rapidity with which these gigantic masses of hydrogen appear and disappear proves that currents, storms, and hurricanes are of constant occurrence in the chromosphere. These changes are doubtless brought about there, as in our own atmosphere, by local variations in temperature. "But," as Kirchhoff remarks, "the differences in temperature which produce these winds may amount to thousands of degrees, and, therefore, the furce of the currents must far exceed that of

\footnotetext{
1 See Aplendix C.
} 
the most violent terrestrial tornadoes." Both telescopic and spectroscopic observations of phenomena which have long been known as peculiarities of the solar disc fully bear out this conclusion. You will have anticipated me when I say, that I refer not only to the dark sun-spots of which I have spoken

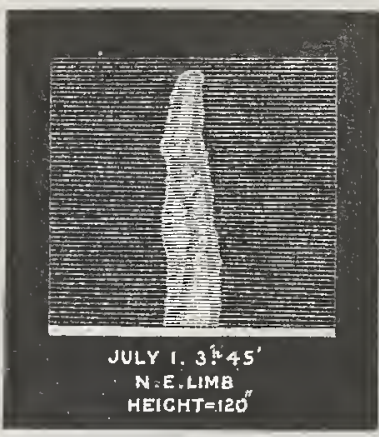

FIG. 87.

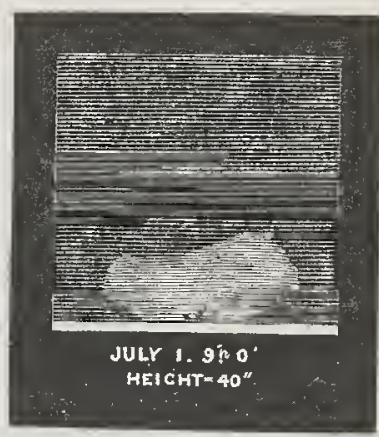

Fiq. 88.

but also to the bright stripes or faculæ which are always more or less visible on the solar surface.

Astronomers formerly supposed that the sun-spots were holes in the photosphere through which the dark body of the sun was

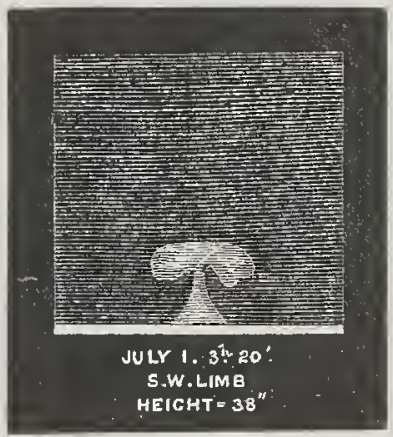

FiG. 89.

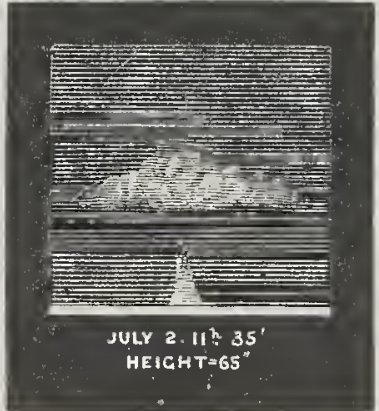

F'IG. 90.

visible. Kirchhoff proved that this theory could not be correct, inasmuch as the interior portion of the sun's body must be white-hot, and he threw out the idea that these spots were clouds floating in the solar atmosphere. Subsequent research has somewhat modified these views of Kirchhoff's, for as I 

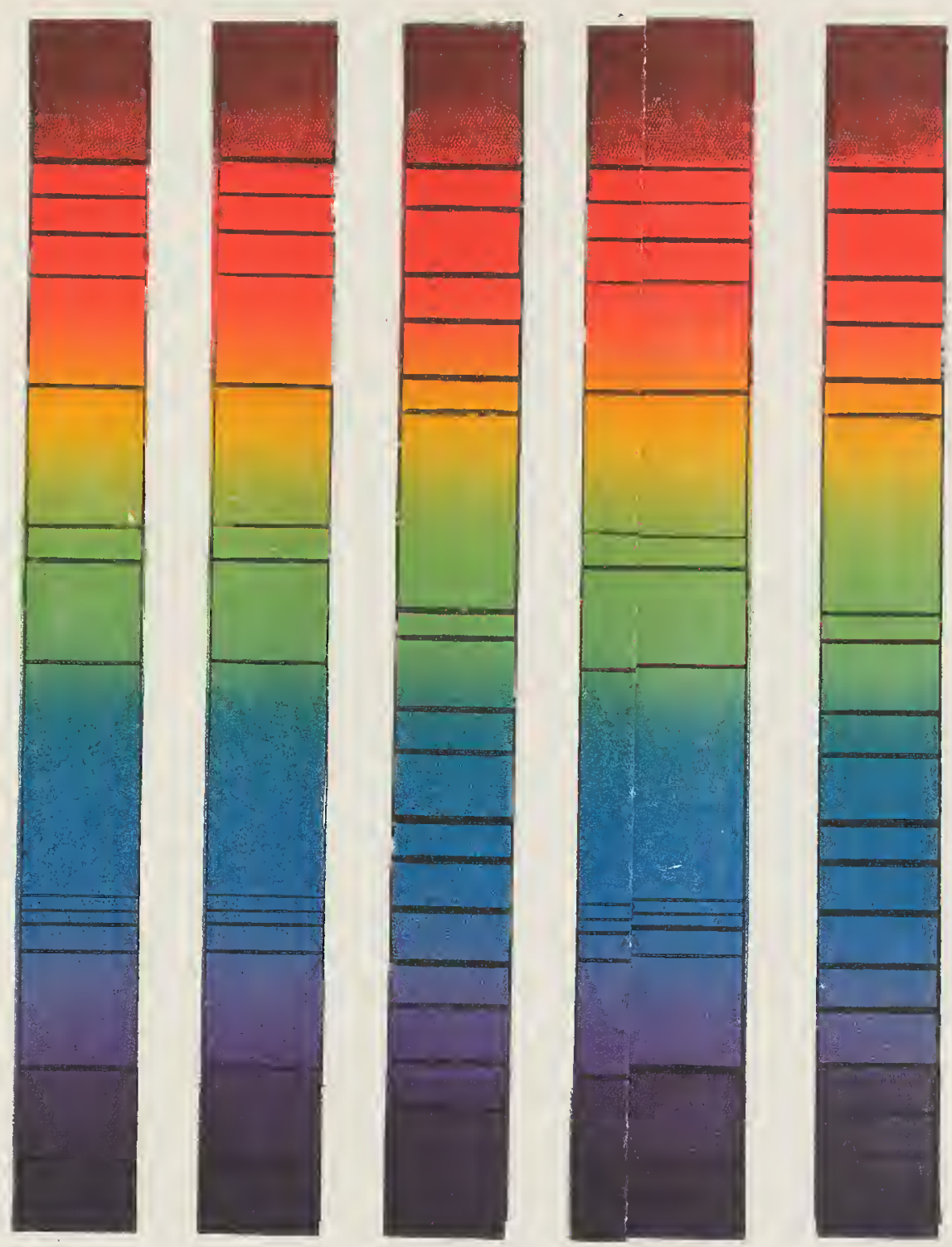



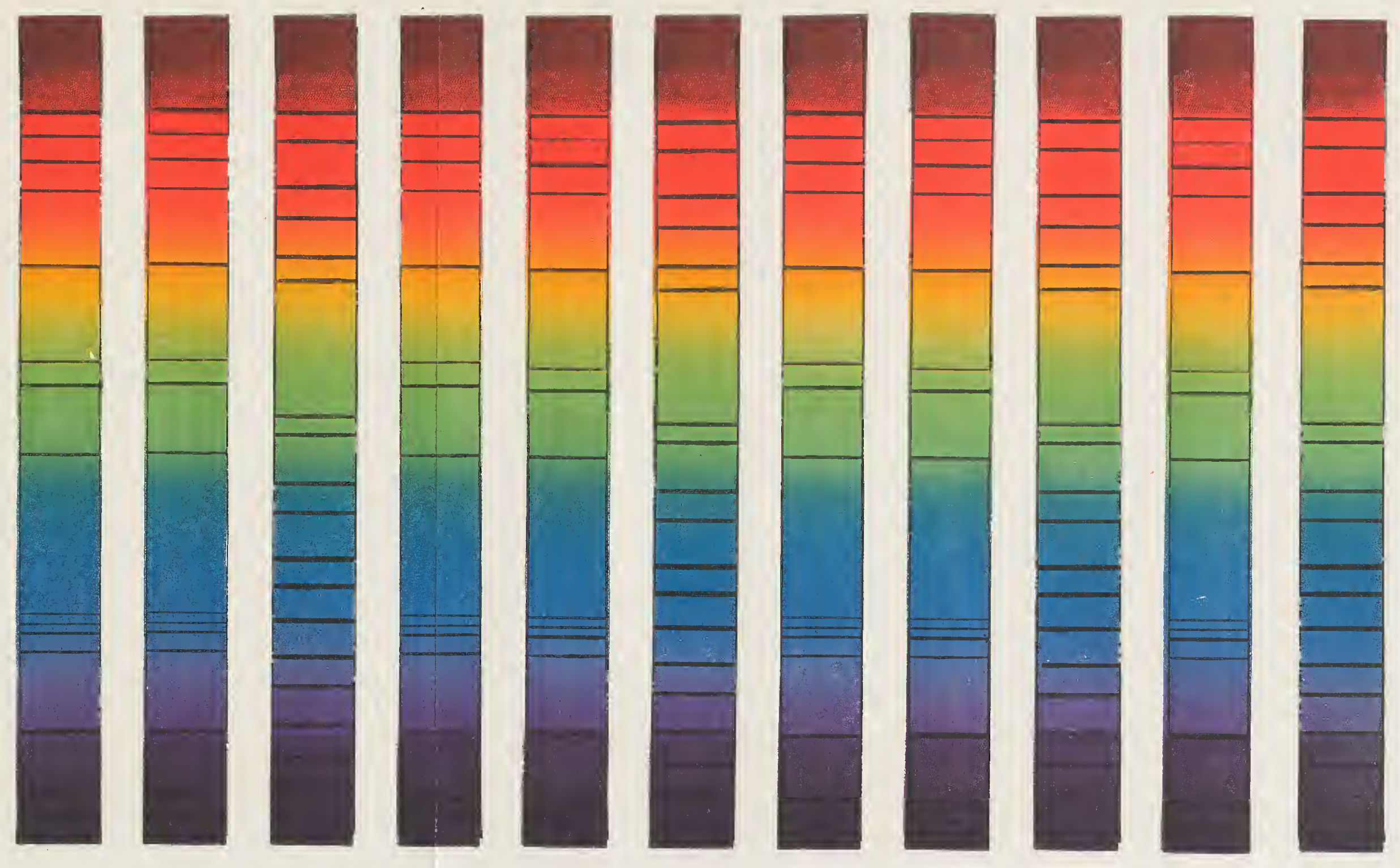



have already stated, sun-spots are really hollows or cavities in the solar atmosphere where the temperature of the glowing gases has been reduced. These spots look to us black because they give off less light than the surrounding portions of the sun's surface, but they are not, therefore, non-luminous; on the contrary they emit much light though they appear dark, just as a candle-flame looks black when held in front of the much more intensely-heated surface of the lime-light. The telescope has long ago told us of the gigantic changes, sometimes extending over even thousands of millions of square miles, which these spots undergo, but it is the spectroscope which admits us into the secrets of the constitution of these most singular phenomena. This instrument has shown us that the gases of the whole solar atmosphere are constantly in a state of the most violent motion, and has proved that a spot is a region of greater absorption. The faculæ, on the contrary, are accompanied by the incandescent vapours of sodium, iron, magnesium, and barium, thrown up by a sort of volcanic action from the lower to the higher regions of the solar atmosphere; for the bright lines, indicative of the presence of these bodies, occasionally make their appearance, not only at the edge of the sun's disc, but also in the centre of the sun's surface near the bright faculæ, or in the hotter ascending currents of the ignited gases. Lockyer even describes a cloud of incandescent magnesium that he saw floating high up above a prominence. What an insight this single observation gives us into the condition of solar physics!

Another most interesting subject has reference to the spectrum of the corona or the halo of silver-white light which surrounds the moon on all sides during a total eclipse of the sun (Fig. 91).

The spectrum of the corona has recently been made the subject of much careful study; but as this examination can only be satisfactorily carried out during the short duration of a total eclipse, and as such total eclipses are not very frequent, our knowledge on this subject is not so precise as that respecting the chromosphere. Nevertheless, thanks to the 
untiring energy of astronomers and spectroscopists of all nations, who have vied with each other in this great work, we lave arrived at certain definite conciusions respecting the corona.

At first indeed it was a matter of doubt whether the coronal halo belonged to the sun or was not rather a purely terrestrial or lunar phenomenon. The numerous photographs of the corona

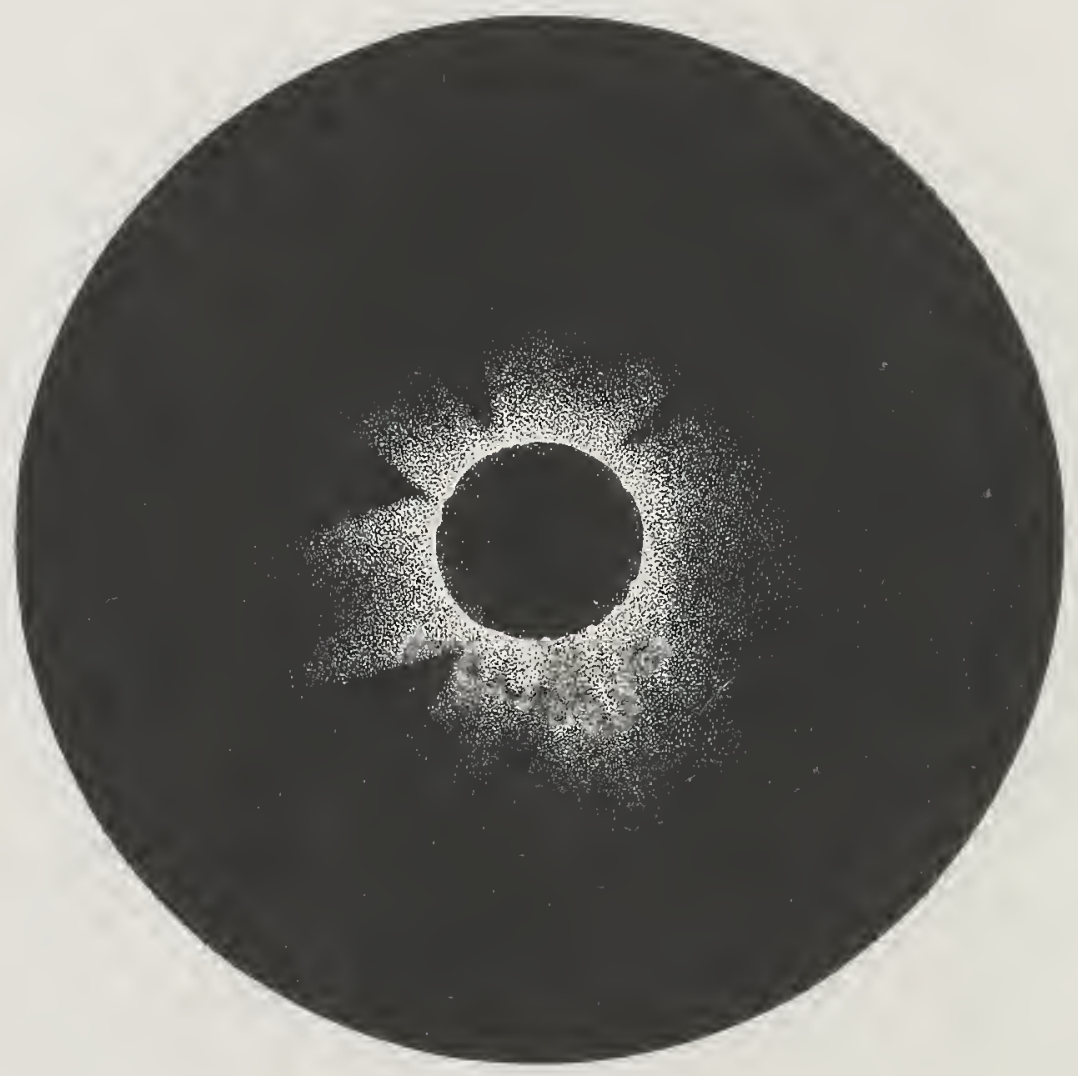

FiG. 91.

however leave no doubt that if any part of the corona is not solar, that part must be very insignificant indeed. Photographs of the same corona, taken at different places, exhibit an identical structure and have shown no sign whatever of any terrestrial influence.

That none of the coronal phenomena are connected with the 
moon is sufficiently proved by the fact that during the eclipse the body of the moon is seen to pass over the lower parts of the corona whilst the outer parts do not change their position, and likewise by the important discovery of Dr. Huggins, to which I shall afterwards allude, that the corona can be photographed in ordinary daylight.

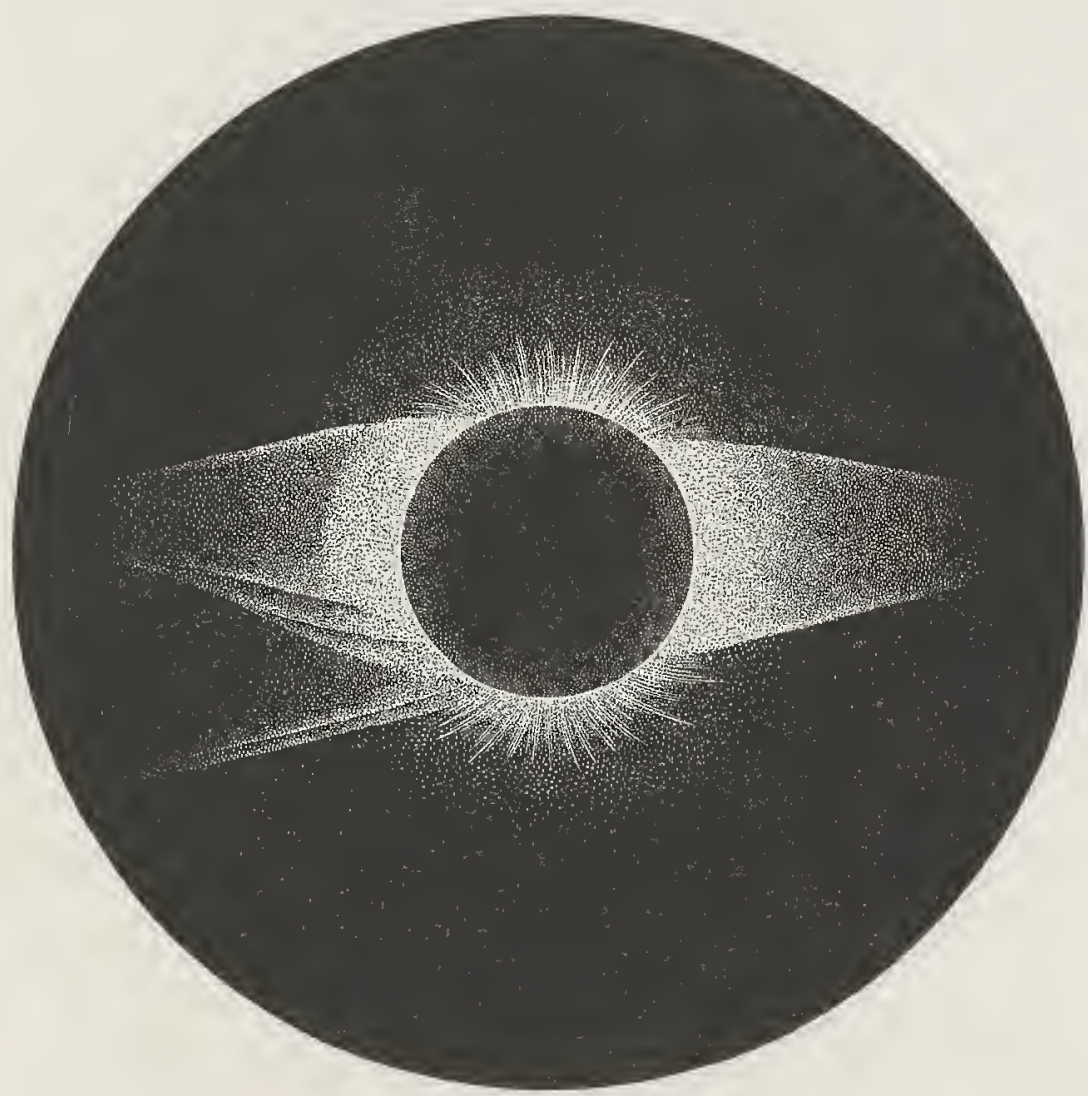

FIG, 92.

The form of the corona is seen to vary much in different eclipses, and a connection between it and the sun-spot period has now been clearly established. Fig. 92 represents a corona such as is seen during a sun-spot minimum. It is a copy of a photograph taken in Colorado by the American observers during the eclipse of 1878. We notice large extensions in the direction of the solat 
equator, while shorter curved rays are observed near the poles. No such symmetry is seen during a corona taken during a period of maximum sun-spot, such as is given in Fig. 91 representing the corona of December 22nd, 1870. An intermediate state between the corona of maximum and that of minimum solar disturbance was seen in the eclipse of 1872 in India and is

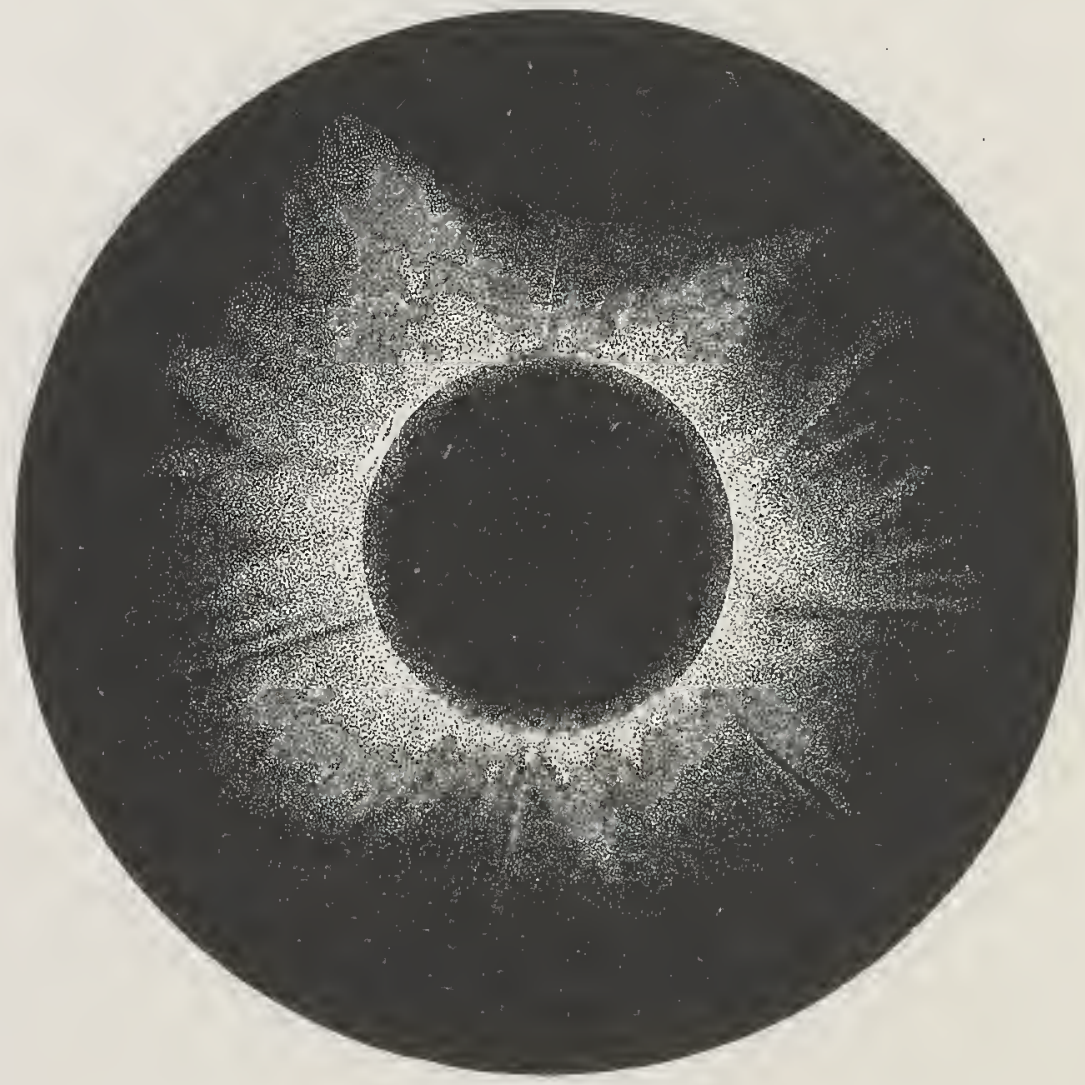

FIG. 93.

represented in Fig. 93. The structural details of the corona as photographed in Egypt during the total eclipse of 1883 during a maximum spot period are well seen in the copper-plate engraving which serves as the frontispiece to this volume.

Let us now pass to the consideration of the spectroscopic discoveries which have been made during total solar eclipses. 
The eclipse of 1868 was chiefly taken up with the study of the prominences; Major Tennant, however, during that eclipse, tried also to examine the corona. He could only see a continuons spectrum. In the following year however Harkness, Young and Pickering, independently discovered at least one bright line in the corona. The wave-length of this line, which does not coincide with the line of any known element, ${ }^{1}$ is $5315 \cdot 9$, but it generally goes by the designation of $\mathrm{K} 1474$ as it occupies that scalenumber on Kirchhoff's map. It is coincident with a dark line in the solar spectrum, and its accurate identification during the short duration of an eclipse will always remain a striking proof of Professor Young's observational skill. The portion of the solar spectrum surrounding the principal coronal line as it appears when seen with great dispersion has been accurately drawn by Professor Young, and is represented in Fig. 94. In this figure two lines marked $\mathrm{x}$ are seen close together. Of these the more refrangible is the coronal line, while the less refrangible is an iron line which at one time was supposed to be coincident with it, until Professor Young separated the two lines. The existence of two other lines, also in the green, has been asserted by some observers, but their position has never been properly fixed. The brightness of the principal green coronal line varies very much during different eclipses, and while it is strikingly brilliant during some, it was not seen during others, as in 1878 , by a great number of the observers, being only slightly brighter than the continuous background.

An important observation by M. Janssen in 1871 proved that the continuous spectrum of the corona is interrupted by some dark Fraunhofer lines. This discovery, since confirmed, proves that the corona shines partly by reflected light, a fact which is also proved by polariscopic observations. A probable explanation of this is that meteoric matter circulating round the sun or falling into it, is present in sufficient quantity to scatter and to

1 It was at one time supposed that the line was eoineident with a weak iron line. To those aequainted with the laws of speetroseopy this eould only indieate an aceidental eoineidence, but it would seem from reeent observations that the iron line is slightly less refrangible. 
reflect ordinary sunlight towards the earth, and thus to give rise to the appearance of all the dark Fraunhofer lines.

Each subsequent eclipse has added to our knowledge, and a great step was mad.e in 1882 in Egypt when, thanks to Captain Abney's dry plates, successful photographs of the spectrum of the corona and prominences were obtained by Dr. Schuster. The continuous spectrum of the corona with the solar group G reversed, confirmed 'Tennant's and Janssen's observations. The photograph
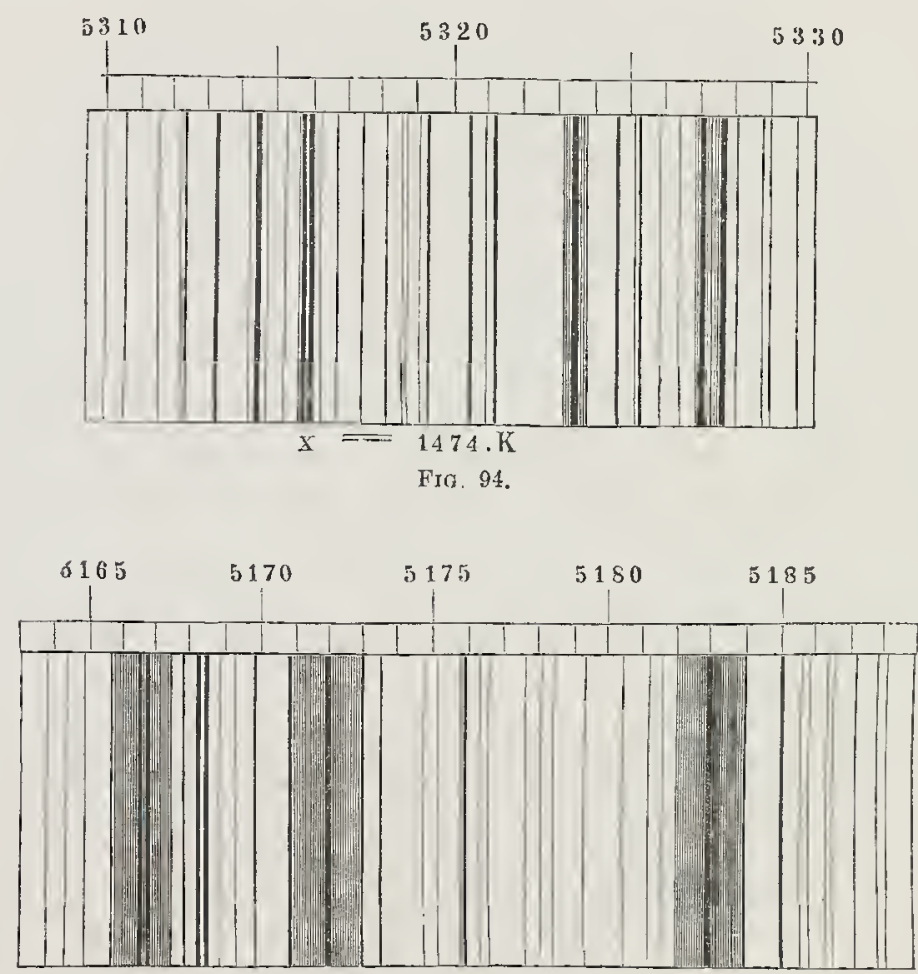

FIG. 95.

seen in Fig. 96 also exhibits the complicated line-spectrum of the corona, and in addition to the one green line, which up to that date was known to be present, we now have a photographic record of about thirty more. ${ }^{I}$ The same eclipse has also given us the following valuable information on the spectrum of prominences. In the first place, the ultra-violet hydrogen lines

I Abney and Schuster, Phil. Trans. 1884, part ii. 
were discovered to be present, besides a number of unknown lines, whilst lines due to calcium appeared even stronger than

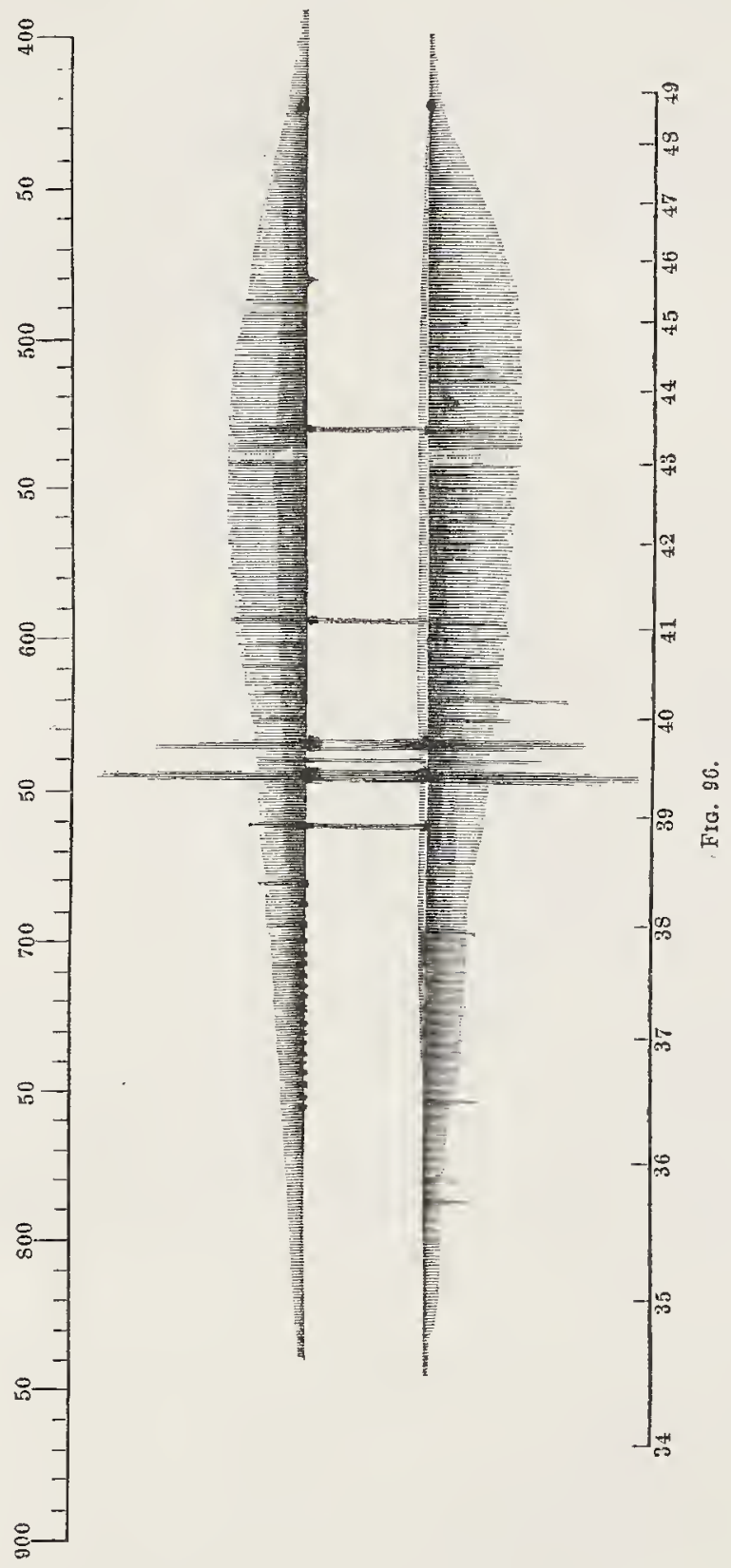


those of hydrogen, a fact suspected in the eclipse of 1875 but not proved until the above year.

A great deal of work remains yet to be done during total solar eclipses, but as time goes on we shall be able to do more and more of the eclipse work in ordinary daylight. In corroboration of this opinion I may mention Dr. Huggins's recent most important success of obtaining photographs of, at any rate, the inner and brighter part of the corona even in our London atmosphere. The first of the two plates on the opposite page gives an accurate representation of the appearance of the corona photographed by him in ordinary daylight, within a few weeks of the total eclipse of May 6, 1883, of which a drawing is given in the second of the two plates from a photograph taken in the South Sea Islands by Messrs. Lawrance and Woods. The general structural resemblance of these, places beyond a doubt the reality of Dr. Huggins's discovery, whilst a comparison of the four coronas, as photographed, shows evidence of a gradual change in the rifts so characteristic of this interesting phenomenon.

From what I have already said, you will see that the spectroscope has now become an instrument as essential to the astronomer as it is to the chemist. You will, however, be more forcibly convinced of the power of this new aid to astronomy, when I explain, as I hope to do in the next lecture, that it has not only given us information as to the chemical and physical condition of the stars, comets and nebulæ, but that it has actually enabled us to ascertain with accuracy the rate with which the ignited gases of the solar atmosphere rush forward and to measure the speed of motion, towards or from the earth, of the fixed stars. 



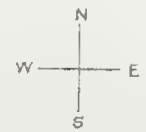

0
$c$
5
0

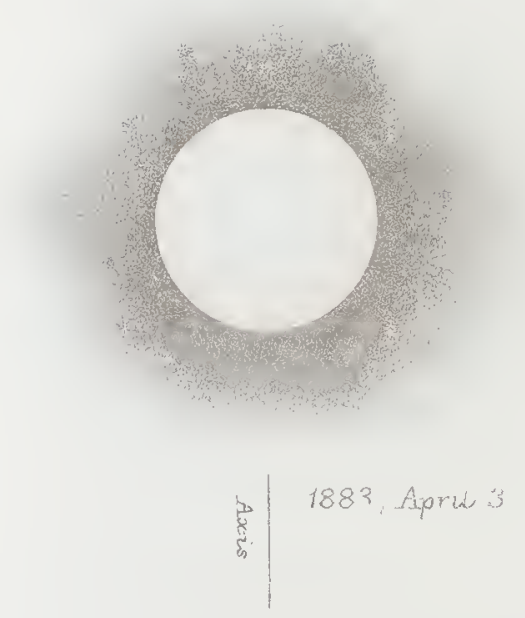

के

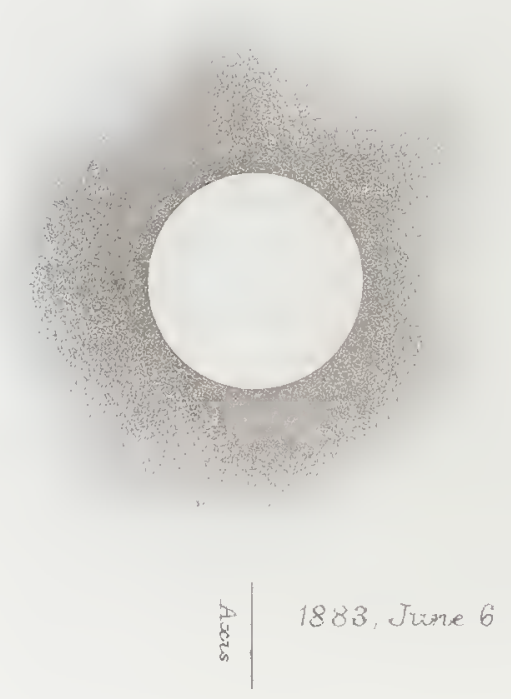

$\infty$
है
a

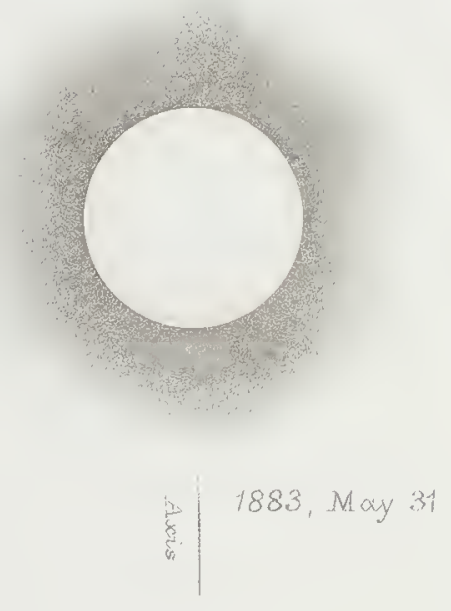

$\infty$
है।
क

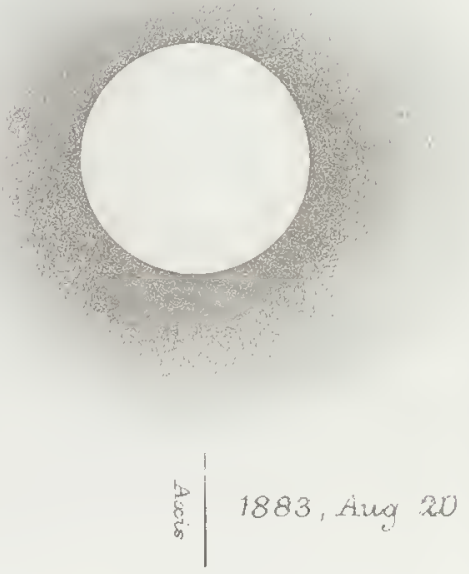



Diagrammatic drawing of the main outhne features of the Photograph of the Corona taken during the Ficlipse of 1883 May 6. at Carotine Istand, by Mess's Lawrance and Woods.

$N$

है।

W

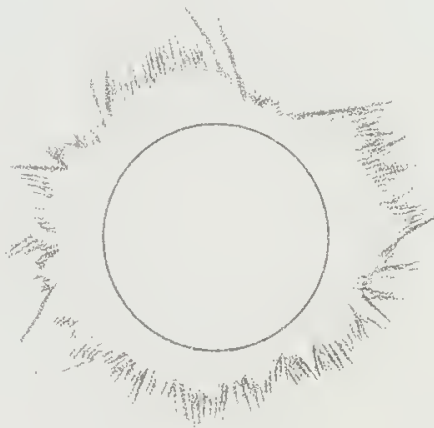

E

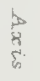

Drawn by M? Westey from a positive made by Captarn Abney, R.F. F.R.S. by permission of the Royal Society. 


\section{LECTURE V.-APPENDIX A.}

\section{PROFESSOR BALFOUR STEWART ON RADIATION AND ABSORPTION. ${ }^{1}$}

WE liave hitherto chiefly confined our remarks to the radiation of carbon at different temperatures; and taking that substance as a type of solids, and comparing its radiation with that from incandescent gases, we have found a very great and striking difference between the two classes of spectra, that of carbon being continuous, while those of gases are discontinuous. We slıall now endeavour to connect the radiative properties of bodies with their absorptive properties.

Let us begin with the temperature of boiling water, or $100^{\circ}$ Cent. Let us now, therefore, suppose that we have a large thermometer at this temperature hung up in a room having the temperature of melting ice. The thermometer will lose heat in two ways: by convection on account of the air which surrounds it, and which is continually carried off and renewed, and also by radiation. But in order to confine our thoughts to the latter process, let us suppose that the chamber is a vacuum. Now, in the first place, let the outside of the glass bulb of the thermometer be coated with a thin coating of polished silver, and let us ascertain how much heat it radiates in one minute. Next let the bulb be coated with lamp-black, the same experiment being repeated, that is to say, the thermometer at $100^{\circ}$ Cent. being allowed to cool for one minute in a vacuum chamber of $0^{\circ}$. It will be found that the bulb now radiates in a minute very much more heat than it did when coated with silver. Next let the glass bulb be left uncovered, and the thermometer

1 See Edinb. Roy. Soc. Trans., xxii. (1861). 
will still be found to radiate almost as much as when the bulb was covered with lamp-black. Finally, let it be covered with white paper, and its radiation will still be found to be almost equally great. We are thus entitled to say that at $100^{\circ}$ Cent. a blackened surface, or one of glass or white paper, radiates much more than a surface of polished silver, and we may thus construct a table of the comparative radiating powers of bodies heated to $100^{\circ}$ Cent., at the top of which we may put a lampblack surface, a surface of glass, and one of white paper, and much lower down one of silver; which is a very bad radiator. Our table of radiating substances for heat of low temperature will therefore stand thus :--

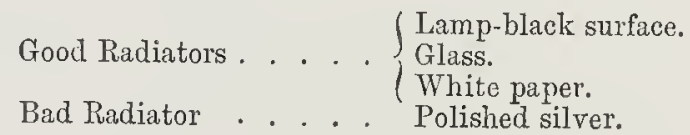

Suppose now that the thermometer is at $0^{\circ}$, and is carried into a vacuum chamber of the temperature of $100^{\circ}$, this being the reverse of the previous process. In the first place, let the bulb, as before, be coated on the outside with a coating of silver; it will absorb a certain quantity of heat in one minute; observe how much. Next, blacken the bulb with lamp-black, repeat the experiment, and measure the absorption which takes place in one minute as before; the absorbing power of the thermometer will now be considerably increased. Again, if the coating be entirely removed, and nothing left above the glass bulb, it will be found that the absorbing power of the glass bulb is almost as great as that of the blackened bulb, and the same result will be obtained if the bulb be covered with white paper. We may thus construct a table of the comparative absorbing powers of various bodies for heat of $100^{\circ}$, at the head of which we may place a lamp-black surface, a surface of glass, and one of white paper, and much further down one of polished silver.

Our table of bodies which absorb heat of $100^{\circ}$ will therefore stand thus :-

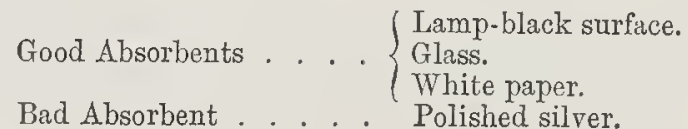


If these two tables, the one of radiators and the other of absorbents, be now compared together, they will be found to be identical; so that the blackened thermometer at $100^{\circ}$ will, in the first case, cool much more rapidly than the silvered one when transferred to the chamber at $0^{\circ}$, on account of its superior radiation, and will also, in the second case, starting from $0^{\circ}$, become heated much more rapidly than the silvered one when transferred to a chamber of $100^{\circ}$. In fine, good radiators are also good absorbents, bad radiators bad absorbents. It is worthy of remark, before proceeding further, that surfaces behave very differently in their absorbing power for different rays. White paper and glass, as we have seen, are both very strong absorbents of low temperature heat, while both of them are manifestly non-absorbents of luminous rays.

Extending now these considerations to visible rays proceeding from bodies of high temperature, they furnish us with some very interesting and instructive experiments, which will now be described.

Experiment 1.-Take a porcelain plate of black and white pattern (the black of the pattern will of course be a strong absorbent of luminous rays, while the white will be a less powerful absorbent). Heat it to a good red or white heat in the fire, and when so heated take it out and rapidly carry it to a dark place; the black will appear much more brilliant than the white, presenting a very curious reversal of the pattern.

Experiment 2.-Take a piece of polished platinum foil, and make an ink-mark upon it. Bring this foil to a red heat with the flame of a Bunsen's burner in a dark room, and the ink-mark will shine out much more brightly than the polished platinum.

Experiment 3.-Make a white mark on a black poker with a piece of chalk; when heated to a good red heat, examine it in the dark, and the chalk will shine out less brightly than the rest of the poker.

These experiments might be multiplied indefinitely, all tending to show that bodies which, when cold, are good absorbents, are, when hot, good radiators; and the observation may be extended to plates of various thicknesses as well as to mere surfaces. 
Thus a polished plate of rock-salt absorbs very little heat of low temperature (Art. 295), and when heated to $100^{\circ}$ Cent. it is found also to give out very little heat. In like manner a piece of transparent colourless glass which absorbs very little light will, when heated in the fire, and quickly examined in the dark, be found to give out very little light; while on the other hand, a piece of opaque glass treated in the same way will give out a great deal of light. In like manner a film or stratum of air is well known to absorb little light or heat of any kind, and so when heated it hardly gives out any.

We may now generalize our conclusions by the statement that opaque and non-reflecting solid or liquid particles are at once good radiators and good absorbents for most hinds of rays; while, on the other hand, polished metallic surfaces, and more especially films of gas, such as air, absorb and radiate very little either of light or heat.

It has been mentioned incidentally that surfaces or plates do not behave in the same manner in regard to different kinds of rays: let me now dwell at greater length on this point, for it is perhaps the most important of the whole subject. White paper, it was seen, was a strong absorbent for heat of low temperature, while it is evidently not so for luminous rays, for the very reason that it appears white. In like manner the uncovered glass of the bulb of a thermometer was found to be a strong absorbent for low temperature lieat, but it is evidently not so for luminous rays. To prove this, we have only to hold a thermometer in the sun, and we shall be dazzled with the light reflected from its bright mercurial surface, which only reaches the eye after it has twice passed through the glass.

Even within the limits of the visible spectrum we have, as a common occurrence, substances which absorb certain rays and allow others to pass. For what is it that makes the leaves of plants appear green? Is it not that they absorb all the various constituents of sunlight except the green which they allow to be reflected? In fine, all coloured substances are substances which behave in a partial manner with respect to the visible 
rays, and if we had no such partial absorption, we should be deprived of one great source of beauty in nature.

Coloured glasses afford a very familiar illustration of this selective or partial absorption. A green glass absorbs all, or nearly all, the red rays which fall upon it, allowing the green to pass; on the other hand, a red glass absorbs nearly all the green, and allows the red to pass.

Thus we see that surfaces or plates which behave in one way with respect to dark rays, may behave differently with regard to luminous ones; nay further, a substance that behaves in one way with regard to a luminuus ray of one colour, may behave in a different way with regard to a luminous ray of another colour. We have stated generally, that good radiators are good absorbents; but in view of the fact that bodies select or choose the rays which they absorb, this statement must be extended, and we now assert that bocties when eold absorb the same kind of rays that they give out when heated.

It will be desirable to give, in the first place, some experimental proofs of this before attempting to explain the principles upon which the statement rests.

Experiment 1.-Rock salt when heated to $100^{\circ}$ gives out that peculiar kind of heat which is greedily absorbed by a cold plate of rock sali. To prove this, heat a thin plate of rock salt to $100^{\circ}$, and allow the heat from it to fall upon an appropriate instrument for measuring such heat, but only after it has passed through a cold plate of the same material: now this cold plate will be found to have stopped at least three quarters of the heat which falls upon it, while it will only stop a very small percentage of any other kind of heat.

Experiment 2.-Red glass stops the green rays. Now heat a piece of ruby-coloured glass to a white heat in the fire; if examined in the dark it will be found to give out a greenish liglit, being the same sort of light that it absorbs. Next, heat a piece of green or blue glass, which absorbs red rays, and its light, when viewed in the dark, will be found to be particularly red, being, as before, the kind of light which it absorbs when cold. 
Experiment 3.- Make a spectrum of the electric light after the method already described, and hold burning sodium between the electric lamp and the slit; it will be found to produce a comparatively dark band in the spectrum. Next, stop the electric discharge while the sodium is left still burning; the same band will now appear luminous, that is to say, the sodium, which being comparatively cold when compared to the temperature of the electric light stops one of its rays, gives out, when heated, this very ray on its own account.

All these experiments tend to show, as a matter of fact, that bodies, when cold, or comparatively so, absorb the same rays which they give out when heated.

Our readers must now permit us to transport them in imagination to a white-hot chamber, kept uniformly at this temperature: such, for instance, as one of those chambers in whicl glass vessels are annealed. We will suppose it to be shut in closely with walls on all sides, with the exception of a small opening through which we can either introduce anything into the chamber, or, if we choose, see what is going on inside.

Let us introduce polished platinum marked with ink, or coal, or black and white porcelain, or red glass, or green glass, or transparent glass, or black glass. When left sufficiently long, until they have acquired the temperature of the walls of the chamber, if we look in through the small hole, we shall see no apparent difference between the light coming from these various substances and that from the walls of the chamber, in fact, everything will appear to be of the same uniform white heat. If, however, we hastily withdraw these various substances, and, without allowing them time to cool, examine them in the dark, we shall find, as already mentioned, a great variety in the appearances which they present; the colourless glass and the polished platinum will give out very little light, the coal and the black of the porcelain a great deal.

These two facts may be reconciled with one another in the following manner. Let us take the transparent glass; this gives out very little light on its own account, but, on the other hand, it stops very little of that which reaches the eye from the white- 
hot wall behind it, being eminently transparent for such light. If we suppose that the rays from the wall are as much recruited by the light given out by the glass on its own account as they are absorbed by its substance, then we shall have an explanation of the fact that the combined radiation of the glass and the wall is no greater than the wall itself, had there been no glass there. The polished platinum, in like manner, gives out little light on its own account, but when in the white-hot chamber it reflects copiously the light which reaches it from the wall, so that, to an observer viewing it through the small opening, it will have so completely supplemented its deficient radiation by its great reflection, that altogether it will appear equally bright with the wall itself.

Applying this explanation to the various substances which we have introduced into the white-hot chamber, we see at once why they cause no change in the intensity of the light that reaches the eye placed at the opening. For although, no doubt, it is only in the case of the black substance, such as coal or black porcelain, that all the light comes from the substance itself, yet, in the other case, what the substance wants in radiating power it makes up by allowing to pass either through its substance, as in the case of transparent glass, or from its surface, as in the case of polished platinum and white porcelain, what is deficient in its own radiation.

But let us now further consider for a moment the red and green glass which we have introduced into this chamber. As we view them from the opening, we are at a loss to distinguish which is the red and which is the green,--they have so absolutely and entirely lost their colour. Nor have we far to seek for an explanation of this. The red glass absorbs the whitish or greenish rays from the heated chamber behind it, but, in return, it gives out on its own account an equal amount of rays, and these of precisely the same kind as it has absorbed, so that the light from the wall behind, in passing through the glass, is just as much recruited as it is absorbed, and this equality holds for every individual kind of ray which goes to compose this light, and thus it happens that the combined radiation of the wall 
and the red glass is precisely the same both in quantity and in quality as if there were no glass.

The same principles apply to the green glass. It absorbs the reddish rays from the wall, but it gives out an equivalent both in quality and quantity for the rays which it absorbs, so that the absorption is virtually cancelled, and the combined result of wall and green glass is, as before, the same as if there were no glass. We thus see that all substances of all kinds, when placed in a room of uniform temperature, and allowed to remain until they have attained the temperature of the enclosure, will absorb just as much as they give out, and that this equality between absorption and radiation will hold good for every individual ray of which the heterogeneous radiation of the heated wall is composed. (By individual rays, we mean the various rays into wlich the whole radiation may be split up by means of the spectroscope.) All that we have now said has been built upon the hypothesis that the substances are in an enclosure, let us say a white-hot one, of the same temperature as themselves, and if we cannot easily command such a field of white-heat, yet the centre of a good fire is a very near approximation ; and if we introduce into such a fire a number of pieces of variously coloured glass, and exclude from the room all sunlight or gaslight, we shall find their colour vanish when once they have reached the temperature of the fire.

\section{APPENDIX B.}

\section{ON MAPS OF THE SOLAR SPECTRUM.}

We owe the first detailed map of the solar spectrum to Professor Kirchlıoff; but this map, though admirable as far as it goes, was unfortunately drawn to an arbitrary scale; and can, therefore only be used in conjunction with other maps which give the wave-lengths of the principal lines. Such a map was supplied by Angström, who carefully measured the wave-lengths of the principal Fraunhufer lines and published an Atlas of the solar spectrum, which still forms the standard for all our wavelength determinations. 
The improvement in spectroscopic instruments was, however, so rapid that a more detailed map even than Angström's and Kirchhoff's became necessary. H. C. Vogel ${ }^{1}$ published a large map drawn by him in conjunction with Dr. Müller representing the spectrum between wave-lengths 4,800 and 5,400. A few years afterwards Fievez, using a combination of prisms and a grating to separate the lines, seems to have obtained a very perfect spectrum and published a map, reaching from 6,600 to $4,500 .^{2}$ This was subsequently extended to wave-length $7,700{ }^{3}$ Professor Piazzi Smyth, ${ }^{4}$ who has given much attention to the study of the solar spectrum, has published measurements representing the state of the solar spectrum as seen by him in 1877 and 1878. His account has no regular map attached to it, but the measurements of each line are given together with a diagramatic sketch of its appearance. The measurements have been reduced to inverse wave-lengths in British inches, which renders their comparison with those published by other observers rather tedious. A number of such careful sets of measurements is especially useful, as the question of the absolute permanency of the solar spectrum is not as yet decided.

By means of photography we are able to study those portions of the solar spectrum which lie outside the range of visibility. Mascart and Cornu ${ }^{5}$ have given us valuable maps of the ultraviolet portions, and we owe to Captain Abney photographs of the dark lines in the infra-red. ${ }^{6}$ Professor Langley has also studied the infra-red region by means of his bolometer.

Even in the visible parts of the solar spectrum photography affords a ready means of accurately fixing the position of the dark lines. The photographs of Mr. Rutherfurd of the regions between $\mathrm{F}$ and $\mathrm{H}$ are excellent but not reduced to a wave-length scale. Much is expected of the promised but not yet published photographs of Rowland, extending over the greater part of the visible spectrum.

I Publicationen des Astrophysikalisehen Observatoriums zu Potsdam, vol. i.,

1879. 2 Annales de l'Ubservatoire Royal de Bruxelles, vol. iv., 1882.

3 Ibidem, vol. v., 1883.

4 Transaction Roy. Soc. Ed., xxix. p. 285, 1880.

5 Comptes Rendus, lxxxvi. 101, 983 .

6 Phil. Trans. 1880, p. 653. 
We possess also some very valuable detailed measurements of certain specially interesting parts of the solar spectrum. Thus Thollon ' and Young (see Fig. 95) and Piazzi Smyth ${ }^{2}$ have carefully drawn the dark lines in the neighbourhood of the $(b)$ group. Piazzi Smyth's paper on the subject contains a most interesting comparison of the lines as seen by different observers and with different instruments. An inspection of the plate given by him affords an excellent opportunity of noticing the gradual improvement which has taken place in our optical instruments, since the solar spectrum was first studied. Fig. 94 represents Professor Young's drawing of the region of the solar spectrum surrounding the principal line of the solar corona.

The region of the two yellow sodium lines has also been carefully studied by different observers, but the drawings are not so easily compared, as the majority of the lines in this part are due to our atmosphere; their intensity and width depends, therefore, on atmospheric conditions.

The admirable photographs by Mr. Lockyer ${ }^{3}$ of the interesting portion of the solar spectrum lying between the solar lines $\mathrm{H}$ and $\mathrm{K}$ also deserve special mention.

\section{APPENDIX C.}

\section{ON LOYKYER AND JANSSEN'S DISCOVERY.}

The discovery of the possibility of observing daily the prominences on the solar limb, is of such importance that the following short historical account may still be of interest. $\mathrm{Mr}$. Lockyer ${ }^{4}$ so long ago as the year 1866 suggested that it might be possible by the use of the spectroscope to obtain evidence of the presence of these prominences, though they had escaped all other means of observation except on the rare occasion of total solar eclipses. After many fruitless attempts to realise his

1 C. R., Ixxxviii. p. 80 (1879).

3 Phil. Trans. 1881, p. 561.
2 Tr. Roy. Soc. Ed., xxxii. (1883.)

4 Proc. Roy. Soc., October 11, 1866. 
hopes, Mr. Lockyer at last succeeded, on October 20, 1868, in obtaining the spectrum of a solar prominence; and he thus announces his important observation to the Royal Society, through Dr. Sharpey:-

"Sir,-I beg to anticipate a more detailed communication by informing you that, after a number of failures, which made the attempt seem hopeless, I have this morning perfectly succeeded in obtaining and observing part of the spectrum of a solar prominence.

"As a result I have established the existence of three bright lines in the following positions:-

I. Absolutely coincident with $\mathrm{C}$.

II. Nearly coincident with $F$.

III. Near D.

"The third line (the one near $D$ ) is more refrangible than the two darkest lines by eight or nine degrees of Kirchhoff's scale. I cannot speak with exactness, as this part of the spectrum requires remapping.

"I have evidence that the prominence was a very fine one.

"The instrument employed is the solar spectroscope, the funds for the construction of which were supplied by the Government Grant Committee. It is to be regretted that its construction has been so long delayed.

$$
\text { "I have, \&c., }
$$

“J. NORMAN LOCKYER.

"The Secretary of the Royal Society."

M. Janssen was sent by the French Government to observe the total eclipse at Guntoor in India, and on August 18, when examining the bright lines exhibited by the spectra of the prominences visible during the totality, the thought struck him that it might be possible to see these lines when the sun was unobscured, and on trying the experiment on the next day he succeeded in his endeavour, "so that," he writes, "for the last seventeen days I have been working as in a perpetual eclipse." 
The results of his observations were communicated (Oct. 26, 1868) to the French Academy in the following words:-

"La station de Guntoor a été sans doute la plus favorisée : le ciel a ćté beau, surtout pendant la totalité, et mes puissantes luncttes de près de trois mètres de foyer m'ont permis de suivre l'étude analytique de tous les phénomènes de l'ćclipse.

"Immédiatement après la totalité, deux magnifiques protubérances ont apparu: l'une d'elles, de plus de trois minutes de hauteur, brillait d'une splendeur qu'il est difficile d'imaginer. L'analyse de sa lumière m'a immédiatement montré qu'elle était formée par une immense colonne gazeuse incandescente, principalement composée de gaz hydrogène.

"L'analyse des régions circumsolaires, où M. Kirchhoff place l'atmosphère solaire, n'a pas donné des résultats conformes à la théorie formulce par ce physicien illustre; ces résultats me paraissent devoir conduire à la connaissance de la véritable constitution du spectre solaire.

"Mais le résultat le plus important de ces observations est la découvert d'une méthode, dont le principe fut conçu pendant l'ćclipse même, et qui permet l'étude des protubérances et des régions circumsolaires en tout temps, sans qu’il soit nécessaire de recourir à l'interposition d'un corps opaque devant le disque du soleil. Cette méthode est fondée sur les propriétés spectrales de la lumière dess protubérances, lumière qui se résout en un petit nombre de faisceaux très-lumineux, correspondant à des raies obscures du spectre solaire.

"Dès le lendemain de l'éclipse la méthode fut appliquée avec succès, et j’ai pu assister aux phénomènes préséntes par une nouvelle éclipse qui a durée toute la journée. Les protubérances de la veille étaient profondément modifiées. Il restait à peine quelques traces de la grande protubérance et la distribution de la matière gazeuse était tout autre.

"Depuis ce jour jusqu'au 4 septembre, j’ai constamment étudié le soleil à ce point de vu. J’ai dressé des cartes des protubérances, qui montrent avec quelle rapidité (sonvent.cn quelques minutes) ces immenses masses gazeuses se déforment 
et se déplacent. Enfin, pendant cette période, qui a été comme une éclipse de dix-sept jours, j'ai recueilli un grand nombre de faits, qui s'offraient comme d'eux-mêmes, sur la constitution physique du soleil.

"Je suis heureux d'offrir ces résultats à l'Académie et au Bureau des Longitudes, pour répondre à la confiance qui m'a été témoignée et à l'honneur qu'on m'a fait en me confiant cette importante mission."

Mr. Lockyer soon afterwards extended his researches by showing the existence of a continuous layer round the sun, which exhibits bright lines. The following is the account of the discovery as described by himself $:^{1}$

"The author, after referring to his ineffectual attempts since 1866 to observe the spectrum of the prominences with an instrument of small dispersive power, gave an account of the delays which had impeded the construction of a larger one (the funds for which were supplied by the Government Grant Committee early in 1867), in order that the coincidence in time between his results and those obtained by the Indian observers might not be misinterpreted.

"Details are given of the observations made by the new instrument, which was received incomplete on the 16th of October. These observations include the discovery, and exact determination of the lines, of the prominence spectrum on the 20th October, and of the fact that the prominences are merely local aggregations of a gaseous medium which entirely envelopes the sun. The term chromosphere is suggested for this envelope, in order to distinguish it from the cool-absorbing atmosphere on the one hand, and from the white light-giving photosphere on the other. The possibility of variations in the thickness of this envelope is suggested, and the phenomena presented by the star in corona are referred to.

"It is stated that, under proper instrumental and atmospheric conditions, the spectrum of the chromosphere is always visible

1 Proc. Roy. Soc., xvii. p. 131. 
in every part of the sun's periphery: its height, and the dimensions and shapes of several prominences, observed at different times, are given in the paper. One prominence, three minutes high, was observed on the 20 th October.

"Two of the lines correspond with Fraunhofer's C and F; another lies $8^{\circ}$ or $9^{\circ}$ (of Kirchhoff's scale) from D towards $\mathrm{E}$. There is another bright line, which occasionally makes its appearance near c, but slightly less refrangible than that line. It is remarked that the line near $\mathrm{D}$ has no corresponding line ordinarily visible in the solar spectrum. The author has been led by his observations to ascribe great variation of brilliancy to the lines. On the 5 th of November a prominence was observed in which the action was evidently very intense; and on this occasion the light and colour of the line at $\mathrm{F}$ were most vivid. This was not observed all along the line visible in the field of view of the instrument, but only at certain parts of the line, which appeared to widen out.

"The author points out that the line $\mathrm{F}$ invariably expands (that the band of light gets wider and wider) as the sun is approached, and that the $\mathrm{C}$ line and the $\mathrm{D}$ line do not; and he enlarges upon the importance of this fact, taken in connection with the researches of Plücker, Hittorf, and Frankland on the spectrum of hydrogen-stating at the same time that he is engaged in researches on gaseous spectra which, it is possible, will enable us to determine the temperature and pressure at the surfaces of the chromosphere, and to give a full explanation of the various colours of the prominences which have been observed at different times.

"The paper" also refers to certain bright regions in the solar spectrum itself.

"Evidence is adduced to show that possibly a chromosphere is, under certain conditions, a regular part of star economy; and the outburst of the star in corona is especially dwelt upon."

Another important step consisted in the invention of a method enabling us to see not only the bright lines of the prominences, but the actual form of the prominence. A large share of this 
discovery is due to Dr. Huggins, ${ }^{1}$ as shown by the following abstracts from his papers :-

"In the report of my Observatory at the last anniversary (p. 88 of the last volume), it is stated that 'during the last two years numerous observations have been made for the purpose of obtaining a view of the red prominences seen during a solar eclipse. If these bodies are gaseous, their spectra would consist of bright lines. With a powerful spectroscope, the light reflected from our atmosphere near the sun's edge would be greatly reduced in intensity by the dispersion of the prisms, while the bright lines of the prominences, if such be present, would remain but little diminished in brilliancy. This principle has been carried out by various forms of prismatic apparatus, and also by other contrivances, but hitherto without success.' The observations of the eclipse of August last having shown the position in the spectrum of the bright lines of the red flames, Mr. Lockyer and M. Janssen succeeded independently, by a similar method, in viewing the spectra of these objects.

"My object in this note is to describe one of the "other contrivances' mentioned in the report.

"The apparatus consisted of screens of coloured glasses and other absorptive media, by which I was able to isolate portions of the spectrum. It appeared highly probable, that if the parts of the spectrum which then alone remained were identical with those in which the bright lines of the flames occur these objects would become visible.

"For this inquiry I obtained a great variety of coloured glasses and other absorptive media. I first examined them with a prism to learn the absorptive power which they exercised on different parts of the spectrum. I then combined them in various ways. These glasses were sometimes employed before the eye, but more frequently by projecting the image of the sun's edge upon a screen, after the light had been sifted by the coloured media. In making these experiments, means were taken that the whole of the sun's image should be got rid of, in order that the eye, kept

1 Monthly Notices of the Roy. Art. Soc., Nov. 13, 1868. 
in comparative darkness, might be more sensitive to the greatly feebler illumination of the objects sought for. As I had no knowledge of the position in the spectrum of the bright lines, it would have been by accident only if I had succeeded in obtaining a view of the flames.

"Now that the positions of these lines are known, this method appears to be very promising. Perhaps the light about the red line at $\mathrm{C}$ will be most easily isolated. I have a deep ruby glass which cuts off all the spectrum except the extreme red. I have since the observations only been able to make one attempt, when the state of the atmosphere was unfarourable.

"It is obvious that by this method tlie form and appearance of these flames could be observed, and the objects measured with accuracy."

A few months afterwards Dr. Huggins succeeded in observing the form of a solar prominence. He describes his method in the following words: ${ }^{1}$ -

"Last Saturday, February 13, 1869, I succeeded in seeing a solar prominence so as to distinguish its form. A spectroscope was used; a narrow slit was inserted after the train of prisms before the object-glass of the little telescope. This slit limited the light entering the telescope to that of the refrangibility of the part of the spectrum immediately about the bright line coincident with c. The slit of the spectroscope was then widened sufficiently to admit the form of the prominence to be seen. The spectrum then became so impure that the prominence could not be distinguished. A great part of the light of the refrangibilities removed far from that of $\mathrm{C}$ was then absorbed by a piece of deep ruby glass. The prominence was then distinctly seen."

Finally Lockyer showed how to dispense with the red glass ${ }^{2}$ as follows :-

"For some time past I have been engaged in endeavouring to obtain a sight of the prominences, by using a very rapidly uscillating slit; but although I believe this method will eventually

I Iroc. Roy. Soc, xvii. p. 302.

2 Ibidem, p. 350. 
succeed, the spectroscope I employ does not allow me to apply it under sufficiently good conditions, and I am not at present satisfied with the results I have obtained.

"Hearing, however, from Mr. De la Rue, on February 27th, that Mr. Huggins had succeeded in anticipating me by using absorbing media and a wide slit (the description forwarded to me is short and vague), it immediately struck me, as possibly it had struck Mr. Huggins, that the wide slit is quite sufficient without any absorptive media; and during the last few days I have been perfectly enchanted with the sight which my spectroscope has revealed to me. The solar and atmospheric spectra being hidden, and the image of the wide slit alone visible, the telescope or slit is moved slowly, and the strange shadow-forms flit past. Here one is reminded, by the fleecy, infinitely delicate cloud films, of an English hedgerow with luxuriant elms; here, of a densely intertwined tropical forest, the intimately interwoven branches threading in all directions, the prominences generally expanding as they mount upwards, and changing slowly, indeed almost imperceptibly. By this method, the smallest details of the prominences and of the chromosphere itself are rendered perfectly visible and easy of observation."

\section{APPENDIX D.}

RESULTS OF RECENT SOLAR ECLIPSE OBSERVATIONS.

The following is a short account of the principal results obtaincd during the different total solar eclipses since the year 1860 :-

18th July, 1860.-The prominences were for the first time photographed by Mr. De la Rue at Rivabellosa in Spain.

18th Angust, 1868.-During the eclipse, the spectroscope was employed for the first time and the spectrum of the prominences was found to consist of bright lines by various observers (Janssen, Rayet, Tennant, Pogson).

7th August, 1869.-Pickering and Eastman saw the continuous spcctrum of the corona; Eastman and Young independently 
discovered the green line of the corona, and Young determined its position.

22nd December, 1870.-Young noticed the momentary appearance, at the beginning of totality, of a large number of bright lines at the sun's limb; these lines corresponding in all probability to the dark Fraunhofer lines seen under ordinary circumstances. Brothers succeeded for the first time in obtaining a good photograph of the solar corona.

12th December, 1871.-Janssen discovered the dark Fraunhofer lines in the spectrum of the solar corona. Lockyer saw the hydrogen lines in the lower parts of the corona.

Excellent photographs were obtained by Messrs. Waterhouse and Hennessey at Dodabetta, and by Mr H. Davis in Lockyer's party at Baikul.

Lockyer and Resphighi observed the solar corona through a prism without lenses, and saw the image of the moon surroundea by bright rings corresponding to the hydrogen lines $\mathrm{C}$ and $\mathrm{F}$, and to the green line of the corona.

16th April, 1874.-The numerous bright lines observed by Young at the beginning of totality in 1870 were seen again by Stone. The hydrogen lines were seen in the inner parts of the corona. The green line and Fraunhofer's dark lines being visible in the outer parts only.

6th April, 1875.-The corona was photographed in Siam by Schuster with Lockyer's arrangement through a prism. The photographs confirmed some of the previously discovered facts, and, in addition, showed for the first time that the most intense radiation of the prominences lies in the extreme violet.

28th July, 1878.-Certain streamers in the corona were traced to very great distances, as seen from Pike's Peak-no less than ten diameters, from the sun, and the continuity between the outer parts of the corona and the zodiacal light was for the first time suggested.

18th May, 1882.-The first photograph of the spectrum of the corona was taken in Egypt by Schuster on Abney's plates. The violet radiation of the prominences was proved to be due to the presence of calcium. A large number of new lines in the spectrum of the corona and of the prominences were discovered. 


\section{APPENDIX E.}

PROFESSOR C. A. YOUNG'S INVESTIGATIONS ON CHROMOSPHERIC LINES.

The method by means of which Lockyer observed the spectrum of the protuberances in daylight showed him also that bright lines can nearly always be seen round the entire limb of the sun. Tacchini has for many years carefully mapped the lines which appear in the upper regions of the chromosphere, but it is to Professor Young that we are indebted for a knowledge of the great number of lines visible in the lower regions, that is to say, close to the solar surface. To observe these lines, very perfect instrumental appliances are necessary, but even these are of no avail except under very favourable atmospheric conditions. During the summer, 1871, Professor Young observed 103 chromospheric lines, and this result seemed so promising that he took his telescopes during the following summer to Sherman, which is situated at an altitude of 8,300 feet. The clearer air at this station enabled him to add a considerable number of chromospheric lines and the following extract from his paper (American Journal of Science, vol. iii. 1872) contains the catalogue of lines thus observed:-

Catalogue of Bright Lines ix the Spectrum of the Chromosphere. 1872.

\begin{tabular}{|c|c|c|c|c|c|c|}
\hline No. & P.C. & K. & A. & F. & B. & $\mathrm{E}$. \\
\hline $1+$ & $1+$ & $5340^{*}$ & $7055 \cdot ?$ & 100 & 12 & \\
\hline $2^{+}$ & $2 \uparrow$ & $654: 3$ & 6676.9 & 25 & 50 & Fe. $\mathrm{Ba}\left({ }_{\mathrm{w}}\right)$ \\
\hline$\overline{3}$ & $3 \uparrow$ & C. $694 \cdot 1$ & $6561 \cdot 8$ & 100 & 100 & $\mathrm{H}$. \\
\hline 4 & & $711 \cdot 4$ & 6515.5 & 15 & 4 & \\
\hline 5 & 4 & $718 \cdot 7$ & $6496^{\circ} 0$ & 18 & 5 & $\mathrm{Ba}$ \\
\hline 6 & & $731 \cdot 7$ & $6461 \cdot 7$ & 5 & 2 & $\mathrm{Ca}$. \\
\hline 7 & 5 & $734 \cdot 0$ & $6453 \cdot 8$ & 10 & 6 & \\
\hline 8 & & $740 \cdot 9$ & $6438 \cdot 1$ & 5 & 2 & $\mathrm{Ca}, \mathrm{Cd}$ \\
\hline $9 \ddagger$ & 6 & $744: 3$ & $6429 \cdot 9$ & 20 & 4 & \\
\hline $10^{\top}$ & & $750 \cdot 1$ & $6415 \cdot 6$ & 5 & 2 & \\
\hline 11 & & 7569 & $6399 \cdot 0$ & 5 & 2 & Fe. \\
\hline
\end{tabular}




\begin{tabular}{|c|c|c|c|c|c|c|}
\hline No. & P.C. & K. & A. & $\mathrm{F}$. & B. & E. \\
\hline 12 & & $759 \cdot 3$ & $6392 \cdot 6$ & 5 & 1 & Fe. \\
\hline 13 & & $767 \cdot ? *$ & $6373 \cdot ?$ & 5 & 2 & \\
\hline 14 & 7 & $768 \cdot ? *$ & $6371 \cdot ?$ & 5 & $\overline{3}$ & \\
\hline 15 & & $778 \cdot 3$ & $6346^{\circ} 1$ & 10 & 4 & Ruth. Tr. \\
\hline $16+$ & 8 & $823 \cdot 5$ & $6245 \cdot 4$ & 8 & 5 & Fe. \\
\hline $17 \div$ & 9 & $827 \cdot 6$ & $6237 \cdot 3$ & 8 & 2 & \\
\hline 18 & & $830 \cdot 2$ & $6231 \cdot 5$ & 5 & 1 & Fe. \\
\hline 19 & & $836 \cdot 5$ & $6218 \cdot 3$ & 3 & 1 & Ti. \\
\hline 20 & & $839 \cdot 2$ & $6214 \cdot 1$ & 3 & 1 & Ti. \\
\hline 21 & & $845 \cdot 7$ & $6199 \cdot 6$ & 2 & 2 & Fe. \\
\hline 22 & & $849 \cdot 7$ & 6190.5 & 10 & 2 & $\begin{array}{l}\mathrm{Fe} . \\
\mathrm{Ca}\end{array}$ \\
\hline $\begin{array}{l}23 \\
24\end{array}$ & & $\begin{array}{l}859 \cdot 7 \\
863 \cdot 9\end{array}$ & $\begin{array}{l}6168 \cdot 3 \\
6161 \cdot 2\end{array}$ & $\begin{array}{l}3 \\
8\end{array}$ & $\begin{array}{l}1 \\
3\end{array}$ & $\begin{array}{l}\mathrm{Ca} . \\
\mathrm{Ca} .\end{array}$ \\
\hline 25 & & $\{870 \cdot 9$ & $6148^{\circ} 1$ & 3 & 2 & Fe. E( $(w)$ \\
\hline 26 & & $\{871 \cdot 4$ & $6146 \cdot 8$ & 3 & 2 & \\
\hline 27 & 10 & $874: 3$ & $6140 \cdot 6$ & 25 & 10 & Ba. \\
\hline 28 & & 876.5 & $6136 \cdot 1$ & 2 & 1 & \\
\hline 29 & & $877 \cdot 0$ & $6135 \cdot 6$ & 2 & 1 & Fe. \\
\hline 30 & & $884 \cdot 9$ & $6121 \cdot 2$ & 5 & 3 & Ca. Co. \\
\hline 31 & & $890 \cdot 2$ & 61099 & 2 & 1. & \\
\hline 32 & & $894 \cdot 9$ & $6101 \cdot 7$ & 3 & 2 & Ca. $\operatorname{Li.}_{T i} \operatorname{Zn}\left({ }_{w}\right)$ \\
\hline 33 & & $903 \cdot 1$ & $6083 \cdot 1$ & 3 & 2 & Fe Ti. \\
\hline $\begin{array}{l}34 \\
35\end{array}$ & & $\begin{array}{l}912 \cdot 1 \\
933 \cdot 8\end{array}$ & $6064 \cdot 5$ & $\begin{array}{l}5 \\
2\end{array}$ & $\begin{array}{l}2 \\
1\end{array}$ & $\begin{array}{l}\text { Ba. } \\
\text { Ba. }\end{array}$ \\
\hline 36 & & $949 \cdot 4$ & $\begin{array}{l}6018^{\circ} \\
5990 \cdot 0\end{array}$ & 10 & 4 & \\
\hline 37 & & $9920^{\circ}$ & $5913 \cdot 2$ & 2 & 1 & Fe. \\
\hline 38 & $11 \dagger$ & $D_{1} 1002 \cdot 8$ & $5895 \cdot 0$ & 50 & 30 & Na. \\
\hline 39 & $12 \dagger$ & $D_{2} 1006 \cdot 8$ & $5889 \cdot 0$ & 50 & 30 & $\mathrm{Na}$ \\
\hline $40 t$ & & $1011 \cdot 2$ & 58830 & 2 & 1 & Fe. \\
\hline $41+$ & $13 \dagger$ & $\mathrm{D}_{3} 1016.5 *$ & $5874 \cdot 9$ & 100 & 90 & \\
\hline 42 & & $1031 \cdot 8$ & $5852 \cdot 7$ & 8 & 2 & $\mathrm{Ba}$. \\
\hline 43 & & $1135^{\circ} 1$ & $\begin{array}{l}5708 \cdot 3 \\
5687.2\end{array}$ & $\begin{array}{l}1 \\
2\end{array}$ & $\begin{array}{l}1 \\
1\end{array}$ & $\begin{array}{l}\text { Fe. } \\
\text { Na. }\end{array}$ \\
\hline $\begin{array}{l}44 \\
45\end{array}$ & & $\begin{array}{l}1151^{\circ} 1 \\
1154^{\circ}\end{array}$ & $\begin{array}{l}5687 \cdot 2 \\
5683 \cdot 5\end{array}$ & $\begin{array}{l}2 \\
5\end{array}$ & 3 & \\
\hline 46 & & $1155 \cdot 8$ & $5681 \cdot 5$ & 2 & 1 & Na. Fe. $\left.N_{(w}\right)$ \\
\hline 47 & & $1165 \cdot 7$ & $5667 \cdot 8$ & 2 & 2 & $S\left({ }_{w}\right)$ \\
\hline 48 & & $1167^{\circ} 0$ & $5666^{\circ} 0$ & 1 & 1 & \\
\hline 49 & & $1170 \cdot 6$ & $5661 \cdot 5$ & 15 & 2 & Fe. Ti. $\left.\mathbf{E}_{(\mathrm{w}}\right)$ \\
\hline 50 & & $1175^{\circ} 0$ & $5656 \cdot 7$ & 8 & 3 & $S\left({ }_{w}\right) N\left({ }_{w}\right)$ \\
\hline 51 & & $1176 \cdot 6$ & $5654 \cdot 4$ & 2 & 1 & Fe. \\
\hline 52 & & $1187 \cdot 1$ & $5640 \cdot 2$ & 1 & 1 & $S(w)$ \\
\hline $\begin{array}{l}53 \\
54\end{array}$ & & $\begin{array}{l}1189 \cdot 3 \\
12006\end{array}$ & $\begin{array}{l}5637 \cdot 3 \\
5623 \cdot 2\end{array}$ & $\begin{array}{l}1 \\
2\end{array}$ & 1 & $\mathrm{Fe}$ \\
\hline $\begin{array}{l}54 \\
55\end{array}$ & & $\begin{array}{l}12006 \\
1207 \cdot 3\end{array}$ & $\begin{array}{l}5623 \cdot 2 \\
5614 \cdot 5\end{array}$ & 2 & 1 & Fe. \\
\hline 56 & & $1229 \cdot 6$ & $5587 \cdot 6$ & 2 & 2 & $\mathrm{Ca}$. \\
\hline 57 & & $1231 \cdot 3$ & $5585 \cdot 5$ & 2 & 1 & Fe. \\
\hline 58 & $14 \dagger$ & $1274 \cdot 2$ & $5534^{\circ} 1$ & 50 & 12 & Ba. Fe. Sr. \\
\hline 59 & 15 & $1281 \cdot 3$ & $5525 \cdot 9$ & 40 & 5 & Fe. \\
\hline 60 & & $1287 \cdot 5$ & $5518 \cdot 7$ & 15 & 2 & Bar \\
\hline
\end{tabular}




\begin{tabular}{|c|c|c|c|c|c|c|}
\hline No. & P.C. & $\mathrm{K}$. & A. & $\mathrm{F}$ & B. & E. \\
\hline 61 & & 12989 & $5505 \cdot 8$ & 2 & 1 & $\mathrm{Fe}$. \\
\hline 62 & & $1303 \cdot 5$ & $5500 \cdot 5$ & 2 & 1 & Fe. Ta. \\
\hline 63 & & $1306 \cdot 7$ & $5496 \cdot 6$ & 2 & 1 & Fe. $\mathrm{E}(\mathrm{w})$ \\
\hline 64 & & 13206 & $5480^{\circ} 2$ & 2 & 1 & Ti. Sr. \\
\hline 65 & & $1324 \cdot 8$ & $5475 \cdot 9$ & 1 & I & $\mathrm{Ni}$. \\
\hline 66 & & $1328 \cdot 7$ & $5472 \cdot 3$ & 3 & 1 & \\
\hline 67 & & $1337^{\circ} 0$ & $5462 \cdot 3$ & 1 & 1 & Fe. $N(w)$ \\
\hline 68 & 16 & $1343 \cdot 5$ & $5454 \cdot 7$ & 10 & 4 & Fe. \\
\hline 69 & 17 & $1351 \cdot 1$ & $5445 \cdot 9$ & 10 & 4 & Fe. Ti. $\operatorname{Br}\left({ }_{w}\right)$ \\
\hline 70 & & $1360 \cdot 9$ & $5435 \cdot 4$ & 5 & 2 & $\left.\mathrm{Zn} \cdot \operatorname{Br}_{(\mathrm{w}}\right)^{(\mathrm{w} /}$ \\
\hline 71 & & $1362 \cdot 9$ & $5433 \cdot 0$ & 2 & 2 & Fe. \\
\hline $72+$ & 18 & $136 \pm 3$ & $5431 \cdot 8$ & 8 & 5 & \\
\hline $73^{+}$ & 19 & $1367 \cdot 0$ & $5428 \cdot 8$ & 8 & 3 & Fe. Ti. \\
\hline 74 & 20 & $1372 \cdot 1$ & $5424 \cdot 5$ & 25 & 6 & Ba. Ti. $\left.S_{(w}\right)$ \\
\hline 75 & 21 & $1377 \cdot 4$ & $5417 \cdot 9$ & 5 & 2 & Ti. Mn. \\
\hline 76 & & $1380 \cdot 5$ & 5414.5 & 2 & 2 & Fe. \\
\hline 77 & 22 & $1382 \cdot 5$ & $5412 \cdot 4$ & 4 & 2 & $\operatorname{Mn}\left({ }_{w}\right)$ \\
\hline 78 & & $1384 \cdot 7$ & 54100 & 2 & 1 & Fe. Ni. \\
\hline 79 & & $1385 \cdot 7$ & $5409 \cdot 0$ & 2 & 2 & $\mathrm{Cr}$ \\
\hline 80 & & $1389 \cdot 4$ & $5404 \cdot 8$ & 2 & I & Fe. \\
\hline 81 & 23 & 13909 & $5403 \cdot 1$ & 5 & 3 & Fe. Ti. \\
\hline 82 & & $1394 \cdot 2$ & $5399 \cdot 6$ & 2 & 1 & $\mathrm{Mn}$ \\
\hline 83 & 24 & $1397 \cdot 5$ & $5396 \cdot 1$ & 4 & 2 & Fe. Ti. \\
\hline 84 & & $1401 \cdot 6$ & $5392 \cdot 2$ & 2 & I & Fe. Ce. \\
\hline 85 & & $1412 \cdot 5$ & $5380 \cdot 2$ & 3 & 2 & Ti. \\
\hline 86 & $25 \dagger$ & $1421 \cdot 5$ & $5370 \cdot 5$ & 10 & 3 & Fe. \\
\hline 87 & & $14230^{\circ}$ & $5369 \cdot 0$ & 1 & 1 & Fe. \\
\hline 88 & & $1425 \cdot 4$ & $5366 \cdot 5$ & 1 & 1 & Fe. \\
\hline 89 & & $1428^{\circ} 2$ & $5364 \cdot 0$ & 1 & 1 & Fe. \\
\hline 90 & $26 \dagger$ & $1430^{\circ} 1$ & $5361 \cdot 9$ & 20 & 10 & Fe. \\
\hline 91 & & $1438 \cdot 9$ & $5352 \cdot 4$ & 4 & 2 & Fe. Co.Ce. \\
\hline 92 & & $1446 \cdot 7$ & $5345 \cdot 0$ & $\mathrm{I}$ & 1 & \\
\hline 93 & & $1450 \cdot 8$ & $5340 \cdot 2$ & 1 & 2 & Fe. Mn. $O\left({ }_{w}\right)$ \\
\hline 94 & 27 & $1454 \cdot 7$ & $5335 \cdot 9$ & 5 & 2 & Ti. $\mathrm{Zn}\left({ }_{\mathrm{w}}\right)$ \\
\hline 95 & & $1461 \cdot 5$ & $5329 \cdot 1$ & 6 & 4 & \\
\hline 96 & 28 & $\int 1462 \cdot 8$ & $5327 \cdot 1$ & 5 & 2 & $\mathrm{Fe}$. \\
\hline 97 & 29 & $\{1463: 3$ & $5327 \cdot 6$ & 5 & 2 & Fe. \\
\hline 98 & & $1464 \cdot 8$ & $5325 \cdot 1$ & 6 & 2 & \\
\hline 99 & & $1471 \cdot 9^{*}$ & $5318 \cdot 0$ & I & 1 & \\
\hline $100+$ & $31 \dagger$ & 14739 & $5315 \cdot 9$ & 90 & 50 & Fe.? $\mathrm{O}\left({ }_{\mathrm{w}}\right)$ ? \\
\hline $101^{\top}$ & & $1476 \cdot 8$ & $5313 \cdot 1$ & 3 & 1 & \\
\hline 102 & & $1497 \cdot 3$ & 52920 & I & 1 & $\mathrm{Cu} . \mathrm{Br}\left({ }_{\mathrm{w}}\right)$ \\
\hline 103 & 32 & $1505 \cdot 3$ & $5283 \cdot 4$ & 20 & 10 & $\operatorname{Ti}\left({ }_{w}\right)^{(w /}$ \\
\hline 104 & $33 \uparrow$ & 1515.5 & $5275 \cdot 0$ & 30 & 15 & \\
\hline 105 & $34^{\prime}$ & $\mathrm{E}_{1} 1522 \cdot 7$ & $5269 \cdot 5$ & 15 & 4 & Fe. Ca. \\
\hline 106 & 35 & $\mathrm{E}_{2} 1523 \cdot 7$ & 5268.5 & 12 & 3 & Fe. \\
\hline 107 & $36 \dagger$ & $1527 \cdot 7$ & $5265 \cdot 8$ & 10 & 4 & Fe. Co. \\
\hline 108 & & $1530 \cdot 2$ & $5263 \cdot 3$ & 1 & $\mathrm{I}$ & Ca. $\operatorname{Br}\left({ }_{\mathrm{v}}\right)$ \\
\hline 109 & & $1538.5 *$ & $5256 \cdot 2$ & 2 & 1 & Sr. \\
\hline
\end{tabular}




\begin{tabular}{|c|c|c|c|c|c|c|}
\hline No. & P.C. & $\mathrm{K}$. & A. & F. & B. & E. \\
\hline 110 & & $1541 \cdot 9$ & $5254 \cdot 1$ & 1 & 2 & Fe. Mn. \\
\hline 111 & & $1547 \cdot 7$ & $5249 \cdot 7$ & 3 & 1 & Fe. $\mathrm{Z}\left({ }_{w}\right) \operatorname{Br}\left({ }_{w}\right)$ \\
\hline 112 & & $1551 \cdot 6$ & $5246 \cdot 3$ & 3 & 1 & Fe. \\
\hline 113 & 37 & $1561 \cdot 0$ & 52390 & 4 & 2 & Fe. \\
\hline 114 & 38 & $1564 \cdot 2$ & $5236 \cdot 3$ & 4 & 2 & \\
\hline 115 & $39 \dagger$ & $1567 \cdot 5$ & 52336 & 10 & 8 & $\operatorname{Mn} . \mathrm{Zn}\left({ }_{\mathrm{w}}\right)$ \\
\hline 116 & 40 & $1569 \cdot 6$ & $5232 \cdot 1$ & 1 & 3 & Fe. \\
\hline 117 & & $1575 \cdot 4$ & $5227 \cdot 5$ & 1 & 1 & Sr.? \\
\hline 118 & 41 & $\int 1577 \cdot 4$ & $5226 \cdot 2$ & 10 & 3 & Fe. \\
\hline 119 & & $1578 \cdot 1$ & $5225 \cdot 5$ & 2 & 3 & $\operatorname{Sr} \cdot \operatorname{Br}\left({ }_{w}\right)$ \\
\hline 120 & 42 & $1580^{\circ} 1$ & $5224: 3$ & 2 & 2 & $\mathrm{Ti}$. \\
\hline 121 & & $1589 \cdot 1$ & $5216 \cdot 5$ & 2 & 1 & $\mathrm{Fe}$. \\
\hline 122 & & $1590 \cdot 7$ & $5215 \cdot 5$ & 3 & 2 & Fe. \\
\hline 123 & & $1592 \cdot 3$ & $5214 \cdot 4$ & 2 & $\overline{1}$ & Fe. \\
\hline 124 & & $1597 \cdot 9 *$ & $5210 \cdot 5$ & 1 & 1 & \\
\hline 125 & & $1598 \cdot 9$ & $5209 \cdot 5$ & 1 & 2 & $\mathrm{Ti}$. \\
\hline 126 & 43 & $1601 \cdot 5$ & $5207 \cdot 6$ & 10 & 6 & Fe. Cr. \\
\hline 127 & 44 & $1604 \cdot 4$ & $5205 \cdot 2$ & 10 & 6 & $\operatorname{Cr} . \mathrm{E}\left({ }_{\mathrm{w}}\right)$ \\
\hline 128 & 45 & $1606 \cdot 4$ & $5203 \cdot 7$ & 10 & 6 & Cr. Fe. \\
\hline 129 & 46 & $1609 \cdot 2$ & $5201 \cdot 5$ & 5 & 3 & Fe. \\
\hline 130 & 47 & $1611 \cdot 3$ & $5199 \cdot 7$ & 4 & 2 & $\mathrm{~S}\left({ }_{\mathrm{w}}\right) \mathrm{E}\left({ }_{\mathrm{w}}\right)$ \\
\hline 131 & & $1613 \cdot 9$ & $5197 \cdot 9$ & 1 & 1 & Fe. \\
\hline 132 & $48 \dagger$ & 16156 & $5197 \cdot 0$ & 15 & 10 & \\
\hline 133 & & $1617 \cdot 4$ & 51950 & 1 & 1 & Mn. \\
\hline 1.34 & & $1618 \cdot 9$ & $5194 \cdot 1$ & 2 & 2 & Fe. \\
\hline 135 & & $1627 \cdot 2$ & $5188 \cdot 2$ & 10 & 5 & Fe. Ca. \\
\hline 136 & & $1628 \cdot 2$ & $5187 \cdot 3$ & 1 & 1 & 'Ti. \\
\hline 137 & & $1631 \cdot 5$ & $5185 \cdot 1$ & 5 & 2 & Fe. Ti. \\
\hline 138 & $49+$ & $b_{1} 1634 \cdot 1$ & 51830 & 50 & 30 & $\mathrm{Mg}$. \\
\hline 139 & $50 \dagger$ & $b_{2} 1648 \cdot 8$ & $5172 \cdot 0$ & 50 & 35 & $\mathrm{Mg}$. \\
\hline 140 & $51 \dagger$ & $\mathrm{b}_{3} 1653 \cdot 7$ & $5168 \cdot 3$ & 40 & 30 & Fe. Ni. $\operatorname{Br}\left({ }_{w}\right)$ \\
\hline 141 & $52 \dagger$ & $b_{4} 1655 \cdot 6$ & $5166^{\circ} \cdot 7$ & 30 & 20 & Fe. Mg. \\
\hline 142 & & $1666^{\circ} ?$ & $5160^{\circ} ?$ & 1 & 1 & \\
\hline 143 & & $1671 \cdot 5$ & $5154 \cdot 8$ & 3 & 1 & $\mathrm{Na}$. \\
\hline 144 & 53 & 16737 & 5152.5 & 3 & 1 & Na. Cu.? \\
\hline 145 & 54 & $1677 \cdot 9$ & $5150 \cdot 1$ & 2 & 2 & Fe. $\operatorname{Br}\left({ }_{w}\right)$ \\
\hline 146 & & $1689 \cdot 5$ & $5142 \cdot 2$ & 1 & 2 & $S\left({ }_{w}\right)$ \\
\hline 147 & & $1701 \cdot 8$ & $5133 \cdot 0$ & 1 & 1 & Fe. \\
\hline 148 & & $1704 \cdot 7$ & 51308 & 1 & 1 & Fe. \\
\hline 149 & & $1707 \cdot 9$ & $5128 \cdot 6$ & 1 & 1 & $\mathrm{Ti}$. \\
\hline 150 & & $1710 \cdot 7$ & $5126 \cdot 7$ & 1 & 1 & Fe. Ti. \\
\hline 151 & & $1712 \cdot 2$ & $5125 \cdot 5$ & 1 & 2 & \\
\hline $152+$ & & 17134 & $5124^{\circ} 4$ & 1 & 1 & Fe. \\
\hline 153 & & $1715^{\cdot 2}$ & $5123 \cdot 2$ & 1 & 1 & $\mathrm{Fe}$. \\
\hline 154 & & $1717 \cdot 9$ & $5121 \cdot 0$ & 1 & 1 & $\mathrm{Fe}$. \\
\hline 155 & & $1719 \cdot 4$ & $5119 \cdot 9$ & 1 & 1 & Ti. \\
\hline $156+$ & & $1727 \cdot 3$ & $5114 \cdot 9$ & 1 & 1 & $\mathrm{Ni}$. \\
\hline $157^{\circ}$ & & $1734^{\circ} 6$ & $5108 \cdot 8$ & 2 & 2 & $\operatorname{Ti}\left({ }_{w}\right)$ \\
\hline 158 & & $1737 \cdot 7$ & $5107 \cdot 0$ & 1 & 1 & Fe. \\
\hline
\end{tabular}




\begin{tabular}{|c|c|c|c|c|c|c|}
\hline No. & P.C. & $\mathrm{K}$. & A. & $\mathrm{F}$. & B. & E. \\
\hline 159 & & $1750 \cdot 4$ & $5098 \cdot 1$ & 1 & 1 & Fe. \\
\hline 160 & & $1752 \cdot 8$ & 5096.5 & 1 & 1 & Fe. $S\left({ }_{w}\right)$ \\
\hline 161 & & $1765^{\circ} 0^{*}$ & $5087 \cdot 0$ & 2 & 1 & $\mathrm{E}(\mathrm{w})^{(\mathrm{w})}$ \\
\hline 162 & & $1771 \cdot 5$ & 5083.5 & 1 & 1 & $\mathrm{Zn}(\mathrm{w})$ \\
\hline 163 & 55 & $1778 \cdot 5$ & $5077 \cdot 9$ & 1 & 2 & Fe. \\
\hline 164 & & $1823 \cdot 6$ & $5047 \cdot 8$ & 2 & 2 & $\mathrm{Fe}$ ? $\mathrm{Zn}\left({ }_{\mathrm{w}}\right)$ \\
\hline 165 & & $1833 \cdot 4$ & $5041 \cdot 2$ & 2 & 2 & $\mathrm{Fe}$ Ca. \\
\hline 166 & & $1834: 3$ & $5040 \cdot 1$ & $\overline{2}$ & 2 & Fe. \\
\hline 167 & & $1848 \cdot 9$ & $5030 \cdot 1$ & 4 & 3 & $S\left({ }_{w}\right)$ \\
\hline 168 & & $1856 \cdot 9$ & $5023 \cdot 5$ & 3 & 1 & $\mathrm{~S}\left(\begin{array}{l}\mathrm{w} / \mathrm{w} \\
\mathrm{w}\end{array}\right)$ \\
\hline 169 & $56 \dagger$ & $1867 \cdot 1$ & $5017 \cdot 6$ & 30 & 15 & Fe. Ni. \\
\hline 170 & $57 \dagger$ & 1870.6 & $5015 \cdot 0$ & 30 & 10 & $\operatorname{Ti}\left({ }_{\mathrm{w}}\right)$ \\
\hline 171 & & $1905 \cdot 1$ & $4993 \cdot 3$ & 2 & 1 & Fe. $N\left({ }_{w}\right)$ \\
\hline $172+$ & & c. $1961 \cdot 0$ & $4956 \cdot 7$ & 1 & 2 & Fe. \\
\hline $173^{\top}$ & $58 \dagger$ & 1989.5 & $4933 \cdot 4$ & 30 & 8 & Ba. \\
\hline 174 & $59 \dagger$ & $2001 \cdot 6$ & $4923 \cdot 1$ & 10 & 12 & $\mathrm{Fe} . \mathrm{S}\left({ }_{\mathrm{w}}\right) \mathrm{Zn}\left({ }_{\mathrm{w}}\right)$ \\
\hline 175 & $60 \dagger$ & $2003 \cdot 2$ & $4921 \cdot 3$ & 30 & 8 & $\mathbf{S}_{(w)}$ \\
\hline 176 & 61 & $2007 \cdot 2$ & $4918 \cdot 2$ & 20 & 3 & Fe. \\
\hline 177 & & 2016.0 & $4911 \cdot 2$ & 3 & 2 & $\mathrm{Zn}\left({ }_{\mathrm{w}}\right)$ \\
\hline 178 & $62 \dagger$ & $2031 \cdot 1$ & $4899 \cdot 3$ & 30 & 6 & Ba. La. $\mathrm{E}\left({ }_{\mathrm{w}}\right)$ \\
\hline 179 & $63 \dagger$ & $2052.5 ? \%$ & $4882 \cdot 9$ & 10 & 4 & Ce. \\
\hline 180 & & $2067 \cdot 8$ & $4869 \cdot 4$ & 5 & 1 & \\
\hline 181 & $64 \dagger$ & $F 2080^{\circ} 0$ & $4860 \cdot 6$ & 100 & 80 & H. \\
\hline 182 & & $2087 \cdot 6$ & $4854 \cdot 7$ & 5 & 2 & Fe. Ni. E( $\left({ }_{w}\right)$ \\
\hline 183 & & $2094 \cdot 0$ & $4848 \cdot 1$ & 3 & 2 & Ca. $O\left(_{w}\right)$ \\
\hline 184 & & $2116 \cdot ? *$ & $4826 \cdot 5$ & 1 & 1 & \\
\hline 185 & & $2121 \cdot 2$ & $4822 \cdot 8$ & 10 & 2 & $\mathrm{Mn}$. \\
\hline 186 & & $2142 \cdot 4$ & $4804 \cdot 1$ & 3 & 1 & Ti. $\left.\left.S_{(w}\right) O_{(w}\right)$ \\
\hline 187 & & $2171 \cdot 5$ & $4778 \cdot 7$ & 3 & 2 & $\left.\operatorname{Co.} N_{(w}\right)^{w \prime}$ \\
\hline 188 & & $2229 \cdot 1$ & $4730 \cdot 8$ & 1 & 1 & Fe. \\
\hline $189 \ddagger$ & & $2251 \cdot 3 *$ & $4712 \cdot 5$ & 2 & 2 & Ce. $\mathrm{O}(\mathrm{w})$ \\
\hline 190 & & $2309 \cdot 5$ & $4666 \cdot 3$ & 3 & 1 & Fe. Ti. \\
\hline 191 & & $2314 \cdot 3$ & 46633 & 2 & 1 & \\
\hline 192 & & $2323 \cdot 0$ & $4656 \cdot 0$ & 2 & 1 & Ti. \\
\hline 193 & 65 & $2358 \cdot 1$ & $4629 \cdot 0$ & 15 & 8 & Ti. $\mathrm{N}\left({ }_{\mathrm{w}}\right)$ \\
\hline 194 & & $2359 \cdot 5 *$ & $4628 \cdot 2$ & 2 & 1 & Ce. \\
\hline 195 & & $2369 \cdot 7$ & $4620 \cdot 3$ & 1 & 1 & \\
\hline 196 & & $2410 \cdot 2$ & $4589 \cdot 4$ & 1 & 1 & \\
\hline 197 & & $2412 \cdot 8$ & $4587 \cdot 5$ & 2 & 2 & \\
\hline 198 & 66 & $2419 \cdot 3$ & $4583 \cdot 2$ & 15 & 6 & \\
\hline 199 & & $2429 \cdot 5$ & $4576 \cdot 0$ & 4 & 2 & : \\
\hline 200 & 67 & $2435 \cdot 5$ & $4571 \cdot 4$ & 10 & 4 & $\mathrm{Ti}$ \\
\hline 201 & 68 & $2443 \cdot 9$ & $4564: 8$ & 10 & 3 & \\
\hline 202 & 69 & 24466 & $4563 \cdot 2$ & 10 & 5 & Ti. \\
\hline 203 & & $2452 \cdot 1$ & $4559 \cdot 5$ & 8 & 2 & \\
\hline 204 & & $2454 \cdot 1$ & $4558 \cdot 1$ & 8 & 1 & \\
\hline 205 & 70 & $2457 \cdot 9$ & $4555 \cdot 3$ & 10 & 5 & Fe. Ti. \\
\hline 206 & 71 & $2461 \cdot 2$ & $4553 \cdot 4$ & 10 & 5 & Ba. \\
\hline 207 & & $2463 \cdot 4$ & $4551 \cdot 8$ & 1 & 1 & Ti. $\left.S_{(w}\right)$ \\
\hline
\end{tabular}




\begin{tabular}{|c|c|c|c|c|c|c|}
\hline No. & P.C. & K. & A. & $\mathrm{F}$. & B. & E. \\
\hline 208 & 72 & $2+67 \cdot 6$ & $4548 \cdot 9$ & 10 & 8 & Ti. \\
\hline 209 & & $2480 \cdot 8$ & $4539 \cdot 2$ & 2 & 1 & Ce. \\
\hline 210 & 73 & $2486 \cdot 6$ & 4535.5 & 2 & 2 & Ti. Ca. \\
\hline 211 & 74 & $2489 \cdot 4$ & $4533 \cdot 2$ & 5 & 5 & Fe. \\
\hline 212 & & $2490 \cdot 5$ & $4532 \cdot 1$ & 3 & 2 & Ti. Ca. \\
\hline 213 & 76 & $2502 \cdot 2$ & $4524 \cdot 4$ & 3 & 2 & Ba. Fe, \\
\hline 214 & 77 & $2505 \cdot 6$ & $4522 \cdot 0$ & 3 & 3 & 11. $S\left({ }_{w}\right)$ \\
\hline $\begin{array}{l}215 \\
216\end{array}$ & & $2517 \cdot 0$ & $4514 \cdot 0$ & 2 & 1 & \\
\hline 217 & & $2527 \cdot 0$ & $\begin{array}{l}4513^{\circ} \\
4506^{\circ} 0\end{array}$ & $\begin{array}{l}1 \\
2\end{array}$ & 1 & \\
\hline 218 & 78 & $2537 \cdot 1$ & $4500 \cdot 3$ & 15 & 6 & Ti. \\
\hline 219 & 79 & $2552 \cdot 4$ & $4490 \cdot 9$ & 20 & 8 & $\mathrm{Mn}$. \\
\hline 220 & 80 & $2555 \cdot 0$ & $4489 \cdot 4$ & 15 & 3 & Fe. Mn. \\
\hline 221 & 81 & $2566 \cdot 3$ & $4480 \cdot 9$ & 5 & 2 & Mg. \\
\hline $224+$ & $82 \dagger$ & f $2581^{\circ} 2$ & $4471 \cdot 2$ & 100 & 25 & Ce. \\
\hline $223^{\circ}$ & 83 & $2585^{\circ} 4$ & $4468 \cdot 5$ & 20 & 5 & Ti. $O\left({ }_{w}\right)$ \\
\hline 224 & & $2620 \cdot 8$ & $4446{ }^{\circ} 3$ & 10 & $\begin{array}{l}1 \\
2\end{array}$ & Ti. \\
\hline $\begin{array}{l}225 \\
226\end{array}$ & 84 & $\begin{array}{l}2625 \cdot 2 \\
9633 \cdot 0\end{array}$ & $\begin{array}{l}4443 \cdot 0 \\
4436 \cdot 7\end{array}$ & 1 & $\begin{array}{l}2 \\
1\end{array}$ & Mn? \\
\hline 227 & & $2639^{\circ} 6$ & 4433.5 & 1 & 1 & \\
\hline 228 & & $2651.5 *$ & $4426 \cdot 0$ & 2 & 3 & \\
\hline 229 & & $2653 \cdot 2$ & $4425^{\circ} 0$ & 2 & 2 & Ca. \\
\hline 230 & & $2664: 9$ & $4418 \cdot 0$ & 2 & 1 & $O\left({ }_{w}\right)$ \\
\hline 231 & & 266599 & $4417 \cdot 5$ & 3 & 1 & 'l'i. \\
\hline
\end{tabular}

232

233

234

235

236

237

238

239

240

241.

242

243

244

245

246

247

248

249

250

251

252

253

254

$2670 \cdot 0$

$4414 \cdot 7$

$4407 \cdot 7$

$4404^{\circ} 2$

$2686 \cdot 8$

$26960^{\circ}$

$2698 \cdot 2$

$4398 \cdot 5$

43965

$4394 \cdot 6$

$2715^{\circ} 2$

$4388 \cdot 5$

$4384 \cdot 7$

$4383 \cdot 5$

$4382 \cdot 8$

$4380 \cdot 4$

$4379^{\circ} 1$

$4375 \circ 5$

$4374 \cdot 2$

$4359 \cdot 1$

$4351 \cdot 8$

$4340 \cdot 1$

$4338 \cdot 2$

$4335 \cdot 1$

$4324^{\circ} 0$

$4320 \cdot 1$

4313.5

$2830 \cdot 7$

$2843 \cdot 0$

$4307 \cdot 2$

255

G. $2854 \cdot 2$

$4302 \cdot 1$

256

$2867 \cdot 7$

$4298 \cdot 0$

Fe. Mn. $\left.\mathrm{O}_{\mathrm{w}}\right)$

Fe. Ca.

$\mathrm{Fe}$.

Ti. Ce. $\left.\mathrm{O}_{(\mathrm{w}}\right)$

$\mathrm{Fe}$ ?

Ca. Ce.

Fe. Cr.

Ca.

$\mathrm{Fe}$.

$\mathrm{E}\left({ }_{\mathrm{w}}\right)$

Cr.

C..

H.

Cr.

La.

Ti. $O\left({ }_{w}\right)$

$\mathrm{Ti}$.

Ca. Fe.

$\mathrm{Ca} . \mathrm{Fe}$.

$\mathrm{Ca} . \mathrm{Fe}$. 


\begin{tabular}{|c|c|c|c|c|c|c|}
\hline No. & P.C. & $\mathrm{K}$. & A. & F. & B. & E. \\
\hline 257 & 97 & 2894.5 & $4289 \cdot 4$ & 1 & 1 & Cr. Ca. $\left.\mathrm{Ce}_{(\mathrm{w}}\right)$ \\
\hline 258 & 98 & $2928 \cdot 5$ & $4274 \cdot 6$ & 2 & 1 & Cr. Ca. \\
\hline 259 & 99 & $8961 \cdot 2$ & $4260 \cdot 0$ & 2 & 1 & Fe. \\
\hline 260 & 100 & 29962 & $4245 \cdot 2$ & 30 & 3 & Fe. \\
\hline 261 & & $3018 \cdot 0$ & $4235 \cdot 5$ & 30 & 5 & Fe. \\
\hline 262 & & $3022 \cdot 8$ & $4233 \cdot 0$ & 15 & 5 & Fe. Ca. \\
\hline 263 & 101 & $3040^{\circ} 0$ & $4226 \cdot 3$ & 3 & 3 & Ca. Sr. \\
\hline 264 & 102 & $3061 \cdot 8$ & $4215 \cdot 3$ & $4 C$ & 7 & Ca. Sr. \\
\hline 265 & & $3155 \cdot 5$ & $4178 \cdot 8$ & 1 & 1 & \\
\hline 266 & & $3187^{\circ} 0$ & $4166 \cdot 7$ & I & 1 & $\mathrm{Ca}$. \\
\hline 267 & $103 \dagger$ & h. 3363.5 & $4101 \cdot 2$ & 100 & 50 & H. \\
\hline 268 & & $3431 \cdot 0$ & $4077 \cdot 0$ & 25 & 2 & Ca. \\
\hline 269 & & $3526 \cdot 0$ & $4045^{\circ} 0$ & 3 & 2 & Fe. \\
\hline 270 & & $3703 \cdot 3$ & $3990^{\circ} ?$ & 2 & 1 & \\
\hline 271 & & $3769 \cdot 5$ & $3970^{\circ} ?$ & 2 & 1 & $\mathrm{Fe}$. \\
\hline $272+$ & & $\mathrm{H}_{1} 3778.5$ & $3967 \cdot 9$ & 75 & 3 & Fe.Ca. \\
\hline 273 & & $\mathrm{H}_{2} 3882 \cdot 5$ & $3932 \cdot 8$ & 50 & 1 & Fe.Ca. \\
\hline
\end{tabular}

Noxes.

1. The position assigned to this line, first observed by Respighi (a faet of whieh 1 was ignorant when the Preliminary Catalogue was published), rests upon two series of mierometrie measurements, referring it to four neighbouring dark lines - the probable error is about $\frac{1}{20}$ th of a division of Kirehhoff's seale.

9. No. 6 in P.C. Position there given, 743 ?

16 and 17. Nos. 8 and 9 of P.C. Position given as 816.8 and $827^{\circ} 6$, by a mistake in identifying lines upon the map.

40. I have never myself seen this line reversed. Professor Emerson, however, saw it several times. It was first reported by Rev. S. J. Perry, in "Nature," vol. iii. p. 67.

41. The position of this line has been independently determined by three series of mierometrie eomparisons with neighbouring lines. My result agrees exaetly with that of Huggins.

72. Eironeously given in P.C. as $1363^{\circ} 1$, whieh line does not reverse, or at least was never seen reversed at Sherman.

100. The principal line in the speetrum of the eorona. The eorresponding line in the speetrum of iron is feeble, and on several oeeasions when the neighbouring lines of iron $(1463, \&$ e. ) have been greatly disturbed, this has wholly failed to sympathise. Henee 1 have marked the Fe with a?. Watts indieates a strong line of oxygen at $5315 \mathrm{~A}$.

152 and 156. Observed only on one day, but verified by Professor Emerson.

172. Called "little C" by Mr. Stoney.

179. Given by Loekyer as K 2054. Its position is a little uneertain; it seems to eoineide with neither of the dark lines at 2051 and 2054, but lies between them, a little nearer to 2051.

189. Rather a band than a line.

222. The position of this line, which, however, like 189, is rather a band, was determined by two series of eareful mierometrieal measurements. It was diseovered by Rayet in January, 1869; sinee ealled "f" by Lorenzoni.

272 and 273. These lines were both reversed (by a narrow bright strine running down the eentre of the broad hazy band) as eonstantly, whenever the 
seeing was good, as $h$ or $\mathrm{C}$ itself. The observation was difficult, however, and required the most sertupulous exclusion of foreign light, and a careful adjustment of the slit in the plane of the snlar image formed by these particular rays.

They were also found to be regularly reversed upon the body of the sun itself, in the penumbra and immediate neighbourhood of every important spot.

"In the catalogue, the first column contains simply a reference number : a + refers to a note at the end of the catalogne.

"The numbers in the second column refer to the 'Preliminary Catalogue,' containing 103 lines, which was published a year ago in the American Journal of Science. In this column a $f$ indicates that some other observer has anticipated me in the determination and publication of the line. As I have depended for my information almost solely upon the Comptes Rendus and the Proceedings of the Royal Society (which gave the observations of Lockyer, Janssen, Rayet, and Secchi), it is quite possible that some other lines ought to be marked in the same manner.

"The third column, headed $K$, gives the positions of the lines on Kirchhoff's scale, the numbers above (C being derived from Thalén's continuation of Kirchhoff's maps. In this column an asterisk denotes that the map shows no corresponding dark line, a? that the exact position, not the existence, of the line is for some reason slightly uncertain.

"The fourth colrmn, hearled A, gives the wave-length of the line in ten-millionths of a millimetre, according to Ångström's atlas.

"The numbers in this and the preceding column were taken, not from the niaps themselves, which present slight inaccuracies on account of the shrinking and swelling of the paper during the operation of printing, but from the numerical catalogues of Kirchhoff and Angström which accompany their respective atlases. In the Preliminary Catalogue the numbers were derived from the maps; hence some slight discrepancies in the tenths of division.

"The fifth column, marked F, contains a rough estimate of the percentage of frequency with which the lines were seen during the six weeks of observation; and the sixth column, B, a similar estimate of their maximum brightness compared with that of the lyydrogen line C.

"The variations of brilliance, however, when the chromosphere was much disturbed, were so considerable and so sudden, that no very great weight can be assigned to the numbers given. Nor is it to be inferred that lines which have in the table the same index of brightness were always equally bright. On certain occasions one set of lines would be particularly conspicuous; on others, another.

"With two or three exceptions, indicated in the notes, no lines have been catalogued which were not seen on at least two different days. In the few cases where lines observed only on one occasion have been admitted to the list, the observations were at the time carefully verified by my assistant, Professor Emerson, so as to place their correctness beyond a doubt. Many other lines were 'glimpsed' at one time and another, but not seen steadily enough or long enough to admit of satisfactory determination.

"The last column of the catalogue contains the symbols of the chemical elements corresponding to the respective lines. The materials at my disposal are the maps of Kirchhoff and Àngström, Thalén's map of the portion of the solar spectrum above G, and Watts' 'Index of Spectra.' Since the positions of the lines in the latter work are given only to the nearest unit of 'Angström's scale,' I have marked the coincidences indicated by it with a (w), considering them less certain than those shown by the maps." 


\section{APPENDIX F.}

\section{SUNLIGHT AND THE EARTH'S ATMOSPHERE. ${ }^{1}$}

$\mathrm{WE}_{\mathrm{E}}$ have been compared to creatures living at the bottom of the sea who frame their deceptive traditional notions of what the sun is like from the feeble changed rays which sift down to them. Though such creatures could not rise to the surface, they might swim up towards it, and if these rays grew hotter, brighter, and bluer as they ascended, it would be almost within the capacity of a fish's mind to guess that they are still brighter and bluer at the top.

Since we children of the earth, while dwelling on it, are always at the bottom of a sea, though of another sort, the most direct method of proof I spoke of, is merely to go up as far as we can and observe what happens, though as we are men, and not fishes, something more may fairly be expected of our intelligence than of theirs.

We will not only guess, but measure and reason, and in particular we will first, while still at the bottom of the mountain, draw the light and heat out into a spectrum, and analyse every part of it by some method that will enable us to explore the invisible as well as record the visible. Then we will ascend many miles into the air, meeting the rays on the way down, before the sifting process has done its whole work, and there analyse the light all over again, so as to be able to learn the different proportions in which the different rays have been absorbed, and, by studying the action on each separate ray, to prove the state of things which must have existed before this sifting-this selective absorption-began.

1 Extracted from a lecture delivered at the Royal Institution, April 17, 1885, by Professor S. P. Langley. Communicated by the author to "Nature." 
It may seem at first that we cannot ascend far enough to do mucl good, since the surface of our aërial ocean is hundreds of miles overhead; but we must remember that the air grows thinner as we ascend, the lower atmosphere being so much denser, that about one-half the whole substance or mass of it lies within the first four miles, which is a less height than the tops of some mountains. Every high mountain, however, will not do, for ours must not only be very high, but very steep, so that the station we choose at the bottom may be almost under the station we are afterwards to occupy at the top.

Besides, we are not going to climb a lofty lonely summit like tourists to spend an hour, but to spend weeks; so that we must have fire and shelter, and, above all, we must have dry air to get clear skies. First I thought of the Peak of Teneriffe, but afterwards some point in the territories of the United States seemed preferable, particularly as the Government offered to give the Expedition, through the Signal Service, and under the direction of its head, General Hazen, material help in transportation and a military escort, if needed, anywhere in its own dominions. No summit in the eastern part of the United States rises much over 7,000 feet, and though the great Rocky Mountains reach double this, their tops are the home of fog and mist, so that the desired conditions, if met at all, could only be found on the other side of the Continent in Southern California, where the summits of the Sierra Nevadas rise precipitously out of the dry air of the great wastes in lonely peaks, which look eastward down from a height of nearly 15,000 feet upon the desert lands.

This remote region was, at the time I speak of, almost unexplored, and its highest peak, Mount Whitney, had been but once or twice ascended, but was represented to be all we desired could we once climb it. As there was great doubt whether our apparatus, weighing several thousand pounds, could possibly be taken to the top, and we had to travel 3,000 miles even to get where the chief difficulties would begin, and make a desert journey of 150 miles after leaving the cars, it may be asked why we committed ourselves to such an immense journey to face such unknown risks of failure. The answer must be that 
mountains of easy ascent and 15,000 feet high are not to be found at our doors, and that these risks were involved in the nature of our novel experiment, so that we started out from no love of mere adventure, but from necessity, much into the unknown. The liberality of a citizen of Pittsburgh, to whose encouragement the enterprise was due, had furnished the costly and delicate apparatus for the expedition, and that of the transcontinental railroads, enabled us to take this precious freight along in a private car, which carried a kitchen, a steward, a cook, and an ample larder besides.

In this we crossed the entire continent from ocean to ocean, stopped at San Francisco for the military escort, went 300 miles south so as to get below the mountains, and then turned eastward again on to the desert, with the Sierras to the north of us, after a journey which would have been unalloyed pleasure except for the anticipation of what was coming as soon as we left our car. I do not indeed know that one feels the triumphs of civilisation over the opposing forces of Nature anywhere more than by the sharp contrasts which the marvellous luxury of recent railroad accommodation gives to the life of the desert. When one is in the centre of one of the great barren regions of the globe, and, after looking out from the windows of the flying train on its scorched wastes for lonely leagues of habitless desolation, turns to his well furnished dimner-table, and the fruit and ices of his dessert, he need not envy the heroes of Oriental story who were carried across dreadful solitudes in a single night on the backs of flying genii. Ours brought us over 3,000 miles to the Mojave desert. It was growing hotter and hotter when the train stopped in the midst of vast sandwastes a little after midnight. Roused from our sleep, we stepped on to the brown sand and saw our luxurious car roll away in the distance, experiencing a transition from the conditions of civilisation to those almost of barbarism, as sharp as could be well imagined. We commenced our slow toil northward with a thermometer at $110^{\circ}$ in the shade, if any shade there be in the shadeless desert which seemed to be chiefly inhabited by rattlesnakes of an ashen grey colour, and a peculiarly venomous bite. There is no water 
save at the rarest intervals, and the soil at a distance seems as though strewed with sheets of salt, which aids the delusive show of the mirage. These are, in fact, the ancient beds of dried-up salt lakes or dead seas, some of them being below the level of the ocean; and such a one on our right, though only about twenty miles wide, has earned the name of "Death Valley," from the number of human beings who have perished in it. Formerly an emigrant train, when emigrants crossed the continent in caravans, had passed through the great Arizona deserts in safety until, after their half-year's journey, their eyes were gladdened by the snowy peaks of the Sierras looking delusively near. The goal of their long toil seemed before them; only this one more valley lay between, and into this they descended, thinking to cross it in a day-but they never crossed it. Afterwards the long line of waggons was found with the skeletons of the animals in the harness, and by them those of men, women, and little children dead of thirst, and some relics of the tragedy remained at the time of our journey. I cite this as an indirect evidence of the phenomenal dryness of the region-a dryness which, so far, served our object, which was, in part, to get rid as much as possible of that water-vapour which is so well known to be a powerful absorber of the solar heat.

Everything has an end, and so had that journey, which finally brought us to the goal of our long travel, at the foot of the highest peak of the Sierras, Mount Whitney, which rose above us in tremendous precipices, that looked hopelessly insurmountable and wonderfully near. The whole savage mountain region in its slow rises from the west, and its descent to the desert plains in the east, is more like the chain called the Apennines in the moon, than anything I know on the earth. The summits are jagged peaks like Alpine "needles," looking in the thin air so delusively near, that, coming on such a scene unprepared, one would almost say they were large grey stones a few fields off, with an occasional little white patch on the top, that might be a handkerchief or a sheet of paper dropped there. But the telescope showed that the seeming stones were of the height of many Snowdons piled on one another, and the white patches 
of occasional snow-fields, looking how invitingly cool, from the torrid heat of the desert, where we were encamped by a little rivulet that ran down from some unseen ice-lake in that upper air. Here we pitched our tents and fell to work (for you remember we must have two stations, a low and a high one, to compare the results), and here we laboured three weeks in almost intolerable heat, the instruments having to be constantly swept clear of the red desert dust which the hot wind brought. Close by these tents a thermometer covered by a single sheet of glass, and surrounded by wool, rose to $237^{\circ}$ in the sun, and sometimes in the tent, which was darkened for the study of separate rays, the heat was absolutely beyond human endurance. Finally, our apparatus was taken apart and packed in small pieces on the backs of mules, who were to carry it by a ten days' journey through the mountains to the other side of the rocky wall which, though only ten or twelve miles distant, arose miles above our heads; and, leaving these mule trains to go with the escort by this longer route, I started with a guide by a nearer way to those white gleams in the upper skies, that had daily tantalised us below in the desert with suggestions of delicious, unattainable cold. That desert sun had tanned our faces to a leather-like brown, and the change to the cooler air as we ascended was at first delightful. At an altitude of 5,000 feet we came to a wretched band of nearly naked savages, crouched around their camp-fire, and at 6,000 found the first scattered trees; and here the feeble suggestion of a path stopped, and we descended a ravine to the bed of a mountain stream, up which we forced our way, cutting through the fillen trees with an axe, fighting for every foot of advance, and finally passing what seemed impassable. It was interesting to speculate as to the fate of our siderostat mirrors and other precious freight, now somewhere on a similar road, but quite useless. We were committed now, and had to make the best of it-and, besides, I had begun to have my attention directed to a more personal subject. This was, that the colder it grew the more the sun burnt the skin-quite literally burnt, I may say, so that by the end of the third day my face and hands, case-hardened, as I thought, in the 
desert, began to look as if they had been seared with red-hot irons, here in the cold where the thermometer had fallen to freezing at night; and still as we ascended the paradoxical effect increased : the colder it grew about us, the hotter the sun blazed above.

We have all heard probably of this curious effect of burning in the midst of cold, and some of us may have experienced it in the Alps, where it may be aided by reflection from the snow, which we did not have about us at any time except in scattered patches, but here by the end of the fourth day my face was scarcely recognisable, and it almost seemed as though sunbeams up here were different things, and contained something which the air filters out before they reach us in our customary abodes. Radiation here is increased by the absence of water-vapour too, and on the whole this intimate personal experience fell in almost too well with our anticipations that the air is an even more elaborate trap to catch the sunbeams than had been surmised, and that this effect of selective absorption and radiation was intimately connected with that change of the primal energies and primal colour of the sun which we had climbed towards it to study.

On the fourth day, after break-neck ascents and descents, we finally ascended by a ravine, down which leaped a cataract, till, at nightfall, we reached our upper camp, which was pitched by a little lake, one of the sources of the waterfall, at a height of about 12,000 feet, but where we seemed in the bottom of a valley, nearly surrounded as we were by an amphitheatre of rocky walls which rose perpendicularly to the height of Gibraltar from the sea, and cut off all view of the desert below or even of the peak above us.

The air was wonderfully clear, so that the sun set in a yellow rather than an orange sky, which was reflected in the little ice-rimmed lakes and from occasional snow-fields on the distant waste of lonely mountain summits on the west.

The mule train, sent off before by another route, had not arrived when we got to the mountain camp, and we realised that we were far from the appliances of civilisation by our inability 
to learn about our chief apparatus; for here, without post or telegraph, we were as completely cut off from all knowledge of what might be going on with it in the next mountain ravine as a ship at sea is of the fate of a vessel that sailed before from the same port. During the enforced idleness we ascended the peak, nearly 3,000 feet above us, with our lighter apparatus, leaving the question of the ultimate use of the heavy ones to be settled later. There seemed little prospect of carrying it up, as we climbed where the granite walls had been split by the earthquakes, letting a stream of great rocks, like a stone river, flow down through the interstices by which we ascended, and, in fact, the heavier apparatus was not carried above the mountain camp.

The view from the very summit was over numberless peaks on the west to an horizon fifty miles away, of unknown mountain-tops, for, with the exception of the vast ridge of Mount Tyndall, and one or two less conspicuous ones, these summits are not known to fame, and, wonderful as the view may be, all the charm of association with human interest which we find in the mountain landscape of older lands is here lacking.

It was impossible not to be impressed with the savage solitude of this desert of the upper air, and our remoteness from man and his works, but I turned to the study of the special things connected with my mission. Down far below the air seemed filled with reddish dust that looked like an ocean. This dust is really present everywhere ( $I$ have found it in the clear air of Etna), and though we do not realise its presence in looking up through it, to one who looks down on it, the dwellers on the earth seem indeed like creatures at the bottom of a troubled ocean. We had certainly risen towards the surface, for about us the air was of exquisite purity, and above us the sky was of such a deep violet blue, as I have never seen in Egypt or Sicily, and yet even this was not absolutely pure, for separately invisible, the existence of fine particles could yet be inferred from their action on the light near the sun's edge, so that even here we had not got absolutely above that dust shell which seems to 
encircle our whole planet. But we certainly felt ourselves not only in an upper, but a different region. We were on the ridge of the continent, and the winds which tore by had little in common with the air below, and were bearing past us (according to the geologists) dust which had once formed part of the soil of China, and been carried across the Pacific Ocean; for here we were lifted into the great encircling currents of the globe, and, "near to the sun in lonely lands," were in the right conditions to study the differences between his rays at the surface and at the bottom of that turbid sea where we had left the rest of mankind. We descended the peak and hailed with joy the first arrival of our mule trains with the requisite apparatus at the mountain camp, and found that it had suffered less than might be expected, considering the pathless character of the wilderness. We went to work to build piers and mount telescopes and siderostats, in the scene shown by the next illustration on the screen, taken from a sketch of my own, where these rocks in the immediate foreground rise to thrice the height of St. Paul's. We suffered from cold (the ice forming three inches deep in the tents at night) and from mountain sickness, but we were too busy to pay much attention to bodily comfort, and worked with desperate energy to utilise the remaining autumn days, which were all too short.

Here, as below, the sunlight entered a darkened tent, and was spread into a spectrum, which was explored throughout by the bolometer, measuring, on the same separate rays which we had studied below in the desert, all of which were different up here, all having grown stronger, but in very different proportions. On the screen is the spectrum as seen in the desert, drawn on a conventional scale, neither prismatic nor normal, but such that the intensity of the energy shall be the same in each part, as it is represented here by these equal perpendiculars in every colour. Fix your attention on these three as types, and you will see better what we found on the mountain, and what we inferred as to the state of things still higher up, at the surface of the aëreal sea.

You will obtain, perhaps, a clearer idea, however, from the 
following statement, where I use, not the exact figures used in calculation, but round numbers, to illustrate the process employed. I may premise that the visible spectrum extends from $\mathrm{H}$ (in the extreme blue) to A (in the deepest red), or from near 40 (the ray of forty-one hundred thousandths of a millimetre in wave-length) to near 80. All below 80 , to the right, is the invisible infra-red spectrum.

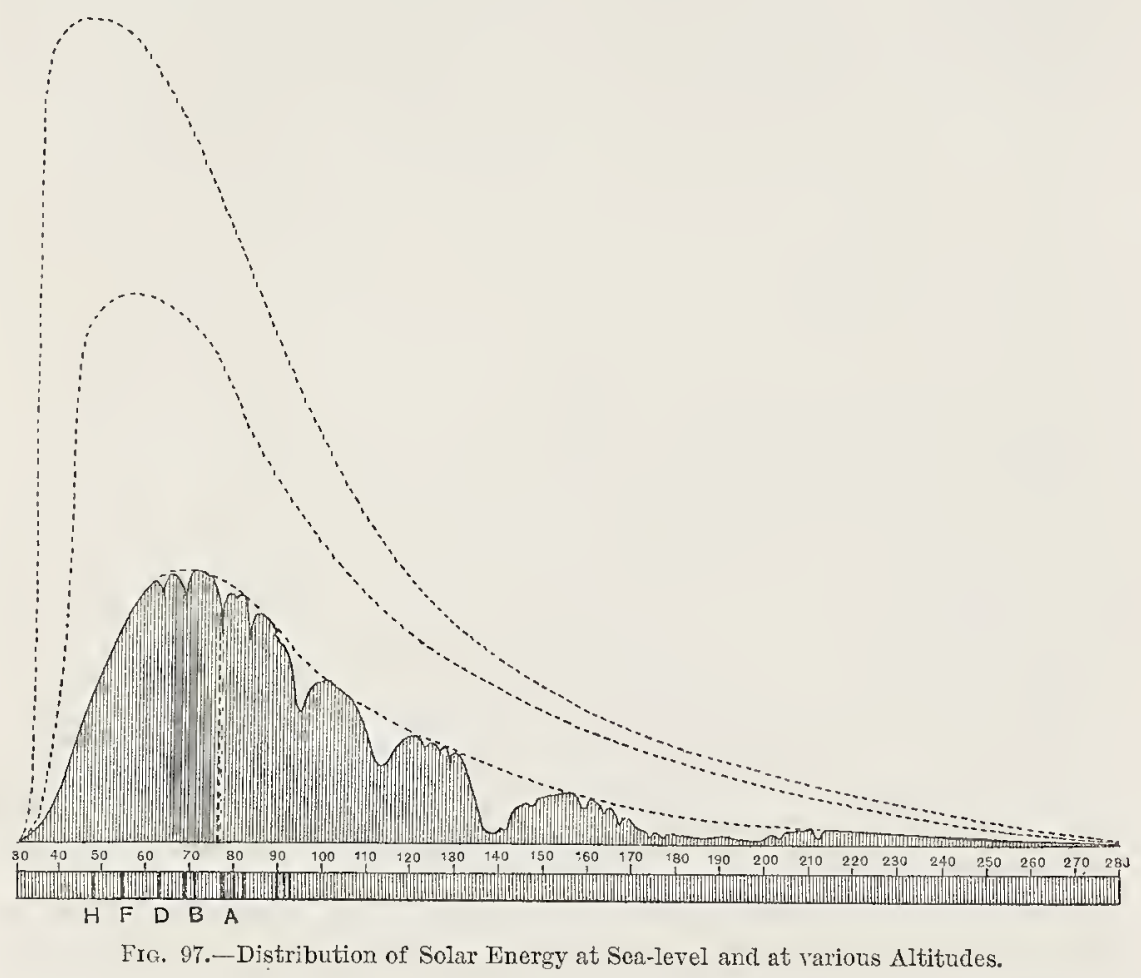

Now, the shaded curve above the spectrum represents the amount of energy in the sun's rays at the foot of the mountain, and was obtained in this way:--Fix your attention for a moment on any single part of the spectrum, for instance, that whose wave-length is 60 . If the heat in this ray, as represented by the bolometer at the foot of the mountain, was (let us suppose) $2^{\circ}$, on any arbitrary scale we draw a vertical line, 2 inches or 2 feet high over that part of the spectrum. If the heat at another point, such as 40 , were but a $\frac{1}{5}$, a line would be drawn 
there a quarter of an inch high, and so on, till these vertical lines mark out the shaded parts of the drawing, the gaps and depressions in whose outline correspond to the "cold bands" already spoken of. Again, if on top of the mountain we measure all these over once more, we shall find all are hotter, so that we must up there make all our lines higher, but in very different proportions. At 60, for instance, the heat (and light) may have grown from $2^{\circ}$ to $3^{\circ}$, or increased one-half, while above 40 the heat (and light) may have grown from $\frac{1}{5}^{\circ}$ to $1^{\circ}$, or increased five times. These mountain measurements give another spectrum, the energies in each part of which are defined by the middle dotted line, which we see indicates very much greater energy, whether heat or light, in the blue end than below. Next, the light or heat which would be observed at the surface of the atmosphere is found in this way. If the mountain top rises through one-half the absorbing mass of this terrestrial atmosphere (it does not quite do so, in fact), and by getting rid of that lower half, the ray 60 has grown in brightness from two to three, or half as mucle again; in going up to the top it would gain half as much more, or become $4: \frac{1}{2}$, while the ray near 40 , which has already increased to five times what it was, would increase five times more, or to 25. Each separate ray increasing thus nearly in some geocentric progression (though the heat, as a whole, does not), you see how we are able, by repeating this process at every point, to build up our outer or highest curve, which represents the light and heat at the surface of the atmosphere. These have grown out of all proportion at the blue end, as you see by the outer dotted curve, and now we have attained, by actual measurement, that evidence which we sought, and by thus reproducing the spectrum outside the atmosphere, and then recombining the colours by like methods to those you have seen on the screen, we finally get the true colour of the sun, which tends, broadly speaking, to blue.

It is so seldom that the physical investigator meets any novel fact quite unawares, or finds anything except that in the field where he is seeking, that he must count it an unusual experience to come unexpectedly on even the smallest discovery. 
This experience I had on one of the last days of work on the spectrum on the mountain. I was engaged in exploring that great invisible heat region, still but so partially known, or, rather, I was mapping in that great "dark continent" of the spectrum, and by the aid of the exquisite sky and the new instrument (the bolometer) found I could carry the survey further than any had been before. I substituted the prism for the grating, and measured on in that unknown region till I had passed the Ultima Thule of previous travellers, and finally came to what seemed the very end of the invisible heat spectrum beyond what had previously been known. This was in itself a return for much trouble, and I was about rising from my task when it occurred to me to advance the bolometer still farther, and I shall not forget the surprise and emotion with which I found new and yet unrecognised regions below-a new invisible spectrum beyond the farthest limits of the old one.

I will anticipate here by saying, that after we got down to lower earth again the explorations and mapping of this new region was continued. The amount of solar energy included in this new extension of the invisible region is much less than that of the visible spectrum, while its length upon the wavelength scale is equal to all that previously known, visible and invisible, as you will see better by this view, having the same thing on the normal as well as the prismatic scale. If it be asked which of these is correct, the answer is " both of them." Both rightly interpreted mean just the same thing, but in the lower one we can more conveniently compare the ground of the researches of others with these. These great gaps I was at first in doubt about, but more recent researches at Alleghany make it probable that they are caused by absorption in our own atmosphere, and not in that of the sun.

We would gladly have stayed longer, in spite of physical discomfort, but the formidable descent and the ensuing desert journey were before us, and certainly the reign of perpetual winter around us grew as hard to bear as the heats of the desert summer had been. On September 10 we sent our instruments and the escort back by the former route, and, ourselves, 
unencumbered, started on the adventurous descent of the eastern precipices by a downward climb, which, if successful, would carry us to the plains in a single day. I at least shall never forget that day, nor the scenery of more than Alpine grandeur which we passed in our descent, after first climbing by frozen lakes in the northern shadow of the great peak, till we crossed the eastern ridges, through a door so narrow that only one could pass it at a time, by clinging with hands and feet as he swung round the shoulder of the rocks - to find that he had passed in a single minute from the view of winter to summer, the prospect of the snowy peaks behind shut out, and instantly exchanged for that below of the glowing valley and the little oasis, where the tents of the lower camp were still pitched, the tents themselves invisible, but the oasis looking like a green scarf dropped on the broad floor of the desert. We went still downward by scenery unique in my recollection. This view of the ravine on the screen is little more than a memorandum made by one of the party in a few minutes' halt part-way down, as we followed the ice-stream between the tremendous walls of the defile which rose 2,000 feet, and between which we still descended, till, toward night, the ice-brook had grown into a mountain torrent, and, looking up the long vista of our day's descent, we saw it terminated by the Peak of Whitney, once more lonely in the fading light of the upper sky.

This site, in some respects unequalled for a physical observatory, is likely, I am glad to say, to be utilised, the President of the United States having, on the proper representation of its value to science, ordered the reservation for such purposes of an area of 100 square miles about and inclusive of Mount Whitney.

There is little more to add about the journey back to civilisation, where we began to gather the results of our observation, and to reduce them-to smelt, so to speak, the metal from the ore we had brought home-a slow but necessary process, which has occupied a large part of two years.

The results stated in the broadest way mean that the sun is blue-but mean a great deal more than that; this blueness in 
itself being perhaps a curious fact only, but in what it implies of practical moment.

We deduce in connection with it a new value of the solar heat, so far altering the old estimates that we now find it capable of melting a shell of ice sixty yards thick annually over the whole earth, or, what may seem more intelligible on its practical bearings, of exerting over one horse-power for each square yard of the normally exposed surface. We have studied the distribution of this heat in a spectrum whose limits on the normal scale our explorations have carried to an extent of rather more than twice what was previously known, and we have found that the total loss by absorption from the atmosphere is nearly double what has been heretofore supposed.

We have found it probable that the human race owes its existence and preservation even more to the heat-storing action of the atmosphere than has been believed.

The direct determination of the effect of water-vapour in this did not come within our scope; but that the importance of the blanketing action of our atmospheric constituents has been in no way overstated may be inferred when I add, that we have found by our experiments that if the planet were allowed to radiate freely into space without any protecting veil, its sunlit surface would probably fall, even in the tropics, below the temperature of freezing mercury.

I will not go on enumerating the results of these investigations, but they all flow from the fact, which they in turn confirm, that this apparently linipid sea above our heads, and about us, is carrying on a wonderfully intricate work on the sunbeam, and on the heat returned from the soil, picking out selected parts in hundreds of places, sorting out incessantly at a task which would keep the sorting demons of Maxwell busy, and as one result, changing the sunbeam on its way down to us in the way we have seen.

I have alluded to the practical utilities of these researches, but practical or not, I hope we may feel that such facts as we have been considering about sunlight and the earth's atmosphere may be stones useful in the future edifice of science, and that if 
not in our own hands then in those of others, when our day is over, they may find the best justification for the trouble of their searches, in the fact that they prove of some use to man.

May I add an expression of my personal gratification in the opportunity with which you bave honoured me of bringing these researches before the Royal Institution, and of my thanks for the kindness with which you have associated yourselves for an hour, in retrospect at least, with that climb toward the stars which we have made together, to find, from light in its fulness, what unsuspected agencies are at work to produce for us the light of common day. 



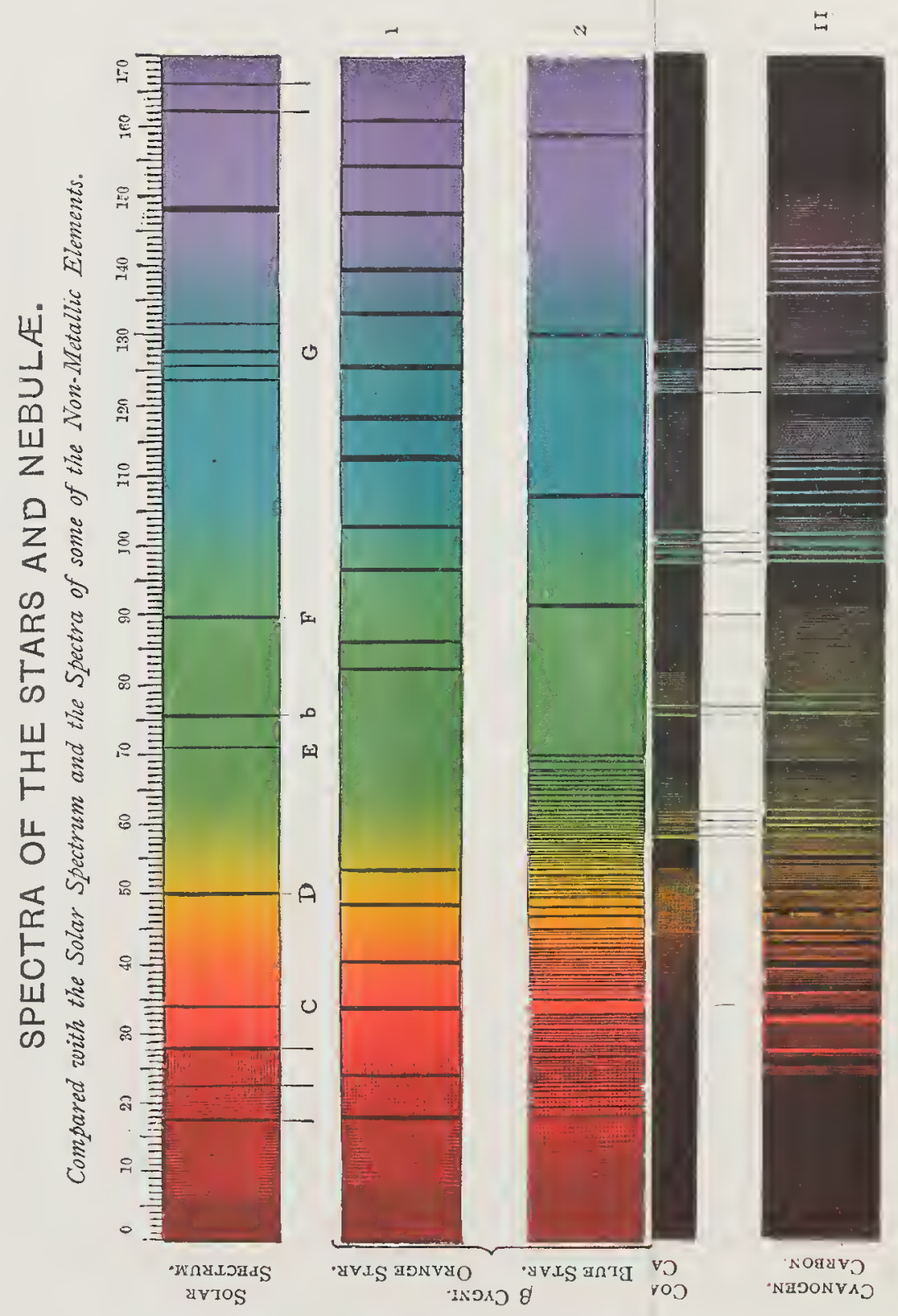




\section{LECI'URE VI.}

Planet- and Moon-light. - Planetary Spectra.-Stellar Cliemistry. Huggins and Miller.-Spectra of the Fixed Stars.--Difficulties of Observation.-Methods employed.-Variable Stars.-Double Stars.Temporary Bright Stars.-Photographs of Star Spectril.-Nebulæ.Comets.-Motion of the Stars (Husgins).-Determinations of Velocity of Solar Storms (Lockyer).

Appendix A.-Extract from a Memoir "On the Spectra of some of the Fixed Stars."

Appexdix B.-On the Spectrum of Mars, with some Remarks on the Colour of that Planet.

Appendix C.-On the Occurrence of Bright Lines in Stellar Spectra, and on the Spectra of Variable Stars.

Appexdix D.-Further Observations on the Spectra of some of the Stars and Nebulæ, with an attempt to determine therefrom whether these bodies are moving towards or from the Earth, also Observations on the Spectra of the Sun and of Comet IL. 1868.

Appendix E.-On the Spectra of the Great Nebula in Orion, and on the inotions of some Stars towards or from the Earth.

Append1x F.-Pho'sographic Spectra of Comets and Nebulæ.

A PPENDIx G.-On the Spectra of Lightning.

IN the last lecture I endeavoured to point out to you the principles upon which Professor Kirchhoff arrived at the remarkable conclusion that certain metals well known on earth are contained in the solar atmosphere. I have to-day to bring before you facts which are still more interesting, with regard to the chemical composition of the stars, comets, and nebulix; and if in the former lectures I lad to couple the names of two great German philosophers, I have to-day to bring before your notice the researches of three distinguished English men 


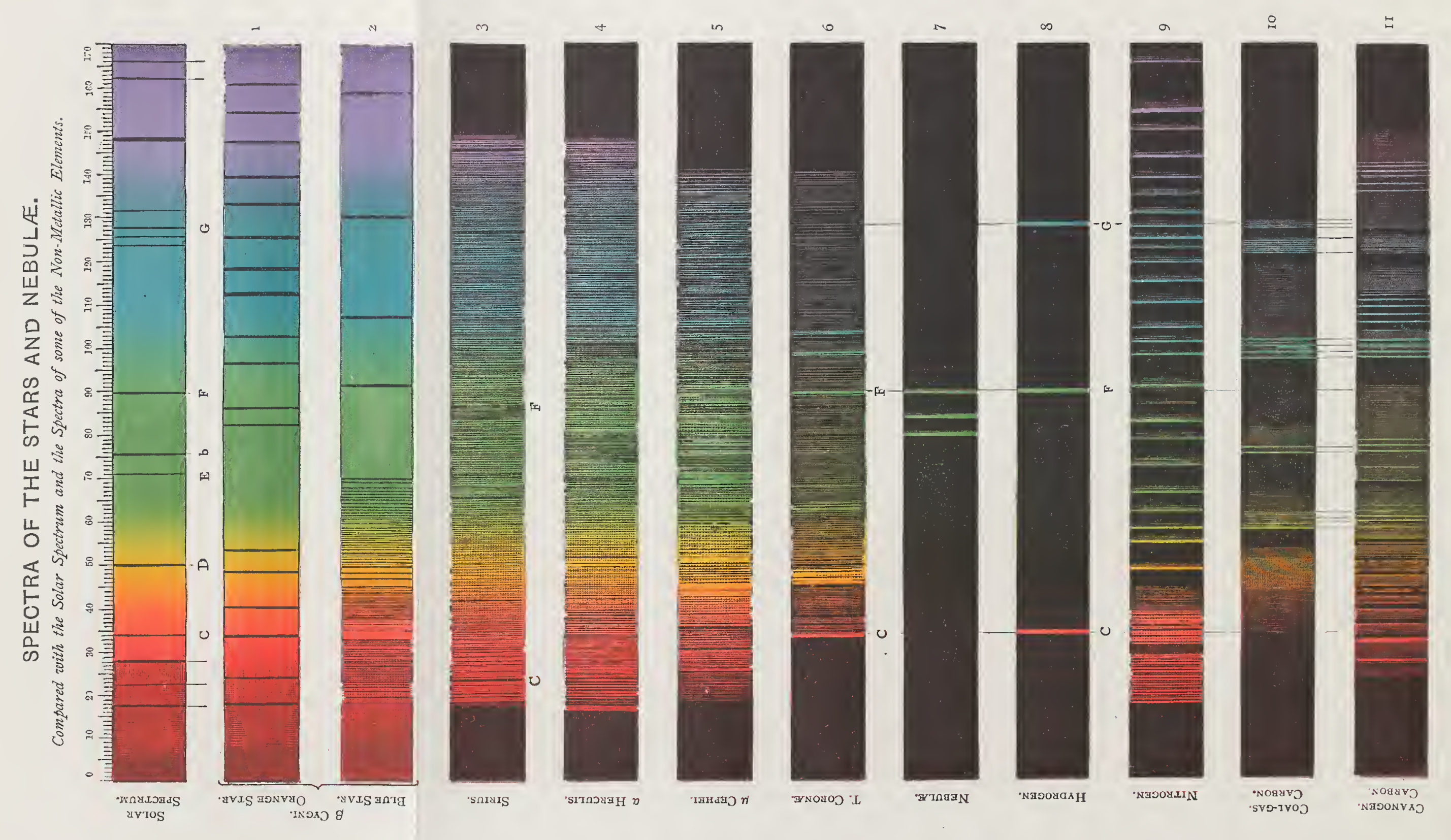



of science-Dr. Huggins, Dr. Miller, and Mr. Lockyer-to whom we are indebted for a great part of our knowledge of celestial chemistry.

Although the moon and planets, shining by borrowed light, do not reveal to the spectroscope the nature of the material of which they are composed, like the sun and stars, yet something may be learned by an examination of the spectra of these bodies. You will remember that some of the dark lines in the solar spectrum are caused by absorption in our own atmosphere : now if an atmosphere of a similar kind exist round the moon or planets, the atmospheric absorption lines must appear more intense in the light reflected from these luminaries than they do in the light which passes through our air alone. With regard to the moon, the observations of Dr. Huggins and Dr. Miller have been negative. No signs of a lunar atmosphere presented themselves. A still more delicate means of ascertaining whether the moon possesses an atmosphere was employed by Dr. Huggins. On January 4th, 1865, he observed the spectrum of a star at the moment the dark edge of the moon passed over it. If an atmosphere existed in the moon, the observer would see the starlight by refraction after the occultation had occurredjust as the setting sun is visible to us after it has actually disappeared below the horizon. The variously coloured rays are, however, differently refrangible; and if any appreciable amount of atmosphere existed round the inoon, the red rays being least so would die out soonest, and the spectrum of the star would be seen progressively to diminish in intensity, beginning from the red end. Dr. Huggins observed nothing of this kind, all the rays of the stellar spectrum disappearing simultaneously: and the conclusion must be drawn that the moon is devoid of any appreciable atmosphere.

As regards planetary spectra we owe our principal information to Huggins in England and Vogel in Germany. Beginning with the brightest planets, Venus and Jupiter, we learn that, in the case of the first of these, no intensifying of the atmospheric absorption lines could be observed by Huggins though Vogel thought that he could discover some evidence of this nature. 
With Jupiter on the other hand the strengthening of some lines is very decided, indicating the existence of an absorptive atmosphere, similar to our own, round this planet. Vogel, moreover, has observed the presence of a band, w.l. 6179, which is not known in the earth's atmosphere, and this tells us of some peculiar gas or vapour which has not yet been identified. Photographs of the spectrum of Jupiter have not revealed any absorption lines in the violet or ultra-violet due to the atmosphere of that planet, a negative result which also holds good in the case of all the other planets which have been thus examined. Observations on the Mars spectrum showed the existence of groups of lines in different parts of the spectrum corresponding to telluric lines, and probably indicating the presence of aqueous vapour.

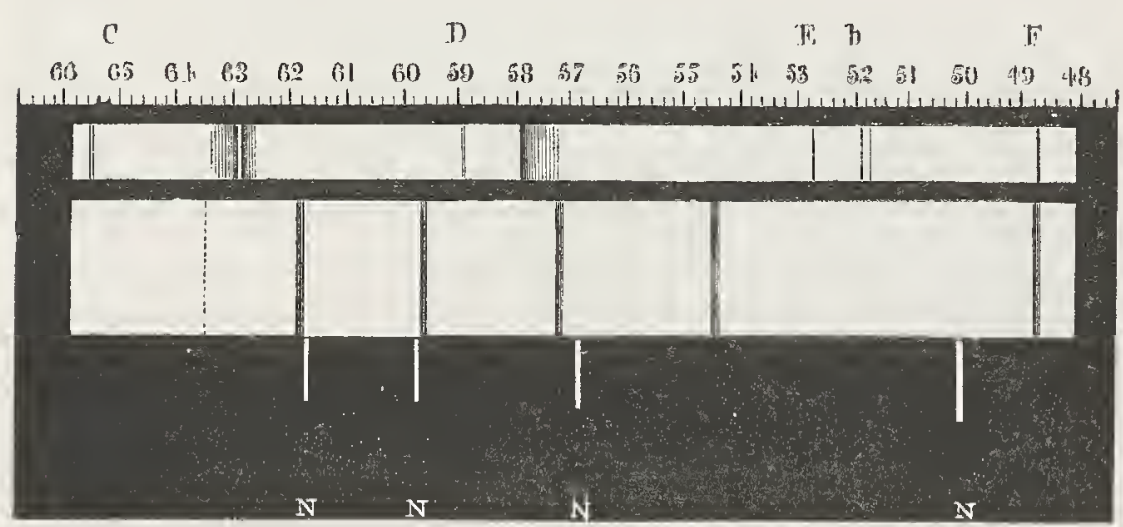

FIG. 98 .

In the case of the planet Mercury, which is, of course, always seen near the horizon, the difficulty of observation is considerably increased, so that no very certain results as to the existence of an atmosphere in this planet have as yet been obtained. Of the more distant planets Saturn appears, according to Vogel, to give much the same spectrum as Jupiter, but in its singular ring the characteristic red band is absent. The spectrum of Uranus is most remarkable; its general appearance as drawn by Dr. Huggins is represented by Fig. 98. The narrow spectrum placed above the broader one of the planet shows the relative 
positions of the chief solar lines, and of the two strongest absorption bands produced by our atmosphere, viz. the group of lines a little more refrangible than $\mathrm{D}$, and that midway between $\mathrm{C}$ and $\mathrm{D}$. The numbers give the wave-lengths in millionths of a millimetre. Vogel has measured the position of these five strongest absorption bands in the spectrum of Uranus and finds them to be 6180 , $5960,5738,5425$, and 4861 , and these you will see agree fairly well with Huggins' map. The spectrum of Neptune appears to be identical with that of Uranus.

I must now pass on to the subject proper of this day's discourse, which is to consider the properties of the light from the fixed stars. The more we learn about this subject, the more I think we must be surprised at the accuracy of the observing powers of those philosophers who have given us this information. By means of this instrument (made by $\mathrm{Mr}$. Browning, and a facsimile of the one used by Dr. Huggins, Fig. 99) we have been placed in possession of facts respecting the composition of the atmospheres, and the physical constitutions of these stars, as accurate as the knowledge we possess concerning the composition of the solar atmosphere. It would be inıpossible for me to give you, even if time permitted, an accurate description of the method employed by Dr. Huggins. (See Appendix A.) Suffice it to say, that at the end of his telescope he has placed this spectroscope, containing two prisms $(h h)$; and that, by very accurate adjustment, he is able to bring the image of the star on the slit of his spectroscope (d). Yon may imagine how difficult these observations are, when you remember that the light of the star emanates from a point-that is to say, the star has no sensible magnitude; that the image of the star has to be kept steady upon a slit only the $\frac{1}{300}$ part of an inch in breadth; and, moreover, that the effect of the earth's motion has to be counteracted. When you add to this, that the amount of light which even the brightest stars give is excessively feeble, that this line of light must be still further weakened by being spread out by a cylindrical lens $(a)$ into a band; and when you remember that in our climate on a few only of those nights in which the stars appear to the naked eye to shine brilliantly, 
is the air steady enough to prevent the flickering and confusion of the spectra fatal to these extremely delicate observations, I think you will easily understand how exceedingly difficult these researches must have been, and I am sure you will acknowledge the debt of gratitude which the world owes to those gentlemen who, by devoted labours, have brought the subject to this interesting issue.

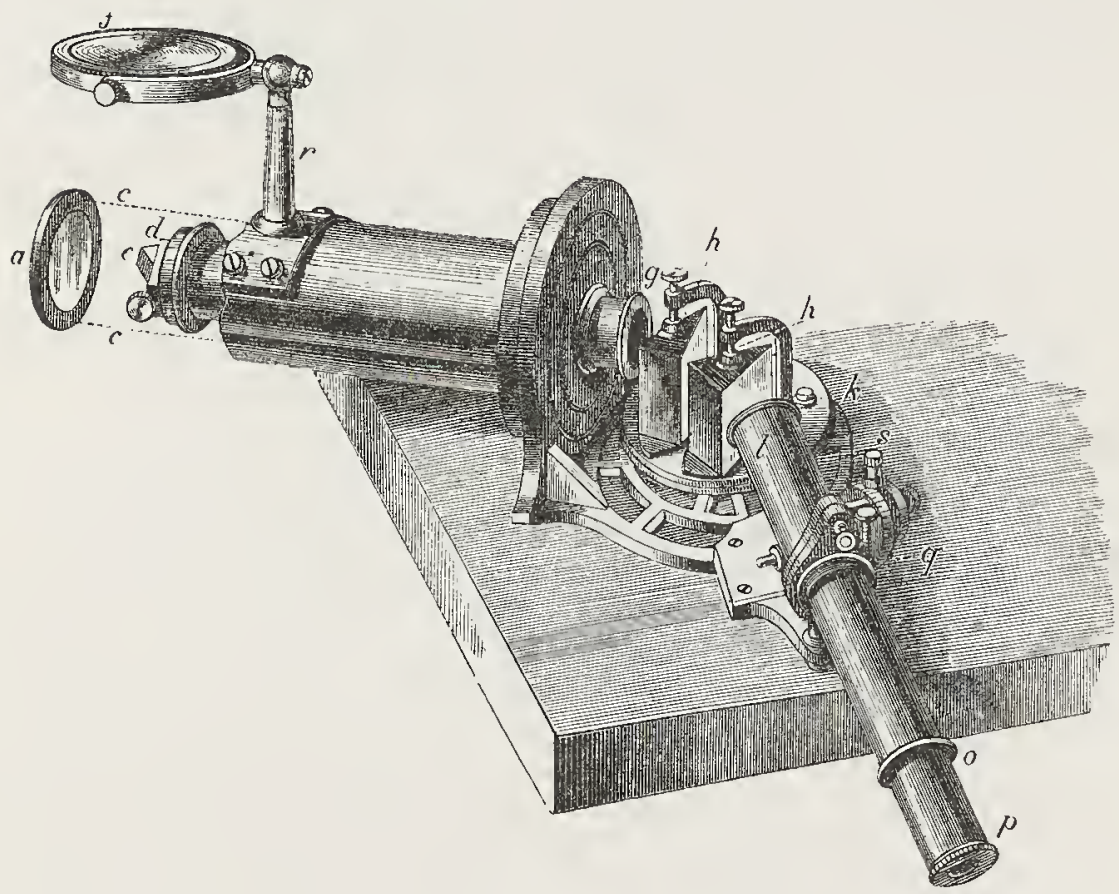

Fic. 99.

In order to get a knowledge of the chemical composition of the stars, or to ascertain what chemical elements are present in them, it is necessary to use excessively delicate arrangements, by which not only the light from the star is allowed to pass through the prisms and to be received on the retina, but also that emitted by the various substances, the presence or absence of which in the stellar atmosphere it is desired to ascertain. These rays must pass together with the beam of starlight, or rather over or under the starlight, into the eyepiece, through the 
same prism, so that we may be able to compare the position of the dark lines in the stellar spectrum with that of the bright lines in the spectrum of the body under examination. For this purpose a very ingenious arrangement is attached to a part of the telescope-spectroscope. It consists of a movable mirror, ( $f$, Fig. 99), placed above the slit of the spectroscope, by means of which the light of the spark passing from the metallic poles) held between metal holders, is reflected by the small prism (e), placed on the slit into the optical arrangement, and is received into the eye, the metal spectrum being ranged close above that derived from the star; so that the coincidence or otherwise of the two sets of lines can be accurately observed. In this way alone is it possible to arrive at any trustworthy conclusion respecting the composition of the stars, and the existence of certain metals in the stellar atmospheres.

The first result which we lave to notice, then, is that the spectra of various stars differ very widely indeed from one another. As I mentioned to you, Fraunhofer in the year 1814 showed that the stellar spectra were not the same, and that they did not contain the same lines as the spectrum of the sun. I have here coloured drawings which will indicate to you, to begin with, the different nature of these stellar spectra: but these drawings do not pretend to give the exact positions of the various lines in the spectra, but only approximately to represent their general appearance. Here (see Nos. 1 and 2 on the Chromoith. Plate facing this lecture), for example, is a picture of the two stars composing $\beta$ Cygni, in each of which, as you see, the relative position of the lines is totally different; and moreover the arrangement of the lines here is quite different from that of the lines in the solar spectrum.

Dr. Huggins specially describes the spectra of two particular stars, of which we have here an exact diagram (Fig. 100). The upper drawing represents the spectrum of Aldebaran, and the lower of Betelgeux, the star known as $a$ in the constellation of Orion. This drawing is made on a similar plan to Kirchhoffs' diagrams of the dark lines in the solar spectrum. The 


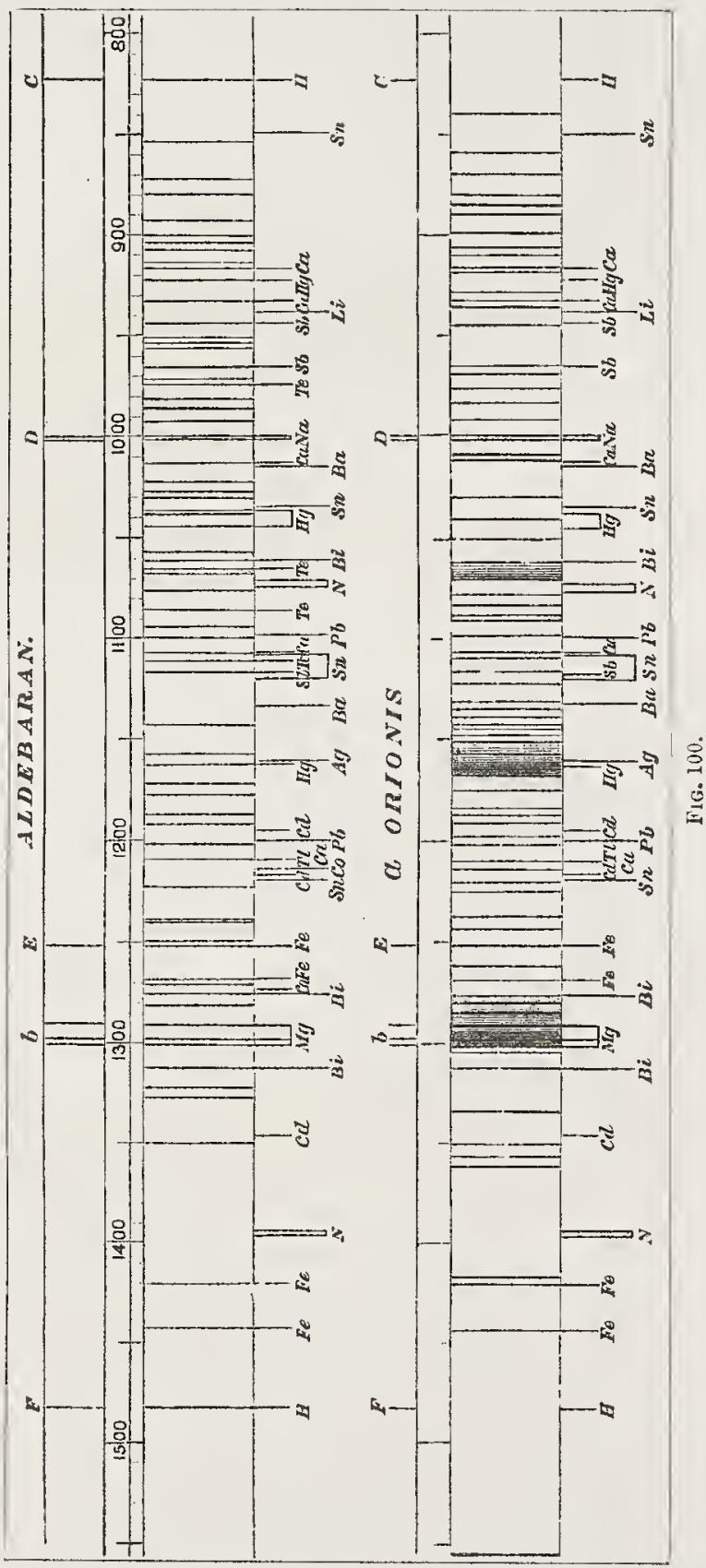


longer lines represent the dark bands in the stellar spectrum, the shorter ones beneath represent the bright lines of the metals with which the star spectrum was compared, the symbols of the elements thus examined being added. In the first place, then, the result at which we have arrived is that the constitution of the starlight, although not identical with the light given off by the sun, is yet similar - that is to say, the light of a fixed star gives a continuous spectrum, interspersed by dark shadows or bands; and hence the conclusion we come to is that the physical constitution of the fixed stars is similar to that of our sun, that their light also emanates from intensely white-hot matter, and passes through an atmosphere of absorbent vapours -in fact, that the stars are suns of different systems. We find, for instance, in these two particular stars to which I am now referring, the $\mathrm{D}$ line caused by sodium exists: the three lines which we know as $b$ are produced by luminous vapour of magnesium. The lines of these substances exactly agree in position with the dark stellar lines; hence both sodium and magnesium are present in the atmosphere of these far distant stars. We also find in Aldebaran that two hydrogen lines, $C$ and $F$, are present; but if we look at the spectrum of a Orionis, we find that the hydrogen lines $\mathrm{C}$ and $\mathrm{F}$ are wanting. Hence we come to the conclusion that hydrogen is present in the atmosphere of the sun and in that of Aldebaran, but that it is wanting in that of Betelgeux. And so I might show you that silver is not present in Aldebaran, nor seen in a Orionis, but that four bright lines of calcium, also seen in the sun's spectrum, are present in both stars. The lines observed in these two stars are at least seventy in number, and Huggins and Miller have found that in Aldebaran we have evidence of the presence of no less than nine e!ements: namely (1) hydrogen, giving the lines $\mathrm{C}$ and $\mathrm{F} ;(2)$ the metal sodium, giving the double line $\mathrm{D}$; (3) magnesium, giving the lines $b$; (4) calcium, giving four lines; (5) iron, giving four lines, and $\mathrm{E}$; (6) bismuth, giving four lines (bismuth is not found in the sun); (7) tellurium, four lines ; (8) antimony is also found, three lines; arid (9) mercury, four lines. Thus the element tellurium, whose name implies a purely eartlily origin, 
is found in the star, although it does not exist in the sun, and is very rare on this earth. There are only two stars-Betelgeux, to which I have just referred, and another star called $\beta$ Pegasi -in which the hydrogen lines are wanting; all the other stars contain hydrogen.

We have, then, now arrived at a distinct understanding of the physical condition of the fixed stars : they consist of a whitehot nucleus, giving off a continuous spectrum, surrounded by an incandescent atmosphere, containing the absorbent vapours of the particular metals. These results are interesting, as bearing on Laplace's nebular theory, because they show that the visible universe is mainly composed of the same elementary constituents, although certain of the stars differ from one anotber widely in their chemical constitution.

The next question to which the attention of the observers was directed was the different character of the light produced by certain of the stars. It is well known that the stars are variously coloured: some shine with a bright white light, others with a yellow light, others with a blue light. Could spectrum analysis give any explanation of the variety of colours exhibited by these different stars? This is proved to be possible, as I shall show you by reference to some diagrams from Huggins' drawings, for most of what I have to say to-day will be the result of his investigations. Here we have, in the first place, a spectrum of a white star, of the star which we all well know as Siritus. In this coloured drawing (No. 3 on the Chromolith. facing the beginning of this lecture) we find a representation of what $\mathrm{Dr}$. Huggins observed in the spectrum of Sirius: you will notice that we have a continuous spectrum with dark lines, and we find that these dark lines or shadows are interspersed pretty generally throughout the length of the spectrum, so that, when all the light enters the eye at once, it produces upon the retina the effect of white light.

We next take an orange-coloured star, known as a Herculis, which is a double star. Here (Chromolith. No. 4) we have a totally different spectrum, and the lines which are most marked in this spectrum exist in the green, blue, and deep red. The 
light is comparatively free from shadows in the yellow and orange portion: and hence, the light from the red, green, and blue portions of this star being weakened, the star shines with a yellow light. This, then, illustrates to us the explanation given by spectrum analysis of the cause of these differently coloured stars in the heavens. We have, however, yet to learn the nature of the substances which produce many of these dark bands in the stellar spectra, and cause the peculiar colour which the stars exhibit.

Another very interesting and well-known astronomical fact next attracts our attention, viz. the existence of certain twin or double stars. It appears that amongst these twin stars, which invariably differ in colour, the blue, green, and purple stars are faint telescopic stars, never found alone, but associated under the protection, as it were, of a brighter red or orange star. Does the same explanation which has been given of the variety of the differently coloured stars also apply to these double stars? We have here (No. 2 on the Chromolith.) a diagram of the combined spectra of the two double stars existing in $\beta$ Cygniabove, the orange star; below, the blue star. This one is orange because there are so many dark lines in the blue and red, whilst there are none at all in the orange portion of the spectrum. In the blue star, on the other hand, we have a vast number of very fine lines existing in the red and in the orange, and a much smaller number existing in the blue: hence the light of this star produces upon the retina the effect of blue light.

Padre Secchi ${ }^{1}$ observing under the clear skies of Rome has investigated the spectra of many hundred stars. He finds it possible to arrange all these stars in four groups, each characterized by a special form of spectrum. Group 1 contains the white stars, Sirius, a Lyræ, Vega, \&c., whose spectra are especially characterized by four black lines, coincident with the bright lines of hydrogen. Group 2 cunsists of the yellow stars, having spectra intersected by numerous fine lines resembling those of our sun : in this group Sechi reckons Pollux, Capella, $\gamma$ Aquilæ,

1 Astronomische Nachrichten, Jan. 28, 1869. 
and our sun. The third group contains the red and orange stars, $a$ Orionis, a Herculis, $\beta$ Pegasi, \&c., the spectra of which are divided into eight or ten parallel columnar clusters of alternate dark and bright bands, increasing in intensity towards the red. Group 4 is made up of the small red stars, whose spectra are distinguished by a succession of three bright zones, increasing in intensity towards the violet. Out of 316 stars examined, Secchi found that 164 belonged to the first type and 140 to the second, whilst the few remaining constituted the third and fourth classes.

Considering the great difficulty of making observations on stellar spectra you will readily understand that the adoption of the photographic method of recording the position of the bright stellar lines has become a matter of the greatest consequence. We owe the working out of this inethod almost exclusively to Dr. Huggins, and the results of the application of photography to the study of stellar spectra confirm in a remarkable manner the conclusions previously arrived at, viz. that the white stars give simpler spectra and, therefore, appear to be hotter than the red and yellow ones. Thus the spectrum of Arcturus, a red star, shows a great analogy with that of our sun, whilst those of the white star's like Lyra and Sirius are totally different from the solar spectrum, being characterized by a few strong lines, apparently almost exclusively due to hydrogen. Fig. 101 represents the appearance of different star spectra showing chiefly the hydrogen lines, as photographed by Dr. Huggins.

Dr. Henry Draper, whose premature death science has to deplore, has also taken some valuable photographs of star spectra; Vogel has likewise classified stellar spectra but in a somewhat different way; we may, however, remember that all such groupings have an arbitrary character.

A very interesting and remarkable observation was made in the month of May, 1866. All at once, in the constellation of the Northern Crown, a star, which was entirely or almost entirely unknown, and which was at any rate a star of very small size, suddenly blazed out and attained a magnitude almost equal tr) that of the largest stars seen in the heavens. The examination 


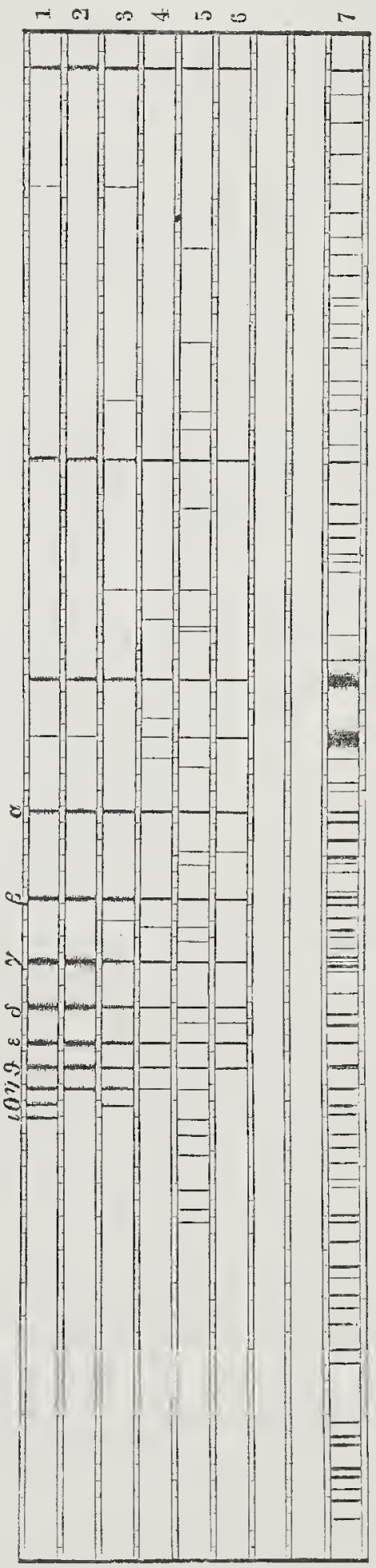

of the spectrum of this particular star naturally excited the liveliest interest, and Huggins and Miller were fortunate enough to be able to investigate spectroscopically at frequent intervals this very remarkable phenomenon, and to their astonishment they found that the spectrum of this star, of which I here show you a diagram (Chromolith. No. 6), differed altogether in its character from the ordinary stellar spectra, inasmuch as superposed on, or in addition to, the ordinary stellar spectrum which you see exhibited here (viz., one consisting of dark lines upon a bright ground), there were in this particular star, bright lines. Now what do bright lines indicate? They indicate the presence of certain gaseous bodies; and the result of the examination of the position of these particular bright lines showed them to be coincident with the bright lines produced by hydrogen.

As this star made its appearance suddenly, so it soon gradually began to diminish in brilliancy, and at last died out, returning, as it were, to its original telescopic dimensions 
of about the tenth magnitude. How was this diminution of the brightness of the star to be explained? The cause of the diminution was revealed to us by the spectroscope, inasmuch as these bright lines were found to dwindle and fade away, and it was observed after a lapse of twelve days, when the star had diminished in brilliancy from the second to the eighth magnitude, that these bright lines became quite invisible. I had the good fortune to see through Dr. Huggins' telescope the very spectrum the drawing of which is now cast upon the screen. The lines when I happened to see them had, however, nearly faded away, but they were still visible. The conclusion to which we must come with regard to this violent outburst is that it was probably due to a rapid ignition of hydrogen, of a similar kind to, though enormously larger than, the sudden outbreaks of incandescent gases seen in the red prominences of our sun. ${ }^{1}$

Another point to which Dr. Huggins directed his attention was the examination of those most interesting and singular astronomical phenomena, the nebulæ. The first nebula which he examined with his spectroscope was one of that class of luminous bodies termed p'anetary nebulæ, in the constellation Draco. On the 20th of August, 1864, Dr. Huggins turned his telescope on to this particular nebula. I am afraid I cannot give you any idea of the delicacy of such observations.

$1 \mathrm{Mr}$. Baxendell's careful estimates of the varying brightuess of this star (Manch. Proc. Nov. 27, 1866) led him to conclude that the intensity of its liglit on Angust 20th, when it reached its minimun, was only $\frac{1}{6} \frac{1}{6}$ th part of that emitted at its maximum on May 12th. From the recent observations of Lockyer and Janssen (see Lecture V.) we learn that the red prominences in the sun are also caused by glowing hydrogen, so that we have a new reason for believing that the sun may belong to the family of variable stars. The qucstion at once suggests itself to the mind, Could a similar conflagration burst out in our system? Of the eff'cets there can be no doubt. The intensity of the sun's rays being increased nearly eight-hundredfold, our solid globe would be dissipated in vapour almost as snon as a drop of water in a furnace. The temperafure in the sunlight would rise at once to that only attainable in the focus of the largest buming-glass, and all life in our planct would instantly cease. In thus speculating on such a possible termination to our terrestrial history, it must be well understood that the probability of such an event occurring is undoubtedly infinitcly small, and that the rcsearches of geologists do not lead us to suppose that any approach to such an occurrence has ever taken place in former geologic ages, although it is not irrational to suggest that ccrtain geological indications of secular variations of terrestrial tcmperature may have becn nroduccd by changes occurring in tle heating powcr of the sun. 
Those, however, of my audience who have seen such a planetary nebula through a telescope will know that the light which those bodies give off is less than that given off by perhaps even the smallest fixed star; and the difficulty of obtaining a spectrum and of examining the nature of this light is, therefore, exceedingly great. ${ }^{1}$ What, however, was Dr. Huggins' astonishment, on bringing the image of this nebula on to the slit of his spectroscope, to observe that he no longer had to do with a class of bodies of the nature of stars !--that instead of having a band of light intersected by dark lines indicating the physical constitution of the body to be that corresponding to the sun and stars, he found the light from this nebula consisted simply of three isolated bright lines, of which we have here (Fig. 102 and in No. 7 of the Chromolith.) a very rough representation.

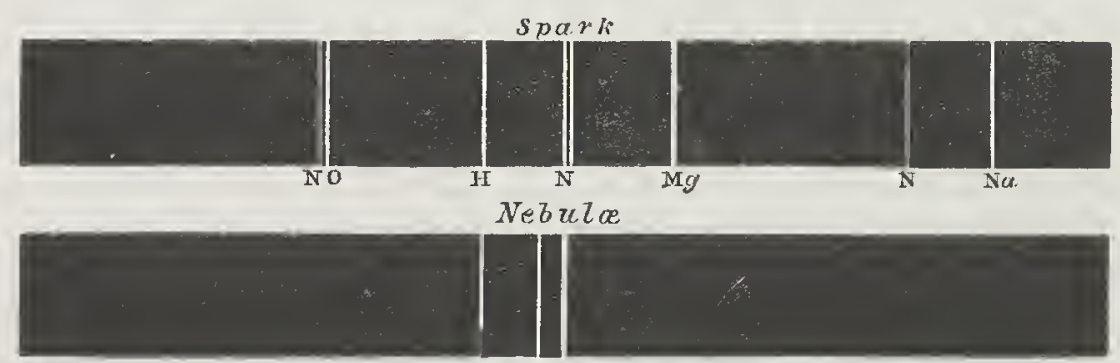

F'c. 102.

If the spectrum of this nebula had been continuous, it would have been very difficult to see it. It was only because the light given off consisted of three bright lines that he was enabled to examine this spectrum at all. You will have already anticipated me in the conclusion that these most curious bodies do not consist of a white-hot nucleus, enveloped in an atmosphere, passing through which the light is absorbed, giving us dark lines; but, on the contrary, that these nebulæe are in the

1 Dr. Huggins gives an idea of the extreme faintness of the more distant nebule. "The light of some of those visible in a moderately large instrument has been estimated to vary from $x_{5}^{\frac{1}{5} 50}$ to $\frac{1}{10} 000$ of the light of a single sperm candle consuming 158 grains of material per hour, viewerl at a distance of a quarter of a mile; that is, such a candle a quarter of a mile off is 20,000 times more brilliant than the nebula!" 
condition of luminous gases, and that it really is nebulous or, strictly speaking, gaseous matter with which we have here to do.

The history of these nebulæ is one into which I cannot enter. You all know that the names of Herschel and of Rosse are associated with the most accurate and careful examination of these particular bodies, and that it is especially to the late Lord Rosse that we are indebted for the very careful examination, by means of his magnificent telescope, of these inost singular bodies. It now became a matter of the very greatest interest to examine the character of the light given off by the other nebulæ. I will indicate to you the appearance of some of these nebulæ, though very roughly, by means of the drawings. The nebula in Aquarius

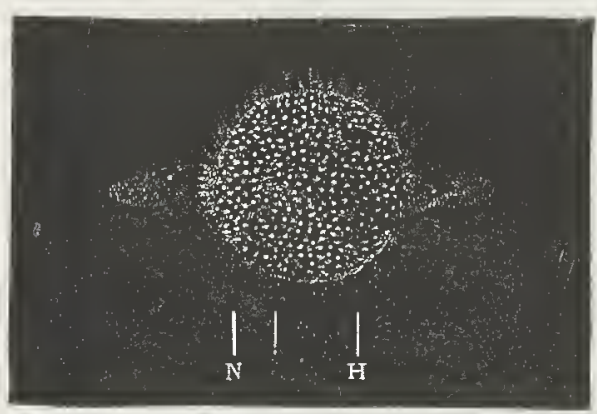

FIG. 103

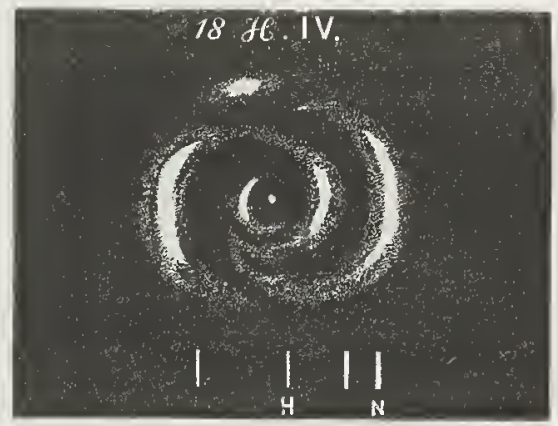

FiG. 104.

is seen in Fig. 103. 'The drawing of this nebula gives you but a faint notion of its appearance in the telescope. I may also show you another nebula (Fig. 104) having a spiral form, and whose spectrum exhibits a fourth bright line. Dr. Huggins then found, on examining the character of the lines which these nebulæ give off, that the spectrum was likewise distinguished by the same three distinct bright lines. The questions will occur' to every one, Do all the nebulæ give similar spectra? and especially, Do those which the telescope had certainly resolved. into a close aggregation of bright points give gaseous spectra?

Dr. Huggins has examined the spectra of about seventy nebulæ, and he finds that these can be divided into two great groups. One group (about one-third of the whole number) 
consists of the nebulæ giving spectra of three bright lines similar to those which I have shown you, or else containing only one or two of these bright lines. "Of these seventy nebulæ, about one-third belong to the class of gaseous bodies: the light of the remaining nebulæ and clusters becomes spread out by the prism into a spectrum which is apparently continuous." To the class of nebulæ giving continuous spectra the well-known nebula in Andromeda belongs. This singularly shaped body is visible to the naked eye (Fig. 105), and is not unfrequently mistaken for a comet. It was observed as early as the year 1612, by Simon

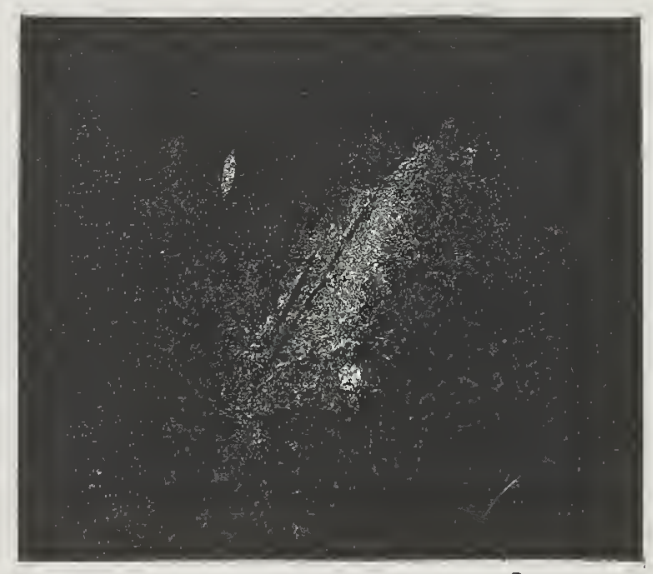

FiG. 105

Marius. The spectrum of this nebula, though apparently continuous, possesses some curious characteristics, the whole of the red and a portion of the orange being wanting, besides the brighter parts exhibiting an unequal and mottled appearance.

It next becomes a most important point to ascertain the chemical nature of the three bright lines in the spectra of the gaseous nebulæ. Dr. Huggins finds that the brightest of the lines of the nebula coincides with the strongest of the lines which are peculiar to nitrogen, whilst the faintest of the lines was found to coincide with the green line $(F)$ of hydrogen. The middle line of the three does not coincide with a line of any known element. 
The upper part of this drawing (Fig. 106) is intended to represent a portion of the solar spectrum. Here you see the dark line $\mathrm{F}$, due to hydrogen, and the lines formed by magnesium, corresponding with the letter $b$. Below are the lines corresponding with some of the bright lines of hydrogen, barium, nitrogen, and magnesium, whilst between them are the three lines observed in these nebulæ.

There is but little doubt that the presence of the line coincident with $\mathrm{F}$, really proves the existence of hydrogen in this nebula; but it is much more difficult to decide whether the coincidence of the green line with one component of the double

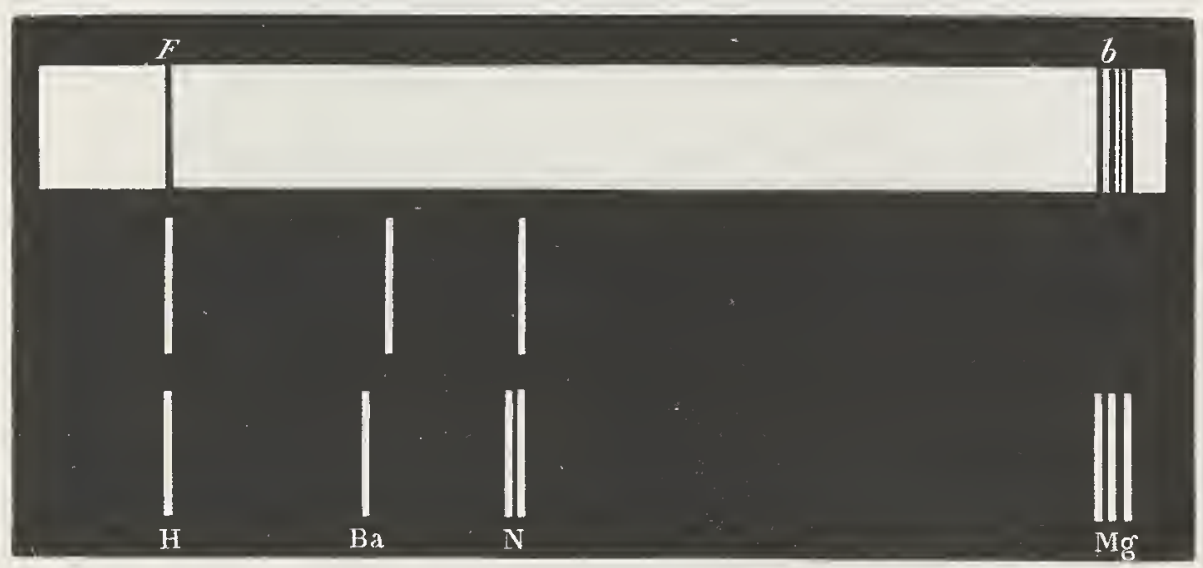

Fig. 106.

nitrogen line may not be purely accidental, for we should certainly expect both components to be present, if the line was due to the presence of nitrogen.

I am almost afraid to take up your time in exhibiting to you many of these diagrams; still I must not omit to show you one of the well-known nebulæ in the sword-handle of Oiron (see Fig. 107), which was discovered by no less a personage than the astronomer Huyghens in 1656. I will read to you Sir Jolın Herschel's description of this nebula: "The general aspect of the less numerous and cirrous portion is simply nebulous and irresolvable; but the brighter portion immediately adjacent to the trapezium forming the square front of the head is shown 
with the eighteen-inch reflector broken up into masses, whose mottled and curdling light evidently indicates, by a sort of granular texture, its consisting of stars, and when examined under the great light of Lord Rosse's reflector, or the exquisite defining power of the great achromatic at Cambridge, U.S., is evidently perceived to consist of clustering stars. There can therefore be little doubt as to the whole consisting of stars too minute to be discerned individually, even with those powerful aids, but which become visible as points of light when closely adjacent in the more crowded parts."

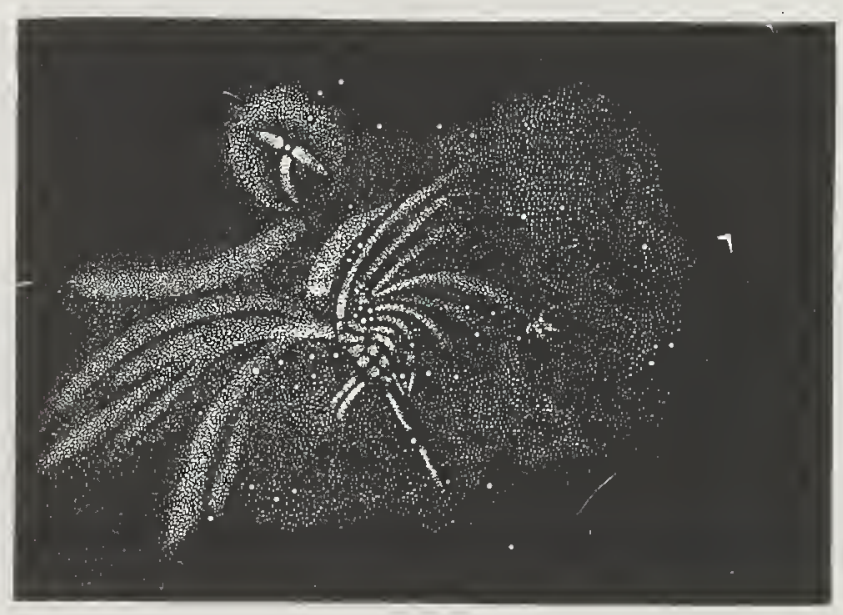

F1G. 107.

It becomes a matter of the greatest interest to learn the conclusions to which the spectroscope leads us, concerning the nature of these resolvable portions of this nebula. Here you have Dr. Huggins' own words on this important subject: "The results of telescopic observation on this nebula seem to show that it is suitable for observation as a crucial test of the correctness of the usually received opinion, that the resolution of a nebula into bright stellar points is a certain and trustworthy indication that the nebula consists of discrete stars after the order of those which are bright to us. Would the brighter portions of the nebula adjacent to the trapezium, which have been resolved into stars, present the same spectrum as the 
fainter and outlying portions? In the brighter parts would the existence of closely aggregated stars be revealed to us by a continuous spectrum, in addition to that of a true gaseous matter?" The answer of the spectroscope comes to us in no doubtful tone: "The light from the brightest parts of the nebula near the trapezium was resolved by the prisms into three bright lines, in all respects similar to those of the gaseous nebula.... The whole of this great nebula, as far as lies within the power of my instrument, emits light which is identical in its characters; the light from one part differs from the light of another part in intensity alone."

Recently Dr. Huggins has actually succeeded in photographing the spectrum of this nebula. The position of the lines seen in the photograph is shown in the lower part of Fig. 108. The upper part represented the typical spectrum of white stars.

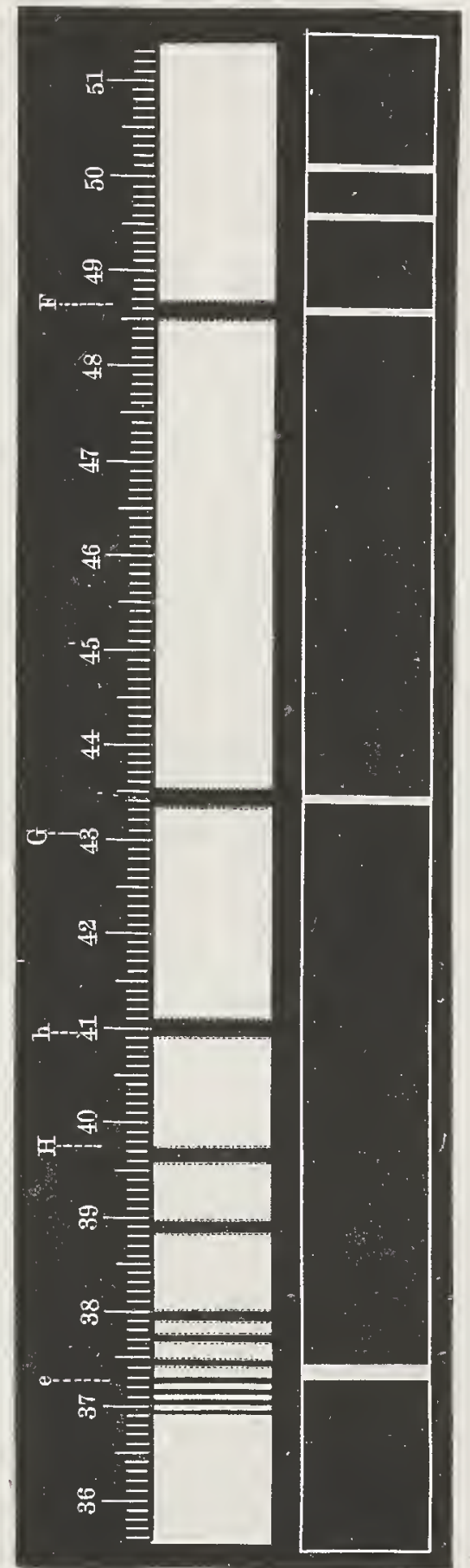

Y 2 
The true nature of comets is involved in even greater obscurity than that of the nebulæ. Dr. Huggins has examined some of these singular bodies. Brorsen's comet, for instance, is a recurring comet, having a period of rotation of five and half years, and its spectrum consisted in 1868 of three bright bands, the central one of which lay between $F$ and $b$, and in addition a very faint continuous spectrum was seen. These observations settled the physical character of the comet: it consists of a mass of glowing gas, and is self-luminous, a portion only feebly reflecting the sunlight. From the drawing (Fig. 109) it will be seen that the light from Brorsen's comet differs from the light emitted by the nebulæ, inasmuch as the lines in the comet spectrum are not identical in position with the lines yielded by the nebulæ. Nor, in fact, are these particular lines-roughly represented here-identical in position with the bands of any known substance. (See Fig. 109.) At the bottom of the diagram the lines of the nebulæ are given; next we have the spark spectra of nitrogen, hydrogen, magnesium, and sodium; at the top we see the solar lines; and between these lie the particular lines of the comet; hence this comet contains something not found in the nebulæ, whose lines do not coincide with any substance known on earth, so far as examination has yet proceeded; and, therefore, we really do not yet know of what this comet consists. These observations, I am sure you will all admit, open out to us subjects of the deepest interest.

Dr. Huggins has also published an account of his observations on the second comet of 1868, known as Winnecke's comet, a drawing of which is seen in Fig. 110. Spectroscopic investigation has shown that this comet contains luminous carbon, or carbon compounds : its spectrum, together with that of Brorsen's comet, is seen in Fig. 109. This gives that modification of the carbon spectrum which we obtain when the electric spark is passed through olefiant gas, the coincidence of the bright lines of Winnecke's comet with those of the spark taken in olefiant gas being clearly seen. In order to obtain an exact comparison of the lines of the comet with those of incandescent olefiant gas, the arrangement shown in Fig. 111 was employed. This consists of a glass bottle, $a$, converted into a gasholder containing the 
olefiant gas. This was connected with the glass tube $b$, through which the gas passed, and into which two platinum wires, $e$ and $f$, had been soldered. This tube being then placed before the

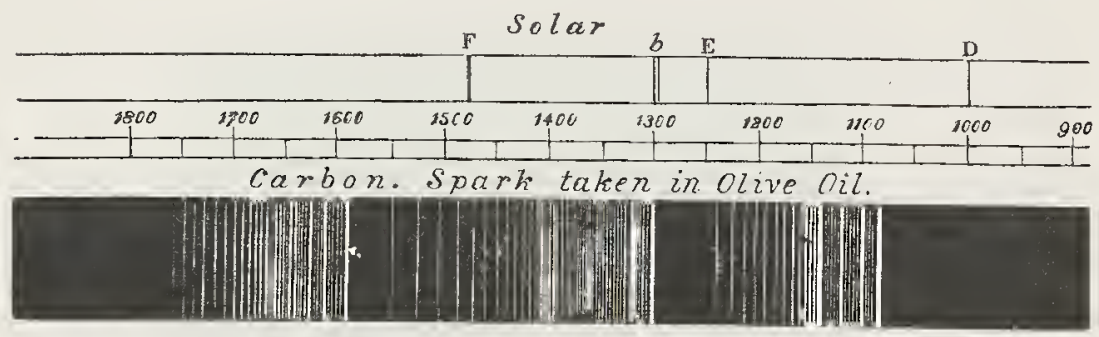

Carbon. Spark taken in Olefiant Gas.

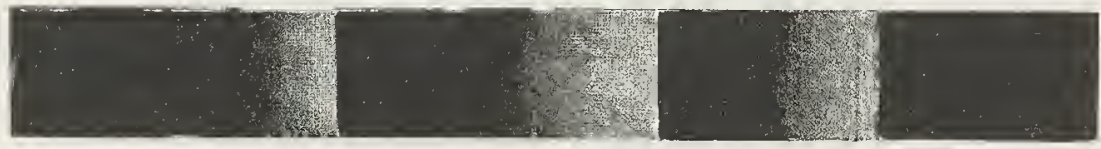

Comet II. 1868.

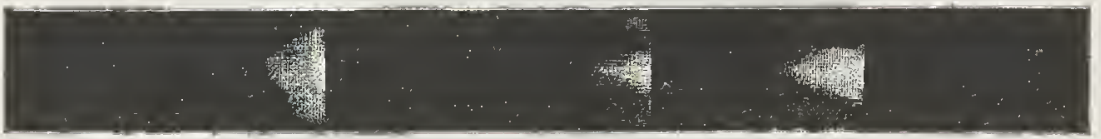

Brorsen's Comet, 1868.
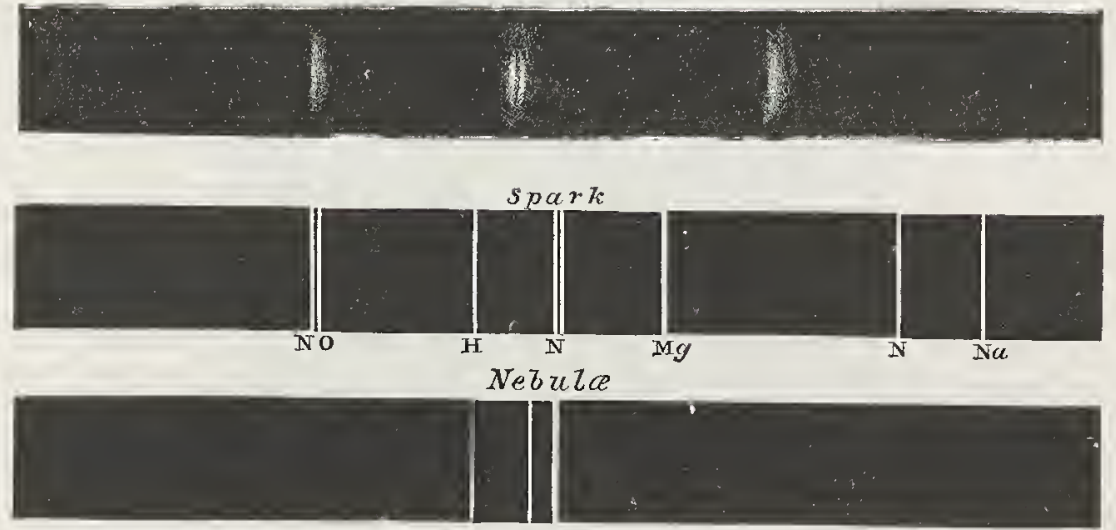

Frg. 109

mirror of the spectroscope (c, Fig. 99) the light of the spark, passing through the gas in the tube by means of the wires, was 
reflected into the instrument $d$, and its spectrum was seen immediately below that of the comet. The two sets of bands were not only found to agree precisely in position, but they

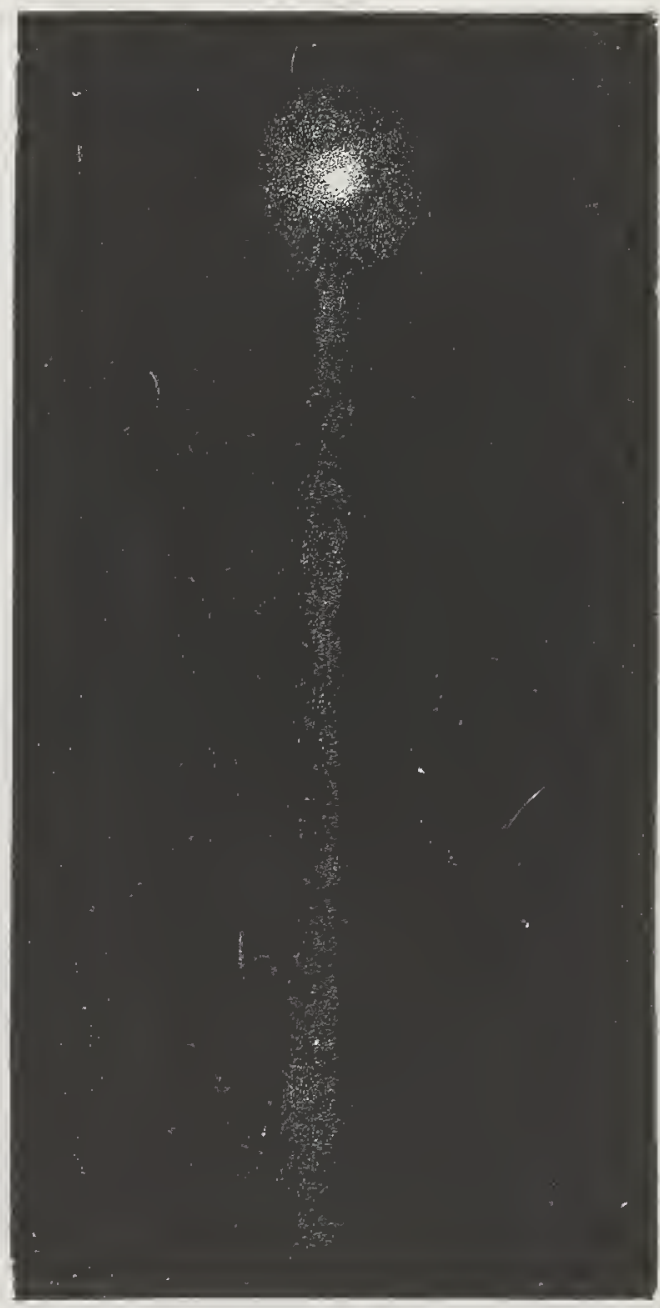

FIG. 110

corresponded in their general characters, and in their relative brightness. Hence we can scarcely doubt that carbon is really the cause of these bright lines. 
We know now that this spectrum is due to the element carbon and not to any of its compounds, but in what way the carbon is rendered luminous is doubtful. An electric discharge through hydrocarbons partially dissociates these compounds, and shows a carbon spectrum, and hence many astronomers now believe that an electric discharge is the cause of the luminosity of comets. When hydrocarbons burn in air or oxygen, the same spectrum is also seen, the element carbon being apparently luminous while it passes from combination with hydrogen, into that with oxygen.

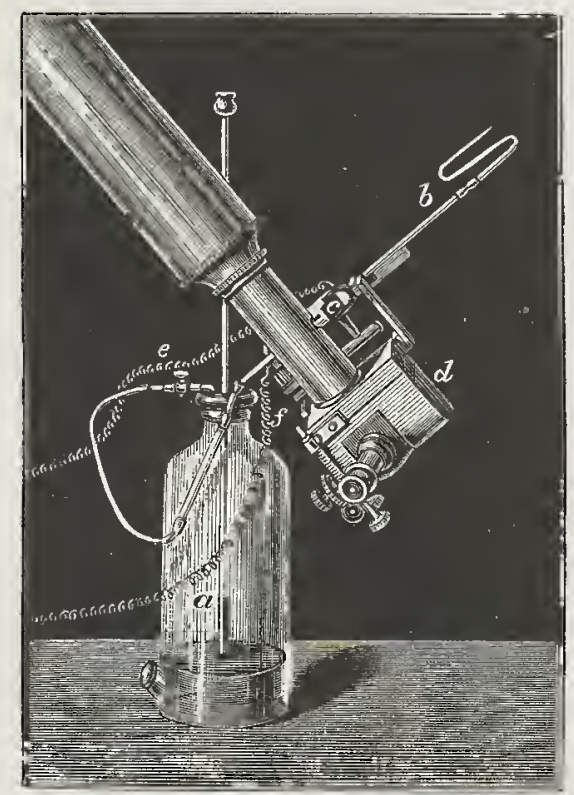

FIG. 111.

A number of other comets have since been found also to give the carbon spectrum, as for instance Encke's comet examined by Young in 1871; and Coggia's comet examined by Huggins in 1874. Different and very interesting results were obtained by the spectroscopic examination of the Comet I, 1882 (Wells). The three ordinary comet bands were faint, but as the comet approached the sun, and became, therefore, brighter, the yellow sodium lines became visible, and gradually increased in 
intensity, the carbon bands disappearing. Unfortunately we possess no observations of the spectrum of this comet during its recession from the sun. Dr. Huggins obtained a photograph of the comet spectrum between the lines $\mathrm{F}$ and $\mathrm{G}$, about the time at which its spectrum was found to change. Fig. 112 represents the

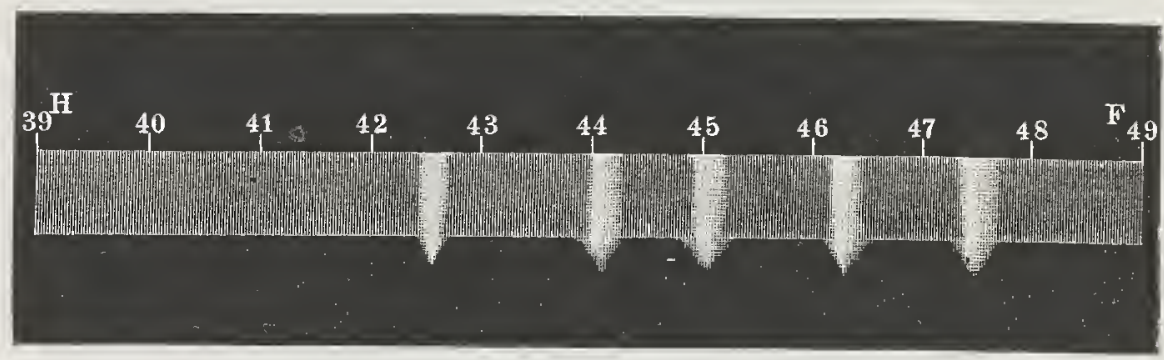

FIG. 11?.

lines appearing on the photograph between the above-mentioned points. The wave-lengths of the bands, which are not as yet identified, were found by Dr. Huggins to be as follows: 4769 , $4634,4507,4412,4253$.

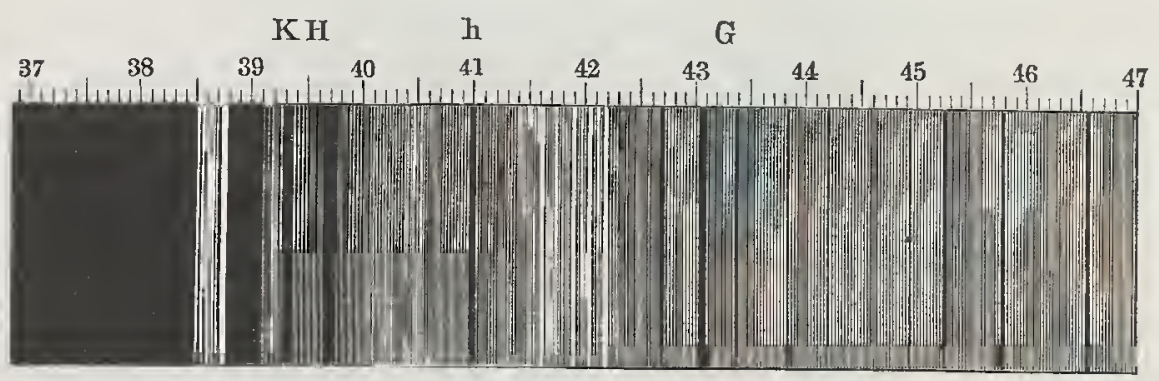

FIG. 113.

Fig. 113 is a drawing from a photograph, also taken by Dr. Huggins, of the spectrum of another comet (Comet II, 1881). The spectrum shows in the first place the dark Fraunhofer lines, proving that part of the light, sent out by the comet, was merely reflected sunlight. But in addition, bright lines appeared, which proved to be identical with certain bands seen when an electric spark passes through cyanogen gas. 
I have still to speak of another result obtained by Dr. Huggins. Not only are we in a position to determine the constitution of the stars and of the nebulæ, but, strange as it may appear, we can actually, by spectroscopic observations, get some ideas respecting the relative motions of these bodies and our earth. It is impossible in the time at my disposal to explain to you the mode in which philosophers or physicists have arrived at the truth of the conclusion, originally propounded by Doppler in 1841, that, when one luminous body is approaching another very rapidly, the kind of light which is received on the retina from that body moving at a very great speed differs in some respects from the light which the retina would receive were that body at rest. An illustration from sound may perhaps render this matter more plain. If in a railway train you listen to the whistle of the engine of another train which is meeting you, you will notice that as the two trains approach the pitch of the note of the whistle alters. ${ }^{1}$ This is because (owing to the sound being produced by the vibration of the particles of the air), when the two trains are approaching each other, the waves of sound are, as it were, forced together and fall more rapidly upon the ear than they would do if the two trains were in a state of rest. The same thing happens with regard to light. If the one object which is luminous is approaching the retina very rapidly, the vibrations causing light will fall more frequently on the retina than if the bodies were at rest; and then the position of the dark lines will be shifted in the direction of the most refrangible rays; whilst, if the bodies were separating, the shifting would take place in the direction of the red or least refrangible rays. Dr. Huggins has actually found that in some of these stars there is a slight disturbance in the position of the hydrogen line $\mathrm{F}$ : he first proved that it is really hydrogen which is present, and then he showed that there is a slight deviation observed between the hydrogen line and the line existing in the star; and hence he comes to the conclusion that there is motion of recession between the earth and what we call a "fixed star."

1 An exact experiment of this kind was made in 1845 by Ballot of Utrecht, in which the alteration of tone for a given velocity was determined. 
Here you see a diagram (Fig. 114) showing the slight deviation which the line F exhibits in Sirius' light. You observe that the narrow line of hydrogen from the vacuum-tube does not coincide with the middle of the Sirius $\mathbf{F}$ line, but crosses it at a distance from the middle, which may be represented by saying that the want of coincidence is apparently equal to about one-third or one-fourth of the interval separating the two $\mathrm{D}$ lines. Hence the $\mathrm{F}$ line has been distinctly deviated towards the red rays, and the vibrations proceeding from the star must have been retarded in their passage; or, in other words, there is a motion of recession between our earth and Sirius of such a nature that the wave-length of the $F$ ray has been increased by the 0.109 millionth part of a millimetre. The velocity of this recession

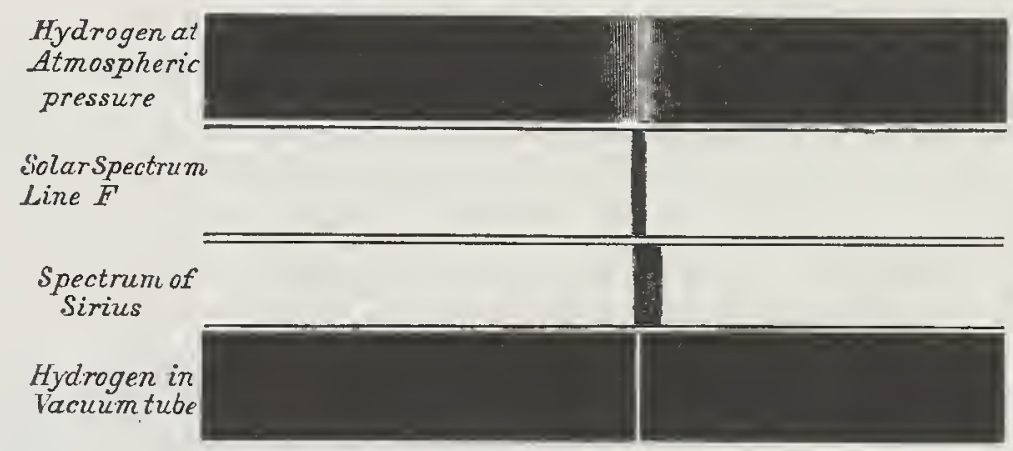

FrG. 114

can easily be calculated. Light travels at the rate of 185,000 miles per second; the ware-length of the $\mathrm{F}$ line is 486.5 millionths of a millimetre: now the velocity with which the two bodies move away from each other stands to the velocity of light in the same proportion as the observed difference of wavelength does to the wave-length of the particular ray; or-

$$
486.5: 0 \cdot 109:: 185000: x=41 \cdot 4 \text {. }
$$

Hence we conclude that this motion of recession between the earth and the star Sirius is 41.4 miles per second, or that if the earth were stationary, instead of moving in its orbit, as it did at the time of the cxperiment, away from Sirius, there would be 
a proper motion of recession of the star of twenty-nine miles per second.

Astronomers have, as you are aware, long ago shown by telescopic observation that the whole of our solar system is moving in space in the direction of the constellation Hercules. Such observations can, however, only be made when the motion to be noticed is at right-angles to the line of vision, whilst the deductions which $I$ have just described as being made with the spectroscope can only be arrived at when the motion is in the direction of the line of vision. We see, therefore, that by a happy combination of these two methods we are enabled to ascertain the actual rate of motion of the stars in space. Before we can, however, attain an exact knowledge of these motions, the delicacy of our spectroscopes must be much improved. This has already been partially accomplished by Zöllner in his reversing spectroscope, by means of which a displacement equal to the $\frac{7}{200}$ part of the distance between the $\mathrm{D}$ lines can be seen. With this instrument Zöllner was not only able to measure the proper motion of the stars, but he has even rendered visible the shifting in the Fraunhofer's lines which must occur from the rotation of the sun on its axis, and thus he was able to distinguish between those which are really solar lines and those caused by absorption in our own atmosphere, which naturally cannot exhibit a shifting in position from the motion of the sun on its axis. The amount of deviation for a point on the solar equator as observed in this way by Dr. Vogel was found to vary from 0.014 to 0.015 millionth of a millimetre, corresponding to a velocity of from 0.35 to 0.42 geographical mile in the second, the generally accepted velocity being 0.27 mile. ${ }^{1}$

Dr. Huggins, in $1872,{ }^{2}$ communicated to the Royal Society some further observations made with a new and magnificent instrument upon this most interesting subject. He finds not only that lis previous conclusions respecting the proper motion of Sirius are confirmed, but by his improved and more delicate methods of measurement he has succeeded in ascertaining that many other so-called fixed stars are in fact moving,

1 Phil. Mag. Jan. 1872.

${ }^{2}$ June 13, 1872. 
some towards and some away from our planet. Thus Sirius, Betelgeux, Rigel, Castor, Regulus, $\delta$ Ursæ Major, and others which are situated in a part of the heavens opposite to Hercules, towards which the sun is advancing, are moving from the earth, whilst stars such as Arcturus, Vega, and $a$ Cygni, situated in the neighbourhood of this region, show a motion of approach. These drifting motions of certain stars had previously been considcred by Mr. Procter; and now spectroscopic investigation shows that such drifting towards and away from our system does really occur. One of the most remarkable instances pointed out by Mr. Procter is that four stars $(\beta, \gamma, \delta$, and $\epsilon)$ of the Great Bear have a community of proper motions, whilst $a$ and $\eta$ of the same constellation have a proper motion in the opposite direction. Now spectroscopic analysis shows that the stars $\beta, \gamma, \delta, \epsilon$, and $\zeta$ have a common motion of recession, whilst the star $a$ is seen to be approaching the earth.

This subject has more recently been taken up at Greenwich and now forms part of the regular work of the Observatory, and the results of the Greenwich observations on this question may be found in the monthly notices of the Royal Astronomical Society.

In the last lecture we learnt that the solar atmosphere, and especially the outlying portion, chiefly consisting of hydrogen gas, is constantly most violently agitated by storms of white-hot calcium and hydrogen, which blow with such fierceness, that, compared with these, our most destructive tornadoes are mere summer breezes. We saw also that these storms give rise to the peculiar appearances which we observe on the solar surface, viz. the sun-spots and the faculw. Lockyer has determined the velocity with which the glowing hydrogen rushes forwards or backwards in these storms by observing the peculiar alterations in breadth and position which the $\mathrm{F}$ line exhibits. These alterations in refrangibility can only be seen when the motion is one of approach to or of recession from the observer's eye; and hence upon the sun's disc we can only observe an upward or downward motion, whilst in the chromosphere or on the sun's limb we may obtain evidence of lateral or cyclonic movements, although we 
cannot there see any upward or downward currents. Now, in looking at the sun's surface tbrough his spectroscope, Lockyer saw the line $\mathrm{F}$ sometimes appear as a dark line, and sometimes flash out as a bright line on a darker ground. In the former case the $F$ line is seen to be bent and in several places shifted towards the red end of the spectrum, whilst at other points a displacement towards the violet end is noticed. Sometimes the line disappears altogether, and before it vanishes it is seen to swell or bulge out, or to terminate in a knotty bulbous form. Then it again becomes invisible as the slit passes over the faculæ, especially when they are near some small spots, and again is seen to swell out several times in the course of a few
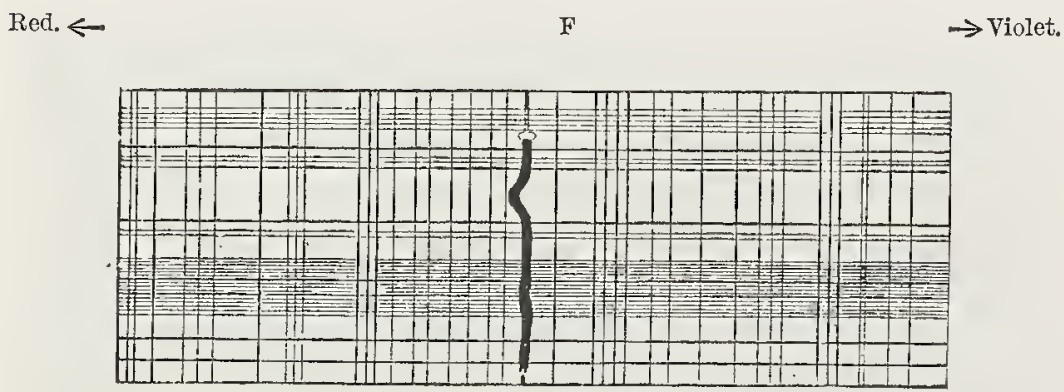

Fra. 115.-Deviation of the $\mathrm{F}$ line in a Spot-Spectrum (Lockyer).

seconds, then to shift towards the violét, and lastly to appear as a straight bright line, without any thickening; over a small spot.

These variations in the position and darkness or luminosity of the $\mathrm{F}$ line are attempted to be represented in Fig. 115, giving the appearance of a spot-spectrum: the dark horizontal lines show that a general absorption occurs where the spots are darkest. In the neighbourhood of one of these spots a facula is situated; and there the $F$ line is seen to be bright and bulbous.

The other Fraunhofer's lines are, as you see, represented by vertical lines in the drawing (Fig. 115). These serve, according to Lockyer, as milestones ${ }^{1}$ by which to measure the velocity

1 Alteration of wave-length have also been detected in the sodium, iron, and magnesium lines in a spot-spectrum. 
upwards or downwards, with which these eruptive masses of hydrogen are moving, for these shiftings and twistings of the $F$ line are of course nothing else than rapid alterations in the wave-length of the $\mathrm{F}$ rays.

If, therefore, a shifting towards the violet is observed equal to the $\frac{1}{10000000}$ part of a millimetre (see No. 1, Fig. 116), this shows that the incandescent hydrogen is rushing upwards with a velocity of thirty-eight miles per second; whilst a like deviation towards the red, as in No. 3 in the figure, proves that a downward current is blowing with an equal velocity. Sometimes the bright line is seen at the violet side of its normal position, whilst a dark line is found shifted towards the red : this proves that an

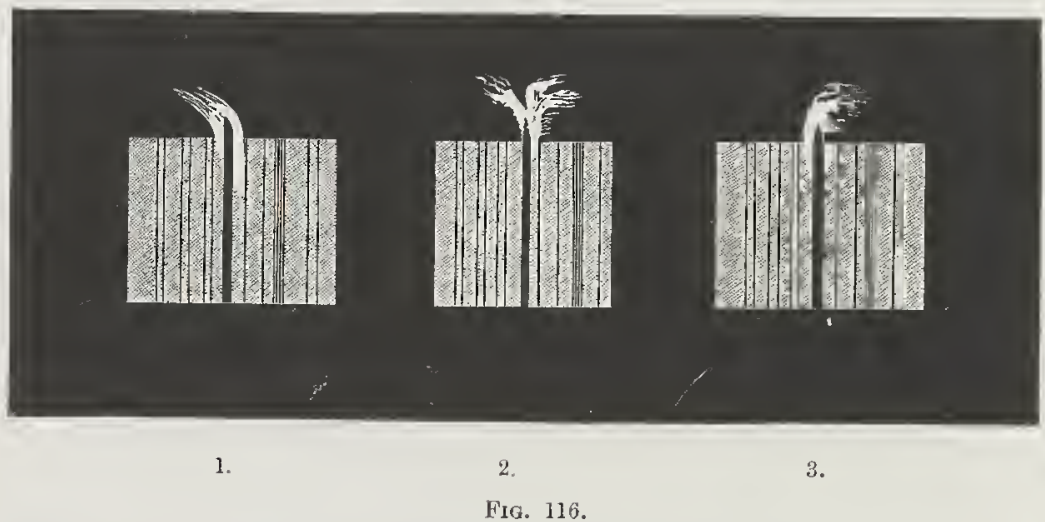

upward rush of intensely-heated incandescent hydrogen occurs on the one side, and a downward rush of cool absorbing yas on the other.

Fig. 117 represents an observation by Professor C. A. Young, in which two red-lines are seen displaced towards the violet in the neighbourhood of a sun-spot.

The lateral motions near the limb are observed by means of the shifting of the bright lines in the spectrum of the chromosphere. The velocity of these eyclones is almost incredible. Lockyer observed such a circular storm on the 14th March, 1869 (No. 2, Fig. 116). The slit of his spectroscope was about $\frac{\bar{s}}{50}$ of an inch in width; and as the diameter of the image of the sun 
cast by his telescope was only 0.94 of an inch, he could observe a strip on the sun's surface some 1,800 miles wide.

If we now assume that the solar circular storm spreads over a width of 1,500 miles and is moving at a fearful rate, we must be able by help of the spectroscope to distinguish between those portions whicl move towards us and those portions which are moving in the opposite direction. The drawing (Fig. 116) shows that this is indeed the case. When the slit was placed upon the centre of this cyclone, the bright line which, in fact, is a continuation of the dark $F$ line, was seen to be shifted to a distance which corresponds to a velocity of forty miles per second. When the slit was directed towards the edge of the storm, it was clear that on the one side the current was moving

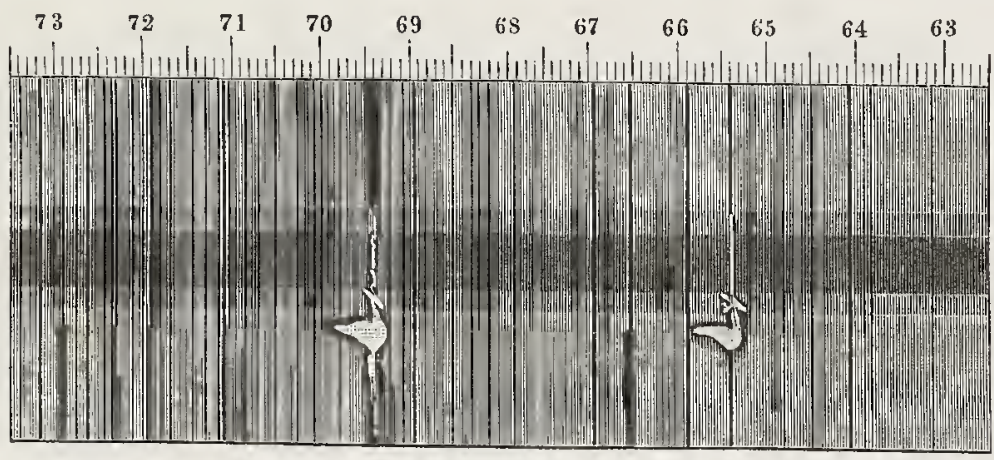

Fig. 117.

towards us, and on the other side away from us, because the deviation in the first case was towards the violet, and in the second towards the red end of the spectrum. The great rapidity with which the prominences appear and disappear shows that the hydrogen gas of which these flames are composed is often in a state of the most violent eruption. From the observation of the $\mathrm{F}$ line in a prominence on May 12, 1869, the following conclusions were drawn: (1) a portion of this flame was in a position of rest as regards us, as is seen from the fact that near the photosphere the bright $F$ line was exactly coincident with the ordinary dark line $\mathrm{F}$ of the solar spectrum; (2) the bright continuation of this line being displaced in a slanting direction 
towards the violet end of the spectrum shows that a portion of the hydrogen was moving towards the earth, but with different velocities, the upper layers moving more rapidly than the lower portions. Lockyer on this occasion saw the $\mathrm{F}$ line triple, and the greatest lateral displacement of the line corresponded to the enormous velocity of 120 miles per second! A still more striking instance of the almost incredible velocities which occur in the movements in the solar atmosphere was observed by Professor Young of Dartmouth College on 7th Sept. 1871. At noon on that day he noticed an enormous protuberance of hydrogen cloud on the eastern limb of the sun. It had remained with little change since the noon of the preceding day. It was made up mostly of filaments nearly horizontal with its lower surface at a height of some 15,000 miles; but was connected with the layer of red hydrogen by three or four vertical columns, brighter and more active than the rest. Rcturning to the telescope half an hour later, Professor Young found, in place of the quiet cloud a mass of detached vertical masses rapidly ascending. Some of them had already reached a height of 100,000 miles, and they continued to rise, with a motion almost perceptible to the eye, until in ten minutes the uppermost were more than 200,000 miles above the solar surface; the velocity of ascent was therefore 166 miles per second !

These spectroscopic results become, if possible, still more interesting when they are combined with telescopic observations. On April 21, 1869, Lockyer was observing a spot near the sun's limb, and at 7 h. $30 \mathrm{~m}$. A.M. a protuberance in full activity was seen in the field of view. The hydrogen lines were very bright, and, as the spectrum of the spot was visible at the same time, it was easy to see that the red flame was moving more quickly than the spot, and that the prominence was fed, so to speak, from the preceding edge of the spot. The violent eruption had torn up a portion of the upper layer of the photosphere beyond the usual limits of the chromosphere, and high up in the hydrogen flame floated a cloud of magnesinm vapour! At 8h. $30 \mathrm{~m}$. there was comparative quiet, but after an hour had elapsed the action had commenced afresh, and the cxistence of a violent 
cyclone was plainly seen. On the same day, and nearly at the same time, a photograph of the sun was taken at Kew, in which the violent changes observed by Lockyer could be clearly traced, the limb of the sun being torn away exactly at the place where the spectroscope had shown a cyclone was situated.

Who could have dreamt a few short years ago that we should so soon attain such an insight into the processes of creation? And yet, great though the results of spectrum analysis already are, they are but a tithe of the numerous questions which this branch of discovery has opened up,-questions of such number and magnitude, that nany generations of men will pass away before they are all satisfactorily answered.

In conclusion, I may say that I feel it would be idle in me to attempt to add any words as to the importance and grandeur of the subjects which in these lectures I have so imperfectly brought before you. I leave the facts to speak for themselves, and I have only to thank you most heartily for the kind attention with which you have honoured me. 


\section{LECTURE VI.-APPENDIX A.}

\section{EXTRACT FROM A MEMOIR ON THE SPECTRA OF SOME OF THE FIXED STARS. ${ }^{1}$}

BY W. HUGGINS, F.R.A.S., AND W. MILLER, M.D., LL.D., TREAS. AND V.P.R.S. ${ }^{2}$

\section{$\S$ I. Introduction.}

1. THE recent discovery by Kirchhoff of the connection between the dark lines of the solar spectrum and the bright lines of terrestrial flamcs, so remarkable for the wide range of its application, has placed in the hands of the experimentalist a method of analysis which is not rendered less certain by the distance of the objects the light of which is to be subjected to examination. The great success of this method of analysis as applied by Kirchhoff to the determination of the nature of some of the constituents of the sun rendered it obvious that it would be an investigation of the highest interest, in its relation to our knowledge of the general plan and structure of the visible universe, to endeavour to apply this new method of analysis to the light which reaches the earth from the fixed stars. Hitherto the knowledge possessed by man of these immensely distant bodies has been almost confined to the fact that some of them, which observation shows to be united in systems, are composed of matter subjected to the same laws of gravitation as those which rule the members of the solar system. To this may be added

I Phil. Trans. 1864.

2 The late Professox of Chemistry, King's Collerge, London. 
the high probability that they must be self-luminous bodies, analogous to our sun, and probably in some cases even transcending it in brilliancy. Were they not self-luminous, it would be impossible for their light to reach us from the enormous distance at which the absence of sensible parallax in the case of most of them shows they must be placed from our system.

The investigation of the nature of the fixed stars by a prismatic analysis of the light which comes to us from them, however, is surrounded with no ordinary difficulties. The light of the bright stars, even when concentrated by an object-glass or speculum, is found to become feeble when subjected to the large amount of dispersion which is necessary to give certainty and value to the comparison of the dark lines of the stella spectra with the bright lines of terrestrial matter. Another difficulty, greater because it is in its effect upon observation more injurious, and is altogether beyond the control of the experimentalist, presents itself in the ever-changing want of homogeneity of the earth's atmosphere through which the stellar light has to pass. This source of difficulty presses very heavily upon observers who have to work in a climate so unfavourable in this respect as our own. On any but the finest nights the numerous and closely approximated fine lines of the stellar spectra are seen so fitfully that no observations of value can be made. It is from this cause especially that we have found the inquiry, in which for more than two years and a quarter we have been engaged, more than usually toilsome; and indeed it has demanded a sacrifice of time very great when compared with the amount of information which we have been enabled to obtain.

2. Previously to January, 1862, in which month we commenced these experiments, no results of any investigation undertaken with a similar purpose had been published. With other objects in view, two observers had described the spectra of a few of the brighter stars, viz. Fraunhofer in 1823, ${ }^{1}$ and Donati, whose memoir, "Intorno alle Strie degli Spettri stellari," was published in the "Annali del Museo Fiorentino" for $\mathbf{1 8 6 z}$.

Fraunhofer recognized the solar lines $\mathrm{D}, \mathrm{E}, b$, and $\mathrm{F}$ in the

${ }^{1}$ Gilbert's Annalen, vol, lxxiv. p. 374 . 
spectra of the moon, Venus, and Mars: he also found the line D in Capella, Betelgeux, Procyon and Pollux; -in the two former he also mentions the presence of $b$. Castor and Sirius exhibited other lines. Donati's elaborate paper contains observations upon fifteen stars; but in no case has he given the positions of more than three or four bars, and the positions which he ascribes to the lines of the different spectra relatively to the solar spectrum do not accord with the results obtained either by Fraunhofer or by ourselves. As might have been anticipated from his well-known accuracy, we have not found any error in the positions of the lines indicated by Fraunhofer.

3. Early in 1862 we had succeeded in arranging a form of apparatus in which a few of the stronger lines in some of the brighter stars could be seen. The remeasuring of those already described by Fraunhofer and Donati, and even the determining the positions of a few similar lines in other stars, however, would have been of little value for our special object, which was to ascertain, if possible, the constituent elements of the different stars. We therefore devoted considerable time and attention to the perfecting of an apparatus which should possess sufficient dispersive and defining power to resolve such lines as $\mathrm{D}$ and $b$ of the solar spectrum. Such an instrument would bring out the finer lines of the spectra of the stars, if in this respect they resembled the sun. It was necessary for our purpose that the apparatus should further be adapted to give accurate measures of the lines which should be observed, and that it should also be so constructed as to permit the spectra of the chemical elements to be observed in the instrument simultaneously with the spectra of the stars. In addition to this, it was needful that these two spectra should occupy such a position relatively to each other as to enable the observer to determine with certainty the coincidence or non-coincidence of the bright lines of the elements with the dark lines in the light from the star.

Before the end of the year 1862 we had succeeded in constructing an apparatus which fulfilled part of these conditions. With this some of the lines of the spectra of Aldebaran, a Orionis, and Sirius were measured; and from these measures, 
diagrams of these stars, in greater detail than had then been published, were laid before the Royal Society in February, 1863. After the note was sent to the Society, we became acquainted with some similar observations on several other stars by Rutherfurd, in Silliman's Journal for 1863.1 About the same time figures of a few stellar spectra were also published by Secchi. ${ }^{2}$ In March 1863 the Astronomer Royal presented a diagram to the Royal Astronomical Society, in which are slown the positions of a few lines in sixteen stars. ${ }^{3}$

Since the date at which our note was sent to the Royal Society our apparatus has been much improved, and in its present form of construction it fulfils satisfactorily several of the conditions required.

\section{\$I. Description of the Apparatus, and Methods of Observation employed.}

4. This specially constructed spectrum apparatus is attached to the eye end of a refracting telescope of 8 inches aperture and 10 feet focal length, which is mounted equatorially in the Observatory of Mr. Huggins at Upper Tulse Hill. The objectglass is a very fine one, by Alvan Clark, of Cambridge, Massachusetts; the equatorial mounting is by Cooke of York; and the telescope is carried very smoothly by a clock motion.

As the linear spectrum of the point of light which a star forms at the focus of the object-glass is too narrow for the observation of the dark lines, it becomes necessary to spread out the image of the star; and to prevent loss of light, it is of importance that this enlargement should be in one direction only; so that the whole of the light received by the object-glass should be concentrated into a fine line of light as narrow as possible, and having a length not greater than will correspond to the breadth of the spectrum (when viewed in the apparatus), just sufficient to enable the eye to distinguish with ease the dark

\footnotetext{
1 Vol. xxxv. p. 71.

2 Astronomische Nachrichten, No. 1405, Narch 3, 1863.

3 Monthly Notices, Roy, Astron. Soc, vol. xxiii. p. 190.
} 
lines by which it may be crossed. No arrangement tried by us has been found more suitable to effect this enlargement in one direction tlian a cylindrical lens, which was first employed for this purpose by Fraunhofer. In the apparatus by which the spectra described in our "Note" of February 1863 were observed the cylindrical lens employed was plano-convex, of 0.5 inch focal length. This was placed within the focus of the object-glass, and immediately in front of the slit of the collimator.

The present form of the apparatus is represented in Fig. 99 (page 309), where the cylindrical lens is marked $a$. This is plano-convex, an inch square, and of about 14 inches focal length. The lens is mounted in an inner tube $b$, sliding within the tube $c$, by which the apparatus is adapted to the eye end of the telescope. The axial direction of the cylindrical surface is placed at right angles to the slit $d$, and the distance of the lens from the slit within the converging pencils from the object-glass is such as to give exactly the necessary breadth to the spectrum.

In consequence of the object-glass being over-corrected, the red and especially the violet pencils are less spread out than the pencils of intermediate refrangibility; so that the spectrum, instead of having a uniform breadth, becomes slightly narrower at the red end, and tapers off in a greater degree towards the more refrangible extremity. ${ }^{1}$

In front of the slit $d$, and over one-half of it, is placed a right-angled prism $e$, for the purpose of reflecting the light which it receives from the mirror $f$, through the slit. In the brass tube $c$ are two holes: by one of these the light is allowed to pass from the mirror to the reflecting prism $e$; and by means

I The experiment was made by so placing the cylindrical lens that the axial direction of its convex cylindrical surface should be parallel with the direction of the slit. The line of light is in this case formed by the lens; and the length of this line, corresponding to the visible breadth of the spectrum, is equal to the diameter of the cone of rays from the object-glass when they fall inpon the slit. With this arrangement, the spectrum appears to be spread out, in place of being contracted at the two extremities. Owing to the large amount of dispersion to which the light is subject, it was judged madvisable to weaken still further the already feeble illumination of the extremities of the spectrum; and in the examination of the stellar spectra the position of the cylindrical lens with its axis at right angles to the slit, as mentioned in the text, was therefore adopted. 
of the other access to the milled head for regulating the width of the slit is permitted. Behind the slit, and at a distance equal to its focal length, is placed an achromatic collimating lens, $g$, made by T. Ross: this has a diameter of 0.6 inch and a focal length of $4 \frac{1}{2}$ inches. These proportions are such that the lens receives the whole of the light which diverges from the linear image of the star, when this is brought, exactly within the jaws of the slit.

A plano-concave cylindrical lens of about 14 inches negative focal length was also tried. The slight advantage which this possesses over the convex form is more than balanced by the inconvenience of the increased length given to the whole apparatus. The dispersing portion of the apparatus consists of two prisins, $h$, each having a refracting angle of about $60^{\circ}$ : they were made by T. Ross, and are of very dense and homogeneous flint glass. The prisms are supported upon a suitable mounting, which permits them to be duly levelled and adjusted. Since the feebleness of the light from the stars limits the observations for the most part to the central and more luminous portions of the spectrum, the prisms have been adjusted to the angle of minimum deviation for the ray D. A cover of brass, $k$, incloses this part of the apparatus; and by this means the prisns are protected from accidental displacement and from dust.

The spectrum is viewed through a small achromatic telescope, $l$, furnished with an object-glass of 0.8 inch diameter and 6.75 inches focal length. This telescope has an adjustment for level at $m$. The axis of the telescope can be lowered and raised, and the tube can be also rotated around the vertical axis of support at $n$. At the focus of the object-glass are fixed two wires, crossing at an angle of $90^{\circ}$. These are viewed, together with the spectrum, by a positive eyepiece, $p$, giving a magnifying power of 5.7 diameters. As the eyes of the two observers do not possess the same focal distance, a spectacle lens, corresponding to the focal difference between the two, was fitted into a brass tube, which slipped easily over the eyepiece of the telescope, and was used or withdrawn as was necessary. 
This telescope, when properly adjusted and clamped, is carried by a micrometer screw, $q$, which was constructed and fitted to the instrument by Cooke and Sons. The centre of motion about which it is carried is placed approximately at the point of intersection of the red and the violet pencils from the last prism: consequently it falls within the last face of the prism nearest the small telescope. All the pencils therefore which emerge from the prism are, by the motion of the telescope, caused to fall nearly centrically upon its object-glass. The micrometer screw has 50 threads to an inch; and each revolution is read to the hundredth part, by the divisions engraved upon the head. This gives a scale of about 1,800 parts to the interval between the lines A and $\mathrm{I}$ of the solar spectrum. During the whole of the observations the same part of the screw has been used; and the measures being relative, the inequalities, if any, in the thread of this part of the screw do not affect the accuracy of the results. The eye lens for reading the divisions of the micrometer screw is shown at $s$.

The mirror $f$ receives the light to be compared with that of the star spectrum, and reflects it upon the prism $e$, in front of the slit $d$. This light was usually obtained from the induction spark taken between electrodes of different metals, fragments of wires of which were held by a pair of small forceps attached to the insulating ebonite clamp $r$. Upon a movable stand in the Observatory was placed the induction coil, already described by one of $\mathrm{us}^{1}{ }^{1}$ in the secondary circuit of which was inserted a Leyden jar, having 140 square inches of tinfoil upon each of its surfaces. The exciting battery, which, for the convenience of being always available, consisted of four cells of Smee's construction, with plates 6 inches by 3 , was placed without the Observatory. Wires, in connection with this and the coil, were so arranged that the observer could make and break contact at pleasure without removing his eye from the small telescope. 'This was the more important, since, by tilting the mirror $f$, it is possible, within narrow limits, to alter the position of the spectrum of the metal relatively to that of the star. An

I Phil. Trans. 1864, p. 141. 
arrangement is thus obtained which enables the observer to be assured of the perfect correspondence in relative position in the instrument of the stellar spectrum and the spectrum to be compared with it.

5. The satisfactory performance of this apparatus is proved by the very considerable dispersion and admirably sharp definition of the known lines in the spectra of the sun and metallic vapours. When it is directed to the sun, the line $D$ is sufficiently divided to permit the line within it, marked in Kirchhoff's map as coincident with nickel, to be seen. The close groups of the metallic spectra are also well resolved.

When this improved apparatus was directed to the stars, a large number of fine lines was observed, in addition to those that had been previously seen. In the spectra of all the brighter stars which we have examined the dark lines appear to be as fine and as numerous as they are in the solar spectrum. The great bread th in the lines in the green and more refrangible parts of Sirius and some other stars, as seen in the less perfect form of apparatus which was first employed, and which bandlike appearance was so marked as specially to distinguish them, has, to a very great extent, disappeared; and though these lines are still strong, they now appear, as compared with the strongest of the solar lines, by no means so abnormally broad as to require these stars to be placed in a class apart. No stars sufficiently bright to give a spectrum have been observed to be without lines. The stars admit of no such broad distinctness of classification. Star differs from star alone in the grouping and arrangernent of the numerous fine lines by which their spectra are crossed.

6. For the convenience of reference and comparison, a few of the more characteristic lines of twenty-nine of the elements were measured with the instrument. These were laid down to scale, in order to serve as a chart, for the purpose of suggesting, by a comparison with the lines measured in the star, those elements the coincidence of the lines of which with stellar lines was probable.

For the purpose of ensuring perfect accuracy in relative 
position in the instrument between the star spectrum and the spectrum to be observed simultaneously with it, the following general method of observing was adopted: The flame of a small lamp of alcohol, saturated with chloride of sodium, was placed centrally before the object-glass of the telescope, so as to furnish a sodium spectrum. The sodium spectrum was then obtained by the induction-spark, and the mirror $f$ was so adjusted that the components of the double line $\mathrm{D}$, which is well divided in the instrument, should be severally coincident in the two spectia. The lamp was then removed, and the telescope directed to the sun, when Fraunhofer's line D was satisfactorily observed to coincide perfectly with that of sodium in the induction spark. Having thus ascertained that the sodium lines coincided in the instrument with the solar lines D, it was of importance to have assurance from experiment that the other parts of the solar spectrum would also accurately agree in position with those corresponding to them in the spectrum of comparison. When electrodes of magnesium were employed, the components of the triple group characteristic of this metal severally coincided with the corresponding lines of the group $b$; $C$ and $F$ also agreed exactly in position with the lines of hydrogen. The stronger of the Fraunhofer lines were measured in the spectra of the moon and of Venus, and these measures were found to be accordant with those of the same lines taken in the solar spectrum.

Before commencing the examination of the spectrum of a star, the alcohol-lamp was again placed before the object-glass of the telescope, and the correct adjustment of the apparatus obtained with certainty. The first observation was whether the star contained a double line coincident with the sodium line D. When the presence of such a line had been satisfactorily determined, we considered it sufficient in subsequent observations of the same star to commence by ascertaining the exact agreement in position of this known stellar line with the sodium line $\mathrm{D}$.

Since from flexure of the parts of the spectrum apparatus the absolute reading of the micrometer might vary when the 
telescope was directed to stars differing greatly in altitude, the measure of the line in the star which was known to be coincident with that of sodium was always taken at the commencement and at the end of each set of measures. The distances of the other lines from this line, and not the readings of the micrometer, were then finally registered as the measures of their position; and these form the numbers given in the Tables, from which the diagrams of the star spectra have been laid down.

The very close approximation, not unfrequently the identity, of the measures obtained for the same line on different occasions, as well as the very exact agreement of the lines laid down from these measures with the stellar lines subsequently determined by a direct comparison with metallic lines, the positions of which are known, have given the authors great confidence in the minute accuracy of the numbers and drawings which they have now the honour of laying before the Society.

\section{APPENDIX B.}

ON THE SPECTRUM OF MARS, WITH SOME REMARKS ON THE COLOUR OF THAT PLANET.

BY WILLIAM HUGGINS, ESQ., F.R.S.

On several occasions during the late opposition of Mars I made observations of the spectrum of the solar light reflected from that planet.

The spectroscope which I employed was the same as that of which a description has appeared in my former papers. ${ }^{1}$ Two

1 "On the Spectra of some of the Fixed Stars." (Phil. Trans. 1864, p. 415.) During my prismatic researches I have tried, and used occasionally, several other arrangements for applying the prism to the telescope. Some of these instruments are fitted with compound prisms, which give direct vision. I have not found any apparatus equal in delicacy and in accuracy to that which is referred to in the text. 
instruments were used, one of which is furnished with a single prism of dense glass, which has a refracting angle of $60^{\circ}$. The other instrument has two similar prisms.

In a paper "On the Spectra of some of the Fixed Stars," by myself and Dr. W. A. Miller, we state that on one occasion several strong lines of absorption were seen in the more refrangible parts of the spectrum of Mars.

During the recent more favourable opportunities of viewing Mars I again saw groups of lines in the blue and indigo parts of the spectrum. However, the faintness of this portion of the spectrum, when the slit was made sufficiently narrow for the distinct observation of the lines of Fraunhofer, did not permit me to measure with accuracy the position of the lines which I saw. For this reason I was unable to determine whether these lines are those which occur in this part of the solar spectrum, or whether any of them are new lines due to an absorption which the light suffers by reflection from the planet.

I have confirmed our former observation, that several strong lines exist in the red portion of the spectrum. Fraunhofer's C was distinctly seen, and its identity determined by satisfactory measures with the micrometer of the spectrum apparatus. From this line the spectrum, as far as it can be traced towards the less refrangible end, is crossed by dark lines. One strong line was satisfactorily determined by the micrometer to be situated from $\mathrm{C}$ at about one-fourth of the distance from $\mathrm{C}$ to $\mathrm{B}$. As a similar line is not found in this position in the solar spectrum, the line in the spectrum of Mars may be accepted as an indication of absorption by the planet, and probably by the atmosphere which surrounds it. The other lines in the red may be identical, at least in part, with $\mathrm{B}$ and $\mathrm{A}$, and the adjacent lines, of the solar spectrum.

On February 14 faint lines were seen on both sides of Fraunhofer's D. The lines on the more refrangible side of $\mathrm{D}$ were stronger than the less refrangible lines. These lines occupy positions in the spectrum apparently coincident with groups of lines which make their appearance when the sun's light traverses 
the lower strata of the atmosphere, and which are therefore supposed to be produced by the absorption of gases or vapours existing in our atmosphere. The lines in the spectrum of Mars probably indicate the existence of similar matter in the planet's atmosphere. I suspected that these lines were most distinct in the light from: the margin in the planet's dise; but this observation was to some extent uncertain. That these lines were not produced by the portion of the earth's atmosphere through which the light of Mars had passed was shown by the absence of similar lines in the spectrum of the moon, which at the time of observation had a smaller altitude than Mars.

I observed also the spectra of the darker portions of the planet's disc. The spectrum of the dark zone beneath the southern polar spot appeared as a dusky band when compared with the spectra of the adjoining brighter parts of the planet. This fainter spectrum appeared to possess a uniform depth of shade throughout its length. This observation would indicate that the material which forms the darker parts of the planet's surface absorbs all the rays of the spectrum equally. These portions should be therefore neutral, or nearly so, in colour.

I do not now regard the ruddy colour of Mars to be due to an elcctive absorption; that is, an absorption of certain rays only so as to produce dark lines in the spectrum.

Further, it does not appear to be probable that the ruddy tint which distinguishes Mars has its origin in the planet's atmosphere, for the light reflected from the polar regions is free from colour, though this light has traversed a longer column of atmosphere than the light from the central parts of the disc. It is in the central parts of the disc that the colour is most marked. If indeed the colour be produced by the planet's atmosphere, it must be referred to peculiar conditions of it which exist only in connection with particular portions of the planetary surface. The evidence we possess at present appears to support the opinion that the planet's distinctive colour has its origin in the material of which some parts of its surface are composed. Mr. Lockyer's observation, that the colour is most intense when the planet's 
atmosphere is free from clouds, obviously admits of an interpretation in accordance with this view.

This opinion appears to receive support from the photometric observations of Seidel and Zöllner, some of the results of which I will briefly state.

These observations show that Mars resembles the moon in the anomalous amount of variation of the light reflected from it as it increases and decreases in phase; also in the greater brilliancy of the marginal portions of its dise. Further, Zöllner has found that the albedo of Mars, that is, the mean reflective power of the different parts of its disc, is not more than about onehalf greater than that of the lunar surface. Now these optical characters are in accordance with telescopic observation, that in the case of Mars the light is reflected almost entirely from the true surface of the planet. Jupiter and Saturn, the light from which has evidently come from an envelope of clouds, are, on the contrary, less bright at the margin than at the central part of the disc. These planets have an albedo, severally, about four and three times greater than that of the inoon. ${ }^{1}$

The anomalous degradation in the brightness of the moon at the phases on either side of the full, as well as the greater brilliancy of the linb, may be accounted for by the supposition of inequalities on its surface, and also by a partly regular reflective property of its superficial rocks. Zöllner has shown that if these phenomena be assumed empirically to be due to inequalities, then the angle of mean elevation of these inequalities must be taken as $52^{\circ}$. On the same hypothesis the more rapid changes of Mars would require an angle of $76^{\circ}{ }^{2}$

It appears to be highly probable that the conditions of surface which give rise to these phenomena are common to the moon and to Mars. The considerations referred to in a former paragraph suggest that these superficial conditions represent peculiarities which exist at the true surface of the planet. In this connection it is of importance to remark that the darker parts of the disc of Mars gradually disappear, and the

1 Photometrische Untersuchungen, vou Dr. J. C. Zülluer ; Leipzig, 1865.

2 Ibid. pp. 113, 128. 
coloured portions lose their distinctive ruddy tint as they approach the limb.

The observations of Sir J. Herschel ${ }^{1}$ and Professor G. Bond ${ }^{2}$ give a mean reflective power to the moon's surface, similar to that from a "grey, weathered sandstone rock." Zölluer has confirmed this statement. According to him,--

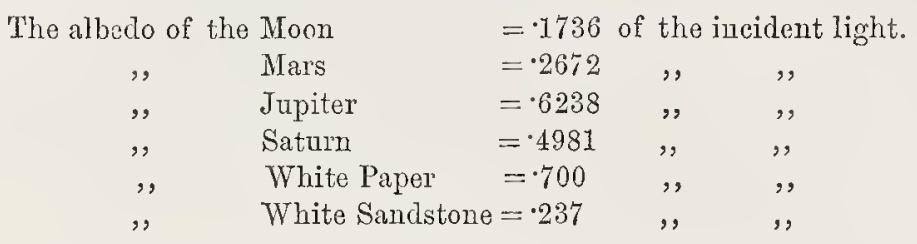

From this Table it appears that Mars takes in for its own use .7328 of the energy which it receives as light. Jupiter's cloudy atmosphere, nearly as brilliant as white paper, rejects more than six-tenths of the light which falls upon it. Therefore, less than four-tenths of the light which this distant planet receives is alone available for the purposes of its economy.

The photographic researches of Mr. De la Rue and others show that the rays of high refrangibility, which are specially powerful in producing chemical action, are similarly affected. ${ }^{3}$ At present we know nothing of the reflective power of the planets for those rays of slower vibration which we call heat.

I "Outlines of Astronomy," p. 272.

2 "On the Light of the Moon and Jupiter," Memoirs Amer. Academy, vol. iii. p. 222. In the same Memoir Professor G. Bond estimates the albedo of Jupiter to be greater than unity. This estimate would require the admission that Jupiter shines in part by native light. (Ibid. p. 284.)

3 Professor G. Bond states that "the Moon, if the constitution of its surfaee resembled that of Jupiter, would photograph in one-fourteenth of the time it actually requires." (ibid. p. 223.) 


\section{APPENDIX C.}

ON THE OCCURRENCE OF BRIGH'T LINES IN STELLAR SPECTRA, AND ON THE SPECTRA OF VARIABLE STARS.

"The spectrum of $y$ Cassiopeiæ appears to be, in some respects at least, analogous to that of 'T. Coronæ. In addition to the bright line near the boundary of the green and blue observed by Father Sechi, there is a line of equal brilliancy in the red, and some dark lines of absorption. The two bright lines are narrow and defined, but not very brilliant. Micrometrical measures made by Dr. Huggins of these lines show that they are doubtless coincident in position with Fraunhofer's $\mathrm{C}$ and $\mathrm{F}$, and with two of the bright lines of luminous hydrogen. In these stars part of the light must be emitted by gas intensely heated, though not necessarily in a state of combustion. The nearly uniform light of $\gamma$ Cassiopeiæ suggests that the luminous hydrogen of this star forms a normal part of its photosphere."-Noticcs, Royal Astronomical Society, vol. xxvii. p. 131.

"Mira Cati, which gives a spectrum apparently identical, or nearly so, with $a$ Orionis, was examined when at its maximum brilliancy, and on several subsequent occasions, after it had commenced its downward course. At the time the star was waning in brightness there was thouglit to be an appearance of greater intensity in several of the groups, but a continued series of observations is desirable before any opinion is hazarded as to the cause of the variation in brightness which has procured for this object the title of 'Wonderful.' At Mr. Baxendell's request the variable $\rho$ Coronæ was examined when at its maximum, but without any successful result. . . . Dr. Huggins has confirmed the observation of MM. Wolf and Raget so far as to the presence of bright lines in the three small stars described by them. He has not determined the positions of these lines."-Ibid. vol. xxviii. p. 87. 


\section{APPENDIX D.}

FURTHER OBSERVATIONS ON THE SPECTRA OF SOME OF THE STARS AND NEBULA, WITH AN ATTEMPT TO DETERMINE THEREFROM WHETHER THESE BODIES ARE MOVING TOWARDS OR FROM THE EARTH; ALSO OBSERVATIONS ON THE SPECTRA OF THE SUN AND OF COMET II. 1868. ${ }^{1}$

BY WILLIAM HUGGINS, ESQ., F.R.S.

\section{$\S$ I. Introduction.}

In a paper "On the Spectra of some of the Fixed Stars," ${ }^{2}$ by myself and Dr. W. A. Miller, Treas. R.S., we gave an account of the method by which we had succeeded during the years 1862 and 1863 in making trustworthy simultaneous comparisons of the bright lines of terrestrial substances with the dark lines in the spectra of some of the fixed stars. We were at the time fully aware that these direct comparisons were not only of value for the more immediate purpose for which they had been undertaken, namely, to obtain information of the chemical constitution of the investing atmospheres of the stars, but that they might also possibly serve to tell us something of the motions of the stars relatively to our system. If the stars were moving towards or from the earth, their motion, compounded with the earth's motion, would alter to an observer on the earth the refrangibility of the light emitted by them, and consequently the lines of terrestrial substances would no longer coincide in position in the spectrum with the dark lines produced by the absorption of the vapours of the same substances existing in the stars.

The apparatus employed by us was furnished with two prisms of dense flint glass, each with a refracting angle of $60^{\circ}$, and permitted the comparisons to be made with so much accuracy

1 Phil. Trans. 1868 , p. 529.

2 Ibid. 1864, p. 413. 
that the displacement of a line, or of a group of lines, to an amount smaller even than the interval which separates the components of - Fraunhofer's D, would have been easily detected. We were therefore in possession of the information that none of the stars, the lines in the spectra of which we had compared with sufficient care, were moving in the direction of the visual ray with a velocity so great, relatively to that of light, as to shift a line through an interval corresponding to a difference of wavelength equal to that which separates the components of $\mathrm{D}$. To produce an alteration of refrangibility of this amount a velocity of about 169 miles per second would be required. The following stars, with some others, were observed with the requisite accuracy :-Aldebaran, $a$ Orionis, $\beta$ Pegasi, Sirius, $a$ Lyræ, Capella, Arcturus, Pollux, Castor.

It appeared premature at the time to refer to these negative results, as it did not seem to be probable that the stars were moving with velocities sufficiently great to cause a change of refrangibility which could be detected with our instrument. The insufficiency of our apparatus for this very delicate investigation does not, however, diminish the trustworthiness of the results we obtained respecting the chemical constitution of the stars, as the evidence for the existence or otherwise of a terrestrial substance was made to rest upon the coincidence, or want of coincidence, in general character as well as position of several lines, and not upon that of a single line.

According to the undulatory theory, light is propagated with equal velocity in all directions, whether the luminous body be at rest or in motion. The change of refrangibility is therefore to be looked for from the diminished or increased distance the light would have to traverse if the luminous abject and the observer had a rapid motion towards or from each other. The great relative velocity of light to the known planetary velocities and to the probable motions of the few stars of which the parallax is known, showed that any alterations of position which might be expected from this cause in the lines of the stellar spectra would not exceed a fraction of the interval between the double line 1 ; for that part of the spectrum. 
I have devoted much time to the construction and trial of various forms of apparatus with which I hoped to accomplish the detection of so small an amount of change of refrangibility. The difficulties of this investigation I have found to be very great, and it is only after some years that I have succeeded in obtaining a few results which I hope will be acceptable to the Royal Society.

The subject of the influence of the motions of the heavenly bodies on the index of refraction of light had already, at the time of the publication of our paper in 1864, occupied the attention of Mr. J. C. Maxwell, F.R.S., who had made some experiments in an analogous direction.

In 1841 Doppler showed that since the iinpression which is received by the eye or the ear does not depend upon the intrinsic strength and period of the waves of light and of sound, but is determined by the interval of time in which they fall upon the organ of the observer, it fullows that the colour and intensity of an impression of light, and the pitch and strength of a sound, will be altered by a motion of the source of the light or of the sound, or by a motion of the observer, towards or from each other. $^{1}$

Doppler endeavoured by this consideration to account for the remarkable differences of colour which some of the binary stars present, and for some other phenomena of the heavenly bodies. That Doppler was not correct in making this application of his theory is obvious from the cousideration that, even if a star could be conceived to be moving with a velocity sufficient to alter its colour sensibly to the eye, still no change of colour would be perceived, for the reason that beyond the visible spectrum, at both extremities, there exists a store of invisible waves which would be at the same time exalted or degraded into visibility to take the place of the waves which had been raised or lowered in refrangibility by the star's motion. No change of colour, therefore, could take place until the whole of those invisible waves of force had been expended, which would

1 "Ueber das farbige Licht der Doppelsterne und einiger anderer Gestirne des Hinmels." (Böhın. Gesell. Abh. ii. 1841.42, S. 465.) 
only be the case when the relative motion of the source of light and the observer was several times greater than that of light.

In 1845 Ballot published a series of acoustic experiments which support Doppler's theory in the case of sound. In the same paper Ballot advances several objections to Doppler's application of his theory to the colours of the stars. ${ }^{1}$

This paper was followed by several papers by Doppler in reply to the objections which were brought against his conclusions. $^{2}$

In 1847 two memoirs were published by Sestini on the colours of the stars in connection with Doppler's theory. ${ }^{3}$

More recently, in 1866, Klinkerfues ${ }^{4}$ published a memoir on the influence of the motion of a source of liglit upon the refrangibility of its rays, and described therein a serics of observations from which he deduces certain amounts of motion, in the case of some of the objects observed by him.

The method employed by Klinkerfues has been critically discussed by Dr. Sohncke. ${ }^{5}$

It may be sufficient to state that, as Klinkerfues employs an achromatic prism, it does not seem possible, by his method of observing, to obtain any information of the motion of the stars; for in such a prism the difference of period of the luminous waves would be as far as possible annulled. It is, however, conceivable that his observations of the light when travelling from E. to W., and from W. to E., might show a difference in the two cases, arising from the earth's motion through the ether.

Father Secchi has quite recently called attention to this

1 "Akustisehe Versuehe auf der Niederländisehen Eisenbalnn nebst gelegentliehen Bemerkungen zur 'Theorie des Herrn Prof. Doppler;" Pogg. Ann. B. Ixvi. S. 321.

2 See Pogg. Ann. B. lxxxi. S. 270 , and B. lxxxvi. S. 371.

3 "Memoria sopra i Colori delle Stelle del Catalogo de Baily osservati dal P. Band. Sestini." Roma, 1847.

${ }_{4}$ "Fernere Mittheilungen über" den Einfluss der Bewegung der Liehtquelle auf die Breehbarkeit eines Strahls." Von W. Klinkerfues, Naehr. K. G. der W. zu Göttingen, No. 4, S. 33.

5 "Ueber den Einfluss der" Bewegung der Liehtqueìe auf die Breehung, kritisehe Bemerkungen zu der Entdeekung des Herrn Prof. Klinkerfues." Vou Herrn Dr. Sohncke, Astron. Nachr. No. 1646. 
subject. ${ }^{1}$ In his paper he states that he has not been able to detect any change of refrangibility in the case of certain stars, of an amount equal to the difference between the components of the double line $\mathrm{D}$. These results are in accordance with those obtained by myself and Dr. Miller in 1868, so far as they refer to the stars which had been examined by us. . . .

\section{\$IT. Description of Apparatus.}

All the experiments were made with my refractor by Alvan Clark, of 8 inches aperture and 10 feet focal length, which is mounted equatorially, and carried very smoothly by a clock motion. As even on nights of unusual steadiness the lines in the spectra of the stars are necessarily, for several reasons, more difficult of minute discrimination of position than are those of the solar spectrum, it is important that the apparatus employed should give an ample amount of dispersion relatively to the degree of minuteness of observation which it is proposed to attempt.

In 1866 I constructed a spectroscope for the special objects of research described in this paper, which was furnished with three prisms of $60^{\circ}$ of very dense flint glass. The solar lines were seen with great distinctness. I found, however, that, in order to obtain a separation of the lines sufficient for my purpose, an eyepiece magnifying ten or twelve diameters was necessary. Under these circumstances the stellar lines were not seen in the continued steady manner which is necessary for the trustworthy determination of the minute differences of position which were to be observed. After devoting to these observations the most favourable nights which occurred during a period of some months, I found that if success was to be obtained it would probably be with an apparatus in which a larger number of prisms and a smaller magnifying power were employed.

The inconvenience arising from the pencils, after passing through the prisms, crossing those from the collimator, when

1 Comptes Rendus, 2 Mars, 1868, p. 398. 
more than three or four prisms are employed, and also, in part, the circumstance that I had in my possession two very fine direct-vision prisms on Amici's principle, which had been made for me by Hofmann of Paris, induced me to attempt to combine in one instrument several simple prisms with one or two compound prisms which gave direct vision. An instrument constructed in this way, as will be seen from the following description, possesses several not unimportant advantages. ${ }^{1}$

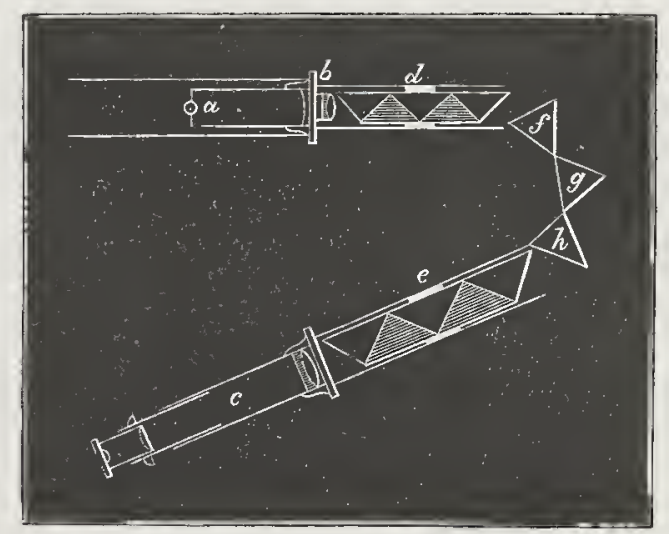

FIG. 118 .

Fig. $118 \alpha$ is an adjustable slit; $b$ an achromatic collimating lens of $4: 5$ inches focal length ; $c$ represents the small telescope with which the spectrum is viewed. The train of prisms consists of two compound prisms, $d$ and $e$, and three simple prisms $f, g, h$. Each of the compound prisms contains five prisms, cemented together with Canada balsam. The shaded portions of the diagram represent the position of the two prisms of very dense flint glass in each compound prism. The compound prism marked $e$ is much larger than the other, and is permanently connected with the telescope $c$, with which it moves. These compound prisms, which were made specially to my order by Hofmann, are of great perfection, and produced severally a dispersion fully equal to two prisms of ordinary dense flint glass. The prisms $f$ and $g$

1 An apparatus in many respects superior to the one here described has been constructed since. - October, 1868. 
were cut for me from a very fine piece of dense glass of Guinand by Messrs. Simms, and have each a refracting angle of $60^{\circ}$. The prism $h$ was made by $\mathrm{Mr}$. Browning from the dense flint glass manufactured by Messrs. Chance : this prism has a refracting ancle of $45^{\circ}$. The great excellence of all these prisms is shown by the very great sharpness of definition of the bright lines of the metals when the induction spark is taken before the slit, even when considerable magnifying power is employed on the small telescope with which the spectrum is viewed. The instrument is provided with a second collimator, of which the object-glass has a focal length of eighteen inches.

The compound prism $e$ is so fixed that it can be removed at pleasure, when the total dispersive power of the instrument is reduced from about six and a half prisms of $60^{\circ}$ to about four and a half prisms of $60^{\circ}$. The facility of being able to reduce the power of the instrument has been found to be of much service for the observation of faint objects, and also on nights when the state of the atmosphere was not very favourable.

The telescope with which the spectrum is viewed is carried by a micrometer screw, which, however, has not been employed for taking measures of the spectra, but only for the purpose of setting the telescope to the part of the spectrum which it is intended to observe. This precaution is absolutely necessary when nebulæ are observed which emit light of two or three refrangibilities only.

For the purpose of the simultaneous comparisons of the light of the heavenly bodies with the lines of the terrestrial elements, the slit was provided, in the usual way, with a small prism placed over one-half of it, which received the light reflected upon it from a small mirror placed opposite the electrodes. The plan of observation formerly employed, and which is described in the paper "On the Spectra of some of the Fixed Stars," was adopted to insure perfect accuracy of relative position in the instrument between the star spectrum and the spectrum to be compared with it, since it is possible, by tilting the mirror, to alter within narrow limits the position of the spectrum of the terrestrial substance relatively to that of the star. Before commencing 
an observation, a small alcohol lamp, in the wick of which bicarbonate of soda was placed, was fixed before the object-glass of the telescope, and then the mirror and the electrodes were so adjusted that the components of the double line D were exactly coincident in both spectra.

This plan was soon found to be very inconvenient, and even in some degree untrustworthy for the more delicate comparisons which were now attempted. An unobserved accidental displacement of the spark, or of the mirror, might cause the two spectra to differ in position by an amount equal to the whole extent of want of coincidence which it was proposed to seek for in this investigation. The observations of many nights have been rejected from the uncertainty as to the possible existence of an accidental displacement.

Another inconvenience, so great as even to seem to diminish the hope of ultimate success, was found to arise from the diffculty of bringing the lower margin of the star spectrum into actual contact with the upper margin of the spectrum of the light reflected into the instrument. The lines in the spectra of the stars are not, on ordinary nights, so steady and distinct as are those of the solar spectrum. Under these difficult circumstances, it is very desirable, as an assistance to the eye in its judgment of the absolute identity or otherwise of the position of lines, that the bright lines of comparison should not merely meet the dark lines in the star spectrum, but that they should overlap them to a small extent. When the two spectra are so arranged as to be in contact, the eye is found to be influenced to some extent by the apparent straightness or otherwise of the compound line formed by the coincident, or nearly coincident, lines in the two spectra. Owing to the unavoidable shortness of the collimator, the lines in a broad spectrum are slightly curved. From this cause the determination of the identity of lines in spectra which are in contact merely is rendered more difficult, and it may be less trustworthy.

The difficulties of observation which have been referred to were in the first instance sought to be overcome by placing the spark before the object-glass of the telescope. In some respects 
this method appears to be unexceptionable, but there are disadvantages connected with it. The bright lines, under these circumstances, extend across the star spectrum, and make the simultaneous observation of dark lines, which are coincident, or nearly so, with them, very difficult. When the spark is taken between electrodes, the consequent disturbance of the air in front of the object-glass is unfavourable to good definition. An important disadvantage arises from the great diminution in the brightness of the spark from the distance (ten feet) at which it is placed from the slit; since in consequence of its nearness to the object-glass the divergence of the light from it is diminished in a small degree only by that lens. It is obvious that, by means of a lens of short focal length placed between the spark and the object-glass, the light from the spark might be rendered parallel, or even convergent; but the adjustment of such a lens, so that the pencils transmitted by it should coincide accurately in direction with the optical axis of the telescope, would be very troublesome. When two Leyden jars, connected as one jar, were interposed, and the spark was taken in air between platinum points, there was visible in the spectroscope only the brightest of the lines of the air spectrum, namely, the double line belonging to nitrogen, which corresponds to the principal line in the spectra of the gaseous nebulæ. When a vacuum-tube containing hydrogen at a low tension was placed before the object-glass, the line corresponding to $\mathrm{F}$ was seen with sufficient distinctness, but the line in the red was visible with difficulty. Some observations, however, have been made with the spark arranged before the object-glass.

The following arrangements for admitting the light from the spark appeared to me to be free from the objections which have been referred to, and to be in all respects adapted to meet the requirements of the case. In place of the small prism, two pieces of silvered glass were securely fixed before the slit at an angle of $45^{\circ}$. In a direction at right angles to that of the slit an opening of about $\frac{x}{10}$ inch was left between the pieces of glass for the passage of the pencils from the object-glass. By means of this arrangement the spectrum of a star is seen accompanied 
by two spectra of comparison, one appearing above and the other below it. As the reflecting surfaces are about 0.5 inch from the slit, and the rays from the spark are divergent, the light reflected from the pieces of glass will have encroached upon the pencils from the object-glass by the time they reach the slit, and the upper and lower spectra of comparison will appear to overlap to a small extent the spectrum formed by the light from the objectglass. This condition of things is of great assistance to the eye in forming a judgment as to the absolute coincidence or otherwise of lines. For the purpose of avoiding some inconveniences which would arise from glass of the ordinary thickness, pieces of the thin glass used for the covers of microscopic objects were carefully selected, and these were silvered by floating them upon the surface of a silvering solution. In order to insure that the induction spark should always preserve the same position relatively to the mirror, a piece of sheet gutta-percha was fixed above the silvered glass: in the plate of gutta-percha, at the proper place, a small hole was niade of about $\frac{1}{20}$ inch in diameter. The ebonite clamp containing the electrodes is so fixed as to permit the point of separation of these to be adjusted exactly over the small hole in the gutta-percha. The adjustment of the parts of the apparatus was made by closing the end of the adapting tube, by which the apparatus is attached to the telescope, with a diaphragm with a small central hole, before which a spirit-lamp was placed. When the lines from the induction spark, in the two spectra of comparison, were seen to overlap exactly, for a short distance, the lines of sodium from the light of the lamp, the adjustment was considered perfect. The accuracy of adjustment has been confirmed by the exact coincidence of the three lines of magnesium with the component lines of $b$ in the spectrum of the moon.

In some cases the spectra produced by the spark are inconveniently bright for comparison with those of the stars and nebulæ. If the spark is reduced in power below a certain point, many of the lines are not then well developed. The plan, therefore, was adopted of diminishing the brightness of the spectrum by a wedge of neutral-tint glass, which can be moved 
at pleasure between the plate of gutta-percha and the sitvered mirror.

Two eyepieces were employed with the apparatus; the one magnifying four diameters, and the other six diameters. . . .

\section{$\$$ III. Observations of Nebulce.}

For the greater convenience of reference and of comparison the spectrum of $37 \mathrm{H}$. IV. Draconis from my paper "On the Spectra of some of the Nebulx"1 has been added. The spectrum of this nebula may be taken as characteristic, in its general features, of the spectra of all the nebulæ which do not give a continuous spectrum. At present $I$ have determined satisfactorily the general characters of the spectra of about seventy nebulæ. This number forms but a part of the much larger list of nebulæ which I have examined, but in the case of many of these objects their light was found to be too feeble for a satisfactory analysis. Of the seventy nebulæ about one-third give a spectrum of bright lines. The proportion which is indicated by this examination, of the nebulæ which gave a spectrum of bright lines to those of which the spectrum is continuous (namely, as one to two), is probably higher than would result from a wider observation of the objects contained in such catalogues as those of Sir John Herschel and Dr. D'Arrest, since many of the objects which I examined were specially selected, on account of the probability (which was suggested by their form or colour) that they were gaseous in constitution.

All the differences which I have hitherto observed between the spectra of the gaseous nebulx may be regarded as modifications only of the typical form of spectrum which is represented in the diagram, since they consist of differences of relative intensity, of the deficiency of one or two lines, or of the presence of one or two additional lines. It is worthy of remark that, so far as the nebulæ have been examined, the brightest of the three lines, which agrees in position in the spectrum with the brightest of the lines of the spectrum of nitrogen, is present in all the

\footnotetext{
I Phil. Trans, 1864, p. 438.
} 
nebulæ which give a spectrum indicative of gaseity. It is a suggestive fact that should not be overlooked, that in no nebula which has a spectrum of bright lines has any additional line been observed on the less refrangible and brighter side of the line common to all the gaseous nebulæ.

The faint continuous spectrum, which in some cases is also seen, has been traced in certain nebulæ, by its breadth, to a distinct brighter portion of the nebula which it is convenient still to distinguish by the term "nucleus," though at present we know nothing of the true relation of the bright points of the nebulæ to the more diffused surrounding portions.

It must not be forgotten that when gases are rendered luminous there may usually be detected a faintly luminous continuous spectrum. In the case of several of the nebulæ, such as the annular nebula of Lyra and the Dumb-bell nebula, no existence of even a faint continuous spectrum has been yet certainly detected.

The determination of the position in the spectrum of the three bright lines was obtained by simultaneous comparison with the lines of hydrogen, nitrogen, and barium. The instrument which I employed had two prisms, each with a refracting angle of $60^{\circ}$, and the positions of the lines were trustworthy within the limits of about the breadth of the double line $D$.

The objects which I proposed to myself, in attempting a reexamination of some of the nebulæ with the large instrument described in this paper, were to determine, first, whether any of the nebulæ were possessed of a motion which conld be detected by a change of refrangibility; secondly, whether the coincidence which had been observed of the first and the third line with a line of hydrogen and a line of nitrogen would be found to hold good when subjected to the test of a spreading out of the spectrum three or four times greater than that under which the former observations were made. It would not, it seemed, be difficult, in the case of the detection of a want of coincidence, to separate the effects of the two distinct sources referred to, from both of which equally a minute difference of refrangibility between the nebular lines and those of terrestrial substances 
might arise. The probability is very great indeed that, in all the nebulæ which give the kind of spectrum of which I am speaking, the two lines referred to are to be attributed to the same two substances, and that therefore, in all these nebulæ, they were originally of the same degree of refrangibility. On the other hand, it is not to be supposed that nebulæ situated in different positions in the heavens would have a similar motion relatively to the earth. An examination of several nebulæ would therefore show to which of these causes any observed want of coincidence was to be attributed.

The great Nebula in Orion.-In my description of this nebula ${ }^{1}$ I stated that the light from all the parts of this strangely diversified object, which were bright enough to be observed with my instrument, was resolved into three bright lines similar to those represented in the diagram.

On the present occasion I applied myself in the first place to as careful a comparison as possible of the brightest line with the corresponding line of the spectrum of nitrogen.

My first observations were made with the light from the induction spark taken in pure nitrogen sealed in a tube at a tension a little less than that of the atmosphere, which was reflected into the instrument, as in my former series of observations, by means of a mirror and a small prism. The precaution was taken to verify the accuracy of the position of the spectrum of comparison relatively to that of the nebula, by placing a small la:np before the object-glass in the way already described.

The coincidence of the line in the nebula with the brightest of the lines of nitrogen, though now subjected to a much more severe trial, appeared as perfect as it did in my former observations. I expected that I night discover a duplicity in the line in the nebula corresponding to the two component lines of the line of nitrogen; but I was not able, after long and careful scrutiny, to see the line double. The line in the nebula was narrower than the double line of nitrogen: this latter may have appeared broader in consequence of irradiation, and it was much brighter than the line in the nebula.

$$
\text { y Proc. Roy. Soc. vol. xiv. p. } 39 .
$$


The following observations are suggestive in connection with the point under consideration. Electrodes of platinum were placed before the object-glass in the direction of a diameter, so that the spark was as nearly as possible before the centre of the lens. The spark was taken in air. I expacted to find the spectruin faint, for the reasons which have been stated in a previous paragraph; but I was surprised to find that only one line was visible in the large spectroscope when adapted to the eye end of the telescope. This line was the one which agrees in position with the line in the nebula; so that under these circumstances the spectrum of nitrogen appeared precisely similar to the spectra of those nebulæ of which the light is apparently monochromatic. This resemblance was made more complete by the faintness of the line; from which cause it appeared much narrower, and the separate existence of its two components could no longer be detected. When this line was observed simultaneously with that in the nebula, it was found to appear but a very little broader than that line. When the battery circuit was completed, the line from the spark coincided so accurately in position with the nebular line that the effect to the eye was as if a sudden increase of brightness in the line of the nebula had taken place. In order to make this observation, and to compare the relative appearance of the lines, the telescope was moved so that the light from the nebula occupied the lower half only of the slit. The line of the spark was now seen to be a very little broader than the line of the nebula, and appeared as a continuation of it in an unbroken straight line. These observations were repeated many times on several nights.

An apparent want of coincidence, which would be represented by 0.02 division of the head of the micrometer screw would be about the smallest difference that could be observed under the circumstances under which these observations were made. At the part of the spectrum where this line of nitrogen occurs the angular interval measured by 02 division of the micrometer corresponds to a difference of wave-length of 0460 millionth of a millimetre. 
At the time the comparisons were made the earth was receding from the part of the heavens in which the nebula is situated by about half its orbital velocity. If the velocity of light be taken at 185,000 miles per second, and the wave-length of the nitrogen line at 500.80 millionths of a millimetre, the effect of half the orbital motion would be to degrade the refrangibility of the line by 0.023 , an alteration of wave-length which would correspond to about 0.01 of the large micrometer head, an interval too small to be detected.

We learn from these observations, that if the line be emitted by nitrogen, the nebula is not receding from us with a velocity greater than ten miles per second; for this motion, added to that of the earth's orbital velocity, would have caused a want of coincidence that could be observed. Further, that if the nebula be approaching our system, its velocity may be as much as twenty miles or twenty-five miles per second; for part of its motion of approach would be masked by the effect of the motion of the earth in the contrary direction.

The double line in the nitrogen spectrum does not consist of sharply defined lines, but each component is nebulous, and remains of a greater width than the inage of the slit. ${ }^{1}$ The breacth of these lines appears to be connected with the conditions of tension and of temperature of the gas. Plicker ${ }^{2}$ states that when an induction spark of great heating power is employed the lines expand so as to unite and form an undivided band. Even when the duplicity exists, the eye ceases to have the power to distinguish the component lines, if the intensity of the light be greatly diminished.

Though I lave been unable to detect duplicity in the corresponding line in the nebula, it might possibly be found to be double if seen under more favourable conditions: I incline to the belief that it is not double. ${ }^{3}$

I Secchi states that with his direct speetroscope this line in the annular nebula in Lyra appears doublc. As the image of the nebula is viewed directly, after elongation by the cylindrical lens, and without a slit, it is probable that the two lines may correspond to the two sides of the elongated annulus of the nebula.

2 Phil. Trans. 1863, p. 13.

3 "On the Spectra of the Chemical Elements," Phil. Trans. 1864, p. 141. 
In my Tables of the lines of the air ${ }^{1}$ I estimated the brightness of each of the components of the double line in the spectrum of nitrogen at 10 , and the components of the double line next in brightness in the orange at 7 and 5 , and those of a third double line on the less refrangible side of $\mathrm{D}$ at 6 and 4 . It was with reference to these two double lines next in apparent brilliancy that I wrote, ${ }^{2}$ in speaking of the line in the nebula"If, however, this line were due to nitrogen, we ought to see other lines as well; for there are specially two strong double lines in the spectrum of nitrogen, one at least of which, if they existed in the light of the nebuln, would be easily visible."

As the disappearance of the whole spectrum of nitrogen, with the exception of the one double line, was unexpectedthough, indeed, in accordance with my previous estimations, I examined the spectrum of nitrogen with a spectroscope furnished with one prism with a refracting angle of $60^{\circ}$, in which the whole of the spectrum from $\mathrm{C}$ to $\mathrm{G}$ is included in the field of view-I then moved between the eye and the little telescope of the spectroscope a wedge of neutral-tint glass corrected for refraction by an inverted similar wedge of crown glass, and which I had found to be sensibly equal in absorbing power on the different parts of the visible spectrum. As the darker part of the ridge was brought before the eye, the two groups in the orange were quite extinguished, while the lines in the green still remained of considerable brightness. The line which under these circumstances remained longest visible, next to the brightest line, was one more refrangible at 2669 of the scale of my map. This observation was made with a narrow slit. When the induction spark was looked at from a distance of some feet with a direct-vision piism held close to the eye, I was surprised to observe that the double line in the orange appeared to me to be the brightest in the spectrum; and when the neutral-tint wedge was interposed, this line in the orange remained alone visible, all the other lines being extinguished.

When, however, in place of the simple prism a small directvision spectroscope provided with a slit was employed, I found

\footnotetext{
1 Phil. Trans. 1864, p. 141.
}

2 Ibid. p. 443. 
it to be possible, by receding from the spark, to find a position in which the double line in the green, with which the line in the nebula coincides, was alone visible, and the spectrum of the spark in nitrogen resembled that of a monochromatic nebula.

It is obvious that, if the spectrum of hydrogen were reduced in intensity, the line in the blue, which corresponds to that in the nebula, would remain visible after the line in the red and the lines more refrangible than $\mathrm{F}$ had become too feeble to affect the eye.

It therefore becomes a question of much interest whether the one, two, three, or four lines seen in the spectra of these nebulæ represent the whole of the light emitted by these bodies, or whether these lines are the strongest lines only of their spectra, which, by reason of their greater intensity, have succeeded in reaching the earth. Since these nebulæ are bodies which have a sensible diameter, and in all probability present a continuous luminous surface, or nearly so, we cannot suppose that any lines have been extinguished by the effect of the distance of these objects from us.

If we had evidence that the other lines which present themselves in the spectra of nitrogen and hydrogen were quenched on their way to us, we should have to consider their disappearance as an indication of a power of extinction residing in cosmical space, similar to that which was suggested from theoretical considerations by Chéseaux, and was afterwards supported on other grounds by Olbers and the elder Struve. Further, as the lines which we see in the nebulæ are precisely those which experiment shows would longest resist extinction, at least so far as respects their power of producing an impression on our visual organs, we might conclude that this absorptive property of space is not elective in its action on light, but is of the character of a general absorption acting equally, or nearly so, on light of every degree of refrangibility. Whatever may be the true state of the case, the result of this re-examination of the spectrum of this nebula appears to give increased probability to the suggestion that followed from my 
former observations, namely, that the substances hydrogen and nitrogen are the principal constituents of the nebulæ of the class under consideration.

I now pass to observations of the third line of the nebular spectrum, the one which I found to coincide with the line of hydrogen which corresponds to Fraunhofer's F. The substance in the nebulæ which is indicated by this line appears to be subject to much greater variation in relative brilliancy, or to be more affected by the conditions under which it emits light; for while the brightest line is always present, the line of which I am speaking seems to be wholly wanting in some nebulæ, and to be of different degrees of relative brightness in some other nebulæ.

In the nebula of Orion this line is relatively stronger than in $37 \mathrm{H}$. IV. Draconis, and some other nebulæ. I have suspected that the relative brightness of this line varies slightly in different parts of this nebula. It may be estimated perhaps in the nebula of Orion at about the brightness of the second line. The second line suffers in apparent brilliancy from its nearness to the brightestline, and may, without due regard to this circumstance, be estimated as brighter than the third line.

In order to conpare the position of the line with that of the corresponding line in the spectrum of hydrogen, I employed a vacuum-tube containing hydrogen at a very small tension, which was placed before the object-glass of the telescope. Under these conditions the line appears narrow when the slit is narrow, without any sensible nebulosity at the edges. The character of the line is altered, as has been shown by Plicker, when hydrogen at the atmospheric pressure is employed: the line then expands into a nebulous band of considerable width, even with a very narrow slit. Such a condition of the line is obviously unsuitable for the delicate comparisons which it was proposed to attempt.

The narrow, sharply-defined line of hydrogen, when the vacuum-tube was before the slit, was observed to coincide perfectly in position with the third line of the nebula. This observation, which shows the coincidence of these lines with an accuracy three or four times as great as my former observations, 
incrcases in the same ratio the probability that the line in the nebula is really due to luminous hydrogen.

I suspect that, although the third line in this nebula may impress the eye as strongly as the second line, yet it is not so narrow and well-defined as that line. If this suspicion be correct, this condition of the linc might indicate that the hydrogen exists at a rather greater tension than that in the socalled vacuum-tubes, but that it is not nearly so dense as would correspond to the atmospheric pressure at the surface of the earth. As, however, the character of the lines of hydrogen is also greatly modified by temperature, it is not possible to reason with any certainty as to the state of things in this distant object, the light of which we have now under examination.

I am still unable to find any terrestrial line which corrcsponds to the middle line. I have made the additional observation that the line in the nebula is in a very slight degree less refrangible than the line of oxygen at 2060 of the scale of my map. It is in a rather larger degree lcss refrangible than the strong line of barium at 2075 of my scale.

Several other nebulæ have beeu observed with the large spectroscope: I prefer, however, to re-examine thcse objects before I publish any observations of them.

\section{§IV. Observations of Stars.}

The chief difficulties which I have had to encounter have arisen from the unsteadiness of our atmosphere. There is sufficient light from stars of the first and second magnitude for the large spectroscope described in this paper, and, so far as the adjustments of the instrument are concerned, the lines in the spectra of the stars would be well defined. Unless, however, the air is very steady, the lines are seen too fitfully to permit of any certainty in the determination of coincidences of the degree of delicacy which is attempted in the present investigation. I have passed hours in the attempt to determine the position of a single line, and have then not considered that the numerous observations which I had obtained were possessed, even collectively, of 
sufficient weight to establish with any certainty the coincidence of the line with the one compared with it.

I prefer, therefore, to reject a large number of observations which appear unsatisfactory from this cause, and to give in this place a very few of the most trustworthy of the observations which I have made.

Sirius.-The brilliant light of this star and the great intensity of the four strong lines of its spectrum make it especially suitable for such an examination. The low altitude of this star in our latitude limits the period in which it can be successfully observed to about one hour on each side of the meridian.

I have confined myself to comparisons of the strong line in the position of $\mathrm{F}$ with the corresponding line of the spectrum of hydrogen. My first trials were made with hydrogen at the ordinary atmospheric pressure: the width of the band of hydrogen, under these circunstances, was greater than the line of Sirius. This line in Sirius, from some cause, is narrower relatively to the length of the spectrum, when considerable dispersion and a narrow slit are employed, than when the image of the star, rendered linear by a cylindrical lens, is observed with a single prism. ${ }^{1}$

When the large spectroscope was employed, I estimated the breadth of the line to be about equal to that of the double line D. In Kirchhoff's map the line $\mathrm{F}$ of the solar spectrum is represented as a little more than one-fourth of the interval separating the lines $\mathrm{D}$. When the spectroscope attached to the telescope was directed to the moon, the line $\mathrm{F}$ appeared even narrower than it is represented in Kirchhoff's map; I estimated it at about one-sixth of the apparent breadth of the corresponding line in the spectrum of Sirius. The character of the line agrees precisely with the line of hydrogen under certain conditions of tension and temperature.

As it was obviously impossible to determine with the required accuracy the coincidence of the line of Sirius when the much broader band of hydrogen at the ordinary pressure was compared with it, I employed a vacuum-tube fixed before the object-glass.

i See Phil. Trans. 1864, p. 42. 
In all these observations the slit used was as narrow as possible. The air at the time of the present observations was more favourable than usual, and the line in Sirius was seen with great distinctness. The line from the sparis appeared in comparison very narrow, not more than about one-fifth of the width of the line of Sirius. When the battery circuit was completed, the line of hydrogen could be seen distinctly upon the dariz line of Sirius. The observation of the comparison of the lines was made many times, and I am certain that the narrow line of hydrogen, though it appeared projected upon the dark line in Sirius, did not coincide with the middle of the line, but crossed it at a distance from the middle, which may be represented by saying that the want of coincidence was apparently equal to about one-third or one-fourth of the interval separating the components of the double line D. I was unable to measure directly the distance between the centre of the line of hydrogen and that of the line in the spectrum of Sirius, but several very careful estimations by means of the micrometer give a value for that distance of 0.040 of the micrometer head. This value is probably not in error by so much as its eighth part.

Comparisons on many other nights were also made, sometimes with the vacuum-tube before the object-glass, and sometimes with the vacuum-tube placed over the small hole in the guttapercha plate. On all these occasions the numerous comparisons which were made gave for the line in Sirius a very slightly lower refrangibility than that of the line of hydrogen, but on no one occasion was the air steady enough for a satisfactory determination of the amount of difference of refrangibility.

I have not been able to detect any probable source of error in this result, and it, may therefore, I believe, be received as representing a relative motion of recession between Sirius and the earth.

The probability that the substance in Sirius by which this line is produced is really hydrogen is strengthened almost to certainty by the consideration that there is a strong line in the red part of the spectrum which is also coincident with a strong line of hydrogen. There is a third line more refrangible than $\mathrm{F}$, 
which appears to coincide with the line of hydrogen in that part of the spectrum.

As the line in Sirius is more expanded than that of the vacuum-tube, it seemed of importance to have proof from experiment that this line of hydrogen, when it becomes broad, expands equally in both directions. I made the comparisons of the narrow line of the vacuum-tube with the more expanded band which appears when denser hydrogen is employed. For this purpose the intersection of the wires of the eyepiece was brought, as nearly as could be estimated, upon the middle of the expanded line which is produced by dense hydrogen. The vacuum-tube was then arranged before the slit, when the narrow line which it gives was observed to fall exactly upon the point of intersection of the wire. Under these terrestrial conditions the expansion of the line may be considered to take place to an equal amount in both directions. There is very great probability that a similar equal expansion takes place under the conditions which determine the absorption of light by this gas in the atmosphere of Sirius, for the reason that the nebulosity at the edges of the line in the spectrum of that star is sensibly equal on both sides.

I made some attempts to compare the strong line at $\mathrm{C}$ with the corresponding line of hydrogen; but when the large spectroscope was employed, though the lines could be seen with tolerable distinctness, they were not bright enough to admit of a trustworthy determination of their relative position. When one of the compound prisms was removed, the lines were much more easily seen, but under these circumstances the amount of dispersion was insufficient for my present purpose.

The lines of Sirius which, in conjunction with Dr. Miller, I had compared with those of iron, magnesium, and sodium, are not sufficiently well seen in our latitude for comparison when a powerful train of prisms is employed, such as is necessary for this special inquiry.

From these observations it may, I think, be concluded that the substance in Sirius which produces the strong lines is really hydrogen, as was stated by Dr. Miller and myself in our former paper. Further, that the aggregate result of the motions of the 
star and the earth in space, at the time when the observations were made, was to degrade the refrangibility of the line in Sirius by an amount corresponding to 0.040 of the micrometer screw. Now the value of the wave-lengths of 0.01 division of the micrometer at the position of $\mathrm{F}$ is 0.02725 millionth of a millimetre. The total degradation of refrangibility observed amounts to 0.109 millionth of a millimetre. If the velocity of light be taken at $185,000^{2}$ miles per second, and the wave-length of $\mathrm{F}$ at 486.50 millionths of a millimetre (Angström's value is 486.52 , Ditscheiner's 486.49 ), the observed alteration in period of the line in Sirius will indicate a motion of recession existing between the earth and the star of 41.4 miles per second.

Of this motion a part is due to the earth's motion in space. As the earth moves round the sun in the plane of the ecliptic, it is changing the direction of its motion at every instant. There are two positions, separated by $180^{\circ}$, where the effect of the earth's motion is a maximum; namely, when it is moving in the direction of the visual ray, either towards or from the star. At two other positions in its orbit, at $90^{\circ}$ from the former positions, the earth's motion is at right angles to the direction of the light from the star, and therefore has no influence on its refrangibility.

The effect of the earth's motion will be greatest upon the light of a star situated in the plane of the ecliptic, and will decrease as the star's latitude increases, until, with respect to a star situated at the pole of the ecliptic, the earth's motion during the

1 The value in wave-lengths of the divisions of the micrometer for different parts of the spectrum was determined by the aid of the tables of the wavelengths corresponding to every tenth line of Kirchhoff's map by Dr. Wolcott Gibbs (Silliman's Jonrnal, vol. xliii. January 1867). A paper on the same subject by the Astronomcr Royal, presented to the Royal Society, is not yet in print. The Astronomer Royal's paper is contained in the Philosophical Transactions for 1868, Part I. p. 29. The wave-lengths computcd by him differ slightly from those assigned to Kirchhoff's numbers by Dr. Gibbs at the part of the spectrum under consideration in the tcxt. The difference is due in part to the employment, by the Astronomer Royal, of Ditscheiner's later measures. These give for $\mathrm{F}$ the higher value of $486 \cdot 87$. - October 1868 .

2 The new determination of the value of the solar parallax by observations of Mars requires that the usually received velocity of light, 192,000 miles per second, should be reduced by about the one twenty-seventh part. The velocity, when diminished in this ratio, agrces nearly with the result obtained by Foucault from direct expcriment. 
whole of its annual course will be perpendicular to the direction of the light coming to us from it, and will be therefore without influence on its period.

That part of the earth's resolved motion which is in the direction of the visual ray, and which has alone to be considered in this investigation, may be obtained from the following formula:

Earth's motion towards star $=v \cdot \cos \lambda \cdot \sin \left(l-l^{\prime}\right)$,

where $v$ is the earth's velocity, $l$ the earth's longitude, $l^{\prime}$ the star's longitude, and $\lambda$ the star's latitude.

At the time when the estimate of the amount of alteration of period of the line in Sirius was made the earth was moving from the star with a velocity of about twelve miles per second.

There remains unaccounted for a motion of recession from the earth amounting to 29.4 miles per second, which we appear to be entitled to attribute to Sirius.

It may be not unnecessary to state that the solar motion in space, if accepted as a fact, will not materially affect this result, since, according to M. Otto Struve's calculations, the advance of the sun in space takes place with a velocity but little greater than one-fourth of the earth's motion in its orbit. If the apex of the solar motion be situated in Hercules, nearly the whole of it will be from Sirius, and will therefore diminish the velocity to be ascribed to that star.

It is interesting, in connection with the motion of Sirius, deduced from these prismatic observations, to refer to the remarkable inequalities which occur in the rather larger proper motion of that star. In 1851 M. Peters ${ }^{1}$ showed that the variable part of the proper motion of Sirius in right ascension might be represented by supposing that Sirius revolves in an elliptic orbit, round some centre of gravity without itself, in a period of 50.093 years. This hypothesis has acquired new interest, and seems indeed to have received confirmation from direct observation by Alvan Clark's discovery of a small companion to Sirius. 
Professor Safford ${ }^{1}$ and Dr. Auwers ${ }^{2}$ have investigated the periodical variations of the proper motion of Sirius in declination, and they have found that these variations, equally with those in right ascension, would be reconcilable with an elliptic orbital motion round a centre not in Sirius. The close coincidence of the observed positions of the new satellite with those required by theory seems to show that it may be the hypothetical body suggested by Peters, though we must then suppose it to have a much greater mass relatively to Sirius than that which its light would indicate.

At the present time the proper motion of Sirius in declination is less than its average amount by nearly the whole of that part of it which is variable. May not this smaller apparent motion be interpreted as showing that a part of the motion of the star is now in the direction of the visual ray? This circumstance is of much interest in connection with the result arrived at in this paper.

Independently of the considerations connected with the variable part of the star's proper motion, it must not be forgotten that the whole of the motion which can be directly observed by us is only that portion of its real motion which is at right angles to the visual ray. Now it is precisely the other portion of it, which we could scarcely hope to learn from ordinary observations, which is revealed to us by prismatic investigations. By combining the results of both methods of research we may perhaps expect to obtain some knowledge of the real motions of the brighter stars and nebulæ.

It seems therefore desirable to compare with the result obtained by the prism the motion of Sirius which corresponds to its assumed constant proper motion. The values adopted by Mr. Main," and inserted by the Astronomer Royal in the Greenwich "Seven-year Catalogue," are -0 ". 035 in R.A. and $+1^{\prime \prime} \cdot 24$ in N.P.D.

1 Proceedings of the American Academy, vol. vi. ; also Astron. Notices, Ann. Arbor, No. 28; Monthly Notices, vol. xxii. p. 145.

2 Astron. Nachrichten, No. 1506; Monthly Notices, vol. xxii. p. 148, and vol. xxv. p. 39.

Nemoirs of the Royal Astronomical Society, vol. xix. 
The parallax of Sirius from the observations of Henderson, corrected by Bessel, $=0^{\prime \prime} \cdot 150$. A recent investigation, by Mr. C. Abbe, ${ }^{1}$ gives for the parallax the larger value of $0^{\prime \prime} \cdot 27$. If the radius of the earth's orbit be taken at its new value of $91,600,000$ miles, the assumed annual constant proper motion in N.P.D. of $1^{\prime \prime} \cdot 24$ would indicate, with the parallax of Henderson, a velocity of Sirius of twenty-four miles nearly per second; with the larger parallax of Mr. Abbe, a velocity of $4.3 \cdot 2$ miles per second. It may be that in the case of Sirius we have two distinct motions; one peculiar to the star, and a second motion which it may share in common with a system of which it may form a part.

Observations and comparisons, similar to those on Sirius, liave been made on a Canis Minoris, Castor, Betelgeux, Aldebaran, and some other stars. I reserve for the present the results which I have obtained, as I desire to submit these objects to a re-examination. It is seldom that the air is sufficiently favourable for the successful prosecution of this very difficult research.

$$
\text { * }
$$

*

*

*

*

\section{$\S$ V. Observations of Comet II. 1868 .}

On June 13 a comet was discovered by Dr. Winnecke, and also independently the same night by M. Becquet, Assistant Astronomer at the Observatory of Marseilles.

I was prevented by buildings existing near my Observatory from making observations of this comet before June 22. On that evening the comet was much brighter than Brorsen's comet, a description of the spectrum of which I recently presented to the Royal Society, ${ }^{2}$ and it gave a spectrum sufficiently distinct for measurement and comparison with the spectra of terrestrial substances.

T'eleseopie Appearance of the Comet.-A representation of the comet as it appeared on June 22 at 11 P.M. is given in Fig. 110, p. 326. The comet consisted of a nearly circular coma, which

1 Monthly Notices of the Royal Astronomical Society, vol, xxviii. p. 2.

2 Proc. hioy. Soc. vol. xvi. p. 386. 
became rather suddenly brighter towards the centre, where there was a nearly round spot of light. The diameter of the coma, including the exterior faint nebulosity, was about $6^{\prime} 20^{\prime \prime}$. The tail, which was traced for more than a degree, was sharply defined on the following edge, but faded so gradually away on the opposite side that no limit could be perceived. No connection was traced between the tail and the brighter central part of the coma. The circular form of the coma was uninterrupted on the side of the tail, which appeared as an extension of the faint nebulosity which formed the extreme margin of the coma.

The bright roundish spot of light in the centre, when examined with eyepieces magnifying from 200 to 600 diameters, presented merely a nebulous light without a defined form.

Spectrum of the Comet.-When a spectroscope furnished with two prisms of $60^{\circ}$ was applied to the telescope, the light of the comet was resolved into three very broad bright bands, which are represented in the diagram. (Fig. 109.)

In the two more refrangible of these bands the light was brightest at the less refrangible end, and gradually diminished towards the other limit of the bands. This gradation of light was not uniform in the middle and brightest band, which continued of nearly equal brilliancy for about one-third of its breadth from the less refrangible end. This band appeared to be commenced at its brightest side by a bright line.

The least refrangible of the three bands did not exhibit a similar marked gradation of brightness. This band, though of nearly uniform brilliancy throughout, was perhaps brightest about the middle of its breadth.

These characters, which are peculiar to the light emitted by the cometary matter, must be distinguished from some appearances which the bands assumed in consequence of the mode of distribution of the light in the coma of the comet. The two more refrangible bands became narrower towards their most refrangible side, as well as diminished in brightness. This appearance was obviously not due to any dissimilarity of the light in the parts of the coma, but to the circumstance that, as the light of the coma became brighter towards the centre, it was 
emitted by a smaller area of the cometary matter. The strong light of the central spot could be traced the whole breadth of the band; but the light surrounding this spot, in proportion as it became fainter and broader, was seen for a shorter distance, so that the light from the faintest parts near the margin of the coma was visible only at the brightest side of the band. Since in the least refrangible band a similar gradation of light did not take place, this band appeared of nearly the same width throughout.

The increasing brightness of the coma up to the brilliant spot in the centre showed itself in this band as a bright axial line fading off gradually in both directions.

On this evening I took repeated measures of the positions of these bands with the micrometer attached to the spectroscope. 'These measures give the following numbers for the commencement and termination of the three bands on the scale adopted in the diagram :-

First band $\left\{\begin{array}{l}1094 . \\ 1196 .\end{array}\right.$ Second band $\left\{\begin{array}{l}1298 . \\ 1440 .\end{array}\right.$ Third band $\left\{\begin{array}{l}1589 . \\ 1700 .\end{array}\right.$

I could not resolve the bands into lines. When the slit was made narrower, the bands became smaller both in breadth and length, from the invisibility of the fainter portions. I suspected, however, the presence of two or three bright lines in the bright central part of the middle band near its less refrangible limit. This part would consist chiefly of light from the bright central spot.

As has been stated, the middle band commences probably with a bright line; for the limit of the band is here abrupt, and distinct. On the contrary, the exact point of commencement and termination of the other bands could not be observed with certainty.

I could perceive no other bands, nor light of any kind beyond the three bands, in the parts of the spectrum towards the red and the violet.

When the marginal portions of the coma were brought upon the slit, the three bands of light could still be traced. When, however, the spectrum became very faint, it appeared to me to 
become continuous; but the light was then so very feeble that it could not be traced beyond the three bands towards the violet or the red.

On this evening I observed the spectrum of the comet in a larger spectroscope, which gives a dispersion equal to about five prisms. In this instrument the middle band was well seen. It retained its nebulous, unresolved character, and the abrupt commencement, as if by a bright line, already mentioned, was distinctly seen.

For convenience of comparison, the spectrum of Brorsen's comet, and that of the gaseous nebulæ, have been added to the diagram, Fig. 109. The spectrum of Brorsen's comet consisted of three bright bands and a faint continuous spectrum. These bands appeared, as represented in the diagram, narrower than those of the comet now under examination. It is not possible to say to what extent this circumstance may bo due to the much feebler light of this comet. Though the bands of Brorsen's comet fall within the limits of position occupied by the broad bands of Comet II., they do not correspond to the brightest parts of these bands. In the middle band I suspected two bright lines which appeared shorter than the band, and may be due to the nuclens. Brorsen's comet differed from the two small comets which I had previously examined ${ }^{1}$ in the much smaller relative proportion of the light which forms a continuous spectrum. In Brorsen's comet the bright middle part of the coma seemed to emit light similar to that of the nucleus; in the other comets the coma appeared to give a continuous spectrum. The three comets resembled each other in the circumstance that the light of the central part was emitted by the cometary matter, while the surrounding nebulosity reflected solar light.

It will be seen in the diagram that the bands of Brorsen's comet and those of Comet II. occupy positions in the spectrum widely removed from those in which the lines of the nebulæ occur. The spectra of gaseous nebulæ consist of true lines, which become narrow as the slit is made narrower.

1 Comet I. 1866, Proceedings, vol. xv.p. 5; and Comet 1867, Monthly Notices of Royal Astronomical Society, vol. xxvii. p. 288. 
The following day I carefully considered these observations of the comet, with the hope of a possible identification of its spectrum with that of some terrestrial substance. The spectrum of the comet appeared to me to resemble some of the forms of the spectrum of carbon which I had observed and carefully measured in 1864. On comparing the spectrum of the comet with the diagram of these spectra of carbon, I was much interested to perceive that the positions of the bands in the spectrum, as well as their general characters and relative brightness, agreed exactly with the spectrum of carbon when the spark is taken in olefiant gas.

These observations on the spectrum of carbon were undertaken in continuation of my researches "On the Spectra of the Chemical Elements." I I have.not presented them to the Royal Society, as they are not so complete as I hope to make them.

Though the essential features of the spectrum of carbon remained unchanged in all the experiments, certain modifications were observed when the spectrum was obtained under different conditions. One of those modifications, which was referred to in my paper "On the Spectra of the Chemical Elements," 2 may be mentioned here. One of the strongest of the lines of carbon is a line in the red a little less refrangible than the hydrogen line, which corresponds to Fraunhofer's c. Now this line is not seen when the carbon is subjected to the induction spark in the presence of hydrogen. Two of the other modifications of the spectrum of carbon are given in Fig. 109. The first spectrum represents the appearance of the spectrum of carbon when the induction spark, with Leyden jars intercalated, was taken between the points of wires of-platinum sealed in glass tubes, and placed almost in contact in olive-oil. In this spectrum are seen the principal strong lines which distinguish carbon. The shading of tine lines which accompanies the strong lines cannot be accurately represented, on account of the small size of the diagram. A spectrum essentially the same is produced when the spark is taken in a current of cyanogen. It may be mentioned that when the heating power of the spark was

1 Phil. Trans. 1864, p. 138.

2 Ibid. p. 145. 
reduced below a certain limit, though the decomposition of the oil still took place, the carbon was not volatilized, and the spectrum was continuous.

The third spectrum in the diagram represents the modification of this typical spectrum when the induction spark is taken in a current of olefiant gas. The bighly-heated vapour of carbon emits light of the same refrangibilities as in the case of the oil; but the separate strong lines, with a similar power of spark, were no longer to be distinguished. The shading, when the carbon was obtained from the olefiant gas, was not composed of numerous fine lines, but appeared as an unresolved nebulous light.

Of course in all these experiments the lines of the other elements present were also seen, but they were known, and could therefore be disregarded.

In the case of the spark in olefiant gas, the three bands in the diagram constitute the whole spectrum, with the exception of a faint band in the more refrangible part of the spectrum.

It was with the spectrum of carbon, as thus obtained, that the spectrum of the comet appeared to agree. It seened, therefore, to be of much importance that the spectrum of the spark in olefiant gas should be compared directly in the spectroscope with the spectrum of the comet. The comparison of the gas with the comet was made the same evening, June 23.

My friend Dr. William Allen Miller visited the Observatory on this evening, and kindly took part in the following observations.

The general arrangements of the apparatus with which the comparison was made is shown in the following diagram (Fig. 119).

A glass bottle converted into a gasholder, $a$, contained the olefiant gas. This was connected by means of a flexible tube $b$, into which were soldered two platinum wires. The part of the tube in front of the points of the wires had been cut away, and the surfaces carefully ground. A small plate of glass closed the opening, being held in its place by a band of vulcanized 
india-rubber. This tube was arranged in its proper position, before the small mirror of the spectroscope $c$, by which the light of the spark was reflected into the instrument, and its spectrum was seen immediately beneath the spectrum of the cornet. The spectroscope employed was furnished with two prisms of $60^{\circ}$.

The brightest end of the middle band of the cometic spectrum was seen to be coincident with the commencement of the corresponding band in the spectrum of the spark. As this limit of

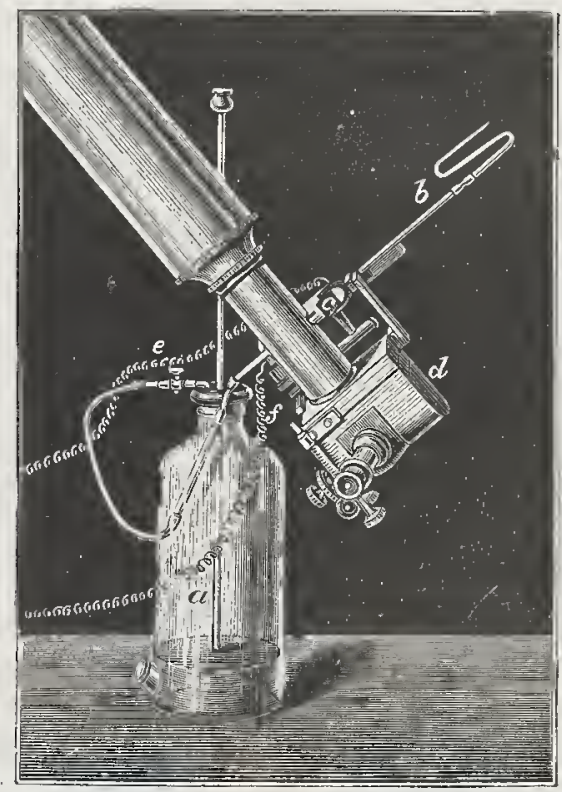

FrG. 119

the band was well defined in both spectra, the coincidence could be satisfactorily observed up to the power of the spectroscope, and may be considered to be determined within about the distance which separates the components of the double line $\mathrm{D}$. As the limits of the other bands were less distinctly seen, the same amount of certainty of exact coincidence could not be obtained. We considered these bands to agree precisely in position with the bands corresponding to them in the spectrum of the spark. 
The apparent identity of the spectrum of the comet with that of carbon rests not only on the coincidence of position in the spectrum of the bands, but also upon the very remarkable resemblance of the corresponding bands in their general characters, and in their relative brightness. This is very noticeable in the middle band, where the gradation of brightness is not uniform. This band in both spectra remained of nearly equal brightness for the same proportion of its length.

On a subsequent evening, June 25, I repeated these comparisons, when the former observations were fully confirmed in every particular. On this evening I compared the brightest band with that of carbon in the larger spectroscope, which gives a dispersion of about five prisms.

The remarkably close resemblance of the spectrum of the comet to the spectrum of carbon necessarily suggests the identity of the substances by which in both cases the light was emitted.

It may be well to state that some phosphorescent and fluorescent bodies give discontinuous spectra, in which the light is restricted to certain ranges of refrangibility. There are, however, several considerations which seem to oppose the idea that the light of comets can be of a phosphorescent character. Phosphorescent bodies are usually so highly reflective that the phosphorescence emitted by them is not seen so long as they are exposed to light. This comet was still in the full glare of the sun, and yet the continuous spectrum corresponding to reflected solar light was of extreme feebleness compared with the three bright bands which we have under consideration. The phenomenon of phosphorescence seems to be restricted to bodies in the solid state, a condition which is not apparently in accordance with certain phenomena which have been observed in large comets, such as the outlow of the matter of the nucleus and the formation of successive envelopes.

There are, indeed, some phenomena of fluorescence, such as that of a nearly transparent liquid becoming an object of some brightness by means of the property which it possesses of 
absorbing the nearly invisible rays of the spectrum, and dispersing them in a degraded and much more luminous form, which are less obviously inconsistent with cometary phenomena than are those of phosphorescence.

The violent commotions and internal changes which we witness in comets when near the sun seem, however, to connect the great brightness which they then assume more closely with the part. of the solar force we call heat. There is also to be considered the fact of the polarized condition of the light of the tail and some parts of the comæ of comets, which shows that a part of their light is reflected.

The observations of the spectrum of Comet II. contained in this paper, which show that its light was identical with that emitted by highly-heated vapour of carbon, appear to be almost decisive of the nature of cometary light. The great fixity of carbon seems indeed to raise some difficulty in the way of accepting the apparently obvious inference of these prismatic observations. Some comets have approached the sun sufficiently near to acquire a temperature high enough to convert even carbon into vapour. ${ }^{1}$ Indeed for these comets a body of great fixity seems to be necessary. In the case of comets which have been submitted to a less fierce glare of solar heat, it may be suggested that this supposed difficulty is one of degree only; for we do not know of any conditions under which even a gas, permanent at the temperature of the earth, could maintain sufficient heat to emit light, a state of things which appears to exist permanently in the case of the gaseous nebulæ.

If the substance of the comet be taken to be pure carbon, it would appear probable that the nucleus had been condensed from the gaseous state in which it existed at some former period. It would therefore probably consist of carbon in a state of excessively minute division. In such a form it would be able to

1 The Comet of 1843 " approached the luminous surface of the sun within about a seventh part of the sun's radius. The heat to which the comet was subjected (a glare as strong as that of 47,000 suns, such as we experience the warmth of) surpassed that in the focus of Parker's great lens in the proportion of $24 \frac{1}{2}$ to 1 without, or $3 \frac{1}{2}$ to 1 with, the concentrating lens. Yet that lens so used melted cornelian, agate, and rock-crystal." - SIR JOHN HERSCHEL, Outlines of Astronomy, 7 th edit. p. 401 . 
take in nearly the whole of the sun's onergy, and thus acquire more speedily a temperature high enough for its conversion into vapour. In the liquid or gaseous state, or in a continuous solid state, this substance appears, from Dr. Tyndall's researches, to be diathermanous. Still, under the most favourable of known conditions, the solar heat, to which the majority of comets are subjected, would seem to be inadequate to the production of luminous vapour of carbon.

It should be stated that olefiant gas when burnt in air may give a similar spectrum of shaded bands. If the gas be ignited at the orifice of the tube from which it issues, the flame is brilliantly white, and gives a continuous spectrum. When a jet of air is directed through the flame, it becomes less luminous, and of a greenish-blue colour. The spectrum is now $\mathrm{n} n$ longer continuous, but exhibits the bands distinctive of carbon. Under these circumstances, for obvious reasons, the bright lines of the hydrogen spectrum are not seen. In this way a spectrum resembling that of the comet may be obtained, with the difference that the fourth more refrangible band, which was not seen in the cometic spectrum, is stronger relatively to the other bands than is the case when the spark is taken in clefiant gas. If we wero to conceive the comet to consist of a compound of carbon and hydrogen, we should diminish in some degree the necessity for the excessively high temperature which pure carbon appears to require for its conversion into luminous vapour: but other difficulties would arise in connection with the decomposition we must then suppose to take place; for we have no evidence, I believe, that olefiant gas or any other known compound of carbon can furnish this peculiar spectrum of shaded bands without undergoing decomposition. If, indeed, it were allowable to suppose a state of combustion, with oxygen or some other element, set up by the solar heat, we should have an explanation of a possible source of a degree of heat sufficient to render the cometary matter luminous, and which the sun's heat would be directly inadequate to produce.

There is one observation made by Bunsen which appears to stand as an exception to the rule that only bodies in the gaseous 
state give, when luminous, discontinuous spectra. Bunsen discovered that solid erbia, when heated to incandescence, gives a spectrum containing bright bands. It is therefore conceivable, though all the evidence we possess from experience is opposed to the supposition, that carbon might exist in some form in which it would possess a similar power of giving a discontinuous spectrum without volatilization. There is the further objection to this hypothesis, that the telescopic phenomena observed in comets appear to show that vaporization does usually take place. However this may be, a state of gas appears to accord with the very small power of reflection which the matter of the coma of this comet possessed, as was shown by the great faintness of the continuous spectrum.

A remarkable circumstance connected with comets is the great transparency of the bright cometary matter. The most remarkable instance is that of Miss Mitchell's comet in 1847, which passed centrally over a star of the fifth magnitude. "The star's light appeared in no way enfeebled: yet such a star would be completely obliterated by a moderate fog extending a few yards from the surface of the earth." 1 We do not know what amount of transparency is possessed by the vapour of carbon, but the absence of a continuous spectrum seems to show that, as it existed in the comet, it was almost perfectly transparent. The light of a star would suffer, therefore, only that kind and degree of absorption which corresponds with its power of radiation, as shown by its spectrum of bright lines. As these occur in the brightest part of the spectrum, we should expect a noticeable diminution of the star's light, if it were not for the luminous condition of the gas, in consequence of which it would give back to the beam light of precisely the same refrangibilities as it had taken, and so enable the part of the field occupied by the image of the star to appear of its original brightness, or nearly so. This state of things would not prevent an apparent diminution of the star's light from the effect upon the eye of the brightness of the surrounding field. In the case of the tails of comets, the great transparency observed is more probably to be referred to

1 Outlines of Astronomy, p. 373. 
the widely-scattered condition of the minute particles of the cometary matter.

I may be permitted to repeat here a paragraph from my paper on the Spectrum of Comet I. $1866 .^{1}$

"Terrestrial phenomena would suggest that the parts of a comet which are bright by reflecting the sun's light are probably in the condition of fog or cloud.

"We know from observation, that the comre and tails of comets are formed from the matter contained in the nucleus. ${ }^{2}$

"The usual order of the phenomena which attend the formation of a tail appears to be that, as the comet approaches the sun, material is thrown off, at intervals, from the nucleus in the direction towards the sun. This material is not at once driven into the tail, but usually forms in front of the nucleus a dense luminous cloud, into which for a time the bright matter of the nucleus continues to stream. In this way a succession of envelopes may be formed, the material of which afterwards is dissipated in a direction opposite to the sun, and forms the tail. Between these envelopes dark spaces are usually seen.

"If the matter of the nucleus is capable of forming by condensation a cloudlike mass, there must be an intermediate state in which the matter ceases to be self-luminous, but yet retains its gaseous state, and reflects but little light. Such a nonluminous and transparent condition of the cometary matter may possibly be represented by some at least of the dark spaces which, in some comets, separate the cloudlike envelopes from the nucleus and from each other."

1 Proe. Roy. Soc. vol. xv. p. 5.

2 The head of Halley's eomet in 1835 in a teleseope of great power "exhibited the appearanee of jets as it were of flame, or rather of luminous smoke, like a gas fan-light. These varied from day to day, as if wavering backwards and forwards, as if they were thrown out of partieular parts of the internal nueleus or kernel, whieh shifted round, or to and fro, by their reeoil, like a squib not held fast. The bright smoke of these jets, however, never seemed to be able to get far out towards the sun, but always to be driven back and foreed into the tail, as if by the aetion of a violent wind setting against them (always from the sun), so as to make it elear that the tail is neither more nor less than the aecumulation of this sort of luminous vapour darted off, in the first instance towards the sun, as it were something raised up and, as it were, exploded by the sun's heat out of the kernel, and then immediately and foreibly turned back and repelled from the sun."Sir Jonn Herscher, Familiar Lectures on Scientific Subjects, p. 115. 
Now considerable differences of colour have been remarked in the different parts of some comets. The spectrum of this comet would show that its colour was bluish-green. Sir W. Herschel describel the head of the comet of 1811 to be of a greenish or bluish-green colour, while the central point appeared to be of a pale ruddy tint. The representations of Hal'ey's comet at its appearance in 1835, by the elder Struve, are coloured bluishgreen, and the nucleus on October 9 is coloured reddish-yellow. He describes the nucleus on that day thus: "Der Kern zeigte sich wie eine kleine, etwas ins Gelbliche spielende, glühende Kohle von länglicher Form." I Dr. Winnecke describes similar colours in the bright Comet of 1862: "Die Farbe des Strahls erscheint mir gelbröthlich; die des umgebenden Nebels (vielleicht aus Contrast) mattbläulich." "Die Farbe der Ausströmung erscheint mir gelblich; die Coma hat bläuliches Licht." 2

Now carbon, if incandescent in the solid state, or reflecting, when in a condition of minute division, the light of the sun, would afford a light which, in comparison with that emitted by the luminous vapour of carbon, would appear as yellowish or approaching to red.

The views of comets presented in this paper do not, however, afford any clue to the great mystery which surrounds the enormous rapidity with which the tail is often projected to immense distances. There are not any known properties peculiar to carbon, even when in a condition of extremely minute division, which would help to a solution of the enigma of the violent repulsive power from the sun which appears to be exerted upon cometary matter shortly after its expulsion from the nucleus, and upon matter in this condition only. It may be that this apparent repulsion takes place at the time of the condensation of the gaseous matter of the coma into the excessively minute solid particles of which the tail probably consists. There is a phenomenon occasionally seen which must not be passed without notice, namely, the formation of faint narrow rays of light, or secondary tails, which start off usually from the brightest side of

1 Beobachtungen des Halleyschen Cometen, S. 41.

2 Mémoires de l’Académic Impériale des Sciences de St. Pétersbourg, t. vii. No. 7. 
the principal tail, not far from the head. Sir John Herschel ${ }^{1}$ considers that "they clearly indicate an analysis of the cometic matter by the sun's repulsive action, the matter of the secondary tails being darted off with incomparably greater velocity (indicating an incomparably greater intensity of repulsive energy) than that which went to form the primary one." The important differences which exist between the spectrum of Brorsen's comet and that of Comet II. 1868 appear to show that comets may vary in their constitution. If the phenomena of the secondary tails were observed in a comet which, like Comet II. 1868, appears to consist of carbon, the analytical action supposed by Sir John Herschel might be to separate between particles of carbon in different conditions, or possibly in a state of more or less subdivision. The enormous extent of space, sometimes a hundred millions of miles in length, over which a comparatively minute portion of cometary matter is in this way diffused, would suggest that we bave in this phenomenon a remarkable instance of the extreme division of matter. Perhaps it would be too bold a speculation to suggest that, under the circumstances which attend the condensation of the gaseous matter into discrete solid particles, the division may be pushed to its utmost limit, or nearly so. If we could conceive the separate atoms to be removed beyond the sphere of their mutual attraction of cohesion, it might be that they would be affected by the sun's energy in a way altogether different from that of which we have been hitherto the witnesses upon the earth.

Though comets may differ in their constitution, reference may be permitted to the periodical meteors, which have been shown to move in orbits identical with those of some comets. If these consist of carbon, we might have some explanation of the appearances presented by these meteors, though their light is doubtless greatly modified by that of the air rendered luminous by their passage, as well as by the degree of temperature to which they are raised. Carbon is abundantly present in some meteorites, but we have no certain evidence at present that the periodical meteors belong to this class of celestial bodies.

1 Tamiliar Lectures on Scientific Subjects, p. 129. 


\section{APPENDIX E.}

ON THE SPECTRUM OF THE GREAT NEBULA IN ORION, AND ON THE MOTIONS OF SOME STARS TOWARDS OR FROM THE EARTH. ${ }^{1}$

BY WILLIAM HUGGINS, LL.D., D.C.L., F.R.S.

In my early observations of the spectrum presented by the gaseous nebulæ, the spectroscope with which I determined the coincidence of two of the bright lines respectively with a line of nitrogen and a line of hydrogen, was of insufficient dispersive power to show whether the brightest nebular line was double, as is the case with the corresponding line of nitrogen.

Subsequently I took some pains to determine this important point by using a spectroscope of greater dispersive power. I found, however, that the light furnished by the telescope of eight inches aperture, to which the spectroscope was attached, was too feeble, even in the case of the brightest nebulæ, to give the line with sufficient distinctness when a narrow slit was used. The results of this later examination are given in a paper I had the honour of presenting to the Royal Society in 1868. I there say: ${ }^{2}$

"I expected that I might discover a duplicity in the line in the nebula corresponding to the two component lines of the line of nitrogen, but I was not able, after long and careful scrutiny, to see the line double. The line in the nebula was narrower than the double line of nitrogen; this latter line may have appeared broader in consequence of irradiation, as it was much brighter than the line in the nebula." When the spark was placed before the object-glass of the telescope, the light was so much weakened that one line only was visible in the spectroscope. "This line was the one which agrees in position with the line in the nebula, so that under these circumstances the spectrum of nitrogen

1 Paper read before the Royal Society, June 13, 1872.

2 Phil. Trans. 1868, pp. 542, 543. 
appeared precisely similar to the spectra of these nebulæ, of which the liglat is apparently monochromatic. This resemblance was made more complete by the faintness of the line; from which cause it appeared narrower, and the separate existence of its two components could no longer be detected. When the line was observed simultaneously with that in the nebula, it was found to appear but a very little broader than that line." I also remark: "The double line in the nitrogen-spectrum does not consist of sharply defined lines, but each component is nebulous, and remains of a greater width than the image of the slit. ${ }^{1}$ The bread th of these lines appears to be connected with the conditions of tension and temperature of the gas. Plücker states that when an induction spark of great heating power is employed, the lines expand so as to unite and form an undivided band. Even when the duplicity exists, the eye ceases to have the power to distinguish the component lines, if the intensity of the light be greatly diminished." I state further: "I incline to the belief that it [the line in the nebula] is not double."

One of the first investigations which I proposed to myself when, by the kindness of the Royal Society, I had at my command a much more powerful telescope, was the determination of the true character of the bright line in the spectrum of the nebula, which is apparently coincident with that of nitrogen. From various circumstances, chiefly connected with the alterations and adjustments of new instruments, I was not able to accomplish this task satisfactorily until within the last few months.

\section{Description of Apparatus.}

It seems to me desirable to give a description of the spectroscopic apparatus with which the observations in this paper were made. In the former paper, to which I have already referred, I gave some reasons ${ }^{2}$ to show that the ordinary method of comparison, by reflecting light into the spectroscope by means of a small prism placed before one half of the slit, is not satisfactory

1 Phil. Trans. 1868, p. 13.

2 Ibid. 1868, pp. 537, 538. 
for very delicate observations unless certain precautions are taken. I then describe an arrangement for this purpose, which, with one or two modifications, is adopted in the collimator constructed for use with the Royal Society's telescope. I give the description from that paper : ${ }^{1}$

"The following arrangement for admitting the light from the spark appeared to me to be free from the objections which have been referred to, and to be in all respects adapted to meet the requirements of the case. In place of the small prism, two pieces of silvered glass were securely fixed before the slit at an angle of $45^{\circ}$. In a direction at right angles to that of the slit an opening of about $\frac{1}{10}$ inch was left between the pieces of glass for the passage of the pencils from the object-glass. By means of this arrangement, the spectrum of a star is seen accompanied by two spectra of comparison, one appearing above and the other below it. As the reflecting surfaces are about 0.5 inch from the slit, and the rays from the spark are divergent, the light reflected from the pieces of glass will have encroached upon the pencils from the object-glass by the time they reach the slit, and the upper and lower spectra of comparison will appear to overlap to a small extent the spectrum formed by the light from the object-glass. This condition of things is of great assistance to the eye in forming a judgment as to the absolute coincidence or otherwise of lines. For the purpose of avoiding some inconveniences which would arise from glass of the ordinary thickness, pieces of the thin glass used for the covers of microscopic objects were carefully selected, and these were silvered by floating them upon the surface of a silvering solution. In order to ensure that the induction-spark should always preserve the same position relatively to the mirror, a piece of sheet gutta-percha was fixed above the silvered glass; in the plate of gutta-percha, at the proper place, a small hole was made of about $\frac{1}{20}$ inch in diameter. The ebonite clamp containing the electrodes is so fixed as to permit the point of separation of these to be adjusted exactly over the small hole in the gutta-percha. The adjustment of the parts of the apparatus

1 Phil. Trans. 1868, p. 538. 
was made by closing the end of the adapting-tube, by which the apparatus is attached to the telescope, with a diaphragm with a small central hole, before which a spirit-lamp was placed. When the lines from the induction-spark in the two spectra of comparison were seen to overlap exactly for a short distance the lines of sodium from the light of the lamp, the adjustment was considered perfect. The accuracy of adjustment has been confirmed by the exact coincidence of the three lines of magnesium with the component lines of $b$ in the spectrum of the moon."

The modifications of this plan consist in the substitution of a thin silver plate polished on both surfaces for the pieces of silvered glass. The opposite side of the silver plate to that from which the terrestrial light is reflected to the slit reflects the images formed by the object-glass to the side of the tube, where a suitable eyepiece is fixed. This arrangement forms a very convenient finder, for it is easy to cause the image of the star to disappear in the hole in the silver plate. When this is the case the line of light formed by the star falls on the slit, and its spectrum is visible in the spectroscope. This collimator is so constructed that, by means of a coupling screw, any one of three spectroscopes can be conveniently attached to it.

This apparatus performs admirably; but it seemed to me desirable, for observations of great delicacy, to be able to dispense with reflection, and to place the source of the light for comparison directly before the slit. Formerly I accomplished this object by placing the spark or vacuum-tube before the object-glass of the telescope. The great length of the present telescope renders this method inconvenient; but a more important objection arises from the great diminution of the light when the spark is removed to a distance of $15 \mathrm{ft}$. from the slit. I therefore resolved to place the spark or vacuum-tube within the telescope at a moderate distance from the slit. For this purpose holes were drilled in the tube opposite to each other, at a distance of $2 \mathrm{ft} .6$ in. within the principal focus. Before these holes short tubes were fixed with screws; in these tubes slicle suitable holders for carrying electrodes or vacuum-tubes. The spark is 
thus brought at once nearly into the axis of the telescope. The final adjustment is made in the following manner:-A bright star is brought into the centre of the field of an ordinary eyepiece; the eyepiece is then pushed within the focus, when the wires or vacuum-tube can be seen across the circle of light formed by the star out of focus. The place of discharge between the electrodes or the middle of the capillary part of the vacuumtube is then brought into the centre of the circle of light. The vacuum-tubes are covered with black paper, with the exception of a space about $\frac{1}{4}$ inch long in the middle of the capillary part; through this small uncovered space alone can the light escape to reach the slit.

The accuracy of both methods of comparison, that by reflection and that by the spark within the tube, was tested by the comparison of the three bright lines of magnesium and the double line of sodium with the Fraunhofer lines $b$ and $D$ in the spectrum of the moon. I greatly prefer the latter method, because it is free from several delicate adjustments which are necessary when the light is reflected, and which are liable to be accidentally displaced.

Spectroscope A is furnished with a single prism of dense glass with a refracting angle $59^{\circ} 42^{\prime}$, giving $5^{\circ} 6^{\prime}$ from $\mathrm{A}$ to $\mathrm{H}$.

Spectroscope Is has two compound prisms of Mr. Grubb's construction, which move automatically to positions of minimum deviation for the different parts of the spectrum. Each prism gives about $9^{\circ} 6^{\prime}$ for minimum deviation from $\mathrm{A}$ to $\mathrm{H}$.

Spectroscope C is furnished with four similar prisms.

The small telescopes of the three spectroscopes are of the same size. Diameter of object-glass $1 \frac{1}{4}$ inch; each is furnished with three eyepieces magnifying $5 \cdot 5,9 \cdot 2$, and $16 \cdot 0$ diameters.

\section{Spectrum of the Nebula of Orion.}

With spectroscopes A and B four lines are seen. ${ }^{x}$

First Line.-With spectroscope $\mathrm{B}$ and eyepiece 1 and 2 , the slit being made very narrow, this line was seen to be very

1 The fourth line was first seen in nebula 18 H. IV. (Phil. Trans. 1864, p. 441). 
narrow, of a width corresponding to the slit, and defined at both edges, and undoubtedly not double. The line of nitrogen when compared with it appeared double, and each component nebulous, and broader than the line of the nebula. This latter line was seen on several nights to be apparently coincident with the middle of the less refrangible line of the double line of nitrogen. This observation was on one night confirmed by observation with the more powerful spectroscope C.

The question suggests itself whether, under any conditions of pressure and temperature, the double line of the nitrogenspectrum becomes single; and further, if this should be found to be the case, whether the line becomes single by the fading out of its more refrangible component, or in what other way the single line comes to occupy the position in the spectrum, not of the middle of the double line, but that of the less refrangible of the lines.

I stated in my former paper that when for any reason the light from the luminous nitrogen is greatly reduced in intensity the double line under consideration is the last to disappear, and consequently a state of things may be found in which the light of nitrogen is sensibly monochromatic when examined with a narrow slit. ${ }^{1}$ Under these circumstances the line of nitrogen appears narrower, and the separate components can be detected with difficulty, if at all.

I stated also that the breath of the component lines appears to be connected with the conditions of density and temperature of the gas. As was to be expected from theoretical considerations, the lines become narrower and less nebulous as the pressure is diminished. My observations of this change seemed to show that the diminution of the breadth of the lines takes place chiefly at the outer sides of the lines, so that in the light from very rarefied gas the double line is narrower, but the space of separation between the components is not as much wider as

1 Phil. Trans. 1868, pp. 540-546. Observations on this point were subsequently made by Frankland and Loekyer (Proe. Roy. Soc. vol, xvii. p. 453). It should be stated that they make no referenee to my observations, though they refer to a purely hypothetical suggestion contained in the same paper. 
would be the case if the lines had equally deereased in width on the sides towards each other.

When the pressure of the gas is reduced to about 15 inches of mercury, the line-spectrum fades out to give place to Plïcker's spectrum of the first order. During this process a state of things occurs when, for reasons already stated, the spectrum becomes sensibly monoehromatic when viewed with a narrow slit and a spectroscope of several prisms. The line is narrower, and remains double, and has the characters described in the preeeding paragraph.

As the pressure is diminished, the double line fades out entirely, and the spectrum of the second order gives place to the spectrum of the first order. When, however, the pressure becomes exceedingly small, from $0 \cdot 1$ inch to 0.05 inch, or less, of mercury, there is a condition of the discharge in which the line again appears, while the other lines remain very faint. Under these conditions I have always been able, though with some difficulty on account of the faint light when the necessary dispersive power (spectroscope $\mathbf{B}$ with second or third eyepiece) and a narrow slit are used, to see the line to be double, but it is narrower than when the gas is more dense, and may be easily mistaken for a single line. I have not yet been able to find a eondition of luminous nitrogen in which the line has the same characters as those presented by the line in the nebula, where it is single and of the width of the slit.

Upon the whole I am still inclined to regard the line in the nebula as probably due to nitrogen.

If this should be found to be the case, and that the nebular line has originally the refrangibility of the middle of the double line of nitrogen, then we should have evidence that the nebula is moving from the earth. The amount of displacement of the nebular line from the middle of the nitrogen double line corresponds to a velocity of 55 niles per seeond from the earth. At the time of observation the part of the earth's orbital motion, which was from the nebula, was 14.9 miles per second. From the remaining 40 miles per second would have to be deducted the probable motion from the nebula due to the motion of the 
solar system in space. This estimation of the possible motion of the nebula can be regarded as only approximate.

If the want of accordance of the line in the nebula with the middle of the double line of nitrogen be due to a recession of the nebula in the line of sight, there should be a corresponding displacement of the third line as compared with that of hydrogen. For reasons which will be found in a subsequent paragraph, I have not been able to make this comparison with the necessary accuracy.

In my former paper ${ }^{1}$ I gave reasons against supposing so large a motion in the nebula; these were based on the circumstance that the nebular line falls upon the double nitrogen line, which the present observations confirm. I was not then able to use a slit sufficiently narrow to show that the nebular line is single and not coincident with the middle of the double line of nitrogen.

I am still pursuing the investigation of the parts of this inquiry which remain unsettled.

Second Line.-This line was found by my former comparison to be a little less refrangible than a strong line in the spectrum of barium. Three sets of measures give for this line a wavelength of 4,957 on Angström's scale; this would show that the line agrees nearly in position with a strong line of iron. At present I am not able to suggest to what substance this line belongs.

This line is also narrow and defined. I suspect that the brightness of this line relatively to the first line varies in different nebulæ.

Third and Fourth Lines.-My former observations show that these lines agree in position with two lines of the spectrum of hydrogen, that at $\mathrm{F}$ and the line near $\mathrm{G}$.

These lines are very narrow, and are defined; the hydrogen, therefore, must be at a low tension.

The brightness of these lines relatively to the first and second lines varies considerably in different nebulæ; and I suspect they may also vary in the same nebulæ at different times, and even

1 Phil. Trans. 1868, pp. 542, 543. 
in different parts of the same nebula, but at present I have not sufficient evidence on these points. ${ }^{1}$ I regret that, in consequence of a continuance of bad weather, I have not yet been able to obtain decisive observations as to the possible motion of the nebula in the line of sight. With spectroscope $B$ and eyepiece 2 , the lines appear to be coincident with those of hydrogen. In consequence of the uncertainty of the character of the first line, which is single, while that of nitrogen is double, this determination can now only be made by means of the comparison of the third line with that of hydrogen. This third line becomes very faint from great loss of light unavoidable in a spectroscope that gives a sufficient dispersive power, and the comparison can only be attempted when the sky is very clear and the nebula near th $\theta$ meridian.

\section{On the Motions of some Stars towards or from the Earth.}

In the early part of 1868 I had the honour of presenting to the Royal Society some observations on a small change of refrangibility which I had observed in a line in the spectrum of Sirius as compared with a line of hydrogen, from which it appeared that the star was moving from the earth with a velocity of about twenty five miles per second, if the probable advance of the sun in space be taken into account. ${ }^{2}$

1 Since writing this sentence I have seen a note by Professor D'Arrest in the "Astronomische Nachrichten," No. 1,885. Speaking of the nebula H. IV. 37, he says:- "Sein Spectrum ist ausser von Huggins bisher nur noch von Dr. H. Vögel untersucht worden. In No. 1,864, Astron. Nachr. theilt Letzterer mit, trotz er im Jahre 1871, im Widerspruch mit Huggins' Angabe, die Linie Neb. $(3)=(2)$, bisweilen sogar $(2)<(3)$ gefunden haben. Auch Huggins war dagegen im Jahre 1864 positiv (2)> (3). Ist Vögcl's Beobachtung, wie ich nicht bczweifle, zuverlässig, so wird seine Vermuthung einer Veränderung hier in der That begrïndet sein, denn diesen Winter, 11amentlich im Februar und März 1872, fand ich wiederum, so wie es Huggins früher gesehen hat, unzweifelhaft $(2)>(3)$. Die relative Intensität der drei Lichtarten habe ich mehrfach in Zahlen geschätzt und crhielt, z. B. in den letzten Nächten :

$$
\begin{aligned}
& \text { (1) März 6. März 13. } \\
& \text { (2) . . . . . } 58 \\
& \text { (3) . . . . . . } 49 \quad 52
\end{aligned}
$$

2 Phil. Trans. 1868, pp. 529-550. As a curious instance in which later methods of observations have been partially anticipated, a reference may be made to an in renious paper in the Plilosophical Transactions for 1783 , vol. lxxiv., by the 
It is only within the last few months that I have found myself in possession of the necessary instrumental means to resume this inquiry, and since this time the prevalence of bad weather has left but few nights sufficiently fine for these delicate observations.

Some time was occupied in obtaining a perfectly trustworthy method of comparison of the spectra of stars with those of terrestrial substances, and it was not until I had arranged the spark within the tube, as described at the beginning of this note, that I felt confidence in the results of my observations.

It may be well to state some circumstances connected with these comparisons which necessarily make the numerical estimations given further on less accurate than I could wish. Even when spectroscope $\mathrm{C}$, containing four compound prisms, and a magnifying power of 16 diameters, are used, the amount of the change of refrangibility to be observed appears very small. The probable error of these estimations is therefore large, as a shift corresponding to five miles per second (about $\frac{1}{40}$ of the distance of $\mathrm{D}^{1}$ to $\mathrm{D}^{2}$ ), or even a somewhat greater velocity, could not be certainly observed. The difficulty arising from the apparent smallness of the change of refrangibility is greatly increased by some other circumstances. The star's light is faint when a narrow slit is used, and the lines, except on very fine nights, cannot be steadily seen, in consequence of the movements in our atmosphere. Further, when the slit is narrow, the clock's motion is not uniform enough to keep the spectrum steadily in view; for these reasons I found it necessary to adopt the method of estimation by comparing the shift with a wire of known thickness, or with the interval between a pair of close lines. I found that, under the circumstances, the use of a

Rev. John Mitchell, entitled "On the Means of discovering the Distance, Magnitude, \&c., of the Fixed Stars, in consequence of the Diminution of the Velocity of their Light." "The author suggests that by the use of a prism "we might be able to discover diminutions in the vclocity of light, as perhaps a hundredth, a two hundredth, a five hundredth, or even a thousandth part of the whole." But he then goes on to reason on the production of this diminished velocity by the attraction produced on the material particles of light by the mattcr of the stars, and that the diminutions stated above would bc "occasioned by sphcres whose diameter should be to the sun, provided they were of the same density, in the several proportions of $70,50,30$, and 22 to 1 respectively." 
micrometer would have given the appearance only of greater accuracy. I wish it therefore to be understood that I regard the following estimations as provisional, as I hope, by means of apparatus now being constructed, to be able to get more accurate determinations of the velocity of the motions.

Sirius.-The comparison of the line at $\mathrm{F}$ with the corresponding line of hydrogen was made on several nights from January 18 to March 5. Spectroscope $\mathrm{C}$ and eyepieces 2 and 3 were used. These observations confirm the conclusion arrived at in my former paper, that the star is moving from the earth; but they ascribe to the star a velocity smaller than that which I then obtained.

'These observations on different days show a change of refrangibility corresponding to a velocity of from twenty-six miles to thirty-six miles per second. The part of the earth's orbital motion from the star varied on these days from ten miles to fourteen miles per second. We may take, therefore, eighteen to twenty-two miles per second as due to the star.

The difference of this estimate, which is probably below rather than in excess of the true a mount, from that which $I$ formerly made may be due in part or entirely to the less perfect instruments then at my command. At the same time, if Sirius be moving in an elliptic orbit, as suggested by Dr. Peters, that part of the star's proper motion which is then in the direction of the visual ray, would constantly vary. ${ }^{1}$

Betelgeux (a Orionis).-In the early observations of Dr. Miller and myself on this star, we found that there are no strong lines coincident with the hydrogen lines at $\mathrm{C}$ and $\mathrm{F}$. The line $\mathrm{H} a$ falls on the less refrangible side of a group of strong lines, and If $\beta$ occurs in the space between two groups of strong lines, where the lines are faint. On one night of unusual steadiness

1 H. Vögel at Bothkamp seems to have repeated my observations on Sirius with the necessary care. He says (Astron. Nachr. No. 1,864):- "Mit der eben beschriebenen Anordnung gelang es Herrm Dr. Lohse und mir am 22 März (1871) bei ganz vorzüglicher Luft die Nichtcoincidenz der drei Wasserstoftlinicn $\mathrm{H} \alpha$, If $\beta$, und $H \gamma$, der Geissler'schen Rohre mit den entsprechenden Iinien des Sirius-spectrums zu sehen . . . mit Berücksichtigung der Geschwindigkeit der Erde zur Zeit der Beobachtung berechnet sich die Geschwindigkeit mit welcher sich Sirius von der Erde bewegt zu 10.0 Meilen in der Secunde, wogegen Procyon sich 13.8 Mcilen in der Sccunde von unserer Frde entfernen wiirde. 
in the air, when the finer lines in the star's spectrum were seen with more than ordinary distinctness, I was able with the more powerful instruments now at my command to see a narrow defined line in the red apparently coincident with $\mathrm{H} a$, and a similar line at the position of $\mathrm{H} \beta$. These lines are much less intense than the lines $\mathrm{C}$ and $\mathrm{F}$ in the solar spectrum; there are certainly no bright lines in the star's spectrum at these places.

The most suitable lines in this star for comparison with terrestrial substances for ascertaining the star's motion are the lines of sodium and of magnesium. The double character of the one line agreeing exactly with that of sodium, and the further circumstance that the more refrangible of the lines is the stronger one, as is the case in the sodium spectrum and in the solar spectrum, and the relative distances from each other and comparative brightness of the three lines, which correspond precisely to the triple group of magnesium, can allow of no doubt that these lines in the star are really produced by the vapours of these substances existing there, and that we may therefore safely take any small displacement of either set of lines to show a motion of the star towards or from the earth. The lines due to sodium are perhaps more intense, but are as narrow and defined as the lines $\mathrm{D}_{1}, \mathrm{I}_{2}$ in the solar spectrum; they fall, however, within a group of very fine lines; this circumstance may possibly account for the nebulous character which has been assigned to them by some observers.

The bright lines of sodium were compared with spectroscope $B$ and eyepiece 3 ; they appeared to fall very slightly above the pair in tlie star, showing that the stellar lines had been degradod by the star's motion from the earth. The amount of displacement was estimated at about one-fifth of the distance of $D_{1}$ from $\mathrm{D}_{2}$, which is probably rather smaller than the true amount. This estimation would give a velocity of separation of thirtyseven miles per second. At the time of observation the earth was moving from the star at about fifteen miles per second, leaving twenty-two miles to be due to the star.

When magnesium was compared, a shift in the same direction 
and corresponding in extent to about the same velocity of recession, was observed; but in consequence of other lines in the star at this place, the former estimation, based on the displacement of the lines of sodium, was considered to be more satisfactory.

Rigel. - The lines of hydrogen are strong in the spectrum of this star, and are suitable for comparison.

The line of $\mathrm{H} \beta$ is not so broad as it appears in the spectrum of Sirius, but is stronger than $\mathbf{F}$ in the solar spectrum: this line was compared by means of spectroscope $C$ and eyepieces 2 and 3. The line of terrestrial hydrogen falls above the middle of the line in the star; the star is therefore receding from the earth. The velocity of recession may be estimated as rather smaller than Sirius, probably about thirty miles per second, the earth at the time of observation moving from the star with a velocity of fifteen miles, leaving about fifteen miles as due to the star. This estimate is probably rather smaller than the true velocity of the star.

Castor.-The spectra of the two component stars of this double star blend in the spectroscope into one spectrum. The line $\mathrm{H} \beta$ is rather broad, nearly as much so as the same line in the spectrum of Sirius.

The narrow line of rarefied hydrogen was compared in spectroscope B with eyepiece 3 ; it appeared to fall on the more refrangible side of the middle of the line in the star, leaving more of the dark line on the side towards the red. The shift seemed to be rather greater than that in Sirius, and may probably be taken at from forty to forty-five miles per second; but the earth's orbital motion was nearly seventeen from the star, thus leaving about twenty-five miles for the apparent velocity of the star. This result rests at present on observations on one night only, but they seemed at the time to be satisfactory.

Regulus.-The line at $\mathrm{F}$ rather broad. The corresponding line of hydrogen falls on the more refrangible side of the middle of the dark line in the star. The air was unfavourable on all the evenings of comparison; a rough estimate gives a velocity of 
from twelve to seventeen miles for the velocity of recession between the star and the sun.

$\beta$ and $\delta$ Leonis.- These stars were compared with hydrogen; they appear to be moving from the earth, but want of steadiness in the air prevented me from making a satisfactory estimate of their velocity. I suspected their motion to be smaller than that of Regulus.

$\beta, \gamma, \delta, \epsilon, \zeta$ Ursce Majoris.-All these stars have similar spectra, in which the line $\mathrm{F}$ is strong, though there are small differences in the breadth of the line. They were compared with hydrogen, and appear to be moving from our system with about the same velocity. Probably their motion may be taken to be not far from thirty miles per second. The eartli's motion at the time of observation was from nine to thirteen miles for these stars, leaving a probable velocity of recession of seventeen to twenty miles per second. In the case of the double star $\zeta$, the spectrum consisted of the light of both stars.

$\eta$ Ursce Majoris was only compared with hydrogen. I believe it shows a motion from the earth, but the observations of this star are at present less satisfactory.

a Virginis and a Coronce Borealis.-These stars were compared with hydrogen. I suspect that they are receding, but I have not had nights sufficiently fine to enable me to make satisfactory observations of these stars.

In addition to these stars some observations (which are less satisfactory on account of the unfavourable state of the weather at the time) appear to show that the stars Procyon, Capella, and possibly Aldebaran, are moving from the earth.

The stars which follow have a motion of approach.

Arcturus.--In the spectrum of this star the lines of hydrogen, of magnesium, and of sodium are sufficiently distinct for comparison. I found the comparison could be most satisfactorily made with magnesium.

The bright lines of magnesium fall on the less refrangible side of the corresponding dark lines in the star's spectrum, showing that the star is approaching the earth. I estimated the shift at about $\frac{1}{5}$ to $\frac{1}{4}$ of the interval between $\mathrm{Mg}_{2}$ and $\mathrm{Mg}_{3}$; 
this amount of displacement would indicate a velocity of approach of fifty miles per second. To this velocity must be added the earth's orbital motion from the star of 5.20 miles per second, increasing the star's motion to fifty-five miles per second.

When I can get favourable weather, I hope to obtain independent estimations from the lines of sodium and of hydrogen.

a Lyroe.- In the spectrum of Vega the line corresponding to If $\beta$ is strong and broad. Comparisons were made on several nights, but on one evening only was the air favourable. The observations are accordant in showing that the narrow bright line from a Geissler's tube falls on the less refrangible side of the middle of the line in the star, thus leaving more of the line on the side towards the violet, The estimations give a motion of approach between the earth and the star of from forty to fifty miles per second, to which must be added 3.9 miles after the earth's motion from the star.

a Cygni.-The hydrogen line at $F$ in the spectrum of this star is narrower than in the spectrum of Sirius and of a Lyræ, though probably rather broader than the same line in the solar spectrum. I have at present observations made on two evenings only-on both of which the state of the air was unfavourable-of the comparison of this line with that of terrestrial hydrogen. They give to the star a motion of approach of about thirty miles per second, which would have to be increased by nine miles, the velocity at the time of the earth from the star.

Pollur..-The lines of magnesium and those of sodium are very distinct in the spectrum of this star. As the air was not very steady at the time of my observations, I found it more satisfactory to use for comparison the lines of magnesium, which are rather stronger than those of sodium. The three lines of magnesium appeared to be less refrangible than the corresponding dark lines in the spectrum of the star by about one-sixth of the interval from $\mathrm{Mg}_{2} \mathrm{Mg}_{3}$. This estimation would represent a velocity of approach equal to about thirty-two miles per second. The earth's motion from the star was $17 \cdot 5$ miles, which increases the apparent velocity of approach to forty-nine miles per second. 
On one evening only was the air favourable enough for a numerical estimate, but the observations were entered in my observatorybook as satisfactory.

a Ursce Majoris. - This spectrum of the star is different from the spectra of the other bright stars of this constellation. The line at $\mathrm{F}$ is not so strong, while the lines at $b$ are more distinct, and are sufficiently strong for comparison with the bright lines of magnesium. The bright lines of this metal fall on the less refrangible side of the dark lines, and show a motion of approach of from thirty-five to fifty miles per second. The earth's motion of 11.8 miles from the star must be added.

$\gamma$ Leonis and $\epsilon$ Bootis.-In both these double stars the compared spectrum due to the light of both component stars was observed. Both stars are most conveniently compared with magnesium. I do not consider my observations of these stars as quite satisfactory, but they seem to show a movement of approach; but further observations are desirable.

The stars y Cygni, a Pegasi, y Pegasi, and Andromedce were compared with hydrogen on one night only. It is probable that these stars are approaching the earth, but I wish to re-observe them before any numerical estimate is given of their motion.

$\gamma$ Cassiopeice.-On two nights I compared the bright lines which are present in its spectrum at $\mathrm{C}$ and $\mathrm{F}$ with the bright lines of terrestrial hydrogen. The coincidence appeared nearly perfect in spectroscope $\mathrm{C}$ with eyepieces 2 and 3 ; but on the night of least definition I suspected a minute displacement of the bright line towards the red when compared with $\mathrm{H} \beta$. As the earth's orbital motion from the star at the time was very small, about 3.25 miles per second, which corresponds to a shift that could not be detected in the spectroscope, it seems probab!e that $\gamma$ Cassiopeiæ has a sinall motion of recession.

In the calculation of the estimated velocities the wave-lengths employed are those given by Angström in his "Recherches sur le Spectre solaire," Upsal, 1868. The velocity of light was taken at 185,000 miles per second.

The velocities of approach and of recession which have been assigned to the stars in this paper represent the whole of the 
motion in the line of sight which exists between them and the sun. As we know that the sun is moving in space, a certain part of these observed velocities must be due to the solar motion. I have not attempted to make this correction, because, though the direction of the sun's motion seems to be satisfactorily ascertained, any estimate that can be made at present of the actual velocity with which he is advancing must rest upon suppositions, more or less arbitrary, of the average distance of stars of different magnitudes. It seems not improbable that this part of the stars' motions may be larger than would result from Otto Struve's calculations, which give, on the supposition that the average parallax of a star of the first magnitude is equal to $0^{\prime \prime} \cdot 209$, a velocity little greater than one-fourth of the earth's annual motion in its orbit.

It will be observed that, speaking generally, the stars which the spectroscope shows to be moving from the earth (Sirius, Betelgeux, Rigel, Procyon) are situated in a part of the heavens opposite to Hercules, towards which the sun is advancing; while the stars in the neighbourhood of this region, as Arcturus, Vega, $a$ Cygni, show a motion of approach. There are in the stars already observed exceptions to this general statement; and there are some other considerations which appear to show that the sun's motion in space is not the only, or even in all cases, as it may found, the chief cause of the observed proper motions of the stars. $^{1}$

There can be little doubt but that in the observed stellar movements we have to do with two other independent motions, namely, a movement common to certain groups of stars, and also a motion peculiar to each star.

Mr. Procter has brought to light strong evidence in favour of the drift of stars in groups having a community of motion, by his

1 As the velocities assigned to the stars are, for the reasons already stated, provisional only, I feel some hesitation in drawing from them the obvious conclusions which they would suggest. The velocities given in the Tables for those stars which are moving in direction in accordance with the sun's motion towards Hercules, do not bear to each other the relation which they should have if they were mainly produced by the sun's motion. Even for thesc stars, therefore, we must look elsewhere for the cause to which they are chicfly due. 
graphical investigation of the proper motions of all the stars in the catalogues of Mr. Main and Mr. Stone. ${ }^{1}$ The probability of the stars being collected into such systems was early suggested by Mitchell and the elder Herschel. ${ }^{2}$ One of the most remarkable instances pointed out by Mr. Procter are the stars $\beta, \gamma, \delta, \epsilon$ of the Great Bear, which have a community of proper motions, ${ }^{3}$ while $a$ and $\eta$ of the same constellation have a proper motion in the opposite direction. Now, the spectroscopic observations show that the stars $\beta, \gamma, \delta, \epsilon, \zeta$, have also a common motion of recession, while the star $a$ is approaching the earth. The star $\eta$ indeed appears to be moving from us, but it is too far from $a$ to be regarded as a companion to that star.

Although it was not to be expected that a concurrence would always be found between the proper motions which indicate the apparent motions at right angles to the line of sight and the radial motions as discovered by the spectroscope, still it is interesting to remark that in the case of the stars Castor and Pollux, one of which is appproaching and the other receding, their proper motions also are different in direction and in amount; and further, that $\gamma$ Leonis, which has an opposite radial motion to $a$ and $\beta$ of the same constellation, differs from these stars in the direction of its proper motion.

I See "Preliminary Paper on certain Drifting Motions of Stars," Proc. Roy. Soc. vol. xviii. p. 169 .

${ }^{2}$ Sir William Herschel writes:- "Mr. Mitchell's admirable idca of the stars being collected into systems appears to be extremely well founded, and is every day more confirmed by obscrvations, though this does not take away the probability of many stars being still as it werc solitary, or, if I may use the expression, intersystematical. . . . A star, or sun such as ours, may have a proper motion within its own system of stars; whilc at the same time the whole starry system to which it belongs may have another proper motion totally different in quantity and direction." Herschel further says, "And should there be found in any particular part of the heavens a concurrence of proper motions of quite a different direction, we shali then begin to form some conjectures which stars may possibly belong to ours and which to other systems."-Phil. Trans. 1783, pp. $276,27 \%$.

3 Mr. Procter, speaking of these stars, says :-- "Their drift is, I thiuk, most significant. If, in truth, the parallelism and equality of motion are to be regarded as accidental, the coincidence is onc of most remarkable character. But such an interpretation can hardly be looked upon as admissible when we remember that the peculiarity is only one of a series of instances, some of which are scarcely less striking."- "Other Worlds than Ours," p. 269: and papcr in Proc. Roy. Soc. vol. xviii. p. 170. 
TABLE 1.-STARS MOVING FrOM THE SUN.

\begin{tabular}{|c|c|c|c|c|c|}
\hline Star. & & Compared with & $\begin{array}{l}\text { Apparent } \\
\text { nuotion. }\end{array}$ & Earth's motion. & $\begin{array}{l}\text { Motion from } \\
\text { sun. }\end{array}$ \\
\hline 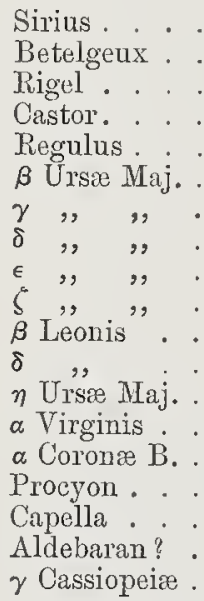 & 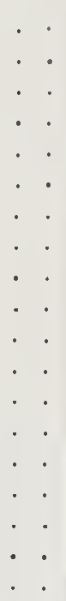 & $\begin{array}{l}\mathrm{H} \\
\mathrm{Na} \\
\mathrm{H} \\
\mathrm{H} \\
\mathrm{H} \\
\mathrm{H} \\
\mathrm{H} \\
\mathrm{H} \\
\mathrm{H} \\
\mathrm{H} \\
\mathrm{H} \\
\mathrm{H} \\
\mathrm{H} \\
\mathrm{H} \\
\mathrm{H} \\
\mathrm{H} \\
\mathrm{H} \\
\mathrm{Mg} \\
\mathrm{H}\end{array}$ & $\begin{array}{l}26 \text { to } 36 \\
37 \\
30 \\
40 \text { to } 45 \\
30 \text { to } 35 \\
30\end{array}$ & $\begin{array}{l}-10 \text { to } 14 \\
-15 \\
-15 \\
-17 \\
-18 \\
-9 \text { to } 13\end{array}$ & $\begin{array}{l}18 \text { to } 22 \\
22 \\
15 \\
23 \text { to } 28 \\
12 \text { to } 17 \\
17 \text { to } 21\end{array}$ \\
\hline
\end{tabular}

TABLE II.--STARS APPROACHING THE SUN.

\begin{tabular}{|c|c|c|c|c|c|}
\hline Star. & & Compared with & $\begin{array}{l}\text { Apparent } \\
\text { motion. }\end{array}$ & Earth's motion. & $\begin{array}{c}\text { Motion } \\
\text { towards sun. }\end{array}$ \\
\hline 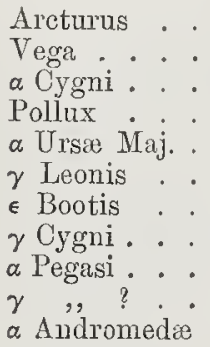 & $\begin{array}{l}: \\
: \\
: \\
: \\
: \\
: \\
: \\
:\end{array}$ & $\begin{array}{l}\mathrm{Mg} \\
\mathrm{H} \\
\mathrm{H} \\
\mathrm{Mg} \\
\mathrm{Mg} \\
\mathrm{Mg} \\
\mathrm{Mg} \\
\mathrm{H} \\
\mathrm{H} \\
\mathrm{H} \\
\mathrm{H}\end{array}$ & $\begin{array}{l}50 \\
40 \text { to } 50 \\
30 \\
32 \\
35 \text { to } 50\end{array}$ & $\begin{array}{l}+5 \\
+39 \\
+\quad 9 \\
+17 \\
+11\end{array}$ & $\begin{array}{l}55 \\
44 \text { to } 54 \\
39 \\
49 \\
46 \text { to } 60\end{array}$ \\
\hline
\end{tabular}

It scarcely needs remark that the difference in breadth of the line $H \beta$ in different stars affords us information of the difference of density of the gas by which the lines of absorption are produced. A discussion of the observations in reference to this point and to other considerations on the physical condition of the stars and nebulæ, I prefer to reserve for the present. 


\title{
APPENDIX F.
}

\author{
I. \\ ON THE PHOTOGRAPHIC SPECTRUM OF COMET " $b$ " 1881.
}

BY WILLIANI HUGGINS, D.C.L., LL.D., F.R.S. ${ }^{1}$

In the years 1866 and 1868 I applied the spectroscope to the light of comets, and in the latter year I showed that the three bright bands in the visible part of the spectrum agree with the similar bright bands which are seen when an induction spark is taken in olefiant gas. ${ }^{2}$ The same bands are also seen in the flames of many compounds of carbon. I was inclined at that time to consider that these bands were due to the vapour of carbon. Subsequent investigations which have been made on the spectra of the compounds of carbon, appear to make it probable that these bands are the spectrum of a compound of carbon with hydrogen. The observations (1868) showed the presence of carbon, probably in combination with hydrogen, in the cometary matter.

Since that time until the present year, no comet has appeared sufficiently bright to allow of the observations on its spectrum being extended to the ultra-violet region. The apparatus with which I had successively photographed the spectra of stars was especially suited to this purpose. ${ }^{3}$ It consists essentially of a spectroscope, furnished with a prism of Tceland spar and lenses of quartz, placed so that the slit shall be in the principal focus of a mirror 18 inches in diameter, equatorially mounted and driven by an electrically controlled clock.

On the evening of June 24th (1881), I directed this instrument, armed with a very sensitive gelatine plate, to the head of Comet $b$, so that the nucleus should be upon one half of the slit. After an exposure of one hour, the open half of the slit was

1 Report of the British Association, 1881. 2 Phil. Trans. 1868, p. 556.

\footnotetext{
3 Phil. Trans. 1880 , p. 669.
} 
closed, the shutter withdrawn from the other half, and the instrument was then directed to Arcturus for fifteen minutes.

After development the plate presented a very distinct spectrum of the comet, together with that of the star, for comparison.

The spectrum of the comet consists of two spectra superposed upon each other: a continuous spectrum, which extends from about $F$ to a little distance beyond $\mathrm{H}$. In this continuous spectrum can be seen the Fraunhofer lines $G, h, H, K$, and many others. This spectrum is therefore due to reflected solar light.

The second spectrum consists of two sets of bright lines, and a suspicion of the presence of a third set. These lines are obviously to be referred to original light from the coinet.

The strongest set consists of two bright lines in the commencement of the ultra-violet region. Measures, made by the aid of the comparison star-spectrum give for these bright lines the wave-lengths 3883 and 3870 . The less refrangible line is much stronger, and a faint luminosity can be traced from it to a little beyond the second line 3870. There can be, therefore, no doubt that these lines represent the brightest end of the ultra-violet group, which appears under certain circumstances in the spectra of the compounds of carbon. Professors Liveing and Dewar have found for the strong line at the beginning of the group the wave-length 38827 , and for the second line 3870.5 .

I am also able to see upon the continuous solar spectrum, a distinct, though fainter, impression of a group of lines between G and $h$. There can be little doubt that this group is the one for the least refrangible limit of which the wave-length 4220 is given by Professors Liveing and Dewar.

An increase of brightness in the continuous spectrum is also seen between $h$ and $H$, which may be due to other bright lines, but the photograph is not strong enough to admit of any certain conclusion on this point.

On June 25, a second photograph was obtained with an exposure of an hour and a half. This photograph, notwithstanding the longer exposure, is fainter, but shows distinctly the bright lines in the ultra-violet, and the continuous spectrum. 
These photographs confirm the results of my earlier observations on comets-that part of their light is reflected sunlight, and part is original light; and, further, that carbon is present in the cometary matter. 'The new bright groups in the comet's spectrum which the photographs have revealed to us, are certainly characteristic of substances containing carbon.

In their paper "On the Spectra of the Compounds of Carbon" 1 Professors Liveing and Dewar bring forward evidence to show that these two groups indicate the presence of cyanogen, and are not to be seen in hydrocarbons unless nitrogen is also present. If this be the case the photograph supplies us with strong evidence of the presence of nitrogen in the comet, in addition to the carbon and hydrogen shown to be there by the bright groups in the visible region of the spectrum. It is of great interest in connection with this result, now that Schiaparelli has shown us the close relationship of meteors and coniets, to mention the results of Professor Graham's experiments on the occluded gases from the meteoric iron of Lenarto. ${ }^{2}$ This iron gave nearly three times its volume of gas, consisting chiefly of hydrogen, with small quantities of carbonic oxide and nitrogen.

Professor Wright's examination of the stony meteorites shows the oxides of carbon, chiefly the di-oxide, to be present in largest quantity, but he obtained also a small percentage of hydrogen and nitrogen. ${ }^{3}$ Other kinds of meteors are known which contain hydrocarbons, even in considerable quantity. It is scarcely necessary to add that, under suitable conditions, the spectra of the gases from some meteorites. will be similar to that observed from the light of comets.

Messis. Liveing and Dewar's experiments would seem to show that a high temperature must be present in the comet, if the cyanogen is formed there, but if cyanides should be found in meteorites this necessity would not exist.

Whatever the views that may be entertained as to the forms of combination in which the carbon exists, there can be no

^ Proc. Roy. Soc. val. xx. p. 494.

2 Proc. Roy. Soc. xv. p. 502, 1867.

3 Amer, Journ. Scien, vol. x. July, 1875. 
doubt whatever of the presence of carbon in comets. I should mention that Mr. Lockyer regards the two bright groups seen in the photograph, and the three groups in the visible spectrum, to be due to the vapour of carbon at different heat-levels. ${ }^{1}$

$\mathrm{It}$ is of importance to mention the strong intersity in the photograph of the lines 3883 and 3870 as compared with the continuous spectrum, and the faint bright group beginning at 4220. At this part of the spectrum, therefore, the light emitted by the comctary matter exceeded by many times the reflected solar light.

On August 21, I attempted to obtain, with an exposure of one hour, a photograph of the spectrum of a large comet which has appeared since Comet c, 1881. The evening was not very favourable, and the comet was at a low altitude and not so brilliant as Comet $b$. I am not able to see on the plate more than a faint trace of the brightest lines (W. L. 3883 and 3870) of the spectrum obtained from the former comet.

II.

ON THE PHOTOGRAPAIC SPECTRUM OF COMET (WELLS) $1,1882$. BY WILLIAM HUGGFNS, D.C.L., LL.D., F.R.S."

Last year I had the honour of presenting to the British Association an account of a photograph of the spectrum of the brightest comet of that year, accompanied by some remarlis on the unity of type of spectrum of all the comets to which the spectroscope had been applied since 1864 .

The bright comet of the present year presents, for the first time, a spectrum differing essentially from the hydrocarbon type to which all the other comets belonged.

Observations of the visible region of the spectrum had already shown the probable absence of the usual hydrocarbon groups, one or two observers suspecting only the brightest of

1 Proc. Roy. Snc. vol. xxx. p. 461.

2 Report of the British Association, 1882. 
the groups. The comet gave a brilliant continuous spectrum, and also a bright double line coincident with that of sodium at $\mathrm{D}$ of the spectrum, and some other bright lines.

On May 31, I obtained a photograph of the spectrum of this comet. The plate was exposed for one hour and a quarter. Through the second half of the slit I took on the same plate a spectrum of a Ursa majoris for the determination in position of the lines of the comet's spectrum.

The photographic plate slowed a strong continuous spectrum extending from about $\mathrm{F}$ to a little beyond $\mathrm{H}$.

In the continuous spectrum of the comet of last year the Fraunhofer lines were clearly visible, but in the stronger continuous spectrum of the present comet I was not able to distinguish any of these solar lines.

The slit was indeed more open than was the case last year, and this circumstance would make these lines less distinct, but the lines $\mathrm{G}$ and $\mathrm{H}$ are well seen in the star's spectrum taken under the same conditions. We may conclude therefore that the part of the comet's original light which gives a continuous spectrum is much stronger relatively to the reflected solar light in this comet than was the case in the comet of last year, and for this reason the Fraunhofer lines are not to be seen.

In agreement with eye observations made in the visible region, this photograph does not contain the strong ultra-violet group assigned to cyanogen; also the bright groups of the comet of last year between $G$ and $h$, and between $h$ and $H$, do not appear to be present.

In the continuous spectrum at least five places of greater brightness can be distinguished, which undoubtedly represent groups of bright lines, though they are not sufficiently distinct in the photograph to admit of resolution into lines. The correctness of this interpretation is rendered much more probable by the circumstance that these groups, as is shown in the diagram, project beyond the strong continuous spectrum on one side. The head of the comet was in sharp focus upon the slit, and the continuous spectrum with defined edges corresponds to the nucleus, which in this comet was very distinct. The side of 
the spectrum on which these suspected groups project corresponds to the light of the corona on the side of the nucleus next the sun. We learn, therefore, that the light of this part of the coma consisted chiefly, in this region of the spectrum, of these gronps; as, on the plate, only a very faint continuous spectrum can be seen between these projected parts of the bright spaces.

It is not possible to measure with accuracy the beginnings and endings of these groups. Measures as accurate as the circumstances would permit have been taken, therefore, of the brightest parts of the groups. The wave-lengths of these brightest parts are:-

$$
\begin{array}{r}
\lambda 4253 \\
" 4412 \\
" 4507 \\
" 4634 \\
\# 4769
\end{array}
$$

As the lines of sodium were strong in the visible part, it may be that some of the light producing some of these groups may be due to that substance.

Mr. Hind has kindly furnished me with the distance of this comet from the sun at the time the photograph was taken. The comet was then $42,380,000$ miles from the sun, while the comet of last year was distant $69,420,000$ when I obtained the photograph of its spectrum. Considering the fact that the presence of sodium and the absence of the hydrocarbon groups were observed some time previously to the taking of the photograph, when the comet was much farther from the sun, we cannot attribute this difference of spectrum directly to greater heat through a nearer approach to the sun, but must regard it as arising from a different chemical constitution of the cometary stuff. Professor A. Herschel and Dr. von Konkoly showed long ago that the spectra of the periodic meteors are different for different swarms, and it is not surprising that the nuclei of comets should differ chemically.

If the light of comets is due to electric discharges, then, although these discharges would owe their existence to solar 
heat, their heating effect upon the cometary matter might not be strictly proportional to the nearness of the comet to the sun. In this case too the absence of the hydrocarbon lines might not necessarily indicate the freedom of the cometary matter from these substances. It has long been known how preferential is an electric discharge when several substances are present at the same time, and 1Dr. Hasselberg has recently shown by some laboratory experiments that in the presence of sodium vapour the hydrocarbon spectrum fades out. Notwithstanding these considerations, it is probable that the comet of this year owed the exceptional character of its spectrum to some unusual chemical condition of the meteoric matter which forms its nucleus.

[P.S.-November 14.-The spectrum of the great comet which was discovered near the sun in September was similar in the visible region to that of comet Wells. Now that this comet has receded from the sun the lines of sodium are fainter, and the hydrocarbon groups seen in former comets have made their appearance. These observations support the view taken in this paper, but suggest that the chemical state of the matter of these two comets is not essentially different from that of former comets, but is probably a modification of the same fundamental constitution.-W. H.]

III.

ON THE PHOTOGRAPHIC SPECTRUM OF THE GRFAT NEBULA IN ORION.

BY WILLIAM HUGGINS, D.C.L., T.T.D., F.R.S. ${ }^{1}$

In September $1864^{2}$ I had the honour to announce to the Royal Society that I had discovered that certain of the nebulæ (up to that time, eight in number), give a spectrum of bright lines. In one nebula four bright lines were observed.

1 Report of the British Association, 1882.

2 Phil. Trans. 1564, 1. 437. 
In January 1865, I found that the light of the Great Nebula of Orion is resolved into the same set of bright lines. Further observations on the spectrum of the nebula in Orion, and the spectra of other nebulæ, were made subsequently with more powerful apparatus. ${ }^{2}$

The result of these observations was to show that in the visible region, the spectrum of the Great Nebula in Orion consists of four bright lines, the positions of which are $\lambda 5005$, 4957,4861 , and 4:340. The least refrangible line appeared, in the instrument used, to be coincident with the less refrangible component of the brightest double line of the spectrum of nitrogen. The third and fourth lines agreed in position with the lines $\beta$ and $\gamma$ of the hydrogen spectrum, with which they were compared directly in the spectroscope.

During the last few years I have made several attempts to extend our knowledge of the spectra of the nebulæ into the more refrangible region beyond the reach of the eye by means of photography. On March 7 last (1882), I succeeded in obtaining a photograph of the spectrum of the Great Nebula in Orion.

The apparatus was the same with which I had photographed the spectra of stars, and the spectrum of comet $b, 1881$. It consists essentially of a spectroscope furmished with a prism of Iceland spar, and lenses of quartz, placed so that the slit shall be in the principal focus of a metallic speculum 18 inches in diameter, and driven by an electrically controlled clock.

A gelatine plate was used very sensitive from about Fraunhofer's $b$ of the spectrum, to a long distance in the ultra-violet.

The exposure was limited by the coming up of clouds to about 45 minutes. The opening of the slit was made wider than in my work on the stars. The slit was kept upon a bright part of the nebula near the trapezium in the "Fish's mouth" of the nebula.

The photographic plate shows a spectrum of bright lines 1 Proc. Roy. Soc. vol. xiv. p. 39.

2 Phil. Trans. 1868, p. 540, and Proc. Roy. Soc. vol. xx. p. 380. 
sufficiently distinct to admit of measurement; there is also present an exceedingly faint continuous spectrum.

In my eye-observations of the visible regions of the spectrum, I was nearly always conscious of the presence of a faint continuous spectrum, but in no part of the nebula did this continuous spectrum become strong relatively to the bright hines, which were present throughout the whole extent of the nebula.

The great range of the spectrum to which the plate was sensitive enabled me to see upon it all the lines which had been previously observed with the eye. In addition to these known lines the plate shows a relatively strong line in the ultra-violet region, which is new to us.

As the slit was wide, this line appears broad on the plate, but for the same reason so do also the known lines. Eye-observations have shown these lines to be extremely narrow and defined at the edges. There is reason to believe that this new line, though. relatively intense, is similar in character to the other lines, namely, narrow and defined. The great advantage of this control from lines common to the photographic plate and to observations by eye, is seen further in the following circumstance. The spaces occupied on the plate by the broad lines do not appear to be quite uniform, but somew hat less strong in the middle; but the results of the eye-observations show conclusively, as might be suspected for other reasons, that this appearance does not indicate duplicity of the lines, but simply a want of absolute precision of focal adjustment.

The broad character on the plate of this now line does not permit of quite the same accuracy of determination of position as would have been possible with a narrower slit. There is little doubt, however, that this new line agrees in position with the line $\zeta$ of the typical spectrum of the white stars. ${ }^{1}$ For this reason this typical spectrum has been placed in the diagram by the side of the photographic spectrum of the nebula. The wave-length of the new line is therefore $\lambda 3730$.

There can be little doubt that this typical spectrum is due to hydrogen, and that this new line in the nebula, as well as the 1 Phil. Trans. 1880, p. 672. 
two less refrangible lines which had been observed by the eye, are produced by this substance.

It is of much interest to compare the extreme differences of character of these lines of hydrogen which are common to the white stars and to the nebula. In the stars these lines are very broad and winged at the edges, while in the nebula they are of exceeding thinness and wholly free from a winged condition, indicating great differences in the temperature and density of the gas as it exists in these two orders of celestial bodies.

I cannot say positively whether the lines of hydrogen between $\mathrm{H}_{\gamma}$ and the line $\zeta$ at 3730 , which are present in the stars, are altogether absent in this nebula. If they exist in the spectrum of the nebula they must be exceedingly faint relatively to those present. I suspect a want of uniformity of the film at this part, and also beyond $\lambda$ 3730, and this may indicate possibly the presence of very faint lines. Under the most favourable conditions of illumination, I am almost certain of faint lines at the positions of $h$ and $\mathrm{H}$. In the diagram only those lines about which there can be no uncertainty are inserted, and the new line is made broad for the purpose of indicating its great intensity relatively to the other lines.

In my laboratory experiments, I have succeeded in obtaining spectra of hydrogen in which some of the lines in this region are either absent or very greatly reduced in intensity, but I have not yet obtained a spectrum which represents a state of things precisely similar to that which obtains in this nebula.

\section{APPENDIX G.}

ON SPECTRA OF LIGETNING. BY ARTHUR SCHUSTER, PH.D., F.R.A.S. ${ }^{1}$

All observers of lightning-spectra agree in having seen the line-spectrum of nitrogen; but most of them have seen, in arldition to this, sometimes a continuous spectrum, sometimes a band spectrum, the chemical origin of which is unknown.

1 Read at the Meeting of the Physical Society on February 22, 1879. 
The following historical summary may give an idea of our knowledge on that point.

Prof. Kundt ${ }^{1}$ observed a line spectrum consisting of one or two lines in the red, some very bright ones in the green, and some less bright ones in the blue. He mentions that the lines are not always seen together. Lines which in one flash appeared especially bright were not seen in another flash. The greater number of flashes, however, gave a different spectrum altogether. In the place of bright lines a great number of bands were seen; and Prof. Kundt even distinguishes two different band spectra.

Mr. John Herschel ${ }^{2}$ observed a variable continuous spectrum crossed by bright lines, which also had a variable intensity. He gives the measurements of two lines, which agree very well with nitrogen-lines.

M. Laborde ${ }^{3}$ observed some lines, especially one near E, which sometimes appeared alone. He also saw a continuous spectrum.

Dr. H. Vögel ${ }^{4}$ describes lines only; but in his list I find two which do not coincide with any bright lines in the spectrum of the electric spark taken in atmospheric air; they do, however, coincide with two bands which I have observed in some flashes of lightning, as I shall show.

Dr. J. P. Joule 5 also observed some spectra of lightning. Frequently there was only one bright line visible, this being coincident with the brightest nitrogen-line. At other times there were several bright lines visible, sometimes with and sometimes without the green nitrogen-line. A continuous spectrum was also observed.

Mr. H. R. Procter ${ }^{6}$ gives some measurements of lines which do not lay claim to any accuracy. He observed also a band spectrum, which he finds not to be the band spectrum of nitrogen.

From conversation with Prof. A. Young, I learned that he also had seen a line spectrum, a band spectrum, and a continuous spectrum.

1 Pogg. Ann. cxxxv. p. 315.

3 Les Mondes, viii. p. 219.

Nature, vol. xvi. p. 161.
2 Proe. Roy. Soc. xvii. p. 61 .

4 Pogg. Ann. cxliii. p. 653.

6 Nature, rol. xvi. pp. 161-220. 
During my stay in Colorado last summer, I had some good opportunities of studying the spectra of lightning. It was my intention to get some reliable measurements of the band spectrum which I, in common with most observers, have seen; and in order to have greater chance of succeeding, I confined myself to one part of the spectrum only. The part I chose extended from $\lambda=5000$ to $\lambda=5800$, and covered, therefore, most of the yellow and green. I used a directrvision spectroscope, with a slit movable by means of a micrometer-screw. A bright line in the principal focus of the telescope formed a fiducial mark. Under ordinary circumstances, the slit is moved until the line to be measured forms a continuation of the bright line which reaches down into the centre of the field. I found, however, that the bands I wanted to measure were nearly as broad as the thin glass bar which carries the bright line; and I used the bar therefore simply as pointer. The measurements were always made at night; and the spectroscope was left indisturbed until the following morning, when the Fraunhofer lines in the neighbourhood were measured, so that the wave-lengths of the measurements could be interpolated.

It is of course impossible to put a pointer on a band during the instantaneous flash; but a succession of flashes allows us to put the pointer successively nearer and nearer until we see it in coincidence with the band. In this way several readings of each band were obtained. The dispersive power of the spectroscopes was such that, with a higher-power eyepiece than the one used in this investigation, the nickel-line could be seen between the two sodium-lines. The distance between the two sodiumlines was such that the two readings of the slit differed by ten divisions of the micrometer, or one-tenth of a whole revolution. With sunlight I can measure easily to the tenth part of the distance between the sodium-lines. I obtained measurements on three different nights. Unfortunately the best nights for the work occurred before the Total Solar Eclipse, which had taken me out to Colorado. A desire to save my eyes prevented me from making as good use of these nights as I otherwise should have done. 
July 25th, West Las Animas.-The whole horizon seemed to be almost constantly illuminated with lightning, generally sheetlightning. I observed about thirty or forty different flashes. I often saw the bright nitrogen-lines 5002 and 5681. I did not take any measurements of these lines; but there can hardly be a doubt as to their position. I saw in the part of the spectrum which I was observing three bands, which, however, did not always appear together. The measurements reduced to wavelengths will be given further on. Two measurements of the bands $\beta$ and $\gamma$ were obtained, but one only of the band $a$. The greatest difference between the two measurements amounts to three times the distance between the sodium-lines. This difference must be partly accounted for by the difficulty of the observation, partly by the fact that the spectroscope had only just been unpacked after the journey; and it was found next day that it was considerably out of adjustment. The micrometerscrew, also, owing to the heat and dust, had a considerable backlash; it was taken to pieces next day and cleaned, which greatly improved it.

August 3rd, Manitou.-Clouds were coming from the west over Pike's Peak; and strong flashes of lightning, partly sheet lightning, partly forked lightning, were observed. Only two measurements were secured. One of the bands measured was ß. Prof. Arthur Wright, who was present, observed that one spot of the sky was illuminated during some flashes with a strong blue light, looking like a fluorescent light. I pointed the spectroscope to that spot, and observed a single broad band in the green. I moved the pointer on it as well as I could; but not being able to get another flash to verify the measurement, I had to take the reading. The position of this band, which I call $\delta$, is very doubtful.

August 18th, Salt-Lake City.-I only obtained one measurement of the band $\gamma$. The kind of lightning observed differed considerably from that of the preceding nights. The lightning was nearly all forked lightning; and the bright nitrogen-line came out very strongly. The bands were but seldom seen. In one flash I saw a series of lines in the green which I had never 
seen before. My impression is that they were at about equal distances from each other, decreasing in strength towards the red; so that the whole made an impression similar to that of a fluted band, such as those seen in the spectrum of aluminium oxide, but shading off towards the red.

In addition to the line and band spectra, I have on many occasions seen a continuous spectrum only.

The following Table contains all the measurements I have taken. I have added in the Jast column numbers contained in Dr. Vögel's list of lines. It will be seen that these coincide with two of the bands I have seen.

\begin{tabular}{|c|c|c|c|c|}
\hline Band. & Date. & $\lambda$ & Mlean. & Vögel. \\
\hline$\alpha$. & July 25 & 5592 & 5592 & \\
\hline$\beta$. & $\begin{array}{l}\text { July } 25 \\
\text { July } 25 \\
\text { Aug. } 3\end{array}$ & $\begin{array}{l}5348 \\
5329 \\
5325\end{array}$ & 5334 & 5341 \\
\hline$\gamma$. & $\begin{array}{l}\text { July } 25 \\
\text { July } 25 \\
\text { Aug. } 18\end{array}$ & $\begin{array}{l}5175 \\
5193 \\
5177\end{array}$ & 5182 & 5184 \\
\hline$\delta$. & Aug. 3 & 5260 & 5260 & \\
\hline
\end{tabular}

In trying to identify these bands with known spectra we meet with an unexpected difficulty. Two of them unfortunately admit of two different interpretations. At first sight I was struck by the close agreement of $a$ and $\gamma$ with two bands of carbonic oxide. These bands fade away towards the blue; and their sharp edges have a wave-length of 5607 and 5197. Observing with the same spectroscope, and widening the slit as I did in observing the lightning, I can produce the same impression of an unshaded band; and taking a measurement of the centres of the bands under these circumstances, I obtain $\lambda=5579$ and $\lambda=5180$, which agree within the limits of possible errors with the above values.

The ordinary spectrum of air, however, contains a band at 
5178 ; so that, as far as mere position is concerned, one might well be taken for the other. I was, however, under the impression that I had sometimes seen this band without the chief nitrogen double line 5002-5; and as the yellow band of carbonic oxide was also apparently present, I stated with considerable confidence when I first wrote out this paper that I had observed the spectrum of carbonic oxide. It was only when I came to work out the position of the band $\delta$ that I began to have serious doubts as to the accuracy of this conclusion. The position of the band $\delta$, as I have said, is very doubtful; I even thought it was possible that I had taken a very bad measurement of either $\beta$ or $\gamma$, and felt at first inclined to reject it altogether. On working out its wave-length, however, I found that it was coincident with one of two strong bands, which are found at the negative pole of vacuum-tubes filled with oxygen. Now the second of the two bands is nearly coincident with the yellow band of carbonic oxide; so that, of the two bands which $I$ at first thought belonged to that gas, one might be due to nitrogen, the other to oxygen, as seen at the negative pole.

The explanation of the band $\beta$ is obvious. It is the brightest of the two green lines in the low-temperature spectrum of oxygen. Its wave-length, when seen under a pressure of about a millimetre, is 5329; but under higher pressures it widens more on the less-refrangible side than towards the blue, and may well appear as a band with its centre at 5334 or even 5341 , as given by Vögel.

I have not been able to obtain this band from atmospheric air in vacuum-tubes, although I have tried the experiment under various pressures. If the so-called continuous discharge is allowed to pass, the band spectrum of nitrogen alone appears; if the disruptive discharge passes, the high temperature spectrum of oxygen is superadded to the line spectrum of nitrogen. As regards the two bands $a$ and $\gamma$, it does not seem to me to be possible at present to decide between the two interpretations which I have given. On the one hand, it seems improbable that the slight traces of carbonic acid known to exist in the 
atmosphere should reveal their presence in the spectrum; but, on the other hand, it is to be remarked that oxygen vacuumtubes, which show the band $\beta$, always reveal the slightest trace of carbonic oxide. It is exceedingly difficult, though quite possible, to obtain the band $\beta$ without the bands $a$ and $\gamma$. The measurement of $\delta$, however uncertain, renclers it probable that the spectrum of the negative pole in oxygen forms part of the spectrum of lightning; and on the whole I should feel inclined to attribute the band a to oxygen. I have shown in my paper on the spectrum of oxygen that this spectrum of the negative pole is due to an allotropic modification of oxygen (possibly ozone), and I have been able to obtain it, though only temporarily, in the positive part of the discharge. As regards the band $\gamma$, I have some difficulty in attributing it to nitrogen, and still think it probably due to carbonic oxide. During the observations I certainly felt convinced that it did not belong to the same spectrum as the chief lines of nitrogen, and $I$ made a note that, on the contrary, it generally appeared together with $\beta$. It seemed sometimes to be present alone, and often to form the most prominent part of the whole spectrum. As the lines of the capillary part of an oxygen-tube are also present at the negative pole, together with the bands distinctive of that pole, I can best express my observations on the band spectrum of lightning by saying that it resembles in a remarkable way the spectrum which is found at the negative pole of a vacuum-tube filled with oxygen which is slightly contaminated with carbonic oxide. 


\section{INDEX.}

A.

ABNex, CAPtain, his dry plates used during eclipse, 262

photography of infra-red rays by, 24, 29, 197, 209, 273

and Lieutenant-Colonel Festing on molecular vibrations as affected by chenical combination, 175

their researches as to organic radicals, 198

Absorption and radiation, correlation between, 265, 268, 388 selective, 183, 291 general law of, 269

Absorption spectra of Bessemer flames, 217 of colouring matters differently dissolved, 180 of compounds, 172

inversion of in erbium and didymium, 227

of the same substance differently dissolved, 192

of sun-spots, 246

in ultra-red and ultra-violet rays, 197

Actinic clouds, how produced, 31

Actinometer, Rosene's, described, 58

Adulteration of wine, spectroscopic detection of, 203

Air, constaut presence of dust in, 297

spectrum of, 127, 424

$$
\text { of sodium salts in, 78, } 95
$$

Albedo of various planets compared, 350

Aldebaran, possible recession of from the earth, 405 spectrum of, 310,312

Aldehyde, spectrum of, 177

Alizarine colours, identity of with madder dyes, 191

Alkali metals, spectra of, 71 spectrum of, 193

an unknown one detected, 102

Alkaline earths, luminous sulphides of, 210 spectra of, 83,94

Allotropic conditions of nitrogen probable, 127, 130

Alloys of gold and copper, quantitative analysis of, 138 zinc and cadmium, spectrum of, 135

and copper, variation of spectrum of, 135 
Altitude as affecting solar energy, 299

Alumina, phosphorescent spectrum of. 209

Aluminium not detected in the sun, 244

Andromeda, continuous spectrum of nebula in, 320

Angström, Professor, his "Atlas of the Solar Spectrum," 272

atmospleric lines mapped by, 185

on the electric spark, 115

on spectra of compounds, 144

on wave-lengths of the dark lines, 38

on widening of lines, 157

Antimony not detected in solar atmosphere, 244

present in Aliebaran, 312

Antiseptic effect of sodium chloride in air, 95

Approximation between the earth and certain fixed stars, 405

Aquarius, telescopic appearance of nebula in, 319

Aqueous vapour, bands peculiar to, 127, 184

Arcturus, its analogy with the sun, 315

estimated velocity of its approach, 405

Aromatic group of bodies, crucial line of, 177

Arragonite, phosphorescent spectrum of, 208

Arsenic not detected in solar atmosphere, 244

Astronomy, spectroscopic aid to, 231, 338, 353

Atlantic Ocean, lithium in water of, 97

Atmosphere, the earth's, absorptive action of, 35, 185, 291, 339

heat-storing action of, 303

presence of sodium in, 78,95

lunar, none appreciable, 306

planetary, $306^{\circ}$

solar, incandescent, height of, 249

metal vapours in, 240

storms in, 332

temperature of, 255

Atmospheric lines, 185, 306

terrestrial substances present in, 243

Atomic weight and wave-length, connection between, 175, 179

Audible musical notes, range of, 8

B.

BADEN, cæsium and rubidium found in waters of, 80

Ballot, of Utrecht, as to the effect of motion on tone, 329 note

Balmain, Mr., his luminous paint, 210

Band-spectrum, associated with low-temperature observations, 130 its change to line-spectrum, 167

Bands, appearance of, in order of volatility of substance, 83

bright, in spectra of gases, 66,75

dark, of absorption, 183, 187

fluted, or channelled, 128, 168

in lightning spectra, 421

of radicals, 177

replaced by lines at high temperatures, 150

shifting of in different compounds of the same body, 174, 189 
Barium, comparison of salts of, 179

compounds of as affected by temperature, 151

flames coloured green by, 67

present in solar atmosphere, 244

spectrum of, 83,150

Barrow, observations on the Bessemer flame at, 220

Baxendell, Mr., on the variable star of the Northern Cross, 317 note

Becquerel, M., on fluorescent bodies, 206

on infra-red rays, 24

on phosphorescent vapours, 209

Beet-root, rubidium found in, 89

Bence Jones, Dr., on the diffusion of lithium, 79

his spectroscopic examination as to the rate of circulation of blood, 82

Benzene, absorption of most refrangible rays by, 174, 198

Bessemer flame, Roscoe's examination of, 201

Dr. Watts's examination of, 215

process described, 198

spectrum, changes in, 216

essentially that of oxide of manganese, 220

Betelgeux, hydrogen absent from, 312

recession of from the earth, 332

Binary stars, their difference of colour, 355

spectra of, 314

Bismuth, presence of in Aldebaran, 312

in solar atmosphere, 245

Bisulphide of carbon, prisins of, used for high dispersion, 23, 212, 225

Blood, arterial and venous, spectra of, 191, 194

rate of circulation of, spectroscopically determined, 82

Blue colour of ozone, 184

of sun, 300,302

lines of lithium and strontium compared, 148

rays, chenical action strongest in the, 24

Newton's experiment as to the refrangibility of, 40

photography in the, 28

spread out in prism-spectrum, 17

Bodies when cold absorb the rays they give out when heated, 269

Bolonieter, Professor Langley's, 21 measurement of rays by, 298, 301

Bourbonne les Bains, cæsium and rubidium in waters of, 89

Boyle's law referred to, 164

Brass, compound spectrum of, 117

Bread, lithium present in, 79

Brewster, Sir David, atmospheric lines observed by, 185

his experiments on didymium compounds, 188

on "internal dispersion," 206

Bright lines in spectra of comets, 411

his theory as to primary colours, 7

of gases, 66,75

of metal vapours, 115

of nebulæ, 318, 392, 417

of stars, 352

in spectrum of solid erbium, 227, 388 
Bromides of metals, spectra of, 173

Bromine, spectroscopic characteristics of, 187

its spectrum varies with varying pressure, 182

Brorsen's comet, observations of', 324

spectrum of, 381

Bunsen, Professor, on changes in absorption bands, 174

his discovery of cæsium and rubidium, 87

his experiments on didymium compounds, 188

mapping of spectra by, 76, 106

on the measurement of solar chemical action, 32

his spectroscope described, 71

on the spectrum of erbium, 227,383

and Kirchhoff on mode of using the spectroscope, 103

and Roscoe on the chemical power of magnesium light, 51

Bunsen-burner used for examining coloured flames, 68

C.

CADMrum present in solar atmosphere, 245

Cæsium discovered by Bunsen, 87

compounds, spectra of, 109

spectrum of, 75,89

Calcium compounds, spectra of compared, 179

effect of temperature on spectrum of, 150

flames coloured red by, 67

present in fixed stars, 312

in red solar prominences, 253,263

in solar atmosphere, 312

spectrum of, 75,83

California, Southern, dryness of climate, 294

Capella probably receling from the earth, 405

Capron, Rand, on the rain-band, 186

Carbo-hydrogen compounds, spectra of, 146

Carbon burnt out of cast iron by Bessemer process, 198, 201, 221

experiments as to spectrum of, 382

fluted spectrum of, 128

possible third spectrum of in Bessemer flame, 219

presence of in comets, 324, 327, 382, 413

in some meteorites, 391,413

probable condition of in comets, 386,390

vapour of at different heat levels in comets, 414

Carbonic oxide gas, blue flame of, 70

peculiar bands of, when in solution in blood, 194

Cassiopeiæ, $\gamma$, motion of recession in, 407

spectrum of, 352

Cast-iron converted into cast-steel by Bessemer process, 198

Castor, estimated velocity of recession of, 404,409

Cerium, present in solar atmosphere, 245

Chemical action of light, 24, 32, 52

produced by rays of all colours, 31, 64

strongest in the blue rays, 27, 197

circulation in body, rapidity of, 82

composition of fixed stars, 309 
Chemistry, celestial, 240, 306

Chloride of sodium in atmosphere, $78,95,146$

Chlorine, bromine and iodine, spectroscopic distinction between, 187 condensed, spectrum of, 182 explosive combination of, with liydrogen, 25,27

Chlorophyll, spectrum of alcoholic solution of, 193

Chromium, its presence in solar atmosphere, 243 salts, absorption spectra of, 172,189

Chromosphere, solar, spectrum of, 277,283

$$
\text { storms in, } 255,332
$$

Ciamician on chlorine, bromine and iodine spectra, 182 on widening of lines, 158, 161

Circulation of blood, spectroscopic determination of rate of, 82

Clark, Alvan, his discovery of companion star to Sirius, 376

Cloud, solar, of incandescent magnesium, 257

Clouds in atmosphere of Jupiter and Saturn, 350

Coal-tar colcurs, identity of, with some madder dyes, 191

Cobalt oxide, spectrum of, 191

possible presence of, in solar atmosphere, 244

sal s, shifting of bands in spectrum of, 175, 189

Coffee, rubidium found in, 89

Coggia's comet, carbon present in, 327

Collimator to spectroscope described, 71

Colorado, study of lightning spectra in, 422

Colour, Newton's definition of the sense of, 50 of rays not changed by re-refraction, 48 result of decomposition of white light, 4

Colours, complementary, together produce effect of whiteness, 6 differing wave-lengths of, 8

Coloured flames, 67

stars, spectra of, 313

substances, relation of absorption to radiation in, 268

vapours, 184

Colouring matters, absorption bands of, 180

spectra of, 191

Comet " $b$, , 1881, photographic spectrum of, 411

Brorsen's, spectrum of, 324,381

Coggia's, carbon present in, 327

Encke's, carbon present in, 327

Halley's, described, 389 , note 390

Miss Mitchell's, transparency of, 388

Wells', I., 414

Winnecke's, 378 carbon present in, 386

Comets, colours of, 390

differing constitution of, 391

examination of, 324

formation of tail of, 389

probable condition of carbon in, 386, 390

relation between meteors and, 413

Cometary light partly reflected sunlight, 386, 413

possibly due to electric discharges, 416

Complementary colours, experiments with, 6 
Compound bodies, spectra of, 129, 138, 145, 172, 178

Compression of gases, effect of, 165

Concave surface, gratings ruled on, by Prof. Rowland, 213

Conflagration, solur, possible effect of on the earth, 317 note

Continuous spectra, generally yielded by solids and liquids, 65, 129 faint, of luminous gases, 364

obtainable from flames, 136

Convection, loss of heat from, 265 from certain nebula, 320

Converter of Bessemer process described, 200

Copper, green flames produced by, 69

in solar atmosphere, 245

spectrum of, 117

Cormu, MI., on vapour of unequal temperatures, 244

Corona, connection of with sun-spot period, 259

photographs of, 258,282

a solar phenomenon, 258

and zodiacal light, suggested continuity between, 282

Crewe, Bessemer process at, 216

Crookes, Mr., discovery of thallium by, 75, 80, 91

Cruorine, red and purple, 194

Curves, method of taking, in mapping spectra, 223

Cyanogen, possibly present in cometary matter, 328, 413

Cyclones, solar, 255, 332

Cygni, a, estimated velocity of approach, 406

$\beta$, spectra of the two stars composing, 310

D.

DARK heating rays, 22,31

lines and bands of solar spectrum, 35, 122, 231

in stellar spectra, Fraunhofer as to, 39

optical effect of, 314

of vapour spectra, 183

widening of, in sunspot spectra, 247

Daylight, ordinary, eclipse work done in, 251, 264

"Death Valley" in Southern California described, 294

Decarbonization of iron, spectroscopic determination of point of, 216

Decomposition of elements in solar atmosphere, suggested by Mr. Lockyer, 245

Defirition, loss of, in large spectroscopes, 212

De la Rue, Mr., his photographs of solar prominences, 249

Delicacy of spectrum analysis, $76,94,102$

Detection of radicals, mode of, 177

Diamond, phosphorescent spectrum of, 207

Didymium cliloride, spectrum of, 190

salts, Bunsen's observations on, 174

elective absorption exercised by, 188

peculiar spectra of, 228

Diffraction gratings described, 15

of light, 9

their object, 212 
Direct vision spectroscope described, 195

Disc, revolving, experiment with as to colours, 6

viewed by electric spark, 7

Discontinuous spectrum of incandescent gases, 66

of red solar prominences, 251

Ditte on relations of elementary spectra, 181

Doppler's theory as to the effect of motion on light, 329,355

Double stars, differences of colour in, 355

spectra of, $310,314,407$

Doublets and triplets of lines in certain spectra, 171

Draco, Dr. Huggins's observations on the nebula in, 317

Draper on incandescent solids, 65

on the maximum heating point of the spectrum, 20

Dry plates, Captain Abney's, 262

Dupré, Dr., on the rate of circulation of blood, 82

Dürkheim, cæsium and rubidium found in waters of, 80,87

Dust, constant presence of, in air, 297

E.

EAR, human, capacity of, for distinguishing sounds, 8

Earth, distribution of chemical rays on surface of, 33

length of radius of orbit, 378

motion of, in space, 375

motions of certain stars relatively to, $329,353,392,400$

Eclipses of sun, observations of tabulated, 281

red prominences seen during, 248

corona photographed during, 258

spectroscopic discoveries made duxing, 260, 274

Electric spark, colour of in Geissler's tubes, 127, 144

gases examined by, 124

heavy metals volatilized by, 113

rotating disc viewed by, 7

spectrum of, 144

temperature of, unknown, 70, 138

two spectra shown by, 115, 123

possible decomposition of, in solar atmosphere, 245

Element, relation of its spectrum to that of its compounds, 172

Elements discovered by spectrum analysis, 2

metallic, spectra of, 113

non-metallic, spectra of, 123

relations between spectra of, 113, 181

terrestrial, present in fixed stars, 313

sum, 243

Encke's comet, presence of carbon in, 327

Energy, solar, increase of, according to altitude, 299

Equality between absorption and radiation at like temperatures, 239, 267 388

Exbium chloride, spectrum of, 191

solid, bright lines in spectrum of, 65 note, 227,388

Erhard on absorption spectra of chromium salts, 172

Ether, luminiferous, 7 
Ethyl compounds, one band for all, 198 radicals, mode of detection, 177

Exchanges, law of, 239, 265

optical reaction of erbium no exception to, 227

Explosive combination of chlorine and hydrogen, 25

F.

FACULA on solar surface, 256

Falsification of wines detected by spectroscopy, 203

Falwarczny, Dr., lithium detected in blood by, 98 , note?

Faraday on the material nature of the electric spark, 114

Ferric ferrocyanide, exceptional spectrum of, 173

Festing, Lt.-Col., and Capt. Abney, experiments on infra-red rays, 198 quoted as to absorption, 175

Fievez, his map of the solar spectrum, 273

Fireworks, coloured by chemical substances, 69

Fixed stars, motions of, 329, 353, 400

physical condition of, 313

spectra of, 308, 341

Flames, Bessemer, critical aspect of, 200

certain, continuous spectra given by, 136

coloured, $67,85,140$

of soda salts incapable of chemical action, 28

of sodium seen black against a larger sodimm flame, 236

solar, observed during eclipse, 248

Flavo-purpurine, spectrum of, 193

Fluor spar, phosphorescence of, 206

Fluorescence, 205

spectrum of, 208

Fluorides of metals, exceptional behaviour of, 179

Fluted band spectra, 128

Fox-Talbot, Mr., on the spectra of coloured flames, 85

on metallic spectra, 118

Frankland and Lockyer, Messrs., on widening of lines, 163

Fraunhofer, diffraction gratings constructed by, 15

his lines described, 36,230

explained by Kirchhoff's discovery, 243

his observations on stellar spectra, 39, 310, 339

G.

GALtioM discoverel by M. Lecoq de Boisbaudran, 80, 92

Galvanism, measurement of heat rays by means of, 21

Gas, movements of molecules of, 168

Gases, continuous spectra sometimes yielded by, 129, 136

effect of compression on, 165

electric spark seen in different, 124

incandescent, spectra of, $66,71,152$

little heat absorbed or radiated by, 268

rays absorbed of the same refrangibility as those emitted by, 238

Gaseous bodies, quality of light emitted by, not affected by temperature, 69 
Gaseous nature of comets, 324

of nebulæ, 318,323

of red prominences, 251

Gassiot, Mr., his spectroscope, 211

Geissler's tubes, 124, 127, 144

Genesis of spectra, Schuster on, 169

Gladstone, Dr., on spectra of compounds of the same base, 172, 189

Glass, coloured, selective absorption illustrated by, 269

colourless, freely absorbs and radiates heat, 266

does not freely absorb light, 268

impermeable to rays of highest refrangibility, 123

refraction of light by, 9

Glow-worm, phosphorescence of, 210

Gold apparently not present in solar atmosphere, 244 and copper alloy, quantitative analysis of, 138

Graham, Prof., his experiments on meteoric iron, 413

Graphite, conversion of, in Bessemer process, 199

Grating diffraction, described, 15 use of, 212

Great Bear, movements of stars in, 332, 409

Green ray, indivisibility of, 7

sun, causes of appearance of, 35

Greenwich, observations on star motions made at, 332

Grouping of stars into systems, 409

Guntoor, M. Janssen's eclipse observations at, 252, 275

$\mathrm{H}$.

HAMOGLOBIN and hæmatin, 194

Halley's comet described, 389 note

Haloid compounds, connection between spectral lines and atomic weight in, 179

Harkness, Mr., his discovery of unknown line in corona, 261

Hartley and Huntington, Messrs., on absorption spectra, 173 on the ultra-violet rays, 198

Hasselberg, Dr., experiments on the hydro-carbon spectrum, 417

Heat, absorbed and radiated, constant balance of, 239, 266

Heavy metals, spectra of, 113

Heidelberg, observations on chemical rays at, 54

Helmholtz and Maxwell, on complementary colours, 6

Hercules, motion of solar system towards, 331

Herculis, $a$, spectrum of, 313

Herschel, Sir John, on Halley's comet, 389 note

on lightning spectra, 421

on the nebula in Orion, 321

on spectra of coloured flames, 85, 140

Herschel, Sir William, his discovery of the infra-red rays, 19

on the grouping of stars into systems, 409

High dispersion of rays, gratings used for, 17, 212

power spectroscopes, 211

"Homogeneal light," Newton's experiments on, 49

Huggius, Dr., air spectrum mapped by, 127

his application of photography to stellar spectra, 315 
Huggins, Dr., on lunar atmosphere, 306

on the red prominences, 253

on spectra of comets, 411,414

of metals, 120

of nebulæ, 317, 322, 392, 417

of stars, $313,341,357$

on spectrum of Mars, 347

and Dr. Reynolds, on reversed absorption bands of erbium, 228

Hydrocarbons, Dr. Hasselberg's experiments as to spectrum of, 417

presence of, in comets, 411

Swan's investigations as to spectra of, 146

Hydrochloric acid gas, formation of, 25

Hydrogen, absent from certain fixed stars, 313

appearance of, in electric spark, 124

and chlorine, combination of, 25,27

compounds, spectra of, 176

incandescent, in solar protuberances, 254

in variable star of Northern Crown, 315

line $\mathrm{C}$ in Bessemer flame affected by weather, 218

$F$ in nebula of Andromeda, 320

in spectrum of Sirius, 372

variation of, in solar spectrum, 278, 332

in spectra of nebulæ, 370

(h) at high temperatures, 126, 252, 262

lines, bright, coincidence of, with dark solar lines, 126

in corona, 282

in star and nebula spectra compared, 420

variation of, in nebula spectra, 370

widening of, 136,157

in meteoric iron, 413

present in most fixed stars, 312

solar storms in, 332

spectrum described, 125

I.

ICELAND spar, prism of, 411

Ignition effected by dark rays, 24

Images, retinal, 32

in the blue rays, 26

Incandescent gases, 66

solids, continuous spectrum of, 65

vapours of, elements, 71,151

Index of spectra, by Dr. Marshall Watts, 121, 123

Indium discovered by Professors Reich and Richter, 80, 93 spectrum of: 75

Infra-red rays, extension of, in Prof. Langley's observations, 301

their heating power, 19, 24

phosphorescence destroyed by, 209 photography of, 24, 197

Insolation vessel described, $53 n$.

Intensity, solar, measurements of, 19, 52, 298 
Interference of light, 9

"Internal dispersion," Sir D. Brewster on, 206

Interpolation forinula given, 224

Inversion of lines in didymium and erbium spectra, 227

Iodine, its analogies with bromine and chlorine, 182

in bisulphide of carbon, as a "ray-filter," 23

spectroscopic distinction of, 187

Iron, discovery of coincidence of dark and bright lines of, 240

ineteoric, occlusion of gases in, 413

presence of, in certain stars, 2,312

in st?n, $2,240,243$

pyrites, presence of thallium in, 80,92

Iso-purpurine, spectrum of, 193

$\mathrm{J}$.

JANssen, M., on bands of aqueous vapour, 184

discovery of Fraunhofer's lines in corona, 261

on the red protuberances, 251,276

Johnstone Storey, on harmonic ratios, 170

Joule, Dr. J. P., on lightning spectra, 421

Jupiter, observations as to atmosphere in, 307,350

K.

KELP, presence of lithium in, 97

Kettle of boiling water photographed in darkness, 31

Kew Observatory, measurements of solar activity taken at, 32

Kirchhoff, his discovery as to the dark solar ljnes, 233, 241

his historical sketch of spectrum analysis, 140

spectra of elements mapped by, 120

his spectroscope, 74,211

on substances in the solar atmosphere, 229

on variations of spectra, 151

his view as to sun-spots, 256

and Bunsen on crsinm and rubidium, 109

Kuihne, Professor, on the "visual purple" of the eye, 32

Kundt, Professor, on absorption bands of colouring matters, 180 on lightning spectra, 421

L.

LABORDE, M., on lightning spectra, 421

Lamp, monochromatic, introduced by Brewster, 84

Lamp-black, radiation and absorption in, 266

Lamy, M., his examination of thallium, 92

Langley, Professor, his bolometer, 21, 298, 301

on distribution of heat in solar spectrum, 21

on radiation as affected by atmosphere, 35

on solar energy at various altitudes, 299

heat, 303

on sunlight, 291 
Law of Exchanges defined, 239, 265

optical reaction of erbium no exception to, 227

Lecoq de Boisbaudran, M., his discovery of gallium, 80, 93

his map of the nitrogen spectrum, 128, 169 of the spectra of barium salts, 179

Lenarto, gases in meteoric iron from, 413

on relations between elementary spectra, 181

Lens, lithium salts taken with food found in the, 82

Leonis, $\beta$ and $\delta$, estimated velocity of recession of, 405

$\gamma$, a double star, 407

its radial and proper motions, 409

Liebig quoted as to unsupported theories, $187 n$.

Lielegg, Professor, on the Bessemer spectrum, 215

Light, absorbed, converted into heat, 205

into molecular motion, 31

appearance of, when decomposed by diffraction grating, 15, 212

of burning magnesium, chemical activity of, 51 by prism, 4,40 , \&c.

chemical action of, $24,32,52$

coloured, 4, \&c.

cometary, nature of, 386,413

decomposition of, Newton on the, 4,40

diffraction of, 9

of electric discharge, 114

emitted by gases, quality of constant, 66,69

incandescent solids, 69

"homogeneal," Newton's experiments on, 49

intensity of, as affected by altitude, 299

interference of, 9

measurements of, 27,33

nature of, 7

phosphorescent, 206

refrangibility of, 4,40 , \&c.

undulatory theory of, 7

variously coloured, in certain stars, 313,355

velocity of, $9,330,375 n ., 407$

vibrations of, 169,329

waves of, measurements of, 8,38

Lightning, spectra of, 420

yellow sodium line seen in, 127

Lines affected by pressure, 136, 163

by temperature, 134, 167

atmospheric, 185,306

bright, characteristic, of gases, 66,75

of solid erbium, $65 n$., 227,388

chromospheric, catalogue of, 283

dark, in solar spectrum, 35, 231, 240

coincidence of, with bright metallic lines, 233,240

Fraunhofer's map of, 36, 230

long and short, 134

refrangibility of, 158

reversal of, 161, 235, 238

shifting of, 160 
Lines, shifting of, Dr. Inggins's conctusions from, 329

telluric, 185

widening of, 136, 157, 246

Lippich on molecular changes of gas under pressure, 164

Lithium band, red, 81

blue, at high temperatures, 82,131

spectrum of, 75,96

compared with that of strontium, 148

wide distribution of, 97

Liveing and Dewar, Professors, on carbon spectra, 413

on the reversibility of long lines, 245

on sodium vapour as affected by temperature, 164

on the widening of magnesium lines, 161

Lockyer, Mr., on decomposition of elements in solar atmosphere, 245

on the carbon lines of comet spectra, 414

on the chromosphere, 277

red prominences seen by him on unobscured sun, 251, 254, 274,280

on reversibility of long lines, 244

his sectional examination of spectra, 132, 155

on solar storms, 332

on the widening of sodium lines, 167

London atmosphere, corona photographed in, 264

Low temperature examinations of calcium, \&c., 150

Luminiferous ether, 7

observations, band spectra associated with, 130

Luminous gas, modes of obtaining solids in the state of, 71 paint, 210

Lunar atmosphere, Dr. Huggins on, 306

Lyra, Secchi's observations on the annular nebnla of, $367 n$. spectruin of, 315

Lyræ, a, estimated rate of approach, 406

M.

MADDER root, absorption spectrum of, 191

Magnesium, burning, chemical rays of, 51

present in Aldebaran, 312

solar cloud of, incandescent, 257

vapour in solar prominences, 336

widening of lines in, 161

Manganese, oxide of, bright lines of Bessemer flame due to, 202, 219, 221 present in solar atmosphere, 244

Mapping spectra, Bunsen's method of, 76, 106

Maps of atmospheric lines, 185

Prof. Stokes's method of, 223

metallic spectra, 120

solar spectrum, 272

Marine origin of salt in atmospluere, 78,95

Mars, spectrum of, 307,347

Marshall Watts, Dr., on the Bessemer flame, 202

his index of spectra, 12I, 123 
Mascart and Cornu, their maps of the ultra-violet rays, 273

Mass of vapour, effect of changes in, 152

Matter, change gradual between gaseous and liquid condition of, 130

extreme subdivision of, in tails of, comets, 391

Maximuin chemical action of rays, 27

heating power of rays, 19, 22

Measurement of chemical action of light, 33, 299

Measurements, spectroscopic, 222

of dark solar lines, 38

on Mount Whitney, 298

of stellar motions, 400

of waves of light, 14

Melville, Thomas, soda flame observed by, 84

Mercury not detected in solar atmosphere, 244

present in Aldebaran, 312

widening of the most refrangible lines of, 159, 161

Mercury, the planet, observations on, 307

Metals, absorption bands in vapours of, 188

of the alkaline earths, spectra of, 71, 100

bright lines of, 117

apparent coincidence of, 120

and their compounds, no connection between spectra of, 180

fluorides of, exceptional behaviour of, 179

organic, 198

present in solar atmosphere, 242-245

proper, spectra of, 113-123

reversal of the lines of, 238

Meteoric iron, occluded gases in, 413 nickel and cobalt present in, 243

matter, circulation of, round sun, 261

stolies, presence of lithium in, 79

Meteorites, carbon occasionally present in, 391

Metcors, hydrocarbons present in, 413

periodical, 391

their connection with comets, 413

their spectra vary in different groups, 416

Methyl compounds, one band for all, 198

Mexico, slowness of photographic processes in, 33

Mica, lithium found in some specimens of, 97

Microscopic spectrun analysis in legal examinations, 197

Micro-spectroscopc, the, 195

Milk, lithium present in, 80

Miller, Prof. W. A., on lunar atmosphere, 306

on spectra of coloured flames, 87,145

Millimetre scale, mode of making, $104 n$.

Mineral waters, elements present in, $79,80,88$

Mira Cœti, spectrum of, 352

Mitchell, Miss, her comct, 388

Mitchell, Rev. J., on measurement of star distallces, 400

on the grouping of stars into systems, $409 n$.

Nitscherlich, Alexancier, on spectra of compounds, 178

Molecular arrangement, spectrum as affected by, 130, 136 changes, change from band to line spectrum due to, 167 
Molecular motion, translation of light into, 31

theory of gases, with reference to widening of lines, 164, 168

vibrations, as affected by chemical combinations, 175

Monochromatic lamp, introduced by Brewster, 84

Moon, absence of atmosphere in, 306

$$
\text { nature of each ray of colour, } 7
$$

corona not connected with, 258

inequalities of surface of, 350

Moonlight, dark lines in spectrum of, 38

Motion, community of, in groups of stars, 408

of luminous bodies detected by spectroscope, 248

molecular, 31, 164, 168

in solar atmosphere, 332-337

of stars relatively to the earth, $329,353,400$

Mount Whitney, Professor Langley's researches at, 292

Mountain measurements of solar energy, 299

Müller, J., on the displacement of lines, 160

on the distribution of heat in spectrum, 21

NEBULA in Andromeda, 320

N.

$$
\begin{aligned}
& \text { Aquarius, } 319 \\
& \text { Draco, } 317,363 \\
& \text { Orion, } 321,365 \\
& \text { spectrum of, } 392
\end{aligned}
$$

Nebulæ, gaseous nature of, $\mathbf{3 1 8}, 370$

observations of, 363

spectra of, 317,417

Nebular theory, bearing of stellar observations on the, 313

Newton, dark lines of spectrum not observed by, 35

his discovery of the frangibility of light, 3

his "Opticks," extracts from, 40-50

Nickel probably present in solar atmosphere, 243

Nitrates, spectra of, 173,190

Nitric acid, absorption band of, 173, 190

Nitrogen, appearance of the electric spark in, 124

in gaseous nebulæ, $320,363,370,398$

in lightning spectra, 420, 423

probable allotropic conditions of, 127, 130 spectra of, 127, 152

Nobert, his diffraction gratings, 15

Non-metallic elements, spectra of, 123

Northern Crown, variable stars in, $315,317 n$.

Notes, musical, audible range of, 8

Nucleus of comets, growth of the tail from, 389

O.

Occlusion of gases in meteoric iron, 413

Octaves of sound and colour compared, 8, 18

Olefiant gas, spectrum of, 387,411

of carbon as modified by, 324, 383 
Olive oil, spectrum of carbon as modified by, 324,382

Opacity of sodium vapour to sodium flame, 235

Opaque particles, free radiation and absorption of heat from, 268

"Opticks," Newton's, extracts from, 40-50

Optogram obtained on the visual purple, 32

Orbit, value of radius of earth's, 378

of Sirius, Dr. Peters on the, 376, 402

Organic liquids, colourless, absorption spectra of, 190

"metals" 198

Orion, great nebula in, Sir J. Herschel on the, 321

Dr. Huggins on the, 322, 365, 392, 396-400, 417

spectrum of $a$ in, $310,312,352,402$

Orthoclase, lithium present in, 97

Overlapping of spectra with diffraction gratings, 16, 213

Oxide of manganese, bright lines of Bessemer flame due to, 202, 220, 221

Oxygen, changes in spectra of, 152

its effect in the examination of organic bodies, 176

four spectra of, 130

two line spectra of, 153

widening of lower temperature lines in, 161

Oxyhæmoglobin, spectrum of, 194

Ozone, blue tint of, 184

PaInT, luminous, 210

P.

Pará, Brazil, measurement of solar intensity at, 34

Parallax, solar value of, $375 n$.

Pegasi, $\beta$, hydrogen wanting in spectrum of, 208

Period of sun-spots, connected with form of corona, 259

Periodical meteors, differing spectra of, in different groups, 416

orbits of, identical with those of certain comets, 391

Peters, M., on the proper motion of Sirius, 376, 402

Phosphate of lime, phosphorescent spectrum of, 208

Phosphorescence, confined to solids, 385

connection of, with fluorescence, 206

destroyed by the infra-red rays, 209

Phosphorite, phosphoric spectrum of, 208

Phosphoroscope described, 206

Photochemistry of the retina, 32

Photography in the blue rays, 28

of cometary spectra, 328,411

of the corona, 258

extent of solar rays shown by, 18

by magnesium light, 50

of nebula spectra, 323,417

of planetary spectra, 307,348

of red solar protuberances, 248, 253

in the red rays, 30

of stellar spectra, 315

of ultra-violet rays, 197

of ultra visible rays, 31,273

Photosphere, solar, distinguished from chromosphere, 277 
Phyllocyanine, spectrum of, 193

Physics, solar, spectroscopic aids to, 229, 248

Physiology aided by the spectroscope, 83

Pickering, Mr., his discovery of unknown line in corona, 261

Planetary spectra, 306, 348

Planets, reflecting power of, compared, 350

Plattner, his analysis of the mineral pollux, 90

Plücker and Hittorf, Messrs., on the spectrum of nitrogen, 127 on the widening of lines, 158

Poisoning by carbonic oxide, spectroscopic detection of, 194 by thallium, spectroscopic detection of, 92

Poles of electric spark, greatest heat at, 134

Potash, flames coloured violet by, 67

Potassium, analogy between it and cæsium and rubidium, 91, 109 compounds, spectrum of, 99 permanganate, absorption bands of, 189, 191 present in solar atmosphere, 245 spectrum of, 75

Pollux, estimated velocity of its approach, 406, 409

Pollux (mineral), cæsium found in, 90

Pressure, effect of, on spectra of gases, 136, 182 heightened in nucleus of sun-spot, 246

a probable cause of widening of lines, 163

Prism, compound, M. Thollon's, 212

of Iceland spar, for cometary researches, 411 of quartz, 123

of rock salt, 21

Prisms of bisulphide of carbon, 212, 225

Procter, Mr., on movements of stars in Grcat Bear, 332, 409

Procyon, probably receding from the earth, 405

Prominences, solar, 248

daily observations of, 274

discontinuous spectrum of, 251

form of, 278

Piazzi Smyth's measurements of, 273

photography of, 281

Purple film of retina, 32 rapid changes in, 335

Purpurine, spectrum of, differs according to solvent, 193

Pyrites, iron, presence of thallium in, 80,92

Q.

QUANTitative spectrum analysis attempted, 138

Quantity of incandescent substance as affecting the spectrum, 135

Quartz, lenses and prisms of, 123, 411

permeable to rays of high refrangibility, 123

Quinine, sulphate of, its use in observing ultra-violet rays, 53, 123

R.

RADIATING layer, spectra of gases influenced by thickness of, 155

Radiation and absorption, correlation between, 265,268

increased by absence of water vapour, 296, 303 
Radiation, solar, as affected by earth's atmosphere, 35, 185, 291

Radicals, detection of, 177

organic, in organic compounds, 198

Radius of earth's orbit, value of, 378

"Rain-band" described, 185

Rainbow colours produced by prism, 4

Ratio, direct, between absorption and radiation, 270

harmonic, in various spectra, 170

Ray-filter devised by Dr. Tyndall, 23

Rays, chemical action of, 24, 31, 52

dark heating, 22, 31

gases absorb such as they emit, 236

from heated bodies absorbed by them when cold, 269

infra-red, heating powers of, 19, 24

Langley's observations on, 301

phosphorescence destroyed by, 209

photography of, 24, 147

intensity of, proportioned to refrangibility, 156

reflexibility of, proportioned to refrangibility, 46

refrangibility of, differing, $4,9,43$

ultra-violet, 18, 27, 197, 209, 232

variously coloured, estimated wave-lengths of, 232

Ray Lankester, Prof., on blood colouring matters, 195

Rayleigh, Lord, on maximum heating rays, 22

Recession between the earth and various fixed stars, 330, 332, 375, 402 404

Red colour of Mars, to what canse due, $\mathbf{3 4 9}$

heat of solids, light emitted by, 64

rays, heating power of, 19

stars, 315

photography in, 30

wines falsified by colouring matters, 203

Reflected light in comets, 411, 413

in the corona, 261

in planets, 350

Reflexibility of rays, Newton's experiments on, 46

Refrangibility of light, 9

of lines, 158

Newton's experiments on, 4, 40

Regulus, estimated velocity of recession of, 404

Reich and Richter, Messrs., indium discovered by, 75, 93

Relations of elementary spectra, 181

Resomance in sound, its analogy in light, 240

Retina, colour impressions on the, 7

photographic action of light on the, 32

Reversal of lines, 161, 235, 238

Reversion spectroscope, Zöllner's, 160

Rigel, estimated velocity of recession of, 404

Rocksalt, observations with prism of, 21

its selective absorption of heat, 268

Roscoe, Prof., his actinometer described, 58

his examination of the Bessemer flame, 215

and Clifton, on metal vapours as affected by temperature, 148 
Rotating disc, viewed by electric spark, 7

Rowland, Professor, his diffraction gratings, 15, 213

Rubia tinctorum, dyes prepared from, superseded by coal-tar colours, 191

Rubidium discovered by Bunsen, 87 compounds, spectrum of, 109

line, widening of, 161

spectrum of, 75

wide diffusion of, 89

Ruling of diffraction gratings, 15

Russell, Prof., on shifting of absorption bands, 175, 189

Rutherfurd, Mr., his diffraction gratings, 15

his photographs of the solar spectrum, 273

S.

SALiva, sodium salts in, 78

Salt, constant presence of, in atmosphere, 78, 95

Saturn, cloudy atmosphere of, 350 spectrum of, 307

Schuster, Prof., on the changes of spectra, 152, 154

on the genesis of spectra, 169

his photographs of corona spectrum, 262

on the relation between coniets and meteors, 413

on spectra of lightning, 420

Sea-water the source of sodium in air, 78,95

Secchi, Padre, on stellar spectra, 314

Selective absorption, 183,291

exercised by the atmosphere, 35, 185, 291, 339

Selenium, blue flames produced by, 69

illustrated by coloured glass, 269

Shifting of absorption bands, 174, 189

motion a cause of, 329

Silicates, presence of potash in, 100

Silicon burnt off in Bessemer process, 199

Silver, polished, bad as radiator and absorbent, 265

salts of, decomposed by exposure to light, 27, 32

spectrum of, 118

widening of lines in, 161

Sirius, discovery of companion star to, 376

Fraunhofer's examination of light from, 231

spectrum of, $313,315,345,372$

velocity of recession from the earth, 330, 375, 402

Smyth, Prof. Piazzi, his measurements of solar spectrum, 273

on the rain-band, 186

his large spectroscope, 212

Soda, flames coloured yellow by, 67

Sodium chloride as an antiseptic in the air, 95

compounds, universal presence of, $78,82,146$

line, its use in measuring stellar spectra, 346, 403

lines, bright, coincident with dark solar lines $\mathrm{D}, 233$

reversal of, 234

scale of spectroscope adjusted to, 222 
Sodium lines, widening of, 158, 160

presence of, in fixed stars, 2, 312

reaction, delicacy of, 96

in solar atmosphere, 243

spectrum, 68,75

high temperature spectrum of, 131

salts, spectra of, 94

vapour, effect of on the hydrocarbon spectrum, 417

Solar atmosphere, faculæ in, 256

opaque to the light given by sudium flame, 235

presence of known substances in, 229, 240

temperature of, 255

tempestuous motion in, 255, 332

energy as affected by altitude, 299

flames observed during eclipse, 248

heat, 19, 205

height of, as observed by Lockyer, 254

intensity, measurements of, 52

prominences, form of, 248,280

rapid changes in, 335

spectrum of, 251,279

spectrum as observed by Newton, 4,40

dark lines in, 35, 231, 240

maps of, 272

system, its motion in space, 331, 408 rate of motion towards Hercules, 376

Solids, action of heat on, 64

incandescent, spectra of, generally continuous, 65, 129

Solvents of colouring matters, their various effects, 181

Sonstadt, Mr. E., on the preparation of magnesium, 52

Sorby, on spectroscopic evidence of the age of wine, $205 n$.

Soret on blood spectra, 194

on spectra of nitric acid and some nitrates, 190

Sound and light, vibrations of, compared, 8, 169, 240, 329

Spark, electric, colour of, in Geissler's tubes, 127, 144

Lockyer's examination of, 132

nature of, 114

spectra of, 115,144

Spark-holder described, 133, 226

Spectra, absorption, 174, 184-189

of alkali metals, 71,83

of alloys, 135, 138

band and line common to nearly all bodies, 130

of coloured flames, 85, 140

of colouring matters, 191

of comets, 324, 381, 411

of compounds, $100,129,138,178,144,146^{\circ}$

continuous from certain flames, 136

from heated solids, 65,129

of different elements, relations of, 181

discontinuous, of gases, 66

of double stars, $310,314,407$

of elements as related to those of their compounds. 172 
Spectra of fixed stars, 39, 231, 310, 313, 338, 352, 402

fluted band, 128

of gases, 66, 71

sometimes continuous, 129, 136

genesis of, 169

of hydrocarbons, 146

mapping of, $76,106,223$

of metals, 113, 115

mode of examining, 119

of meteors, 416

of nebulæ, $317,319,396$

of nitrates, 173,190

of non-metals, 123

phosphorescent, 207

photography of, 197

of planets, 306, 347

of radicals, 177

of solutions of salts, 172

of sun-spots, 247, 333

variation of, 135

Spectrum analysis, as compared with chemical, 101, 109

early investigation concerning, 140

practical applications of, 198

principles on which based, 71

Spectrum of air, 127 wide scope of, 2

aldehyde, 177

alizarine, 193

alumina, 207

arragonite, 208

barium, 83,150

compounds, 151

Bessemer flame, 215

blood, 191

brass, 117

bromine, 187

cæsium, 75, 109

compounds, 173

calcium, 75,83

carbon, 128, 219

chlorophyll, 193

chromium salts, 172, 189

chromosphere, solar, 277, 283

cobalt, 191

copper, 117

corona, solar, 261

diamond, 207

didymium, 174

double stars, 314

salts of, 190

electric spark, 115, 123, 144

erbium, 141, 227, 388

ferric ferrocyanide, 173 
Spectrum of flavo-purpurine, 193

fluor spar, 208

hydrogen, 125, 136, 176

iodine, 187

iron, 240

iso-purpurine, 193

lightning, 420

lithium, 75, 96

madder, 191

magnesium, 161

moon, 38

nitrogen, 127

olefiant gas, 387,411

oxygen, 130,152

oxyhæmoglobin, 194

phosphorite, 208

phyllocyanine, 193

potassium, 99

purpurine, 193

rubidium, 75, 88

silver, 118, 161 compounds, 109

sodium, 75,403 salts of, 94

spiegeleisen, 203,220

spinelle, 208

strontium, 77, 83, 131

sun, 40,230

compounds, 151

thallium, 75

uranium nitrate, 191, 208, $189 n$.

vanadium, 121

wine, 203

yttrium, 118, 121

zine, 117

zirconium, $189 n$.

Spectroscope, direct vision, 195

high power, 210

mode of using, 103

various forms of, $71,117,222,341,357,393$

Spectroscopy, historical sketch of, 84

Speculum metal used for diffraction gratings, 15, 213

Spiegeleisen, its use in the Bessemer process, 218

Spinelle, phosphorescent spectrum of, 208

Starlight, its similarity to sunlight, 312

Stars, binary, 314, 355, 407

bright lines in spectra of, 352

coloured, spectra of, $313,315,355$

dark lines in spectra of, 39,231

displacement of lines in spectra of, 160

motion of, in space, $331,353,400$

spectra of, $306,338,371$

systems of, 409 
Stars, variable, 315,352

Steam, telluric lines in, 185

Steel contains less carbon than iron, 198

Steinheil, his spectroscope described, 72

Stewart, Prof. Balfour, on radiation and absorption, 265

Stokes, Professor, on fluorescence, 206 on blood spectra, 194

on lines in the ultra-violet rays, 123, 232

his measurements of wave-lengths, 223

on the solar lines D and the sodium lines, 240

on spectra of colouring matters, 193

Striated appearance of electric discharge through gases, 125

Strontium, lines in spectrum of, 77,83

flames coloured crimson by, 67

spectrum as affected by temperature, 131, 150

Struve, M. Otto, on the advance of the solar system in space, 376

Sulphate of thallium, decomposition of, 92

Sulphides of alkaline earths, luminous, 210

Sulphur, burning, temperature of, 69

Sun, blueness of, 300,302

corona of, 258

chromosphere of, 277,283

faculæ in atmosphere of, 256

green, causes of appearance of, 35

mode of examining special parts of, 246

photosphere of, 277

red prominences of, 248

repulsive action of, on cometary matter, 390

rotation of, shown by spectrum, 331

possibly a variable star, $57,317 n$.

Sunlight, physical properties of, 7

Professor Langley on, 291

Sun-spots, connection of, with corona, 259

former suppositions concerning, 256

lowered temperature of, 246

widening of lines in spectra of, 247

Swan, Professor, his collimator, 71

on the sodium spectrum, 146

on the spectra of hydrocarbons, 146

Systems of stars, community of motion in, 408

T.

TAILS of Comets, rapidity of their projection, 390

transparency of, 388

Talbot on spectra of coloured flames, 141

Tartaric acid, lithium obtained from manufactories of, $98 n .2$

Tea, lithium present in, 79

Telescope combined with spectroscope by Dr. Huggins, 308

Telluric lines, 185

Tellurium, its presence in Aldebaran, 312

Temperature of electric spark unknown, 70, 138 
Temperature of electric spark highest at the poles, 134

low, of burning sulphur, 69

quality of light emitted by gases does not vary according to, 69

emissive and absorptive powers equal at the same, 239

by solids varies according to, 65,69

of solar atmosphere, 255

vapour of certain metals as affected by, 148

raised by absorption of light, 205

type of spectrum affected by, 130, 148, 154

Terrestrial substances, occurrence of, in solar atmosphere, 243

Thalén on wave-lengths in metal spectra, 120

Thallium, discovery of, 91

spectrum of, 75,91

Thames water, lithium present in, 80

Thermometer, infra-red rays discovered by means of, 19

Thorpe, Professor, his measurements of tropical chemical rays, 34

Tilherode, thallium first detected at, 91 .

Tobacco, presence of lithium in, 98,100

potassium, 100

rubidium, 89

Tone as affected by motion, $329 \mathrm{n}$.

Transparency of cometary matter, 388

Tropics, variation of solar chemical intensity in, 34

Troost and Hautefemille, comparisons of various spectra, 182

Twin stars, spectra of, 314, variation of colour in, 355

Tyndall, Professor, on "actinic clouds," 31 his "ray-filter," 23

U.

ULTRA-RED, see infra-red

Ultra-violet rays, 18, 197, 232

absorption lines in, 198, 232, 262

abundance of, in magnesium light, 51

bands of carbon spcctrum in, 129

metallic spectra in, 123

bodies rendered phosphorescent by exposure, 209

chemical action of, $24,27,64$

detection of heating power in, 21

Undulatory theory of light, 7 photography of, 197

Uranium nitrate, spectrum of, 191, 208

Uranus, spectrum of, 307

$$
\text { in solar atmosphere, } 245
$$

Ursa Major, motions of stars in, 332, 405, 409

Ursæ Majoris, $\alpha$, estimated velocity of its approach, 407

V.

VACUUM-TUBES, use of, 124

Vanadium in solar atmosphere, 245

spectrum of, 121 
Van der Willigen, his spectroscopic experiments, 144

Vapour, aqueous, bands peculiar to, 184

radiation from earth checked by, 303

density of, a cause of expansion of lines, 165

luminous, distinctive of each element, 71

of magnesium in solar prominences, 336

of metals, absorption bands in, 188

of sodium, its effect on the hydrocarbon spectrum, 417

Vapours, coloured, 184

colourless, effect of light on, 31

phosphorescent, 209

Variable stars, spectra of, 315,352

sun possibly one of the, $317 n$.

Variation of spectra, Kirchhoff on, 151

Vegetables, rubidium found in, 89

Velocity of approach and recession in stars, 405

light waves, $9,330,375 n .407$

of movements in solar atmosphere, 336

of sun's motion in space, 376

of vibrations of sound, 8

Venus, atmosphere of, 306

dark lines in spectrum of, 38

Vibrations of light, 7

and sound compared, $8,169,240,329$

Visual purple of the eye, 32

Vögel, H. C., on lightning spectra, 421

on planetary spectra, 306

on Sirius, 402

and Dr. Miller, their map of solar spectrum, 273

Volatility of substances, varying rate of, 83, 100, 118

Volatilization of the heavy metals, 113

W.

WATrs, Dr. Marshall, on the Bessemer flame, 219

on high temperature sodium lines, 131

Waves of light measured, 38

Wave-lengths of light and sound compared, 8

of lines, how ascertained, 222

in metal spectra, 120

Weather as affecting hydrogen line in Bessemer spectrum, 218

Wheal Clifford mine, lithium in waters of, 79

Wheatstone, Sir Charles, on metal spectra, 115

his spectroscopic experiments, 144

White heat of solids, all rays of light given off by, 65

light, Newton on the decomposition of, 3

paper freely absorbs and radiates heat, 266

does not freely absorb light, 268

stars, 313,314

Whiteness, effect of, produced by mixing complementary colours, 6

Newton's experiments as to, 47

Whitney, Mount, U.S. reserved for scientific purposes, 302 
Widening of lines, Ångström quoted as to, 157

in hydrogen spectrum, 136, 157

in spectrum of mercury, 159

possible causes of, 162-167

in sun-spot spectra, 247

Wine, presence of lithium in, 79

spectroscopic examination of, 203

Winnecke's comet, 324,378

Wollaston, Dr., dark lines of solar spectrum observed by, 35 on the electric spark, 114

Wright, Professor, on the composition of stony meteorites, 413

Wülner, on widening of lines under pressure, 158

$Y$

Young, Professor C. A., on chromospheric lines, 283

on displacement of solar lines, 334

on lightning spectra, 421

on movements in solar atmosphere, 336

on sun-spot spectra, 247

on unknown line in corona spectrum, 261

Yttrium spectroscopically distinguished from erbium, 118

Yucatan, photography difficult in, 34

Z.

Z.TXC blende, gallium discovered in, 93

indium discovered in, 80, 93

lines, various lengths of, 134

in solar atmosplıere, 244

spectrum of, 117

Zirconium, spectrum of, $189 n$.

Zodiacal light and corona, continuity between, suggested, 282

Zöllner, M., on changes in intensity dependent on quantity, 157

his drawings of the red protuberances, 254

his measurements of star motions, 331

on widening of sodium lines, 160

\section{ADDENDA.}

Crookes, William. On Radiant Matter Spectroscopy. Phil. Trans., 1883; Part II. Proc. Roy. Soc. xxxviii. 414.

Welsbach, C. A. von. Decomposition of Didymium. Nature, vol. xxxii. p. 435 .

Lecoq de Boisbaudran. On a New kind of Metallic Spectra. Comptes Rendus, c. 1437 ; Chemical News, vol. lii. p. 4. 



